\title{
VERZEICHNIS
}

\section{DER BIS ENDE 1912 AN DEN TECHNISCHEN HOCHSCHULEN DES DEUTSGHEN REICHES ERSCHIENENEN SCHRIFTEN}

MIT UNTERSTUTZUNG DES KGL.PREUSZISCHEN MINISTERIUMS DER GEISTL. UND UNTERRICHTSANGELEGENHEITEN SOWIE DER TECHNISCHEN HOCHSCHULEN HERAUSGEGEBEN VON DEM BIBLIOTHEKAR DER DANZIGER HOCHSCHULE

DR. PAUL TROMMSDORFF

KOMMISSIONSVERLAG VON JULIUS SPRINGER BERLIN 1914 



\section{VERZEICHNIS}

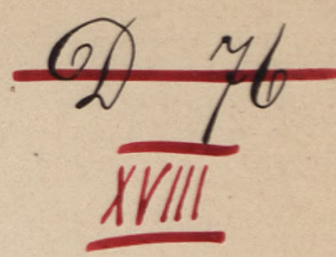

DER BIS ENDE 1912 AN DEN

\section{TECHNISCHEN HOCHSCHULEN DES DEUTSCHEN REICHES ERSCHIENENEN SCHRIFTEN}

MIT UNTERSTÜTZUNG DES KGL.PREUSZISCHEN MINISTERIUMS DER GEISTL. UND UNTERRICHTSANGELEGENHEITEN SOWIE DER TECHNISCHEN HOCHSCHULEN HERAUSGEGEBEN VON DEM BIBLIOTHEKAR DER DANZIGER HOCHSCHULE

DR. PAUL TROMMSDORFF

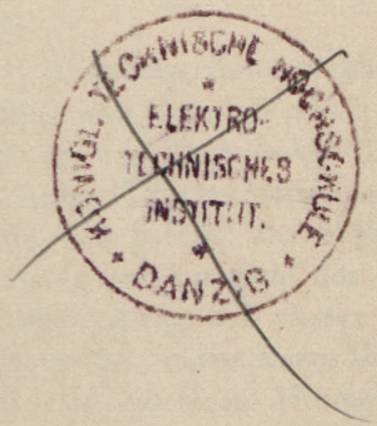

KOMMISSIONSVERLAG VON JULIUS SPRINGER BERLIN 1914 
(1ii)

II 71758

$\frac{B-1}{D / R-77919} \frac{6}{65}$ 


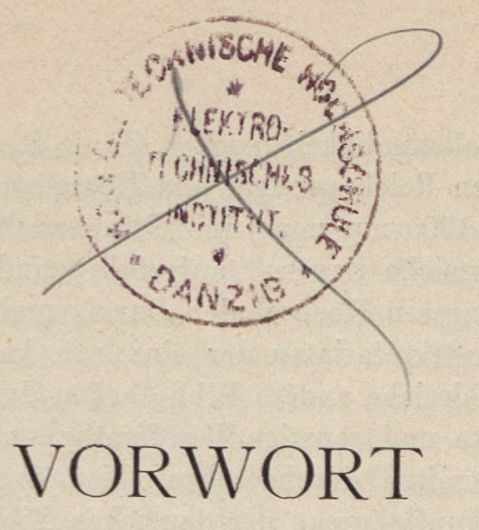

Das vorliegende Verzeichnis verdankt seine Entstehung einer Anregung des jetzigen Bibliothekars an der Universitätsbibliothek Göttingen Dr. Joh a n n es F ü chsel. Mit der Einrichtung der Bibliothek der Technischen Hochschule Danzig betraut, legte er dem Senat am 24. Mai 1907 den Plan vor, eine Zusammenstellung der bis hahin erschienenen Doktor-Ingenieur-Dissertationen und ein Jahresverzeichnis der künftig erscheinenden Schriften der deutschen Technischen Hochschulen herauszugeben. Nachdem ich im folgenden Jahre die Leitung der Bibliothek übernommen hatte, beantragte ich am 23. Juli 1908, auf einen älteren, auch von Füchsel erwogenen Plan zurückgreifend, die Schriften der Technischen Hochschulen vielmehr in das von der Königlichen Bibliothek in Berlin herausgegebene ,Jahresverzeichnis der an den deutschen Universitäten erschienenen Schriften" aufzunehmen, womit sich der vorgesetzte Herr Minister am 30. November 1909 einverstanden erklärte. Inzwischen aber hatte der Beirat für Bibliotheksangelegenheiten in Preußen Änderungen in der Versendung der Universitätsschriften und in der Drucklegung des Verzeichnisses vorgeschlagen, die langwierige Verhandlungen erforderten. Infolgedessen wurde erst am 12. September 1913 verfügt, daß die seit dem 1. Januar 1913 erschienenen Schriften der Technischen Hochschulen in das Jahresverzeichnis aufzunehmen seien. Diese späte Entschließung hat auch die Fertigstellung und Drucklegung des vorliegenden Verzeichnisses stark verzögert. Es umfaßt nunmehr die bis zum 31. Dezember 1912 herausgekommenen Schriften der deutschen Technischen Hochschulen, findet also seine unmittelbare Fortsetzung in dem Jahrgang 29 des ,Jahresverzeichnisses der an den deutschen Universitäten und Technischen Hochschulen erschienenen Schriften“".

Statt der von Füchsel geplanten Beschränkung auf die Doktor-Ingenieur-Dissertationen, über die eine kürzlich von $\mathrm{C}$ a rl $\mathrm{W}$ a l t h e $\mathrm{r}^{1}$ ) bearbeitete Bibliographie unterrichtet, sind hier sämtliche an den Technischen Hochschulen des Deutschen Reiches seit ihrer Begründung erschienenen Druckschriften zusammengestellt. Der Begriff „,Hochschulschriften" ist möglichst weit gefaßt; als solche wurden alle Schriften angesehen, die auf Veranlassung oder unter der Autorität der Hochschulen, ihrer Abteilungen oder Institute erschienen sind, gleichgültig, auf wessen Kosten sie gedruckt wurden. Ausgeschlossen wurden Auszüge aus Satzungen, kurze Mitteilungen ohne besonderes Interesse, undatierte Neudrucke und von einzelnen Hochschulen veranlaßte Abdrücke solcher Bestimmungen, die sich auf alle preußischen Hochschulen beziehen.

Die hiernach in Betracht kommenden Schriften lückenlos zu verzeichnen, erwies sich als eine recht schwierige Aufgabe. Nur für die Doktor-Ingenieur-Dissertationen war

1) Carl Walther: Bibliographie der an den deutschen Technischen Hochschulen erschienenen Doktor-Ingenieur-Dissertationen in sachlicher Anordnung. 1900 bis 1910. Berlin: Springer in Komm. 1913. 
eine sichere Prüfung der Vollständigkeit möglich, da sie den Promotionsordnungen gemäß halbjährlich im „Deutschen Reichsanzeiger und Königlich Preußischen Staatsanzeiger", in Bayern außerdem (seit 1905 nur noch) im ,,Ministerialblatt für Kirchen- und Schulangelegenheiten im Königreiche Bayern" aufgeführt sind. Die übrigen Schriften, abgesehen von denen der jüngsten Hochschulen Danzig und Breslau, waren weder in den Bibliotheken noch in den Sekretariaten der einzelnen Anstalten vollständig zu finden. Es wurden daher noch zahlreiche andere Bibliotheken durchforscht, und außerdem die Programme, Jahresberichte und sonstige Veröffentlichungen der Hochschulen auf die Erwähnung von Druckschriften durchgesehen. Von den meisten der so ermittelten Schriften konnte das Auskunftsbureau der deutschen Bibliotheken ein Exemplar nachweisen. Einige ältere Satzungen der preußischen Hochschulen fanden sich in den Akten des Kultusministeriums. Zu dem Verzeichnis der Schriften des Collegium Carolinum in Braunschweig hat das Generallandesarchiv in Wolfenbüttel Ergänzungen geliefert.

Innerhalb der einzelnen Abschnitte sind die Schriften chronologisch geordnet, die Satzungen, Ordnungen und Bestimmungen nach dem Tage des Erlasses oder der Genehmigung, die Habilitationsschriften nach dem Tage (die Dresdener nach dem Semester) der Habilitation, die Dissertationen nach dem Datum des Diploms, die Schriften der übrigen Gruppen nach dem Jahre des Erscheinens.

Die Titelaufnahme erfolgte im allgemeinen nach den für das Jahresverzeichnis der Universitätsschriften maßgebenden Grundsätzen. Der Drucker wurde jedoch, wie in dem Berliner ${ }^{1}$ ) und dem Breslauer ${ }^{2}$ ) Universitätsschriftenverzeichnis, weggelassen, auch wurde auf die praktisch belanglose Angabe des Druckortes verzichtet, und das Erscheinungsjahr, sofern es in einer datierten Druckschrift fehlte, nicht ergänzt. Durch weitere Vereinfachungen konnte in den Abschnitten ,Satzungen, Ordnungen, Bestimmungen" und "Periodische Schriften" größere Übersichtlichkeit erzielt werden. Ganz unwesentliche orthographische und stilistische Änderungen bei Neudrucken und späteren Ausgaben sind hier nicht berücksichtigt, ohne weiteres verständliche Abkürzungen wie Kgl., Herzogl., Halbj., W.-S., S.-S. verwendet, die Monatsnamen bei Datierungen durch Ziffern wiedergegeben; auch wurden Format und Umfang der Schriften nicht vermerkt.

Der größte Wert ist auf Vollständigkeit und Genauigkeit der bibliographischen Anmerkungen gelegt worden. Von jeder Schrift wurde zu ermitteln versucht, ob und wo sie außerdem (in gleicher, erweiterter oder gekürzter Form) erschienen ist, Bei diesen Angaben sind Verleger und Jahreszahl, wenn sie mit dem Erscheinungsvermerk übereinstimmen, nicht wiederholt. Bei Dissertationen, die von dem akademischen Lehrer, unter dessen Leitung die Arbeit entstand, verändert oder im Zusammenhang mit anderen Arbeiten veröffentlicht sind, wurde in der Regel mit ,Vgl." auf den Ort des anderweitigen Erscheinens hingewiesen.

Endlich ist überall notiert, wo eine Schrift zu finden ist, die die Bücherei der Technischen Hochschule Danzig nicht besitzt. ${ }^{3}$ ) Als Fundort wurden in erster Linie die Bibliothek oder das Sekretariat der betreffenden Hochschule, sonst nach Möglichkeit eine am Ort der Hochschule befindliche öffentliche Bibliothek genannt.

1) Verzeichnis der Berliner Universitätssehriften 1810-1885. Berlin: Weber in Comm. 1899; über die Weglassung der Angabe des Druckers vgl. S. VIII.

$\left.{ }^{2}\right)$ Karl Pretzsch: Verzeichnis der Breslauer Universitätsschriften 1811-1885. Breslau : Korn 1905.

3) Die Bücherei der Technischen Hochschule Danzig ist bemüht ihre Sammlung der Hochschulschriften so vollständig wie möglich auszugestalten und wird daher für jedes Angebot ihr fehlender Schriften sehr dankbar sein. 
Leider konnte das Verzeichnis nicht so eingerichtet werden, daß jeder einzelne Titel herausgeschnitten ${ }^{1}$ ) für Katalogisierungszwecke ohne weiteres benutzbar ist. Bei den Dissertationen und Habilitationsschriften wurden zwar die Hochschule und die Art der Schrift in der für die Angabe des Datums ohnehin erforderlichen Schlußzeile vermerkt; bei den übrigen Veröffentlichungen mußte aber darauf verzichtet werden, da es sonst vielfach unmöglich gewesen wäre, gleichartige Schriften übersichtlich zusammenzufassen.

Ein Sachregister, wie es das Jahresverzeichnis der Universitätsschriften aufweist, ist nicht beigegeben, da das vorliegende Verzeichnis nicht so sehr den Inhalt der Schriften erschließen, als vielmehr einen Überblick über die Tätigkeit der einzelnen Hochschulen bieten soll. Dieser Verzicht erschien auch deshalb unbedenklich, weil über die das Hauptinteresse beanspruchenden Schriften, die Doktor-Ingenieur-Dissertationen, für die Jahre 1900 bis 1910 die vortrefflich geordnete Walthersche Bibliographie vorliegt, deren Fortsetzung in Aussicht gestellt ist. Die Schriften jeder Hochschule sind jedoch möglichst klar gruppiert, die Dissertationen innerhalb einzelner Abteilungen noch nach sachlichen Gesichtspunkten geordnet.

Bei der Ausarbeitung des Verzeichnisses bin ich von allen Technischen Hochschulen, von Bibliotheksverwaltungen und sonstigen Behörden durch Nachforschungen in Akten und Bücherbeständen, Übersendung von Schriften und Erteilung von Auskünften bereitwilligst unterstützt worden. Die bibliographischen Angaben rühren bei vielen Schriften von den Verfassern, bei einigen chemischen Dissertationen von den Referenten her. Für die Drucklegung hat der vorgesetzte Herr Minister einen erheblichen Zuschuß und jede einzelne Hochschule einen Kostenbeitrag gewährt. Die Korrektur hat Herr Oberbibliothekar Dr. J a hr von der Königlichen Bibliothek in Berlin mitgelesen und dabei auf Grund seiner reichen Erfahrung manche Unebenheiten beseitigt. Allen diesen Förderern, insbesondere aber Seiner Exzellenz Herrn Ministerialdirektor Dr. Dr.= $\mathfrak{n}$ g. N a u m a n n, der dieser Arbeit von Anfang an lebhaftes Interesse entgegengebracht hat, spreche ich auch

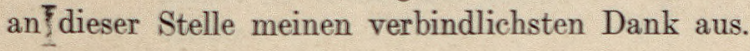

Danzig-Langfuhr, den 30. März 1914.

1) Einseitig bedruckte, zum Ausschneiden und Aufkleben der einzelnen Titel geeignete Exemplare des Verzeichnisses sind zum Preise von $6 \mathrm{M}$ erhältlich. 



\section{INHALT}

Anzahl der verzeichneten Schriften Abkürzungen

\section{AACHEN}

1. Satzungen, Ordnungen, Bestimmungen Allgemeines und Vermischtes ... Prüfungsordnungen . . . . . .

2. Periodische Schriften .......

3. Gelegenheits- und vermischte Schriften Reden zur Kaisergeburtstagsfeier. . 4 Verschiedene Schriften ..... 4

4. Doktor-Ingenieur-Dissertationen

I. Abteilung für Architektur . . .

II. Abteilung für das Bau-Ingenieurwesen ..........

III. Abteilung für das Maschinen-Ingenieurwesen .......

IV. Abteilung für Bergbau und Hüttenkunde, für Chemie und Elektrochemie . . . . . . . . .

V. Abteilung für allgemeine Wissenschaften, insbesondere für Mathemathik und Naturwissenschaften

\section{BERLIN}

1. Satzungen, Ordnungen, Bestimmungen

Bauakademie ....... 13

Gewerbeakademie ...... 14

Technische Hochschule

Allgemeines und Vermisehtes . . 14

Prüfungsordnungen . . . . . 16

2. Periodische Schriften

Bauakademie ........ 17

Gewerbeakademie...... 18

Technische Hochschule... 18

3. Gelegenheits- und vermischte Schriften

Bauakademie ........ 18

Gewerbeakademie ...... 19

Technische Hochschule

Reden zum Geburtsfeste des Kaisers
Seite

17
8
8
8
9
9

Reden und Schriften bei verschiedenen Anlässen. . . . . . 20

Schriften einzelner Abteilungen und Institute . . . . . . 22

4. Habilitationsschriften . . . . . . . 22

5. Doktor-Ingenieur-Dissertationen

I. Abteilung für Architektur . . . 23

II. Abteilung für Bau-Ingenieurwesen 23

III. Abteilung für Maschinen-Ingenieurwesen ........ 24

IV. Abteilung für Schiff- und Schiffsmaschinen-Bau ...... 30

V. Abteilung für Chemie und Hüttenkunde .........

\section{BRAUNSCHWEIG}

1. Satzungen, Ordnungen, Bestimmungen

Collegium Carolinum ..... Polytechnische Schule und Technische Hochschule Allgemeines und Vermischtes . . 37 Prüfungsordnungen . . . . . . 38

2. Periodische Schriften . . . . . 38

3. Gelegenheits- und vermischte Schriften 39 Collegium Carolinum

Zur Geschichte der Anstalt . . . 39

Reden und Oden bei Feiern des Herzogshauses . . . . . . 40

Wissenschaftliche Abhandlungen . 43

Verschiedene Schriften .... 45

Polytechnische Schule und Technische Hochschule

Zur Geschichte der Anstalt . . . 46

Verschiedene Schriften ..... 47

4. Habilitationsschriften . . . . . . 48

5. Doktor-Ingenieur-Dissertationen

I. Abteilung für Architektur ... 48

II. Abteilung für Ingenieurbauwesen 49

III. Abteilung für Maschinenbau einschließlich Elektrotechnik und Textilindustrie ....... 49

IV. Abteilung für Chemie $\ldots \ldots \quad 51$ 


\section{BRESLAU}

1. Satzungen, Ordnungen, Bestimmungen Allgemeines und Vermischtes . . . Prüfungsordnungen . . . . . . .

2. Periodische Schriften ........

3. Gelegenheits- und vermischte Schriften

4. Doktor-Ingenieur-Dissertationen

I. Abteilung für Maschinen-Ingenieurwesen und Elektrotechnik .

II. Abteilung für Chemie und Hüttenkunde ..........

\section{DANZIG}

1. Satzungen, Ordnungen, Bestimmungen Allgemeines und Vermisehtes ... . Prüfungsordnungen . . . . . . .

2. Periodische Schriften .......

3. Gelegenheits- und vermischte Schriften Reden zur Feier des Geburtstages des Kaisers ... . . . . . . Verschiedene Schriften ..... 58

4. Habilitationsschriften . . . . . . 58

5. Doktor-Ingenieur-Dissertationen

I. Abteilung für Architektur . . .

II. Abteilung für Bauingenieurwesen

III. Abteilung für Maschineningenieurwesen und Elektrotechnik . . .

IV. Abteilung für Schiff- und Schiffsmaschinenbau ...... 60

V. Abteilung für Chemie . . . . 60

\section{DARMSTADT}

1. Satzungen, Ordnungen, Bestimmungen Allgemeines und Vermischtes . . . 61 Prüfungsordnungen . . . . . . 62

2. Periodische Schriften . . . . . . 63

3. Gelegenheits- und vermischte Schriften Reden zur Feier des Geburtstages des Großherzogs ......... 64

Verschiedene Schriften ...... 64

4. Habilitationssehriften . . . . . . 66

5. Doktor-Ingenieur-Dissertationen

I. Abteilung für Architektur . . . 68

II. Abteilung für Ingenieurwesen . 69

III. Abteilung für Maschinenbau einschlieBlich Papierfabrikation . . 70

IV. Abteilung für Elektrotechnik . . 71

V. Abteilung für Chemie einschließlich Elektrochemie und Pharmazie ..........

\section{DRESDEN}

Seite

1. Satzungen, Ordnungen,-Bestimmungen Allgemeines und Vermischtes ... 74

Prüfungsordnungen . . . . . . 76

2. Periodische Schriften ... . . . 77

3. Gelegenheits- und vermischte Schriften 79

4. Habilitationsschriften . . . . . . 80

5. Doktor-Ingenieur-Dissertationen

I. Hochbau-Abteilung . . . . . .

II. Ingenieur-Abteilung für Ban-Ingenieurwesen mit Einschluß der Geodäsie . . . . . . . . .

III. Mechanische Abteilung für Maschinenbau, Elektrotechnik und Fabrikbetrieb . . . . . . .

IV. Chemische Abteilung für chemische Technik und Fabrikbetrieb

In Verbindung mit der Bergakademie zu Freiberg ......... .

\section{HANNOVER}

1. Satzungen, Ordnungen, Bestimmungen Allgemeines und Vermischtes . . . 99

Prüfungsordnungen . . . . . . . 99

2. Periodische Schriften . . . . . . 100

3. Gelegenheits- und vermischte Schriften 101

4. Doktor-Ingenieur-Dissertationen

I. Abteilung für Arehitektur . . . 102

II. Abteilung für Bau-Ingenieurwesen 103

III. Abteilung für Maschinen-Ingenieurwesen ......... 104

IV. Abteilung für chemisch-technische und elektrotechnische Wissenschaften ........ 105

\section{KARLSRUHE}

1. Satzungen, Ordnungen, Bestimmungen Allgemeines und Vermischtes . . . 109

Prüfungsordnungen . . . . . 110

2. Periodische Schriften ........ 111

3. Gelegenheits- und vermischte Schriften

Reden beim Direktorats- (1895ff. : Rektorats-) Wechsel ... . . 112

Festschriften zur Feier des Geburtstages des Großherzogs . . . . . 112

Verschiedene Schriften . . . . . 113

4. Habilitationsschriften . . . . . . 115

5. Diplom-Dissertationen . . . . . . 118 
6. Doktor-Ingenieur-Dissertationen Seite

II. Abteilung für Architektur . . . 120

III. Abteilung für Ingenieurwesen . 121

IV. Abteilung für Maschinenwesen . 121

V. Abteilung für Elektrotechnik . . 122

VI. Abteilung für Chemie . . . . . 124

VII. Abteilung für Forstwesen . . . 129

\section{MÜNCHEN}

1. Satzungen, Ordnungen, Bestimmungen

Allgemeines und Vermischtes . . . 129

Prüfungsordnungen . . . . . 130

2. Periodische Schriften ... . . . 131

3. Gelegenheits- und vermischte Schriften 134

4. Habilitationsschriften . . . . . . 13ॅ

5. Doktor-Dissertationen . . . . . 135

I. Allgemeine Abteilung . . . . 135

II. Bauingenieur-Abteilung. . . . . 138

III. Architekten-Abteilung . . . . 138

IV. Maschineningenieur-Abteilung . . 139

V. Chemische Abteilung . . . . 142

VI. Landwirtschaftliche Abteilung . 153
STUTTGART

Seite

1. Satzungen, Ordnungen, Bestimmungen

Allgemeines und Vermischtes .. . 154

Prüfungsordnungen . . . . . 155

2. Periodische Schriften . . . . . 158

3. Gelegenheits- und vermischte Schriften

Reden zum Geburtsfest des Königs. 159

Verschiedene Schriften . . . . 159

4. Habilitationsschriften . . . . . 160

5. Doktor-Ingenieur-Dissertationen

I. Abteilung für Architektur . . . 162

II. Abteilung für Bauingenieurwesen ........ 162

III. Abteilung für Maschineningenieurwesen einschlieBlich der Elektrotechnik ........ 163

IV. Abteilung für Chemie einschlieBlich des Hüttenwesens und der Pharmazie ........ 164 
. 


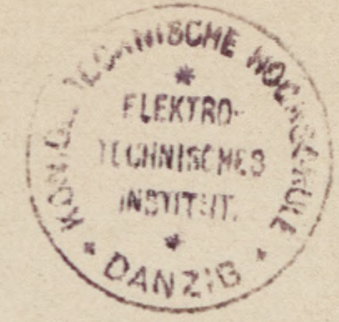

\section{ANZAHL DER VERZEICHNETEN SCHRIFTEN}

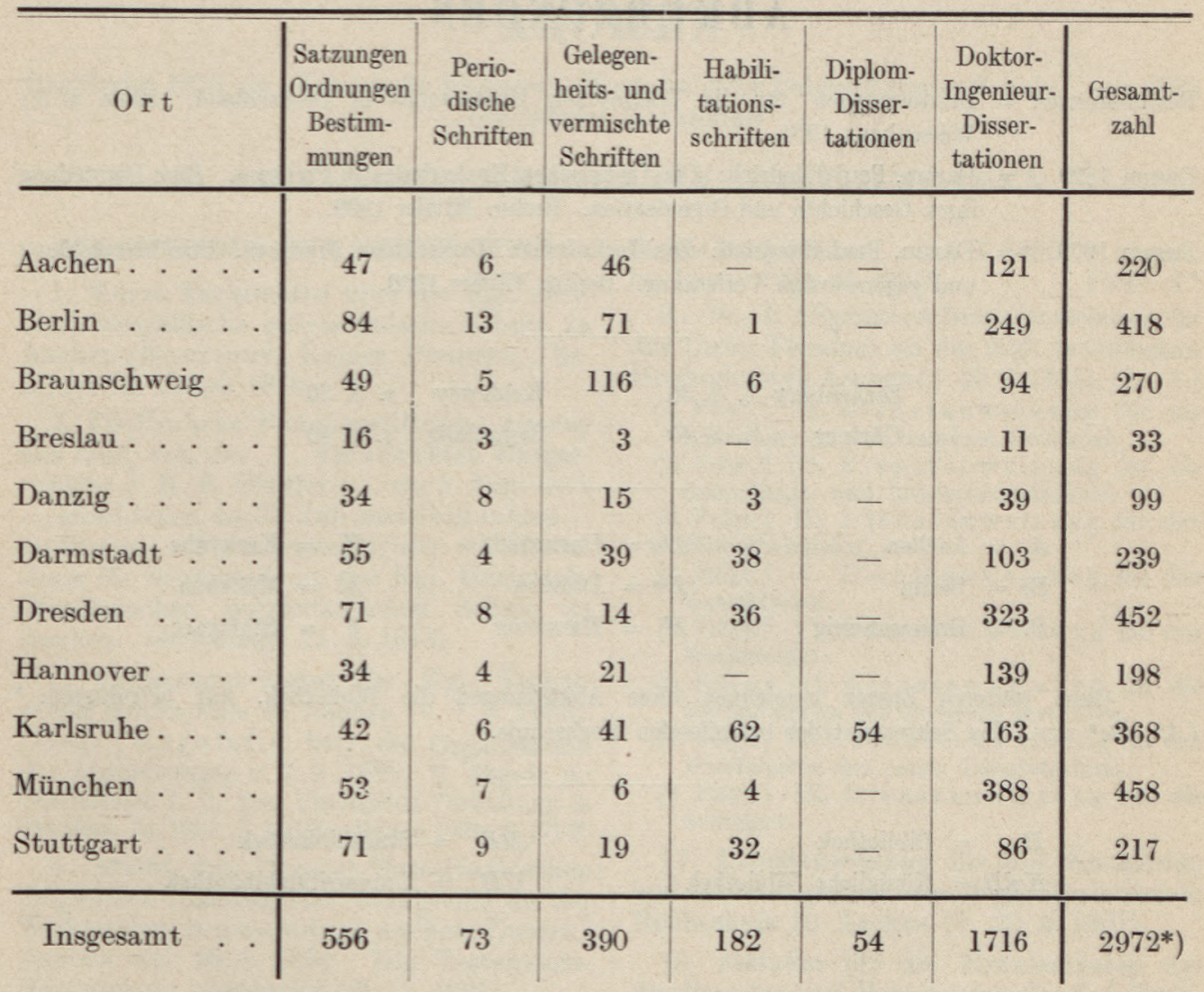

*) Unter den Nummern 1-2969 des Verzeichnisses fehlen Nr 54, 683, 759, 807, 1088, 1328-1333, 2780, 2781, 2832; dazugekommen sind Nr 98 a. b, 264 a - e, 378 a -d, 933 a, 1168 a, 1270 a, 2014 a, 2074 a. b. 


\section{ABKÜRZUNGEN}

Bestimmungen $=$ Bestimmungen für die Technischen Hochschulen in Deutschland. Halle a. S.: Waisenhaus 1904.

Damm 1899 = Damm, Paul Friedrich: Die Technischen Hochschulen in Preussen. Eine Darstellung ihrer Geschichte und Organisation. Berlin: Mittler 1899.

Damm 1909 = Damm, Paul Friedrich: Die Technischen Hochschulen Preußens. Thre Entwicklung und gegenwärtige Verfassung. Berlin: Mittler 1909.

\begin{tabular}{ll|ll} 
Eschenburg & s. S. 36 & Koldewey & s. S. 36 \\
Gärtner & s. S. 40 & Sammlung & s. S. 40
\end{tabular}

$$
\begin{array}{l|l|l}
A=\text { Aachen } & D a=\text { Darmstadt } & K=\text { Karlsruhe } \\
B e=\text { Berlin } & D r=\text { Dresden } & M=\text { München } \\
B r=\text { Braunschweig } & H=\text { Hannover } & S=\text { Stuttgart }
\end{array}
$$

Ohne weiteren Zusatz bezeichnen diese Abkürzungen die Bibliothek, mit beigefügtem * ( $A^{*}, B e^{*}$ usw.) das Sekretariat der betreffenden Hochschule.

$B \quad=$ Bibliothek

$K g l B=$ Königliche Bibliothek

$$
\begin{aligned}
& S t B=\text { Stadtbibliothek } \\
& U B=\text { Universitätsbibliothek }
\end{aligned}
$$




\section{AACHEN}

Gegründet 1870 als „Königliche Rheinisch-Westphälische Polytechnische Schule“, seit 1880 „Technische Hochschule“

\section{SATZUNGEN ORDNUNGEN BESTIMMUNGEN}

\section{ALLGEMEINES UND VERMISCHTES}

1. Kurze Nachriehten über die Kgl. rheinisch-westfälische polytechnische Schule zu Aachen 〈Rheinprov., Königr. Preussen〉. Eröffnet im Jahre 1870.

2. Pfeiffer'sche Familienstiftung. Auszug aus dem, von der ... verwittweten Bürgermeister J. H. B. Pfeiffer . . . am 5. Juni 1872 ...errichteten, am 31. Juli desselbenJahres... eröffneten ... Testaments [!] (betr. ein Stipendium für Studirende an der Kgl. RheinischWestfälischen polytechnischen Schule zu Aachen. Genehmigt 21. 5. 1873).

3. Verfassungs-Statut der Kgl. Technischen Hochschule zu Aachen (v. 7. 9. 1880). [Folgt:] Regulativ, betr. die Organisation der Abtheilungen v. 7. 9. 1880. Abgedr. in: Centralblatt f. d. ges. Unterrichts-Verwaltung in PreuBen. Jg.1881. S.156 ff.; Damm 1909. S.171ff.

4. Statut der Deusner-Hasselbach'schen Stipendien-Stiftung (für Studirende an der Technischen Hochschule zu Aachen. Unterz.: Aachen am 28.9. 1881. Die TestamentsExecutoren; genehmigt 22.11. 1881).

5. Urlaubsordnung für die Professoren und Dozenten der technischen Hochschule zu Aachen (v. 28. 4. 1882). ' Abgedr. in: Damm 1909. S. $191 \mathrm{f}$.

6. Bibliothek-0rdnung für die $\mathrm{Kgl}$. Technische Hochschule zu Aachen, genehmigt 9. 1. 1883. T Abgedr. in: Damm 1909. S. $207 \mathrm{ff}$.

7. Habilitations-0rdnung für die Technische Hochschule zu Aachen (v. 24. 4. 1884). - Abgedr. in: Damm 1909. S. 193 ff.

8. Vorsehriften für die Studirenden der Kgl. Technischen Hochschule zu Aachen (v. 12.8. 1884). - [Dasselbe. Folgt:] Vorschriften für die Hospitanten ... v. 5. 4. 1886. ๆ Abgedr. in: Damm 1909. S. 199 ff. $1886: A^{*}$

9-16. I. Allgemeine Dienstanweisung für das Unter-Personal an der $\mathrm{Kgl}$. technischen Hochschule zu Aachen (v. 23.1.1895). [1-8.]

[1. Folgt:] II. Dienstanweisung für die Kastellane an den einzelnen Gebäuden.

[2. Folgt:] III. Dienstanweisung für die Sammlungs- und Laboratoriumsdiener.

[3. Folgt:] IV. Dienstanweisung für den Bibliothekdiener.

[4. Folgt:] V. Dienstanweisung für den Kassenboten.

[5. Folgt:] VI. Dienst anweis ung für den Werkmeister.

[6. Folgt:] VII. Dienstanweisung für die Mechaniker an den einzelnen Instituten.

[7. Folgt:] VIII. Dienstan weisung für den Maschinisten des neuen Laboratoriums.

[8. Folgt:] IX. Dienstanweisung für die Schreiner.

17. Dienstanweisung für den Kanzlisten und Hausinspektor der Kgl. Technischen Hochschule zu Aachen (v. 23. 5. 1901).

18. Statuten für die Krankenkasse der Studirenden und Hospitanten der Kgl. Technischen Hochschule zu Aachen (v. 12.6.1901).

19. Bestimmungen über den Erlaß des Honorars und die Verleihung von Stipendien und Unterstützungen an der Kgl. Technischen Hochschule zu Aachen (v. 14. 4. 1903).

20. Satzungen für die Krankenkasse der Studierenden und Hörer der $\mathrm{Kgl}$. Technischen Hochschule (v. 22. 3. 1906). - Abgedr. in: Damm 1909. S. 217.

21. Satzungen der Hülfskasse der $\mathrm{Kgl}$. Technischen Hochschule zu Aachen (v. 15. 1. u. 10.4. 1907). - Abgedr. in: Damm 1909. S. $209 \mathrm{ff}$. 
22-28. I. Allgemeine Dienstanweisung für das Unterpersonal an der Kgl. Technischen Hochschule zu Aachen(v.13.11.1911). [1-7.]

[1. Folgt:] II. Dienstanweisung für die Kastellane an den einzelnen Gebäuden.

[2. Folgt:] III. Dienstanweisung für die Sammlungs- und Laboratoriumsdiener.

[3. Folgt:] IV. Dienstanweisung für den Bibliothekdiener.

[4. Folgt:] V. Dienstanweisung für den Bureau- und Kassenboten.

[5. Folgt:] VI. Dienstanweisung für den Werkmeister.

[6. Folgt:] VII. Dienstanweisung für die Mechaniker an den einzelnen Instituten.

[7. Folgt:] VIII. Dienstanweis ung für den Maschinisten des neuen Laboratoriums.

29. Dienstanweisung für den Hausinspektor der Kgl. Technischen Hochschule zu Aachen (v. 13. 11. 1911).

\section{PRÜFUNGSORDNUNGEN}

30. Entwurf einer Diplom-Prüfungs-Ordnung für die $\mathrm{Kgl}$. rheinisch-westphälische polytechnische Schule zu Aachen. [1873.] $A^{*}$

31. Kgl. rheinisch-westphälische polytechnische Schule zu Aachen. Specielle Bestimmungen über die Diplom-Prüfungen der verschiedenen Fächer. $\langle\mathrm{Nach}$ den Beschlüssen des Lehrer-Collegiums in denPlenarSitzungen v. 14., 16., 18. u. 25. 1. 1873.) $A^{*}$

32. Ordnung der Diplom-Prüfungen an der Kgl. Rheinisch-Westphälischen polytechnischen Schule zu Aachen (v. 20.5. 1873). Abgedr. in: Programm f. 1873/741879/80 [Nr 48].

A Privatbibliothek v. Prof. Aug. Hertwig

33. Verfügungen betr. die Bauführer- und Baumeister-Prüfungen, welche bis zum 6. Mai 1878 der Direction des Polytechnikums zu Aachen zugegangen, oder bekannt geworden waren.

34. Ordnung für die Diplomprüfungen an der Kgl. Technischen Hochschule zu Aachen (v.21.12.1887). Abgedr. in: Centralblattf. d. ges. Unterrichts-Verwaltung in Preußen. Jg. 1888. S. 199 ff.; Programm f. $1893 / 94-1899 / 1900$. [Nr 48]; Damm 1899. S. 136 ff.

\section{Abteilung für Architektur}

35. Diplomprüfungs-0rdnung der Abteilung für Architektur der Kgl. Technischen Hochschule zu Aachen. Durch Erlaß v. 13. 2. 1903 provisorisch in Kraft gesetzt. T Abgedr. in: Bestimmungen. S. $39 \mathrm{ff}$.

\section{Abteilung für das Bau- Ingenieurwesen}

36. Diplomprüfungs-0rdnung der Abteilung für Bau-Ingenieurwesen der Kgl: Technischen Hochschule zu Aachen. Durch Erlaß v. 13. 2. 1903 provisorisch in Kraft gesetzt. Abgedr. in: Bestimmungen. S. $47 \mathrm{ff}$.

37. Diplomprüfungs-0rdnung der Abteilung für Bau-Ingenieurwesen der Kgl. Technischen Hochschule zu Aachen. Vom 28. 7. 1910. (Nebst Deckbl. zu den ,Übergangsbestimmungen" auf S. 8. 1911.)

\section{Abteilung für das Maschinen- Ingenieurwesen}

38. Diplomprïfungs- Ordnung der Abteilung für Maschinen-Ingenieurwesen der Kgl. Technischen Hochschule zu Aachen. Durch Erlaß v. 13. 2. 1903 provisorisch in Kraft gesetzt. - Abgedr. in: Bestimmungen. S. $55 \mathrm{ff}$.

39. Diplomprüfungs-0rdnung der Abteilung für Maschinen-Ingenieurwesen der $\mathrm{Kgl}$. Technischen Hochschule zu Aachen. Vom 12. 6. 1907.

40. Diplomprüfungs-0rdnung der Abteilung für Maschinen-Ingenieurwesen der Kgl. Technischen Hochschule zu Aachen. Vom 28. 7. 1910.

Abteilung für Bergbau und Hüttenkunde, für Chemie und Elektrochemie

41. Diplomprüfungs-0rdnung der Abteilung für Bergbau, Hüttenkunde, Chemie und Elektrochemie der Kgl. Technischen Hochschule zu Aachen. Durch Erlaß v. 13. 2. 1903 provisorisch in Kraft gesetzt. - Abgedr. in: Bestimmungen. S. $65 \mathrm{ff}$.

42. Diplomprüfungs - Ordnung für die Fachrichtung des Bergbaues in der Abteilung für Bergbau- und Hüttenkunde, Chemie und Elektrochemie der Kgl. Technischen Hoch. schule zu Aachen. Vom 17. 7. 1906.

43. Diplomprüfungs- Ordnung für die Fachrichtungen der Hüttenkunde in der Abteilung für Bergbau- und Hüttenkunde, Chemie und Elektrochemie der Kgl. Technischen Hochschule zu Aachen. Vom 17.7.1906.

44. Diplomprüfungs- Ordnung für die Fachrichtung der Chemie in der Abteilung für Bergbau- und Hüttenkunde, Chemie und Elektrochemie der Kgl. Technischen Hochschule zu Aachen. Vom 17. 7. 1906. 
Abteilung für allgemeine Wissenschaften

45. Kgl. Technische Hochschule in Aachen. Ordnung für die Diplom-Prüfung in den Handelswissenschaften (v. 23. 1. 1900). $A^{*}$

46. Handelshochschule, angelehnt an die $\mathrm{Kgl}$. Technische Hochschule in Aachen.
Ordnung für die Diplomprüfung in den Handelswissenschaften (v. 12.4. 1905).

47. Prüfungsordnung für Feuerversicherungsingenieure in der Abteilung für allgemeine Wissenschaften der Kgl. Technischen Hochschule zu Aachen (v. 28. 7. 1910).

\section{PERIODISCHE SCHRIFTEN}

48. Programm der Kgl. Rheinisch-Westphälischen Polytechnischen Schule (1879/80: Technischen Hochschule) zu Aachen für den Cursus 1870/71-1879/80. 18[70]-79.

Fortges. u. d. T.:

Kgl. Technische Hochschule zu Aachen. Abgekürztes Programm für das Studienjahr $1880 / 81$.

Fortges. u. d. T.:

Programm der Kgl. Technischen Hochschule zu Aachen (1899/1900-1902/03: mit angelehntem ,zweijährigen Kursus für Handelswissenschaften"; 1903/1904 1907/1908: mit angelehnter Handelshochschule). für das Studienjahr 1881/18821912/1913. 1881-1912.

1870/71. Anl.: Special-Programme der ordentlichen Vorträge.

1871/72. Anl.: Allgemeine Rathsehläge für diejenigen, welche ein Polytechnikum besuchen wollen (v. Juli 1871).

1872/73. Anl.: Allgemeine Rathschläge... (v. Juni 1872).

1874/75. Anl.: Special-Programm der Vorträge und Uebungen.

1879/80. Anl.: Die Chemischen Laboratorien der kgl. rheinisch-westfälischen Technischen Hochschule zu Aachen. Mit 2 Bl. Zeichn. 1879.

1899/1900-1902/03. Anl.: Lehrplan des,,Zweijährigen Kursus für Handelswissenschaften“.

1903/04. 1904/05. 1906/07. 1907/08. Anl.: Lehrplan der Handelshochschule. 1870/71 nebst Anl. 1871/72. 1872/73 nebst Anl. 1878/79. 1880/81: $A$; 1874/75: $A^{*}$

49. Studienpläne für das Bergfach an der Kgl. Technischen Hochschule zu Aachen nebst Erläuterungs-Bericht 1880/81.

50. Technische Hochschule zu Aachen. Die Einführung des ersten Rektors am 15. November 1880 und die Übergabe des Rektorates am 2. Juli 1883. 1883.

Fortges. u. d. T.:

Technische (1892 ff.: Kgl. Technische) Hochschule zu Aachen. Die Übergabe des
Rektorates am 1. Juli 1886. 1892. 1895. 1898. 1901. 1904. 1886-1904.

\section{Darin:}

1883. Wüllner, [A dolph]: Die direkte Verwendung der Physik in der Technik und vor allem diejenige der Elektricität. [Ausz. in: Elektrotechn. Zeitschrift. Jg. 4. S. $304 \mathrm{ff}$.]

1886. Dürre, [Ernst Friedrich]: Entwicklung des Hüttenwesens.

1892. Heinzerling, [Friedrich]: Entwickelung der Technik des Brückenbaues.

1895. Intze, [Otto]: Welche Mittel bietet die Technische Hochschule den Studierenden, um den Anforderungen der Praxis zu genügen?

1898. Mangoldt, [Hans] v.: Die Entladung einer Leidener Flasche.

1901. Bräuler, [Ludwig]: Bilder aus der Vergangenheit und Gegenwart des Eisenbahnwesens.

1904. Borchers, [Wilhelm]: Ist das Hüttenwesen ein Zweig der technischen Chemie? [Auch einzeln u. d. T.: Ansprache bei Uebernahme des Rektorats der Königlichen Technischen Hochschule zu Aachen am 1. Juli 1904.]

51. Einrichtung eines zweijährigen Kursus für Handelswissenschaften, angelehnt an die Kgl. technische Hochschule zu Aachen. Programm für das Studienj. 1898/99. 1898. Fortges. u. d. T.:

Lehrplan des ,Zweijährigen Kursus für Handelswissenschaften" (1903/1904. 1904/ 1905: der Handelshochschule; 1905/1906 -1907/1908: Programm der Handelshochschule), angelehnt an die $\mathrm{Kgl}$. technische Hochschule zu Aachen für das Studienj. 1899/1900-1907/1908. 19[00]-07.

52. Personal-Verzeichniss der Kgl. Technischen Hochschule zu Aachen fürs W.H. 1900-1901. S.-H. 1901 [usw.]-W.-H. 1912-1913.

53. Kgl. Technische Hochschule zu Aachen. Studienpläne für wirtschaftswissenschaftliche Ausbildung und (1910/1911 ff.: Spezialstudienpläne) für Feuerversicherungsingenieure. Studienj. 1908/1909-1912/13. 1908-12. 


\section{GELEGENHEITS- UND VERMISCHTE SCHRIFTEN}

\section{REDEN ZUR KAISERGEBURTSTAGS- FEIER}

Im Jahre 1888 fand keine Kaisergeburtstagsfeier statt. Die im Jahre 1892 gehaltene Rede von Hans von Mangoldt ,Rückblick auf die Geschichte der Rechenkunst" erschien in: Aachener Anzeiger, Politisches Tageblatt. Jg. 14. 1892, Nr. 32. 34. 38. Die Rede von Gustav Herrmann v. J. 1896 s. $\mathrm{Nr} 87$, die Rede von Nikolaus Holz v. J. 1901 s. Nr 92. Die Reden von Georg Frentzen v. Jahre 1899 ,Über die Entwickelung der Arehitektur im neuen deutschen Reiche in den letzten Jahrzehnten" und von Max Schmid v. Jahre 1905 erschienen nicht im Druck.

55. Struck, E[mil]: Friedrich List, als Nationalökonom und Patriot. 1886. 16 S. $8^{0}$

56. Wüllner, A[dolph]: Die Entwicklung der Grundanschauungen in der Physik im Laufe unseres Jahrhunderts. 1887. 14 S. $8^{\circ}$

57. Sehulz, W[ilhelm]: Die Entwickelung des Bergbaus im brandenburg-preuBischen Staate. 1889. 24 S. $8^{0}$

58. Herrmann, [Gustav]: Die deutsche Allgemeine Ausstellung für Unfallverhütung. 1890. 19 S. $8^{0}$

59. Herrmann, [Gustav]: Die gewerbliche Arbeit. 1891. 22 S. $8^{0}$

60. Heinzerling, [Friedrich]: Das Vermittelungs-Gesetz. [1893.] 23 S. $8^{0}$

61. Schmid, [Max]: Friedrich der Grosse als Bauherr. [1894.] 15 S. $8^{\circ}$

62. Borght, R[iehard] van der: Krieg und Volkswirtschaft. [1895.] 23 S. $8^{0}$

63. Intze, [0tto]: (Die neuesten Ausführungen auf wasserwirtschaftlichem Gebiete). [1897.] 16 S., 25 Taf. $8^{\circ} \quad A$ StB

64. Borght, R[ichard] van der: Sociale Friedenspoiitik der letzten 10 Jahre in Deutschland. [1898.] 27 S. $8^{\circ}$ i Auch bei 0 . Müller in Aachen.

65. Bredt, J[ulius]: Die Doctor-Promotion an technischen Hochschulen und die Bedeutung der wissenschaftlichen Arbeit für dio organisch-chemische Technik. (Berlin: Springer 1900.) $5 \mathrm{Bl} .4^{0}$ - Erg. aus: Zeitschrift f. angewandte Chemie. Jg. 14.

66. Borchers, W[ilhelm]: (Versuche zu den höchsten Wärmegraden auch ohne Zuhilfenahme von Elektricität zu gelangen.) [1902.] 19 S. $8^{0}$

67. Jürgens, E[nno]: (Die Stellung der Mathematik im Leben der Völker.) [1903.] 20 S. $8^{0}$
68. Lengemann, [August]: (Die Entwicklung, der gegenwärtige Stand und die $\mathrm{Zu-}$ kunftsziele der bergmännischen Ausbildung in Deutschland.) [1904.] $28 \mathrm{~S} .8^{\circ}$ - Ohne die Einl. u. d. SchlnB in: Glückauf. Jg. 40. S. $192 \mathrm{ff}$.

69. Kähler, W[ilhelm]: Nationalökonomie und Ingenieurbildung. [1906.] 18 S. $8^{0}$ ๆ Ausz. in: Hochschul-Nachrichten. Jg. $16.1905 / 06$.

70. Hertwig, A[ugust]: Die Stellung der Technik im geistigen Leben des 19. Jahrhunderts. [1907.] 15 S. $8^{0}$

71. Hirseh, [August]: Die Wiedereinführung von Schiffahrtsabgaben auf den natürlichen Wasserstrassen. [1908.] $20 \mathrm{~S} .8^{0}$

72. Wüst, [Fritz]: Die Entwicklung der deutschen Eisenindustrie in den letzten Jahren. Halle: Knapp 1909. 33 S. $4^{0}$ - Aus: Metallurgie. Jg. 6; auch in: Mitteilungen aus d. Eisenhüttenmänn. Institut d. Kgl. Techn. Hochschule Aachen. Bd 3.

73. Kähler, W[ilhelm]: Staat und Vaterland. Grundsätzliche Gedanken über staatsbürgerliche Bildung. [1910.] 16 S. $8^{\circ}$ - Auch bei J. A. Mayer in Aachen.

74. Wallichs, [Adolf]: Die Maschine als Kulturwert. [1911.] 16 S. $8^{0}$ - Abgedr. in: Zeitschrift d. Verbandes deutseher DiplomIngenieure. Jg. 2.

75. Stark, J[ohannes]: Die kulturgeschichtliche Bedeutung der physikalischtechnischen Idee. [1912.] 12 S. $8^{0}$

\section{VERSCHIEDENE SCHRIFTEN}

76. Die Eröffnungs-Feier der Königlichen Rheinisch-Westphälischen polytechnischen Schule zu Aachen. Ein Gedenkblatt für die Festgenossen. Nebst einer artistischen Beil. 1870. 74 S., 1 Taf. $8^{\circ} \quad A$

77. Fest-Rede bei der Eröffnung der Königlichen rheinisch-westphälischen Polytechnischen Schule zu Aachen am 10. October 1870, vom Dir. Baurath [August] von Kaven. Aachen: J.A. Mayer $1870.23 \mathrm{~S} .8^{\circ} A^{*}$

78. Katalog der Bibliothek der Königlichen Rheinisch-Westphälischen Polytechnischen Schule zu Aachen. Als Ms. gedr. 1872. 238 S. $8^{\circ}$

79. ... Nachtrag-Katalog der Bibliothek der Königlichen (1: rheinisch-westfälischen) Technischen Hochschule zu Aachen. (2-4. Vorr.: Herm. Peppermüller.) $1-4$. $1879-1904.8^{\circ}$ 
1. 〈Umfassend die Acquisitionen seit Ende 1872 bis dahin 1878. $1879.393 \mathrm{~S}$.

2. UUmfassend die Erwerbungen seit 1879 bis Anfang 1898.) 1898. X, $851 \mathrm{~S}$.

3. 〈Umfassend die Erwerbungen seit Anfang 1898 bis Ende 1899.) 1900. VII, $186 \mathrm{~S}$.

4. UUmfassend die Erwerbungen seit Anfang 1900 bis Ende 1903.) 1904. VIII, $298 \mathrm{~S}$.

80. Gewerbe- und Kunst-Ausstellung in Düsseldorf 1880. Gruppe XIX. Schulwesen. No. 2382. Verzeichnis der Gegenstände: welche ausgestellt sind von der Königlichen Rheinisch-Westfälischen Technischen Hochschule zu Aachen nebst Mitteilungen über die Anstalt. Mit 1 Zeichn. (1880.) 56 S., 1 Taf. $8^{0}$

81. Der Reibungswinkel. Eine Festgabe zur 3. Säcular-Feier der Univ. Würzburg am 1. Aug. 1882. Gewidmet von der Kgl. Technischen Hochschule zu Aachen. Auf Wunsch des Rectors u. Senats dargeboten von Gustav Herrmann. 1882. 25 S. $4^{0}$

82. Allgemeine Deutsche Ausstellung auf dem Gebiete der Hygiene und des Rettungswesens, Bєrlin 1883. Königliche Technische Hochschule zu Aachen. Disciplin für gewerbliche Gesundheitspflege und Fabrikwesen. Special-Catalog der ausgestellten Gegenstände nebst Mittheilungen über die Disciplin. Aussteller: Docent Gewerberath [Franz] Reiche 1. Gruppe 25: Handel und Industrie. Kollektiv-Ausstellung des Kgl. Preußischen Ministeriums für Handel und Gewerbe. 18 S., 1 Taf. $8^{0}$

83. Zur Auflösung linearer Gleichungssysteme und numerischen Berechnung von Determinanten. Eine Festgabe zur 5. Säcular-Feier der Univ. Heidelberg am 2. Aug. 1886. Gewidmet von der Kgl. Technischen Hochschule zu Aachen. Auf Wunsch des Rectors u. Senats dargeboten von Dr. Enno Jürgens. $1886.20 \mathrm{~S} .4^{0}$

84. Rede zum Gedächtniß an Kaiser Wilhelm I. geh. bei der akademischen Trauerfeier in der Aula der technischen Hochschule zu Aachen Donnerstag, den 22. März 1888 vom Rektor Prof. Dr. [ErnstFriedrich] Dürre. 8 Bl. $8^{0}$

85. Kaiser Friedrich III. Rede, geh. anlässlich der Trauerfeier in der Aula der Kgl. Technischen Hochschule zu Aachen am 30. Juni 1888 von Prof. Dr. [Adolph] Wüllner. 20 S. $8^{0}$

86. Untersuchungen über die Oxymethylenderivate der Ketone. Eine Festgabe zur Feier des zweihundertjährigen Bestehens der Univ. Halle-Wittenberg am
2. Aug. 1894. Gewidmet von der Kgl. Technischen Hochschule zu Aachen. Auf Wunsch des Rectors u. Senats dargeboten von L[udwig] Claisen. 1894. $59 \mathrm{~S} .4^{0}$ T Auch in: J. Iiebig's Annalen d. Chemie. Bd 281.

87. Festrede zur Feier der 25. Wiederkehr des Tages der Neubegründung des Deutschen Reiches und zur Vorfeier des Geburtstages Sr. Majestät des Kaisers ... Wilhelm II., geh. in der Aula d. Technischen Hochschule zu Aachen am Sonnabend, 18. Jan. 1896, vom Geh. Regierungsrat Prof. Gust[av] Herrmann (Über den Einfluß, den die technischen Wissenschaften und die Entwickelung der Technik auf die Verhältnisse unserer Zeit ausgeübt haben). $18 \mathrm{~S} .8^{0}$

88. Festrede zur Feier des hundertsten Geburtstages Kaiser Wilhelms I. geh. am 22. März 1897 in der Aula der Kgl. Technischen Hochschule zu Aachen von Prof. Dr. A[dolph] Wiillner. 20 S. $8^{0}$

89. Feier zur Einweihung des Neubaues für Elektrotechnik und Bergbau der Königlichen Technischen Hochschule zu Aachen in Gegenwart ... des ... Ministers ... Dr. Bosse am 15. Mai 1897. Eine Gedenkschrift für die Festteilnehmer. Mit einer Photogr. des Neubaues u. 2 Zeichn. 49 S. $4^{0}$

90. Rede bei der akademischen Feier zum Andenken an den ersten Reichskanzler Fürst Otto von Bismarck geh. am 12. Jan. 1899 in der Aula der Kgl. Technischen Hochschule zu Aachen von Prof. Dr. [Richard] van der Borght. 12 S. $8^{0}$

91. Bilder aus der Entwickelung der reinen und angewandten Mathematik während des neunzehnten Jahrhunderts mit besonderer Berücksichtigung des Einflusses von Carl Friedrich Gauss. Festrede bei der aus Anlass der Jahrhundertwende veranstalteten akadem. Feier der Kgl. Technischen Hochschule zu Aachen. Geh. am 11. Jan. 1900 vom Rektor Prof. Dr. H[ans] v. Mangoldt. 22 S. $8^{0}$

92. Festrede zur Begehung des zweihundertjährigen Bestehens des Königreiches Preussen und zur Vorfeier des zweiundvierzigsten Geburtstages Sr. Majestät des deutschen Kaisers ... Wilhelm II. Geh. am 18. Jan. 1901 in der Aula d. Kgl. Technischen Hochschule zu Aachen von Prof. N[ikolaus] Holz (Über einige bemerkenswerte Dinge auf dem Gebiete des Bauwesens in Norwegen, insbesondere in Südnorwegen). $22 \mathrm{~S}$.. 39 Taf. $8^{0}$ 
93. Der zweijährige Kursus für Handelswissenschaften, angelehnt an die $\mathrm{Kgl}$. Technische Hochschule zu Aachen, seine Ziele und seine Einrichtungen, dargest. von Dr. W[ilhelm] Kähler,Prof. d. Nationalökonomit an der Kgl. Technischen Hochschule zu Aachen. Auf Veranlassung des Kuratoriums des Kursus als Ms. gedr. [1901.] 10 S. $8^{0}$

94. Wie studiert man an einer Handelshochschule? Ein Briefwechsel. Studien-Anleitung für die Studierenden des zweijährigen Kursus für Handelswissenschaften, angelehnt an die Kgl. Technische Hochschule zu Aachen, hrsg. von Dr.W[ilhelm] Kähler, Prof. d. Nationalökonomie an d. Kgl. Technischen Hochschule zu Aachen. Mit Einverständnis des Kuratoriums des Kursus als Ms. gedr. [1901.] $12 \mathrm{~S} .8^{0}$

95. Königliche Technische Hochschule zu Aachen. Denksehrift, verf. aus Anlass der Industrie- u. Gewerbe-Ausstellung für Rheinland, Westfalen und benachbarte Bezirke, verbunden mit einer deutsch-nationalen Kunstausstellung Düsseldorf 1902 . 88 S. $4^{0}$

Enth. u. a.: H[ans] v. Mangoldt u. [Ludwig] Bräuler: Die Königliche Technische Hochschule. - Otto Grotrian: Liste der aus dem Elektrotechnischen Institute hervorgegangenen Veröffentlichungen. - Julius] Bredt: Organisches Laboratorium. - Arwed Wieler: Das Botanische Institut. - [Hermann] Peppermüller: Bibliothek. - P[eter] Polis: Bericht über wissenschaftliche Arbeiten am Meteorologischen Observatorium zu Aachen. - Friedrich Heinzerling: [1.] Steife doppelte Spreng-, Häng- und HängsprengWerke im Holzbrücken-Bau. [2.] Eiserne Treppendächer. - Wilhelm Borchers: Einrichtungen und Ziele des neuen Laboratoriums für Metallhüttenwesen, Elektro- und Thermo-Metallurgie. A[rnold] Sommerfeld: Zur Theorie der Eisenbahnbremsen. - Wilhelm Kähler: Der gegenwärtige Stand der Handelshochschul-Bewegung in
Deutschland und der an die Kgl. Technische Hochschule zu Aachen angelehnte zweijährige Kursus für Handelswissenschaften.

96. Das neue Institut für Metallhüttenwesen und Elektrometallurgie an der Königlichen Technischen Hochschule zu Aachen. Dr. Wilhelm Borehers, Prof. f. Metallhüttenkunde u. Elektrometallurgie. Abschnitt: Elektrische Messinstrumente, bearb. von Dr. H[einrich] Danneel, Privatdoz. f. physikal. Chemie. Halle: Knapp 1903.61 S., 1 Taf. $4^{0}$

97. Otto Intze +28 . Dezember 1904. Gedächtnisrede geh. am 11.Jan. 1905 in der Aula der Kgl. Technischen Hochschule zu Aachen von W[ilhelm] Borehers. $8 \mathrm{~S} .4^{0}$

98. Festschrift Ad ol phWüllner gewidmet zum siebzigsten Geburtstage 13. Juni 1905 von der Königl. Technischen Hochschule zu Aachen, ihren früheren und jetzigen Mitgliedern. Mit d. Bildn. A. Wüllners in Heliogr., 8 Taf. u. 91 Fig. im Text. Leipzig: Teubner 1905. VIII, 264 S., 9 Taf. $8^{0}$

98a. Mitteilungen aus dem Eisenhüttenmännischen Institut der Königl. Techn. Hochschule Aachen. Hrsg. von Prof. Dr. F[ritz] Wüst. Bd [1]-5. Halle: Knapp $1906-13.4^{0}$

[1.] (Enth. 22 Abhandlungen.) 1906. 173 S., 8 Taf.

2. (Enth. 14 Abhandlungen.) 1908. $172 \mathrm{~S} ., 28 \mathrm{Taf}$.

3. Mit $238 \mathrm{Abb}$. u. 1 farbigen Taf. (Enth. $9 \mathrm{Ab}-$ handlungen.) 1909. $165 \mathrm{~S} ., 32 \mathrm{Taf}$.

4. Mit 372 Abb. (Enth. 21 Abhandlungen.) 1911. 230 S., 55 Taf.

5. Mit 205 Abb. (Enth. 13 Abhandlungen.) 1913. 160 S., 9 Taf., 23 Bl. Diagramme.

98b. Zum Gedächtnis von Friedrich Gustav Herrmann. (Verf.: 0[tto] Köchy u. 0[tto] Grotrian. Dat. Aachen Dez. 1907.) $5 \mathrm{Bl} .4^{0}$

\section{DOKT OR - INGENIE UR - DISSERTATIONEN}

\section{ABTEILUNG FÜR ARCHITEKTUR}

99. Höhle, Heinrich: Basilika und Zentralanlage. Leitideen der kirchlichen Planentwickelung von Konstantin dem Großen bis zum Ausgang der Romanik. 1906. 75 S. $8^{0}$

Aachen, Dr.-Ing.-Diss. v. 28. 8. 1906

100. Hecker, Hermann, Architekt d. Rheinischen Vereins zur Förderung d. Arbeiterwohnungswesens, Düsseldorf: DieWohnungsfrage und das Problem architektonischen
Gestaltens. Eine ästhetisch-wirtschaftliche Studie. Aachen: Jacobi 1909. 259 S. $8^{0}$ - Auch als Buch.

Aachen, Dr.-Ing.-Diss. v. 15. 7. 1909

101. Sehmidt, R[obert], Beigeordneter d. Stadt Essen 〈1912〉: Denkschrift betreffend Grundsätze zur Aufstellung eines General-Siedelungsplanes für den Regierungsbezixk Düsseldorf 〈rechtsrheinisch〉. (1912.) $102 \mathrm{~S} ., 15$ Taf. $4^{\circ}$

Aachen, Dr -Ing.-Diss. v. 12. 6. 1913 


\section{ABTEILUNG FÜR DAS BAU- INGENIEURWESEN}

102. Dondorff, J[akob], Reg.-Bauführer: Die Knickfestigkeit des geraden Stabes mit veränderlichem Querschnitt und veränderlichem Druck, ohne und mit Querstützen. 1907. 47 S., I Taf. $8^{0}$ - Anch bei Baedeker in Düsseldorf 1908.

Aachen, Dr.-Ing.-Diss. v. 20. 3. 1908

103. Pirlet, Joseph: Fehleruntersuchungen bei der Berechnung mehrfach statisch unbestimmter Systeme. 1909. 100 S. $8^{0}$

Aachen, Dr.-Ing.-Diss. v. 12. 8. 1909

104. Kayser, 0[tto], Reg.-Baumeister a. D., Direktor d. städt. Vorortbahnen zu Cöln: Die belgischen Kleinbahnen. Berlin: Springer 1911. 86 S., 9 Taf., $1 \mathrm{Kt} .8^{0} \cdot$ Auch als Buch.

Aachen, Dr.-Ing.-Diss. v. 11. 10. 1911

\section{ABTEILUNG FÜR DAS MASCHINEN- INGENIEURWESEN}

\section{Maschinen-Ingenieurwesen}

105. Immenkötter, Theodor: Über Heizwertbestimmungen mit besonderer Berücksichtigung gasförmiger und flüssiger Brennstoffe. 1905 . VII, 97 S. $8^{0}$ o Auch bei Oldenbourg in München; Abschn. 2 ff. auch in: Schilling's Journal f. Gasbeleuchtung. Jg. 48.

Aachen, Dr.-Ing.-Diss. v. 10. 7. 1905

106. Rummel, K[urt]: Der Einfluss der Vergaserdüse auf das Mischungsverhältnis bei Motoren für flüssige Brennstoffe <speziell für Automobilmotoren〉. 1906. $32 \mathrm{~S} .4^{0}$ - Aus: Der Motorwagen. Jg. 9. 10. 1906. 07. Aachen, Dr.-Ing.-Diss. v. 26. 9. 1906

107. Weidmann, Carl: Utber eine zwangläufige Verbrennung bei Verbrennungsmaschinen. (Berlin: Springer) 1906. 96 S., 4 Taf. $8^{0}$ ๆ Buchausg. 1905 erw. u. d. T.: Zwangläufige Regelung der Verbrennung bei Verbrennungsmaschinen.

Aachen, Dr.-Ing.-Diss. v. 7. 12. 1906

108. Zahn, Walter: Zur Theorie der Bandagen-Schwungräder. 1909. 41 S. $4^{0}$ - Aus: Verhandlungen d. Vereins zur Beförderung d. Gewerbfleißes. Jg. 87. 1908.

Aachen, Dr.-Ing.-Diss. v. 16. 1. 1909

109. Essieh, Eugen: Über Steuerungsgetriebe mit Wälzhebeln. $1909.30 \mathrm{~S} .4^{0}$ - Aus: Verhandlungen d. Vereins zur Beförderung d. Gewerbfleißes. Jg. 88.

Aachen, Dr.-Ing.-Diss. v. 20. 10. 1909
110. Voissel, Peter: Resonanzerscheinungen in der Saugleitung von Kompressoren und Gasmotoren. 1910. 57 S., 6 Taf. $4^{0}$ - Etwas gekürzt in: Mitteilungen über Forschungsarbeiten auf $d$. Gebiete d. Ingenieurwesens. H. 106. 1911; Ausz. in: Zeitschrift d. Vereines deutscher Ingenieure. Bd 56. 1912, Halbj. 1.

Aachen, Dr.-Ing.-Diss. v. 9. 7. 1910

111. Thelen, Karl: Beitrag zur Entwicklung und zum gegenwärtigen Stande der Vakuumverdampfung. 1911. 94 S. $8^{0}$ I Ein Teil zuerst in: Zeitschrift f. chem. Apparatenkunde. Jg. 3. 1908.

Aachen, Dr.-Ing.-Diss. v. 20. 3. 1911

112. Hellenschmidt, G[eorg]: Die Gemischbildungen der Gasmaschinen. Berlin: Springer 1911. IV, 50 S., 1 Taf. $8^{0}$ - Auch als Buch.

Aachen, Dr.-Ing.-Diss. v. 12. 4. 1911

113. Liepe, Siegfried: Die Verwendung der Brinell'schen Kugeldruckprobe zu Kraftund Schlagarbeitsmessungen. 1911. $52 \mathrm{~S} .4^{0}$ - Fast unverändert in: Verhandlungen d. Vereins zur Beförderung d. Gewerbfleißes. Jg. 91. 1912. Aachen, Dr.-Ing.-Diss. v. 25. 4. 1911

114. Barth, Curt: Die Grundlagen der Zahnradbearbeitung unter Berücksichtigung der modernen Verfahren und Maschinen. Berlin: Springer 1911. 63 S. $4^{0} \uparrow$ Auch als Buch.

Aachen, Dr.-Ing.-Diss. v. 12. 7. 1911

115. Bergmann, Arnold: Untersuchungen an Lamellen-Senksperrbremsen. [1911.] 26 S. $4^{0}$ ๆ Aus: Dingler's Polytechn, Journal. Jg. 92 . Bd 326 .

Aachen, Dr.-Ing.-Diss. v. 12. 7. 1911

116. Moldenhauer, Erich: Wirtschaftliche Schachtförderung aus großen Teufen. 1911. 38 S. $4^{0}$ - Um Zahlentaf. $10-18$ verm. aus: Glückauf. Jg. 47.

Aachen, Dr.-Ing.-Diss. v. 20. 12. 1911

117. Nettmann, Paul: Untersuchung über den Einfluß einer Axial-Belastung auf den Gleitmodul bei Drehung. Berlin: Krayn 1912. 35 S. $8^{0}$ - Auch in: Die Turbine. Jg. 9. 1912/13; soll auch erscheinen als Teil von: Nettmann, Der Torsionsindikator; engl. Übers. in Vorbereitung für: Cassier's Engineering Monthly.

Aachen, Dr.-Ing.-Diss. v. 14. 5. 1912

118. Beckmann, Ernst: Hammer und Presse. 1912. 31 S., 4 Bl. Tab., 21 Taf. $8^{\circ}$ Aachen, Dr.-Ing.-Diss. v. 28. 9. 1912

\section{Elektrotechnik}

119. Spielmann, F[riedrich]: Die Stromverteilung in Kurzschlußwickelungen. 1903. 24 S. $4^{0}$

Aachen, Dr.-Ing.-Diss. v. 30. 6. 1903 
120. Bauwens, $\mathbf{F}[\mathrm{ranz}]:$ Die Winkel-Verschiebung der magnetischen Axe im stromlosen Anker bei Rotation. 1904. 21 S., 9 Taf. $4^{0}$

Aachen, Dr.-Ing.-Diss. v. 20. 4. 1905

121. Bartz, Karl: Untersuchungen über die Kraftlinienstreuung von Spulen. [1908.] $93 \mathrm{~S} ., 15$ Taf. $4^{0}$

Aachen, Dr.-Ing.-Diss. v. 20. 3. 1908

122. Kühle, J[osef]: Über die Fortpflanzungsgeschwindigkeit des Magnetismus in lokal erregten Eisenstäben und die Frage der magnetischen Viskosität oder Trägheit. 1908. $48 \mathrm{~S} .4^{0}$

Aachen, Dr.-Ing.-Diss. v. 31. 7. 1908

123. Werner, $\mathbf{C h}[$ ristian] $\mathbf{A}[$ lbert]: Die mechanische Beanspruchung raschlaufender Magneträder 〈Turbogeneratoren〉. Halle: Knapp 1908. IV, 95 S. 8 $\uparrow$ Auch als Buch. 1909.

Aachen, Dr.-Ing.-Diss. v. 4. 1. 1909

124. Hellmann, Emil, $\mathrm{Kgl}$. Oberlehrer: Der magnetische Widerstand von Lufträumen zwischen parallelen quadratischen und rechteckigen Endflächen von Eisenkernen. 1910. 35 S. $4^{0}$

Aachen, Dr.-Ing.-Diss. v. 2. 9. 1910

\section{ABTEILUNG FÜR BERGBAU UND HÜTTENKUNDE, FÜR CHEMIE UND ELE KTR OCHEMIE}

\section{Bergbau}

125. Denker, Wilhelm, c. Gewerbeinsp. i. Gummersbach: Die bergmännischen Sprengarbeiten im Lichte der Unfallstatistik. 1904. 28 S., 2 Tab. $4^{0}$ - Auch in: Glückauf. Jg. 40.

Aachen, Dr.-Ing.-Diss. v. 1. 2. 1904

126. Köhler, Gustav: Die „Rücken“ in Mansfeld und in Thüringen sowie ihre Beziehungen zur Erzführung des Kupferschieferflötzes. Leipzig: W.Engelmann 1905. 29 S., 13 Taf. $4^{0}$. Auch als Buch.

Aachen, Dr.-Ing.-Diss. v. 23. 9. 1905

127. Freise, $\operatorname{Fr}[\mathbf{i e}] \mathrm{d}[\mathrm{rich}]$ : Stratameter und Bohrlochneigungsmesser. 1906. 69 S., 4 Taf. $8^{0}$ Auch bei Craz \& Gerlach in Freiberg i. S.; Ausz. in: Oesterr. Zeitschrift f. Berg- u. Hüttenwesen. Jg. 54 u. in Organ d. Vereins d. Bohrtechniker. Vereinsj. 13. (Beibl. z. Allg. österreich. Chemiker- u. Techniker-Zeitung. Jg. 24.)

Aachen, Dr.-Ing.-Diss. v. 29. 3. 1906

128. Esser, Friedrich: Elektrostatische Aufbereitung der Erze. Halle: Knapp 1907. $27 \mathrm{~S} .4^{0}$ I Verkürzt in: Metallurgie. Jg. 4. Aachen, Dr.-Ing.-Diss. v. 26. 1. 1907
129. Putseh, Albert: Die Mineralien der Eifel und der angrenzenden Gebiete. 1905. XVI, 115 S. $8^{0}$

Aachen, Dr.-Ing.-Diss. v. 16. 2. 1907

130. Seiler, Carl: Uber die aussergewöhnlichen Silberverluste bei der Aufbereitung von silberhaltigem Bleiglanz und ihre Verringerung auf mechanischem Wege. 1906. 52 S., 4 Taf: $8^{0}$

Aachen, Dr.-Ing.-Diss. v. 14. 3. 1907

131. Randhahn, Walther: Der Wettbewerb der deutschen Braunkohlen-Industrie gegen die Einfuhr der böhmischen Braunkohle. Jena: Fischer 1908. 119 S., 1 Kt. $8^{0}$ - Auch als: Mitteilungen d. Gesellschaft f. wirtschaftl. Ausbildung. N. F. H. 3.

Aachen, Dr.-Ing.-Diss. v. 25. 6. 1908

132. Fluhr, Robert: Geologie und Hydrologie im engeren Einzugsgebiet der Streu unter besonderer Berücksichtigung bodentechnischer Fragen. 1908. 66 S. $8^{0}$

Aachen, Dr.-Ing.-Diss. v. 21. 9. 1908

133. Hilgenstoek, [Karl], Bergass., Dahlhausen $\langle\mathrm{Ruhr}\rangle$ : Untersuchungen über wechselnde Kohlenfestigkeit und ihren Einfluß auf das Lohnwesen. Ein Beitrag zur Frage der Lohntarife im Steinkohlenbergbau. 1909. 25 S. $4^{0}$ Aus: Glückauf. Jg. 45 .

Aachen, Dr.-Ing.-Diss. v. 8. 11. 1909

134. Forstmann, [Richard], Bergass., Essen: Untersuchungen über die Austrocknung der Grubenbaue durch große Wettermengen und Versuche mit verschiedenen Mitteln zur Kohlenstaubbekämpfung. 1910. 52 S. $4^{0}$ - Aus: Glückauf. Jg. 46; auch im Verlag des "Glückauf".

Aachen, Dr.-Ing.-Diss. v. 10. 3. 1910

135. Glinz, K[arl], Bergass. a. D.: Aufgaben und Lösungen auf dem Gebiete der maschinellen Fortbewegung und Lagerung im Grubenbetriebe gewonnener Massengüter, insbesondere Eisenerz und Kohle, auf Tagesanlagen und deren Ausbildung hierfür. 1909. 32 S., 2 Taf. $4^{0}$ - Ausz. in: Stahl u. Eisen. Jg. 30. 1910, Halbj. 2.

Aachen, Dr.-Ing.-Diss. v. 20. 5. 1910

136. Schöppe, Willi: Über kontaktmetamorphe Eisen-Mangan-Lagerstätten am Aranyos-Flusse, Siebenbürgen. 1910. 35 S., 1 Taf. $4^{0}$ - Auch in: Zeitschrift f. prakt. Geologie. Jg. 18. Aachen, Dr.-Ing.-Diss. v. 2. 9. 1910

137. Obersehuir, Ewald, Bergass.: Die Heranziehung und Sesshaftmachung von Bergarbeitern im Ruhrkohlenbecken. Kritik der bisher getroffenen Massnahmen und 
Vorschläge zur Gewinnung eines sesshaften Arbeiterstammes. $1910.103 \mathrm{~S} .8^{0}$

Aachen, Dr.-Ing.-Diss. v. 15. 10. 1910

138. Viersehilling, Aloys: Die Eisen- und Manganerzlagerstätten im Hunsrück und im Soonwald. Berlin: Krahmann 1910. 43 S., 1 Taf., $1 \mathrm{Kt} .4^{0}$ - Aus: Zeitschrift f. prakt. Geologie. Jg. 18.

Aachen, Dr.-Ing.-Diss. v. 24. 11. 1910

139. Lange, Theodor: Uber die Amphibolite des nordwestlichen Thüringer Waldes. 1910. 52 S., 3 Taf. $8^{\circ}$ T Aus: Jahrbuch d. Kgl. PreuB. Geolog. Landesanstalt f. 1911. Bd 32 .

Aachen, Dr.-Ing.-Diss. v. 7. 3. 1911

140. Herwegen, Leo: Das Schwimmsandproblem und seine Anwendung auf verschiedene Abteufmethoden in schwimmendem Gebirge. 1911. 24 S. $4^{0}$ - Etwas geändert in: Braunkohle. Jg. 10. 1911/12.

Aachen, Dr.-Ing.-Diss. v. 14. 7. 1911

141. Walbrecker, Walther: Studien und Versuche über das Gefrierverfahren. (1910.) $21 \mathrm{~S} .4^{0}$ - Mit geringfügigen Änderungen aus: Glïckauf. Jg. 46.

Aachen, Dr.-Ing.-Diss. v. 15. 2. 1912

142. Westermann, [Heinrich], Bergass. Dr., Dortmund: Die Wirtschaftlichkeit und Zweckmäßigkeit des Unterwerksbaues und Oberwerksbaues im rheinisch-westfälischen Steinkohlenbergbau. [1912.] 36 S. $4^{0}$ ๆ Aus: Glückauf. Jg. 48.

Aachen, Dr.-Ing.-Diss. v. 27. 6. 1912

143. Dittmann, Kurt Emil: Das Tertiär am Nordostabfall der Eifel. 1912. 43 S., 6 Bl. Taf. $4^{0}$

- Aachen, Dr.-Ing.-Diss. v. 18. 9. 1912

144. Lehmann, Max: Ueber aussergewöhnliche Hauptschacht-Fördersysteme, im besonderen über die Schachtförderung mittels Becherwerkes. Halle: Knapp 1912. 56 S. $4^{0}$

Aachen, Dr.-Ing.-Diss. v. 5. 11. 1912

145. Kirsehmann, W[alter], Bergass., Elberfeld: Die Lagerungsverhältnisse des oberen Allertales zwischen Morsleben und Walbeck. Berlin: Krahmann 1912. 34 S. $4^{0}$ I Aus: Zeitschrift f. prakt. Geologie. Jg. 21. 1913.

Aachen, Dr.-Ing.-Diss. v. 16. 1. 1913

\section{Hüttenkunde}

146. Günther, Emil: Verfahren zur Gewinnung von Kupfer und Nickel aus kupferund nickelhaltigen Magnetkiesen. 1903. 32 S. $4^{0}$ - Auch bei Craz \& Gerlach in Frei- berg i. S. u. als: Mitteilungen über Forschungsarbeiten auf d. Gebiete d. Ingenieurwesens. H. 10; Ausz. in: Zeitschriftd.Vereines deutscher Ingenieure. Bd 47, Halbj. 2.

Aachen, Dr.-Ing.-Diss. v. 14. 1. 1903

147. Thomas, Friedrich: Über die Einwirkung des Ferrisulfats auf Kupferkies. 1903. 31 S. $4^{0}$ - Aus: Metallurgie. Jg. 1. 1904. Aachen, Dr.-Ing.-Diss. v. 2. 2. 1903

148. Stockem, Lorenz: Studien über die Abscheidung der wichtigeren Alkali- und Erdalkalimetalle aus ihren geschmolzenen Haloidsalzen. 1903. 16 S. $4^{0}$ \& Erw. in: Metallurgie. Jg. 1. 1904.

Aachen, Dr.-Ing.-Diss. v. 30. 6. 1903

149. Bruch, Reinhard: Versuche, Schmiedeeisen mit Leuchtgas, Petroleumdampf, Acetylen und Kohlenoxydgas zu kohlen. 1904. 37 S., 3 Taf. $4^{0}$

Aachen, Dr.-Ing.-Diss. v. 18. 4. 1904

150. Schlösser, Paul: Die Temperkohle und der Einfluss von Kohlenstoff, Silicium, Mangan, Schwefel, Phosphor und Kupfer auf die Bildung derselben im Eisen. 1904. 40 S., 2 Taf. $4^{0}$ I Ausz. mit Änderungen in: Stahl u. Eisen. Jg. 24, Halbj. 2 u. in: Mitteilungen aus d. Eisenhüttenmänn. Institut d. Kgl. Techn. Hochschule Aachen. 1906 [Nr 99].

Aachen, Dr.-Ing.-Diss. v. 18. 4. 1904

151. Geiger, Carl: Beiträge zur Kenntnis der zwei Kohlenstofformen im Eisen ,Temperkohle " und ,Graphit". 1904. 52 S. $4^{0}$ T Ausz. in: Stahl u. Eisen. Jg. 25. 1905, Halbj. 2; Mitteilungen aus d. Eisenhütttenmänn. Institut d. Kgl. Techn. Hochschule Aachen. 1906 [Nr 99].

Aachen, Dr.-Ing.-Diss. v. 17. 10. 1904

152. Geilenkirchen, Theodor: Ueber Verwendung von kalt erblasenem Roheisen zur Flusseisen-Darstellung. [1904.] 102 S. $8^{0}$ I Ausz. in: Stahl u. Eisen. Jg. 25. 1905, Halbj. 1. Aachen, Dr.-Ing.-Diss. v. 17. 10. 1904

153. Huppertz, Wilhelm: Versuche über die Herstellung von Titan und Titanlegierungen aus Rutil und Titanaten im elektrischen Ofen. 1904. 41 S. $4^{0}$ - Aus: Metallurgie. Jg. 1.

Aachen, Dr.-Ing.-Diss. v. 17. 10. 1904

154. Ott, G[otthilf], Hüttening., Augsburg: VergleichendeUntersuchungen von rheinischwestfälischem Giesserei- und Hochofenkoks. 1905. 67 S., 1 Taf. $8^{0}$ T Ausz. in: Stahl u. Eisen. Jg. 26. 1906, Halbj. 2.

Aachen, Dr.-Ing.-Diss. v. 17. 10. 1904

155. Peetz, L[udwig]: Scheidung von Zinn und Blei aus Zinn-Blei-Legierungen. 1904.

30 S. $4^{0}$ I Aus: Metallurgie. Jg. 1. Aachen, Dr.-Ing.-Diss. v. 17. 10. 1904 
156. Wolff, Paul: Das Verhalten des Koksschwefels im Hochofen. 1904. 68 S. $8^{0}$ - Ausz. in: Stahl u. Eisen. Jg. 25. 1905, Halbj.1; Mitteilungen aus d. Eisenhüttenmänn. Institut d. Kgl. Techn. Hochschule Aachen. 1906 [Nr 99].

Aachen, Dr.-Ing.-Diss. v. 17. 10. 1904

157. Nathusius, Hans: Beziehungen zwischen den magnetischen und anderen Eigenschaften des Gußeisens. (1905.) 34 S., 2 Taf. $4^{0}$. I Ausz. in: Stahl u. Eisen. Jg. 25, Halbj. 1; Mitteilungen aus d. Eisenhüttenmänn. 'Tnstitut d. Kgl. Techn. Hochschule Aachen. 1906 [Nr 99].

Aachen, Dr.-Ing.-Diss. v. 27. 7. 1905

158. Brandt, Paul: Das Verblasen von Kupferstein mittels mit Sauerstoff angereicherten Windes. 1905. $20 \mathrm{~S} .4^{0}$ ๆ Aus: Metallurgie. Jg. 2.

Aachen, Dr.-Ing.-Diss. v. 18. 9. 1905

159. Lehmer, C[arl]: Elektrisches Verschmelzen sulfidischer Erze und Hüttenprodukte unmittelbar auf Metall. 1906. 24 S. $4^{0}$ - Aus: Metallurgie. Jg. 3.

Aachen, Dr.-Ing.-Diss. v. 19. 2. 1906

160. Pütz, Paul: Der Einfluss des Vanadins auf Eisen und Stahl. 1906. 39 S., 1 Taf. $4^{0}$ - Abgeändert in: Metallurgie. Jg. 3; Mitteilungen aus $\mathrm{d}$. Eisenhüttenmänn. Institut $\mathrm{d}$. $\mathrm{Kgl}$. Techn. Hochschule Aachen. Bd 2. 1908 [Nr 99].

Aachen, Dr.-Ing.-Diss. v. 11. 12. 1906

161. Graumann, Carl Artur: Studien über das Verhalten von Eisenoxyden zu Zinkblende. 1906. 17 S. $4^{0}$ - Erw. aus: Metallurgie. Jg. 4. 1907.

Aachen, Dr.-Ing.-Diss. v. 28. 12.1906

162. Petersen, 0tto: Beitrag zum Einfluss des Siliziums auf das System EisenKohlenstoff. Halle: Knapp 1907. 34 S. $4^{0}$ - Ausz. in: Metallurgie. Jg. 3. 1906; Mitteilungen aus d. Eisenhüttenmänn. Institut d. Kgl. Techn. Hochschule Aachen. Bd 2. 1908 [Nr 99].

Aachen, Dr.-Ing.-Diss. v. 1. 6. 1907

163. Goerens, Paul: Über die Vorgänge bei der Erstarrung und Umwandlung von Eisenkohlenstofflegierungen und deren Beobachtung auf metallographischem Wege. Halle: Knapp 1907. 46 S. $4^{0}$. Auch als Buch aus: Metallurgie. Jg. 3. 4. 1906. 07; auch in: Mitteilungen aus d. Eisenhüttenmänn. Institut d. Kgl. Techn. Hochschule Aachen. Bd [1.] 2. 1906. 08 [Nr 99].

Aachen, Dr.-Ing.-Diss. v. 19. 6. 1907

164. Schütz, Ernst: Über die Affinität des Schwefels zu einer Reihe von Metallen. (Eine Nachprüfung der Reihe von Fournet.) Halle: Knapp-1907. 21 S. $4^{0}$ - I Aus: Metallurgie. Jg. 4.

Aachen, Dr.-Ing.-Diss. v. 29. 7. 1907
165. Oberhoffer, Paul: Über die spezifische Wärme des Eisens. Halle: Knapp 1907. 45 S. $4^{0}$ - Etwas gekürzt in: Metallurgie. Jg. 4 ; Mitteilungen aus d. Eisenhüttenmänn. Institut d. Kgl. Techn. Hochschule Aachen. Bd 2. 1908 [Nr 99].

Aachen, Dr.-Ing.-Diss. v. 4. 10. 1907

166. Harkort, Hermann: Beitrag zum Studium des Systems Eisen-Wolfram. Halle: Knapp 1907. 66 S. $4^{0}$ - Auch in: Metallurgie. Jg. 4.

Aachen, Dr.-Ing.-Diss. v. 29. 1. 1908

167. Quasebart, Karl: Vergasungsversuche mit dem Morgan-Generator. Halle: Knapp 1908. 26 S., 3 Taf. $4^{0}$ ๆ Aus: Metallurgie. Jg. 5 .

Aachen, Dr.-Ing.-Diss. v. 9. 6. 1908

168. Stadeler, August: Beitrag zur Kenntnis des Mangans und seiner Legierungen mit Kohlenstoff. Halle: Knapp 1908. 26 S., 4 Taf. $4^{0}$ - Aus: Metallurgie. Jg. 5; auch in: Mitteilungen aus d. Eisenhüttenmänn. Institut d. Kgl. Techn. Hochschule Aachen. Bd 2 [Nr 99]. Aachen, Dr.-Ing.-Diss. v. 19. 6. 1908

169. Beck, Erieh: Studien über die Darstellung von Legierungen nitridbildender Metalle. Halle: Knapp 1908. 22 S. $4^{0}$

- Aus: Metallurgie. Jg. 5.

Aachen, Dr.-Ing.-Diss. v. 21. 9. 1908

170. Laval, Leo: Experimentelle Untersuchung des Thomasprozesses. Halle: Knapp 1908. 55 S. $4^{0}$ ๆ Aus: Metallurgie. Jg. 5; auch in: Mitteilungen aus d. Eisenhüttenmänn. Institut d. Kgl. Techn, Hochschule Aachen. Bd 3. 1909 [Nr 99].

Aachen, Dr.-Ing.-Diss. v. 21. 9. 1908

171. Müller, Albert: Über die Darstellung des Elektrolyteisens, dessen Zusammensetzung und thermische Eigenschaften. Halle: Knapp 1909. 24 S., 1 Taf. $4^{0}$ ๆ Aus: Metallurgie. Jg. 6; auch in: Mitteilungen aus d. Eisenhüttenmänn. Institut d. Kgl. Techn. Hochschule Aachen. Bd 3 [Nr 99]. Autoreferat in: Stahl u. Eisen. Jg. 29, Halbj. 1.

$$
\text { Aachen, Dr.-Ing.-Diss. v. 6. 4. } 1909
$$

172. Maurer, Ed[uard]: Untersuchungen über das Härten und Anlassen von Eisen und Stahl. Halle: Knapp 1909. 40 S., 4 Taf. $4^{0}$ - Verkürzt in: Metallurgie. Jg. 6. Aachen, Dr.-Ing.-Diss. v. 10. 5. 1909:

173. Warlimont, Felix: Über die Oxydation der Sulfide und die Dissoc[i]ation der Su[l]fate von Eisen, Kupfer und Nickel und ein auf diese Versuche sich stützendes einfaches Verfahren zur Verhüttung Kupfer und Nickel führender Magnetkiese. Halle: Knapp 1909. 22 S. $4^{0}$ if Aus: Metallurgie. Jg. 6.

Aachen, Dr.-Ing.-Diss. v. 25. 8. 1909 
174. Becker, Hermann: Über das Glühfrischen mit gasförmigen Oxydationsmitteln. Halle: Knapp 1910. 24 S., 2 Taf. $4^{0}$ - Aus: Metallurgie. Jg. 7; auch in: Mitteilungen aus d. Eisenhüttenmänn. Institut d. Kgl. Techn. Hochschule Aachen. Bd 4. 1911 [Nr 99]. Aachen, Dr.-Ing.-Diss. v. 7. 3. 1910

175. Gutowsky, Nikolaus: Zur Theorie des Schmelz- und Erstarrungsprozesses der Eisen-Kohlenstofflegierungen. Halle: Knapp 1910. 18 S., 5 Taf. $4^{0}$ - Aus: Metallurgie. Jg. 6. 1909; auch in: Mitteilungen aus d. Eisenhüttenmänn. Institut d. Kgl. Techn. Hochschule Aachen. Bd 4. 1911 [Nr 99].

Aachen, Dr.-Ing.-Diss. v. 18. 3. 1910

176. Springorum, Friedrich: Experimentelle Untersuchungen des Hoeschprozesses. Halle: Knapp 1910. 26 S., 6 Bl. Fig. $4^{0}$ - Aus: Metallurgie. Jg. 7; auch in: Mitteilungen aus d. Eisenhuittenmänn. Institut d. Kgl. Techn. Hochschule Aachen. Bd 4. 1911; gekürzt in: Stahl u. Eisen. Jg. 30, Halbj. 1 [Nr 99].

Aachen, Dr.-Ing.-Diss. v. 2. 4. 1910

177. Liesching, Theodor: Uber den Einfluss des Schwefels auf das System EisenKohlenstoff. Halle: Knapp 1910. 16 S., 1 Taf. $4^{0}$ - Etwas verkürzt in: Metallurgie. Jg. 7; Mitteilungen aus d. Eisenhüttenmänn. Institut d. Kgl. Techn. Hochschnle Aachen. Bd 4. 1911 [Nr 99].

Aachen, Dr.-Ing.-Diss. v. 20. 9. 1910

178. Weyl, Fritz: Uber Zementation im luftleeren Raum mittels reinen Kohlenstoffs. Halle: Knapp 1910. 21 S., 4 Taf. $4^{0}$ I Aus: Metallurgie. Jg. 7; auch in: Mitteilungen aus d. Eisenhüttenmänn. Institut d. Kgl. Techn. Hochschule Aachen. Bd 4. 1911; kurzer Ausz. in: Stahl u. Eisen. Jg. 30, Halbj. 2 [Nr 99].

Aachen, Dr.-Ing.-Diss. v. 20. 9. 1910

179. Thomas, Felix: Über den Einfluss von Wasserdampf, bezw. Kohlenwasserstoffen, auf die Röstung der Zinkblende. Halle: Knapp 1910.16 S. $4^{0}$ - Aus: Metallurgie. Jg. 7.

Aachen, Dr.-Ing.-Diss. v. 5. 10. 1910

180. Felser, Hans L[udwig]: Der Einfluss der Seigerung auf die Festigkeit des Flusseisens. 1910. $25 \mathrm{~S} ., 8 \mathrm{Bl}$. Taf. $4^{0}$ - Auch in: Metallurgie. Jg. 7 u. in: Mitteilungen aus d. Eisenhüttenmänn. Institut d. Kgl. Techn. Hochschule Aachen. Bd. 4. 1911 [Nr 99]

Aachen, Dr.-Ing.-Diss. v. 24. 11. 1910

181. Gillhausen, W[erner] G[iesbert]: Untersuchungen über die Wärme- und Stoffbilanz beim Hochofen. Halle: Knapp 1910. 49 S., 1 Taf. $4^{0}$ - Aus: Metallurgie. Jg. 7; auch in: Mitteilungen aus d. Eisenhüttenmänn. Institut d. Kgl. Techn. Hochschule Aachen. Bd 4. 1911 [Nr 99].

Aachen, Dr.-Ing.-Diss. v. 5. 12.1910
182. Müller, Paul: Die elektrische Leitfähigkeit der Metallegierungen im flüssigen Zustande. Halle: Knapp 1911. 57 S. $4^{0}$ -Vorläufige Mitteilung in: Metallurgie. Jg. 7. 1910. Aachen, Dr.-Ing.-Diss. v. 9. 2. 1911

183. Monnartz, Philipp: Beitrag zum Studium der Eisenchromlegierungen unter besonderer Berücksichtigung der Säurebeständigkeit. Halle: Knapp 1911. 34 S. $4^{0}$

- Aus: Metallurgie. Jg. 8.

Aachen, Dr.-Ing.-Diss. v. 23. 2. 1911

184. Krupp, Otto, Bergass. Dr. jur.: Die Versorgung der niederrheinisch-westfälischen Hochofenwerke mit Eisenerz und die schwedische Eisenerzfrage. 1910. 23 S., 2 Anl. $4^{0}$ - Aus: Glückauf. Jg. 47. 1911.

Aachen, Dr.-Ing.-Diss. v. 10. 3. 1911

185. Láng, Georg: Über den Einfluss des Mangans auf die Eigenschaften des Flusseisens. Halle: Knapp 1911. 20 S., 1 Taf. $4^{0}$ - Aus: Metallurgie. Jg. 8; auch in: Mitteilungen aus d. Eisenhüttenmänn. Institut d. Kgl. Techn. Hochschule Aachen. Bd 5. 1913 [Nr 99].

Aachen, Dr.-Ing.-Diss. v. 24. 3. 1911

186. Schütz, Wilhelm, Hüttenass.: Das Verhalten von Baryumsulfat und Calciumsulfat gegen die wichtigeren Metallsulfide bei Gegenwart freier Kieselsäure. Halle: Knapp 1911. 22 S. $4^{0}$ ・ Aus: Metallurgie. Jg. 8. Aachen, Dr.-Ing.-Diss. v. 27. 4. 1911

187. Hesse, Robert: Das Verschmelzen stark bleihaltiger Kupfersteine. Halle: Knapp 1911. 31S. $4^{0}$ - Aus: Metallurgie. Jg. 8. Aachen, Dr.-Ing.-Diss. v. 17. 5. 1911

188. Niedt, $\mathbf{H}[\mathrm{ermann}]$ : Untersuchungen über die Zusammensetzung des Gasstromes im Hochofen. Halle: Knapp 1911. 56 S., 7 Taf. $4^{0}$ - Aus: Metallurgie. Jg. 8; auch in: Mitteilungen aus d. Eisenhüttenmänn. Institut d. Kgl.Techn. Hochschule Aachen. Bd 5. 1913 [Nr 99]. Aachen, Dr.-Ing.-Diss. v. 18. 9. 1911

189. Joisten, Anton: Einfluss der thermischen Behandlung auf die Korngrösse und die Festigkeitseigensehaften des Eisens. Halle: Knapp 1911. 26 S., 2 Taf. $4^{0}$ T Ausz. aus d. ersten Teil in: Metallurgie. Jg. 7. $1910 \mathrm{u}$. in: Mitteilungen aus d. Eisenhüttenmänn. Institut d. Kgl. Techn. Hochschule Aachen. Bd 4. 1911 [Nr 99]. Aachen, Dr.-Ing.-Diss. v. 28. 11. 1911

190. Wark, N[icolas] J[ean]: Bestimmung der Löslichkeitslinie des Eisencarbids $\left\langle\mathrm{Fe}_{3} \mathrm{C}\right\rangle$ in $\gamma$-Eisen. Untersuchungen über die Polyederstruktur in Eisen-Kohlenstofflegierungen. Halle: Knapp 1911. 18 S., 3 Taf. $4^{0}$ II Aus: Metallurgie. Jg. 8; auch in: Mitteilungen aus d. Eisenhüttenmänn. Institut d. Kgl. Techn. Hochschule Aachen. Bd 5. 1913 [Nr 99]. Aachen, Dr.-Ing.-Diss. v. 17. 2. 1912 
191. Münker, E[mil]: Ueber den Finfluß geringer Mengen Phosphor, Mangan und Zinn auf die physikalischen Eigenschaften von Kupfer. Halle: Knapp 1912. 16 S., 6 Taf. $4^{0}$ - Aus: Metallurgie. Bd 9; auch in: Mitteilungen aus d. Eisenhüttenmänn. Institut d. Kgl. Techn. Hochschule Aachen. Bd 5. 1913 [Nr 99].

Aachen, Dr.-Ing.-Diss. v. 29. 3. 1912

192. Barth, 0tto: Die Erhöhung der chemischen Widerstandsfähigkeit mechanisch noch gut bearbeitbarer für Konstruktionszwecke verwendbarer Legierungen. Halle: Knapp 1912. 29 S., 1 Taf. $4^{0}$ ๆ Etwas gekürzt in: Metallurgie. Bd 9.

Aachen, Dr.-Ing.-Diss. v. 22. 4. 1912

193. Paglianti, Pietro: Úber den Einfluß des Siliciums auf die Eigenschaften des Flußeisens. Halle: Knapp 1912. 18 S., 4 Taf. $4^{0}$ I Aus: Metallurgie. Bd 9; auch in: Mitteilungen aus d. Eisenhüttenmänn. Institut d. Kgl. Techn. Hochschule Aachen. Bd 5. 1913 [Nr 99].

Aachen, Dr.-Ing.-Diss. v. 22. 4. 1912

194. Eulenstein, $\operatorname{Fr}[i t z]$ : Betrachtungen über die Wärmebilanz eines Siemens-Zinkofens. Halle: Knapp [1912]. 30 S., 1 Bl. Fig. $4^{0}$ - Auch als Buch; aus: Metallurgie. Bd 9.

Aachen, Dr.-Ing.-Diss. v. 14. 5. 1912

195. Pedersen, Harald: Studien über Vereinfachung der Verhüttung eisen- und kupferhaltiger sulfidischer Nickelerze und Hüttenprodukte. Halle: Knapp 1912. 21 S., 2 Taf. $4^{0}$ ๆ Aus: Metall u. Erz. Jg. 10. 〈N. F. 1.〉 1913.

Aachen, Dr.-Ing.-Diss. v. 18. 7. 1912

196. Meuthen, A[dam]: Kalorimetrische Untersuchung des Systems Eisen-Kohlenstoff. Halle: Knapp 1912. 22 S., 1 Taf., 2 Bl. Fig. $4^{0}$ - Aus: Ferrum. Jg. 10. 1912/13; auch in: Mitteilungen aus d. Eisenhüttenmänn. Institut d. Kgl. Techn. Hochschule Aachen, Bd 5. 1913 [Nr 99].

Aachen, Dr.-Ing.Diss. v. 2. 10. 1912

197. Rauschenplat, G[ünzel] von: Die elektrische Leitfähigkeit der Metallegierungen im flüssigen Zustande. Halle: Knapp 1912. $44 \mathrm{~S} .4^{0}$ - Aus: Metallurgie. Jg. 9.

Aachen, Dr.-Ing.-Diss. v. 30. 10. 1912

198. Styri, Haakon: Über basisches Verschmelzen von Kupferstein und Verarbeitung eines oxydischen Kupfererzes. Halle: Knapp 1912. 33S. $4^{0}$ ๆ T. 1 auch in: Metallurgie. Jg. 9; T. 2.3 auch in: Metall u. Erz. Jg. 10. 〈N. F. 1.〉 1913.

Aachen, Dr.-Ing.-Diss. v. 21. 2. 1913
Chemie und Elektrochemie

199. Pfeil, Jos[ef]: Ueber Dihydro-campholyto-lactam und Dihydro-campholytolacton. Ein Beitrag zur Begründung der Bredt'schen Camphersäure-Constitutionsformel. 1901. $42 \mathrm{~S} .8^{0}$

Aachen, Dr.-Ing.-Diss. v. 27. 7. 1901

200. Fischer, Arthur: Über die elektrolytische Bestimmung und Trennung von Antimon und Zinn aus ihren Sulfosalzlösungen nebst einem Anhange über die Trisulfidmethode des Antimons. 1904. 59 S. $8^{0}$ - Aus: Zeitschrift f. anorgan. Chemie. Bd 42.

Aachen, Dr.-Ing.-Diss. v. 17. 10. 1904

201. Amberg, Richard: Ueber chemische Eigenschaften und das Verbindungsgewicht des Palladiums. 1905. $74 \mathrm{~S} .8^{\circ}$ - Aus: Liebig's Annalen d. Chemie. Bd 341.

Aachen, Dr.-Ing.-Diss. v. 2. 10. 1905

202. Weiwers, J[ohann]: Ueber den unvergärbaren Zucker im Wein. 1906. 33S. $4^{0}$ - Aus: Apothekerzeitung. Jg. 21.

Aachen, Dr.-Ing.-Diss. v. 30. 6. 1906

203. Salm, Eduard: Studie über Indikatoren. Leipzig: W. Engelmann 1906. 37 S., 1 Taf. $8^{0}$ I Aus: Zeitschrift f. phys. Chemie. Bd 57. 1907.

Aachen, Dr.-Ing.-Diss. v. 7. 12. 1906

204. Christie, M[aleolm] Grahame: Studien über das Verhalten der SteinkohlenstickstoffVerbindungen bei höherer Temperatur in bezug auf deren Konstitution. 1908. 56 S., 1 Taf. $8^{0}$

Aachen, Dr.-Ing.-Diss. v. 20. 3. 1908

205. Plenske, Ernst: Über Mikrostruktur und Bildung der Porzellane. [1908.] 45 S., 1 Taf. $4^{0}$

Aachen, Dr.-Ing.-Diss. v. 5. 5. 1908

206. Hensen, Caspar: Kritische Untersuchung der Vanadin-Bestimmungsmethoden. 1909. 60 S. $4^{0}$

Aachen, Dr.-Ing.-Diss. v. 22. 4. 1909

207. Hardebeck, C[arl]: Ueber das Verhalten einiger Legierungen zum Gesetze von Wiedemann und Franz. 1909. 40 S., 1 Tab. $8^{0}$

Aachen, Dr.-Ing.-Diss. v. 11. 5. 1909

208. Lambris, Gustav: Studie über die Kohlenstoffaufnahme durch Metalle bei der Elektrolyse aus wässeriger Lösung, mit besonderer Berücksichtigung des Nickels. 1909. $55 \mathrm{~S} .8^{0}$ - Ausz. in: Zeitschrift f. Elektrochemie. Jg. 15.

Aachen, Dr.-Ing.-Diss. v. 3. 11. 1909 
209. May, Richard: Beziehungen des Kamphens zur Apokamphersäure. Ein Beitrag zur Erklärung der Umwandlung des Kamphens in Kampher. 1. Uebergang des Kamphens in dio Karboxylapokamphersäure über die Zwischenstufen: Kamphenilnitrit, Tricyklenkarbonsäure, $\gamma$-Bromapokamphankarbonsäure, Oxyapokamphankarbonsäure und Ketopinsäure. 2. Ueberführung der Apokamphersäure in $\alpha$-Oxyapokamphersäure, Apokamphansäure und Isodehydroapokamphersäure. $1910.80 \mathrm{~S} .8^{0}$ ๆ Auch als: Schriften d. Verbandes Deutscher DiplomIngenieure. 3.

Aachen, Dr.-Ing.-Diss. v. 5. 10. 1910

210. Sandkuhl, Hermann: Utber die Elektroreduktion der Camphocarbonsäure zu cis- und cis-trans-Borneolcarbonsäure, über Bornylencarbonsäure und die Beziehungen der Camphylglykole zu den Borneolcarbonsäuren. 1911. VI, $80 \mathrm{~S} .8^{0}$ ศ Vgl. J. Liebig's Annalen d. Chemie. Bd 366. 1909.

Aachen, Dr.-Ing.-Diss. v. 6. 7. 1911

211. Hilbing, Wilhelm: Ueber $\beta$-Campher aus Bornylencarbonsäure. Derivate der Bornylencarbonsäure. $1912.50 \mathrm{~S} .8^{0}$ ๆ Vgl. Journal f. prakt. Chemie. Bd 192. N. F. Bd 84 . 1911.

Aachen, Dr.-Ing.-Diss. v. 16. 3. 1912

212. Hallmann, Karl: Vergleichende Untersuchung über Methoden der quantitativen Antimonbestimmung. 1911. 103 S. $8^{0}$

Aachen, Dr.-Ing.-Diss. v. 26. 3. 1912

213. Roderburg, Alexander: Das elektrochemische Verhalten des Wolframs. 1912. 54 S. $4^{0}$

Aachen, Dr.-Ing.-Diss. v. 24. 4. 1912
214. Gelbke, M[ilan]: Lang- und kurzwellige Absorptions- und Fluoreszenzbanden der Karbonylgruppe. 1912. 40 S. $8^{0}$ If Aus: Jahrbuch d. Radioaktivität u. Elektronik. Bd 10. 1913/14; vgl. Phys. Zeitschrift. Jg. 13.

Aachen, Dr.-Ing.-Diss. v. 18. 7. 1912

\section{ABTEILUNG FÜR ALLGEMEINE WISSENSCHAFTEN, INSBES ONDERE FÜR MATHEMATIK UND NATUR- WISSENSCHAFTEN}

215. Föppl, 0tto: Windkräfte an ebenen und gewölbten Platten. Berlin: Springer 1911. 75 S. $4^{0}$ - Aus: Jahrbuch d. Motorluftschiff-Studiengesellschaft. 1911.

Aachen, Dr.-Ing.-Diss. v. 21. 7. 1911

216. Alberty, M[atthias], Reg.-Baumeister: Der Übergang zum Staatsbahnsystem in Preußen, seine Begründung, seine Durchführung und Folgen. Eine wirtschaftspolitische Untersuchung. Jena: Fischer 1911. VIII, 55 S. $8^{0}$ I Vollst. ebd.

Aachen, Dr.-Ing.-Diss. v. 16. 8. 1911

217. Greineder, Friedrich: Die finanzielle Überwachung der Gaswerksunternehmen. 1911. IV, 117 S. $8^{0}$ ๆ Auch bei Oldenbourg in München.

Aachen, Dr.-Ing.-Diss. v. 5. 9. 1911

218. Weihs, Gustav: Über Spannungsund Formänderungszustände in dünnwandigen Hohlreifen. 1911. 89 S., 3 Taf. $8^{0}$ Aachen, Dr.-Ing.-Diss. v. 24. 10. 1911

219. Eiler, Karl: Buchführung und Bilanzen kommunaler Elektrizitätswerke. 1912. VI, $84 \mathrm{~S} .8^{0}$ Auch bei Hahn in Hannover. Aachen, Dr.-Ing.-Diss. v. 7. 10. 1912

\section{BERLIN}

Hervorgegangen 1879 aus der Vereinigung der 1799 gegründeten, 1831-1848 „Allgemeine Bauschule " benannten ,Bau-Akademie "und der 1821 unter dem Namen ,Technische Schule" errichteten, 1827-1866 als "Gewerbe-Institut" bezeichneten "Gewerbe-Akademie"

\section{SATZUNGEN ORDNUNGEN BESTIMMUNGEN}

\section{BAUAKADEMIE}

220. Publikandum wegen der vorläufigen Einrichtung der von Seiner Königlichen Majestät Allerhöchstselbst unter dem Namen einer Königlichen Bau-Akademie zu Berlin gestifteten allgemeinen Bau-UnterrichtsAnstalt. De Dato Berlin, den 6. July, 1799. - Abgedr. in: Berlinische Nachrichten v. Staatsu. gelehrten Sachen. No. 88. 23. Julius 1799. Be Minist. d. geistl. Angel. [IV, 14]
221. Deklaration des Publikandi vom sechsten July 1799, wegen der vorläufigen Einrichtung der von Seiner Königlichen Majestät unter dem Nahmen einer Königlichen Bau-Akademie zu Berlin gestifteten allgemeinen Bau-Unterrichts-Anstalt. Berlin 1803. (Dat. 12. Febr.)

Be Minist. d. geistl. Angel. [IV, 14]

222. Vorschrift für die Ausbildung und Prüfung derjenigen, welche sich dem Bau- 
fache widmen (v. 1. 8. 1849). [Folgt:] Vorsehriften für die Kgl. Bau-Academie zu Berlin v. 1. 8. 1849. Abgedr. in: Zeitschrift f. prakt. Baukunst. Jg. 9.

$B e^{*}$

223. Reglement betreffend die Benutzung der vereinigten Bibliothek der Kgl. BauDeputation und der Kgl. Bau-Akademie ( $\nabla$. 23. 2. 1851).

224. Vorschriften für die Ausbildung und Prüfung derjenigen, welche sich dem Baufache widmen (v. 18. 3. 1855). [Folgt:] Vorsehriften für die Kgl. Bau-Akademie zu Berlin (v. 18. 3. 1855).

$B e^{*}$

225. Bestimmungen über den nach $\S 7$ der Vorschrift für die Kgl. Bau-Akademie vom 18ten März 1855 in dem Lehrgange für künftige Bauführer ertheilten Unterricht im Zeichnen und Entwerfen baulicher Gegenstände, so wie in dem des Ornament- und Maschinenzeichnens (v. 6.6. 1856). Be*

226. Vorschriften für die Ausbildung und Prüfung derjenigen, welche sich dem Baufache im Staatsdienste widmen (v. 3. 9. 1868). [Folgt:] Vorsehriften für die Kgl. Bau-Akademie zu Berlin v. 3. Sept. 1868. - Auch u. d. T.: Reglement f. d. Ausbildung, Prüfung u. Anstellung derjenigen ... bei Heymann in Berlin 1872; 2. Aufl. 1877.

227. Bestimmungen über die Verfassung des Lehrkörpers bei der Kgl. Bau-Akademie zu Berlin (v. 10. 11. 1875). I Abgedr. in: Programm f. 1876/77-1878/79 [Nr 301]; Damm 1909. S. $6 \mathrm{ff}$.

$B e^{*}$

228. Vorsehriften für die Kgl. Bau-Akademie zu Berlin (v. 17. 9. 1876). I Einzeln nicht gedr.; abgedr. in: Programm f. 1876/771878/79 [Nr 301]; Damm 1909. S. 8 ff.

229. Ordnung für die Benutzung der Bibliothek der $\mathrm{Kgl}$. Bau-Akademie zu Berlin (v. 16. 11. 1878).

$B e^{*}$

\section{GEWERBEAKADEMIE}

230. Regulativ für die Organisation des Kgl. Gewerbe-Instituts (v. 5. 6. 1850). - Abgedr. in: F. W. Nottebohm, Chronik d. Kgl. Gewerbe-Akad. 1871 [Nr 318] S. 27 ff. Be KglB

231. Bestimmungen für die Zöglinge des Kgl. Gewerbe-Instituts (v. 15. 12. 1858). Be

232. Regulativ für die Organisation des Kgl. Gewerbe-Instituts (v. 23. 8. 1860). [Folgt:] Spezielle Vorschriften für die Studirenden des $\mathrm{Kgl}$. Gewerbe-Instituts, bezüglich der Aufnahme, der Honorar-Zahlungen, der Repetitionen und der Ausstellung der Zeugnisse v. 18.9. 1860 [u.] Ha us-Ordnung für das Kgl. Gewerbe-Institut v. 18.9. 1860. 11 S. Abgedr. in: Lections-Plan f. 1861/62-1864 [Nr 304] u. in: F. W. Nottebohm, Chronik d. Kgl, Gewerbe-Akad. 1871 [Nr 318]. S. 38 ff.

$B e^{*}$

[Dasselbe.] (Mit Nachtrag zu $\$ 9$ v. 31. 3. 1866.) $12 \mathrm{~S}$. Be Minist.d.geistl. Angel. [Techn. H. Berlin I,16]

233. Verfassungs-Statut der $\mathrm{Kgl}$. Gewerbe-Akademie zu Berlin. 〈Vom 1. 11. 1871). - [Dasselbe.] (Mit Nachtrag v. 26. 9. 1877.) - Abgedr. in: Programm f. $1875 / 76$ [dort versehentlich als v. 1. 11. 1851 bezeichnet]. 1876/77 [Nr 304]; Damm 1909. S. 24 ff.

Ausg. mit Nachtr.: Be

234. Ordnung der Diplom-Prüfungen an der Kgl. Gewerbe-Akademie (v. 20. 5. 1873). - Abgedr. in: Programm f. 1877/78. 1878/79 [Nr 304]; Verhandlungen d. Vereins zur Beförderung d. Gewerbfleißes in Preußen. Jg. 53. 1874. Be Minist. d. geistl. Angel.

235. Regulatir für die Verwaltung der Kgl. Gewerbe-Akademie zu Berlin (v. 2. 4. 1874).

$B e^{*}$

236. Verzeiehniß der Unterrichts-Gegenstände für die Diplom-Vorprüfung (an der Kgl. Gewerbe-Akademie zu Berlin v. 26. 6. 1875). Be Minist. d. geistl. Angel.

237. Verzeichniß der Unterrichts-Gegenstände für die Diplom-Hauptprüfung an der Kgl. Gewerbe-Akademie zu Berlin (v. 26. 6. 1875).

Be Minist. d. geistl. Angel.

238. Bestimmungen über die Benutzung der Bibliothek der Kgl. Gewerbe-Akademie (v. 12. 12. 1878).

$B e^{*}$

\section{TECHNISCHE HOCHSCHULE}

\section{ALLGEMEINES UND VERMISCHTES}

239. Provisorisches Verfassungs-Statut der Kgl. Technischen Hochschule zu Berlin (v. 17. 3. 1879). T Abgedr. in: Centralblatt f. d. ges. Unterrichts-Verwaltung in Preußen. Jg. 1879. S. 251 ff. ; Programm f. 1879/80 [Nr 305]. S. 91 ff.; Entwurf abgedr. in: Denkschrift über das technische Unterrichtswesen [verf. im] Ministerium f. Handel, Gewerbe u. öffentl. Arbeiten. (Sammlung sämtl. Drucksachen des Hauses d. Abgeordneten aus d. 13. Legislaturperiode. 3. Session. 1878/79. Bd 2, Nr 65; auch einzeln.)

$B e^{*}$

240. Verfahren bei der Immatrikulation und bei der Annahme der Vorlesungen an der Kgl. Technischen Hochschule zu Berlin (v. 28. 3. 1879). 
241. Vorsehriften für die Studierenden der Kgl. Technischen Hochschule zu Berlin (v. 3. 3. 1880). 1880. - [Dasselbe] (v. 3. 3. $1880,15.2 .1886$ u. 16. 7. 1888; dat. 28. 3. 1890). - [Neudr. mit Anm. zu $\$ 10$. - [Neudr. mit berichtigtem $\S 10$.$] - [Das-$ selbe] (v. 3. 3. $1880 \ldots$ u. 12. 11. 1906; dat. 14. 11. 1906). ' Abgedr. in: Damm 1909. S. $73 \mathrm{ff}$ Die beiden ersten Ausg.: Be*

242. Bestimmungen betr. Erlass des Honorars, sowie Verleihung von Stipendien und Unterstützungen an der $\mathrm{Kgl}$. Technischen Hochschule zu Berlin (v. 4. 3. 1881, 4. 2. $1887,24.7 .1891$ u. 9.6. 1893). - [Dasselbe] (v. 4. 3. $1881 \ldots$ u. 14. 10. 1898). [Dasselbe] (v. 4. 3. 1881 ... u. 18. 3. 1904). 1. u. 2. $A$ usg.: Be*

243. Urlaubs-0rdnung für die Professoren und Dozenten der Technischen Hochschule zu Berlin (v. 28. 4. 1882). - Abgedr. in: Damm 1909. S. $64 \mathrm{ff}$.

244. Verfassungs-Statut der $\mathrm{Kgl}$. Technischen Hochschule zu Berlin (auf Grund des Allerhöchsten Erlasses v. 28. 7. 1882; ausgefertigt 22. 8. 1882). - [Dasselbe.] (Mit Änderungen v. 11. 6. 1894.) - [Dasselbe.] (Mit Änderungen v. 11. 6. 1894 u. 5. 7. 1905.) - [Dasselbe.] (Mit Änderungen v. 11. 6. 1894, 5. 7. 1905 u. 2. 12. 1906.) - [Dasselbe.] (Mit Änderungen v. 11. 6. 1894 ..., 21. 2. 1910 u. 14. 9. 1911. 1911.) - Abgedr. in: Centralblatt f. d. ges. Unterrichts-Verwaltung in PreuBen. Jg. 1883. S. $228 \mathrm{ff}$; Damm 1909. S. $40 \mathrm{ff}$.

245. Entwurf von Bestimmungen über die Zulassung, die Rechte und Pflichten und die Ausschliessung von Privatdozenten an der technischen Hochschule. [1883.]

Be Minist. d. geistl. Angel.

246. Habilitations-0rdnung für die $\mathrm{Kgl}$. Technische Hochschule zu Berlin (v. 24. 4. 1884). - [Dasselbe.] (Mit Zusätzen zu $\S 3$ u. 12 v. 27. 7. 1892.) - [Dasselbe.] (Mit Abänderung der $\$ 18$ u. 19 v. 3. 12. 1908.) - Abgedr. in: Centralblatt f. d. ges. Unterrichts. Verwaltung in PreuBen. Jg. 1885. S. 603 ff.; Damm 1909. S. $66 \mathrm{ff}$.

1884: $\mathrm{Be}$

247. Statuten der Studierenden der Kgl. Technischen Hochschule zu Berlin (v. 9.1. 1885).

$B e^{*}$

248. Bestimmungen für Ertheilung von Preisaufgaben an Studirende der Kgl. Technischen Hochschule zu Berlin (v.13.4.1886). Be

249. Statuten der Studierenden der Kgl. Technischen Hochschule zu Berlin (v. 1. 2 . 1887).
250. Bestimmungen über die Benutzung der Bibliothek der $\mathrm{Kgl}$. Technischen Hochschule zu Berlin (v. 15.6. 1887. Nebst Deckbl. zu § 1 v. 4. 4. 1895). - [Dasselbe] (v. 15. 6. 1887 u. 24. 11. 1903). T Abgedr. in: Damm 1909. S. 85 ff.

251. Dienstanweisung für die Saaldiener der $\mathrm{Kgl}$. Technischen Hochschule zu Berlin (v. 30.6. 1888).

252. Bestimmungen über die alljährlich für die Studirenden der Kgl. Technischen Hochschule zu Berlin $\mathrm{zu}$ stellenden Preisaufgaben (v. 12.7. 1889).

253. Verzeichniss der Stipendien und sonstigen Vergünstigungen, welche den Studirenden der Kgl. Technischen Hochschule zu Berlin zu Theil werden können. [Um 1890.] - [Dasselbe berichtigt u. ergänzt.] (1911.)

254. Satzungen des Kranken-Vereins der Studierenden der Kgl. Technischen Hochschule zu Berlin (v. 1. 10. 1893). Be

255. [Dasselbe] (v. 1. 10. 1894). [Nebst] Nachtraegliche Aenderungen (v. 1. 4. 1895).

$B e^{*}$

256. Satzungen der Studierenden der $\mathrm{Kgl}$. Technischen Hochschule zu Berlin (v. 6. 10. 1893).

$B e^{*}$

257. Bestimmungen über die alljährlich für die Studirenden der $\mathrm{Kgl}$. Technischen Hochschule zu Berlin zu stellenden Preisaufgaben (v. 4. 5. 1894).

258. Regulativ über die Organisation der Abtheilungen an der Kgl. Technischen Hochschule zu Berlin (v. 23. 11. 1894). - [Dasselbe.] (Mit Zusatz zu $\& 6$ v. 16.5. 1896.) - Abgedr. in: Damm 1909. S. 62 ff.

259. Satzungen der Studierenden der $\mathrm{Kgl}$. Technischen Hochschule zu Berlin (v. 28. 2. 1896).

$B e^{*}$

260. Satzungen der Vereinigung der Studierenden (v. 23. 6. 1899) und des Krankenvereins der Studierenden der Technischen Hochschule zu Berlin (v. 14. 7. 1899). Be

261. Dienstanweisung für die Saaldiener der Kgl. Technischen Hochschule zu Berlin (v. 4. 8. 1900).

262. Satzungen für die Hilfskasse der $\mathrm{Kgl}$ Technischen Hochschule zu Berlin (v. 27. 10. 1900). TAbgedr. in: Damm 1909. S. 89 ff.

263. Satzungen der Vereinigung der Studierenden, des Kranken-Vereins der Studierenden und des Bücher-Aussehusses der Kgl. Technischen Hochschule zu Berlin (v. 14. 3. 1902). 
264. Vorsehriften über die Benutzung der Anschlagstafeln und Säulen in den Gebäuden der Technischen Hochschule zu Berlin < v. 17. 7. 1903).

264a. Satzungen der Akademischen Kranken-Kasse der Kgl. Technischen Hochschule zu Berlin. [Entwurf.] - [Dasselbe] v. 21. 6. 1904. 1904. - Abgedr. in: Damm 1909. S. 95 ff.

264b. Satzungen der Vereinigung der Studierenden und des Bücher-Ausschusses der Kgl. Technischen Hochschule zu Berlin (v. 15. 7. 1904).

264c. Bestimmungen über die Behandlung der Anzeigen von Mitgliedern des Lehrkörpers über Unterrichtsausfall (v. 10. 2. 1905).

264d. Bestimmungen über die alljährlich für die Studierenden der Kgl. Technischen Hochschule zu Berlin zu stellenden Preisaufgaben (v. 9.4. 1907). - [Neudr.] (1911.) - Abgedr. in: Damm 1909. S.284f. 1. Ausg.: Be

264e. Verwaltungsordnung für die Bibliothek der Kgl. Technischen Hochschule zu Berlin. Vom 15. 4. 1910.

\section{PRÜFUNGS ORDNUNGEN}

265. Diplomprüfungs-0rdnung für die Abtheilung III. der $\mathrm{Kgl}$. Technischen Hochschule zu Berlin [v. 31. 5. 1880].

266. Vorsehriften über die Diplom-Prüfung für die Abtheilungen II, III u. IV der Kgl. Technischen Hochschule zu Berlin (v. 21. 12. 1887). 1888. - [Neudr.] 1892. 1894. - Abgedr. in: Centralblatt f. d. ges. UnterrichtsVerwaltung in Preußen. Jg. 1888. S. $176 \mathrm{ff}$. 1892. 1894: $B e^{*}$

267. Vorsehriften über die Diplom-Prüfung für die Abtheilungen II, III, IV u. V der Kgl. Technischen Hochschule zu Berlin (v. 21. 12. 1887). 1894. - [Neudr.] 1897. - Abgedr. in: Damm 1899. S. $111 \mathrm{ff}$.

1894: $\mathrm{Be} ; 1897: \mathrm{Be}^{*}$

268. Gesehäfts-0rdnung für die DiplomPrüfungs-Kommissionen der Abtheilungen II, III und IV der Kgl. Technischen Hochschule zu Berlin (v. 24. 3. 1888). 1888.

\section{Abteilung für Architektur}

269. Diplomprüfungs-0rdnung für die Abtheilung für Architektur der Kgl. Technischen Hochschule zu Berlin. Durch Erlass v. 16. 6. 1902 in Kraft gesetzt. - [Dasselbe.] Durch Erlass v. 4. 7. 1902 in Kraft gesetzt. - Abgedr. in: Bestimmungen. S. $83 \mathrm{ff}$.
270. Geschäfts-0rdnung für die Diplomprüfungen der Abteilung für Architektur der Kgl. Technischen Hochschule zu Berlin. Vom 23. 1. 1904.

271. Diplomprüfungs-0rdnung für die Abteilung für Architektur der Kgl. Technischen Hochschule zu Berlin. Vom 11. 4. 1907.

272. [Dasselbe.] Vom 18. 1. 1909.

273. Geschäfts-0rdnung für die Diplomprüfungen der Abteilung für Architektur der Kgl. Technischen Hochschule zu Berlin. Vom 18. 1. 1909.

\section{Abteilung für Bau-Ingenieurwesen}

274. Diplomprüfungs-0rdnung für die Abtheilung für Bau-Ingenieurwesen der Kgl. Technischen Hochschule zu Berlin. - [Dasselbe.] Mit Abänderungen v. 29. 11.1901. Be*

275. Entwurf vom März 1902. Diplomprüfungs-0rdnung für die Abtheilung für Bau-Ingenieurwesen der $\mathrm{Kgl}$. Technischen Hochschule zu Berlin.

$B e^{*}$

276. Diplom-Prüfungs-0rdnung nebst Uebergangs-Bestimmungen der Abtheilung für Bau-Ingenieurwesen der Kgl. Technischen Hochschule zu Berlin. Vom 4. 7. 1902. (Nebst Deckbl. zu $\S 6$ u. zu § 14 v. 17. 5. 03.) - [Dasselbe mit den entsprechenden Änderungen.] - Abgedr. in: Bestimmungen. S. $91 \mathrm{ff}$.

277. Gesehäfts-0rdnung für die Diplomprüfungen der Abteilung für Bau-Ingenieurwesen der $\mathrm{Kgl}$. Technischen Hochschule zu Berlin. Vom 23. 1. 1904. - [Dasselbe.] Vom 23. 1. 1904 u. 4. 8. 1910.

278. Diplom-Prüfungs-0rdnung der $\mathrm{Ab}$ teilung für Bau-Ingenieurwesen der $\mathrm{Kgl}$. Technischen Hochschule zu Berlin. Vom 2. 6. 1908 .

\section{Abteilung für Maschinen-Ingenieur-} wesen

279. Diplomprüfungs-0rdnung. Abtheilung für Maschinen-Ingenieurwesen der Kgl. Technischen Hochschule zu Berlin. [1. Entwurf. Um 1900.]

$\mathrm{Be}$

280. Diplomprüfungs - Ordnung nebst Uebergangs-Bestimmungen der Abtheilung für Maschinen-Ingenieurwesen der Kgl. Technischen Hochschule zu Berlin. [2. Entwurf. 1900.] 
281. Uebergangs-Vorsehriften über die Diplom-Prüfung der Abtheilung für Maschinen-Ingenieurwesen der $\mathrm{Kgl}$. Technischen Hochschule zu Berlin. 1900. Aus: Diplomprüfungs-Ordnung nebst Uebergangs-Bestimmungen [Nr 280].

$\mathrm{Be}$

282. Diplomprüfungs - Ordnung nebst Ubergangs-Bestimmungen der Abteilung für Maschinen-Ingenieurwesen der Kgl. Technischen Hochschule zu Berlin. Vom 4. 7. 1902.

283. Geschäftsordnung für die Diplomprüfungen der Abteilung für Maschinen-Ingenieurwesen der Kgl. Technischen Hochschule zu Berlin. Vom 23. 1. 1904.

284. Diplomprüfungs - Ordnung nebst Uebergangs-Bestimmungen der Abteilung für Maschinen-Ingenieurwesen der $\mathrm{Kgl}$. Technischen Hochschule zu Berlin. Vom 27. 1. 1904. (Nebst hs. Änderungen zu $\S 6$ u. 14 v. 23. 11. 1905.) - Abgedr. in: Bestimmungen. S. $104 \mathrm{ff}$.

285. Diplomprüifungs-0rdnung der $\mathrm{Ab}$ teilung für Maschinen-Ingenieurwesen der Kgl. Technischen Hochschule zu Berlin. Vom 23. 11. 1905.

286. [Dasselbe.] Vom 3. 2. 1909.

287. [Dasselbe.] Vom 4. 5. 1912.

288. Gesehäftsordnung für die Diplomprüfungen der Abteilung für Maschinen-Ingenieurwesen der $\mathrm{Kgl}$. Technischen Hochschule zu Berlin. Vom 4.5. 1912.

\section{Abteilung für Schiff- und Schiffs- maschinen-Bau}

289. Diplomprüfungs- Ordnung für die $\mathrm{Ab}$ theilung für Schiff- $u$. Schiffsmaschinenbau der Kgl. Technischen Hochschule zu Berlin.
Vom 4. 7. 1902. [Folgt:] UebergangsVorschriften. Abgedr. in: Bestimmungen. S. $121 \mathrm{ff}$.

290. Diplomprüifungs - Ordnung nebst Übergangsbestimmungen der Abteilung für Schiff- und Schiffsmaschinen-Bau der Kgl. Technischen Hochschule zu Berlin. Vom 17. 12. 1904.

291. Geschäftsordnung für die Diplomprüfungen der Abteilung für Schiff- $u$. Schiffsmaschinen-Bau der Kgl. Technischen Hochschule zu Berlin. Vom 17. 12. 1904.

292. Diplomprüfungs-0rdnung der $\mathrm{Ab}$ teilung für Schiff- und Schiffsmaschinen-Bau der Kgl. Technischen Hochschule zu Berlin. Vom 16. 9. 1907. (Nebst Deekbl. zu § 11 u. 15 v. 5. 11. 1909.)

293. [Dasselbe.] Vom 4.3. 1912.

Abteilung für Chemie und Hüttenkunde

294. Diplomprüfungs-0rdnung der $\mathrm{Ab}$ teilung für Chemie und Hüttenkunde der Kgl. Technischen Hochschule zu Berlin. Vom 4.7.1902. - Abgedr. in: Bestimmungen. S. $141 \mathrm{ff}$.

295. [Dasselbe.] Vom 22. 6. 1904.

296. Geschäftsordnung für die Diplomprüfungen der Abteilung für Chemie und Hüttenkunde der Kgl. Technischen Hochschule zu Berlin. Vom 22. 6. 1904.

297. Diplomprüfungs-0rdnung derAbteilung für Chemie und Hüttenkunde der $\mathrm{Kgl}$. Technischen Hochschule zu Berlin. Vom 4. 7. 1908.

298. [Dasselbe.] Vom 20. 1. 1912.

\section{PERIODISCHE SCHRIFTEN}

\section{BAUAKADEMIE}

299. VerzeiehniB und Ordnung der Vorlesungen bei der $\mathrm{Kgl}$. Bau-Akademie zu Berlin vom 1. Okt. 1799 bis 1. April 1800; vom 1. April bis 1. Okt. 1800, also für die erste Hälfte des Unterrichts-Jahres 1800/1801 [usw.] - vom 1. Okt. 1807 bis Ende März 1808 .

Außer dem nicht nachweisbaren Verzeichnis $v$. 1. 4. bis 1.10.1802: Be Minist.d.geistl. Angel. [IV, 15]

300. Anordnung des Unterrichts, welcher in der Kgl. Bau-Akademie ... ertheilt wird.
Vom 1. Okt. 1830 bis Ende Febr. 1831; vom 1. April bis Ende Aug. 1831.

Be Minist. d. geistl. Angel. [III, 11]

301. Verzeichniß der Unterrichts-Gegenstände, welche auf der Kgl. Bau-Akademie zu Berlin gelehrt werden. W.-H. v. Michaelis 1849 bis Ostern 1850. S.-H. v. Ostern bis Michaelis 1850 . W.-H. v. Michaelis 1850 bis Ostern 1851.

Fortges. u. d. T.:

Verzeichniß der Unterrichts-Gegenstände der $\mathrm{Kgl}$. Bau-Academie zu Berlin für das S.-H. 1851. W.-H. 1851/2 [usw.]-S.-H. 1876. 
Fortges. u. d. T.:

Programm der Kgl. Bau-Akademie zu Berlin für das Jahr 1876-77. 1877-78. 1878-79. $1849 / 50-1851$. 1861. 1862. 1866/67. 1867. 1868/69-1871/72. 1872/73-1875. 1876. 1876/77. 1877/78: $\mathrm{Be} ; 1851 / 52$ - 1860/61. 1861/62. 1862/63 -1866. 1867/68. 1868. 1872. 1878/79: $\mathrm{Be}^{*}$

302. Stundenplan der Kgl. Bau-Akademie zu Berlin pro W.-S. 1876-77; pro S.-S. 1877; für das Jahr 1877-78; für das Studienhalbj. 1878-79.

1876/77. 1877: $\mathrm{Be} ;$ 1877/78. 1878/79: $\mathrm{Be}^{*}$

303. Special-Programme für die Vorlesungen und Uebungen an der $\mathrm{Kgl}$. BauAkademie zu Berlin. Studienj. 1878-1879. Nach den Angaben der Herren Docenten auf Beschluss des Lehrer-Collegiums als Internum der Kgl. Bau-Akademie gedr.

\section{GEWERBEAKADEMIE}

304. Lections-Plan für das Kgl. GewerbeInstitut (1866/67 ff.: die Kgl. Gewerbe-Akademie) zu Berlin. W.-S. 1858/59. S.-S. 1859 [usw.]-S.-S. 1867.

Fortges. u. d. T.:

Verzeichniß der Unterrichts-Gegenstände der Kgl. Gewerbe-Akademie zu Berlin für das W.-S. $1867 / 68$. S.-S. 1868 [usw.]-S.-S. 1875.

Fortges. u. d. T.:

Programm der Kgl. Gewerbe-Akademie zu Berlin für das Studienj. 1875/76-1878/79.

$1859-1860 / 61$. (Folgt: Lections-Plan für die zum Kgl. Gewerbe-Institut gehörige MusterzeichnenSchule $1859-1860 / 61$.)

1858/59-1860. 1861/62. 1865/66. 1868/69. 1869/70-1878/79: Be; 1860/61. 1862-1865. $1866-1868$. 1869: Be $; 1861: K$

\section{TECHNISCHE HOCHSCHULE}

305. Kgl.Technische Hochschule zu Berlin Programm für das Studienj. 1879-80-19121913.

1883/84. 1893/94: $\mathrm{Be}$
306. Special-Programme der für die Hörer der Abtheilung für Bau-Ingenieurwesen bestimmten Kollegien der Kgl. Technischen Hochschule zu Berlin. Studienj. 1880-1881. Nach den Angaben der Herren Docenten auf Beschluss des Kollegiums der II. Abtheilung gedr. 1880.

307. Statistik der Kgl. Technischen Hochschule zu Berlin pro (1890 ff.: für das) W.-S. 1882-83. S.-S. 1883 [usw.]-S.-S. 1900. - 1881/82 u. 1882 abgedr. in Centralblatt d. Bauverwaltung. Jg. 1. 1881. S. 330. Jg. 2. 1882. S. 184 u. in: Deutsche Bauzeitung. Jg. 15. 16, 1881. 82; $1900 / 01 \mathrm{ff}$. Statistische Uebersichten der Studirenden in: Personal-Verzeichniss f. 1900-01 ff. [Nr 310.] $1883 / 84$ 1884. 1885/86. 1890/91. 1892. 1895/96: Be

308. Preis-Aufgaben für die Studirenden der Kgl. Technischen Hochschule zu Berlin pro 1886-87. [1887/88. 1888/89 in: Urtheile über die Bearbeitungen ... der 1886 bzw. 1887 gestellten Preisaufgaben. Nr 309.] Fortges. u. d. T.:

Kgl. Technische Hochschule zu Berlin. Preisaufgaben für das Jahr 1889-90 - 1912-13. 1889/90-1894/95. 1898/99. 1900/01: Be; 1896/97. 1902/03-1904/05: Be*

309. Kgl. Technische Hochschule zu Berlin. Urtheile über die Bearbeitungen, welche zur Lösung der im Jahre... gestellten Preisaufgaben eingereicht worden sind (1886: und Anzeige der neuen Preisaufgaben für das Jahr 1887-88; 1887: und Preisaufgaben für das Jahr 1888-89). 1886-1911. 1886. 1892: Be; 1899: $\mathrm{Be}^{*}$

310. Personal-Verzeichniss der Kgl. Technischen Hochschule zu Berlin fürs W.-H. 1900-1901. S.-H. 1901 [usw.]-W.-H. 1912/13. $1907 / 08$ ff. [Nebst Verzeichnis der] HonorarA ssistenten für das [W.-H. 1907/08. S.-H. 1908 usw.]-W.-H. 1912/13.

311. Kgl. Technische Hochschule zu Berlin. Bericht über die Kgl. Technische Hochschule zu Berlin für das Rektoratsjahr vom 1. Juli ... bis zum 30. Juni .... 1910/11. 1911/12. [Frühere Berichte im Programm. Nr 305.]

\section{GELEGENHEITS- UND VERMISCHTE SCHRIFTEN}

\section{BAUAKADEMIE}

312. VerzeichniB der in der Bibliothek der Königl. Ober-Baudeputation vorhandenen Werke. Berlin, im Dez. 1848. 153 S. $8^{\circ}$
313. VerzeichniB der in der vereinigten Bibliothek der Königlich Technischen BauDeputation und der Königlichen Bau-Akademie vorhandenen Werke. Berlin, im März 1858. 1858. VIII, 196 S. $8^{\circ}$ 
314. Verzeichniß der Dozenten, Verwaltungs-Beamten und der Studirenden der Königlichen Bau-Akademie zu Berlin. Nach amtl. Quellen zsgest. u. hrsg. vom Ausschuß der Studirenden der Kgl. Bau-Akademie. März 1876. 49 S. $8^{\circ}$

Be

\section{GEWERBEAKADEMIE}

315. Verzeichniß der im Gewerbehause aufgestellten Königlichen Bibliothek. Berlin, im Jan. 1843. 266 S. $8^{\circ}$

$\mathrm{Be}$

316. Katalog der Bibliothek des Königlichen Gewerbe-Instituts zu Berlin. 1866. VII, $371 \mathrm{~S} .8^{\circ}$

317. I. Nachtrag zum Katalog der Bibliothek der Königlichen Gewerbe-Akademie zu Berlin. 1867. VII, 28 S. $8^{\circ}$ $\mathrm{Be}$

318. Chronik der Königlichen GewerbeAkademie zu Berlin. Festschrift zur Feier des fünfzigjährigen Bestehens der Anstalt. Auf Veranlassung des Herrn Ministers f. Handel, Gewerbe u. öff. Arbeiten Grafen von Itzenplitz bearb. von F[riedrich] W[ilhelm] Nottebohm, vortragendem Rath im Minist. f. Handel, Gewerbe u. öff. Arbeiten. 1871. 83 S., 2 Taf. $4^{\circ}$

319. Verzeichniss der Sammlung von Gipsabgüssen der Königlichen GewerbeAkademie. 1. 2. u. 3. Abth. 1872. 44 S. $8^{\circ}$

320. Erklärendes Verzeichniss der Sammlung von Gipsabgüssen, Bronzen und Majoliken der Königl. Gewerbe-A kademie. (Vorr.: Albert Grell.) 1875. 124 S. $8^{\circ}$

\section{TECHNISCHE HOCHSCHULE}

\section{REDEN ZUM GEBURTSFESTE DES KAISERS}

Im Jahre 1888 fand keine Kaiser-Geburtstagsfeier statt; die im Jahre 1901 gehaltene Rede s. Nr 365 .

321. Hauek, Guido: Die Grenzen zwischen Malerei und Plastik und die Gesetze des Reliefs. 1885. $20 \mathrm{~S} .4^{\circ}$ - Mit einigen Anderungen in: PreuB. Jahrbücher. Bd 56.

$B e$

322. Dobbert, Eduard: Die Kunstgeschichte als Wissenschaft und Lehrgegenstand. 1886. 16 S. $4^{\circ}$ - Abgedr. in: E. Dobbert: Reden u. Aufsätze kunstgeschichtl. Inhalts. 1900.

323. Rüdorff, Friedrieh: Die Fortschritte der Chemie in den letzten fünfundzwanzig Jahren. 1887. $16 \mathrm{~S} .4^{\circ}$
324. Schliehting, Julius: Die Aufgaben der Hydrotechnik. 1889. 24 S. $4^{0} \quad B e$

325. Jacobsthal, E[duard]: Rückblicke auf die baukünstlerischen Prinzipien Schinkels und Böttichers. 1890. 20 S. $4^{0}$

326. Reuleaux, F[ranz]: Deutschlands Leistungen und Aussichten auf technischem Gebiete. 1891. 14 S. $4^{0}$

327. Doergens, R[ichard]: Ueber die Ursachen der heutigen socialen Mißstände. 1892. 22 S. $4^{0}$

328. Lampe, E[mil]: Die Entwickelung der Mathematik im Zusammenhange mit der Ausbreitung der Kultur. 1893. 26 S. $4^{\circ}$ I Abgedr. in: Zeitsehrift f. math. u. naturwiss. Unterricht. Jg. 24.

329. Rietschel, Hermann: Der Stand der wissenschaftlichen und praktischen Wohnungs-Hygiene in Beziehung zur Luft. 1894. 19 S. $4^{0}$ - Abgedr. in: Gesundheits-Ingenieur. Jg. 17.

330. Slaby, A[dolf]: Das Gesetz von der Erhaltung der Energie und seine Bedeutung für die Technik. 1895. $13 \mathrm{~S} .4^{0}$ ๆ Abgedr. in: Zeitschrift d. Vereines deutscher Ingenieure. Bd 39.

331. Müller-Breslau, Heinrich: Vom Kriege hinter der Front 1870-71. 1896. $24 \mathrm{~S} .4^{\circ}$ i Etwas geändert u. mit Abb, versehen abgedr. in: Centralblatt d. Bauverwaltung. Jg. 16.

332. Hauck, Guido: Ueber innere Anschauung und bildliches Denken. 1897. $20 \mathrm{~S} .4^{0}$

333. Witt, 0tto N[ikolaus]: Die Lebensbedingungen der modernen chemischen Industrie. 1898. $16 \mathrm{~S} .4^{0}$

334. Goering, A[dolf]: Ueber die verschiedenen Formen und Zwecke des Eisenbahnwesens. 1899. 21 S. $4^{0}$

335. Riedler, A[lois]: Ueber die geschichtliche und zukünftige Bedeutung der Technik. [1900.] $16 \mathrm{~S} .4^{0}$ / Abgedr. in: Die Hundertjahrfeier d. Kgl. Techn. Hochschule zu Berlin. 1900 [Nr 362.]

336. Bubendey, [Johann Friedrich]: Die Grenzen der Seeschiffahrt. 1902. 20 S. $4^{\circ}$

337. Kammerer, [0tto]: Ist die Unfreiheit unserer Kultur eine Folge der Ingenieurkunst? [1903.] $14 \mathrm{~S} .4^{0}$ - A Abgedr. in: Zeitschrift d. Vereines deutscher Ingenieure. Bd 47, Halbj. 1.

338. Hettner, G[eorg]: Alte mathematische Probleme und ihre Klärung im neunzehnten Jahrhundert. [1904.] $18 \mathrm{~S} .4^{\circ}$ 
339. Miethe, [Adolf]: Die geschichtliche Entwicklung der farbigen Photographie. [1905.] $15 \mathrm{~S} .4^{\circ}$

340. Flamm, [0swald]: Wissenschaftliches Arbeiten auf schiffbautechnischen Gebieten. [1906.] 17 S. $4^{\circ}$

341. Grantz, [Max]: Kulturelle Bedeutung der Wasserwirtschaft und Entwicklung der Wasserwirtschaft in Preussen. [1907.] $19 \mathrm{~S} .4^{\circ}$ - Abgedr. in: Baugewerks-Zeitung. Jg. 39.

342. Kammerer, [0tto]: Werkzeug und Arbeitsteilung. [1908.] 16 S. $4^{\circ}$ - Abgedr. in: Zeitschrift d. Vereines deutscher Ingenieure. Bd 52, Halbj. 1.

343. Borrmann, R[iehard]: Die Bauschule von Berlin. [1909.] 18 S. $4^{\circ}$

344. Mathesius, W[alter]: Die Entwicklung der Eisenindustrie in Deutschland. [1910.] 24 S. $4^{\circ}$ - Aus: Stahl u. Eisen. Jg. 30.

345. Witt,otto N[ikolaus]: Rückblicke und Ausblicke auf dem Gebiete der technischen Chemie. 1911. $17 \mathrm{~S} .4^{\circ}$ Abgedr. in: Die chem. Industrie. Jg. 34.

346. Seheffers, G[eorg]: Allgemeine Bildung in Vergangenheit und Gegenwart. 1912. $18 \mathrm{~S} .4^{\circ}$

\section{REDEN UND SCHRIFTEN BEI VER- SCHIEDENEN ANLÄSSEN}

347. Königliche Technische Hochschule zu Berlin. Verhandlungen der Versammlung von Delegirten der Deutschen Technischen Hochschulen am 31. März, 1. und 2. April 1880 zu Berlin. Nach stenogr. Aufzeichnungen zsgest. $46 \mathrm{~S}$. $8^{\circ}$

348. Gedäehtniss-Feier der Königlichen Technischen Hochschule für die hundertjährige Wiederkehr des Geburtstages von Peter Christian Wilhelm Beuth am 21. Januar 1882 . $11 \mathrm{~S}$. $8^{\circ}$

349. Festsehrift der Königlichen Technischen Hochschule zu Berlin zur Feier der Einweihung ihres neuen Gebäudes am 2. November 1884. 1884. VIIC, 337 S., 14 Taf. $4^{\circ}$

Enth.: E[duard] Dobbert: Bau-Akademie, Gewerbe-Akademie und Technische Hochschule zu Berlin. [Auch einzeln ersch.; 2. Aufl. s. Nr 359.] Julius] Weingarten: Über die Theorie der aufeinander abwickelbaren Oberflächen. [Als S.-A. bei Calvary in Berlin.] - H[ermann] W[ilhelm] Vogel: Über die Photographie farbiger Gegenstände in den richtigen Tonverhältnissen. [Abgedr. in: Vogel, die
Photographie farbiger Gegenstände. Berlin 1885. S. 1-21.] - H[ein rich] Weber: Zur Theorie der Modular-Gleichungen. - Julius Lessing: Die kunstgewerblichen Alterthümer im Beuth-SchinkelMuseum zu Berlin. - E[mil] Winkler: Über die Belastungs-Gleichwerthe der Brückenträger. Ein Beitrag zur Theorie der Brücken. - C [arl] Fink: Zur Theorie der Gaskraftmaschinen. - E[mil] Dietrich: Die Gewinnung der Baumaterialien für die Steinstrassen. - Friedrich Adler: Der Ursprung des Backsteinbaues in den baltischen Ländern. - Guido Hauck: Mein perspectivischer Apparat. - Christian Havestadt: Über das Verhalten der Tidewelle in Flussmündungen und Meeresbuchten. - A[lfred] Dietrich: Einiges über die Konstruktion von Kreuzerschiffen. $\mathrm{E}$ [duard] Jacobsthal: Araceenformen in der Flora des Ornaments. [Ohne die farbigen Taf., aber durch einige Holzschn. u. in d. Anm. durch einige Zusätze verm. in: Der Zeichenlehrer. Jg.1. 1888/89; daraus auch als S.-A.] - Julius Weeren: Beiträge zur Kenntnis der Constitution des Eisens.

350. Rede bei der Feier des 25jährigen Regierungsjubiläums Seiner Majestät des Kaisers und Königs in der Aula der Königlichen Technischen Hochschule zu Berlin am 4. Januar 1886 geh. von dem zeitigen Rektor Eduard Dobbert. 1886. 16 S. $4^{\circ}$ - Abgedr. in: E. Dobbert: Reden u. Aufsätze kunstgeschichtl. Inhalts. 1900. S. 1 ff.

351. Rede bei der Gedächtniß-Feier für weiland Seine Majestät den in Gott ruhenden Kaiser und König Wilhelm in der Aula der Königlichen Technischen Hochschule zu Berlin am 22. März 1888 von dem zeitigen Rektor Georg Meyer. 1888. 15 S. $4^{\circ}$

352. Rede bei der Gedächtniß-Feier für weiland Seine Majestät den in Gott ruhenden Kaiser und König Friedrich in der Aula der Königlichen Technischen Hochschule zu Berlin am 30. Juni 1888 geh. von dem zeitigen Rektor Georg Meyer. 1888, 12 S. $4^{\circ}$

353. Feier zur Enthüllung der Büsten der verstorbenen Professoren Hermann Spielberg und Dr. Emil Winkler in der Halle der Königlichen Technischen Hochschule zu Berlin am 11. März1891. Ansprachen zum Gedächtniss der beiden Verblichenen geh. von den Prof. Fritz Wolff u. A[dolf] Goering. $1891.17 \mathrm{~S} .8^{\circ}$

354. Feier zur Uebergabe der Büste des verstorbenen Professors Dr. Karl Boetticher in der Halle der Königlichen Technischen Hochschule zu Berlin am 30. November 1894. Ansprachen geh.von Prof. [Eduard] Jacobsthal (Über Boettichers Leistungen für die Baukunst) und Prof. [Adolf] Slaby. 1894. $17 \mathrm{~S} .8^{\circ}$ 
355. Rede bei der Feier des 25jährigen Gedenktages der Proklamirung des Deutschen Reiches am 18. Januar 1896 in der Aula der KöniglichenTechnischen Hochschule zu Berlin geh. von E[mil] Lampe. 1896. $25 \mathrm{~S} .4^{\circ}$

356. Gedächtnißrede zur Feier des 100. Geburtstages Seiner Majestät des hochseligen Kaisers Wilhelms des Großen geh. in der Königlichen Technischen Hochschule zu Berlin von Hermann Rietsehel, Prof. 1897. $17 \mathrm{~S} .4^{\circ}$

357. Rede bei der Gedenkfeier für Seine Durchlaucht den verewigten Reichskanzler Fürsten von Bismarck am 9. März 1899 in der Aula der Königlichen Technischen Hochschule zu Berlin geh. von dem z. Prorektor Otto N[ikolaus] Witt. $1899.12 \mathrm{~S} .4^{\circ}$

358. Die Technischen Hochschulen und ihre wissenschaftlichen Bestrebungen. Red€ zum Antritt des Rektorates der K gl. Technischen Hochschule zu Berlin geh. in dor Aula am 1. Juli 1899 von $\mathbf{A}\left[\right.$ lois] Riedler. 1899. 17 S. $4^{\circ}$ - Abgedr. in: Zeitschrift d. Vereines dentscher Ingenieure. Bd 43.

359. Chronik der Königlichen Technischen Hochschule zu Berlin 1799-1899. Berlin: Ernst 1899. 268 S., 11 Taf. $4^{\circ}$

Enth.: Eduard Dobbert: Bauakademie, Gewerbeakademie und Technische Hochschule bis 1884. Histor. Skizze. (2. verb. Aufl.) [1. Aufl. s. Nr 349.] - Alfred G[otthold] Meyer: Die Technische Hochschule von 1884 bis 1899. Auf Grund von Einzelberichten bearb.

360. Ueber den Zusammenhang der $\mathrm{Ma}$ schinentechnik mit Wissenschaft und Leben. 3. Teil der Festreden am 19. Okt. 1899 in der Festhalle der Kgl. Technischen Hochschule zu Berlin anlässlich ihrer Jahrhundertfeier von 0tto Kammerer, Prof. 1899. 9 S. $4^{0}$ - Abgedr. in: Die Hundertjahrfeier d. Kgl. Techn. Hochschule zu Berlin [Nr 362]. S. $138 \mathrm{ff}$.

361. Feier zur Úbergabe der Büste des verstorbenen Geheimen Regierungs-Raths, Professors $\mathrm{H}$ [ermann] Wiebe in derHalle des Hauptgebäudes der Königlichen Technischen Hochschule zu Berlin am 1. Dezember 1899. Ansprachen geh. von dem Maschinenfabrikanten E[duard] Becker sen., d.Prof. O[tto] Kammerer u. d. Rektor d.Techn.Hochschule, Prof. A [lois] Riedler. 1899. $8 \mathrm{Bl} .4^{\circ}$

362. Die Hundertjahrfeier der Koeniglichen Technischen Hochschule zu Berlin 18. - 21.October 1899. (Verf.: Alfred G[ otthold] Meyer.) 1900.210 S. $2^{\circ}$
363. Rede zur Feier der Jahrhundertwende in der Halle der Königlichen Technischen Hochschule zu Berlin am 9. Januar 1900 geh. von dem zeitigen Rektor A[lois] Riedler. 24 S. $4^{0}$ I In ihrem wesentl. Inh. abgedr. in: Zeitschrift d. Vereines deutscher Ingenieure. Bd 44. 1900, Halbj. 1. S. 97 ff.

364. Gedäehtniss-Feier für den verstorbenen Professor Dr. Eduard Dobbert in der Aula der Königlichen Technischen Hochschule zu Berlin am 1. März 1900 veranstaltet von der Technischen Hochschule in Gemeinschaft mit der Königlichen Akademie der Künste und der akademischen Hochschule für die bildenden Künste in Berlin. 1900. $19 \mathrm{~S} .8^{\circ}$ - Etwas gekürzt in: E. Dobbert: Reden u. Aufsätze kunstgeschichtl. Inhalts. 1900. S. V ff.

365. Berlin, die Stadt der Hohenzollern. Rede bei $d$. Feier des zweihundertjährigen Jubiläums des Königreichs Preußen und des Geburtstages des Kaisers am 18. Jan. 1901 geh. in der Kgl. Technischen Hochschule zu Berlin von dem zeitigen Rektor Fritz Wolff. 1901. 14 S. $4^{\circ}$

366. Die Aufgaben des Diplom-Ingenieurs. Rede zum Antritt des Rektorates der Kgl. Technischen Hochschule zu Berlin geh. am 30. Juni 1902 von 0[tto] Kammerer. 1902. 11 S. $4^{0}$ - Abgedr. in: Zeitschrift d. Vereines deutscher Ingenieure. Bd 46, Halbj. 2.

367. 1799. 1899. Festsehrift der Hundertjahrfeier der Königl. Techn. Hochschule zu Berlin. (Hrsg. vom Ausschuß der Studierenden. 1903.) (Umschlagt.: Königl. Technische Hochschule zu Berlin. Die Hundertjahrfeier 1899.) $132 \mathrm{~S} .4^{\circ} \mathrm{Be}$

368. Guido Hauck. Rede zur Gedächtnisfeier für Guido Hauck am 17. Mai 1905 in der Halle der Technischen Hochschule zu Charlottenburg geh. von E[mil] Lampe in Berlin. 〈Sonderabdr. aus d. XIV. Bde des Jahresberichts der Deutschen MathematikerVereinigung.) Nebst der Rede am Sarge geh. am 28. Jan. 1905 von A [dolf] Parisius in Großbeeren. Mit einem Bildn. von G. Hauck als Titelbild. 1905. $32 \mathrm{~S} ., 1$ Taf. $8^{\circ}$

369. Schillers Bedeutung für das MaschinenZeitalter. Festrede bei der Schillerfeier der Technischen Hochschule zu Berlin geh. in der Aula am 8. Mai 1905 von 0[tto] Kammerer. Berlin u. München: Oldenbourg 1905. 12 S. $4^{0}$ - Auch in: Zeitschrift d. Vereines deutscher Ingenieure. Bd 49, Halbj. 1.

370. Die Enthüllungsfeier des HauckDenkmals in der Halle des Hauptgebäudes der Königlichen Technischen Hochschule zu 
Berlin am 14. November 1906. Mit einem Bilde des Denkmals. 1906. 14 S., 1 Taf. $8^{\circ}$ 371. Die Imponderabilien des Hochschulstudiums. Rede zum Antritt des Rektorates der Kgl. Technischen Hochschule zu Berlin geh. am 1. Juli 1909 von W[alter] Mathesius. $8 \mathrm{~S} .4^{\circ}$

372. Die Ausbildung des Ingenieurs. Von Prof. E[mil] Josse (derzeitigem Rektor der Kgl. Technischen Hochschule Charlottenburg. Bei der Übernahme des Rektorats am 1. Juli [1912] geh. Ansprache.) 6 S. $8^{\circ}$ - Aus: Hochschul-Nachrichten. Jg. 22.

\section{SCHRIFTEN EINZELNER ABTEILUNGEN UND INSTITUTE}

373. Katalog der Abtheilung A der Bibliothek der Königlichen Technischen Hochschule zu Berlin <aufgestellt im Bau-Akademie-Gebäude, Schinkelplatz 6 $>$. 1879. VI, 315 S. [Nebst] Nachtrag I. 1880. 20 S.; II. 1881. $23 \mathrm{~S}$; III. 1882.13 S. $4^{\circ}$ Nachtr. 1: Be

374. Das Mineralogische Museum der Königlichen Technischen Hochschule Berlin. Ein Beitrag zur topogr. Mineralogie, sowie ein Leitfaden zum Studium der Sammlungen, von Dr. Julius Hirsehwald, Prof. d. Mineralogie u. Geologie u. Vorsteher d. Mineralog. Instituts an d. Techn. Hochschule. Mit einem Grundrisspl. d. Sammlung. Berlin: Friedländer (1885). XVII, 243 S., 1 Taf. $8^{\circ}$ Be

375. Katalog der Bibliothek der Königlichen Technischen Hochschule zu Berlin. 1885. X, 882 S. [Nebst] Nachtrag I. 1888. X, 194 S. ; Nachtrag II. 1890. X, 154 S. $4^{\circ} B e$

376. Mittheilungen aus dem MaschinenLaboratorium der $\mathrm{Kgl}$. Technischen Hochschule zu Berlin. (1. 2.: Hrsg. zur Jahrhundertfeier der Hochschule.) Von Prof. E[mil] Josse, Vorsteher d. Masch. Labor. H. 1-5. München u. Leipzig: Oldenbourg 1899-1913. $4^{0}$

1. Die Maschinen, die Versuchseinrichtungen u. Hülfsmittel d. Maschinen-Laboratoriums. Mit 73 Textfig. u. 2 Taf. 1899.78 S., 2 Taf.

Mit 39 Textfig. 1899. $49 \mathrm{~S}$.
3. Neuere Erfahrungen u. Versuche mit Abwärmekraftmaschinen. Mit 20 Textfig. 1901. $42 \mathrm{~S}$.

4. Neuere Wärmekraftmaschinen. Versuche u. Erfahrungen mit Gasmaschinen, Dampfmaschinen, Dampfturbinen ete. Mit 87 Textabb. u. 1 Taf. 1905. VIII, 108 S., 1 Taf.

5. Über Kondensation, insbesondere für Dampfturbinen. Versuche über die Wärmeübertragung von Dampf an Kühlwasser. Kesselfeuerungsversuche mit Teeröl. Mit 137 Textfig. 1913.

377. Katalog der Bibliothek der Königlichen Technischen Hochsehule zu Berlin. 1900. X, 1218 S. [Nebst] I. Nachtrag. 1907. $\mathrm{XI}, 383 \mathrm{~S} .4^{\circ}$

378. Das Königliche Materialprüfungsamt der Technischen Hochschule Berlin auf dem Gelände der Domäne Dahlem beim Bahnhof Gross-Lichterfelde West. Denkschrift zur Eröffnung bearb. von d. Dir. A[dolf] Martens, Prof., u. d. Bauleitenden M[ax] Guth, Kgl. Landbauinspektor. Mit zahlreichen Textfig. u. 6 Taf. Berlin: Springer 1904. IV, 380 S., 5 Taf. $4^{\circ}$

378a. Inhaltsverzeiehnis (Alphabetisches Register) der für die Bibliothek der Königlichen Technischen Hochschule zu Berlin in der Zeit vom 1. April 1904-1. April 1905 angekauften und geschenkten Werke. 1905. $31 \mathrm{~S} .4^{0}$

$\mathrm{Be}$

378 b. Bemerkungen zu dem neuen Plane für den Elektrotechnischen Unterricht. (Unterz.: Charlottenburg, 1. 11. 1905. Der Abteilungs-Vorsteher. W[alter] Reichel.) 1 Bl. $4^{\circ}$

378c. Bautechnische Gesteinsuntersuchungen. Mitteilungen aus d. Mineralog.geolog. Institut der Kgl. Technischen Hochschule Berlin. Hrsg. von Prof. Dr. J [ulius] Hirschwald. Jg. 1. 1910, H. 1. 2; Jg. 2. 1911, H. 1. 2; Jg. 3. 1912, H. 1. Berlin: Borntraeger 1910-12. $4^{\circ}$

378d. Königl. Technische Hochschule zu Berlin. Studienreise der Abteilung für Bauingenieurwesen nach Dänemark, Schweden und Norwegen 17. Juli bis 3. August 1910. Dieser Führer ist für die Teilnehmer als Ms. gedr. 32 S., 25 Taf. $8^{\circ}$

\section{HABILITATIONSSCHRIFTEN}

379. Hecht, Hermann, Dr. phil.: Untersuchungen über einige zwischen Porzellanund Feldspath-Steingut bestehende Be- ziehungen. Berlin: Thonindustrie-Zeitung 1897.23 S. $8^{\circ}$ ๆ Aus: Thonindustria-Zeitung. Jg. 21. Berlin. Te. H., Hab.-Schr. v. 25. 3.1897 


\section{DOKTOR-INGENIEUR-DISSERTATIONEN}

\section{ABTEILUNG FÜR ARCHITEKTUR}

380. (Heller, Wilhelm), Architekt, Breslau: Die historischen Merkmale der thüringischen und slavischen Holzarchitektur beim deutschen Bauernhaus. 1908. 56 S., 17 Taf. $8^{\circ}$

Berlin, Dr.-Ing.-Diss. v. 17. 7. 1978

381. Nova, Max: Die Stadttore der Mark Brandenburg im Mittelalter. Berlin: Wasmuth 1909. IV, $88 \mathrm{~S} .4^{\circ}$ - Auch als: Beiträge zur Bauwissenschaft. H. 15.

Berlin, Dr.-Ing.-Diss. v. 26. 3. 1909

382. Behr, Heinrich v.: Die Porta Nigra in Trier. (Trier: Lintz in Komm.) [1908.] 86 S. $4^{\circ}$ - Aus: Zeitschrift f. Bauwesen. Jg. 58. Berlin, Dr.-Ing.-Diss. v. 14. 5. 1909

383. Seheibner, Richard: Duderstadt. Einbeck. Gandersheim. Ein Beitrag zur Geschichte des städtischen Bürgerhauses Niedersachsens. Dresden: Kühtmann 1909. 159 S. $4^{0}$ - Buchausg. 1910 u. d. T.: Das städtische Bürgerhaus Niedersachsens. Duderstadt. Einbeck. Gandersheim.

Berlin, Dr.-Ing.-Diss. v. 16. 7. 1909

384. Hölseher, Uvo, Reg.-Baumeister: Das Hohe Tor von Medinet Habu. Eine baugeschichtliche Untersuchung. 1909. 38 S., 1 Taf. $8^{\circ}$ - Vollst. als: Wissenschaftl. Veröffentlichung d. Deutschen Orient-Gesellschaft. 12.

Berlin, Dr.-Ing.-Diss. v. 15. 10. 1909

385. Ruppel, F[riedrieh], Baurat: Deutsche und ausländische Krankenanstalten der Neuzeit. [1909.] VIII, 149 S. $4^{\circ} \bullet$ Auch bei Leineweber in Leipzig.

Berlin, Dr.-Ing.-Diss. v. 11. 2. 1910

386. Meyer, Kurt: Die Baugeschichte des Doms zu Brandenburg a. H. [1910.] 61 S., 1 Taf. $4^{\circ}$ - Teilweise in: Zeitschrift f. Geschichte d. Architektur. Jg. 1. 1907/08.

Berlin, Dr.-Ing.-Diss. v. 11. 3. 1910

387. Rappaport, Philipp A[ugust]: Steigende Strassen. Eine Studie zum deutschen Städtebau. Berlin: Wasmuth 1911. 55 S., 2 Taf. $4^{\circ}$ - Auch als Buch.

Berlin, Dr.-Ing.-Diss. v. 24. 3. 1911

388. Das Hamburger Bürgerhaus. Seine Bau- und Kunstgeschichte. T. 1. Erbe, Albert, Bauinspektor, Hamburg: Die architektonische Entwicklung des Äußeren. T. 2. Ranek, Christoph, Bauinspektor, Hamburg: Die Hausanlage, die Konstruktion und der innere Ausbau. (Hamburg: Boysen \& Maasch 1911.) 92 S., 16 Taf. gr. $2^{\circ} \odot$ Buch. ausg. mit 27 Taf.

Berlin, Dr.-Ing.-Diss. v. 26. 5. 1911
389. Kuhlow, Kurt: Das Königliche Schloß Charlottenhof bei Potsdam baugeschichtlich und kunstgeschichtlich dargestellt unter besonderer Berücksichtigung der Handzeichnungen König Friedrich Wilhelms IV. Berlin: Kühn 1911. 98 S., 12 Taf. $4^{0}$ - Buchausg. 1912.

Berlin, Dr.-Ing.-Diss. v. 14. 7. 1911

390. Liemke, Otto, Reg.-Baumeister: Das Kloster Haina im Mittelalter. Ein Beitrag zur Baugeschichte der Cistercienser Deutschlands. (Mit $150 \mathrm{Abb}$.) (1911.) 7[2] S. $4^{0}$ Berlin, Dr.-Ing.-Diss. v. 14. 7. 1911

391. Rosintal, J[osef]: Pendentifs, Trompen und Stalaktiten. Beiträge zur Kenntnis der islamischen Architektur. [1912.] VIII, $87 \mathrm{~S} .8^{\circ}$ - Auch bei Hinrichs in Leipzig.

Berlin, Dr.-Ing.-Diss. v. 15. 3. 1912

392. Strach, Georg: Der keltische und römische Einfluß auf die Entwicklung des Stadtgrundrisses im Elsaß. Berlin: Decker 1912. VI, 114 S. $8^{0}$ - Buchhausg. u. d. T.: Der keltische und römische Einfluß auf den Städtebau im Elsa B.

Berlin, Dr.-Ing.-Diss. v. 12. 7. 1912

\section{ABTEILUNG FÜR BAU-INGENIEUR- WESEN}

393. Reissner, $\mathbf{H}[$ ans]: Schwingungsaufgaben aus der Theorie des Fachwerks. 1902. 28 S. $8^{\circ}$ - Auszugsweise u. um einen 2. T. verm. in: Zeitschrift f. Bauwesen. Jg. 53. 1903.

Berlin, Dr.-Ing.-Diss. v. 20. 6. 1902

394. Blum, 0tto, Reg.-Baumeister: Reibungsbahnen und Bahnen gemischten Systems. Ein Vergleich ihrer wirtschaftlichen Verhältnisse. 1903. $41 \mathrm{~S} .4^{0}$ ๆ Aus: Zeitschrift f. Bauwesen. Jg. 53.

Berlin, Dr.-Ing.-Diss. v. 12. 6. 1903

395. Oder, M[oritz]: Betriebskosten der Verschiebebahnhöfe. Berlin: Springer 1904. $95 \mathrm{~S} ., 1$ Taf. $8^{\circ}-$ Auch als Buch; aus: Archiv f. Eisenbahnwesen. Jg. 27. 28. 1904. 05. Der gröBte Teil ins Russ. übers. u. d. T.: [Russ.] Ekkploatacionnye raschody na sortirovočnych staneijach. Perevod $\mathrm{s}$ německago Inženera $\mathrm{S}$. D. Karejši. (Izvěstija Obščago Bjuro Soběšě. S ězdov. [Mitteilungen d. gemeinschaftl. Bureaus der beratenden Versammlungen.] 1905. No. $4-5$ ); daraus auch als S.-A. S.-Peterburg 1905: P. P. Sojkin.

Berlin, Dr.-Ing.-Diss. v. 14. 10. 1904 
396. Dietrich, Max: Die Entwicklung des Strassenbahngleises infolge Einführung des elektrischen Betriebes. Berlin: Berliner Union Verlagsgesellschaft 1906. 51 S. $4^{0}$ - Aus: Eisenbahntechn. Zeitschrift. Jg. 12.

Berlin, Dr.-Ing.-Diss. v. 13. 10. 1905

397. Harwig, Gerhard, Reg.-Bauführer: Untersuchungen über die Anwendungsmöglichkeit eines von Hand erzeugten elektrischen Stromes für die Sicherung der Zugfahrt und Zugfolge. 1905. 78 S., 5 Taf. $8^{\circ}$ - Auch bei Heydenreich in Charlottenburg.

Berlin, Dr.-Ing.-Diss. v. 23. 3. 1906

398. Klose, Georg: Ueber den Einfluss des Einbaues der Strassenbahngleise auf die Pflasterarten der Verkehrsstrassen in Großstädten. Berlin: J. Engelmann 1907. 26 S. $4^{0}$

- Auch als Buch; aus: Zeitschrift f. Transportwesen u. Straßenbau. Jg. 21.

Berlin, Dr.-Ing.-Diss. v. 8. 3. 1907

399. Havestadt, Christian, Reg - Bauführer: Uber die Verwendung von Heberverschlüssen bei Kammerschleusen. 1907. 70 S., 2 Taf. $8^{\circ}$ - Auch bei Ernst in Berlin 1908.

Berlin, Dr.-Ing.-Diss. v. 1. 11. 1907

400. Wienecke, Carl: Kritische Betrachtungen über die Versuche mit Balken aus Eisenbeton. (Als Ms. gedr.) Berlin: Ernst 1908. VIII, $118 \mathrm{~S} .4^{\circ}$

Berlin, Dr.-Ing.-Diss. v. 12. 6. 1908

401. Bernhard, Friedrich: Untersuchungen über die Ursachen der Bildung des Staubes auf Steinschlagstrassen und über Versuche zur Bekämpfung desselben. [1908.] 63 S. $4^{0}$ - Aus: Gesundheit. Jg. 33; auch bei Leineweber in Leipzig.

Berlin, Dr.-Ing.-Diss. v. 17. 7. 1908

402. Probst, Emil, (Zivil-Ing.): Einfluß der Armatur und der Risse im Beton auf die Tragsicherheit. (Mit 77 Abb. im Text u. 9 Taf. Berlin: Springer 1907.) IV, 144 S.,9 Taf. $4^{0}$ - Mitteilungen aus d. Kgl. Materialprüfungsamt zu GroB-Lichterfelde West. 1907. Erg. H. 1.

Berlin, Dr.-Ing.-Diss. v. 26. 2. 1909

403. Mann, Ludwig: Statische Berechnung steifer Vierecknetze. Berlin: Ernst 1909. 47 S. $4^{0}$ - Aus: Zeitschrift f. Bauwesen. Jg. 59.

Berlin, Dr.-Ing.-Diss. v. 25. 6. 1909

404. Sehenek, Rudolf, Kreisbaumeister a. D.: Beiträge zur Bestimmung der Erweichungskoeffizienten natürlicherBausteine. Berlin: Borntraeger 1910. 43 S. $4^{\circ}$ - Aus: Bautechn. Gesteinsuntersuchungen. Mitteilungen aus d. Mineralog.- geolog. Institut d. Kgl. Techn. Hochschule Berlin. Jg. 1 [Nr 378c].

Berlin, Dr.-Ing.-Diss. v. 13. 5. 1910

405. Birkenstock, otto: Untersuchung der Kontinuität der Längsträger zwei- gleisiger Balkenbrücken. Berlin: Krayn 1910. 47 S. $8^{\circ}$

Berlin, Dr.-Ing.-Diss. v. 15. 7. 1910

406. Kyrieleis, Wilhelm: Über Grundwasserabsenkung bei Fundierungsarbeiten. Berlin: Springer 1911. IV, 114 S., 3 Taf. $8^{\circ}$

- Erw. 1913 u. d. T.: Grundwasserabsenkung... Berlin, Dr.-Ing.-Diss. v. 15. 7. 1910

407. Struve, Henry: Einfluss von Niederungen und Eindeichungen auf den Verlauf von Hochwasserwellen, erläutert an Beispielen der unteren Oder. 1911. $49 \mathrm{~S}$, 5 Taf. $8^{\circ}$ Erw. als: Sammlung wasserwirtschaftl. Schriften. Bd 3.

Berlin, Dr.-Ing.-Diss. v. 11. 11. 1910

408. Mareus, Henri: Beitrag zur Theorie mehrfach gestützter Stabzüge. Berlin: Springer 1911. 122 S. $8^{\circ}$ - Buchausg. u. d. T.: Studien über mehrfach gestützte Rahmen- und Bogenträger.

Berlin, Dr.-Ing.-Diss. v. 26. 5. 1911

409. Henneking, Carl, Stadtbaurat, Magdeburg: Die Abwässerreinigung mittels intermittierender Bodenfiltration in Nordamerika, insbesondere im State Massachusetts und ihre Bedeutung im Hinblick auf die Reinigungsmethoden durch Berieselung und künstliche biologische Reinigung. [Berlin: Hirschwald 1912.] 74 S. $8^{\circ}$ - Unter auszugsweiser Wiedergabe des in: Mitteilungen aus d. Kgl. Prüfungsanstalt f. Wasserversorgung u. Abwässerbeseitigung zu Berlin. H. 12. 1909 erschienenen Reiseberichts.

Berlin, Dr.-Ing.-Diss. v. 12. 7. 1912

\section{ABTEILUNG FÜR MASCHINEN- INGENIEURWESEN}

\section{Maschineningenieurwesen}

410. Rülf, Benno: Der Reguliervorgang bei Dampfmaschinen. 1902. 15 S. $4^{0}$ - Vollst. bei Springer in Berlin; aus: Zeitschrift d. Vereines deutscher Ingenieure. Bd 46, Halbj. 2.

Berlin, Dr.-Ing.-Diss. v. 24. 1. 1902

411. Roth, Paul: Die Festigkeitstheorien und die von ihnen abhängigen Formeln des Maschinenbaues. 1902. 45 S. $8^{\circ}$ ๆ Mit einigen Änderungen in: Zeitschrift f. Mathematik u. Physik. Bd 48. 1903.

Berlin, Dr.-Ing.-Diss. v. 11. 7. 1902

\section{Elektrotechnik}

412. Jordan, Franz: Die Verwendung von Druckluft bei elektrisch betriebenen Hebezeugen. 1903. 24 S., 2 Taf. $8^{\circ}$ - Auch in: Dinglers Polytechn. Journal. Jg. 84. Bd 318. Berlin, Dr.-Ing.-Diss. v. 22. 5. 1903 
413. Wendt, Karl: Untersuchungen an den Gaserzeugern der Tiegelgußstahlfabrik „Poldihütte" zu Kladno in Böhmen. 1904. 27 S. $4^{\circ}$ - In kürzerer Form in: Zeitschrift d. Vereines deutscher Ingenieure. Bd 48, Halbj. 2 u. in: Mitteilungen über Forschungsarbeiten auf d. Gebiete d. Ingenieurwesens. H. 31. 1906.

Berlin, Dr.-Ing.-Diss. v. 12. 2. 1904

414. Sehlesinger, Georg: Die Passungen im Maschinenbau. 1904. 41 S., 1 Tab. $4^{\circ}$ - Auch in: Mitteilungen über Forschungsarbeiten auf d. Gebiete d. Ingenieurwesens. H. 18; Ausz. in: Zeitschrift d. Vereines deutscher Ingenieure. Bd 48, Halbj. 2.

Berlin, Dr.-Ing.-Diss. v. 26. 2. 1904

415. Werner,Siegfried G[eorg]:Kurvenführungen im Werkzeugmaschinenbau. 1905. $35 \mathrm{~S} .4^{\circ}$ - Aus: Verhandlungen d. Vereins zur Beförderung d. Gewerbfleißes. Jg. 84; auch in: Zeitschrift f. Werkzeugmaschinen u. Werkzeuge. Jg. 9. $1904 f 05$.

Berlin, Dr.-Ing.-Diss. v. 26. 2. 1904

416. Stauber, G[eorg]: Regulierung von Gasmaschinen. 1904. $11 \mathrm{~S} .4^{\circ}$

Berlin, Dr.-Ing.-Diss. v. 24. 6. 1904

417. Giller, Willy: Vergleich zwischen den verschiedenen Betriebsarten von Schleusenanlagen. München \& Berlin: Oldenbourg 1904. 79 S., 6 Taf. $8^{\circ} \cdot$ Auch als Buch.

Berlin, Dr.-Ing.-Diss. v. 15. 7. 1904

418. Heine, Bruno: Ober die Erzeugung elektrischer Energie mit Hilfe von Kanalisations-Klärschlamm. $1904.37 \mathrm{~S} .4^{\circ}$

Berlin, Dr.-Ing.-Diss. v. 15. 7. 1904

419. Schürmann, Eugen: Über Schwerlast-Drehkrane im Werft- und Hafenverkehr. 1904. VI, 79 S., 12 Taf. $8^{\circ}$ ฯ Auch bei Oldenbourg in München.

Berlin, Dr.-Ing.-Diss. v. 15. 7. 1904

420. Sehwabach, Max C[arl] G[ünther]: Dynamische Theorie der Versch windelafetten und kinematische Schußtheorie. $1904.69 \mathrm{~S} .4^{\circ}$ - Auch in: Verhandlungen d. Vereins zur Beförderung d. Gewerbfleißes. Jg. 84. 1905.

Berlin, Dr.-Ing.-Diss. v. 2. 12. 1904

421. Bauersfeld, Walther: Uber die automatische Regulierung der Turbinen. Berlin: (Springer) 1905. $186 \mathrm{~S} .8^{\circ}$ - Buchausg. mit erw. Einl. u. d. T.: Die automatische Regulierung ...

Berlin, Dr.-Ing.-Diss. v. 16. 12. 1904

422. Biel, R[udolf]: Ueber den Druckhöhenverlust bei der Fortleitung tropftarer und gasförmiger Flüssigkeiten. 1907. $64 \mathrm{~S}$. $4^{0}$ - Auch als: Mitteilungen über Forschungsarbeiten auf d. Gebiete d. Ingenieurwesens. H. 44; Ausz. in: Zeitschrift d. Vereines deutscher Ingenieure. Bd 52. 1908, Halbj. 1.

Berlin, Dr.-Ing.-Diss. v. 16. 12. 1904
423. Bendemann, $\mathbf{F}[$ riedrich]: Ueber den Ausfluß des Wasserdampfes und über Dampfmengenmessung. 1906. 65 S. $4^{0}$ ๆ Auch in: Mitteilungen über Forschungsarbeiten auf $\mathrm{d}$. Gebiete d. Ingenieurwesens. H. 37. 1907.

Berlin, Dr.-Ing.-Diss. v. 13. 5. 1905

424. Crain, Rudolf: Schraubenräder mit geradlinigen Eingriffsflächen. Berlin: (Springer) $1907.47 \mathrm{~S} .4^{0}$ - Aus: Werkstattstechnik. Jg. 1.

Berlin, Dr.-Ing.-Diss. v. 14. 7. 1905

425. Rötseher, Felix: Versuche an einer 2000pferdigen Riedler-Stumpf - Dampfturbine. 1906.59 S. $4^{0}$ - Auch als: Mitteilungen über Forschungsarbeiten auf d. Gebiete d. Ingenieurwesens. H. 50. 1908; Ausz. in: Zeitschrift d. Vereines deutscher Ingenieure. Bd 51. 1907, Halbj. 1.

Berlin, Dr.-Ing.-Diss. v. 15. 12. 1905

426. Möller, Paul: Untersuchungen an Drucklufthämmern. 1906. 33 S. $4^{0}$ ๆ Auch in: Mitteilungen über Forschungsarbeiten auf $d$. Gebiete d. Ingenieurwesens. H. 37. 1907; Ausz. in: Zeitschrift d. Vereines deutscher Ingenieure. Bd 50, Halbj. 2.

Berlin, Dr.-Ing.-Diss. v. 12. 1. 1906

427. Gensecke, Wilhelm: Untersuchung einer mittelbaren Dampfmaschinenregelung. 1907. $68 \mathrm{~S} .4^{\circ}-$ Auch als: Mitteilungen über Forschungsarbeiten auf d. Gebiete d. Ingenieurwesens. H. 53. 1908; Ausz. in: Zeitschrift d. Vereines deutscher Ingenieure. Bd 51, Halbj. 2.

Berlin, Dr.-Ing.-Diss. v. 23. 2. 1906

428. Heinel, C[arl]: Zahlenstoff und Winke für Bau und Betrieb von Kältemaschinenanlagen. 1907. XIV, 190 S., 19 Taf. $8^{\circ}$ Vollst. bei Oldenbourg in München u. d. T.: Bau u. Betrieb v. Kälte-Maschinenanlagen. Zahlenstoff $u$. Winke f. Ingenieure... 1906. (Oldenbourgs Techn. Handbibliothek. Bd 8.)

Berlin, Dr.-Ing.-Diss. v. 14. 12. 1906

429. Rieppel, Paul: Versuche über die Verwendung von Teerölen zum Betrieb des Dieselmotors. 1907. $37 \mathrm{~S} .4^{0}$ I Auch in: Mitteilungen über Forschungsarbeiten auf d. Gebiete d. Ingenieurwesens. H. 55. 1908; Ausz. in: Zeitschrift d. Vereines deutscher Ingenieure. Bd 51, Halbj. 1.

Berlin, Dr.-Ing.-Diss. v. 25. 2. 1907

430. Drawe, R(udolf): Ausbildung und vergleichende Bewertung der Regelung grosser Viertakt-Gasmaschinen. 1907. 24 S., 15 Taf. $4^{\circ}$

Berlin, Dr.-Ing.-Diss. v. 8. 3. 1907

431. Kuseh, Max: Die Betriebssicherheit und Wirtschaftlichkeit von kleineren Heißdampflokomobilen, Sauggasanlagen und 
Dieselmotoren. 1907. 50 S. $4^{\circ}$ - Auch in: Verhandlungen d. Vereins zur Beförderung d. Gewerbfleißes. Jg. 86 .

Berlin, Dr Ing.-Diss. v. 22. 3. 1907

432. Bertsehinger, Hermann: Die Wirtschaftlichkeit von Schiffshebewerken. 1908. 26 S. $4^{\circ}$ - Aus: Zeitschrift d. Vereines deutscher Ingenieure. Bd 51. 1907, Halbj. 2.

Berlin, Dr.-Ing.-Diss. v. 25. 3. 1907

433. Mattersdorff, Wilhelm: Untersuchung der den städtischen Verkehr bestimmenden Einflüsse und Nutzanwendung der Ergebnisse bei Verkehrsschätzungen. (Berlin: Springer) [1907]. 42 S., 4 Taf. $4^{\circ}$

- Buchausg. u. d. T.: Städtische Verkehrsfragen. Untersuchung der...

Berlin, Dr.-Ing.-Diss. v. 28. 6. 1907

434. Müller, Arno 0tto: Messung von Gasmengen mit der Drosselscheibe. 1907. 29 S. $4^{\circ}$ - Auch in: Mitteilungen über Forschungsarbeiten auf d. Gebiete d. Ingenieurwesens. H. 49. 1908; Ausz. in: Zeitschrift d. Vereines deutscher Ingenieure. Bd 52, Halbj. 1.

Berlin, Dr.-Ing.-Diss. v. 28. 6. 1907

435. Doehne, Konrad: Die Bewegungsverhältnisse vonSteuergetrieben mitSch wingdaumen. 1908. 69 S. $4^{0}$ - Aus: Verhandlungen d. Vereins zur Beförderung d. Gewerbfleißes. Jg. 87.

Berlin, Dr.-Ing.-Diss. v. 13. 12. 1907

436. Kürth, Alfred: Ueber die Beziehung der Kugeldruckhärte zur Streckgrenze und zur Zerreißfestigkeit zäher Metalle. 1908. $45 \mathrm{~S} .4^{0}$ - Auch in: Mitteilungen über Forschungsarbeiten auf d. Gebiete d. Ingenieurwesens. H. 65/66. 1909; Ausz. in: Zeitschrift d. Vereines deutseher Ingenieure. Bd 52, Halbj. 2.

Berlin, Dr.-Ing.-Diss. v. 13. 3. 1908

437. Wenger, A[lbert]: Bestimmung des Maximalwertes des thermo-dynamischen Wirkungsgrades und der günstigsten Stufenzahl bei Dampfturbinen. Berlin: Springer 1908. 84 S., 2 Taf. $8^{\circ}$ - Auch als Buch.

Berlin, Dr.-Ing.-Diss. v. 13. 3. 1908

438. Jastrow, F[ritz]: Maschinelle Abwasserreiniger. 1908. 63 S. $8^{\circ}$ - Auch bei Heymann in Berlin.

Berlin, Dr.-Ing.-Diss. v. 27. 3. 1908

439. Osthoff, Max Heinrich Philipp, Reg.Bauführer: Die Lentz-Ventilsteuerung an Lokomotiven. 1908. $45 \mathrm{~S} .4^{\circ}$ - Auch in: Dinglers Polytechn. Journal. Jg. 90. 1909. Bd 324.

Berlin, Dr.-Ing.-Diss. v. 8. 5. 1908

440. Hilpert, August: Die Verwendbarkeit der Azetylen-Sauerstoff-Schweissung im Maschinenbau. 1908. 34 S. $4^{0}$

Berlin, Dr.-Ing.-Diss. v. 26. 6. 1908
441. Purper, Emil: Der Einfluss der verschiedenen Stützkonstruktion bei Turm. Drehkranen. 1908. 52 S., 2 Taf. $8^{\circ}$

Berlin, Dr.-Ing.-Diss. v. 17. 7. 1908

442. Sobbe, Carl: Beiträge zur Technologie des Schmiedepressens. (Berlin: Springer) 1908. 36 S. $4^{0}$ - Aus: Werkstattstechnik. Jg. 2.

Berlin, Dr.-Ing.-Diss. v. 17. 7. 1908

443. Zillgen, J[oseph]: Die Verkürzung der Fahrzeit im Schnellzugbetriebe und die Mittel zu ihrer Durchführung. 1908. 26 S. $4^{\circ}$

- Buchausg. bei Gebr. Grunert in Berlin.

Berlin, Dr.-Ing.-Diss. v. 17. 7. 1908

444. Hanszel, Hubert: Versuche an einer Dreifach-Expansions-Dampfmaschine. Beitrag zur Frage der Heizung der Dampfmaschine. 1910. $82 \mathrm{~S} ., 1 \mathrm{Tab} .4^{0}$ - Auch als: Mitteilungen über Forschungsarbeiten auf $d$. Gebiete d. Ingenieurwesens. H. 101. 1911; Ausz. in: Zeitschrift d. Vereines dentscher Ingenieure. Bd 56. 1912, Halbj. 1.

Berlin, Dr.-Ing.-Diss. v. 22. 1. 1909

445. Krüger, Walter: Untersuchungen über die Anstrengung dickwandiger Hohl zylinder unter Innendruck. 1908. 65 S., 2 Taf. $4^{0}$ - Auch als: Mitteilungen über Forschungsarbeiten auf d. Gebiete d. Ingenieurwesens. H. 87. 1910; Ausz. in: Zeitschrift d. Vereines deutscher Ingenieure. Bd 53. 1909, Halbj. 2.

Berlin, Dr.-Ing.-Diss. v. 22. 1. 1909

446. Riebensahm, Paul: Úber die Ausbildung der Laufräder schnellaufender Niederdruck-Zentrifugalpumpen. 1909. $19 \mathrm{~S}$., 1 Taf. $4^{\circ}$ - Auch beiOldenbourg in München; aus: Zeitschrift f. d. gesamte Turbinenwesen. Jg. 6 . Berlin, Dr.-Ing.-Diss. v. 22. 1. 1909

447. Korthals, Wilhelm: Der Lauf von Fahrzeugen durch Krümmungen sowie das Verhalten der Lenkachsen. 1909. 47 S. $8^{\circ}$ - Der 1. Teil einer Abhandlung über das im Titel angegebene Thema; mehr bis 1913 nicht ersch.

Berlin, Dr.-Ing.-Diss. v. 26. 2. 1909

448. Meyer, Friedrich: Die Technik der Verbrennung und Energie-Gewinnung aus städtischen Abfallstoffen. Leipzig: Leineweber 1910. 24 S. $4^{0}$ ` Aus: Gesundheit. Jg. 35; auch als Buch.

Berlin, Dr.-Ing.-Diss. v. 26. 2. 1909

449. Ellon, Kurt: Versuche zur Bestimmung der Strömung im Laufrad und Saugrohr einer Francis-Schnellläuferturbine. 1910. $35 \mathrm{~S} .4^{0}$ - Auch in: Mitteilungen über Forschungsarbeiten auf $d$. Gebiete $d$. Ingenieurwesens. H. 102.1911.

Berlin, Dr.-Ing.-Diss. v. 16. 7. 1909 
450. Klein, Georg: Untersuchung und Kritik von Hochdruckmessern. 1909. VI, $78 \mathrm{~S} .4^{\circ}$ - Ausz. in: Zeitschrift d. Vereines deutscher Ingenieure. Bd 54. 1910, Halbj. 2.

Berlin, Dr.-Ing.-Diss. v. 16. 7. 1909

451. Tuekermann, Ernst: Regelungen von Zweitact-Gross-Gasmaschinen. (1910.) 48 S. $8^{\circ}$ Auch in: Zeitschrift f. Elektrotechnik u. Maschinenbau. Bd 13. (Elektrotechn. u. polytechn. Rundschau. Jg. 27.)

Berlin, Dr.-Ing.-Diss. v. 16. 7. 1909

452. Barten, Ernst, Gewerbe-Refer. : Notwendigkeit, Erfolge und Ziele der technischen Unfallverhütung. [1909.] $100 \mathrm{~S} .8^{\circ}$

\section{- Auch bei Troschel in GroB-Lichterfelde.}

Berlin, Dr.-Ing.-Diss. v. 29. 10. 1909

453. Nicolaus, Georg, Kaiserl. Bauinsp. Die technischen Anforderungen des Wertpapierdruckes. (Berlin: Springer in Komm.) [1910.] 12 S. $4^{\circ}$ - Aus: Zeitschrift d. Vereines deutscher Ingenieure. Bd 54, Halbj. 2.

Berlin, Dr.-Ing.-Diss. v. 26. 11. 1909

454. Claus, Carl: Der Umschlagverkehr in Baumaterialien auf den Berliner Wasserstraßen und die Zweckmäßigkeit der Verwendung mechanischer Entladevorrichtungen für den Ziegeltransport. Berlin: Krayn [1910]. 71 S., 1 Taf. $8^{\circ}$ T Auch als: Schriften d. Verbandes Deutscher Diplom-Ingenieure. 2; Ausz. in: Technik u. Wirtschaft. Jg. 3.

Berlin, Dr.-Ing.-Diss. v. 17. 12.1909

455. Hochsehild, Heinrich: Versuche über die Strömungsvorgänge in erweiterten und verengten Kanälen. $1910.58 \mathrm{~S} .4^{\circ}$ - Auch als: Mitteilungen über Forschungsarbeiten auf d. Gebiete d. Ingenieurwesens. H. 114. 1912; Ausz. in: Zeitschrift d. Vereines deutscher Ingenieure. Bd 57. 1913, Halbj. 1.

Berlin, Dr.-Ing.-Diss. v. 17. 12. 1909

456. Sehneider, John J[oseph]: Die Kugelfallprobe. 1910. 50 S. $4^{\circ}$ - Auch in: Mitteilungen über Forschungsarbeiten auf d. Gebiete d. Ingenieurwesens. H. 104. 1911; Ausz. in: Zeitschrift d. Vereines deutscher Ingenieure. Bd 54, Halbj. 2. Berlin, Dr.-Ing.-Diss. v. 17. 12. 1909

457. Hoening, Carl: Untersuchung über die Bedingungen ruhigen Laufs von Drehgestellwagen für Schnellzüge. Berlin: Springer 1910. 57 S. $8^{\circ}$ Buchansg. u. d. T.: Die Bedingungen... Berlin, Dr.-Ing.-Diss. v. 11. 2. 1910

458. Pockrandt, Willy: Versuche zur Ermittelung der günst[i] gsten Arbeitsweise der Rundschleifmaschine. 1910.65 S. $4^{\circ}$ - Auch als: Mitteilungen über Forschungsarbeiten auf d. Gebiete d. Ingenieurwesens. H. 105. 1911; Ausz. in: Zeitschrift d. Vereines deutscher Ingenieure. Bd 54, Halbj. 2.

Berlin, Dr.-Ing.-Diss. v. 11. 2. 1910
459. Koch, Waldemar, Dr. phil.: Die Industrialisierung Chinas. [1910.] 86 S. $8^{\circ}$ - Auch bei Springer in Berlin; Ausz. in: Technik u. Wirtschaft. Jg. 3 .

Berlin, Dr.-Ing.-Diss. v. 11. 3. 1910

460. Finkelstein, Alfons: Prüfung der Arbeitsgenauigkeit von Werkzeugmaschinen. Berlin: Springer [1911]. 35 S. $4^{\circ}$ - Auch als Buch; aus: Werkstattstechnik. Jg. 4. 5. 1910. 11.

Berlin, Dr.-Ing.-Diss. v. 13. 5. 1910

461. Berlowitz, Max: Der Wärmedurchgang in Maischbottichen. 1910. 14 S. $4^{\circ}$ - Aus: Gesundheits-Ingenieur. Jg. 33.

Berlin, Dr.-Ing.-Diss. v. 27. 5. 1910

462. Geitmann, Hans: Die wirtschaftliche Bedeutung der deutschen Gaswerke. 1910. IV, 136 S. $8^{\circ}$ ศ Um 6 S. erw. bei Oldenbourg in München.

Berlin, Dr.-Ing.-Diss. v. 27. 5. 1910

463. Bieńkowski, Stanislaw von: Untersuchungen über Arbeitseignung und Leistungsfähigkeit der Arbeiterschaft eines großindustriellen Betriebes. [1910.] $45 \mathrm{~S} .8^{\circ}$ -Auch in: Schriften d.Vereins f. Sozialpolitik. Bd 134. Berlin, Dr.-Ing.-Diss. v. 10. 6. 1910

464. Peiseler, Gottlieb: Anwendung der modernen Organisationsgrundlagen auf Klein- und Mittelbetriebe. Ausgeführt an einem Beispiel aus der Kleineisenindustrie. Berlin: Springer [1911]. 45 S. $4^{0}$ ๆ Aus: Werkstattstechnik. Jg. 4. 5. 1910. 11.

Berlin, Dr.-Ing.-Diss. v. 24. 6. 1910

465. Höniger, Walter: Ein Verfahren zur Ermittlung des Verlaufs der veränderlichen Stoßkraft bei Stauchversuchen. 1910. 26 S., 20 Taf. $4^{\circ}$ - Gekürzt in: Zeitschrift d. Vereines deutscher Ingenieure. Bd 56. 1912, Halbj. 2.

Berlin, Dr.-Ing.-Diss. v. 28. 10. 1910

466. Chrzanowski, Wieslaw von: Ge schwindigkeitsregelung der Dampffördermaschinen. [1910.] 57 S. $4^{0}$

Berlin, Dr.-Ing.-Diss. v. 2. 12. 1910

467. Cordier, Wilhelm von: Strömungsuntersuchungen an einem Rohrkrümmer. [1911.] 89 S., 8 Taf. $8^{\circ}$

Berlin, Dr.-Ing.-Diss. v. 16. 12. 1910

468. Kerner, Fritz: Unfallsicherheit und Betriebsökonomie im Kraftmaschinenbetrieb. [1911]. $45 \mathrm{~S} .8^{\circ}$ - Mit unwesentl. Ânderungen in: Zeitschrift f. Gewerbe-Hygiene, Unfall-Verhütung u. Arbeiter-Wohlfahrts-Einrichtungen. Jg. 18.

Berlin, Dr.-Ing.-Diss. v. 3. 2. 1911

469. Nádai, Árpád: Untersuchungen der Festigkeitslehre mit Hilfe des thermoelektrischen Temperaturmessverfahrens. Berlin: Ebering [1911]. 55 S. $4^{\circ}$ - Auch als Buch. Berlin, Dr.-Ing.-Diss. v. 3. 2. 1911 
470. Cranz, Hermann: Versuche mit Schmierringen bei höheren Tourenzahlen. [1912.] 65 S. $4^{\circ}$

Berlin, Dr.-Ing.-Diss. v. 12. 5. 1911

471. Sanio, Paul: Ueber die Wirtschaftlichkeit moderner Trockenbagger und verwandter Bodenförderungsanlagen. Berlin: Sturm [1911]. 135, XXIX S. $8^{\circ}$ - Auch als Buch.

Berlin, Dr.-Ing.-Diss. v. 26. 5. 1911

472. Christlein, Paul: Untersuchungen über das allgemeine Verhalten des Geschwindigkeitskoeffizienten von Dampfturbinenelementen 〈Düsen, Leitapparate und Laufschaufeln $>$ bei verschiedenen Betriebsbedingungen mit besondererBerücksichtigung von Ausführungen des praktischen Dampfturbinenbaues. 1911. $36 \mathrm{~S} .4^{\circ}$ - Mit einigen Änderungen in: Zeitschrift f. d. gesamte Turbinenwesen. Jg. 9. 1912.

Berlin, Dr.-Ing.-Diss. v. 14. 7. 1911

473. Kock, Friedrich: Aufnahme von Resonanzkurven unter Anwendung eines Kurvenzeichners. 1912. 33 S. $4^{0}$

Berlin, Dr.-Ing.-Diss. v. 27. 10. 1911

474. Wolf, Hermann: Die Materialbewegung im Eisenhüttenbetrieb. Eine Untersuchung über die Belastung der Erzeugnisse durch den Transport. [1911.] 48 S., 4 Taf. $8^{\circ}$

Berlin, Dr.-Ing.-Diss. v. 27. 10. 1911

475. Karbe, Werner: Die Arbeitsweise und Berechnung der Mammutpumpen <Druckflüssigkeitsheber $\rangle$. 1912. $45 \mathrm{~S} .8^{\circ}$ - Aus: Journal f. Gasbeleuchtung. Jg. 55.

Berlin, Dr.-Ing.-Diss. v. 15. 12. 1911

476. Selter, Fritz, Reg.-Rat: Zur Kritik der Lohntarifverträge auf Basis der Stücklohnung in Grossbetrieben des Maschinenbaues $u$. verwandter Industrien mit differenzierter Fabrikation. [1912.] $46 \mathrm{~S}$. $8^{\circ}$ - Auch u. d. T.: Ueber die Einführung von Tarifverträgen in den Grossbetrieben des Maschinenbaues $\mathrm{u}$. verwandter Industrien. Berlin: Seydel [in Komm.] 1911; zum Teil in: Werkstattstechnik. Jg 5. 1911.

Berlin, Dr.-Ing.-Diss. v. 15. 12. 1911

477. Breitung, Georg: Untersuchung von Geschwindigkeitsmessern. [1912.] $18 \mathrm{~S} .4^{0}$ - Aus; Zeitschrift d. Mitteleurop. MotorwagenVereins. Jg. 11.

Beriin, Dr.-Ing.-Diss. v. 16. 2. 1912

378. Bergmann, Georg: Indikator für Einspritz-Vergaser. Die Pitot'sche GleichWechselstrom-Düse. Indikator für Lokomotiv-Blasrohre etc. Berlin: Krayn [1912]. 28 S., 1 Taf. $4^{\circ}$

Berlin, Dr.-Ing.-Diss. v. 10. 5. 1912
479. Ludwig, Hans: Der Azetylen-Sauerstoff-Schweißbrenner, seine Wirkungsweise und seine Konstruktionsbedingungen. Berlin Springer [1912]. 30 S. $4^{\circ}$ T Auch als: Berichte $d$. Versuchsfeldes $\mathrm{f}$. Werkzeugmaschinen an d. Techn. Hochschule Berlin. H. 2.

Berlin, Dr.-Ing.-Diss. v. 21. 6. 1912

480. Pfahl, Werner: Kräfteverteilung und Greifen bei Selbstgreifern. 1912. 16 S. $4^{\circ}$ A Aus: Zeitschrift d. Vereines deutscher Ingenieure. Bd 56, Halbj. 2.

Berlin, Dr.-Ing.-Diss. v. 21. 6. 1912

481. Becker, Gabriel: Wagentechnische Untersuchung eines 35 PS-Kraftlastwagens $\left\langle o h n e\right.$ Motoruntersuchung〉. 1912. 44 S. $4^{\circ}$ - Aus: Wissenschaftl. Automobil-Wertung. Berichte VI-X d. Laboratoriums f. Kraftfahrzeuge an d. Kgl. Techn. Hochschule zu Berlin. Von A. Riedler. 1912.

Berlin, Dr.-Ing.-Diss. v. 12. 7. 1912

482. Hoefer, Kurt: Untersuchungen über die Strömungsvorgänge im Steigrohr eines Druckluft-Wasserhebers. $1912 . \quad 57$ S. $4^{0}$ - Auch in: Mitteilungen über Forschungsarbeiten auf d. Gebieted. Ingenieurswesens. H. 138. 1913; Ausz. in: Zeitschrift d. Vereines deutscher Ingenieure. Bd 57. 1913, Halbj. 1.

Berlin, Dr.-Ing.-Diss. v. 12. 7. 1912

483. Schwetje, Heinrich: Beiträge zur Kenntnis der Reibungsverhältnisse zwischen $\mathrm{Holz}$ und Eisen, insbesondere bei Bremseinrichtungen für Leistungsmessung. Berlin: Krayn 1912. 50 S. $4^{0}$

Berlin, Dr.-Ing.-Diss. v. 12. 7. 1912

484. Szitniek, Robert: Einfluß der Spaltbreite und der Deckelstellung auf die Kraftanzeige einer Meßdose. T. 1. 56 S.; T. 2. (Versuchsreihen u. Zahlentafeln.) $28 \mathrm{Bl}$. 1912. $4^{0}$ - Ausz. in: Mitteilungen über Forschungsarbeiten auf d. Gebiete d. Ingenieurwesens. H. 138. 1913.

Berlin, Dr.-Ing.-Diss. v. 12. 7. 1912

485. Fehrmann, Karl: Über TriebwerkVerluste in Kraftwagen. [1912.] $20 \mathrm{~S} .4^{\circ}$ - Erw. in: Sitzungsberichte d. Vereins zur Beförderung d. Gewerbfleißes. 1907.

Berlin, Dr.-Ing.-Diss. v. 29. 11. 1912

486. Reiehel, Walter: Betrachtungen und Versuche über die Verwendung des Drehstromes für den Betrieb elektrischer Bahnen, insbesondere des hochgespannten Drehstromes. 1903. 73 S., 8 Taf. $4^{\circ}$ I Um einen Abschnitt C erw. bei Oldenbourg in München u. d. T.: Die Verwendung des Drehstroms insbesondere des hochgespannten Drehstroms für den Betrieb elektrischer Bahnen.

Berlin, Dr.-Ing.-Diss. v. 13. 2. 1903 
487. Kramer, Erwin: Die Sachgemässheit der Bremsen elektrischer Strassenbahnen und die Mittel zur sachgemässen Steigerung ihrer Leistungsfähigkeit. 1904. 36 S. $4^{\circ}$ - Aus: Elektr. Bahnen. Jg. 2.

Berlin, Dr.-Ing.-Diss. v. 13. 11. 1903

488. Lieber, Max: Untersuchung des Drehfeldes eines asynchronen DreiphasenMotors mit Phasenanker. 1904. 40 S., 15 Taf. $8^{\circ}$

Berlin, Dr.-Ing.-Diss. v. 26. 2. 1904

489. Wagner, Gustav: Stroboskopischer Schlüpfungsmesser für asynchrone Wechselund Drehstrommotoren. Berlin: Springer 1904. 16 S., I Taf. $4^{0}$ - Buchausg. u. d. T.: Ein neuer stroboskopischer Schlüpfungsmesser...; in kürzerer Form in: Annalen f. Gewerbe u. Bauwesen. Bd 55 .

Berlin, Dr.-Ing.-Diss. v. 11. 3. 1904

490. Arldt, Conrad: Ueber die bei elektrischen Anlagen an Bord von Schiffen zu verwendende Stromart. 1904. $31 \mathrm{~S} .4^{\circ}$ - Aus: Schiffbau. Jg. 5. 1903/04.

Berlin, Dr.-Ing.-Diss. v. 3. 6. 1904

491. Wommelsdorf, Heinrich: Die Kondensatormaschine mit Doppeldrehung, Mitteilung ihrer Anordnung, Theorie und Wirkungsweise. 1904. $58 \mathrm{~S} .8^{\circ}$ - Nur Abschn. 1 u. 2 ; Abschn. 3 u. 5 in: Annalen d. Physik. Folge 4, Bd 15; Abschn. 4 in: Physik. Zeitschrift. Jg. 5.

Berlin, Dr.-Ing.-Diss. v. 15. 7. 1904

492. Morck, E[manuel]: Theorie der Wechselstromzähler nach Ferrarisschem Prinzip und deren Prüfung an ausgeführten Apparaten. 1905. 116 S. $8^{\circ}$ ' Auch bei Enke in Stuttgart u. als: Sammlung elektrotechn. Vorträge. Bd 8 , H. $8 / 10$.

Berlin, Dr.-Ing.-Diss. v. 10. 2. 1905

493. Hilpert, Georg: Über die Trägheit der von elektrischer Energie beeinflußten Massen und ihre einfache Ermittlung auf graphischem Wege. 1905. 17 S., 9 Taf. $4^{\circ}$ - Auch in: Elektr. Bahnen u. Betriebe. Jg. 4. 1906.

Berlin, Dr.-Ing.-Diss. v. 14. 7. 1905

494. Koch, R[iehert] von: Über die Entwicklungsmöglichkeiten des Induktionsmotors für Einphasen-Wechselstrom. Berlin: (Springer) 1905. $104 \mathrm{~S} .8^{\circ}$ - Auch als Buch.

Berlin, Dr.-Ing.-Diss. v. 14. 7. 1905

495. Meyer, Georg I[sidor]: Beitrag zur Kenntnis der Abschmelz-Sicherungen. 1906. $44 \mathrm{~S} ., 4$ Taf. $8^{\circ}$ - Vollst. bei Oldenbourg in München u. d. T.: Zur Theorie der Abschmelzsicherungen.

Berlin, Dr.-Ing.-Diss. v. 11. 5. 1906
496. Becker, Leonhard: Betrachtungen über die Verluste bei Ilgner-Förderanlagen und Bestimmung der wirtschaftlichsten Schlüpfung ihrer Anlaßmotoren. 1907. $30 \mathrm{~S}$. $4^{0}$ - Auch in: Elektr. Kraftbetriebe u. Bahnen. Jg. 5.

Berlin, Dr.-Ing.-Diss. v. 22. 3. 1907

497. Roemmelt, Josef: Beiträge zur Berechnung magnetisch betätigter Kupplungen und Bremsen. 1907. 53 S., 9 Taf. $8^{\circ}$

Berlin, Dr.-Ing.-Diss. v. 18. 10. 1907

498. Lichtenstein, Leon: Beiträge zur Theorie der Kabel. Untersuchungen über die Kapazitätsverhältnisse der verseilten und konzentrischen Mehrfachkabel. 1908. 40 S. $4^{\circ}$ - Auch bei Oldenbourg in München; Ausz. in: Elektr. Kraftbetriebe u. Bahnen. Jg. 6.

Berlin, Dr.-Ing.-Diss. v. 22. 11. 1907

499. Sehapira, Carl: Ueber den Wirkungsgrad der Hochfrequenz-Lampe mit unterteiltem Lichtbogen. [1907.] 34 S. $8^{\circ}$ - Gekürzte Bearbeitung in: Jahrbuch d. drahtlosen Telegraphie u. Telephonie. Bd 2. 1909.

Berlin, Dr.-Ing.-Diss. v. 13. 12. 1907

500. Alexander, Johann: Drehstrommotoren mit Kommutator für regelbare Drehzahl. 1908. 48 S., 14 Taf. $8^{\circ}$

Berlin, Dr.-Ing.-Diss. v. 17. 7. 1908

501. Majerezik, Wilhelm: Die Berechnung elektrischer Freileitungen nach wirtschaftlichen Gesichtspunkten. Berlin: Springer [1910]. $59 \mathrm{~S} .8^{\circ}$ - Auch als Buch.

Berlin, Dr.-Ing.-Diss. v. 8. 4. 1910

502. Seidner, Michael: Theorie und Konstruktion der Teillochwicklungen für Mehrphasengeneratoren. Wien: Selbstverl. 1910. $13 \mathrm{~S} .4^{\circ}$ - Aus: Elektrotechnik u. Maschinenbau. Jg. 28.

Berlin, Dr.-Ing.-Diss. v. 24. 6. 1910

503. Müller, Paul: Gegenstrom- und Kurzschlussbremsung bei ReihenschlussKommutator-Motoren. 1911. 18 S. $4^{\circ}$ - Ausz. in: Elektr. Kraftbetriebe u. Bahnen. Jg. 9. Berlin, Dr.-Ing.-Diss. v. 14. 10. 1910

504. Steil, Edmund: Untersuchungen über Solenoide und über ihre praktische Verwendbarkeit für Straßenbahnbremsen. 1911. $41 \mathrm{~S} ., 1 \mathrm{Taf}, 4^{\circ}$ - Auch in: Mitteilungen über Forschungsarbeiten auf $d$. Gebiete $d$. Ingenieurwesens. H. 121. 1912.

Berlin, Dr.-Ing.-Diss. v. 3. 2. 1911

505. Weil, Robert: Beanspruchung und Durchhang von Freileitungen. Berlin: Springer 1911. $106 \mathrm{~S} ., 2$ Taf. $8^{\circ}$ - Buchausg. 1910. Berlin, Dr.-Ing.-Diss. v. 10. 3. 1911 
506. Euler, Karl: Untersuchung eines Zugmagneten für Gleichstrom. Berlin: Springer 1911. 92 S., 1 Taf. $8^{\circ}$ - Auch als Buch.

Berlin, Dr.-Ing.-Diss. v. 24. 3. 1911

507. Holm, Fritz: Untersuchungen über magnetische Hysteresis. 1912.29 S. $4^{\circ}$ - Auch in: Mitteilungen über Forschungsarbeiten auf d. Gebiete d. Ingenieurwesens. H. 134. 1913; Ausz. in: Zeitschrift d. Vereines deutscher Ingenieure. Bd 56, Halbj. 2.

Berlin, Dr.-Ing.-Diss. v. 2. 2. 1912

\section{ABTEILUNG FÜR SCHIFF- UND SCHIFFSMASCHINEN-BAU}

\section{Schiffbau}

508. Sehultz, Clarence B[enjamin]: Uber die Vergrösserung der Handelsdampfer mit Rücksicht auf ihre Rentabilität. 1903. 111 S., 1 Tab. $8^{\circ}$

Berlin, Dr.-Ing.-Diss. v. 26. 6. 1903

509. Thele, Walter: Vibrationserscheinungen neuerer Schnelldampfer. 1907. $16 \mathrm{~S}$. $4^{0}$ Aus: Schiffbau. Jg. 8. 1906/07; Ausz. in: Zeitschrift d. Vereines deutscher Ingenieure. Bd 51. Berlin, Dr.-Ing.-Diss. v. 22. 3. 1907

510. Matthaei, Wilhelm 0[tto]: Kritische Beleuchtung der Yachtmessverfahren. 1907. 91 S., 1 Taf. $8^{\circ}$

Berlin, Dr.-Ing.-Diss. v. 31. 5. 1907

511. Sachsenberg, Ewald: Über den Widerstand von Schleppzügen. [1907.] 31 S., 10 Taf. $4^{0}$

Berlin, Dr.-Ing.-Diss. v. 28. 6. 1907

512. Foerster, Ernst: Die Bedeutung der flüssigen Feuerung für Konstruktion, Betrieb und Rentabilität eines transatlantischen Schnelldampfers. Berlin: Schiffbau 1907. 23 S., 3 Taf. $4^{0}$ - Aus: Sehiffbau. Jg. 9. 1907/08.

Berlin, Dr.-Ing.-Diss. v. 18. 10. 1907

513. Weitbrecht, Martin: Der über seine starre Unterlage überhängende, nicht eingespannte Balken sowie die Druckverteilung unter dem Ablaufschlitten eines Schiffes während des Stapellaufes mit Berücksichtigung der elastischen Formänderungen des Schiffskörpers. Berlin: Schiffbau 1908. 12 S., 3 Taf. $4^{\circ}$ - Aus: Schiffbau. Jg. 9. $1907 / 08$.

Berlin, Dr.-Ing.-Diss. v. 12. 6. 1908

514. Siemann, Riehard: Elastische Formänderung des Schiffskörpers. [1909.] 16 S.,

2 Taf. $4^{0}$ - Aus: Schiffbau. Jg. 11. 1909/10. Berlin, Dr.-Ing.-Diss. v. 11. 6. 1909
515. Moll, Friedrich: Untersuchung über die Ursachen des Unterganges der verschollenen Fischdampfer. (Berlin: Marfels) [1910]. $16 \mathrm{~S} .4^{0}$ - Aus: Schiffban. Jg. 11. $1909 / 10$.

Berlin, Dr.-Ing.-Diss. v. 16. 7. 1909

516. Gümbel, Ludwig: Fabrikorganisation mit spezieller Berücksichtigung der Anforderungen der Werftbetriebe. Berlin: Springer (1910). S. $329-436,10$ Taf. $4^{\circ}$ T Aus: Jahrbuch d. Schiffbautechn. Gesellschaft. Bd 11.

Berlin, Dr.-Ing.-Diss. v. 28. 1. 1910

517. Sehoeneich, Hugo: Die Beanspruchungen des Rudergeschirrs auf Seeschiffen. [1910.] $49 \mathrm{~S} ., 3$ Taf. $4^{0}$

Berlin, Dr.-Ing.-Diss. v. 8. 4. 1910

518. Horn, Fritz: Die dynamischen Wirkungen der Wellenbewegung auf die Längsbeanspruchung des Schiffskörpers. 1910. 118 S., 3 Taf. $4^{0}$ - Auch bei Springer in Berlin.

Berlin, Dr.-Ing.-Diss. v. 24. 6. 1910

519. Rothe, Johannes: Das Froudesche Gesetz, Untersuchungen über seine Anwendbarkeit zur Ermittlung des Schiffswiderstandes. Berlin: (Marfels) [1911]. 23 S. $4^{0}$ - Aus: Sehiffbau. Jg. 12. 1910/11.

Berlin, Dr.-Ing.-Diss. v. 3. 2. 1911

520. Roeh, Eugen: Handelsschiffbau und Klassifikationsgesellschaften. [1911.] $37 \mathrm{~S}$, 2 Taf. $4^{\circ}$

Berlin, Dr.-Ing.-Diss. v. 12. 5. 1911

521. Sehmidt, Reinhold: Ein schiffbautechnisch-kritischer Beitrag zurVermessungsfrage. Berlin: Marfels 1911. 27 S. $4^{0}$ - Aus: Schiffbau. Jg. 13. 1911/12.

Berlin, Dr.-Ing.-Diss. v. 12. 5. 1911

522. Asthöwer, Walter: Gleitgeschwindigkeit und Widerstand von Schleppkähnen. Nach Versuchen auf dem Rheinstrom. [1912.] $19 \mathrm{~S} ., 8$ Taf. $4^{\circ}$

Berlin, Dr.-Ing.-Diss. v. 15. 12. 1911

523. Tillmann, Max, Baumeister d. Schiffbaufachs: Anker und Ankerketten im Seeschiffsbetrieb <Arbeitsvorgänge und Größenbestimmung >. 1912. 22 S., 6 Taf. $4^{\circ}$ Berlin, Dr.-Ing.-Diss. v. 15. 12. 1911

524. Commentz, Carl: Die Grundlagen der Rentabilität von Frachtschiffen und ihre Beeinflussung durch die moderne Technik. Charlottenburg: Deutsche Schiffahrt [1912].

IV, 108 S., 7 Taf. $8^{\circ}$

Berlin, Dr.-Ing.-Diss. v. 21. 6. 1912 


\section{Schiffsmaschinenbau}

525. Pröll, Arthur: Kraft- und Festigkeits - Verhältnisse bei SchiffsmaschinenSteuerungen. 1906. 16 S. $4^{\circ}$ - Aus: Schiffbau. Jg. 7. 1905/06.

Berlin, Dr.-Ing.-Diss. v. 11. 1. 1907

526. Staueh, Adolf: Ueber den elektrischen Antrieb des Schiffssteuers. Berlin: Schiffbau 1908. 35 S. $4^{\circ}$ - Aus: Schiffbau. Jg. 9. 10. 1907/08. 08/09.

Berlin, Dr.-Ing.-Diss. v. 17. 7. 1908

527. Praetorius, Paul, Marinebaumeister: Die fortlaufende indikatorischeUntersuchung von Rudermaschinen während der Rudermanöver. Eine Methode zur Feststellung der beim Manövrieren von Schraubenschiffen erzeugten Rudermomente bzw. Ruderdrücke. [1909.] 25 S., 3 Taf. $4^{\circ} \bullet$ Ein Teil in: Sehiffbau. Jg. 8. 1906/07.

Berlin, Dr.-Ing.-Diss. v. 12. 3. 1909

528. Jahn, Johannes: Ventilsteuerungen für Schiffsmaschinen. [1909.] $29 \mathrm{~S} . \quad 4^{\circ}$ - Aus: Schiffbau. Jg. 11. 1909/10.

Berlin, Dr.-Ing.-Diss. v. 26. 3. 1909

529. Linnenbrügge, Hans: Über eine neue graphische Methode zur Untersuchung der Schiffsschaufelräder. [1910.] 35 S., 20 Taf. $8^{\circ}$

[Berlin,] Dr.-Ing.-Diss. v. 25. 6. 1909

530. Schmidt, Rudolf: Verwendung des Heissdampfes in für Sattdampf gebauten Schiffsmaschinenanlagen. [1910]. 29 S. $4^{0}$ Berlin, Dr.-Ing.-Diss. v. 11. 3. 1910

531. Besig, Karl, Kgl. Oberlehrer in Stettin: Die Dampfturbine als Schiffsmotor. Vergleichsrechnung für verschiedene Systeme (Zoelly, Rateau, Curtis, Parsons, Melms-Pfenninger $\rangle$. [1911.] 75 S., 8 Taf. $8^{\circ}$

- Auch bei Springer in Berlin.

Berlin, Dr.-Ing.-Diss. v. 13. 1. 1911

\section{ABTEILUNG FÜR CHEMIE UND HÜTTENKUNDE}

\section{Chemie}

532. Bandow, Erieh: I. Beiträge zur Kenntnis des Isonarkotins. II. Ueber zwei neue Basen aus Hydrocotarnin. 1901. $45 \mathrm{~S} .8^{\circ}$

Berlin, Dr.-Ing.-Diss. v. 14. 6. 1901

533. Hoyer, Emil: I. Ueber einige Abkömmlinge des 2.3-Dibrom- $\alpha$-Naphtochinons.
II. Ueber einige Abkömmlinge des Anhydrobisdiketohydrindens $\langle$ Bïndons $\rangle$. 1901.43S. $8^{\circ}$ - Vgl. Berichte d. Deutschen Chem. Gesellschaft. Jg. 34

Berlin, Dr.-Ing.-Diss. v. 14. 6. 1901

534. Glawe, Alfred: Ueber Dihalogenindone. Ihr Verhalten gegen Schwefelsäure, Salpetersäure und Natriumalkoholate. 1902. 75 S. $8^{\circ}$ V Vgl. Berichte d. Deutschen Chem. Gesellschaft. Jg. 35.

Berlin, Dr.-Ing.-Diss. v. 20. 6. 1902

535. Boeters, Oskar, Kontreadmiral z. D.: Ueber Reaktionen der Dihalogenthymochinone. 1902, $46 \mathrm{~S} .8^{0}$ ๆ Vgl. Berichte d. Deutschen Chem. Gesellschaft. Jg. 35.

Berlin, Dr.-Ing.-Diss. v. 10. 10. 1902

536. Collett, Emil: Ueber Anilidoaldehyde. 1903. $52 \mathrm{~S} .8^{\circ}$

Berlin, Dr.-Ing.-Diss. v. 9.1. 1903

537. Briick, 0swald: Ueber die Konstitution der Dibromphtalsäure. Ueber einige Derivate der 4.5.Dibromphtalsäure. 1904. 68 S. $8^{0}$

Berlin, Dr.-Ing.-Diss. v. 13. 3. 1903

538. Ozorovitz, Naftaly: Zur Kenntnis der Dioxyfluoresceïne. 1903. 60 S. $8^{\circ}$ - Vgl. Berichte d. Deutschen Chem. Gesellschaft. Jg. 36 .

Berlin, Dr.-Ing.-Diss. v. 24. 4. 1903

539. Kollmann, Ernst: Über die Gautier'sche Verbindung $\mathrm{P}_{5} \mathrm{H}_{3} \mathrm{O}$. 1903. $47 \mathrm{~S} .8^{\circ}$

Berlin, Dr.-Ing.-Diss. v. 22. 5. 1903

540. Wenzel, Georg: Über die Einwirkung von Halogen und Schwefelkohlenstoff auf Natriummethylenverbindungen. 1903. 32 S. $8^{\circ}$

Berlin, Dr.-Ing.-Diss. v. 26. 6. 1903

541. Book, Gilbert: Ueber die Reaktion von Aceton gegen Nitroopiansäure und einige neue Chinaldinderivate. $1903.45 \mathrm{~S} .8^{\circ}$ - Vgl. Berichte d. Deutschen Chem. Gesellschaft. Jg. 35. 1902.

Berlin, Dr.-Ing.-Diss. v. 17. 7. 1903

542. Engelskirchen, Peter: Beiträge zur Kenntnis der Salze der Kiesel- und Titanfluorwasserstoffsäure. 1903.46 S. $8^{\circ}$

Berlin, Dr.-Ing.-Diss. v. 17. 7. 1903

543. Wolff, Albert: Ueber das Verhalten organischer Persulfate beim Erhitzen. Ein Fall von Pseudomorphose. 1903. 55 S. $8^{\circ}$ If Vgl. Berichte d. Deutschen Chem. Gesellschaft. Jg. 37. 1904.

Berlin, Dr.-Ing.-Diss. v. 17. 7. 1903 
544. Herz, Paul: Ueber den Bidioxymethylenindigo, seinen Auf- und Abbau. 1905. 34 S. $8^{\circ}$

Berlin, Dr.-Ing.-Diss. v. 14. 7. 1905

545. Kropf, Fritz: Über Kondensationen des Cotarnins. 1905. $33 \mathrm{~S} .8^{\circ}$ - Vgl. Berichte d. Deutschen Chem. Gesellschaft. Jg. 37. 1904.

Berlin, Dr.-Ing.-Diss. v. 14. 7. 1905

546. Luft, Max: Ueber cyklische Basenderivate des 4-Aminoantipyrins. 1906.

$36 \mathrm{~S} .8^{0}$ ๆ Vgl. Beriehte d. Deutschen Chem. Gesellschaft. Jg. 38. 1905.

Berlin, Dr.-Ing.-Diss. v. 12. 1. 1906

547. Faber, Paul: Beiträge zur Kenntnis des sechswertigen Titan. 1906. $38 \mathrm{~S} .8^{\circ}$ T Ausz. in: Zeitsehrift f. analyt. Chemie. Jg. 46. 1907.

Berlin, Dr.-Ing.-Diss. v. 23. 3. 1906

548. Müller, Julius: Beiträge zur Kenntnis der Metaphosphate. 1906. 42 S. $8^{\circ}$

Berlin, Dr.-Ing.-Diss. v. 23. 11. 1906

549. Celichowski, Kasimir: Uber $\beta$-Methyläskuletin und Äskuletin- $\beta$-carbonsäure. 1907. 41 S. $8^{0}$

Berlin, Dr.-Ing.-Diss. v. 22. 3. 1907

550. Auerbach, Herbert: Spektroskopische Untersuchungen über das Verhalten der Metallsalze in Flammen von verschiedener Temperatur. 1907. 47 S. $8^{\circ}$ - Mit unwesentl. Ånderungen in: Zeitschrift f. wissensehaftl. Photographie, Photophysik u. Photochemie. Bd 7. 1909.

Berlin, Dr.-Ing.-Diss. v. 13. 12. 1907

551. Smissen, Heinrich van der: Beiträge zur Kenntnis der chemischen Eigenschaften des Kalziummetalls. 1907. 35 S. $8^{0}$ Berlin, Dr.-Ing.-Diss. v. 13. 12. 1907

552. Haslinger, Carl: Über die Einwirkung auf Äthylamin auf Isatine. 1908. 52 S. $8^{\circ}$ - Vgl. Berichte d. Deutschen Chem. Gesellschaft. Jg. 40. 1907.

Berlin, Dr.-Ing.-Diss. v. 26. 6. 1908

553. Mardus, Georg: Elektrolytische Raffination von Blei aus borfluorwasserstoffsaurer Lösung. 1908. 56 S. $8^{\circ}$

Berlin, Dr.-Ing.-Diss. v. 26. 6. 1908

554. Sehulte, Wilhelm: Über die Abscheidung des Antimons aus seiner Sulfantimoniatlösung. 1908. $76 \mathrm{~S} .8^{\circ}$ - Ausz.

in: Metallurgie. Jg. 6. 1909.

Berlin, Dr.-Ing.-Diss. v. 26. 6. 1908

555. Dahmen, Reiner: Ueber den Aufbau von Diphenylaminderivaten aus p-Nitrochlorbenzol. 1908. $59 \mathrm{~S} .8^{\circ}$ - Vgl. Berichte d. Deutschen Chem. Gesellschaft. Jg. 41. Berlin, Dr.-Ing.-Diss. v. 17. 7. 1908
556. Trucksaess, Hans: Ueber Glaukophansäure. 1908. $49 \mathrm{~S} .8^{\circ}$ - Vgl. Berichte d. Deutschen Chem. Gesellschaft. Jg.40. 1907. Berlin, Dr.-Ing.-Diss. v. 17. 7. 1908

557. Wentzel, Fritz: Beiträge zur optischen Sensibilisation der Chlorsilbergelatine. 1908. 117 S. $8^{\circ}$ - Ausz. in: Zeitschrift f. wiss. Photographie, Photophysik u. Photochemie. Bd 7. 1909.

Berlin, Dr.-Ing.-Diss. v. 17. 7. 1908

558. Thielseh, Max: Über das symmetrische Trichlornitrobenzol und verwandte Verbindungen. 1909. $40 \mathrm{~S} .8^{\circ}$

Berlin, Dr.-Ing.-Diss. v. 22. 1. 1909

559. Michaelis, Kurt: Ueber die Reaktion der Gruppe $\langle\mathrm{CO}-\mathrm{CX}=\mathrm{CX}\rangle$ in Chinon- und Indonkernen. Ueber die Reaktionen zwischen asymmetrisch substituierten $\mathrm{Hy}$ drazinen und Harnstoffderivaten. [1909.] 63 S. $8^{\circ}$

Berlin, Dr.-Ing.-Diss. v. 26. 2. 1909

560. Danaila, Negoitza: Ueber die Konstitution der Phenol- und Dimethylanilinisatine und ihrer Farbabkömmlinge. [1909.] 67 S. $8^{\circ}$ Vgl. Berichte d. Deutschen Chem. Gesellschaft. Jg. 40. 1907; Comptes rendus hebdomadaires des séances de l'Académie des Sciences. T.149. S. 793; Le Moniteur Scientifique du Docteur Quesneville. T. 72. Sér. 4, T. 24, P. 1. 1910. S. $22 \mathrm{ff}$.

Berlin, Dr.-Ing.-Diss. v. 12. 3. 1909

561. Hollaender, Josef: Studien in der Cumarinreihe. 1909. 66 S. $8^{0}$

Berlin, Dr.-Ing.-Diss. v. 12. 3. 1909

562. Rindl, Max: Studien über Trinitrochlornaphtaline. [1909.] $53 \mathrm{~S} .8^{\circ}$

Berlin, Dr.-Ing.-Diss. v. 11. 6. 1909

563. Quensell, Hermann: Ueber Glycerinester der Stearolsäure und Behenolsäure. [1909.] $70^{\circ} \mathrm{S} .8^{\circ}$ ๆ Vgl. Berichte d. Dentschen Chem. Gesellschaft. Jg. 42.

Berlin, Dr.-Ing.-Diss. v. 25. 6. 1909

564. Renker, Max: Über Bestimmungsmethoden der Cellulose. 1909. 106 S. $8^{\circ}$ - Ausz. in: Zeitschrift f. angewandte Chemie. Jg. 23, 1. 1910; 2. verb. Aufl. bei Borntraeger in Berlin als: Schriften d. Vereines d. Zellstoff- u. Papier-Chemiker. H. 1. 1910.

Berlin, Dr.-Ing.-Diss. v. 16. 7. 1909

565. Schmiedt, Friedrieh: Beiträge zur elektrolytischen Oxydation des Chroms. [1909.] 56 S. $8^{\circ}$

Berlin, Dr.-Ing.-Diss. v. 26. 11. 1909

566. Wolff, Justus: Über 2.6-Dichlorp-phenylendiamin. 1909. 35 S. $8^{\circ}$

Berlin, Dr.-Ing.-Diss. v. 26. 11. 1909 
567. Landsberger, Felix: Über Nitroeumarine und ihre Konstitutionsermittelung. 1910. $63 \mathrm{~S} .8^{\circ}$

Berlin, Dr.-Ing.-Diss. v. 27. 5. 1910

568. Lampe, Erieh Hermann: Beiträge zur Alkalichlorid-Elektrolyse. Utber den Einfluss der Salze des Urans, des Wolframs, des Molybdäns, des Vanadins und der Phosphorsäure. [1910.] 65 S. $8^{\circ}$

Berlin, Dr.-Ing.-Diss. v. 10. 6. 1910

569. Singer, Felix: Ueber künstliche Zeolithe und ihren konstitutionellen $\mathrm{Zu}$ sammenhang mit andern Silicaten. [1910.] $72 \mathrm{~S} .8^{\circ}$ Ausz. in: Transactions of the American Ceramic Society. Vol. 33; Sprechsaal. Jg. 44. 1911. S. 325 ff.

Berlin, Dr.-Ing.-Diss. v. 24. 6. 1910

570. Kellermann, Heinrich: Ueber die Darstellung des metallischen Cers und seine Verbindungen mit Arsen und Antimon. [1910.] 47 S. $8^{\circ}$ - Zum Teil erw. od. gekürzt in: Kellermann: Die Ceritmetalle u. ihre pyrophoren Legierungen. Halle: Knapp 1912. Monographien über chem.-techn. FabrikationsMethoden. Bd 27.)

Berlin, Dr.-Ing.-Diss. v. 15. 7. 1910

571. Loebel, Avram: Utber die Einwirkung von Organomagnesiumverbindungen auf $o$ Aldehydophenoxyessigsäure und die Überführung der entstehenden Produkte in Derivate des Cumarons. 1910. 48 S. $8^{\circ}$

Berlin, Dr.-Ing.-Diss. v. 15. 7. 1910

572. Stockfiseh, Karl: Einwirkung von Alkylmagnesiumhaloiden auf Anhydroecgonin- und d- $\psi$-Eegoninester. $1910.36 \mathrm{~S} .8^{\circ}$

Berlin, Dr.-Ing.-Diss. v. 15. 7. 1910

573. Zablinsky, Karl: Beiträge zur Kenntnis des Metanicotins. 1910.33 S. $8^{\circ}$ Berlin, Dr.-Ing.-Diss. v. 2. 12. 1910

574. Braun, 0tto: Studien über Acetonaphtole. $1910.72 \mathrm{~S} .8^{\circ}$

Berlin, Dr.-Ing.-Diss. v. 16. 12. 1910

575. Sell, Hans: Uber die Umwandlung von Kalkstickstoff in Cyanid. [1911.] 45 S. $8^{\circ}$

Berlin, Dr.-Ing.-Diss. v. 16. 12. 1910

576. Werner, Paul: Beiträge zum Nachweis von Beimischungen tierischen Fettes zu Pflanzenfett mit Hilfe von Cholesterin und Phytosterin. 1911. 50 S. $8^{\circ}$

Berlin, Dr.-Ing.Diss. v. 16. 12. 1910

577. Billig, Gerhard: Untersuchungen in der Anthrachinon-Reihe. [1911.] $56 \mathrm{~S} .8^{\circ}$

Berlin, Dr.-Ing.-Diss. v. 10. 3. 1911
578. Fodor, Otto: Studien in der Antrachinon-Reihe. [1911.] $66 \mathrm{~S} .8^{\circ}$ ๆ Vgl. J. Liebig's Annalen d. Chemie. Bd 380.

Berlin, Dr.-Ing.-Diss. v. 10. 3. 1911

579. Nicoleseu〈0tin $\rangle$, Cristea: Beiträge zur Kenntnis der auf elektrochemischem Wege gewonnenen Oxydationsprodukte des Wismuts. Berlin: Blanke [1911]. 62 S. $8^{\circ}$

Berlin, Dr.-Ing.-Diss. v. 10. 3. 1911

580. Zsuffa, Milan: Über einige Derivate des Acenaphthenchinons. 1911. 25 S. $8^{\circ}$ T Ein Teil ersch. in: Berichte d. Deutschen Chem. Gesellschaft. Jg. 43. 1910.

Berlin, Dr.-Ing.-Diss. v. 10. 3. 1911

581. Wäser, Bruno: Die Reduktion der Ölsäure auf elektrochemischem Wege. 1911. 48 S. $8^{\circ}$

Berlin, Dr.-Ing.-Diss. v. 12. 5. 1911

582. Beyer, Johannes: Studien über die Gleichgewichtsbedingungen zwischen Wasserstoff-Wasserdampf-Gemischen und Eisenoxyden. 1911. $33 \mathrm{~S} .8^{\circ}-\mathrm{Vgl}$. Berichte d. Deutschen Chem. Gesellschaft. Jg. 44.

Berlin, Dr.-Ing.-Diss. v. 26. 5. 1911

583. Kroll, Adolphe Victor: UUber Ultraphosphate. Leipzig u. Hamburg: Voss 1912. $76 \mathrm{~S} .8^{\circ}$ A Aus: Zeitschrift f. anorgan. Chemie. Bd 76. 77.

Berlin, Dr.-Ing.-Diss. v. 15. 12. 1911

584. Schuller, Aladár: Sensitometrie photographischer Prozesse. [1912.] 64 S. $8^{\circ}$ - Vgl. Schuller: Fotografiai, eljárások szenzitométriája. Budapest: O. Nagel 1911; der Abschnitt "Die Sensitometrie des Pigmentprozesses" etwas abgeändert in: Zeitschrift f. Reproduktionstechnik. Jg. 15. 1913.

Beriin, Dr.-Ing.-Diss. v. 2. 2. 1912

585. Teodorin, Joan: Studien über rumänische Erdölprodukte. Ein Beitrag zur Lehre von der Spaltung der Kohlenwasserstoffe. 1912. 51 S. $8^{\circ}$

Berlin, Dr.-Ing.-Diss. v. 2. 2. 1912

586. Stecher, Emil: Zwei neue elektroanalytische Schnellmethoden. Bildung der Ueberschwefelsäure bei ausserordentlich hohen Stromdichten und der Einfluß anodischer Innenkühlung bei diesen Stromdichten. [1912.] 62 S. $8^{\circ}$ - Ausz. aus T. 1 in: Zeitschrift \&. Elelstrechemie. Jg. 18.

Berlin, Dr.-Ing.-Diss. v. 24. 5. 1912

587. Gebhardt, Adolf: Über Phtaloine 〈Benzoine der Phthalreihe.) Ein Beitrag zur Kenntnis der Vorgänge bei der Benzoinbildung. 1912. $48 \mathrm{~S} .8^{\circ}$ - Soll später auszugsweise veröffentlicht werden in: Berichte d. Deutschen Chem. Gesellschaft; bis 1913 nicht ersch.

Berlin, Dr.-Ing.-Diss. v. 21. 6. 1912 
588. Memmel, Christian: Über Versuche zur Darstellung eines Isomeren des Histidins und die Konstitution des Kondensationsproduktes aus $5\langle 4\rangle$-Methyl-imidazol und Chloral. [1912.] 47 S. $8^{\circ}$ - Teilweise u. gekürzt in: Berichte d. Deutschen Chem. Gesellschaft. Jg. 45. S. $509 \mathrm{ff}$.

Berlin, Dr.-Ing.-Diss. v. 21. 6. 1912

589. Ürményi, Dezsö: Ueber Anthrachinonxanthone. [1912.] $56 \mathrm{~S} .8^{\circ}$ ๑ Vgl. Berichte d. Deutschen Chem. Gesellschait. Jg. 45.

Berlin, Dr.-Ing.-Diss. v. 21. 6. 1912

590. Guggiari, Pedro Bruno: Zur Kenntnis der Farblacke, welche die Hydroxylfarbstoffe mit oxydischen Beizen, speziell mit solchen von selteneren Metallen, bilden. [1912.] 90 S., 3 Tab. $8^{\circ}$ I Vgl. Berichte d. Deutschen Chem. Gesellschaft. Jg. 45.

Berlin, Dr.-Ing.-Diss. v. 12. 7. 1912

591. Buteseu, Dimitrie: Einwirkung von Oxalylchlorid auf substituierte Anthracene. Berlin: Ebering [1912]. 45 S. $8^{0} \bullet$ Vgl. Beriehte d. Deutschen Chem. Gesellschat̂t. Jg. 45. 46. 1912. 13.

Berlin, Di.-Ing.-Diss. v. 8. 11. 1912

\section{Hüttenkunde}

592. Waldeck, Karl: Gasanalytische Untersuchungen an Bleischachtöfen. 1901. 35 S., 1 Taf. $8^{\circ}$

Berlin, Dr.-Ing.-Diss. v. 14. 6. 1901

593. Lukaszezyk, Jacob: Beiträge zur Erz-Aufbereitung. Die Vorteile des schiefen Stosses bei ebenen Stossherden. Freiberg i.S.: [Craz \& Gerlach in Komm.] 1902. 46S., 8 Taf. $8^{\circ}$

Berlin, Dr.-Ing.-Diss. v. 11. 7. 1902

594. Sadlon, Alfred: Beiträge zur Chemie des Zinkblende-Röst - Prozesses. 1903. 48 S. $8^{\circ}$

Berlin, Dr.-Ing.-Diss. v. 22. 5. 1903

595. Böhler, 0tto: Über Wolfram- und Rapidstahl. 1903. 57 S., 4 Taf. $8^{\circ}$

Berlin, Dr.-Ing.-Diss. v. 17. 7. 1903

596. Bublitz, Erich: Untersuchungen über die Einwirkung des Wasserstoffs auf die Sauerstoffverbindungen des Mangans. 1903. 35 S. $8^{\circ}$

Berlin, Dr.-Ing.-Diss. v. 17. 7. 1903

597. Unger, 0tto: Beiträge zur Chemie der Cadmiumgewinnung. 1904. $52 \mathrm{~S} .8^{\circ}$

Berlin, Dr.-Ing.-Diss. v. 15. 7. 1904
598. Philips, Moritz: Beiträge zur Kenntnis des Kupfersilicids. 1904.63 S. $8^{\circ}$ Berlin, Dr.-Ing.-Diss. v. 13. 1. 1905

599. Bräutigam, Max: Studien über die Kohlenwasserstoffe, welche bei der Behandlung kohlenstoff- und manganhaltigen Eisens mit verdünnten Säuren entwickelt werden, und über die Beziehungen dieser Kohlenwasserstoffe zu den Kohlenstoffformen im Eisen. 1905. 52 S. $8^{\circ}$

Berlin, Dr.-Ing.-Diss. v. 15. 12.1905

600. Anton, Alfred: Beiträge zur Kenntnis der Eisenkohlenstoffverbindungen und der Konstitution des Kohlenstoffeisens. 1905. 57 S. $8^{\circ}$

Berlin, Dr.-Ing.-Diss. v. 12. 1. 1906

601. Kassel, Georg: Beiträge zur Kenntnis der Reduktion von Eisenschlacken durch Kohlenoxyd und Wasserstoff. 1906. 46 S. $8^{\circ}$ - Ganz kurzer Ausz. in: Stahl u. Eisen. Jg. 26, Halbj. 2.

Berlin, Dr.-Ing.-Diss. v. 23. 3. 1906

602. Kumpmann, Walter: Beiträge zur Kenntnis der Reduktion von Zinkoxyd durch Wasserstoff und Kohlenoxyd. 1906. 51 S. $8^{\circ}$

Berlin, Dr.-Ing.-Diss. v. 29. 6. 1906

603. Wilms, otto: Über Kohlenstoffnickel. 1907. 54 S., 2 Taf. $8^{\circ}$

Berlin, Dr.-Ing.-Diss. v. 22. 3. 1907

604. Steffe, Hermann: Über die Bildungstemperaturen einiger Eisenoxydul-KalkSchlacken und einiger kalkfreien Eisenoxydul-Schlacken, deren Kenntnis für das Verschmelzen der Bleierze Bedeutung hat 1908. 21 S. $8^{\circ}$

Berlin, Dr.-Ing.-Diss. v. 27. 3. 1908

605. Nugel, Karl: Beiträge zur Kenntnis der Betriebslaugen des Cyanidprozesses. 1908. 30 S. $8^{\circ}$ - Auch in: Metallurgie. Jg. 5 .

Berlin, Dr.-Ing.-Diss. v. 12. 6. 1908

606. Theusner, Martin: Beiträge zur Erweiterung der bisherigen Kenntnisse von der Konstitution natürlicher und künstlicher Schlacken. 1908. 67 S., 1 Taf. $8^{\circ}$ - Ausz. in: Metallurgie. Jg. 5.

Berlin, Dr.-Ing.-Diss. v. 12. 6. 1908

607. Philippi, Heinrieh: Schmelz- und Lösungsversuche in der Reihe Kalk-Kieselsäure. 1908. 54 S. $8^{\circ}$

Berlin, Dr.-Ing.-Diss. v. 26. 6. 1908

608. Saklatwalla, Byramji: Phosphoreisen, seine Konstitution. 1908. 38 S. $8^{\circ}$ I Ausz. in: Metallurgie. Jg. 5.

Berlin, Dr.-Ing.-Diss. v. 26. 6. 1908 
609. Friedmann, Ignatz: Ueber Zinkcyanid und Zinkalkalidoppelcyanidverbindungen in den Arbeitslösungen des Prozesses der Goldgewinnung und ihren Einfluss auf den Verlauf des Prozesses. 1908. 62 S. So

Berlin, Dr.-Ing.-Diss. v. 17. 7. 1908

610. Lepiarezyk, Vietor: Beiträge zur Chemie des Zinkhüttenprozesses. [1909.] $14 \mathrm{~S} .4^{\circ}$ - Ohne die SchluBbemerkungen in: Metallurgie. Jg. 6 .

Berlin, Dr.-Ing.-Diss. v. 8. 1. 1909

611. Hanemann, Heinrich: Über die Reduktion von Silicium aus Tiegelmaterialien durch geschmolzenes kohlehaltiges Eisen. [1909.] 21 S. $8^{\circ}$

Berlin, Dr.-Ing.-Diss. v. 22. 1. 1909

612. Puppe, J[ohann]: Ueber Versuche zur Ermittlung des Kraftbedarfs an Walzwerken. 1909. 191 S., 8 Taf. $4^{\circ}$ - Auch bei Stahleisen in Düsseldorf.

Berlin, Dr.-Ing.-Diss. v. 12. 3. 1909

613. Weiller, Paul: Die Bleisilikate. Halle: Knapp [1909]. 14 S., 2 Taf. $4^{\circ}$ - Vgl. Berichte d. Deutschen Chem. Gessellschaft. Jg. 42.

Berlin, Dr.-Ing.-Diss. v. 14. 5. 1909

614. Kahnert, Paul: Studien über die Durchführung des Roheisen-Erz-Prozesses im Martinofen. [1909.] 40 S. $8^{\circ}$

Berlin, Dr.-Ing.-Diss. v. 11. 6. 1909

615. Kohlmeyer, Ernst J[ustus]: Uber die Calciumferrite, ihre Konstitution und ihr Auftreten in hüttenmännischen Prozessen. Halle: Knapp 1909. $41 \mathrm{~S} ., 1$ Taf. $4^{\circ}$ - Ausz. in: Berichte d. Deutschen Chem. Gesellschaft. Jg. 42 S. 1-31 mit unerhebl. Änderungen in: Metallurgie, Jg. 7. 1910.

Berlin, Dr.-Ing.-Diss. v. 16. 7. 1909

616. Blome, Hermann: Beiträge zur Konstitution der Thomasschlacke. Halle Knapp [1910]. 21 S. $4^{\circ}$ - Auch in: Metallurgie. Jg. 7 .

Berlin, Dr.-Ing.-Diss. v. 13. 5. 1910

617. Klönne, Theodor: Verringerung der Selbstkosten in Adjustagen und Lagern von Stabeisenwalzwerken. Berlin: Springer 1910. 124 S., 2 Taf. $8^{\circ}$ - Auch als Buch. Berlin, Dr.-Ing.-Diss. v. 15. 7. 1910

618. Jung, Adalbert: Studie über die Einwirkung thermischer Behandlung auf die Festigkeitseigenschaften und die Mikrostruktur hypereutektoider Stähle. Berlin: Borntraeger 1911. $46 \mathrm{~S} ., 12$ Taf. $4^{\circ}$ Aus: Internat. Zeitschrift f. Metallographie. Bd 1. Berlin, Dr.-Ing.-Diss. v. 2. 12. 1910
619. Thomsen, Kurt: Beiträge zur Kenntnis der Löslichkeit des Graphits im festen Eisen und der Schmelzerscheinungen des grauen Roheisens. [1911.] 24 S. 5 Taf. $4^{\circ}$

Berlin, Dr.-Ing.-Diss. v. 16. 12. 1910

620. Metz, Norbert: Studien über die im Hochofen zwischen den Eisenerzen und Gasen obwaltenden Verhältnisse. Düsseldorf: Stahleisen 1912. $30 \mathrm{~S} .4^{\circ}$ - Ausz. in: Stahl u. Eisen. Jg. 33. 1913, Halbj. 1.

Berlin, Dr.-Ing.-Diss. v. 24. 2. 1911

621. Steinweg, Eugen: Die Konstitution des vierbasischen Kalkphosphates und seine Reduzierbarkeit durch kohlenstoffhaltiges und reines Eisen. Halle: Knapp [1911]. 18 S. $4^{0}$ - Aus: Metallurgie. Jg. 9. 1912.

Berlin, Dr.-Ing.-Diss. v. 12. 5. 1911

622. Kurek, Franz: Beiträge zur Kenntnis der Zementation des Eisens mittels Gasen. [1911.] 21 S. $4^{0}$ - Ausz. in : Stahl u. Eisen. Jg. 32. 1912.

Berlin, Dr.-Ing.-Diss. v. 26. 5. 1911

623. Dieckmann, Theodor: Uber einige Mono- und Bi-Arsenide des Eisens, Mangans und Chroms, über ihre chemischen und magnetischen Eigenschaften, sowie über die magnetischen Eigenschaften einiger ManganWismut-Legierungen. 1911. 36 S. $8^{0}$

Berlin, Dr.-Ing.-Diss. v. 14. 7. 1911

624. Mathesius, Walther: Studie über die magnetischen Eigenschaften von Manganund Nickelstahl. [1911.] 30 S., 1 Taf. $4^{0}$ - Vgl. Stahl u. Eisen. Jg. 32. 1912, Halbj. 1; Zeitschrift f. Elektrochemie u. angewandte physik.
Chemie. Jg. 18. 1912.

Berlin, Dr.-Ing.-Diss. v. 14. 7. 1911

625. Schraube, Gustav: Uber das Gleichgewicht des Generatorgases. [1912.] 39 S. $8^{\circ}$

Berlin, Dr.-Ing.-Diss. v. 14. 7. 1911

626. Schäfer, Rudolf: Über den Schwefel bei der Roheisendarstellung. [1912.] $90 \mathrm{~S} .4^{\circ}$ - Abschnitt 3, 1 mit geringfügigen Änderungen in Ferrum. Jg. 10. 1912/13

Berlin, Dr.-Ing.-Diss. v. 16. 2. 1912

627. Liedgens, Josef: Ueber den Einfluß des Arsens auf die Eigenschaften des Flußeisens. Düsseldorf: Stahleisen 1912. $22 \mathrm{~S} .4^{0}$ - Ausz. in: Stahl u. Eisen. Jg. 32, Halbj. 2.

Berlin, Dr.-Ing.-Diss. v. 24. 5. 1912

628. Nielsen, 0tto: Beiträge zum System Kalk - Phosphorsäure - Kieselsäure. Halle: Knapp 1912. 19 S. $4^{\circ}$ ๆ Auch in: Ferrum. Jg. 10. 1912/13.

Berlin, Dr.-Ing.-Diss. v. 12. 7. 1912 


\section{BRAUNSCHWEIG}

Gegründet 1745 unter dem Namen „Collegium Carolinum“ als allgemeine höhere Bildungsanstalt, 1808 in eine Militärschule umgewandelt, 1814 wiederhergestellt, 1835 mit einer technischen Abteilung ausgestattet, 1862 in eine "Polytechnische Schule" erweitert, seit 1877 "Herzogliche technische Hochschule Carolo-Wilhelmina“"

\section{SATZUNGEN ORDNUNGEN BESTIMMUNGEN}

\section{COLLEGIUM CAROLINUM $1745-1862$}

Vgl. J. J. Eschenburg: Entwurf einer Geschichte des Collegii Carolini in Braunschweig. Berlin u. Stettin 1812; darin S. 147 ff.: Verzeichniß der Druckschriften, welche das Collegium Carolinum betreffen. - Friedrich Koldewey: Braunschweigische Schulordnungen von den ältesten Zeiten bis zum Jahre 1828. Bd 1. Schulordnungen der Stadt Braunschweig. Berlin 1886. (Monumenta Germaniae Paedagogica. Bd 1,1.)

629. Gesetze für dieienigen, welche ins Collegium Carolinum aufgenommen werden. 1745. - 2. Ausg. 1745. - Abgedr. in: Koldewey. S. $217 \mathrm{ff}$.

Beide Ausg.: $\mathrm{Br}$

630. Serenissimi gnädigste Declaration, den, dem Collegio Carolino in Braunschweig verliehenen Burgfrieden betreffend, de dato Salzthalen, den 10. Julii 1745. 2 Bl. $8^{0}-$ [Dasselbe. 1 Bl. Plakatform ohne Titel.] - Abgedr. in: Fernerweite Nachricht [Nr 686]; Eschenburg. S. 155 ff.; Koldewey. S. 225 ff.

8: Br; Plakat: Br LandschB

631. [Serenissimi gnädigste Verordnung, das Leihen an die Studiosos Carolini betr. Beg.:] Von Gottes Gnaden Carl, Herzog zu Braunschw. und Lüneb. \&c.... (Dat. Gegeben Salzthalen, den 10ten Julii 1745.) 1 Bl. $8^{0}-$ [Dasselbe. 1 Bl. Plakatform. Beg.:] Von... zu Braunschweig und Lüneburg \&c. ... Abgedr. in: Fernerweite Nachricht [Nr 686]; Eschenburg. S. 158; Koldewey. S. 227. $\quad 8^{\circ}$ : Br; Plakat: $B r$ LandschB

632. [Das Gebet fürs Carolinum. Verf. nach Eschenburg. S. 26: Johann Christoph Köcher. Beg.: ] Walte auch, allmächtiger gütiger Gott. . [1745.] 1 Bl. $8^{0}$ - Abgedr. in: Fernerweite Nachricht [Nr 686]; Koldewey. S. $228 \mathrm{f}$.

Wolfenbüttel HzglB

633. Kurzgefaßte Puncte die Aufnahme ins Collegium Carolinum betr. [1745.] 2. Ausg. [u. d. T.:] Kurzgefaßte... Aufnahme in das... [1746.] - Abgedr. in: Koldewey. S. $243 \mathrm{ff}$.

1. Ausg.: Br; 2. Ausg.: Br LandschB

634. [Wiederholung der unter dem 10. 7. 1745 wegen des Creditirens an die Studiosos in dem Collegio Carolino ergangenen Ver- ordnung. Beg.: ] Von Gottes Gnaden Carl, Herzog zu Braunschweig und Lüneburg \&c. Wir haben mit grossem Mißfallen vernommen ... (Dat.: Gegeben in Unserer Vestung Wolfenbüttel, den 5ten Maji 1747.) Br

635. Serenissimi gnädigste. Declaration die Verminderung der von den sogenannten Semi-Carolinern für den Unterricht im Fürstl. Collegio Carolino künftig zu bezahlenden Lections- und Exercitien-Gelder betreffend. D. d. Braunschweig, den 29ten September, 1777. 2 Bl. $8^{0}-$ [Dasselbe. $1 \mathrm{Bl}$. Plakatform ohne Titel.] - Abgedr. in: Braunschweig. Anzeigen. Jahr 33. 1777. Sp. 889 f.; Eschenburg. S. 159 ff.; Koldewey. S. 420 f. 80: Br; Plakat: Br LandschB

636. Serenissimi erneuerte Verordnung, wegen des denen Studiosis auf dem CollegioCarolino hieselbst zu gebenden Credits. De dato Braunschweig, den 16ten Oct. 1783. 12 Bl. $8^{0}$ - [Dasselbe. $1 \mathrm{Bl}$. Plakatform.] 80: $\mathrm{Br}$ Landsch B; Plakat: $\mathrm{Br} \mathrm{StB}$

637. Erneuerte und vermehrte Gesetze des Collegii Carolini. [Verf. nach Eschenburg. S. 38: Carl Christian Gärtner. $] 1784$. $~ A b-$ gedr. in: Koldewey. S. 422 . Br LandschB

638. L o ix du College Carolin renouvellées \& augmentées, à Brunsvic en 1784 . [Verf.: Carl Christian Gärtner. Übers. von Nr 637.]

Br Landsch B

639. The $\mathrm{law}$ s of the Caroline College renovated and augmented. Brunswick 1784. [Verf.: Carl Christian Gärtner.] 1784. [Übers. von Nr 637.] WolfenbüttelHzglB

640. Gesetze für die Studirenden des Collegii Caro!ini in Braunschweig durch höchste Genehmigung bestätigt. 1802. - Abgedr. in : Eschenburg. S. 190 ff.; Koldewey. S. 458 ff.

641. Gesetze für die Studirenden des Collegii Carolini (v. 31. 3. 1823). 1823. $\tau$ Abgedr. in: Koldewey. S. $461 \mathrm{ff}$.

642. Allgemeine Bestimmungen über den Wirkungskreis, die Verhältnisse und Verpflichtungen des Directoriums, der Vorsteher und der Lehrer bei den drei Abtheilungen des Carolinums (v. 25. 3. 1836). 
POLYTECHNISCHE SCHULE UND TECHNISCHE HOCHSCHULE $1862-1912$

\section{ALLGEMEINES UND VERMISCHTES}

643. Bestimmungen für die Benutzung der technischen Bibliothek des Collegii Carolini durch die Studirenden (v. 1. 1. 1867).

644. Sehulgesetze für das Collegium Carolinum. [Wahrscheinlich 1872.]

645. Verwaltungs-0rdnung für das Herzogl. Collegium Carolinum zu Braunschweig (v. 24. 4. 1872). 1872.

646. Mittheilungen über die Herzogl. Polytechnische Schule (Collegium Carolinum> zu Braunschweig. (1877.)

647. Bestimmungen für die Benutzung der Bibliothek und des Lesezimmers der Herzogl. technischen Hochschule (v. 6. 10. 1877).

$\mathrm{Br}$

648. Hausordnung der Herzogl. technischen Hochschule zu Braunschweig (v. 18. 10. 1877).

649. Verfassung der Herzogl. technischen Hochschule ,Carolo-Wilhelmina" zu Braunschweig (v. 9. 3. 1878). 1878. - [Neudr.] 1883.

650. Herzogl. technische Hochschule zu Braunschweig. Laboratoriums-0rdnung [v. 1. 10. 1878].

651. Herzogl. technische Hochschule zu Braunschweig. Gesetze für die Studirenden und Zuhörer [v. 4. 8. 1879].

652. Verfügung des Herzogl. StaatsMinisteriums, betr. Abänderungen einzelner Bestimmungen der Verfassung der Herzogl. technischen Hochschule, bezw. eine Ergänzung derselben (v. 26. 7. 1887).

653. Satzungen der Krankencasse für die Studentenschaft der Herzogl. technischen Hochschule zu Braunschweig (v. 10.10. 1889).

654. Hausordnung der Herzogl. technischen Hochschule zu Braunschweig (v. 1. 5. 1892).

655. Bestimmungen für die Benutzung der Bibliothek und des Lesezimmers durch die Studirenden und Zuhörer der Herzogl. technischen Hochschule (v. 1. 10. 1892).

656. Satzungen der Krankencasse für die Studentenschaft der Herzogl. technischen Hochschule zu Braunschweig. Vom 29. 3. 1893.
657. Verfassung der Herzogl. technischen Hochschule Carolo-Wilhelmina zu Braunschweig (v. 30. 6. 1894). 1894. - (Neudr., April 1906, nebst den von dem Herzogl. Staatsministerium genehmigten Aenderungen). 1906.

658. Satzungen für die Studirenden der Herzogl. Techn. Hochschule Carolo Wilhelmina zu Braunschweig (v. Nov. 1894). 1895. (Enth.: A. Satzungen für die StudentenVersammlungen. B. Satzungen für den Ausschuß der Studirenden. [C.] LesezimmerOrdnung.) $\mathrm{Br}$

659. Satzungen der Krankencasse für die Studentenschaft der Herzogl. technischen Hochschule zu Braunschweig. Vom 26. 2. u. 18. 3. 1895 .

$\mathrm{Br}$

660. Herzogl. technische Hochschule zu Braunschweig. Gesetze für die Studirenden und Zuhörer. 〈Gültig vom 1. 10. 1896.〉

661. Satzungen der Krankencasse für die Studentenschaft der Herzogl. technischen Hochschule zu Braunschweig. Vom 1.7. 1897.

$\mathrm{Br}$

662. Bestimmungen, die Zulassung, die Rechte und die Pflichten der Privatdocenten an der Herzogl. technischen Hochschule zu Braunschweig betr. (v. 1. 10. 1897).

663. Herzogl. Technische Hochschule zu Braunschweig. Gesetze für die Studierenden und Zuhörer. 〈Gültig v. 1. 10. 1898.〉

664. Satzungen der Krankencasse für die Studentenschaft der Herzogl. technischen Hochschule zu Braunschweig. Vom 27. 3. 1899 (nebst Nachtrag zu $§ 8$ v. 8. 7. 1902). - [Neudr. mit in den Text aufgenommenem Nachtr.]

665. Satzungen für die Studirenden der Herzogl. Techn. Hochschule Carolo Wilhelmina zu Braunschweig (v. Dez. 1899). $\mathrm{Br}$

666. Promotions-0rdnung für die Ertheilung der Würde eines Doktor-Ingenieurs durch die Herzogl. Techn. Hochschule Carolo-Wilhelmina zu Braunschweig (v. 18. 10. 1900). 1900. - [Neudr.] 1907. 1909. 1912. Abgedr. in: Bestimmungen. S. $157 \mathrm{ff}$. 1907: K

667. Bestimmungen für die Benutzung der Bibliothek und des Lesezimmers durch die Studierenden und Zuhörer der Herzogl. Technischen Hochschule (v. 1. 10. 1901). [Neudr. mit anderer Schreibung: .... Studirenden ... technischen.] o. J.

668. Bestimmungen, die Zulassung, die Rechte und die Pflichten der Privatdozenten 
an der Herzogl. Technischen Hochschule zu Braunschweig betr. (v. 1. 10. 1903).

669. Satzungen der Krankenkasse für die Studentenschaft der Herzogl. Technischen Hochschule zu Braunschweig (v. 27. 6. 1906). 1906.

670. Satzungen der Studentenschaft der Herzogl. Technischen Hochschule ,CaroloWilhelmina" zu Braunschweig (v. 28.2. 1907). 1907. - [Neudr.] 1909. 1907: $\mathrm{Br}$

671. Satzungen der Krankenkasse für die Studentenschaft der Herzogl. Technischen Hochschule zu Braunschweig. Vom 23. 3. 1909. 1909 .

672. I. Bestimmungen für die Benutzung der Bibliothek der Herzogl. Technischen Hochschule zu Braunschweig durch die Studierenden und Zuhörer (v. 8.4. 1909).
[Folgt:] II. Bestimmungen für die Benutzung des Lesezimmers der Studierenden in der Herzogl. Technischen Hochschule zu Braunschweig v. 8. 4. 1909.

673. Hausordnung der Herzogl. Technischen Hochschule zu Braunschweig (v. 1. 5. 1911).

\section{PRÜFUNGSORDNUNGEN}

674. Vorsehriften über die Diplomprüfungen an der Herzogl. technischen Hochschule zu Braunsthweig. Vom 18. 5. 1893.

675. [Dasselbe.] Vom 1. 7. 1897, bez. 22. 3. 1898 .

676. [Dasselbe] (v. 12. 6. 1902).

677. [Dasselbe.] Vom 3. 9. 1904.

[Neudr. mit Zusätzen zu $§ 1$ u. 2 u. neuem $\S 26 \mathrm{a}$.]

\section{PERIODISCHE SCHRIFTEN}

678. Anzeige der Vorlesungen und Uebungen, welche in dem Collegio Carolino zu Braunschweig zum Theil bereits ihren Anfang genommen haben, zum Theil aber und vornehmlich von Michaelis 1745 bis Ostern 1746 werden angestellet werden.

Fortges. u. d. T.:

Anzeige der Vorlesungen und Uebungen (1746-1776/77:, welche) in dem (1775 ff. : Fürstl.) Collegio Carolino zu Braunschweig ... (1746-1747. 1748-1765/66: werden angestellet werden; 1747/48: sollen angestellet werden; 1766 - 1774: öffentlich gehaiten werden; $1774 / 75-1776 / 77$ : gehalten werden). Von Ostern bis Michaelis 1746. Von Michaelis 1746 bis Ostern 1747. Von Ostern bis Michaelis 1747. Von Michaelis 1747 bis gegen Ostern 1748. Vom 18ten dieses Monats Febr. an bis zum 3ten Aug. 1748 [usw. in ähnlicher Fassung]. - W.-H. 1808/09.

1745/46. [1. Ausg.] - 2. Ausg. [Abgedr. in: Koldewey. S. $229 \mathrm{ff}$.]

1756-1760/61 abgedr. in: Braunschw. Anzeigen. Jahr 12. 1756- Jahr 16. 1760.

1761-1787/88 in: Gelehrte Beyträge zu d. Braunsehw. Anzeigen. 1761-Bd 27. 1787.

1774/75 [u. d. T.:] Nachricht von einigen wichtigen Verbesserungen des Fürstl. Collegii Carolini, nebst der Anzeige der Vorlesungen und Uebungen, welche im gedachten Collegio zu Braunschweig .... gehalten worden. [Abgedr. in: Koldewey. S. $401 \mathrm{ff}$. Die von Eschenburg. S. 32 u. 36 erwähnten Übersetzungen ins Franz. von Eleazar Mauvillon u. ins Engl. von Johann Arnold Ebert nicht nachweisbar.]
1788-1808/09 abgedr. in: Braunschw. Magazin. Bd 1. $1788-B d 21$. 1808. $1745 / 46$ beide Ausg. 1746. 1750. 1774/75: $\mathrm{Br}$ StB; 1746/47-1747/48. 1769/70. 1779/80. 1793. 1793/94: Br; 1748. 1749/50. 1750/51. 1751. 1752. 1753. 1754. 1756-175\%. 1759/60-1760/61. 1762. $1763 / 64-1768$. 1769. 1772/73. 1773/74. 1774. 1775. 1781/82-1783/84. 1784/85. 1785. 1786. 1787. 1789-1792. 1794 -1795/96. 1796/97-1805. 1806. 1806/0\%. 1808. 1808/09: Wolfenbüttel HzglB; 1748/49. 1752/53. 1753/54. 1758-1759. 1761. 1762/63. 1771/72. 1776. $1777 / 78$ - 1779. 1780 - 1781. 1784. 1785/86. 1786/87. 1787/88-1788/89.1792/93. 1805/06. 1807: nicht nachweisbar; 1749.177\%. 1796: Wolfenbüttel Landeshauptarchiv; 1751/52. 1754/55-1755/56. $1757 / 58$. 1761/62. 1763. 1768/69. 1770-1771. 1772. 1773. 1775/76. 1776/77: $B r$ LandschB

679. Anzeige der von den Lehrern des (1825/26. 1826: Fürstl.; 1826/27 ff. : Herzogl.) Collegii Carolini ... zu haltenden Vorlesungen (1834 ff.: und anzustellenden Uebungen). In dem W.-H. von Michaelis 1814 bis Ostern 1815; in dem S.-H. von Ostern bis Michaelis 1815 [usw.] - in dem S. - H. von Ostern bis Michaelis 1862. Fortges. u. d. T. :

Lehrplan der auf dem Herzogl. Collegium Carolinum nach seiner Erweiterung als Polytechnische Schule in dem Studienj. 1862/63 zu haltenden Vorlesungen und anzustellenden Uebungen.

Fortges. u. d. T.:

Anzeige der Vorlesungen und Uebungen an der (1872-73 - 1877-78: Programm der) Herzogl. Polytechnischen Schule, dem Collegium Carolinum in Braunschweig 
(1878-79 ff.: Herzogl. Technische Hochschule Carolo-Wilhelmina zu Braunschweig. Programm) für das Jahr (1866-67 ff.: das Studienj.) 1863-64-1912-1913. 1863-1912. 1814/15-1862. Auch in: Braunsehw. Magazin. Bd 27. $1814-$ Bd 75. 1862.

1835/36. 1836/37. 1837/38. [Nebst] Tabellarische Übersicht der Vorlesungen.

1862f63. Auch in: Braunschweig. Magazin. Bd 75. 1862. S, $457 \mathrm{ff}$.

1815. $1815 / 16.1816 / 17.1818 .1818 / 19$.

1819/20. 1820. 1821/22. 1822/23. 1824. 1826. 1827. 1828. 1828/29. 1830-1850/51: $\mathrm{Br} ; 1851$. 1852/53. 1853/54 1856. 1857-1861: Br StB; 1835/36. 1836/37 Übers. 1837/38 Übers. 1851/52. 1852. 1853. 1862: Wolfenbuittel HzglB; 1856/57: Wolfenbüttel Landeshauptarchiv; 1861/62: nicht nachweisbar.

680. Frequenz-Übersieht (Studienj. 1896/ $1897 \mathrm{ff}$.: Besuchs-Uebersieht) der Herzogl. technischen Hochschule (W.-S. 1894/95 ff.: zu Braunschweig) im Studienj. 1882/83; 1883/84; W.-S. 1884/85; Studienj. 1884/85 [usw.] - Studienj. 1891/92; Studienj. 1892/ 93; W.-S. 1893/94 [usw.] - W.-S. 1895/96; W.-S. 1896/97; Studienj. 1896/97; Studienj. 1897/98; S.-S. 1898; W.-S. 1898/99; Studienj. 1898/99; Studienj. 1899/1900; S.-S. 1900; Studienj. 1900/01; W.-S. 1901/02; S.-S. 1902 [usw.] - W.-H. 1912/13. [Autogr.] W.-S. 1889/90. 1894/95. 1896/97. 1898/99: Br;
W.-S. 1892/93. Studienj. 1895/96. S.-S. 1899. W.-S. 1899/1900. S.-S. 1901: nur hs in Br*

681. Zusammenstellung der Jahres-Frequenz der Herzogl. technischen Hochschule vom Studienjahre 1877/78 bis zum Studienjahre 1883/84 incl. [Hektogr.]

682. Herzogl. Technische Hochschule Carolo-Wilhelmina zu Braunschweig. Die öffentliche Feier der Rektoratsübergabe am 28. Okt. 1904. 2. Nov. 1906. 30. Okt. 1908. 11. Nov. 1910. 22. Nov. 1912. 1904-12. Darin:

1904. Fricke, R[obert]: Über die Bedeutung der allgemeinen Abteilung der technischen Hochschulen. [Abgedr. in: Jahresbericht d. deutschen Mathematiker-Vereinigung. Bd 14. 1905. S. $175 \mathrm{ff}$.]

1906. Müller, R[einhold]: Die Ausbildung der malerischen Perspektive im Zeitalter der italienischen Frührenaissance.

1908. Zeidler, G[eorg]: Die Entwickelung der neuen Bewegung in der Kunst, im besonderen der Raumkunst.

1910. Lüdicke, Arthur: Neuzeitliche Fabrikanlagen. [Gekürzte Zusammenfassung von: Lüdicke: Neuzeitliche Fabrikanlagen u. Einrichtungen. 5 Vorträge geh. gelegentl. der Hochschulkurse f. Ingenieure Ostern 1910 an d. techn. Hochschule zu Braunschweig.]

1912. Beckurts, Heinrich: Über Arzneimittelsynthesen.

\section{GELEGENHEITS- UND VERMISCHTE SCHRIFTEN}

\section{COLLEGIUM CAROLINUM $1745-1862$}

\section{ZUR GESCHICHTE DER ANSTALT}

684. Vorläuffige Nachricht von dem Collegio Carolino zu Braunschweig (dat. 17. April 1745). [Verf. nach Eschenburg. S. 14: Johann Friadrich Wilhelm Jerusalem.] - 2. [unveränd.] Ausg. - 3. Ausg. [Mit geringfügigen Änderungen.] Je 16 S. $8^{0}$ - Abgedr. in: Koldewey. S. $203 \mathrm{ff}$.

1. u. 2. Ausg.: Br; 3. Ausg.: Wolfenbüttel HzglB

685. Entwurf des jährlichen Aufwandes im Collegio Carolino zu Braunschweig. [1746.] $2 \mathrm{Bl}$. $8^{0}$ [Die von Eschenburg. S. 147 erwähnten anderen Drucke nicht nachweisbar.] - Abgedr. in: Koldewey. S. 254 ff. Wolfenbüttel $\mathrm{HzglB}$

686. Fernerweite Nachrieht von dem Collegio Carolino zu Braunschweig. 1746. (Darin: Beylage 1. Serenissimi gnädigste Declaration, denBurgfrieden betr.[Nr630];

2. Serenissimi gnädigste Verordnung, das
Leihen betr. [Nr 631]; 3. Das Gebet fürs Carolinum [Nr 632].) 16 S. $8^{0} \quad \mathrm{Br}$ StB

687. Weitere Nachricht von dem Collegio Carolino und von der Aufnahme in dasselbe. 1750. 31 S. $8^{0}$

\section{$\mathrm{Br}$}

688. Zugabe zu der fortgesezten Nachricht, von dem Collegio Carolino. (Dat. 16. April 1752.) 7 S. $8^{0} \quad \mathrm{Br}$

689. Plan de la dépense annuelle. (Dat.: Brunsvic ce 28. me Oct. 1756.) [Übers. von Nr 685, mit Änderungen.] $2 \mathrm{Bl}$. $8^{0}$

Wolfenbüttel HzglB

690. Nachricht von dem Collegio Carolino in Braunschweig. 1765. [Verf.: Johann Friedrieh Wilhelm Jerusalem.] 31 S. $8^{0}$ - Abgedr. in: Jerusalem: Nachgelassene Schriften. Th. 2. Braunschweig 1793 . S. $71 \mathrm{ff}$.

691. Naehrieht von dem gegenwärtigen Zustande des Collegii Carolini in Braunschweig. 1782. - [Veränderte Fassungen] 1786. 1790. Je 2 Bl. $8^{0}$ 1782. 1786: Wolfenbiittel HzglB; 1790: Wolfenbüttel Landeshauptarchiv 
692. The present State of the Caroline College at Brunswick. 1782. [Übers. von Nr 691.] - [Veränderte Fassung.] 1786. Je 2 Bl. $8^{0}$ 1782: Wolfenbuittel HzglB; 1786: Wolfenbüttel Landeshauptarchiv; die von Eschenburg. S. 148 erwähnte Ausg. von 1790 nicht nachweisbar

693. Avertissement sur l'état présent du Collège Carolin à Brunsvic. 1782. [Übers. von Nr 691.] 2 Bl. $8^{0}$ Wolfenbüttel Landeshauptarchiv; die von Eschenburg. S. 148 erwähnten Ausg. von 1786 u. 1790 nicht nachweisbar

694. Utber Wesen und Zweck des Herzoglichen Collegii Carolini zu Braunschweig, ein Wort der Verständigung an seine Mitbürger, veranlasst durch das neulich erschienene Sendschreiben über Errichtung einer Universität oder eines polytechnischen Instituts hieselbst, von Dr. Vietor Friedrich Lebreeht Petri, Prof. d. alten Literatur u. Mitgliede des Directoriums am Carolino. Braunschweig: G. C. E. Meyer 1831. 44 S. $8^{0} \mathrm{Br}$

695. Nachricht über die Umgestaltung und Erweiterung des Collegii Carolini zu Braunschweig. $2 \mathrm{Bl}$. $8^{0}$ - Aus: Braunschw. Magazin. Bd 48. 1835. S. $297 \mathrm{ff.}$

$\mathrm{Br}$

696. Die höhere technische Lehranstalt oder die technische Abtheilung des Herzoglichen Collegii Carolini zu Braunschweig, nach Zweck, Plan und Einrichtung, unter Mitwirkung ihrer Lehrer dargestellt von dem Vorsteher derselben A[ugust] Uhde, Dr. phil., Prof. d. Math. u. Astron. Braunschweig: Vieweg 1836. IV, 90 S. $8^{0}$

$\mathrm{Br}$

697. Die höhere Handelslehranstalt oder die merkantilische Abtheilung des Herzoglichen Collegii Carolini zu Braunschweig. (Unterz.: Braunschweig, den 3. 10. 1836. Der Vorstand der merkantil. Abth. [Julius Levin Ulrich] Dedekind.) $2 \mathrm{Bl} .8^{0}-$ [Dasselbe u. d. T.:] Die höhere ... zu Braunschweig, in ihren Grundzügen dargest. vom Hofrathe u. Prof. J. L. U. Dedekind. Statt hs. Mitth. (Mit Anm. u. 2 Anl.) 15 S. $8^{0}$ - Ohne die Anl. abgedr. in: Braunschw. Magazin. Bd 49. 1836. S. $325 \mathrm{ff}$. Ausg.v. 2 Bl.: $K$

698. Lehrplan und sonstige Einrichtungen der höheren technischen Lehranstalt des Herzogl. Collegii Carolini zu Braunschweig. 1855. $46 \mathrm{~S} .8^{0}$.

\section{REDEN UND ODEN BEI FEIERN DES HERZOGSHAUSES}

Sammlung $=$ Sammlung der neuesten deutschen Schriften, welche bey Gelegenheit hoher Festivitäten im Collegio Carolino nach und nach herausgekommen sind. Braunschweig (1749).

Wolfenbüttel $\mathrm{HzglB}$
Gärtner = C. C. Gärtner: Sammlung einiger Reden. Braunschweig 1761. Braunschweig StB

699. Ode auf das höchsterfreuliche Geburtsfest des ... Herrn Carl, regierenden Herzogs zu Braunschweig und Lüneburg \&c. ... aufgesetzet von Elias Caspar Reichard. Den 1. Aug. 1745. $4 \mathrm{Bl}$. $4^{0} \quad B$ r LandschB

700. Bey der höchsterfreulichen Feyer des hohen Geburtsfestes des... Herrn Carl, regierenden Herzogs zu Braunschweig und Lüneburg \&c. \&c., welches den 1. Aug. 1746 zum 33. male ... eintrat, bezeugte das Collegium Carolinum in Braunschweig ... seine ... Ehrfurcht ... in zwoen öffentlichen Glückwunschreden; zu deren Anhörung auf den 2. Aug.... vermittelst gegenwärtiger Schrift, worinn von den Vortheilen, welche junge Herren vom Stande aus Erlernung der schönen Wissenschaften erlangen, gehandelt wird, alle... Gönner und Freunde... eingeladen werden. [Beigedr.:] Text zur Musik, welche an dem ... Geburtsfeste des ... Herrn Carl ... bey Gelegenheit zwoer öffentlich gehaltener Reden aufgeführet worden den 1. Aug. 1746. $14 \mathrm{~S} ., 2 \mathrm{Bl}$. $8^{0} \quad$ Wolfenbüttel $\mathrm{HzglB}$

701. An dem hohen und höchsterfreulichen Geburtsfeste des... Herrn C a r l, regierenden Herzogs zu Braunschweig und Lüneburg \&c. \&c. wollten ihre ... Ehrfurcht ... bezeugen die sämtlichen Hofmeister und Studiosi des Collegii Carolini den 1. August 1746. [Ode.] $4 \mathrm{Bl}$. $2^{\circ}$ - [Anderer Druck.] $4 \mathrm{Bl} .4^{0} \quad \mathrm{Br}$ LandschB

702. Natalitia auspicatissima ... Caroli regentis Brunsvicensium ac Luneburgensium ducis ... ipsis Kalendis Augusti 1746 recurrentia ... celebrat Elias Caspar Reichard, Collegii Carolini prof. p. o. $6 \mathrm{Bl} .2^{0}$

\section{Wolfenbüttel HzglB}

703. L'amour des sciences consideré comme la source de la félicité. Ou harangue prononcée le 2. d'aout 1746 dans le grand auditoire du collège Carolin de Bronswick, à l'occasion de l'anniversaire de l'heureuse naissance de ... Charle, duc regnant de Bronswick et de Lunebourg \&c. par Jean Auguste Voigt, étudiant en droit, \& membre du surdit collége. 1746. $12 \mathrm{~S}$. $4^{0}$

Wolfenbüttel HzglB

704. Bey der höchstbeglückten Ankunft des Herrn Carl Wilhelm Ferdinand, ErbPrinzens zu Braunschweig und Lüneburg zeigten die Regungen ihrer .... Freude ... die Hofmeister und Studiosi des Collegii Carolini. 1747. [Ode.] [Beigedr.:] Cantate, 
welche bey der feyerlichen Abend-Musik abgesungen wurde, als über ... Carl Wilhelm Ferdinand, Erb-Prinzen .... Ankunft ihre ... Freude ... bezeugeten sämtliche Hofmeister ... 1747. 4, 2 Bl. $2^{0}$ A Abgedr. in: Sammlung. S. 31 ff. Wolfenbüttel HzglB

705. Bey der höchsterfreulichen Feyer des hohen Geburtsfestes des ... Herrn Carl, regierenden Herzogs zu Braunschweig und Lüneburg \&c. \&c., welches den 1. August 1747 zum 34. male ... erschien, bemühete sich das Collegium Carolinum zu Braunschweig ... die Regungen seiner ... Freude ... in zwoen den 2. August ... öffentlich zu haltenden Glückwunschsreden zu offenbaren, welche anzuhören alle Freunde der Wissenschaften ... hierdurch ... ersuchet werden. (Dat. 1. Aug. 1747.) 15 S. $4^{0}$ Wolfenbüttel HzglB

706. Les serenissimes dues de l'auguste maison de Bronsvick-Lunebourg representés comme des héros dans la république des lettres ou harangue prononcée le 2. d'août de l'an 1747 dans le grand auditoire du collège Carolin de Bronsvick à l'occasion de l'anniversaire de l'heureuse naissance de... Charles duc regnant de Bronsvick et de Lunebourg etc. par Erneste Antoine Heiliger, étudiand en belles-lettres et membre du susdit collège. 1747 . $12 \mathrm{~S} .4^{0} \mathrm{Br}$ Landsch $B$

707. Bey der den 2. Aug. 1747 auf dem Collegio Carolino angestellten Feyer des... Geburtsfestes des ... Herrn $\mathrm{C}$ a r l, regierenden Herzogs zu Braunschweig und Lüneburg \&c. \&c. wurde nachstehendes Singgedichte aufgeführet. $2 \mathrm{Bl}$. $8^{\circ}$

Wolfenbüttel HzglB

708. Discours prononcé le 15 . de mars de l'an 1748 dans le grand auditoire du Collège Carolin de Bronsvick, à l'occasion de l'anniversaire de l'heureuse naissance de ... Madame Philippine Charlotte, duchesse régnante de Bronsvick et de Lunebourg etc. par Guillaume Jules Noble de Plotho, étudiant en belles lettres et membre du dit Collège. [Verf.: Carl Christian Gärtner.] 15 S. $4^{0}-$ Deutsch in: Gärtner. S. 7 ff.

Br Landsch $B$

709. Bey dem hohen Geburthsfeste Ihro Königl. Hoheit der .... Frauen Philippine Charlotte, regierenden Herzoginn zu Braunschweig und Lüneburg \&c., welches den 18. März 1748 von dem Collegio Carolino gefeyert wurde, wartet mit nachfolgender Cantate ... auf Wilhelm Julius Edler von Plotho. 2 Bl. $8^{\circ}$ - Abgedr. in: Sammlung. S. $45 \mathrm{ff}$. Br Landsch $B$
710. An dem hohen und höchsterfreulichen Geburtsfeste des ... Herrn Carl, regierenden Herzogs zu Braunschweig und Lüneburg \&c. \&c. wollten ... ihre ... Ehrfurcht ... bezeugen die sämtlichen Hofmeister und Studiosi des Collegii Carolini den 1. August 1748. [Ode.] $4 \mathrm{Bl}$. $4^{0}$. Abgedr. in: Sammlung. S. 7 ff.; Sammlung verm. Schriften v. d. Verf. d. Bremischen neuen Beyträge. Stück 3. 1749. S. $187 \mathrm{ff}$. Wolfenbüttel HzglB

711. Rede, welche bey dem hohen Geburtsfeste Ihro Königlichen Hoheit der ... Frauen Philippine Charlotte, regierenden Herzoginn zu Braunschweig und Lüneburg \&c. den 13ten März des 1749. Jahres, in dem grossen Hörsaale des . . Collegii Carolini gehalten worden von Johann Friedrich von Lattorff. [Verf.: Carl Christian Gärtner.] 16 S. $4^{0}$ - Abgedr. in: Sammlung. S. 99 ff.; Gärtner. S. 51 ff. Hamburg Stb

712. Cantate auf das hohe Geburtsfest Ihro Königlichen Hoheit der... Frau Philippine Charlotte, regierenden Herzoginn zu Braunschweig und Lüneburg \&c. Den 18. März 1749. - Abgedr. in: Sammlung. S. $51 \mathrm{ff}$. Der nach Eschenburg. S. 153 vorher erschienene Einzeldruck nicht nachweisbar

713. Rede, welche bey dem hohen Vermählungsfeste des ... Herrn Ernst Friedrichs, Erbprinzen und Herzogs von SachsenSalfeld \&c. \&c. mit der ... Frauen Sophia Antoinette, Prinzessin von Braunschweig und Lüneburg \&c. den 26ten April des 1749. Jahrs in dem Collegio Carolino öffentlich gehalten worden von Christian Albrecht Günther Freyherrn von Brockenburg. [Verf.: Carl Christian Gärtner.] 14 S. $4^{0}$. Abgedr. in: Sammlung. S. $129 \mathrm{ff}$; ; Gärtner. S. 27 ff. Wolfenbüttel HzglB

714. Cantate auf die hohe Vermählung des ... Herrn Ernst Friedrichs Erbprinzen und Herzogs von Sachsen \&c. mit der ... Frau Sophia Antoinetta Prinzessin zu Braunschweig und Lüneburg \&c. 1749. - Abgedr. in: Sammlung. S. $57 \mathrm{ff}$; Sammlung verm. Schriften, v. d. Verf. d. Bremischen neuen Beyträge. Stück 6. 1749. S. 425 ff.

\section{Einzeln nicht nachweisbar}

715. Bey dem hohen und höchsterfreulichen Geburtsfeste des ... Herrn Carls, regierenden Herzogs zu Braunschweig und Lüneburg \&c. \&c. bezeugten ... ihre ... Ehrfurcht die sämtlichen Hofmeister und Studiosi des Collegii Carolini, den Isten August 1749. [Ode.] $5 \mathrm{Bl} .2^{0}$ - Abgedr. in: Sammlung. S. $19 \mathrm{ff}$; Sammlung verm. Schriften y. d. Verf. d. Bremischen neuen Beyträge. Stück 6 . 1749. S. $419 \mathrm{ff}$ Wolfenbüttel HzglB 
716. Rede, welche bey dem hohen Geburtsfeste des ... Herrn Carls, regierenden Herzogs zu Braunschweig und Lüneburg \&c. \&c. den 1sten August des 1749sten Jahres in dem ... Collegio Carolino gehalten worden von Carl Abraham Freyherrn von Zedlitz. $18 \mathrm{~S} .2^{0}$ Abgedr. in: Sammlung. S. $63 \mathrm{ff}$.

Wolfenbüttel HzglB

717. Bey dem hohen Geburtsfeste des... Herrn Carls, regierenden Herzogs zu Braunschweig und Lüneburg \&c. \&c. und der dabey im Collegio Carolino gehaltenen Rede wartete mit folgender Cantate ... auf Carl Abraham Freyherr von Zedlitz. 1749. 2 Bl. $2^{0} \quad$ Wolfenbüttel HzglB

718. Ode, womit bey dem hohen Geburtsfeste des ... Herrn Carls, regierenden Herzogs zu Braunschweig und Lüneburg \&c. \&c. den 1. August 1750 seine ... Ehrfurcht bezeigte das Collegium Carolinum. 4 Bl. $2^{0}$

$\mathrm{Br} S t B$

719. Rede, auf das hohe Geburtsfest des ... Herrn Carls, regierenden Herzogs zu Braunschweig und Lüneburg \&c. den [6.] des Augustmonats im 1750sten Jahre in dem grossen Hörsale des Collegii Carolini geh. von Hans Christian Freyherrn von Schwarzenau aus Darmstadt. [Verf.: Carl Christian Gärtner.] 15 S. $4^{0} \bullet$ Abgedr. in: Gärtner. S. $73 \mathrm{ff}$. Wolfenbüttel HzglB

720. Bey dem hohen Geburtsfeste des... Herrn Carls, regierenden Herzogs zu Braunschweig und Lüneburg \&c., welches im Augustmonate des 1750sten Jahres in dem Collegio Carolino gefeyert wurde, wartetemit nachfolgender Cantate ... auf Hans Christian Freyherr von Sehwarzenau. 2 Bl. $4^{0}$ Wolfenbüttel HzglB

721. Rede, welche bey dem hohen Geburtsfeste der ... Frauen Philippine Charlotte, regierenden Herzoginn zu Braunschweig und Lüneburg \&c. den 13ten März des 1751. Jahres, in dem grossen Hörsaale des... Collegii Carolini gehalten worden von Carl Ludwig, Grafen von Giech. [Verf. : Carl (Christian Gärtner.] 15 S. $4^{0}$ - Abgedr. in: Gärtner. S. 95 ff. Wolfenbüttel HzglB

722. Rede auf das hohe Geburtsfest des ... Herrn Carls, regierenden Herzogs zu Braunschweig und Lüneburg \&c., welches den [1.?] August des 1751sten Jahres ... begangen wurde, in dem grossen Hörsaale des Collegii Carolini geh. von Friedrieh Gotthard Freyherrn von Zedlitz, aus Schlesien. 16 S. $4^{0}$

$\mathrm{Br} \mathrm{StB}$
723. Auf das Geburtsfest des ... Herrn Carls, regierenden Herzogs zu Braunschweig und Lüneburg \&c. 1751. Im Namen des Collegii Carolini. Cantate. [Verf.: Nikolas Dietrich Giseke.] - Abgedr. in: Giseke: Poet. Werke hrsg. von C. Ch. Gärtner. Braunschweig 1767. S. $277 \mathrm{ff}$. Einzeln nicht nachweisbar

724. Augustus dux gloriosae memoriae in Carolo duce redivivus. Oratio panegyrica, qua .... principi iuventutis in spem successionis nato, duci Brunsvico-Luneburgensi ... Carolo Wilhelmo Ferdinando ... diem natalitium 17 ad diem 9 Octobr. 1751 ... gratulatur, qui eam ex promta memoria in Collegio Carolino Brunsvici recitavit, Otto a Blome. $15 \mathrm{~S} .2^{0}$ Wolfenbüttel HzglB

725. Rede, auf das hohe Geburtsfest des ... Herrn Carls, regierenden Herzogs zu Braunschweig und Lüneburg \&c. den [1.?] des Augustmonats im 1752. Jahre, in dem grossen Hörsale des Collegii Carolini geh. von Hanns Julius Graf von Schweinitz und Krain Freyherr von Kauder. [Verf.: Carl Christian Gärtner.] 15 S. $4^{0}$ - Abgedr. in: Gärtner. S. $113 \mathrm{ff}$. Br StB

726. Rede auf das hohe Geburtsfest des Herrn Carls, regierenden Herzogs zu Braunschweig und Lüneburg \&c. \&c. ausgearbeitet und den [1.?] Aug. 1752 in dem grössern Hörsaale des Collegii Carolini öffentlich geh. von Carl Friedrich Wilhelm Zinken. 14 S. $4^{0} \quad$ Wolfenbüttel HzglB

727. Rede auf das Geburtsfest Sr. Durchl. des regierenden Herzogs [Carl] zu Braunschweig und Lüneburg. [Beg.: Durchlauchtigster Herzog ... Nur in den traurigen Gegenden, wo ....] - Abgedr. in: Sammlung verm. Schriften, v. d. Verf. d. Bremischen neuen Beyträge zum Vergnügen des Verstandes u. Witzes. Bd 3, Stück 5. 1755. S. 365 ff.

Einzeln nicht nachweisbar

728. Rede auf die hohe Vermählung des ... Herrn Friedrich V., Erbköniges zu Dännemark, Norwegen ... mit ... Juliana Maria, gebornen Herzoginn zu Braunschweig und Lüneburg \&c. \&c. den 8. des Augustmonats 1752 in dem grössern Hörsaale des Collegii Carolini zu Braunschweig geh. von Elias Caspar Reiehard, öff. ord. Lehrer am gedachten Collegio. $8 \mathrm{~S}$. $4^{0}$

Wolfenbüttel HzglB

729. Judas Makkabäus ein musikalisches Gedicht. Nach [Georg Friedrich] Händelischer Musik. Zur unterthänigsten Feyer des Höchsten Geburtsfestes Unsers ... Herzogs zum erstenmale aufgeführt im Col egio Carolino den 2. Aug. 1772. 9 Bl. $8^{0} \quad$ Br LandschB 
730. Principi serenissimo [Kar] II. Herzog von Braunsehweig] die natali suo congratulantur Collegii Carolini cives. 1829. [Ode.] $2 \mathrm{Bl}$. $4^{0}$

\section{WISSENSCHAFTLICHE ABHANDLUNGEN}

731. De philosophia polemica et iudice competente in controversiis philosophicis. Ad praelectiones philosophicas ... Caroli Brunsvic. et Luneb. ducis iussu .... incoandas auditores ... invitaturus quaedam commentatur Ioannes Andreas Fabricius, Philos. et A. L. Magister, prof. Conlegii Carolini, rector gymnasii Cathar. [1745 ?] 15 S. $8^{0} \mathrm{Br}$

732. De pyrrhonismo juridico programmate lectionibus juridicis in Collegio Carolino Brunsvigae instituendis praemisso disserit Bodo Henricus Morgenstern, J. U. L., duc. Brunsvic. et Luneburg. commissar. provinc. et praefect. Eichens. (Dat. Cal. Oct. 1745.) $12 \mathrm{~S} .8^{0}$

$\mathrm{Br}$

733. De cura Graecorum veterum quam in educationem filiorum impenderunt pauca praefatus iuvenes bonis litteris deditos ad audiendas in Carolino antiquitates Graecas invitat humaniter M. Io. David Heumannus, Collegii Carolini prof. extra ordinem publ. et gymnasii ducalis Cathariniani conrector. (Dat. Oct. 1745.) 8 S. $8^{\circ} \quad B r S t B$

734. Lectiones futuro semestri hiemali habendas indicit simulque de mensura virium pauca commentatur Io. Ludor. Oederus, A. M., Coll. Carolini p. p. o. (Dat. 7. Oct. 1745.) 4 Bl. $8^{0} \quad$ Br StB

735. De salutari scholarum ex umbra claustri in lucem urbis evocatione sub ipsa Collegii Carolini Brunsvigae exsurgentis auspicia nonnulla disserit Theodorus Guilielmus Ritmeier, ad d. Andreae pastor et dioeceseos Campensis superintendens. (Dat. 7. Oct. 1745.) 24 S. $8^{0}$

Br StB

736. Ioannis Christophori Harenbergii programma Brunsvicense primum, quo verum et genuinum sensum capitis VIII Danielis ex historia ecclesiastica et civili, ex antiquitatibus Iudaicis et orientalibus, ex geographia, ac reliquis fontibus philologicis in gratiam recentiorum, qui fatorum mirabilium proxime instantium sunt praecones, edisserit, ac simul praelectiones suas indicit, in Carolino, gloriossissime Brunsvici instituto, ad 21 Octobris 1745 inchoandas. (Dat. 1745 , 11. Oct.) $36 \mathrm{~S}$. $8^{\circ}$

Br StB
737. De finibus licentiae poeticae constituendis ad illustrandam M. A. Senecae controversiam X. disserit simulque lectiones suas in Collegio Carolino ab autumno a. 1745 ad tempus vernum a. 1746 publice habendas indicit Elias Caspar Reichard, prof. publ. ord. (Dat. a. d. V. Id. Oct. 1745.) XII S. $8^{0}$

Br StB

738. Programma quo methodum tractandi compendium historiae universalis civilis commonstrat, et scholas suas historicas in recens instituto Collegio Carolino Brunsvicensi sibi demandatas d. XIV. Octobr. 1745 aperit ... Io. Heinr. Schrodt, Colleg. Carolini prof. ord. et Martinei rector. (Dat. Id. Oct. 1745.) XII S. $4^{0} \quad \mathrm{Br}$

739. De praelectionibus in sacros fontes celerioribus quas vulgo vocant, cursoriis earumque natura et usu sub ipsa earundem in Collegio Carolino per instans tempus brumale instituendarum initia paucis praefatur Io. Henr. Blanke, p. C. C. et g. M. cr. (Dat. 20. Oct. 1745.) 14 S. $8^{0}$ Br StB

740. Theologiae pontificiorum liturgicae specimen quo illos veri nominis mediatores sanctos suos existimare et colere demonstrat atque recitationes suas theologicas in illustri Collegio Carolino divini numinis auspiciis instituendas indicat Io. Christoph. Koeeherus D. (Dat. 21. Oct. 1745.) 16 S. $8^{0}$

$\mathrm{Br}$ StB

741. De theologia primorum Christianorum dogmatica, ex monumentis patrum ecclesiasticis collecta, programmatis secundi Brunsvicensis loco disserit et praelectiones suas publicas semestri proximo peragendas, atque in ... Carolino Brunsvici ... expromendas, denuo indicit Io. Christophorus Harenberg, praep. coen. S. Laurentii supra Scheningam, gen. schol. insp., doc. in Carolino honorarius. 1746. Pasch. 96 S. $8^{0} \mathrm{Br} S t B$

742. Lectiones suas aestivales auditoribus suis indicat, atque de vibratione chordarum aliqua praefatur Io. Lud[ov.] Oederus, A. M., prof. pub. ord. (Dat. 1. Mai 1746.) $4 \mathrm{Bl}$. $8^{\circ}$

$\mathrm{Br}$ StB

743. Exercitatio Carolina, qua verum usum variosque abusus aliquot argumentorum pro praestantia religionis Christianae modeste pensitat eamdemque ... Caroli, ducis Brunsvicensis et Luneburgensis ... natali die primo Augusti ... dicatam p. p. Theodorus Guilielmus Ritmeier, superintendens Campensis et pastor Brunsvicensis ad aedem d. Andreae. (Dat. 1746 1. Aug.) 53 S. $8^{0}$ 
744. Programma, in quo de utilitate et necessitate iuris Germanici in cameralibus quaedam disserit ac recitationes suas camerales hiemales in Collegio Carolino Brunovicensi ... indicat D. Georgius Henricus Zinckius [Zincke], iuris et rerum cameralium in Academia Iulia Carolina p. p. o. ut et Collegii Carolini curator. (Dat. III. Non. Oct.) 1746. 24 S. $8^{0}$

Wolfenbüttel $\mathrm{HzglB}$

745. Commentatio de eruditione, quam in Collegio Carolino publicae eruditorum disquisitioni subiicient praeses Io. Christoph. Koecherus, Collegii Carolini curator et prof. publ. ord. et respondens Justus Ludovieus Daniel Lambreeht die XXI. Oct. 1746. 4 Bl., 40 S. $8^{0} \quad$ Wolfenbüttel $\mathrm{HzglB}$

746. Dum ... Ioannes Heinricus Blanke,... Collegii Carolini Brunovicensis prof. publ. ord., et gymnasii ibidem Martiniani conrector ... hanc posteriorem spartam cum alia superiore commutaturus, et fasces rectoris apud gymnasium Catharinianum Brunovicense propediem capessiturus, musis nostris publice valedicere, et ... orationem de bonis litteris sui neglectum vindicantibus $d$. 19. m. decembr. anni 1746 habere constituit, eam, posteaquam pauca quaedam, quam varia ac diversa censorum de scholarum rectore iudicia sint, praefatus est, indicit Io. Heinr. Schrodt, Collegii Carolini p. p. o. et Martinei Brunovicensis rector. (Dat. prid. Id. dec. 1746.) 8 S. $8^{0} \quad B r$ LandschB

747. Stirpis Estensis origines, progenitores serenissimorum ducum BrunsvicoLuneburgicorum vetustissimos septem, programmate Brunsvicensi tertio ex diplomatibus ... edissuerit simulque praelectionum suarum subsequentium, in Collegio Carolino illustri expromendarum, indicem, indixit nundinis hibernis anni 1748 Ioannes Christophorus Harenbergius, prof. Collegii Carolini honorarius. (Dat. 1748. 3. Febr.) $56 \mathrm{~S} .8^{0}$ Wolfenbüttel $\mathrm{HzglB}$

748. De parabolanis veteris ecclesiae Christianae, quorum in codice Theodosii et Iustiniani contracta iniicitur mentio, succincte commentatur ac programmate IV Brunsvicensi praelectionibus suis, in ... Collegio Carolino expromendis, proludit . . anno 1748, mense Sept. Ioannes Christophorus Harenberg. $16 \mathrm{~S}$. $8^{0}$ Wolfenbuittel HzglB

749. Historiam belli tricennalis et pacis Westphalicae singularem, quantum nempe ad solas terras Brunovicenses ac Lunaeburgenses pertinet, ex actis publicis ... in huius anni saecularem memoriam recensere, et insuper recitationes suas publicas historicas in ... Collegio Carolino instante semestri brumali 1748 et 1749 habendas publica hac commentatione indicare voluit Io. Heinr. Sehrodt, Collegii Carolini prof. ord. publ. et Martinei Brunovicensis rector. (Dat. 1748.) 47 S. $8^{0}$

Wolfenbüttel HzglB

750. Disputatio historica politica aeque ac ecclesiastica de primis Tatarorum vestigiis victricibus, Silesiae funestis, quam ... in Carolino Brunsvici publico eruditorum examini submittunt praeses Ioannes Christoph. Harenberg, prof. Collegii Carolini honorarius et autor respondens Ernestus Gottlieb Latzke. 1750. 9. Oct. 84 S. $8^{0}$

Wolfenbüttel $\mathrm{HzglB}$

751. Der Dänen Trost in ihren allerdurchlauchtigsten Königinnen. Eine Abhandlung, womit zu ... Anhörung seiner auf die ... den 8. Jul. 1752 ... vollzogene Vermählung des ... regierenden dänischen Monarchen Friederichs V. mit der ... Prinzeßinn von Braunschweig-Lüneburg Juliana Maria im Collegio Carolino zu Braunschweig den 2. Aug. 1752 zu haltenden feyerlichen Rede ... einladet Elias Caspar Reichard, öff. ord. Lehrer am gedachten Collegio. [Beigedr. :] Singgedicht, welches bey der Rede musikalisch wird aufgeführet werden. $14 \mathrm{~S} ., 1 \mathrm{Bl} .4^{0} \quad B r \operatorname{Landsch} B$

752. Ueber das Verhältniß der Mischung zur Form. Eine Einladungsschrift zu seinen Vorlesungen über Physik und Chemie von Dr. Carl Miehael Marx, ord. Prof. am Collegio Carolino u. am anatom.-chirurg. Institute in Braunschweig. 1824. $10 \mathrm{~S} .8^{0}$

Wolfenbüttel HzglB

753. Scholarum in Collegio Carolino ducali vertente hoc semestri aestivo habendarum et a. d. XIV. Calendas Majas auspicandarum initium indicit Dr. Vietor Frederieus Lebrecht Petri, linguarum veterum prof. publ. ord., Collegii moderatoribus adscriptus. Insunt observationes aliquot in Pindari hymnum Olympicum primum. 1831. 22 S. $8^{\circ}$

$\mathrm{Br}$

754. Anthologia Pindarica theologicomoralis sive locorum Pindari ad rerum divinarum officiorumque doctrinam spectantium per titulos digesta collectio. Scripsit subjectisque $\ldots$ animadversionibus scholarum hibernarum auspicia ... celebraturus Collegii Carolini ducalis civibus proposuit Dr. Vietor Frederieus Lebrecht Petri, ex Collegii moderatoribus, literarum antiquarum prof. publ. ord. 1831. 36 S. $8^{0}$ Wolfenbüttel HzglB 
755. De oratione Corinthiaca falso Dioni Chrysostomo adscripta commentatus est, et emendationes aliquot corruptorum Dionis locorum adjecit et Collegii Carolini ducalis eivibus proposuit, scholarum aestivarum auspicia de more celebraturus, Dr. Adolphus Emperius, Brunopolitanus. 1832. 31 S. $8^{\circ} \mathrm{Br}$

756. Observationes in Lysiam scripsit et Collegii Cárolini ducalis civibus proposuit scholarum hibernarum auspicia ... celebraturus Dr. Adolphus Emperius. 1833. $48 \mathrm{~S}$. $8^{0}$ Woljenbüttel $\mathrm{HzglB}$

757. Commentationum in Jobum fasciculus primus. Scripsit et praemissis de Collegii Carolini indole atque ratione observationibus scholarum hibernarum exitum ... celebraturus ed. Dr. Vietor Frederieus 0rthobius Petri, linguarum antiquarum prof. publ. ord., Collegii moderatoribus adscriptus. 1833. 30 S. $4^{0}$

Wolfenbuittel $\mathrm{HzglB}$

758. Commentationum in Jobum fasciculus secundus. Scripsit et scholarum aestivarum auspicia de more celebraturus literarum sacrarum studiosis Collegii Carolini civibus proposuit Dr. Vietor Frederieus Orthobius Petri, ex Collegii moderatoribus, linguarum antiquarum prof. publ. ord. 1833. 28 S. $8^{\circ}$

$\mathrm{Br}$

\section{VERSCHIEDENE SCHRIFTEN}

760. Memoria viri praenobilissimi ac doctissimi Melchioris Caroli Doerrien, studiosae in Collegio Carolino iuventutis per aliquot tempus morum magistri diligentissimi meritissimi a. d. VIII. Idus Iul. 1746 ... vita defuncti posteritati officiose mandata. (Dat. a. d. VII. Id. Iul. 1746.) VIII S. $8^{0}$

Woljenbüttel $\mathrm{HzglB}$

761. Theses philosophicae a. d. VI. Iul. 1748 in auditorio magno illustris Collegii Carolini disputatae ab Io. Guil. Seidlero, phil. doct. et prof. p. o. et Io. Frider. Meyero, Lauenburgo-Hannoverano. $2 \mathrm{Bl} .4^{0}$ Br Landsch $B$

762. Exsequias funeri iuvenis illustrissimi Caroli Guilielmi S. R. I. comitis de Giech ... d. 14. Martii 1750 ... defuncti ... templo Catharineo Brunsvicensi ... eundas indicit Collegium Carolinum. D. 28. Martii 1750. 4 Bl. $2^{\circ}$ Wolfenbüttel $\mathrm{HzglB}$

763. Manibus felicissimis iuvenis illustrissimi Caroli Ludovici S. R. I. comitis de Giech ... III. Idus Ianuar. 1752 praematura acerbissimaque morte exstincti sa- cram esse hanc tabulam voluit Collegium Carolinum. Ipsis Nonis Febr. 1752. 4 Bl. $2^{0}$ Wolfenbüttel HzglB

764. Bey dem höchstschmerzlichen Absterben des... Herrn Friederich August des Heil. Römischen Reichs Grafen Rutowsky ... bezeugte im Namen des Herzogl. Collegii Carolini durch nachgesetzte Elegie das empfindlichste Mitleid Carl Christian Gärtner. Prof. d. Sittenlehre. 1755. 2 Bl. $2^{0}$

Wolfenbüttel $\mathrm{HzglB}$

765. Passions-Musik, von der Composition des Herrn Capellmeisters [Karl Heinrieh] Graun, am Charfreytage des $1755^{\text {sten }}$ Jahrs, in dem Collegio Carolino aufgeführt. Braunschweig den 28. Martius. $4 \mathrm{Bl}$. $8^{\circ}$ $B r$ Landsch $B$

766. Der Tod Jesu, eine Cantate, nach der Composition des Königl. Preußischen Capellmeisters, Herrn [Karl Heinrich] Grauns, aufgeführet den Sonntag vor Ostern 1757 in dem grossen Saale des Collegii Carolini zu Braunschweig. $16 \mathrm{~S}$. $8^{0} \quad B r$ Landsch $B$

767. Te Deum laudamus nach aer Composition des berühmten Königl. Preußischen Capellmeisters Herrn [Karl Heinrieh] Grauns wegen der erwünschten Zurückkunft der durchlauchtigsten Herrschaften aufgeführt im grossen Saale des Collegii Carolini. Braunschweig, den 12ten März 1758. 4 Bl. $8^{\circ}$ Br LandschB

768. Das befreyte Israel, nach Anleitung des Mosaischen Lobgesanges im 2. Buch Mos. Cap. 15. von Friedrieh Wilhelm Zachariä, und in Musik gesetzt von dem berühmten Herrn Capellmeister [Georg Philipp] Telemann in Hamburg. Im großen Saal des Collegii Carolini aufgeführt Braunschweig, den 19. Jun. 1761. 8 S. $8^{0} \quad B r$ LandschB

769. Der Tod Jesu, eine Cantate, nach der Composition des ehemaligen Königl. Preuss. Capellmeisters, Herrn [Karl Heinrich] Grauns, welche auf besonderes Verlangen den Sonntag vor Ostern 1763 zum drittenmal aufgeführet wird in dem großen Saale des Collegii Carolini zu Braunschweig. 1763. $16 \mathrm{~S}$. $8^{0}$ $\mathrm{Br}$ LandschB

770. Klagen über den empfindlichen Verlust eines zärtlichgeliebten Freundes, Herrn Friedrich Wilhelm von Schliestedt [gest. auf dem Collegio Carolino]. 1764. [Ode.] $2 \mathrm{Bl}$. $8^{\circ} B r \operatorname{LandschB}$

771. Empfindungen bey dem frühen Todedes hoch wolgebohrnen Herrn Frie dric $\mathrm{hW}$ il helm von Schliestedt [gest. auf dem Col legio Carolino]. [1764.] 4 Bl. $8^{\circ} \cdot B r$ Landsch B 
772. Einladung zu der dritten Saecularfeier der Kirchenverbesserung. Abseiten des Fürstl. Collegii Carolini. 1817. 8 S. $8^{0}$ Wolfenbüttel HzglB

773. Ueber die Nothwendigkeit und Zweckmäßigkeit der durch Luther ausgeführten Kirchen-Reformation. Eine Rede am 3. Säcularfeste der Reformation den 1. Nov. 1817 vor den Lehrern und Studirenden des Collegii Carolini und einer höchstverehrlichen Versammlung geh. von Joh. Ferd. Friedr. Emperius, Prof. 40 S. $8^{0}$ Wolfenbüttel HzglB

774. Den Manen ihres unvergesslichen Lehrers des Herrn Hofrathes [Joh. Ferd. Friedr.] Emperius die sämmtlichen Studirenden des Collegiums. 24. Oct. 1822. [Ode.] 2 Bl. $8^{0}$

Wolfenbüttel $\mathrm{HzglB}$

775. Dem Herrn Obrist-Lieutenant [Franz Karl Anton] Schönhut, Lehrer der Mathematik am Herzoglich Braunschweigischen Collegio Carolino bei der Feier seiner funfzigjährigen Amtsführung aus inniger Liebe und Dankbarkeit dargebracht von sämmtlichen Studirenden des Collegii Carolini. Braunschweig den 15. Sept. 1824. [Ode.] 2 Bl. $2^{0}$

$\mathrm{Br}$

776. Die Physikalische Sammlung des Herzoglichen Collegii Carolini in Braunschweig, beschrieben von Dr. C(arl) M(ichael) Marx, ord. Prof. d. Physik u. Chemie am Collegio Carolino u. am anatom.-chirurg. Institute daselbst. Mit Abb. Braunschweig: Vieweg 1831. XII, 134 S., 1 Taf. $8^{0} \quad \mathrm{Br}$

777. Domino celsissimo generosissimo Guilielmo serenissimo duci Brunsvicensi Luneburgensi Olsnensi ... natalitia ... agenda sumtique ante hos viginti quinque annos principatus ... memoriam a. d. VII. Kal. Maj. 1856 . . . gratulantes vota solvunt ... Collegii Carolini ducalis moderatores professores cives. [Ode.] $4 \mathrm{Bl} .4^{0}$

Wolfenbiuttel HzglB

POLYTECHNISCHE SCHULE UND TECHNISCHE HOCHSCHULE $1862-1912$

\section{ZUR GESCHICHTE DER ANSTALT}

778. Lehrplan der polytechnischen Schule in Braunschweig. In Auftrag des Herzogl. Staatsministeriums veröff. vom Directorium der Anstalt: Hofrath Dr. [Julius Levin Ulrich] Dedekind, Prof.Dr. [Johann Heinrieh] Blasius, Prof. [Carl Friedrich Heinrich] Ahlburg. 1862.
(Nebent.: Lehrplan des Herzoglichen Collegii Carolini in Braunschweig nach seiner Erweiterung als polytechnische Schule.) $104 \mathrm{~S} .8^{\circ}$ - Aus: Braunschw. Magazin. Bd 75. S. $357 \mathrm{ff}$.

779. Neubau der Herzoglichen Technischen Hochschule in Braunschweig. Entworfen u. ausgeführt von den Prof. [Constantin] Uhde u. [Carl] Körner. Veröff. im Auftrage des Herzogl. Staatsministeriums. Mit einem Atlas von 18 Taf. Berlin: Wasmuth [1877.] $17 \mathrm{~S} .4^{0} ; 18$ Taf. gr. $2^{0}$

780. Worte beim Abschiede von den Räumen des Collegium Carolinum am 19. October 1877 gesprochen von Prof. Dr. L[ouis]Ph[ilippe] Sy. 1877. $8 \mathrm{~S} .8^{\circ} \mathrm{Br}$

781. Die feierliche Eröffnung des Polytechnikum Carolo-Wilhelminum zu Braunschweig am 16. October 1877. $12 \mathrm{~S} .4^{0} \mathrm{Br}$

782. Allgemeine Ausstellung für Landwirthschaft Köln a. Rhein, Mai bis October 1890. Erläuterungs-Berieht zur CollectivAusstellung der Herzogl. Technischen Hochschule zu Braunschweig. Braunschweig: Vieweg 1890. 34 S., 1 Taf. $8^{0} \quad \mathrm{Br}$

783. Fest-Programm zur Banner-Feier der Herzogl. Techn. Hochschule Carolo-Wilhelmina zu Braunschweig 15.-17. Juli 1892. 17 S., 4 Taf. $4^{0}$

784. Festordnung für die Jubelfeier der Herzogl. technischen Hochschule CaroloWilhelmina zu Braunschweig vom 25. bis 27. Juli 1895. $1 \mathrm{Bl} .4^{0} \quad \mathrm{Br}$

785. Jubelfeier der Herzoglichen technischen Hochschule Carolo-Wilhelmina 25. bis 27. Juli 1895. Verzeichniss der anwesenden Vertreter auswärtiger Hochschulen und der alten Herren der Carolo-Wilhelmina. $2 \mathrm{Bl}$. $4^{0}$

786. Lieder für den Kommers der Jubelfeier der Herzoglich technischen Hochschule Carolo-Wilhelmina zu Braunschweig am 26. Juli 1895. $47 \mathrm{~S} .8^{0} \mathrm{Br}$

787. Das 150 jährige Jubiläum der Herzoglichen Technischen Hochschule CaroloWilhelmina zu Braunschweig im Juli 1895. Festbericht veröff. vom Allgemeinen Jubiläumsausschusse. Mit 11 Taf. 1896. 118 S., 11 Taf. $8^{\circ}$

788. Herzogliche Technische Hochsehule Carolo-Wilhelmina zu Braunschweig. 1907. [IIl. Beschreibung.] 24 S., 1 Taf. $8^{\circ} \mathrm{Br}$

789. Herzogliche Technische Hochschule Carolo-Wilhelmina zu Braunschweig. Einführung und Rede des Rektors Professor Dr. Otto Reinke (Über die Entwicklung 
der landwirtschaftlich-chemischen Technik und deren wichtige Beziehungen zu den technischen Hochschulen) sowie die öffentliche Preisverteilungsfeier am 6. Dezember 1907. 1908. $20 \mathrm{~S} .8^{0}$

\section{VERSCHIEDENE SCHRIFTEN}

790. Festschrift zur Saecularfeier des Geburtstages von Carl Friedrich Gauss dargebracht vom Herzoglichen Collegium Carolinum zu Braunschweig. Über die Anzahl der Ideal-Classen in den verschiedenen Ordnungen eines endlichen Körpers von Riehard Dedekind. 1877 . 55 S. $4^{0}$

791. Katalog der Bibliothek der Herzoglichen Technischen Hochschule Carolo-Wilhelmina zu Braunschweig. Abth. 1. [Mehr nicht ersch.] Mathematik. Physik. Chemie. Mineralogie. Botanik. Zoologie. Berg- u. Hüttenwesen. Land- u. Forstwirthschaft. Technologie. Mechanik u. Maschinenwesen. Bau- u. Ingenieurwesen. Bildende Künste. 1880. 319 S. $8^{0}$

792. Die Feier von Lessing's hundertjährigem Todestage zu Braunschweig. Denkschrift, hrsg. von den Studirenden der Herzoglich technischen Hochschule zu Braunschweig. Inh.: Festbericht der Studiosen A[lbert] Bode u. B[runo]Sauer. Festrede des Dr. Otto Sievers. Poetische Gaben von Friedrich Bodenstedt, Felix Dahn, Oscar von Redwitz, Emil Rittershaus, Ernst Scherenberg. Mit 5 Ill. Braunschweig: Schulbuchh. in Comm. 1881. 41 S. $8^{0}$

793. Die Hermannshöhle bei Rübeland. Geologisch bearb. von Dr. J[ohan] H[ermann] Kloos, Prof. d. Mineralogie u. Geologie, photogr. aufgenommen von Dr. Max Mïiller, a. o. Prof. an d. Herzogl. Techn. Hochschule zu Braunschweig. Hrsg. von der Herzoglich Technischen Hochschule zu Braunschweig. I.Text. (76 S.) II. Taf. (20 Photogr. in Mappe.) Weimar: Deutsche Photographen-Zeitung 1889. $4^{0}$

794. Ueber Ziele und Aufgaben der Elektrochemie. Von Pıof. Dr. Riehard Meyer. 〈Vortrag, geh. bei d. öffentl. Preisvertheilung in der Aula der Herzogl. Technischen Hochschule zu Braunschweig am 14. Dec. 1894.> 18 S. $8^{0}$ - Aus: Naturwiss. Rundschau. Jg. 10. 1895.

$\mathrm{Br}$

795. Fest-Sehrift der Herzoglichen Technischen Hochschule Carolo-Wilhelmina dargeboten den naturwissenschaftlichen Theilnehmern an der 69 . Versammlung deutscher
Naturforscher und Ärzte vom Herzogl. Braunschw. Staats-Ministerium. Bearb. von Mitgliedern des Lehrkörpers der Carolo-Wilhelmina u. hrsg. im Auftrage des geschäftsführenden u. litterarischen Ausschusses von Prof. Dr. Heinr. Beckurts. Mit TextAbb. u. Taf. Braunschweig: Vieweg 1897. 464 S., 2 Taf. $4^{0}$

Enth.: R[ichard] Dedekind: Über Zerlegungen von Zahlen durch ihren grössten gemeinsamen Theiler. - R[einhold] Müller: Beiträge zur Theorie des ebenen Gelenkvierecks. - R[obert] Fricke: Über den arithmetischen Charakter gewisser Netze von unendlich vielen congruenten Vierecken. - H[einrich] Weber: Ableitung der Gleichgewichtsgleichung der Nadel im Rotationsinductor. - M[ax] Möller: Über die fortschreitende Geschwindigkeit von Wellen mit einer longitudinalen Schwingung der Elemente. - A[lfred] Vierkandt: Die Entstehungsgründe neuer Sitten. - F[riedrich] Knapp: Die wissenschaftlichen Anschauungen über die Gerbeprocesse in ihrer Entwicklung. - Richard Meyer: Über einige Beziehungen zwischen Fluorescenz und chemischer Constitution. - H[einrich] Beckurts u. J[ulius] Tröger: Über das ätherische Öl der Angosturarinde.-J[ulius] Troeger: Über die Einwirkung von alkoholischem Kaliumsulfid und alkoholischem Kaliumsulfhydrat auf symmetrische Dibromsubstitute von Sulfonen. - J[ohan] H[ermann] Kloos: Die tektonischen Verhältnisse des norddeutschen Schollengebirges auf Grund der neuesten Tiefbohrungen im Leinethale und bei Hannover sowie die Gliederung des Salzgebirges daselbst. W[ilhelm] Blasius: Neuer Beitrag zur Kenntniss der Vogelfauna von Celebes. - R[udolf] Blasius u. H[einrich] Beckurts: Úber die centrale Wasserversorgung der Gemeinden des Herzogthums Braunschweig. - P[aul] Degen er: Über den Einfluss der Temperatur auf die Acidität einiger Säuren.

796. Die deutsche Baukunst der Zukunft. Vortrag geh. gelegentlich der Preisvertheilung an der Techn. Hochschule in Braunschweig am 16. Dec. 1898 von Prof. Hermann Pfeifer. Berlin: Ernst 1899. 11 S. $8^{0}$ - Aus: Centralblatt d. Bauverwaltung. Jg. 19. $\mathrm{Br}$

797. Probleme der organischen Chemie. Von Prof. Dr. Richard Meyer. 〈Vortrag, geh. bei d. Preisvertheilung in der Aula der Herzogl. Technischen Hochschule zu Braunschweig am 11. Dec. 1901.> 8 S. $4^{0}$ ฯ Aus: Naturwiss. Rundschau. Jg. 17. $1902 . \quad \mathrm{Br}$

798. Katalog der Bibliothek der Herzoglichen Technischen Hochschule Carolo-Wilhelmina zu Braunschweig. Abt. 3. 5. 1907. $8^{0}$ 3. Maschinenwesen einschl. der mechanischen Technologie, Elektrotechnik, sowie der Heizungs-, Lüftungs-, Wasserversorgungs- u. Gasinstallationstechnik. $81 \mathrm{~S}$.

5. Mathematik, Geodäsie, Astronomie u. Mechanik. $75 \mathrm{~S}$. 
799. Herzogliche Technische Hochschule Carolo-Wilhelmina zu Braunschweig. Utber Ursprung und Bedeutung der darstellenden Geometrie von Prof. Dr. H[einrich] E[mil] Timerding. Festrede bei der öffentl. Preisverteilung am 26. Nov. 1909. 1910. 16 S. $8^{0}$

800. Aus dem Pharmazeutischen Institut der Herzogl. Technischen Hochschule CaroloWilhelmina. Den Tfilnehmern der 39. Hauptversammlung des Deutschen Apotheker-Veceins in Braunschweig vom 6. bis 8. Sept. 1910 gewidmet von $\mathrm{H}$ [einrich] Beckurts. Mit 9 Abb. im Text, 1 Grundriss u. 5 Taf. Braunschweig: Vieweg 1910. $121 \mathrm{~S} ., 5$ Taf. $8^{\circ} \mathrm{Br}$
Enth. u. a.: $\mathrm{H}[$ einrich] Beckurts: Das pharmazeutische Institut der Herzoglichen Technischen Hochschule in Braunschweig 18701910. - $O(t$ t o ) Lin de: Die Anfertigung pharmakognostischer Dauerpräparate. - J [ulius] Troeger u. H(ermann) Runne: Beiträge zur Erforschung der Angosturaalkaloide. [Vgl. die Dissertation von Runne. Nr 889.] - Hermann Emde: Zur Beziehung zwischen chemischer Konstitution und physiologischer Wirkung: Substanzen mit lockerer Kohlenstoff-Stickstoffbindung. - $\mathrm{H}$ (einrich) Frerichs: Uber die Darstellung von Chinatinkturen und über den Gehalt derselben an Alkaloiden. - Robert Bohlmann: Die Apotheken der Stadt Braunschweig.

\section{HA BILITATI ON S SCHRIFTEN}

801. Beckmann, Ernst, Dr.: Untersuchungen ueber die Aluminate und basischen Haloïdsalze des Bariums sowie Notizen ueber Barythydrat und die Haloïdsalze des Bariums. $1882.96 \mathrm{~S} .8^{0}$ - Aus: Journal f. prakt. Chemie. Bd134. 135. N.F. Bd26. 27. 1882. 83. Br

Braunsehweig Te. H., Hab.-Schr. v. 23. 5. 1882

802. Vierkandt, A[lfred], Dr.: Die Volksdichte im westlichen Central-Afrika. Mit 4 Kt. Leipzig: Duncker \& Humblot 1895. 110 S., 1 Kt. $8^{0}$ T Aus: Wissensch. Veröffentlichungen d. Vereins f. Erdkunde zu Leipzig. Bd 2.

Braunschweig Te. H., Hab.-Schr. v. 11. 7. 1894

803. Biehringer, Joachim, Dr., I. Assistent am Labor. f. analyt. u. techn. Chemie: Ueber die Farbstoffe der Pyroningruppe. Leipzig: J. A. Barth 1896. 43 S. $8^{0}$ ๆ Aus: Journal f. prakt. Chemie. Bd 162. N. F. Bd54. Br

Braunschweig Te. H., Hab.-Schr. v. 15. 7. 1896
804. Mosler, Hugo, Dr. phil., Dipl.-Ing. Konstruktion und Berechnung von Selbstanlassern für elektrische Aufzüge mit Druckknopfsteuerung. [1904.] VIII, $102 \mathrm{~S} .8^{0}$ T Auch bei Springer in Berlin. $\mathrm{Br}$ Braunschweig Te. H., Hab.-Schr. v. 1. 7. 1903

805. Limmer, Fritz, Dr. phil.: Das Ausbleichverfahren (Farbenanpassungsverfahren〉, eine Möglichkeit der direkten Körperfarbenphotographie. 1911. VIII, $138 \mathrm{~S} .8^{0}$ Vollst. als: Enzyklopädie d. Photographie. H. 77; Nachtrag (S. 139-154) auch einzeln.

Braunschweig Te. H., Hab.-Schr. v. 17. 5. 1909

806. Bergwitz, Karl, Dr. phil., Oberlehrer: Die $\gamma$-Strahlung des Erdkörpers und ihr Anteil an der spontanen Ionisierung der Atmosphäre. Braunschweig: Vieweg 1910. $44 \mathrm{~S} .8^{0}$ - Aus: 16. Jahresbericht d. Vereins f. Naturwissenschaft zu Braunschweig.

Braunschweig Te. H., Hab.-Schr. v. 23. 2. 1910

\section{DOKT OR-INGENIEUR-DISSERTATIONEN}

\section{ABTEILUNG FÜR ARCHITEKTUR}

808. Hinz, R[ichard] $\mathbf{R}$ [einhold], Reg.Baumeister: Vom Einfluß der Bauordnungen in Preußen auf die bauliche Entwicklung der Bauerndörfer. Ein Beitrag zur Förderung des Heimatschutzes. Berlin-Mariendorf: Berliner Bauplan-Vereinigung [1911]. 99 S., 2 Taf. $8^{\circ}$ Braunschweig, Dr.-Ing.-Diss. v. 7. 7. 1910

809. Pöthig, 0tte: Das Problem der deutschen Dachform im Einzel- und Städtebau und die neuen Dachkonstruktionen. Vit 1 Titelbild u. $134 \mathrm{Abb}$. Berlin: Ernst 1911. $288 \mathrm{~S} ., 1$ Taf. $8^{0}$ - Auch als Buch.

Braunschweig, Dr.-Ing.-Diss. v. 22. 12. 1910
810. Bieder, Curt: Platz und Turm, im besonderen Piazza Erbe und Piazza dei Signori in Verona und Vicenza und Neugestaltung des Wilhelmsplatzes in Frankfurt a. Oder. 1911. 61 S., 28 Taf. $4^{0}$ - Soll bei Ernst in Berlin erscheinen; bis 1913 nicht ersch.

Braunschweig, Dr.-Ing.-Diss. v. 24. 3. 1911

811. Former, Alexander, Kgl. Reg.-Baumeister, Braunschweig: Die Wiederherstellung von Bauten im 19. Jahrhundert, insbesondere die Wiederherstellung der Alexanderkirche zu Wildeshausen in Oldenburg im Jahre 1908-09. [1912.] 78 S., 6 Taf. $4^{0}$ - Auch bei Neff in EBlingen.

Braunschweig, Dr.-Ing.-Diss. v. 9. 3. 1912 
812. Ebinghaus, Hugo: Das Ackerbürgerhaus der Städte Westfalens und des Wesertales. Text mit $119 \mathrm{Abb}$. Dresden: Kühtmann 1912. VIII, 128 S. $4^{0}$ - Auch als Buch.

Braunschweig, Dr.-Ing.-Diss. v. 16. 7. 1912

\section{ABTEILUNG FÜR INGENIEURBAU- WESEN}

813. Hartmann, R[obert], Kgl. Reg.-Bauführer: Beitrag zur Wirbelbewegung. Die $\mathrm{U}[\mathrm{e}]$ bertragung des Quergefälles einer in horizontalem Bogen strömenden Flüssigkeitsschicht auf Schichten anderer Höhenlagen ist $\mathrm{zu}$ untersuchen. Die hierbei abgeleiteten Erscheinungen und Vorgänge sind auf den Strombau anzuwenden. 1902. $31 \mathrm{~S}$. $8^{0}$ - Auch in:Zeitschriftf. Gewässerkunde. Bd5. 1903.

Brannschweig, Dr.-Ing.-Diss. v. 28. 5. 1902

814. Diethelm, Hans: Kritische Besprechung der Auflagerkonstruktionen eiserner Balkenbrücken nach Form und Berechnung. 1906. 40 S., 20 Taf. $4^{0}$

Braunschweig, Dr.-Ing.-Diss. v. 16. 3. 1906

815. Sehaller, Ludwig: Einflußlinien für durchlaufende Träger auf drei und mehr Stützen. [1907.] $53 \mathrm{~S} .8^{0}$

Braunschweig, Dr.-Ing.-Diss. v. 29. 6. 1907

816. Müller, Paul: Ein Beitrag zur Theorie der Nebenspannungen in gegliederten Trägern. 1908. 48 S., 12 Taf. $8^{0}$

Braunschweig, Dr.-Ing.-Diss. v. 11. 12. 1907

817. Feyerherm, Paul, Reg.-Bauführer: Beitrag zur Theorie der Eisenbahnkurven. 1908. 47 S. $8^{0}$

Braunschweig, Dr.-Ing.-Diss. v. 23. 5. 1908

818. Leiner, Franz, Reg.-Bauführer: Der Gehängebau. (Eine Darstellung der Entwicklung und Anwendung des Gehängebaues mit kritischen Ansichtsäußerungen und neuen konstruktiven Vorschlägen des Verfassers.) Mit $28 \mathrm{Abb}$. Leipzig: W. Engelmann 1909. X, $78 \mathrm{~S} .4^{0}$ Auch als: Fortschritted. Ingenieurwissenschaften. Gruppe2, H.21.

Braunschweig, Dr.-Ing.-Diss. v. 3. 2. 1909

819. Gerecke, Karl: Untersuchungen zu Knotenpunktausbildungen bei Holzkonstruktionen. 1909. VIII, 88 S., 3 Taf. $8^{0}$ Braunschweig, Dr.-Ing.-Diss. v. 12. 3. 1909

820. Ehlers, H[ermann], Baumeister d. Bau-Deputation i. Hamburg: Ein Beitrag zur statischen Berechnung von Spundwänden unter Berücksichtigung besonderer örtlicher Verhältnisse. 1910 . 34 S. $8^{0}$ - Aus: Zeitschrift f. Architektur u. Ingenieurwesen. Jg. 1910. Bd 56. N. F. Bd 15.

Braunschweig, Dr.-Ing.-Diss. v. 20. 7. 1909
821. Wolf, Josef: Ueber den günstigsten EinfluB einer Anlage von Stauweihern im Gebiet der oberen Werra auf deren Wasserführung. Mit $19 \mathrm{Bl}$. Zeichn. Hannover: Verein f. Schiffbarmachung d. Werra. 1910. IV, 39S. ; 10 Taf., $1 \mathrm{Kt}$. [in besonderer Mappe.] $8^{0}$

Braunschweig, Dr.-Ing.-Diss. v. 24. 11. 1909

822. Gleye, [Rudolf]: Die leitenden Gesichtspunkte zur Durchführung der Kanalisation einer Stadt. Eine wirtschaftlich technische Studie, als Beitrag zur KanalisationsLiteratur. 1910. 88 S., 1 Taf. $8^{0}$ - 2. erw. Aufl. soll demnächst erscheinen; bis 1913 nicht ersch.

Braunschweig, Dr.-Ing.-Diss. v. 16. 12. 1910

823. Schröder, Herbert: Gerbersysteme als Raumfachwerke. Berlin: Krayn [1911]. 72S. $8^{0}$

Braunschweig, Dr.-Ing.-Diss. v. 6. 2. 1911

824. Richter, Fritz: Ueber das Holz als Baumaterial des Wasserbaues, insbesondere über die Höhenlage der Fäulnisgrenze des Holzes und seine Dauerhaftigkeit unter verschiedenen Verhältnissen. 1911. 79 S. $8^{0}$

Braunschweig, Dr.-Ing.-Diss. v. 15. 11. 1911

\section{ABTEILUNG FÜR MASCHINENBAU EINSCHLIESZLICH ELEKTROTECHNIK UND TEXTILINDUSTRIE}

\author{
Maschinen bau
}

825. Nachtweh, Alwin, a. o. Prof. an d. Univ. Halle: Beiträge zur Theorie und Beurteilung der Mähemaschinen. 1903. 47 S., 6 Taf. $4^{0}$ - Auch bei Parey in Berlin 1904; vollst. in: Landwirtschaftl. Jahrbücher. Bd 32 .

Braunschweig, Dr.-Ing.-Diss. v. 30. 7. 1903

826. Lindemann, Wilhelm, Reg.-Bauführer, Berlin: Der Lokomotivrahmen als starrer Balken auf federnden Stützen. Ein Beitrag zur Bestimmung der Lastverteilung von Lokomotiven. 1904. 31 S., 3 Taf. $4^{0}$ - Gekürzt in: Annalen f. Gewerbe u. Bauwesen. Bd 54 .

Braunschweig, Dr.-Ing.-Diss. v. 18. 5. 1904

827. Wedemeyer, 0tto, staatl. gepr. Bauführer, Betriebsassist. d. Eisengiesserei d. Gutehoffnungshütte, Abt. Sterkrade: Uber die Verwendung von Manganerzen als Entschweflungsmittel beim Schmelzen von Gusseisen. 1904. $27 \mathrm{~S} .8^{0}$ - Etwas geändert in: Stahl u. Eisen. Jg. 24, Halbj. 2.

Braunschweig, Dr.-Ing.-Diss. v. 19. 5. 1904

828. Lawaezeck, Franz: Beitrag zur Theorie und Konstruktion der Wage, mit besonderer Berücksichtigung der n-fach übersetzten Hebelwage. Berlin: Dietze 1906. 80 S. $8^{0}$ ศ Aus: Dinglers Polytechn. Journal. Jg. 87. Bd 321 .

Braunschweig, Dr.-Ing.-Diss. v. 14. 5. 1906 
829. Stenzel, Georg: Die Fingerschutzvorrichtungen für Tiegeldruckpressen. 1906. 138 S., 31 Taf. $8^{0}$

Braunschweig, Dr.-Ing.-Diss. v. 26. 7. 1906

830. Sehmidt, Karl: Die Berechnung der Luftpumpen für Oberflächenkondensationen unter besonderer Berücksichtigung der Turbinenkondensationen. Berlin: Springer 1909. 148 S. $8^{0}$ - Auch als Buch.

Braunschweig, Dr.-Ing.-Diss. v: 1. 5. 1909

831. Zacharias, Ludwig: Untersuchungen an zylindrischen Schraubenfedern mit kreisförmigem Querschnitte. 1910. $31 \mathrm{~S} .4^{0}$ - Auch in: Mitteilungen über Forsehungsarbeiten auf $\mathrm{d}$. Gebiete d. Ingenieurwesens. H. 106. 1911.

Braunschweig, Dr.-Ing.-Diss. v. 12. 11. 1909

832. Sehmitz, 0tto H[einrich]: Úber Druckmessungen an hydraulischen Geschützbremsen. Ein Beitrag zur Theorie der Indikatoren. Berlin: Buntrock 1910. 74 S. $8^{\circ}$ - Auch als Buch.

Braunschweig, Dr.-Ing.-Diss. v. 11. 7. 1910

833. Skutsch, Rudolf: Utber den Einfluß der elastischen Nachwirkung auf die Leistungsfähigkeit der Riementriebe. Dortmund: Selbstverl. 1910. 37 S., 2 Taf. $4^{0}$ - Ohne das Vorw. in: Mitteilungen über Forschungsarbeiten anf d. Gebiete d. Ingenieurwesens. H. 120. 1912; Ausz. in: Zeitschrift d. Vereines deutscher Ingenieure. Bd 55. 1911, Halbj. 2.

Braunschweig, Dr.-'ng.-Diss. v. 21. 7. 1910

834. Hofmann, [Adam], Reg.-Baumeister a. D.: Uber Grundlagen für den Bau von Kraft-Wagen. Berlin: R. C. Schmidt \& Co. 1912. 84 S., 5 Taf. $4^{0}$ - Auch als Buch.

Braunsehweig, Dr.-Ing.-Diss. v. 21. 2. 1911

835. Essich, 0tto: Theorie und Berechnung der Turbogebläse und Turbokompressoren. Berlin: Krayn [1911]. 64 S. $8^{0}$

Braunschweig, Dr.-Ing.-Diss. v. 28. 2. 1911

836. Schwarze, Bruno, Reg.-Baumeister: Härteuntersuchungen an Radreifenstoff nach dem Kohn-Brinellschen Kugeldruckverfahren. 1911. IV, 63 S. $8^{0}$ - Auch bei Vieweg in Braunsehweig.

Braunschweig, Dr.-Ing.-Diss. v. 27. 10. 1911

837. Speer, 0skar: Die Sicherheit der Förderseile. 1912. 44 S. $4^{0}$ - Aus: Glückauf. Jg. 48.

Braunschweig, Dr.-Ing.-Diss. v. 1. 3.1912

\section{Elektrotechnik}

838. Baldamus, Max: Die Rentabilitätsgrenzen von Überlandcentralen mit Dampf als Betriebskraft. Wolfenbüttel: Heckner 1902. 46 S., 5 Taf. $4^{0}$

Braunschweig, Dr.-Ing.-Diss. v. 20. 6. 1902
839. Voege, Willi: Untersuchungen über die Strahlungseigenschaften der neueren Glühlampen. 1904. 33 S., 4 Taf. $4^{0}$ - Auch bei Gräfe \& Sillem in Hamburg in Komm.; aus: Jahrbuch d. hamburg. wiss. Anstalten. Jg. 22.

Braunschweig, Dr.-Ing.-Diss. v. 17. 12. 1903

840. Wille, Heinrieh: Pufferbatterien im Strassenbahnbetriebe. $1905.11 \mathrm{~S} .4^{0}$ - Aus: Elektr. Bahnen u. Betriebe. Jg. 3.

Braunschweig, Dr.-Ing.-Diss. v. 26. 4. 1904

841. Neugebohrn, Karl: Untersuchungen über die Änderung des Selbstinduktionskoeffizienten im Magnetfelde. 1904. $20 \mathrm{~S}$., 3 Taf. $4^{0}$

Braunschweig, Dr.-Ing.-Diss. v. 28. 7. 1904

842. Weeken, Wilhelm: Vergleichende Untersuchungen über lineare und drehende magnetische Hysteresis. 1905. $64 \mathrm{~S} .8^{0}$ - Ausz. in: Zeitschrift f. Elektrotechnik. Jg. 23. Braunschweig, Dr.-Ing.-Diss. v. 23. 12. 1904

843. Lulofs, W[arner]: Úber das Verhalten des Ohmschen Widerstandes und des Selbstinduktionskoeffizienten in Abhängigkeit von der Frequenz des durchgeschickten Wechselstromes. 1906. 38 S. $8^{\circ}$

Braunschweig, Dr.-Ing.-Diss. v. 29. 1. 1906

844. Siebert, Wilhelm: Wirbelströme in massiven Polschuhen. 1908. 70 S., 11 Taf. $8^{\circ}$ - Auch bei d. Druckerei-Gesellschaft Hartung \& Co. in Hamburg.

Braunschweig, Dr.-Ing.-Diss. v. 23. 7. 1907

845. Natalis, Friedrich, Obering. d. Siemens-Schuckertwerke i. Berlin: Die selbsttätige Regulierung der elektrischen Generatoren. 1908. $112 \mathrm{~S} ., 4$ Taf. $8^{0}$ - Auch als: Elektrotechnik in Einzeldarstellungen. H. 11.

Braunsehweig, Dr.-Ing.-Diss. v. 19. 11. 1907

846. Wasmus, Adolf: Uber Versuche am Peukertschen Hochfrequenzgenerator. 1909. 32 S. $8^{0}$

Braunschweig, Dr.-Ing.-Diss. v. 27. 3. 1909

847. Subkis, Solomon: Der Einfluss der Koppelung bei langsamen ungedämpften Schwingungen. Leipzig: J. A. Barth 1910. 31 S. $8^{\circ}$ - Aus: Jahrbuch d. drahtlosen Telegraphie u. Telephonie. Bd 5. 1911.

Braunschweig, Dr.-Ing.-Diss. v. 29. 7. 1910

848. Buch, Albert: Ueber Spannungsteilung bei Gleichstrommaschinen. 1912. 41 S. $8^{\circ}$

Braunsehweig, Dr.-Ing.-Diss. v. 15. 3. 1912

849. Erb, Friedrieh: Über die Ausbreitung Hertzscher Wellen an Metallen und Salzlösungen. 1912. $33 \mathrm{~S} .8^{0}$

Braunschweig, Dr.-Ing.-Diss. v. 10. 5. 1912 


\section{Textilindustrie}

850. Schatz, Desiderius, Dir. d. Höheren Webschule zu Zittau i. S.: Einfluss der Appretur auf die physikalisehen Eigenschaften eines halbwollenen Gewebes. 1902. 84 S. $8^{0}$ - Aus: Zeitschrift f. d. ges. Textilindustrie. Jg. 5. 6. 1901/02. 02/03.

Braunschweig, Dr.-Ing.-Diss. v. 20. 3. 1902

851. Zeising, Hermann: Uber SchappeSpinnerei. Leipzig: Leipziger Monatsschrift f. Textil-Industrie. Th. Martin 1910, 48 S. $4^{0}$ - Auch in: Leipziger Monatsschrift f. TextilIndustrie. Jg. 25.

Braunschweig, Dr.-Ing.-Diss. v. 7. 2. 1910

\section{ABTEILUNG FÜR CHEME}

852. Maier, Johann: Studien über Ringcondensationen. 1901. 42 S. $8^{0}$ - Vgl. J. Liebig's Annalen d. Chamie. Bd 327. 1903. S. $17 \mathrm{ff}$.

Brannschweig, Dr.-Ing.-Diss. v. 23. 7. 1901

853. Lutzau, Gustar v.: Ober die Einwirkung von Estern zweibasischer Säuren auf primäre aromatische Amidokörper. 1902 $31 \mathrm{~S} .8^{0}-\mathrm{VgL}$ J. Liebig's Annalen d. Chemie. Bd 347. 1906. S. $17 \mathrm{ff}$.

Braunschweig, Dr.-Ing.-Diss. v. 13. 3. 1902

854. Jaeger, Paul: Studien über Ringkondensationen. 1903. $46 \mathrm{~S} .8^{0}$ - Vgl. J. Liebig's'Annalen d. Chemie. Bd 347. 1906. S. $17 \mathrm{ff}$; Berichte d. Deutschen Chem. Gesellschaft. Jg. 36. S. $1555 \mathrm{ff}$.

Braunschweig, Dr.-Ing.-Diss. v. 23. 3. 1903

855. Eberlein, Wilhelm: Über einige komplexe Salze des Silbers, des Goldes und des Quecksilbers. 1904. 56 S. $8^{\circ}$ - S. $1-47$ auch in: Zeitsehrift f. anorgan. Chemie. Bd 39. S. $197 \mathrm{ff}$.

Braunschweig, Dr.-Ing.-Diss. v. 26. 3. 1903

856. Köppen, Karl von: Bildungsgeschwindigkeit und Dissociation von Schwefelsäureanhydrid bei Anwesenheit von Platin. 1903. 61 S. $8^{0}$ - S. $1-36$ zuerst in: Zeitschrift f. Elektrochemie. Jg. 9.

Braunschweig, Dr.-Ing.-Diss. v. 22. 7. 1903

857. Kunsehert, Franz: Ober komplexe Salze des Kupfers und des Zinks. 1904. $42 \mathrm{~S} .8^{0}$ Auch in: Zeitschrift f. anorgan. Chemie. Bd 41.

Braunschweig, Dr.-Ing.-Diss. v. 22. 7. 1903

858. Spengler, 0sear: Studien über die Konstitution der Phtaleīnsalze. 1904. 35 S. $8^{0}$ - Vgl. Berichte d. Deutschen Chem. Gesellschaft. Jg. 36. 1903.

Braunschweig, Dr.-Ing.-Diss. v. 6. 11. 1903
859. Idaszewski, Kasimir S[igismund]: Versuche über das elektrolytische Verhalten von Schwefelkupfer. 1905. 72 S. $8^{0}$ Auch in: Zeitschrift f. Elektrochemie. Jg. 11.

Braunschweig, Dr.-Ing.-Diss. v. 25. 6. 1904

860. Bock, Paul: Zur Kenntnis der Isobernsteinsäure. 1905.34 S. $8^{0}$ - Ausz. in: J. Liebig's Annalen d. Chemie. Bd 347. 1906.

Braunschweig, Dr.-Ing.-Diss. v. 8. 3. 1905

861. Hartwig, Ludwig: Untersuchungen über die Einwirkung von Harnstoff auf Verbindungen der Cyanessigsäure. 1905. $52 \mathrm{~S} .8^{0}$ - Auch in: Journal f. prakt. Chemie. Bd 180. 181. N. F. Bd 72. 73 . $1905 / 06$.

Braunschweig, Dr.-Ing.-Diss. v. 9. 3. 1905

862. Hartmann, Ernst: Über 1, 3, 6-Trioxynaphthalin. 1905. $32 \mathrm{~S} .8^{0}$ - Vgl. Berichte d. Deutschen Chem. Gesellschaft. Jg. 38.

Braunschweig, Dr.-Ing.-Diss. v. 23. 3. 1905

863. Tögel, Karl: Zur Kenntnis der Grignard'schen Reaktion. 1905. 39 S., 1 Taf. $8^{0}$ - Vgl. J. Liebig's Annalen d. Chemie. Bd 347.1906. Braunschweig, Dr.-Ing.-Diss. v. 21. 7. 1905

864. Witte, Karl: Uber Kondensationsprodukte des Hydrochinons. 1906. 27 S. $8^{0}$ - Vgl. Berichte d. Deutschen Chem. Gesellschaft. Jg. 41. 1908.

Braunschweig, Dr.-Ing.-Diss. v. 25. 1. 1906

865. Scheller, Alfred: Ueber die anormale anodische Polarisation durch Halogene. [1905.] 74 S., 1 Taf. $8^{0}$ - Vgl. Zeitschrift f. anorgan. Chemie. Bd 48. 1906.

Braunschweig, Dr.-Ing.-Diss. v. 20. 2. 1906

866. Pfotenhauer, Herm[ann]: Studien in der Phtaleïngruppe. 1906. $43 \mathrm{~S} .8^{0}$

Braunschweig, Dr.-Ing.-Diss. v. 17. 5. 1906

867. Schellhaass, H[ans] W[alther] Hugo: Die Rolle der Caro'schen Säure bei der elektrolytischen Bildung der Ueberschwefelsäure und ihrer Salze. [1906.] 92 S. $8^{0}$ - Ausz. in: Zeitschrift f. Elektrochemie. Jg. 13. 1907.

Braunschweig, Dr.-Ing.-Diss. v. 26. 7. 1906

868. Marx, Karl: Studien über die Tautomerie des Succinylchlorides. 1907. $42 \mathrm{~S} .8^{0}$ - Vgl. Berichte d. Deutschen Chem. Gesellschaft. Jg. 41. 1908.

Braunschweig, Dr.-Ing.-Diss. v. 28. 1. 1907

869. Puttkammer, Georg: Über Säureadditionsprodukte von Dimethylazobenzolhydrazonen, 1908. $39 \mathrm{~S} .8^{0} \bullet \mathrm{Vgl}$. Journal f. prakt. Chemie. Bd 186. N. F. Bd 78 .

Braunschweig, Dr.-Ing.-Diss. v. 25. 5. 1908

870. Masur, Tobias: Über die Bildung einiger neuer Akridinfarbstoffe aus $\mathrm{Ab}$ kömmlingen des Diaminodiphenylmethans. 1908. $36 \mathrm{~S} .8^{0}$

Braunschweig, Dr.-Ing.-Diss. v. 21. 7. 1908 
871. Lux, Emil: Úber die Beweglichkeit der Wasserstoffatome der Methylengruppe in Verbindungen der allgemeinen Formeln: $\mathrm{RSO}_{2} \mathrm{CH}_{2} \mathrm{CN}, \mathrm{RSO}_{2} \mathrm{CH}_{2} \mathrm{CONH}_{2}, \mathrm{RSO}_{2} \mathrm{CH}_{2}$ $\mathrm{COOC}_{2} \mathrm{H}_{5}$. 1909. $62 \mathrm{~S}$. $8^{0}$ - Etwas gekürzt in: Arehiv d. Pharmazie. Bd 247.

Braunschweig, Dr.-Ing.-Diss. v. 30. 12. 1908

872. Müller, Otto: Beiträge zur Erforschung der Angosturaalkaloide. 1909. 72 S. $8^{\circ}$ - Ausz. in: Apotheker-Zeitung. Jg. 24. S. 678 f. Braunschweig, Dr.-Ing.-Diss. v. 30. 12. 1908

873. Lipski, Jakob: Ueber Synthese des Ammoniaks aus den Elementen. 1909. 57 S. $8^{\circ}$ - Aus: Zeitschrift f. Elektrochemie. Jg. 15.

Braunschweig, Dr.-Ing.-Diss. v. 28. 1. 1909

874. Franke, Max: Styrylaminverbindungen. Verhalten quartärer Styrylammoniumverbindungen gegen nascierenden Wasserstoff. 1909. 52 S. $8^{0}$ - Ausz. in: Archiv d. Pharmazie. Bd 247.

Braunsehweig, Dr.-Ing.-Diss. v. 8. 2. 1909

875. Feinmann, Isidor : Uber einige Aminsäuren, Imide und Isoimide zweibasischer Säuren. 1909. 36 S. $8^{0}$

Braunschweig, Dr.-Ing.-Diss. v. 12. 3. 1909

876. Kissin, Sehmul-Juda: Zur Kenntnis der Phtaleïnoxime. 1909. $32 \mathrm{~S} .8^{0} \bullet \mathrm{Vgl}$ Berichte d. Deutschen Chem. Gesellschaft. Jg. 42. Braunschweig, Dr.-Ing.-Diss. v. 12. 3. 1909

877. Proehnow, Adolf: Beiträge zur Untersuchung des Kakaos und seiner Präparate. 1909. $70 \mathrm{~S} .8^{0}$ - Ausz. in: Archiv d. Pharmazie. Bd 247. 248. 1909. 10.

Braunschweig, Dr.-Ing.-Diss. v. 16. 3. 1909

878. Lindner, Bernhard: Beiträge zur Untersuchung von Tranen. 1909.62 S. $8^{0}$ Braunschweig, Dr.-Ing.-Diss. v. 19. 3. 1909

879. Spies, Fritz: Untersuchungen von nach dem Plasmolyse-Verfahren gewonnenen Hefe-Enzymen. 1909. 83 S. $8^{0}$

Braunschweig, Dr.-Ing.-Diss. v. 9. 6. 1909

880. Desamari, Kurt: Über das „Tribromresochinon." 1909. $60 \mathrm{~S} .8^{0}$ "Vgl. Berichte d. Deutschen Chem. Gesellschaft. Jg. 42. Braunschweig, Dr.-Ing.-Diss. v. 19. 7. 1909

881. Wolfsleben, Kurt: Uber Naphtoresorcin und 1-3-Amidonaphtol. 1909. 48 S. $8^{\circ}$ - Vgl. Berichte d. Deutschen Chem. Gesellschaft. Jg. 44. 1911.

Braunsehweig, Dr.-Ing.-Diss. v. 20. 7. 1909

882. Westerkamp, Arthur: Beiträge zur Kenntnis der Azo-aryl-hydrazinsulfonsäuren. (1910). 46 S. $8^{0}$ - Auch in: Archiv d. Pharmazie. Bd 247. 1909.

Braunschweig, Dr.-Ing.-Diss. v. 23. 11. 1909
883. Titlestad, Nicolay: Photo-VoltaKetten mit Urano-Uranylsulfat. Leipzig: W. Engelmann 1910. 55 S. $8^{\circ}$ - Aus: Zeitschrift $\hat{f}$. physik. Chemie. Bd 72 .

Braunschweig, Dr.-Ing.-Diss. v. 20. 12. 1909

884. Taitelbaum, Itzek: Studien über Brennstoffketten. 1910. 50 S. $8^{0}$ - Aus: Zeitschrift f. Elektrochemie. Jg. 16.

Braunschweig, Dr.-Ing.-Diss. v. 8. 3. 1910

885. Okada, Harukiehi: Studie über den Samen der "Euphorbia elastica“. Braunschweig: Appelhans 1910.70 S. $8^{0}$

Braunschweig, Dr.-Ing.-Diss. v. 6. 7. 1910

886. Schaper, Carl: Uber das Oxydationspotential der Oxalate des Eisens und des Oxalations. 1910. 55 S. $8^{0}$ - Vgl. Zeitschrift f. physik. Chemie. Bd 72 .

Braunschweig, Dr.-Ing.-Diss. v. 21. 7. 1910

887. Sehellbach, Johannes: Uber die Festigkeit der Kohlenstoff-Stickstoffbindung in quartären Ammoniumverbindungen mit der Gruppierung C:C.C.N. 1910. 61 S. $8^{0}$ - Vgl. Arehiv d. Pharmazie. Bd 249. 1911.

Braunschweig, Dr.-Ing.-Diss. v. 23. 7. 1910

888. Runne, Ernst: Kohlenstoff-Stickstoff-Bindung in Aminoketonen und Aminoalkoholen. 1910. $63 \mathrm{~S} .8^{0}$ - Vgl. Arehiv d. Pharmazie. Bd 249. 1911.

Braunschweig, Dr.-Ing.-Diss. v. 29. 7. 1910

889. Runne, Hermann: Beiträge zur Erforschung der Angosturaalkaloide. 1911. 37 S. $8^{\circ}$ - Auch in: Arehiv d. Pharmazie. Bd 249; Ausz. in: Aus d. Pharmazeut. Institut d. Hzgl. Techn. Hochschule Carolo-Wilhelmina. 1910 [Nr 800] u. in: Apotheker-Zeitung. Jg. 25. 1910.

Braunschweig, Dr.-Ing.-Diss. v. 29. 11. 1910

890. Siehling, Konrad: Uber die Natur der Photochloride des Silbers und deren Lichtpotentiale. Leipzig: W. Engelmann 1911. 59 S. $8^{0}$ - Aus: Zeitschrift f. physik. Chemie. Bd 77 .

Braunschweig, Dr.-Ing.-Diss. v. 16. 2. 1911

891. Glüeksberg, Raehmil: Uber neue Bildungsweisen von Farbstoffen der Pyroningruppe aus alkylierten $\mathrm{m}$-Amidophenolen. 1911. 53 S. $8^{0}$ - Vgl. J. Liebig's Annalen d. Chemie. Bd 391. 1912. S. $308 \mathrm{ff}$.

Braunschweig, Dr.-Ing.-Diss. v. 16. 3. 1911

892. Laehmann, Robert: Beitrag zur Kenntnis des Berberins. 1911. 47 S. $8^{\circ}$ - Vgl. J. Liebig's Annalen d. Chemie. Bd 397. 1913. Braunschweig, Dr.-Ing.-Diss. v. 2. 6. 1911

893. Meyer, Karl: Uber die Phtaleīne des $3,5,3^{\prime}, 5^{\prime}$ - Biresorcins. 1911. 31 S. $8^{0}$ - Vgl. Berichte d. Deutsehen Chem. Gesellschaft. Jg. 44.

Braunschweig, Dr.-Ing.-Diss. v. 20. 6. 1911 
894. Ulrieh, Christoph, staatl. geprüfter Nahrungsmittelchemiker: Der Nachweis von Schalen im Kakao und in seinen Präparaten. Detmold: Meyer 1911. 97 S. So - Auch in: Archiv d. Pharmazie. Bd 249.

Braunschweig, Dr.-Ing.-Diss. v. 19. 7. 1911

895. Schiller, Herbert: Ueber den Becquerel-Effekt bej komplexen Eisen- und Uran-Salzen. 1912.83 S., 23 Taf. $8^{0}$ - Ausz. in: Zeitschrift f. physik. Chemie. Bd 80.

Braunschweig, Dr.-Ing.-Diss. v. 21. 7. 1911

896. Rinekleben, Paul: Die Gewinnung von Zymase unter besonderer Berücksichtigung der Plasmolyse frischer Brauereihefe. 1912. 22 S. $4^{0}$ - Aus: Allg. Zeitschrift f. Bierbrauerei u. Malzfabrikation. Jg. 40.

Braunschweig, Dr.-Ing.-Diss. v. 15. 3. 1912

897. Schuster, Siegfried: Untersuchungen über die Ursache der Oxydation von Letternmetallen. 1912. 32 S. $8^{0}$

Braunschweig, Dr.-Ing.-Diss. v. 16. 3. 1912
898. Kroseberg, Wilhelm: Beiträge zur Erforschung der Angosturaalkaloide. [1912.] 42 S. $8^{0}-$ Aus: Archiv d. Pharmazie. Bd 250.

Braunschweig, Dr.-Ing.-Diss. v. 21. 3. 1912

899. Schlie, Klaus: Uber pyrogene Acetylenkondensation. 1912. $36 \mathrm{~S}$., 1 Taf. $8^{0}$ - Vgl. Berichte d. Deutschen Chem. Gesellschaft. Jg. 45. S. $1609 \mathrm{ff}$.

Braunschweig, Dr.-Ing.-Diss. v. 23. 5. 1912

900. Duczmal, Casimir: Umsetzungen von Chloressigsäure mit Phenolcarbonsäuren und Nitrophenolen. 1912. $40 \mathrm{~S} .8^{0}$ i Vgl. Berichte d. Deutschen Chem. Gesellschaft. Jg. 46. 1913.

Braunsehweig, Dr.-Ing.-Diss. v. 23. 7. 1912

901. Fischer, Otto: Spektrographische Studien. 1912. 39 S. $8^{0}$ - Vgl. Berichte d. Deutschen Chem. Gesellschaft. Jg. 44. 46. 1911. 13

Braunschweig, Dr.-Ing.-Diss. v. 23. 7. 1912

\section{B RESLAU}

Gegründet 1910

\section{SATZUNGEN ORDNUNGEN BESTIMMUNGEN}

\section{ALLGEMEINES UND VERMSCHTES}

902. Verfassungsstatut der Kgl. Technischen Hochschule zu Breslau (v. 20. 7. 1910). [19]10. - Abgedr. in: Zentralblatt f. d. ges. Unterrichtsverwaltung in PreuBen. Jg. 1910. S. $754 \mathrm{ff}$. u. in: Programm f. das Studienj. 1910-1911 [Nr 919].

903. Gesehäftsanweisung für die Abteilungs-Kollegien der Kgl. Technischen Hochschule in Breslau (v. 17. 8. 1910). [19]10.

904. Vorsehriften für die Studierenden und Hörer der Kgl. Technischen Hochschule in Breslau (v. 17. 8. 1910). [19]10.

905. Bestimmungen über den Geschäftskreis des Kgl. Kommissars bei der Technischen Hochschule in Breslau (v. 30.8. 1910). [19]10.

906. Habilitations-Ordnung für die $\mathrm{Kgl}$. Technische Hochschule zu Breslau. [19]10.

907. Haus-0rdnung der Kgl. Technischen Hochschule zu Breslau [v. 17. 2. 1911].

908. Urlaubs-0rdnung für die Professoren und Dozenten der Kgl. Technischen Hochschule zu Breslau. Vom 24. 3. 1911.
909. Dienstanweisung für die Saaldiener der Kgl. Technischen Hochsehule zu Breslau (v. 19.6. 1911).

910. Vorläufige Benutzungsordnung für die Hauptbücherei der Kgl. Technischen Hochschule in Breslau (v. 20. 6. 1911).

911. Laboratoriums - Ordnung des Maschinenlaboratoriums der Kgl. Technischen Hochschule in Breslau (v. 11. 9. 1911).

912. Bestimmungen über die Verleihung von Reisestipendien an DiplomIngenieure, welche die Diplomprüfung auf der Technischen Hochschule in Breslau abgelegt haben (v. 12. 2. 1912).

\section{PRÜFUNGSORDNUNGEN}

913. Diplomprïfungs-0rdnung der Abteilung für Maschineningenieurwesen und Elektrotechnik der Kgl. Technischen Hochschule in Breslau. Vom 7. 9. 1911. [Nebst Neudr. der Anlagen.] 1912. 
914. Gesehäfts-0rdnung für die DiplomPrüfungen der Abteilung für Maschineningenieurwesen und Elektrotechnik der $\mathrm{Kgl}$. Technischen Hochschule in Breslau. Vom 7. 9. 1911.

915. Diplomprülungs-Ordnung für Chemiker in der Abteilung für Chemie und Hüttenkunde der Kgl. Technischen Hochschule in Breslau. Vom 7. 9. 1911.
916. Diplomprüfungs-0rdnung für die Fachrichtungen der Hüttenkunde in der Abteilung für Chemie und Hüttenkunde der $\mathrm{Kgl}$. Technischen Hochschule in Breslau. Vom 7. 9. 1911.

917. Gesehäfts-0rdnung für die DiplomPrüfungen der Abteilung für Chemie und Hüttenkunde der $\mathrm{Kgl}$. Technischen Hochschule in Breslau. Vom 7. 9. 1911.

\section{PERIODISCHE SCHRIFTEN}

918. Kgl. Technische Hochschule zu Breslau. Verzeiehnis der Vorlesungen und Ubungen im Studienj. 1910-11.

919. Kgl: Technische Hochschule zu (1911/12 f.: in) Breslau. Programm für das Studienj. 1910-1911-1912-1913.

\section{GELEGENHEITS - UND}

921. Technische Hochschule in Breslau. Festsehrift zur Eröffnung 1. Oktober 1910. Breslau: Hirt. 47 S., 24 Taf. $4^{0}$

922. Denkschrift über die Einweihungsfeier der Königlichen Technischen Hochschule zu Breslau am 29. November 1910.
920. Personal-Verzeichnis der Kgl. Technischen Hochschule zu (1911 ff. : in) Breslau für das W.-H. 1910/11. S.-H. 1911 [usw.]W.-H. 1912/13.

\section{DOKTOR-INGENIEUR-DIS SERTATIONEN}

\section{ABTEILUNG FÜR MASCHINEN- INGENIEURWESEN UND ELEKTR 0- TECHNIK}

924. Fleig, F[duard]: Stromtarife für Großabnehmer elektrischer Energie. Berlin: Springer [1912]. IV, 166 S. $8^{0}$ ฯ Buchausg. 1913.

Breslau, Dr.-Ing.-Diss. v. 29. 1. 1913

\section{ABTEILUNG FƯR CHEMIE UND HÜTTENKUNDE}

\section{Chemie}

925. Lütke, Heinrich: Untersuchungen über das Gesetz von Wiedemann und Franz an Metallegierungen. 1911. 40 S., 5 Taf. $4^{0}$

Breslau, Dr.-Ing.-Diss. v. 19. 5. 1911

926. Sehossberger, Endre: Zur Kenntnis des Carvenens und Terpinolens. Ueber enolCitralacetat, Isogeraniol und Isocitral. 1911. 69 S. $8^{\circ}$

Breslau, Dr.-Ing.-Diss. v. 27. 10. 1911
927. Thiel, Georg, Bergass.: Das Asphaltkalkgebiet des Pescaratales am Nordabhange der Majella 〈Abruzzen〉. 1912. 34 S., 2 Taf. $4^{0}$ - Aus: Zeitschrift f. prakt. Geologie. Jg. 20.

Breslau, Dr.-Ing.-Diss. v. 7. 6. 1912

\section{Hüttenkunde}

928. Buck, Rudolf: Beiträge zur Ausnutzung der Hochofengase. Düsseldorf: Stahleisen 1911. 43 S. $4^{0}$ - Auch in: Mitteilungen aus d. Eisenhüttenmãnn. Institut d. Kgl. Techn. Hochschule Breslau. Bd 1. 1913; Ausz. in: Stahl u. Eisen. Jg. 31, Halbj. 2.

Breslau, Dr.-Ing.-Diss. v. 16. 6. 1911

929. Canaris, C(arl): Ueber den Einfluß des Gießens auf die Qualität von FlußeisenBrammen.Düsseldorf: Stahleisen 1911.20 S. $4^{\circ}$ - Ausz. in: Mitteilungen aus d. Eisenhüittenmänn. Institut d. Kgl. Techn. Hochschule Breslau. Bd 1. 1913 ; S. 8-17 auch in: Stahl u. Eisen. Jg. 32. 1912, Halbj. 2; S. 17 ff. ebd. Halbj. 1 . S. $303 \mathrm{ff.}$ Breslau, Dr.-Ing.-Diss. v. 16. 2. 1912 
930. Becker, Erich: Das Zustandsdiagramm Schwefeleisen - Eisen. Düsseldorf: Stahleisen 1912. 20 S. $4^{0}$ - Ausz. in: Stahl u. Eisen. Jg. 32, Halbj. 1.

Breslau, Dr.-Ing.-Diss. v. 19. 4. 1912

931. Sehulz, Erdmann: Beiträge zur Verhüttung schwefelhaltiger Kiesabbrände im Hochofen. Düsseldorf: Stahleisen 1912. 16 S. $4^{0}$ Auch in: Mitteilungen aus d. Eisenhüttenmänn. Institut d. Kgl. Techn. Hochschule Breslan. Bd 1. 1913; Ausz. in: Stahl u. Eisen. Jg. 32, Halbj. 2. S. $1254 \mathrm{ff}$.

Breslau, Dr.-Ing.-Diss. v. 7. 6. 1912

932. Harnickell, Wilhelm: Beiträge zur Röstung und Aufbereitung der Siegerländer Spateisensteine. Düsseldorf: Stahleisen 1912. $12 \mathrm{~S} .4^{0}-$ Auch in: Mitteilungen aus d. Eisen- hüttenmänn. Institut d. Kgl. Techn. Hochschule Breslau. Bd 1. 1913; Ausz. in: Stahl u. Eisen. Jg. 32, Halbj. 2.

Breslau, Dr.-Ing.-Diss. v. 18. 10. 1912

933. Markgraf, Henry: Experimentelle Untersuchungen über die Verwendung von Braunkohle im Martinbetrieb. Düsseldorf: Stahleisen 1912. 20 S. $4^{0}$ - Auch in: Mitteilungen aus d. Eisenhüttenmänn. Institut d. Kgl. Techn. Hochschule Breslau. Bd1. 1913; vgl. Stahl u. Eisen. Jg. 32, Halbj. 2. S. $1477 \mathrm{ff}$.

Breslau, Dr.-Ing.-Diss* v. 18. 10. 1912

933a. Hüser, Friedrich: Experimentelle Untersuchung des Kuppelofen-Schmelzprozesses. Düsseldorf: Stahleisen 1912. 29 S. $4^{0}$ - Ausz. in: Stahl u. Eisen. Jg. 33. 1913, Halbj. 1. Breslau, Dr.-Ing.-Diss. v. 7. 2. 1913

\title{
DANZIG
}

\author{
Gegründet 1904
}

\section{SATZUNGEN ORDNUNGEN BESTIMMUNGEN}

\section{ALLGEMEINES UND VERMISCHTES}

934. Verfassungsstatut der Kgl. Technischen Hochschule zu Danzig (v. 1. 10. 1904). - [Neudr.] UUnter Berücksichtigung der durch Allerhöchste Kabinettsorder vom 5. 7. 1905 verfügten Abänderungen.> 190.5. - Abgedr. in: Zentralblatt f. d. gesamte Unterrichts-Verwaltung in Preußen. Jg. 1904. S. 528 ff.; Damm 1909. S. $219 \mathrm{ff}$.

935. Gesehäftsanweisung für die Abteilungs-Kollegien der Kgl. Technischen Hochschule zu Danzig (v. 2. 10. 1904). - Abgedr. in: Damm 1909. S. 232 ff.

936. Vorschriften für die Studierenden und Hospitanten der $\mathrm{Kgl}$. Technischen Hochschule zu Danzig (v. 2. 10. 1904). [Nebst] Nachtrag zu $\S 21$ v. 15. 9. 1906. - Abgedr. in: Damm 1909. S. 241 ff.

937. Bestimmungen über den Geschäftskreis des $\mathrm{Kgl}$. Kommissars bei der Technischen Hochschule in Danzig (v. 2. 10. 1904).

938. Habilitations-0rdnung für die Kgl. Technische Hochschule zu Danzig (v. 3. 10. 1904). - Abgedr. in: Damm 1909. S. 234 ff.

939. Hausordnung der $\mathrm{Kgl}$. Technischen Hochschule zu Danzig (v. 13. 2. 1905). - Abgedr. in: Damm 1909. S. $249 \mathrm{f}$.
940. Stipendien für die Provinz Westpreußen. Auszug aus dem Protokoll über die Sitzung des 29. Westpreuß. ProvinzialLandtages am 2. 3. 1905 (betr. Stiftung eines Stipendiums für einen der Prov. Westpreußen angehörigen Studierenden der Technischen Hochschule in Danzig. Unterz.: Danzig, 11. 3. 1905. Der Landeshauptmann der Provinz Westpreußen.) 1910.

941. Satzungen der Heckmann-Stiftung (betr. Gewährung von Stipendien an Studierende der Abteilung für Schiff- und Schiffsmaschinenbau der Kgl. Technischen Hochschule in Danzig v. 21. 6. 1905). 1905.

942. Satzungen der Henrietten-Stiftung (betr. die Unterstützung von Studierenden der Technischen Hochschule in Danzig v. 14. 7. 1905). 1905.

943. Benutzungs-0rdnung für die Bücherei der Kgl. Technischen Hochschule zu Danzig. [1905. Nicht genehmigt.] - [Dasselbe.] Genehmigt 2. 8. u. 9.9. 1905. I Abgedr. in: Damm 1909. S. $250 \mathrm{ff}$.

944. Satzungen der Krankenhilfskasse für die Studentenschaft der Kgl. Technischen Hochschule zu Danzig. [Entwurf. 1905.] [Dasselbe] (v. 11. 9. 1905). 1905. 
945. Satzung der Krankenhilfskasse für die Studentensehaft der Kgl. Technischen Hochschule zu Danzig (v. 11. 9. 1905, Änderungen v. 18.12 .1906 u. 15.4. 1907). - Abgedr. in: Damm 1909. S. $255 \mathrm{ff}$.

946. Stiftungsurkunde der StipendienStiftung ,Conradinum - Technische Hochschule $^{\text {si }}$ (betr. Stipendien für Vollstudierende der Techn. Hochschule zu Danzig. Unterz. : Danzig, 11. 11. 1905. Adolf Unruh. 1910.)

947. Bestimmungen über die Verleihung von Reisestipendien an Diplom-Ingenieure, welche die Diplomprüfung auf der Technischen Hochschule in Danzig abgelegt haben (v. 15. 7. 1906). (1910.) - [Dasselbe] (mit Abänderung v. 13. 2. 1912). โ Abgedr. in: Damm 1909. S. $283 \mathrm{f}$.

948. Seminar für Städtebau. [Mitteilungen.] (1908.)

949. Bestimmungen für die Drucklegung der Doktor-Ingenieur-Dissertationen (v. 17. 12. 1908.) 1909. - [Dasselbe mit einem Zusatz am Schluß.] (1910.)

950. Bestimmungen für die Bücherei der Kgl. Technischen Hochschule Danzig. Vom 30. 6. 1909. 1909 .

951. Benutzungsordnung für die Bücherei der $\mathrm{Kgl}$. Technischen Hochsehule Danzig. 1909. - Aus: Bestimmungen für die Büeherei [Nr 950].

952. Satzung der Krankenhilfskasse für die Studentenschaft der $\mathrm{Kgl}$. Technischen Hochschule Danzig (v. 5. 4. 1910. 1910).

953. Urlaubs-0rdnung für die Professoren und Dozenten der $\mathrm{Kgl}$. Technischen Hochschule zu Danzig. Vom 17. 2. u. 13.6.11. 1911.

954. Satzung der Krankenhilfskasse für die Studentenschaft der Kgl. Technischen Hochschule zu Danzig. Vom 15. 4. 1912. [19]12.

955. Gesehäftsordnung für die Mitgliederversammlung der Krankenhilfskasse für die Studentenschaft der Kgl. Technischen Hochschule zu Danzig. Vom 15. 4. 1912. [19]12.

955. Leistungen der Krankenhilfskasse für die Studentenschaft der Kgl. Technischen Hochschule zu Danzig. Aufgestellt nach $\$ 5$ Abs. 6 der Satzungen in der Mitgliederversammlung v. 15. 5. 12. [19]12.

\section{PRÚPUNGSORDNUNGEN}

\section{Abteilung für Architektur}

957. Diplomprüfungs-0rdnung der $\mathrm{Ab}$ teilung I für Arehitektur an der $\mathrm{Kgl}$. Technischen Hochschule zu Danzig. Vom 12. 9. 1905. [Nebst] Deckblätter (zu S. 8) [u.] Deckblatt (zu § 7). 1905. [07. 09.]

958. Gesehäfts-0rdnung für die Diplomprüfungen der Abteilung für Architektur der $\mathrm{Kgl}$. Technischen Hochschule zu Danzig. Vom 12. 9. 1905. 1905.

\section{Abteilung für $\mathrm{Bauingenieurwesen}$}

959. Diplomprüfungs-0rdnung der Abteilung II für Bauingenieurwesen an der $\mathrm{Kgl}$. Technischen Hochschule zu Danzig. Vom 12. 9. 1905. [Nebst] Deckbl. (zu S. 10 -12). 1905. [07.] - [Dasselbe.] (Unter Berücksichtigung der Deckbl. v. 1907.) [Nebst] Deckbl. (zu \& 7). 1907. [09.] - [Dasselbe.] Abgeändert 19. 6. 1911. 1911.

960. Gesehäfts-Ordnung für die Diplomprüfungen der Abteilung für Bauingenieurwesen der Kgl. Technischen Hochschule zu Danzig. Vom 12.9. 1905. [Nebst] Deckblätter (zu $\$ 7$ u. 8). 1905. [07.] - [Dasselbe.] Abgeändert 19. 6. 1911. 1911.

Abteilung für Maschinen-Ingenieurwesen und Elektrotechnik

961. Diplompriifungs-0rdnung der Abteilung III für Maschineningenieurwesen und Elektrotechnik an der $\mathrm{Kgl}$. Technischen Hochschule zu Danzig. Vom 12.9. 1905. [Nebst] Deckbl. [1 (zu $\$ 8$ u. 17). 2 (zu $\S 3$ v. 2. 10. 09).] 1905. [09.] - [Veränderte Fassung.] 1912. [Nebst] Erläuterungen.

962. Gesehäfts-0rdnung für die Diplomprüfungen der Abteilung für MaschinenIngenieurwesen und Elektrotechnik der $\mathrm{Kgl}$. Technischen Hochschule zu Danzig. Vom 25. 11. 1905. 1905.

\section{Abteilung für Schiff- und Schiffs- maschinenbau}

963. Gesehäfts-0rdnung für die Diplomprüfungen der Abteilung für Schiff- und Schiffsmaschinenbau der Kgl. Technischen Hochschule zu Danzig. Vom 12. 9. 1905. 1905. 
964. Diplomprüfungs-0rdnung der Abteilung IV für Schift- und Schiffsmaschinenbau der $\mathrm{Kgl}$. Technischen Hochschule zu Danzig. Vom 25. 11. 1905. 1905.

965. Diplomprüfungs-0rdnung der Abteilung IV für Schiff- und Schiffsmaschinenbau der $\mathrm{Kgl}$. Technischen Hochschule zu Danzig. Vom 5. 5. 1908. [Nebst] Deckbl. zu $\S 8$ u. 11). 1908. [09.]
Abteilung für Chemie

966. Diplomprüfungs-0rdnung der Abteilung V für Chemie an der $\mathrm{Kgl}$. Technischen Hochschule zu Danzig. Vom 12.9.1905. [Nebst] Deckbl. [1 (zu § 8). 2 (zu § 6).] 1905. [07. 09.]

967. Geschäfts-0rdnung für die DiplomPrüfungen der Abteilung für Chemie der $\mathrm{Kgl}$. Technischen Hochschule zu Danzig. Vom 12. 9. 1905. 1905 .

\section{PERIODISCHE SCHRIFTEN}

968. Kgl. Technische Hochschule (1904/051906/07: zu) Danzig. Aufnahmebedingungen und Honorareinzahlung. Studienj. [19041905.] 1905-1906. 19[04]. 05.

969. Kgl.TechnischeHochschule zu Danzig. Programm für das Studienj. 1904/1905 1912-1913. $1904-12$.

1904/05. [1. Aufl.] - 2. Aufl.-3. Aufl.

970. Personal-Verzeichnis der Kgl. Technischen Hochschule zu Danzig für das W.-H. 1904/1905. S.-H. 1905 [usw.] - W.-H. 1912/13. 19[05]-12.

971. Vorlesumgen und Übungen für Interessenten staats- und handelswissenschaftlicher Fächer an der Kgl. Technischen Hochschule zu Danzig. 1905. 1906/07-1909-1910. $19[05]-09$.

972. Stundenplan der Abteilung II <Bauingenieurwesen $>$ für die zu Ostern eintretenden Studierenden. 1906. F Aus: Programm für 1906/07 [Nr 969].

\section{GELEGENHEITS- UND VERMISCHTE SCHRIFTEN}

\section{REDEN ZUR FEIER DES GEBURTS- TAGES DES KAISERS}

976. Mangoldt, H[ans] von: Neuere Anschauungen über das Wesen der Elektrizität. [1905.] 12 S. $4^{0}$ T Aus: Umschau. Jg. 9.

977. Genzmer, Ewald: Uber die Entwickelung des Wohnungswesens in unseren Großstädten und deren Vororten. Danzig: Kafemann 1906. 25 S. $8^{\circ}$ - Etwas gekürzt in: Gesundheit. Jg. 31.

978. Krohn, R[einhold]: Über die Berufstätigkeit des Ingenieurs. Danzig: Schwital \& Rohrbeck 1907. 28 S. $8^{\circ}$

979. Wagener, A[ugust]: Uber die Ausgestaltung des Unterrichtes und der Prüfungsvorschriften für das Maschineningenieur-
973. Kgl. Technische Hochschule zu Danzig. Stundenplan (für das Studienj. 1907-1908 - 1909-1910). 1907-09. [Für die vorhergehenden $u$. die folgenden Jahre im Programm. Nr 969.]

974. Kgl. Technische Hochschule zu Danzig. Die Feier der Utbergabe des Rektorats am 1. Juli 1907. 1909. 1907-09. Fortges. u. d. T.:

Kgl. Technische Hochschule Danzig. Die Übergabe desRektorats am15. Nov.1911.1911. 1907. (Darin: R[einhold] Krohn: Die geschichtliche Entwicklung des Brückenbaues.)

1909. Darin die Rektoratsrede: ,Die Lage der Baukunst in der Gegenwart und die Erziehung zum Architekten an unseren technischen Hochschulen"v. Adelbert Matthaei.

975. Vorlesungen und Übungen für Studierende der Mathematik und der Naturwissenschaften an der $\mathrm{Kgl}$. Technischen Hochschule zu Danzig 1907-1908-19091910. 1908-09. wesen an der technischen Hochschule Danzig: Schwital \& Rohrbeck 1908. 29 S. $8^{0}$

980. Mentz, W[alter]: Entwickelung und Stand des deutschen Schiff- und Schiffsmaschinenbaues und des Hochschulunterrichtes auf diesen Gebieten. Danzig: Schwital \& Rohrbeck 1909. 18 S. $8^{0}$ - Ausz. in: Humanistisches Gymnasium u. modernes Kulturleben. Dankesgrüße ehemaliger Schüler zur Feier des 350jährigen Bestehens des Erfurter Gymnasiums. 1911.

981. Wohl, A[lfred]: Organische Chemie und die Lehre vom Leben. Danzig: Schwital \& Rohrbeck 1910. 49 S. $8^{0}$

982. Lorenz, [Hans]: Die Theorie in der Technik. Danzig: Schwital \& Rohrbeck 1911. $18 \mathrm{~S} .8^{\circ}$ - Abgedr. in: Physik. Zeitschrift. Jg. 12. 
983. Carsten, A[lbert]: Die Gartenkunst und ikre Beziehungen zur Architektur. Danzig: Schwital \& Rohrbeck 1912. 25 S. $8^{0}$

\section{VERSCHIEDENE SCHRIFTEN}

984. Technische Hochschule in Danzig. Festschrift zur Eröffnung 6. Oktober 1904. [Verf. nach persönl. Mitteilung: Albert Carsten.] 48 S., 24 Taf. $4^{0}$

985. Denkschrift über die Eröffnungsfeier der Königlichen Technischen Hochschule zu Danzig am 6. Oktober 1904. Zsgest. von dem Rektor Prof. Dr. Hans von Mangoldt. 64 S. $8^{0}$

986. Schillers Ringen um eine Weltanschauung. Rede zum Gedächtnis des 100jährigen Todestages Friedrich Schillers, geh. am 9. Mai 1905 in der Aula der Kgl.
Technischen Hochschule zu Danzig von Adelbert Matthaei. Danzig: Kafemann 1905. 28 S. $8^{\circ}$

987. Zeitsehriften-Verzeiehnis der Bücherei der Königlichen Technischen Hochschule zu Danzig. 1906. VIII, 52 S. $8^{\circ}$

988. Bücherei der Königlichen Technischen Hochschule Danzig. Sehiffbau, Schiffsmaschinenbau, Seewesen. [Auswahl katalog.] (Vorr.:[Paul]Trommsdorff.)1910.V, 678. $8^{\circ}$

989. Tagesordnung für die Rektorenkonferenz [in Danzig] am 20. und 21. Mai 1912. [Folgt:] Protokoll der Rektorenkonferenz... $3 \mathrm{~S} .4^{\circ}$

990. Verzeiehnis der von der Bücherei und den Instituten und Sammlungen der Königlichen Technischen Hochschule Danzig laufend gehaltenen Zeitschriften. Sept. 1912. 44 S. $8^{\circ}$

\section{HABILITATIONSSCHRIFTEN}

991. Plato, Wilhelm, Dr. phil.: Erstarrungserscheinungen an anorganischen Salzen und Salzgemischen. 1908. 52 S. $8^{\circ}$ - Umgearb. aus: Zeitschrift f. physik. Chemie. Bd 55. 58. 63. 1906-08.

Danzig Te. H., Hab.-Schr. v. 19. 12. 1907

992. Pröll, A[rthur], Dr.-Ing.: Beiträge zur Theorie der Schiffsschraube. 1909.
S. $785-845.4^{0}$ - Aus: Jahrbuch d. Sehiffbantechn. Gesellschaft. Bd 11. 1910.

Danzig Te. H., Hab.-Schr. v. 25. 2. 1909

993. Brunn, A[Ibert] v. : Uber die Masse des Planetoidenringes. Mit 4Taf, u. 1 Abb. im Text. 1910. S. $101-148,3$ Taf. $8^{\circ}$ - Aus: Schriften d. Naturforseh. Gesellschaft in Danzig. N. F. Bd 12.

Danzig Te. H., Hab.-Schr. v. 8. 11. 1909

\section{DOKTOR-INGENIEUR-DISSERTATIONEN}

\section{ABTEILUNG FÜR ARCHITE KTUR}

994. Meyer, Carl, Kgl. Reg.-Baumeister Die Augustiner-Klosterkirche zu Ravengiersburg. Berlin: Wasmuth 1908. $78 \mathrm{~S} .4^{0}$ ๆ Auch als: Beiträge zur Bauwissenschaft. H. 12. 1909.

Danzig, Dr.-Ing.-Diss. v. 20. 3. 1908

995. Sleumer, Hermann Josef: Die ursprüngliche Gestalt der Zisterzienser-Abteikirche Oliva. Heidelberg: Winter 1909. 31 S., 1Taf. $4^{0}$ (Enth. nur einenTeil derZeichnungen.) - Mit dem gesamten Illustrationsmaterial u. 1Zeittaf. als: Zeitschrift f. Geschichte d. Architektur. Beih. 1.

Danzig, Dr.-Ing.-Diss. v. 17. 11. 1908

996. Gentzen, Felix, Reg.-Bauführer: Die Kanzelhäuser und ähnliche Miethäuser AltDanzigs. Danzig: (Selbstverl.) 1909. 44 S., 11 Taf. $4^{0}$

Danzig, Dr.-Ing.-Diss. v. 4. 5. 1909
997. Brandt, Paul, Reg.-Bauführer: Das rechtstädtische Rathaus zu Danzig. Eine baugeschichtliche Studie. [1910.] $40 \mathrm{~S}$, 3 Taf. $8^{\circ}$

Danzig, Dr.-Ing.-Diss. v. 6. 8. 1909

998. Fischer, Friedrieh: Der Danziger Kirchenbau des 15. und 16. Jahrhunderts. 1910. 38 S. $8^{\circ}$ Auch bei Kafemann in Danzig.

Danzig, Dr.-Ing.-Diss. v. 18. 1.1910

999. Weishaupt, Carl: Alt-St. Marien und Alt-St. Peter und Paul zu Danzig im Typ der reduzierten Basilika. [1910.] $109 \mathrm{~S} .4^{0}$

Danzig, Dr.-Ing.-Diss. v. 18. 1. 1910

1000. Abraham, Riehard, Reg.-Bauführer, Danzig: Die Trinitatiskirche zu Danzig. [Danzig: Kafemann in Komm.] 1910. 37 S. $8^{\circ}$ Danzig, Dr.-Ing.-Diss, v. 3. 5. 1910 [Tag der Promotion: 10. 5. 1910] 
1001. Kallmorgen, Walther: Der Bau von Wendeltreppen aus Backstein im norddeutschen Backsteingebiet. Berlin: Selbstverl. 1910. XXXI, 27 S. $4^{0} \bullet$ Auch bei J. Harder in Altona.

Danzig, Dr.-Ing.-Diss, v. 3. 5. 1910 [Tag der Promotion: 10. 5. 1910]

\section{ABTEILUNG FÜR BAUINGENIEUR- WESEN}

1002. Haimberger, Paul Freiherr von, Markscheider: Beiträge zur Bestimmung der Strahlenbrechung über der Meeresfläche. Freiberg i. S.: Craz \& Gerlach 1910. 45 S. $4^{0}$

Danzig, Dr.-Ing.-Diss. v. 18.1. 1910

\section{ABTEILUNG FÜR MASCHINEN- INGENIEURWESEN UND ELEKTRO- TECHNIK}

\section{Maschinen bau}

1003. Borth, Walther: Untersuchungen über den Verbrennungsvorgang in einem Körting-Leuchtgas-Motor. 1907. $47 \mathrm{~S} .4^{0}$ I Auch in: Mitteilungen über Forschungsarbeiten auf d. Gebiete d. Ingenieurwesens. H. 55. 1908; Ausz. in: Zeitschrift d. Vereines deutscher Ingenieure. Bd 52. 1908, Halbj. 1.

Danzig, Dr.-Ing.-Diss. v. 12. 6. 1907

1004. Hoffmann, Paul: Prüfung von Geschwindigkeitsmessern. 1909. $39 \mathrm{~S} .4^{0}$ - Auch in: Mitteilungen über Forschungsarbeiten suf d. Gebiete d. Ingenieurwesens. H. 100. 1911.

Danzig, Dr.-Ing.-Diss. v. 23. 7. 1909

1005. Mangold, Georg: Die 'Regulierfähigkeit der Dampfturbinen bei stoBfreiem Eintritt. 1911. 80 S. $8^{\circ}$ \% Ausz. in: Zeitschrift f. d. gesamte Turbinenwesen. Jg. 8 .

Danzig, Dr.-Ing.-Diss, v. 15. 3. 1910

1006. Heumann, Hermann, Reg.-Baumeister: Das Windwerk von Hochbahnkranen mit feststehender Winde, wagerechter oder schwach geneigter Fahrbahn und nicht selbstfüllenden Fördergefäßen. 1910. 142 S., 9 Taf. $8^{0}$

Danzig, Dr.-Ing.-Diss. v. 10. 5. 1910

1007. Retschy, Curt: Beiträge zur Herstellung und Untersuchung annähernd geordneter Luftströme. [1912.] $32 \mathrm{~S} .44^{0}$ - Auch in: Der Motorwagen. Jg. 15.

Danzig, Dr.-Ing.-Diss. v. 12. 12. 1911
1008. Kirsch. Albert: Stauchversuche an zylindrischen Bleiröhren. Halle: Knapp 1912. 19 S., 6 Bl. Taf. $4^{0}$ I Auch in: Ferrum. Jg. 10. 1912/13.

Danzig, Dr.-Ing.-Diss. v. 19. 3. 1912

1009. Henkelmann, Josef: Untersuchungen über die Wirtschaftlichkeit einer Ferndampfheizungsanlage. 1912. 42 S. $4^{0}$ - Etwas gekürzt in: Gesundheits-Ingenieur. Jg. 36. 1913.

Danzig, Dr.-Ing.-Diss. v. 16. 7. 1912

\section{Elektrotechnik}

1010. Monasch, Bruno: Utber den Energieverlust im Dielektrikum von Kondensatoren und Kabeln. Wien: Selbstverl. 1906. 55 S., 4 Taf. $8^{0}$

Danzig, Dr.-Ing.-Diss. v. 19. 7. 1906

1011. Geldermann, Arthur: Über eine Methode zur Behandlung unsymmetrischer Kabelsysteme unter Berücksichtigung des konzentrischen mit Bleimantel umpreßten Zwei-Leiter-Kabels als Beispiel. 1906. 56 S. $8^{0}$

Danzig, Dr.-Ing.-Diss. v. 18.12. 1906

1012. Rogowski, W[alter]: Ueber das Streufeld und den Streuinduktionskoeffizienten eines Transformators mit Scheibenwicklung und geteilten Endspulen. 1908. 39 S., 1 Taf. $4^{0}$ - A Auch in: Mitteilungen über Forschungsarbeiten auf d. Gebiete d. Ingenieurwesens. H. 71. 1909; Ausz. in: Zeitschrift d. Vereines deutscher Ingenieure. Bd 53. 1909, Halbj. 1.

Danzig, Dr.-Ing.-Diss. v. 20.12. 1907

1013. David, R(ichard): Theoretische und experimentelle Untersuchungen über künstliche Hochspannungs-Kabel. 1910. 56 S. $4^{0}$ - Auch bei Simion in Berlin u. in: Verhandlungen d. Vereins zur Beförderung d. Gewerbfleißes. Jg. 89.

Danzig, Dr.-Ing.-Diss. v. 27. 11. 1908

1014. Philippi, Erich: Ueber Ausschaltvorgänge und magnetische Funkenlöschung. 1909. VII, 62 S. $4^{0}$ of Auch bei Simion in Berlin 1910 u. in: Verhandlungen d. Vereins zur Beförderung d. Gewerbfleißes. Jg. 88.

Danzig, Dr.-Ing.-Diss. v. 13. 1. 1909

1015. Cauwenberghe, $\mathbf{R}[$ obert] Van, Ingénieur des Constructions Civiles: Beitrag zur allgemeinen Theorie der Asynchronmotoren ohne Kollektor. Die Kaskadenschaltung. Berlin: Selbstverl. (Zu erhalten bei Van Goethem \& Co., Gent 1909.) 122 S., 28 Taf. $8^{0}$

Danzig, Dr.-Ing.-Diss. v. 25. 5. 1909 
1016. Vollmer, K[arl]: Über die Schwankungen der Frequenz und Intensität der Lichtbogenschwingungen. Leipzig: J. A. Barth 1910. 95 S. $8^{\circ}$ I Aus: Jahrbuch d. drahtlosen Telegraphie u. Telephonie. Bd 3.

Danzig, Dr.-Ing.-Diss. v. 4. 8. 1909

1017. Lambertin, G[ustav]: Doppeltarifzähler für Gleichstrom von der Zentrale umzuschalten durch übergelagerten Wechselstrom von 5000 Perioden. 1911. $71 \mathrm{~S}$. 80

Danzig, Dr.-Ing.-Diss. v. 18. 7. 1911

\section{ABTEILUNG FÜR SCHIFF- UND SCHIFFSMASCHINENBAU}

\section{Schiffbau}

1018. Waldmann, Ernst: Einfluss der Schiffsvermessung auf die Stabilität der Schiffe. [1911.] 23 S. $4^{0}$ i Aus: Sehiffbau. Jg. 12. 1910/11.

Danzig, Dr.-Ing.-Diss. v. 10. 1. 1911

\section{Schiffsmaschinenbau}

1019. Helling, Hermann: Die LentzSteuerung an Schraubenschiffsmaschinen. 1911. 45 S. $4^{0}$. Aus: Verhandlungen d. Vereins zur Beförderung d. Gewerbfleißes. Jg. 89. 90. 1910. 11.

Danzig, Dr.-Ing.-Diss. v. 15. 3. 1910

1020. Steuer, Karl: Untersuchung von Dampfdiagrammen auf Grund der Dynamik der Dampfströmung in der Kolbendampfmaschine. 1911. 43 S. $8^{0}$

Danzig, Dr.-Ing.-Diss. v. 13. 7. 1911

\section{ABTEILUNG FÜR CHEMIE}

1021. Claußner, Paul: I. Úber Oxydation der Xylole und des Mesitylens sowie einige Derivate derselben. II. Zur Kenntnis der Oxalessigsäure und verwandter Verbindungen. [1907.] 45 S. $8^{0}$ I Vgl. Berichte d. Deutschen Chem. Gesellschaft. Jg. 40.

Danzig, Dr.-Ing.-Diss. v. 23. 7. 1907

1022. Bornemann, Ferdinand: Über das Osmium. Analytische Bestimmung. Chloride und Oxyde. 1910. 63 S. $8^{0}$ I Ausz. in: Zeitschrift f. anorgan. Chemie. Bd 65.

Danzig, Dr.-Ing.-Diss. v. 18. 1. 1910
1023. Hecht, Leopold: Uber die Natur des Sulfammoniums und ein Beitrag zur spektrometrischen Untersuchung eines Gemisches mehrerer lichtabsorbierender Stoffe. [1910.] 57 S. $8^{0}$ ๆ Vgl. Zeitschrift f. anorgan. Chemie. Bd 70. 1911.

Danzig, Dr.-Ing.-Diss. v. 31. 5. 1910

1024. Koch, Franz: Über das Sulfanilid. Zur Kenntnis des Glycerinaldehyds. [1910.] 52 S. $8^{0}$ - Vgl. Berichte d. Deutschen Chem. Gesellschaft. Jg. 43 .

Danzig, Dr.-Ing.-Diss. v. 25. 10. 1910

1025. Nagelschmidt, Ernst: Zur Kenntnis der substituierten Isophtalsäuren. [1910.] 62 S. $8^{\circ}$ V Vl. Berichte d. Deutschen Chem. Gesellschaft. Jg. 43 .

Danzig, Dr.-Ing.-Diss. v. 25. 10. 1910

1026. Liekfett, Herbert: Chloride, Bromide und Fluoride des Vanadins. [1911.] 65 S. $8^{0}$ - Vgl. Berichte d. Deutschen Chem. Gesellschaft. Jg. 44.

Danzig, Dr.-Ing.-Diss. v. 16. 5. 1911

1027. Goecke, 0tto: Der elektrische Vakuumofen und seine Verwendung. Halle: Knapp 1911. 22 S., 1 Taf. $4^{0}$ ๆ Vgl. Metallurgie. Jg. 8; Zeitschrift f. angewandte Chemie. Jg. 24. S. $1459 \mathrm{ff}$.

Danzig, Dr.-Ing.-Diss. v. 30. 5. 1911

1028. Schiller, Emil: Über Tantal- und Niobpentafluorid, sowie über die Reindarstellung der Tantal- und der Niobsäure. [1911.] 55 S. $8^{0}$ - Vgl. Zeitschrift f. anorgan. Chemie. Bd 72. S. $329 \mathrm{ff}$,

Danzig, Dr.-Ing.-Diss. v. 30. 5. 1911

1029. Heinzelmann, Alfred: Das Uranhexafluorid, ein Beitrag zur Kenntnis des sechswertigen Urans. 1911. 55 S. $8^{0}$ I Vorläufige Mitteilung in: Berichte d. Deutschen Chem. Gesellschaft. Jg. 42. 1909; vgl. Zeitschrift f. anorgan. Chemie. Bd 72.

Danzig, Dr.-Ing.-Diss. v. 27. 6. 1911

1030. Martin, Walter: Ueber reines $\mathrm{Va}$ nadin. [1912.] 55 S. $8^{0}$ I Ausz. in: Zeitschrift f. angewandte Chemie. Jg. 25.

Danzig, Dr.-Ing.-Diss. v. 18. 12. 1911 [Tag der Promotion: 12. 12. 1911]

1031. Gersten, Ewald: Über die Karbide des Eisens, Mangans und Nickels. Freiberg i. S.: Craz \& Gerlach 1912.58 S. $8^{0}$ - Vgl. betr. T.1 Berichte d. Deutschen Chem. Gesellschaft. Jg. 45.

Danzig, Dr.-Ing.-Diss. v. 11. 6. 1912

1032. Schroth, Walter: Ueber Amidester und Amidsäuren von substituierten Isophtalsäuren, ein Beitrag zur Theorie des Benzolkerns. [1912.] 31 S. $8^{0}$

Danzig, Dr.-Ing.-Diss. v. 11. 6. 1912 


\section{DAR MSTADT}

Gegründet 1836 als „Höhere Gewerbschule", 1864 umgewandelt in "Technische Schule“, 1868 erweitert als "Polytechnische Schule"; seit 1877 "Technische Hochschule"

\section{SATZUNGEN ORDNUNGEN BESTIMMUNGEN}

\section{ALLGEMEINES UND VERMISCHTES}

1033. Gesetze für die Schüler der höhern Gewerbschule zu Darmstadt. [1846.] - [Mit veränderter Fassung v. S. 20 u. d. T.:] Gesetze ... höheren ... [1847.] 1847: $K$

1034. Lehrverfassung der vier technischen Fach-Classen an der höheren Gewerbschule zu Darmstadt. 1858.

1035. Besondere Bestimmungen für die Schüler und Zuhörer der technischen Schule zu Darmstadt. 1864.

1036. Disciplinar-Vorsehriften für die Schüler der beiden allgemeinen Classen der Großherzogl. technischen Schule zu Darmstadt (v. 26. 3. 1866).

1037. Diseiplinar-Vorsehriften für die Schüler der Fachkurse der Großherzogl. technischen Schule zu Darmstadt (v. 26. 3. 1866).

1038. Verordnung, die Erhebung der technischen Schule zu Darmstadt zu einer polytechnischen Schule betr. 〈Regierungsblatt Nr. 54 vom 14. 10. 1868.> (Dat. 3. 10. 1868.) [Folgt:] Organische Restimmungen für die polytechnische Schule in Darmstadt. $D a \operatorname{HofB}$

1039. Disciplinarbestimmungen für die Studirenden der polytechnischen Schule zu Darmstadt (v. 28. 9. 1869).

1040. Großherzogl. polytechnische Schule zu Darmstadt. Bibliothek-0rdnung. Auszug. B. Benutzung der Bibliothek Seitens der Docenten des Polytechnikums. [Folgt:] C. Bestimmungen über Anschaffung der Bücher (v. 12. u. 13. 2. 1874). [Autogr.]

1041. Grossherzogl. Polytechnikum Darmstadt. Auszug aus der Bibliothekordnung. Bestimmungen für die Benutzung der Bibliothek seitens der Studirenden (v. 12, u. 13. 2. 1874). [Folgt:] Bestimmungen über die Benutzung des Lesezimmers seitens der Studirenden. - [Anderer Dr. u. d. T.:] Grossherzogl. polytechnische Schule zu Darmstadt. Auszug aus der Bibliothek-Ordnung...

1. Dr.: $D a$ HofB
1042. Grossherzogl. polytechnische Schule zu Darmstadt. Instruction für das Verfahren bei der Aufnahme, bei der Immatrikulation und beim Austritt der Studirenden, sowie beim Eintritt von Hospitanten (v. 10. 9. 1875).

1043. Bekanntmachung, die organischen Bestimmungen der polytechnischen Schule zu Darmstadt und die künftige Benennung dieser Anstalt betr. (v. 10. 10.1877). 〈Aus d. Regierungsblatt Nr. 44 v. 19. 10. 1877. $\rangle$

1044. Grossherzogl. technische Hochschule zu Darmstadt. Instruction für das Verfahren bei der Aufnahme und beim Austritt der Studirenden, sowie beim Eintritt von Hospitanten (v. Juli 1882). - [Veränderte Fassungen] (v. April 1887. Sept. 1895).

1887: $M$

1045. Disciplinar-Bestimmungen für die Studirenden der Grossherzogl. Technischen Hochschule zu Darmstadt. [1885.]

1046. Statuten für die Krankenkasse der Studirenden der Grossherzogl. Technischen Hochschule zu Darmstadt (v. 21. 7. 1891). - [Neudr.] (1895.)

1047. Statuten des Ausschusses der Studierenden der Grossherzogl. Technischen Hochschule zu Darmstadt. Genehmigt durch Direktorialerlass vom Sommer 1894.

1048. Disziplinar-Bestimmungen für die Studierenden der Grossherzogl. Technischen Hochschule zu Darmstadt (v. 20.3. 1894).

1049. Grossherzogl. Technische Hochschule zu Darmstadt. Auszug aus der Bibliothek-0rdnung der Grossherzogl. Technischen Hochschule zu Darmstadt. (B.) Bestimmungen für die Benutzung der Bibliothek seitens der Docenten. ([Folgt:] C. Bestimmungen über Anschaffungen.) 1895 .

1050. Grossherzogl. Technische Hochschule zu Darmstadt. Auszug aus der Bibliothek-0rdnung. Bestimmungen für die $\mathrm{Be}-$ nutzung der Bibliothek seitens der Studierenden. [Folgt:] Bestimmungen für die Benutzung des Lesezimmers seitens der Studierenden. [1895.] 
1051. Auszug aus der Bibliotheksordnung der Großh. Technischen Hochschule zu Darmstadt. (B.) Bestimmungen für Dozenten. ([Folgt:] C. Bestimmungen über Anschaffungen.) 1895. (Neudr. 1912.)

1052. Verordnung, die Verfassung der Technischen Hochschule zu Darmstadt betr. (v. 2. 2. 1895). Aus d. Regierungsblatt Nr. 3 v. 9. 2. 1895. [Folgt:] Verfassung der Großherzogl. Technischen Hochschule zu Darmstadt.

1053. Grossherzogl. Technische Hochschule zu Darmstadt. Bestimmungen, das Verfahren bei Beitreibung der Unterrichtsgelder betr. (v. 12. 10. 1895).

1054. AuInahme-Bedingungen für die Großherzogl. Technische Hochschule zu Darmstadt (v. 17. 6. 1896).

1055. Grossherzogl. Technische Hochschule zu Darmstadt. Normen für die Thätigkeit der Privatdocenten (v. 11. 7 . 1896).

1056. Grossherzogl. Technische Hochschule zu Darmstadt. Bestimmungen über das Verfahren bei Zahlung der Unterrichtsgebühren (v. 24. 2. 1897).

1057. Grossherzogl. Technische Hochschule zu Darmstadt. Promotions-0rdnung für die Erteilung der Würde eines DoktorIngenieurs (v. 18.6. 1900). [Nebst] Nachtrag (zu \$ 1 v. 20. 4. 1906 u. 8. 5. 1907) [u.] Zusatz-Bestimmung zu §1, (v. 6. 4. 1909). If Abgedr. in: Bestimmungen. S. $171 \mathrm{ff}$.

1058. Vertrag (zwischen dem „Nordstern, Unfalls- und Alters-Versicherungs-AktienGesellschaft zu Berlin" und der Großherzogl. Technischen Hochschule zu Darmstadt). Police Nr. 111857. (4. 3. 1902 genehmigt.)

1059. Grossherzogl. Technische Hochschule zu Darmstadt. Normen für die Thätigkeit der Privatdocenten (v. 10. 11. 1902).

1060. Grossherzogl. Technische Hochschule zu Darmstadt. Geschäfts-0rdnung für die beiden Senate und die Fachabteilungen. Vom 17. 7. 1903. 1903.

1061. Grossherzogl. Technische Hochschule zu Darmstadt. Habilitations-0rdnung (v. 19. 3. 1904).

1062. Grossherzogl. Technische Hochschule zu Darmstadt. Statut für die Verwendung der Promotionsgebühren und die Verwaltung der Gebührenkasse (v.9.12.1904).
1063. Aufnahme-Bestimmungen für die Grossherzogl. Technische Hochschule zu Darmstadt (v. 28. 2. 1908). [Folgt:] Anhang über die Prüfungen.

1064. Police Nr. 570161. Vertrag zwischen der Grossherzogl. Technischen Hochschule zu Darmstadt und der Unfall-VersicherungsGesellschaft Nordstern in Berlin (v. 23. 12. 1908, genehmigt 15. 1. 1909).

1065. Satzungen des Ausschusses der Studierenden der Großherzogl. Technischen Hochschule zu Darmstadt. Genehmigt 3. 3. 1909.

1066. Statut für die Krankenkasse der Studierenden und Hörer der Grossherzogl. Technischen Hochschule zu Darmstadt (v. 13. 3. 1909).

1067. Grossherzogl. Technische Hochschule zu Darmstadt. Bestimmungen für die Drucklegung der Doktor-Ingenieur-Dissertationen (v. 18. 6. 1909).

1068. Habilitationsordnung der Großherzogl. Technischen Hochschule Darmstadt (v. 22. 11. 1910).

1069. Aufnahme-Bestimmungen für die Grossherzogl. Technische Hochschule zu Darmstadt (v. 26. 7. 1911). [Folgt:] Anhang, betr. die Prüfungen. [Nebst] AusführungsBestimmungen (v. 24. 10. 1911).

\section{PRÜFUNGSORDNUNGEN}

1070. Grossherzogl. polytechnische Schule zu Darmstadt. Provisorische Prüfungsordnung (v. 25. 5. 1873). - [Anderer Dr. u. d. T.:] Grossherzogl. Polytechnikum Darmstadt... - Abgedr. in: Programm 1872/73 [Nr 1089].

1071. Bestimmungen über die Abhaltung der Abgangsprüfungen an der Grossherzogl. Hessischen technischen Hochschule zu Darmstadt. 1879.

1072. Provisorische Bestimmungen über die Abhaltung der Abgangsprüfung für Consolidations-Geometer an der Grossherzogl. Technischen Hochschule zu Darmstadt. 1883.

1073. Bestimmungen über die Abhaltung der Abgangs-Prüfungen an der Grossherzogl. Technischen Hochschule zu Darmstadt. 1886.

1074. Bestimmungen über die Abhaltung von ausserordentlichen Prüfungen für Ausländer an der Grossherzogl. Technischen Hochschule zu Darmstadt (v. 27. 7. 1888). 1889. - [Neudr.] (1902.) 
1075. Bestimmungen über die Abhaltung der Diplomprüfungen an der Grossherzogl. Technischen Hochschule zu Darmstadt. 1889. - [Neudr.] 1893.

1076. Geschäftsordnung für die DiplomPrüfungscommission an der Grossherzogl. Technischen Hochschule zu Darmstadt ( $v$. 24. 6. 1896).

1077. Bestimmungen über die Abhaltung der Diplomprüfungen an der Grossherzogl. Technischen Hochschule zu Darmstadt (v. 28. 7. 1896). 1896. (Nebst Deckbl. zu § 5.)

1078. Grossherzogl. Technische Hochschule zu Darmstadt. Bestimmungen über die Abhaltung von Semestral-Schlussprüfungen (v. 12. 1. 1898).

1079. Bestimmungen über die Abhaltung der Diplomprüfungen an der Grossherzogl. Technischen Hochschule zu Darmstadt (v. 23. 3. 1899). 1899.

1080. [Dasselbe] (จ. 7. 1. 1903). 1903. - Abgedr. in: Bestimmungen. S. 163.

1081. Diplomprüfungs-0rdnung der Grossherzogl. Technischen Hochschule zu Darmstadt. Vom 20. 11. 1905. (Nebst Neudr. der
S.16-19, genehmigt 27.9. 1912 u. S.20-23, genehmigt 4. 11. 1912.)

1082. Geschäfts-0rdnung für die Diplomprüfungs-Kommission an der Grossherzogl. Technischen Hochschule zu Darmstadt (v. 7. 2. 1906).

1083. Grossherzogl. Technische Hochschule zu Darmstadt. Ordnung für die Fachprüfungen der Abteilung für Architektur. Vom 4. 3. 1907. 1907.

1084. Grossherzogl. Technische Hochschule zu Darmstadt. Ordnung für die Fachprüfungen der Abteilung für Ingenieurwesen. Vom 4. 3. 1907. 1907.

1085. Grossherzogl. Technische Hochschule zu Darmstadt. Ordnung für die Fachprüfungen der Abteilung für Maschinenbau. Vom 4. 3. 1907. 1907.

1086. Grossherzogl. Technische Hochschule zu Darmstadt. Ordnung für die Fachprüfungen der Abteilung für Elektrotechnik. Vom 4. 3. 1907. 1907.

1087. Grossherzogl. Technische Hochschule zu Darmstadt. Ordnung für die Fachprüfungen der Abteilung für Chemie. Vom 30. 5. 1907.1907.

\section{PERIODISCHE SCHRIFTEN}

1089. Programm der (1858-59: Großherzogl. Hessischen) höheren Gewerbschule zu Darmstadt für das Jahr [1858-18599.] 18591860. 1860-1861. 1858-[60].

Fortges. u. d. T.:

Programm der Großherzogl. Hessischen technischen Schule zu Darmstadt. Herbst 1864. 1865. $1864-65$.

Fortges. u. d. T.:

Programm der Grossherzogl. Hessischen Polytechnischen Schule (1878-79ff. : Technischen Hochschule) zu Darmstadt (1909/10ff. : Grossherzogl. Technische Hochschule zu Darmstadt. Programm) für das Jahr (187374 ff. : Studienj.) 1869-1870-1912/13. 1869 - [1912].

1873-74-1875-76. [Nebst] Beil. Nachrichten über die Prüfungen, welche Seitens der polytechnischen Schule veranstaltet und über die Zeugnisse, welche von derselben ausgestellt werden.

1876/77. [Nebst] Anl. I. Nachrichten... II. Vorschriften über die Ausbildung und Prüfung für den Staatsdienst im Bau- und Maschinenfach im Königreich Preussen.

1860/61. 1865: $S$
1090. Programm der Grossherzogl. Hessischen Polytechnischen Schule zu Darmstadt für das Studieni. 1873-1874. Erster Abschnitt nach dem Entwurfe des Lehrerraths der polytechn. Schule.

$D a$ Hof $B$

1091. Die feierliche Übergabe des Rectorats (1907/08 ff. : an) der Grossherzogl. Technischen Hochschule zu Darmstadt für das Studienj. 1896-97. 1898-99. 1900-1901. 1902-1903. 1903-1904. 1905/1906. 1907/08. 1909/10. 1911/12. 1896-1911.

Darin:

1896-97. Berndt, 0tto: Die Entwickelung der Locomotive.

1898-99. Koch, [Alexander]: Die heutigen Anforderungen der Seeschiffahrt an die Seekanäle. 1900-01. Schering, Karl: Ueber die geschichtliche Entwickelung der Hypothesen in der Physik, besonders in der Lehre vom Lichte und von der Elektricität.

1902-03. Pfarr, [Adolf]: Ueber die Ausbeutung der sich im Meere betätigenden Wasserkräfte. [Auch als Sonderabdr.] S.-A.: Be

1903-04. Dingeldey, [Friedrich]: Über die Anwendung der Mathematik auf Astronomie, Physik und Technik. 
1905/06. Gutermuth, [Max Friedrich]: Einfluss von Wissenschaft und Wirtschaft auf die Entwicklung der Technik.

1907/08. Walbe, [Heinrich]: Baukunst und Hochschulunterricht.

1909/10. Schenck, [Heinrich]: Entstehung der Arten.

1911/12. Wickop, G[eorg]: Die Entwicklung der Großherzoglichen Technischen Hochschule zu Darmstadt und ihre zukünftigen Aufgaben in den Fragen der staatsbürgerlichen, ethischen und künstlerischen Erziehung. [Ein Teil mit einigen Ergänzungen u. d. T.: Die Technischen Hochschulen und ihre zukünftigen Aufgaben ... Darmstadt: BergstraeBer 1912.]

1092. Personal-Verzeichniss der Grossherzogl. Hessischen Technischen Hochschule zu Darmstadt (1910/11 ff.: Grossherzogl. Technische Hochschule Darmstadt. PersonalVerzeichnis) für das S.-S. 1896. IV.-S. 189697 [usw.]-W.-S. 1912/13. 1896-1912.

\section{GELEGENHEITS- UND VERMISCHTE SCHRIFTEN}

\section{REDEN ZUR FEIER DES GEBURTSTAGES DES GROSZHERZOGS}

Im Jahre 1903 fiel der Festakt zur Feier des Geburtstages des Großherzogs aus. Die Grobherzogs-Geburtstagsreden des Jahres 1906 von Rudolf Kautzsch: ,Der Sieg der malerischen Tendenz in der bildenden Kunst des 19. Jahrhunderts" und des Jahres 1909 von Konrad Zeissig: „Physikalische Methoden und Resultate der Erdbebenforschung" sind nicht im Druck erschienen.

1093. Lepsius, Richard: Der Rheinstrom und seine Überschwemmungen. 1895. 19 S. $4^{0}$

1094. Harnack, 0tto: Ueber Goethe's Verhältnis zu Shakespeare. 1896. 24 S. $8^{\circ}$ T Etwas gekürzt in: Harnack: Essais u. Studien zur Literaturgeschichte. Braunschweig 1899.

1095. Hofmann, [Karl]: Der Dom zu Worms und seine Wiederherstellung. 1897. 29 S. $8^{0}$

1096. Berghoff-Ising, [Franz]: Ueber Methode und Aufgabe der Volkswirthschaftslehre. 1898.30 S. $8^{0}$

1097. Harnack, 0tto: Ueber die Verwendung historischer Stoffe in der Dichtung. 1899. 17 S. $8^{0}$ ๆ Mit geringfügigen Änderungen in: Harnack: Aufsätze u. Vorträge. Tübingen 1911.

1098. Pützer, Friedrich: Ueber Denkmalskunst. $1900.28 \mathrm{~S} .8^{\circ}$

1099. Wegele, Hans: Die Sicherheit im Eisenbahnwesen. 1902. [Rede 1901.] 27 S., 2 Taf. $8^{0}$

1100. Walbe, Heinrich: Über Bauordnungen. 1902. 32 S. $8^{0}$

1101. Roessler, L[udwig] von: Über mikroskopische Metalluntersuchung (Metallographie>, ihre Bedeutung und Entwickelung. 1905. [Rede 1904.] 19 S. $8^{0}$

1102. Berger, Arnold E[rich]: Die Lehre von der Naturnachahmung. 1906. [Rede 1905.] 27 S. $8^{0}$
1103. Müller, Reinhold: Die geometrische Reliefperspektive in ihrer Anwendung auf die Werke der bildenden Kunst. 1908. [Rede 1907.] $21 \mathrm{~S} .8^{0}$

1104. Horn, Jakob: Die ältesten Beziehungen zwischen der Infinitesimalrechnung und der Mechanik. 1909. [Rede 1908.] 16 S. $8^{0}$

1105. Kayser, Heinrich: Entwicklung und Fortschritte des Eisenbetonbaues. 1911. [Rede 1910.] 23 S., 2 Taf. $8^{0}$

\section{VERSCHIEDENE SCHRIFTEN}

1106. Ueber $\mathrm{Zweck}$ und Einrichtung der neu gegründeten Großh. Real- und höheren Gewerbschule zu Darmstadt. (Verf. : [Theodor] Sehacht.) 1836. 15 S. $8^{0}$

1107. Einladung zu den öffentlichen Prüfungen, die in der Grossherzogl. höheren Gewerbschule Montag den 20. März, Vormittags 9 und Nachmittags $2 \mathrm{Uhr}$, und in der damit verbundenen Realschule den 21sten zur gleichen Tageszeit und den 22sten Nachmittags 2 Uhr abgehalten werden. 1837. (Darin: [Theodor] Schacht: Einige Worte in Betreff der höheren Gewerbschule.) $18 \mathrm{~S}$. $8^{0}$

Da $H \circ f B$

1108. Fernere Nachrichten über die Großherzogl. höhere Gewerb- und Realschule zu Darmstadt, als Einladung zum Examen in den vier Klassen der Realschule den 10. und 11. April 1838. (Verf.: [Theodor] Sehacht.) 16 S. $8^{0}$

Da Hof B

1109. Ueber Zweck und Einrichtung der höhern Gewerbschule des Großherzogthums Hessen und der damit verbundenen Realschule zu Darmstadt. Von ihrem Director Dr. Theod[or] Schacht, Mitglied des Oberstudien- u. Oberschulraths. Nebst 1 lithogr. Beil. Darmstadt: Pabst 1843, 64 S., 1 Taf. $8^{0}$ 
1110. Die höhere Gewerbschule zu Darmstadt nach Zweck und Einrichtung dargest. von dem Director derselben Dr. Edmund Külp, Prof. d. Physik u. Mathematik. Darmstadt: Pabst 1850 . IV, 110 S. $8^{\circ} \mathrm{Da}$ HofB

1111. Zur fünfundzwanzigjährigen Jubelfeier der höheren Gewerbschule zu Darmstadt am 12. Juli 1861. [Liederheft.] 4 Bl. $8^{0}$

1112. Die Feier des fünfundzwanzigjährigen Stiftungstages der höheren GewerbSchule zu Darmstadt am 12. Juli 1861. 32 S. $8^{0}$

1113. Die höhere Gewerbschule zu Darmstadt und ihre Zukunft, beleuchtet von dem Lehrercollegium dieser Anstalt. Als Ms. gedr. 1863. 16 S. $8^{0}$

1114. Katalog der allgemeinen Bibliothek der Grossherzoglich Hessischen Polytechnischen Schule zu Darmstadt. 1876. VI, 159 S. $8^{0}$ - 2. Aufl. s. Nr 1127.

1115. Grossherzogliche Technische Hochschule zu Darmstadt. Adressen-Verzeichniss der ehemaligen Studirenden der höheren Gewerbeschule, der technischen Schule, sowie der polytechnischen Schule, bezw. technischen Hochschule zu Darmstadt. 1885. 88 S. $8^{0}$

Da $H$ of $B$

1116. Festschrift zu der Jubelfeier des fünfzigjährigen Bestehens der Grossherzoglichen Technischen Hochschule zu Darmstadt. 1886. XL, 137 S., 7 Taf. $4^{0}$

Enth.: (Theodor) Landsberg. (Eduard) Sonne: Geschichtliche Entwickelung der Großherzoglichen Technischen Hochschule zu Darmstadt. [Auch einzeln.] - Theodor Landsberg: Beitrag zur Theorie des ebenen Fachwerks. Eduard Sonne: Ueber den Schiffswiderstand bei Fluß- und Kanalkähnen. - Adolph Klaas: Der Beruf des Kultur-Ingenieurs. - Ernst A [dolf] Brauer: Berechnung verjüngter Förder-Drahtseile und deren Spiralkörbe. - Hermann von Pfister: Verhältnis des Kampfes und seiner Mittel beim Auftreten gepanzerter Schiffe gegen Strand-Batterien. - Karl Thiel: Die Zusammensetzung des Mauer-Mörtels der Klosterkirche zu Lorsch. Joseph Klein: Ueber die quantitative Bestimmung der Ameisensäure und über die SchulzeTrommsdorff'sche Bestimmung der organischen Substanz im Wasser. - Ernst Dorn: Bemerkung über einige Apparate zur Untersuchung der atmosphärischen Elektricität. - Gottlieb von $\mathrm{Koch}$ : Untersuchung über das Wachsthum von Antipathes. - Lebrecht Henneberg: Eine Aufgabe der graphischen Statik. - $R u$. dolf Mehmke: Eine kinematische Anfgabe. Friedrich Graefe: Das Gesetz der Anpassung und die Grundlehren der Logik. - Heinrich Wagner: Die Kreuzigungsgruppen am Dom zu
Frankfurt a. M., an der Pfarrkirche zu Wimpfen a. B. und an der St. Ignaz-Kirche zu Mainz. Georg Schaefer: Die Deckengemälde in der Pfarrkirche zu Heusenstamm. - August Noack: Das Menschenbild im Spiegel der Kunst. - Otto Roquette: Goethe und die Gartenkunst.

1117. Jubelfeier des fünfzigjährigen Be stehens der Grossh. Technischen Hochschule zu Darmstadt. Lieder. $[1-3$. $] 8^{0}$

[1.] Vorabend am Donnerstag, den 1. Juli 1886 im Ritsert'schen Saale. 2 Bl.

[2.] Frühschoppen am Samstag, den 3. Juli 1886 im Saalbau. $8 \mathrm{~S}$.

[3.] Fest-Commers am Samstag, den 3. Juli 1886 im grossen Saale des Saalbaus. 15 S. $D a H o f B$

1118. Denkschrift, betreffend die räumlichen Verhältnisse der Großherzoglichen technischen Hochschule zu Darmstadt. (Unterz. : Darmstadt, im Juli 1888. Im Auftrage des Lehrerrathes die Commission.) $18 \mathrm{~S} .4^{0}$ [Autogr.]

Da HofB

1119. Denkschrift, betreffend die räumlichen Verhältnisse der Grossherzoglichen Technischen Hochschule zu Darmstadt nach Errichtung eines Neubaues für das elektrotechnische und phrsikalische Institut. (Unterz. : Darmstadt, den 21. Mai 1889. Die Raumnothcommission.) 7 S. $4^{0}$ [Autogr.]

Da HofB

1120. Technische Hochschule Darmstadt. Denkschrift über die Raumnoth und die durch dieselbe gegenwärtig herbeigeführten Verhältnisse. (Unterz.: Darmstadt, Ende Juli 1890. Der Lehrerrath der Technischen Hochschule.) $2 \mathrm{Bl}$. $4^{0} \quad \mathrm{Da} H o f B$

1121. Darmstadt, am 3. Okt. 1890. Kommissions-Bericht, betr. die Wahl eines Bauplatzes für Neubauten der Grossherzoglichen Technischen Hochschule zu Darmstadt. Hierzu Anl. A: Lageplan-Skizzen. 4 Bl., 1 Taf. $4^{0} \quad$ DaHofB

1122. Grossherzogliche Technische Hochschule zu Darmstadt. Beschlüsse des Lehrerraths, betr. die Wahl eines Bauplatzes für Neubauten der Grossherzoglichen Technischen Hochschule zu Darmstadt. (Unterz.. : Darmstadt, den 1. Nov. 1890. Die Direction der Grossherzogl. Techn. Hochschule.) 1 Bl. $4^{0}$

$\mathrm{DaHofB}$

1123. Grossherzogliche Technische Hochschule zu Darmstadt. Untersuchung über die Raumverhältnisse der Technischen Hochschule nach der bevorstehenden Erbauung der Institute für Physik und Elektrotechnik. (Unterz.: Darmstadt, den 25. Nov. 1890. [Theodor] Landsberg, z. Z. Dir. d. Techn. 
Hochschule.) [Nebst] Anl.: Zusammenstellung der Raumverhältnisse, Baukosten u. s. w. der neun Technischen Hochschulen Deutschlands, aufgestellt von $\mathrm{H}[$ einrich] Wa gner. $6 \mathrm{Bl}$. $4^{0}$

$\mathrm{DaH} H \mathrm{HB}$

1124. Die neuen Gebäude der Grossherzoglichen Technischen Hochschule zu Darmstadt. Festschrift zur feierlichen Einweihung der Neubauten am 28. Oktober 1895 (red. v. [Richard] Lepsius). Mit 21 Taf. 1895. 128 S. $4^{0}$

1125. Ueber die Methoden des Unterrichts auf der Technischen Hochschule. Rede geh. bei d. Festakte zur Feier der Einweihung der neuen Gebäude der Grossherzogl. Techn. Hochschule zu Darmstadt am 28. Okt. 1895 von d. Rector Prof. Dr. Richard Lepsius. Darmstadt: Bergstraesser 1895. 23 S. $8^{6}$

1126. Mittheilungen über die Stadt Darmstadt und den Aufenthalt daselbst. [Hrsg. von der Großherzogl. Technischen Hochschule Darmstadt.] (1900.) - [Neudr. mit Änderungen.] (1901.) - [Veränderte Fassung u. d. T.:] Mitteilungen ... (1909.) Je 2 Bl. $8^{\circ}$
1127. Katalog der Bibliothek der Grossh. Technischen Hochschule in Darmstart. 2. Aufl. 1902. IX, 956 S. $4^{0}$ - 1. Aufl. s. Nr 1114.

1128. Das Elektrotechnische Institut der Großherzoglich Technischen Hochschule Darmstadt. Zur Feier seines 25jährigen Bestehens. Darmstadt, den 1. Nov. 1907. 29 S., 13 Taf. $4^{0}$

1129. Die Grossherzogliche Technische Hochschule zu Darmstadt 1896-1908. Festschrift zur Feier der Eröffnung der Erweiterungsbauten am 23. Juli 1908. 1908. $173 \mathrm{~S} .4^{0}$

1130. Die dem pharmazeutischen Studium dienenden Institute an der Großh. Technischen Hochschule zu Darmstadt. [1908.] 36 S. $4^{0}$ - Zum Teil aus: Die Gr. Techn. Hochsch. zu D. 1896-1908 [Nr 1129].

1131. Die Einrichtungen des Kraftwerkes und Maschinenbaulaboratoriums I der Großherzoglichen Technischen Hochschule Darmstadt unter Leitung des Geheimen Baurat M[ax] F[riedrich] Gutermuth, Prof. des Maschinenbaues. Bearb. unter Mitw. des Institutsvorstandes von d. Assist. Dipl.-Ing. [Adolf] Watzinger und [Hans] Stiefelhagen. Berlin: Springer 1908. 55 S., 13 Taf. $4^{0}$

\section{HABILITATIO N S SCHRIFTEN}

1132. Einhorn, Alfred, Dr.: Über die Beta-Lactone, eine neue Gruppe von organischen Verbindungen. $1885.61 \mathrm{~S} .8^{0}$

Darmstadt Te. H., Hab.-Schr. v. 23. 2. 1885

1133. Klein, Joseph, Dr.: U(e)ber die Anwendbarkeit des dithiokarbaminsauren Ammons in der Analyse. Hamburg: Voss 1887. 22 S. $8^{0}$ - Aus: Repertorium d. analyt. Chemie. Jg. 7.

Darmstadt Te. H., Hab.-Schr. v. 10.12.1887

1134. Hansen, Adolph, Dr.: Die Farbstoffe des Chlorophylls. Mit Brewster's Spektralzeichn, einer eigenen Spektraltaf. u. 2 Holzschn. 1889.88 S., 2 Taf. $8^{0}$ - Auch bei Bergstraesser in Darmstadt.

Darmstadt Te. H., Hab.-Schr. v. 10. 11. 1888

1135. Dingeldey, Friedrich, Dr.: Über einen neuen topologischen Process und die Entstehungsbedingungen einfacher Verbindungen und Knoten in gewissen geschlossenen Flächen. Mit 5 lithogr. Taf. 1889. 31 S., 5 Taf. $8^{0}$ A Auch in: Sitzungsberichte d. math.-naturwiss. Classe d. Kais. Akademie d. Wissenschaften. Bd 98. Abth. 2a.

Darmstadt Te. H., Hab.-Schr. v. 9. 2.1889
1136. Dieffenbach, 0tto, Dr.: Beziehungen zwischen Verbrennungswärme und Konstitution organischer Verbindungen. Leipzig: W. Engelmann 1890. 25 S. $8^{0}$ - Aus: Zeitschrift f. physik. Chemie. Bd 5.

Darmstadt Te. H., Hab.-Schr. v. 11. 7. 1890

1137. Greim, G[eorg], Dr.: Beitrag zur Kenntniss des Kieselschiefers. Mit 1 Taf. in Lichtdr. Würzburg: Stahel 1891. 34 S., 1 Taf. $8^{0}$ - Aus: Verhandlungen d. phys.-medic. Gesellschaft zu Würzburg. N. F. Bd 24.

Darmstadt Te. H., Hab.-Schr. v. 25. 4. 1891

1138. Jännicke, Wilh[elm], Dr. phil.: Die Sandflora von Mainz, ein Relict aus der Steppenzeit. 1892. 25 S. $8^{0}$ - Umgearb. aus: Flora oder allg. botan. Zeitung. N. R. Jg. 47. 1889.

Darmstadt Te. H., Hah.-Schr. v. 22. 4. 1892

1139. Klemm, Gustav, Dr.: Gletscherspuren im Spessart und östlichen Odenwald. 1894. 12 S., 1 Taf. $8^{0}$ - Aus: Notizblatt d. Vereins f. Erdkunde u. d. grossh. geolog. Landesanstalt zu Darmstadt. Folge 4, H. 14.

Darmstadt Te. H., Hab.-Schr. v. 23. 2. 1894 
1140. Baur, Ludwig, Dr.: Aufstellung eines vollständigen Systems von Differentialen erster Gattung in einem cubischen Functionenkörper. 1894. S. $31-61 . \quad 8^{0}$ - Aus: Math. Annalen. Bd 46. 1895.

Darmstadt Te. H., Hab.-Schr. v. 5. 1. 1895

1141. Schilling, August Jakob, Dr. phil.: Der Einfluss von Bewegungshemmungen auf die Arbeitsleistungen der Blattgelenke von Mimosa pudica. Jena: Fischer 1895. 20 S. $8^{0}$ I Aus: Jenaische Zeitschrift f. Naturwissenschaft. Bd 29. N. F. Bd 22.

Darmstadt Te. H., Hab.-Schr. v. 13. 4. 1895

1142. Kolb, Adelbert, Dr.: Ueber Dimethyldiphenylpyrazin und einige Derivate des Phenylacetons. 1896. 45 S. $8^{0}$ - Aus: J. Liebig's Annalen d. Chemie. Bd 291.

Darmstadt Te. H., Hab.-Schr. v. 16. 3. 1896

1143. Loebell, Richard: Der Anti-Necker J. H. Mercks und der Minister Fr. K. v. Moser. Ein Beitrag zur Beurteilung J. H. Mercks. Darmstadt: Klingelhoeffer 1896. 55 S. $8^{0}$ Auch als Buch.

Darmstadt Te. H., Hab.-Schr. v. 13. 5. 1896

1144. Nagel, Willibald, Dr.: Die Entwickelung der Musik in Frankreich und England. Berlin: Reuther \& Reichard 1898. 49 S. $8^{0}$

Darmstadt Te. H., Hab.-Schr. v. 25. 6. 1898

1145. Vaubel, Wilhelm, Dr. phil.: Der Benzolkern. 1898. VI [vielmehr IV], 78 S. $8^{0}$ I Auch bei Rieger in München als: Sterochem. Forschungen. Bd 1, H. 1.

Darmstadt Te. H., Hab.-Schr. v. 25. 6. 1898

1146. Heyl, Georg, Dr. phil.: Über Oxydiphenylenketon und o-Phenylsalicylsäure. Leipzig: J. A. Barth 1899. 32 S. $8^{0}$ - Aus: Journal f. prakt. Chemie. Bd 167. N. F. Bd 59.

Darmstadt Te. H., Hab.-Schr. v. 7. 6. 1899

1147. Camerer, Rudolf, Dr. phil.: Versuche über die Regulirung der Rider-Steuerung Berlin: (Springer in Komm.) 1899. 14 S. $4^{0}$ I Aus: Zeitschrift d. Vereines dentscher Ingenieure. Bd 43.

Darmstadt Te. H., Hab.-Schr. v. 17. 11. 1899

1148. Forch, Carl, Dr. phil. nat.: Über die Oberflächenspannung wässeriger Lösungen. Mit 2 Taf. Leipzig: J. A. Barth 1899. 19 S., 2 Taf. $8^{0}$ il Ohne d. Nachtrag auch in: Annalen d. Physik u. Chemie. Bd 68.

Darmstadt Te. H., Hab.-Schr. v. 27. 12. 1899

1149. Rudolphi, Max, Dr.: Die Molekularrefraktion fester Körper in Lösungen mit verschiedenen Lösungsmitteln. 'Ravensburg: Maier 1900. 57 S. $8^{0}$ - Buchausg. 1901.

Darmstadt Te. H., Hab.-Schr. v. 6.12. 1900
1150. Vetterlein, Ernst, Dr.-Ing.: Die Aufnahme des frühgotischen Chores zu Hirzenach am Rhein. [Nebst] Atlas mit 6 Taf. 1902 . 15 S. $8^{0} ; 6$ Taf. $4^{0}$

Darmstadt Te. H., Hab.-Schr. v. 15. 5. 1902

1151. Feldmann, Clarence, Ingenieur: Asynchrone Generatoren für ein- und mehrphasige Wechselströme. Ihre Theorie und Wirkungsweise. Berlin: Springer 1903. VI, 134 S. $8^{0}$ - Auch als Buch.

Darmstadt Te. H., Hab.-Schr. v. 19. 11. 1902

1152. Goldstein, Julius, Dr.: Die empiristische Geschichtsauffassung David Humes mit Berücksichtigung moderner methodologischer und erkenntnistheoretischer Probleme. Leipzig: Dürr 1902. 57 S. $8^{0}$

Darmstadt Te. H., Hab.-Schr. v. 15. 12. 1902

1153. Keppeler, Gustav, Dr., dipl. Chemiker: Über die Reinigung des technischen Acetylens. 1902. 54 S. $8^{0}$ Aus: Schilling's Journal f. Gasbeleuchtung. Jg. 44. 45. 1901. 02. Darmstadt Te. H., Hab.-Schr. v. 7. 1. 1903

1154. Schwalbe, Carl Gustav, Dr.: Über direkte Substitution bei Schiffschen Basen. 1903. 24 S. $8^{0}$ - Auch in: Zeitschrift f. Farben- u. Textil-Chemie. Jg. 1. 1902.

Darmstadt Te. H., Hab.-Schr. v. 2. 3. 1903

1155. Kučera, Gottlieb, Dr.: Die Oberflächenspannung von polarisiertem Quecksilber. 1903. $87 \mathrm{~S} ., 2$ Taf. $8^{0}$ - Ausz. in: Annalen d. Physik. Folge 4, Bd 11.

Darmstadt Te. H., Hab.-Schr. v. 14. 3. 1903

1156. Schlink, Wilhelm, Dr., Reg.-Bauführer a. D.: Stabilitäts- und Spannungsuntersuchungen von speziellen Fachwerksträgern mittels des erweiterten Systems. 1903. 36 S., 12 Taf. $8^{0}$ T Etwas gekürzt in: Zeitschrift f. Architektur u. Ingenieurwesen. Bd 49. N. F. Bd 8.

Darmstadt Te. H., Hab.-Schr. v. 16. 4. 1903

1157. Schrader, Ernst, Dr.: Zur Grundlegung der Psychologie des Urteils. Leipzig: J. A. Barth 1903. 98 S. $8^{0}$ - Auch als Buch. Darmstadt Te. H., Hab.-Schr. v. 21. 4.1903

1158. Alt, Carl, Dr.: Schiller und die Brüder Schlegel. Weimar: Böhlau 1904. VIII, 130 S. $8^{0}$. Auch als Buch.

Darmstadt Te. H., Hab.-Schr. v. 30. 3. 1904

1159. Gast, Paul, Dr.: Utber Luftspiegelungen im Simplon-Tunnel. Stuttgart: Wittwer 1904. 31 S. $8^{0}$ I Aus: Zeitschrift f. Vermessungswesen. Bd 33.

Darmstadt Te. H., Hab.-Schr. v. 17. 5. 1904

1160. Fritseh, Carl, Dr.: Das Bogenspektrum des Mangans. 1904. VIII, 34 S. $8^{0}$ - Auch in: Annalen d. Physik. Folge 4, Bd 16. 1905. Darmstadt Te. H., Hab.-Schr. v. 5. 8.1904 
1161. Moldenhauer, Wilhelm, Dr.-Ing.: Über die Einwirkung von Sauerstoff und Wasserdampf auf Chlormagnesium. 1906. $23 \mathrm{~S} .8^{0}$ Aus: Zeitschriftf. anorgan. Chemie. Bd51.

Darmstadt Te. H., Hab.-Sehr. v. 21. 12. 1906

1162. Petersen, Waldemar, Dr.-Ing. : Elektrostatische Maschinen. Mit $9 \mathrm{Abb} .1907$. 44 S. $8^{0}$ Eine erw. Bearbeitung nebst sehr vielen Ergänzungen soll demnächst in d, elektrotechn. Fachpresse erscheinen; bis 1913 nicht ersch.

Darmstadt Te. H., Hab.-Schr. v. 5. 7. 1907

1163. Gasser, Max, Dr.: Zur Entwicklung der Basisapparate und Basismessungsmethoden. 1907. 64 S., 5 Taf. $8^{0}$ - Auch bei Lindauer in München.

Darmstadt Te. H., Hab.-Schr. v. 24. 7. 1907

1164. Ans, Jean d', Dr.-Ing.: Untersuchungen über Calciumalkalisulfate. Hamburg u. Leipzig: Voss 1909. 48 S. $8^{0}$ - Durch Literaturverz. erw. aus: Zeitschrift f. anorgan. Chemie. Bd 62.

Darmstadt Te. H., Hab.-Schr. v. 7. 5. 1909

1165. Preuss, E[rnst], Dr.-Ing.: Die Festigkeit von Nickelstahlnieten in Verbindung mit Flußeisenblechen unter besonderer Berücksichtigung des Gleitwiderstandes. Darmstadt: Herbert 1909. 79 S., 1 Taf. $8^{0}$ - Engl. in: Carnegie Scholarship Memoirs. Vol. 1; kurzer Ausz. in: Metallurgie. Jg. 6 и. in: Stahl u. Eisen. Jg. 29.

Darmstadt Te. H., Hab.-Schr. v. 1. 7. 1909
1166. Dammann, Walter H[einrich], (Dr. phil.): Panorama und Tafellandschaft. Anfänge und Frühzeit der Landschaftsmalerei in Hamburg bis 1830. Hamburg: Commeter 1910. 88 S. $8^{0}$ - Hamburgische LiebhaberBibliothek

Darmstadt Te. H., Hab.-Schr. v. 27. 10. 1910

1167. BlaeB, Viktor, Dr.-Ing., Reg.-Baumeister: Die Strömung in Röhren und die Berechnung weitverzweigter Leitungen und Kanäle mit Rücksicht auf Be- und Entlüftungsanlagen, Grubenbewetterung, Gastransport, pneumatische Materialförderung etc. (1910.) V, 146 S., 1 Taf. $8^{0}$ - Nebst Tafelbd bei Oldenbourg in München 1911.

Darmstadt Te. H., Hab.-Schr. v. 1. 12. 1910

1168. Kleinlogel, A[dolf], Dr.-Ing.: Über neuere Versuche mit umschnürtem Beton 〈spiralumwickelte und ringbewehrteSäulen〉. Berlin: Ernst 1912. 48 S., 2 Taf. $4^{0}$ - Um ein Vorw. verm. als: Forscherarbeiten aus d. Gebiete d. Eisenbetons. H. 19.

Darmstadt Te. H., Hab.-Schr. v. 11. 3.1912

1168a. Baerwald, Hans, Dr. phil.: Utber die Erregung der Erdalkaliphosphore durch Kanalstrahlen. Leipzig: J. A. Barth 1912. 57 S. $8^{0}$ Ausz. in: Annalen d. Physik. Folge 4. Bd 39.

Darmstadt Te. H., Hab.-Schr. v: 21. 8. 1912

\section{DOKTOR-INGENIEUR-DISSERTATIONEN}

\section{ABTEILUNG FÜR ARCHITEKTUR}

1169. Vetterlein, Ernst: Das Auftreten der Gotik am Dom zu Mainz. 1902. 32 S., 2 Taf. $8^{0}$

Darmstadt, Dr.-Ing.-Diss. v. 8. 3. 1902

1170. Waag, Hans, Kgl. Reg.-Bauführer: Der Bolongaro-Palast zu Höchst am Main. 1904. 59 S., 7 Taf. $4^{0}$ - Auch bei Knauer in Frankfurt a. M.

Darmstadt, Dr.-Ing.-Diss. v. 3. 9. 1904

1171. Forbát, Emerich: Der Bau der Städte an Flüssen in alter und neuer Zeit. 1904. 16 S., 2 Taf. $4^{0}$ - Aus: Der Städtebau. Jg. 1.

Darmstadt, Dr.-Ing.-Diss. v. 16. 9. 1904

1172. Hercher, Ludwig, Reg.-Baumeister i. Münster i. W.: Grossstadterweiterungen. Ein Beitrag zum heutigen Städtebau. Göttingen: Vandenhoeck \& Ruprecht 1904. 46 S., 1 Taf. $8^{0}$ - Auch als Buch.

Darmstadt, Dr.-Ing.-Diss. v. 26. 9. 1905
1173. Lipp, Ludwig, Großh. Hess. Reg.Baumeister: Beiträge zum ländlichen Schulhausbau. 1907. 56 S., 14 Taf. $4^{0}$ - Auch bei Roth in Gießen.

Darmstadt, Dr.-Ing.-Diss. v. 14. 3. 1907

1174. Göbel, Heinrich, Reg.-Bauführer: Darstellung der Entwicklung des süddeutschen Bürgerhauses. Text mit $311 \mathrm{Abb}$. [Nebst] Atlas mit 30 Taf. Dresden: Kühtmann 1908. 411 S., 1 Taf. $4^{0}$; 30 Taf. gr. $2^{\circ}$ T Buchausg. u. d. T.: Das süddeutsche Bürgerhaus. Eine Darstellung seiner Entwickelung.

Darmstadt, Dr.-Ing.-Diss. v. 23. 12. 1907

1175. Vogts, Hans, Kgl. Reg.-Bauführer: Das Mainzer Wohnhaus im 18. Jahrhundert. 1909. IV, 74 S., 4 Taf. $4^{0}$ I Um einen 2. Teil verm. als: Beiträge zur Geschichte d. Stadt Mainz. 1. 1910. Darmstadt, Dr.-Ing.-Diss. v. 23. 11. 1909

1176. Carius, Arthur: Ornamentik am oberhessischen Bauernhause. Frankfurt a. M. : Keller 1910. 16 S., 27 Taf. $4^{0}$ - Buchausg. vollst. Darmstadt, Dr.-Ing.-Diss. v. 2. 2. 1910 
1177. Siedler, E[duard] Jobst, Reg.-Baumeister a. D.: Die Gärten und Gartenarchitekturen Friedrichs des Grossen. Berlin: Ernst 1911. 28 S., 2 Taf. gr. $2^{0}$ - Aus: Zeitschrift f. Bauwesen. Jg. 61.

Darmstadt, Dr.-Ing.-Diss. v. 4. 2. 1911

1178. Schmitt, Franz: Die Kirchen des Kreises Lauterbach 〈Oberhessen〉. Ein Beitrag zur Entwicklungsgeschichte des protestantischen Kirchenbaues. 1911. 24 S., 12 Taf. $2^{0}$. Soll auch im Buchh. ersch.; bis 1913 nicht erseh.

Darmstadt, Dr.-Ing.-Diss. v. 4. 9. 1911

\section{ABTEILUNG FÜR INGENIEURWESEN}

1179. Völker, Philipp, Reg.-Bauführer: Die Beziehungen zwischen den Auflagerbedingungen u. Stabkräften beim ebenen und räumlichen Fachwerk. [1902.] 32 S. $8^{0}$ - Aus: Bauingenienr-Zeitung. Jg. 2 u.: Das Baugewerbe. Jg. 1.

Darmstadt, Dr.-Ing.-Diss. v. 17. 11. 1902

1180. Boos, Eduard: Berechnung räumlicher Fachwerke mittelst des ProjektionsVerfahrens. [1905.] 28 S., 1 Taf. $8^{0}$

Darmstadt, Dr.-Ing.-Diss. v. 25. 5. 1905

1181. Sachs, L[eon]: Zur Berechnung räumlicher Fachwerke: Allgemeine Formeln für statisch bestimmte und insbesondere statisch unbestimmte Kuppel-, Zelt- und Turmdächer. Mit 3 Taf. Berlin: Ernst 1905. 56 S., 3 Taf. $4^{0}$ - Auch als Buch.

Darmstadt, Dr.-Ing.-Diss. v. 12. 7. 1905

1182. Bohny, Friedrich, Obering. d. Ver. Maschinenfabrik Augsburg u. Maschinenbauges. Nürnberg, A. -G., Z weiganst. Gustavsburg: Theorie und Konstruktion versteifter Hängebrücken. Leipzig: W. Engelmann 1905. VI, 109 S. $4^{0}$ - Auch als Buch.

Darmstadt, Dr.-Ing.-Diss. v. 27, 12.1905

1183. Strieboll, E[rich]: Materialverhältnisse bei Balkenträgern und Bogenträgern mit Zugband. Theoretische Ableitung der Gewichtsformeln - besonders für letztere und Vergleich. 1905. 59 S., 2 Taf. $8^{0}$

Darmstadt, Dr.-Ing.-Diss. v. 27.8. 1906

1184. Schinkel, Max, Reg.-Bauführer: Der elektrische Schiffszug. Eine technische und wirtschaftliche Untersuchung über die Möglichkeit bezw. Zweckmäßigkeit einer Erhöhung der Fahrgeschwindigkeit auf verkehrsreichen Kanälen. 1906. V, 102 S., 7 Taf. $8^{0}$ Auch als: Mitteilungen d. Gesellschaft f. wirtschaftl. Ausbildung. N. F. H. 1.

Darmstadt, Dr.-Ing.-Diss. v. 15. 11. 1906
1185. Schütz, Renatus, Reg.-Bauführer: Beiträge zur zeichnerischen Massenermittlung, Massenverteilung und Förderkostenbestimmung der Erdarbeiten. Berlin: Ernst 1908. 62 S., 3 Taf. $4^{0}$ - Auch als Buch; aus: Zeitschrift f. Bauwesen. Jg. 58 .

Darmstadt, Dr.-Ing.-Diss. v. 10. 8. 1908

1186. Boseh, Joh[ann] Bapt[ist], städt. Bauamtmann i. München: Berechnung der gekreuzt armierten Eisenbetonplatte und deren Aufnahmeträger unter Berücksichtigung der Kraftwirkungen nach zwei Richtungen. [1908.] $50 \mathrm{~S}$. $4^{0}$ of Auch als: Forscherarbeiten auf d. Gebiete d. Eisenbetons. H. 9; Abschnitt 1 auch in: Beton u. Eisen. Jg. 4. 1905. Darmstadt, Dr.-Ing.-Diss. v. 25. 9.1908

1187. Martens, Hans A[lfred], Kgl. Eisenbahn-Bauinsp.: Grundlagen des Eisenbahnsignalwesens für den Betrieb mit Hochgeschwindigkeiten unter Berücksichtigung der Bremswirkung. Wiesbaden: Kreidel 1909. XII, 82 S., 13 Taf. $4^{0}$ - Auch als Buch; Ausz. in: Dinglers Polytechn. Journal. Jg. 90. Bd 324.

Darmstadt, Dr.-Ing.-Diss. v. 8. 11. 1909

1188. Weiss, [Richard], Stadtbaumeister i. Glogau: Das Mangan im Grundwasser und seine Beseitigung. [1910.] 61 S., 3 Taf. $8^{0}$ - Auch in: Der städtische Tiefbau. Jg. 1910. Darmstadt, Dr.-Ing.-Diss. v. 10. 6. 1910

1189. Saller, Heinrich, Kgl. bayer. Direktionsrat: Stoßwirkungen an Tragwerken und am Oberbau im Eisenbahnbetriebe. Mit 6 Abb. im Texte. Wiesbaden: Kreidel 1910. 70 S. $4^{0}$ Auch als Buch.

Darmstadt, Dr.-Ing.-Diss. v. 22. 8. 1910

1190. Esch, Rudolf Julius: Über den Einfluß der Geschwindigkeit der Beförderung auf die Selbstkosten der Eisenbahnen. Eine technisch-wirtschaftliche Untersuchung unter besonderer Berücksichtigung und mit einer Selbstkostenberechnung der PreußischHessischen Staatseisenbahnen. Jena: Fischer 1910. $98 \mathrm{~S} .8^{0}$ - Auch als: Mitteilungen d. Gesellschaft f. wirtschaftl, Ausbildung. N. F. H. 6. 1911.

Darmstadt, Dr.-Ing.-Diss. v. 5, 1. 1911

1191. Willmann, Erich von, Reg.-Baumeister: Über einige Gebirgsdruckerscheinungen in ihren Beziehungen zum Tunnelbau. Mit 20 Textabb. Leipzig: W. Engelmann 1911. 32 S. $4^{0}$ - Auch als: Fortschritte d. Ingenieurwissenschaften. Gruppe 2, H. 26.

Darmstadt, Dr.-Ing.-Diss. v. 14. 7. 1911

1192. Tecklenburg, Kurt, Reg.-Baumeister: Der Betriebskoeffizient der Eisenbahnen und seine Abhängigkeit von der 
Wirtschaftskonjunktur. Berlin: Springer 1911. 100 S., 5 Taf. $8^{0}$ - Auch als Buch; gekürzt in: Archiv f. Eisenbahnwesen. Jg. 34.

Darmstadt, Dr.-Ing.-Diss. v. 22. 11. 1911

1193. Goetz, Franz: Ausbildung der Straßenbahnanlagen mit Rücksicht auf die Bodenbewegungen im Senkungsgebiete des rheinisch - westfälischen Kohlenbergbaus. Essen-R.: Fredebeul \& Koenen 1911. 63 S., 2 Taf. $8^{\circ}$

Darmstadt, Dr.-Ing.-Diss. v. 18. 12. 1911

1194. Geyer, Georg, Dipl.-Ing. d. Maschinenfabrik Augsburg-Nürnberg, A.-G., Werk Gustavsburg: Beitrag zur Ableitung von Einflußlinien für durchlaufende Träger auf $n$ Stützen unter Zugrundelegung des Trägers auf (n-1) Stützen als statisch bekanntes Hauptsystem. 1912. 46 S., 12 Taf. $4^{0}$

Darmstadt, Dr.-Ing.-Diss. v. 31. 7. 1912

\section{ABTEILUNG FÜR MASCHINENBAU EINSCHLIESZLICH PAPIERFABRIKATION}

1195. Camerer, Rudolf, Dr. phil.: Neue Diagramme zur Turbinentheorie. Berlin: Dietze [1902]. 30 S. $8^{0}$ - Auch als Buch; aus: Dinglers Polytechn. Journal. Jg. 83. Bd 317.

Darmstadt, Dr.-Ing.-Diss. v. 15. 12. 1902

1196. Schmoll von Eisenwerth, Adolf: Beitrag zur Theorie und Berechnung der hydraulischen Regulatoren für Wasserkraftmaschinen. Berlin: Dietze 1904. 85 S. $8^{0}$ - Aus: Dinglers Polytechn. Journal. Jg. 85. Bd 319.

Darmstadt, Dr.-Ing.-Diss. v. 28. 6. 1904

1197. Recke, Oskar: Druck und Geschwindigkeitsverhältnisse bei (übergeklebt: des Dampfes in) Freistrahl-Grenzturbinen. (Mit 50 Textfig. Als Ms. gedr. 1904.) $27 \mathrm{~S} .4^{0}$ - Vollst. bei Oldenbourg in München 1907; auch in: Zeitschrift f. d. gesamte Turbinenwesen. Jg. 3. 1906.

Darmstadt, Dr.-Ing.-Diss. v. 18. 3. 1905

1198. König, August: Arbeitsdiagramme von Flachformmaschinen. Berlin: Dietze 1906. 48 S. $4^{0}$ - Auch in: Dinglers Polytechn. Journal. Jg. 87. Bd 321.

Darmstadt, Dr.-Ing.-Diss. v. 14. 8. 1906

1199. Blaess, Viktor, Gr. Reg.-Baumeister: Uber Zentrifugalpumpen und Ventilatoren. 1907. 30 S. $4^{0}$ ๆ Auch in: Zeitschrift f. d. gesamte Turbinenwesen. Jg. 4.

Darmstadt, Dr.-Ing.-Diss. v. 18. 10. 1907

1200. Preuss, Ernst: Die Geschwindigkeit der elastischen Durchbiegungen eines wagerechten, auf zwei Stützen frei aufliegenden Trägers. 1908. 50 S., 1 Taf. $8^{0}$ Darmstadt, Dr.-Ing.-Diss. v. 28. 7. 1908
1201. Wulff, C[onstantin], Reg.-Bauführer: Die Talsperrengenossenschaften im Ruhrund Wuppergebiet. Jena: Fischer 1908. VI, 169 S. $8^{0}$ - Auch als: Mitteilungen d. Gesellschaft f. wirtschaftl. Ausbildung. N. F. H. 4.

Darmstadt, Dr.-Ing.-Diss. v. 18. 9. 1908

1202. Gramberg, Anton: Ueber das Verhalten einer Rateau-Dampfturbine unter wechselnden Betriebsbedingungen. 1908. 43 S. $4^{0}$ - Auch in: Mitteilungen über Forschungsarbeiten auf d. Gebiete d. Ingenieurwesens. H. 76. 1909; Ausz. in: Zeitschrift d. Vereines deutscher Ingenieure. Bd ̋̌3. 1909, Halbj. 1.

Darmstadt, Dr.-Ing.-Diss. v. 6. 10. 1908

1203. Altmann, Eugen: Über die Entwickelung und Bedeutung der Kartelle in der deutschen Eisenindustrie. [1909.] 100 S., $6 \mathrm{Bl}$. Tab. $8^{0}$

Darmstadt, Dr.-Ing.-Diss. v. 27. 2. 1909

1204. Hiemenz, Hans: Der Reguliervorgang beim direkt gesteuerten hydrostatischen Turbinenregulator unter Berücksichtigung der Wirkung der Anschläge am Steuerventil. Berlin: Dietze 1909. $55 \mathrm{~S} .8^{0}$ I Auch in: Dinglers Polytechn. Journal. Jg. 90. Bd 324.

Darmstadt, Dr.-Ing.-Diss. v. 10. 6. 1909

1205. Jaeger, Hans: Über Messungen an Turbinenkanälen. 1909. 42 S. $4^{0}$ - Auch in: Zeitschrift f. d. gesamte Turbinenwesen. Jg. 6.

Darmstadt, Dr.-Ing.-Diss. v. 28. 6. 1909

1206. Luther, Gerhard, Dr. phil.: Der deutsche Mühlenbau. Eine Monographie dieses Gewerbezweiges nach seiner jüngeren Entwickelung. [1909.] 78 S., 1 Taf. $8^{0}$

Darmstadt, Dr.-Ing.-Diss. v. 18. 9. 1909

1207. Mysz, Ernst: Beitrag zur Theorie des Druckversuches. 1909. 54 S. $4^{0}$

Darmstadt, Dr.-Ing.-Diss. v. 18. 9. 1909

1208. Utard, A[nton]: Die bei der Turbinenregulierung auftretenden sekundären Erscheinungen, bedingt durch die Massenträgheit des zufließenden Arbeitswassers. Berlin: Dietze 1909. VIII, 93 S. $4^{0}$ ฯ Auch in: Dinglers Polytechn. Journal. Jg. 90. Bd 324.

Darmstadt, Dr.-Ing.-Diss. v. 18. 9. 1909

1209. Haake, Heinrich: Der Reguliervorgang beim direkt gesteuerten hydrostatischen Turbinenregulator mit nachgiebiger Rückführung 〈Isodromregulator〉. Berlin: Dietze 1910. 49 S. $8^{0}$ o Auch in: Dinglers Polytechn. Journal. Jg. 91. Bd 325.

Darmstadt, Dr.-Ing.-Diss. v. 15. 3. 1910

1210. Watzinger, A[dolf]: Ueber den praktischen Wert der Zwischenüberhitzung bei Zweifachexpansions - Dampfmaschinen. 1910. 79 S. $4^{0}$ - Auch als: Mitteilungen über 
Forschungsarbeiten auf $d$. Gebiete $d$. Ingenieurwissenschaften. H. 92; vgl. Zeitschrift d. Vereines deutscher Ingenieure. Bd 54, Halbj. 2. S. $1344 \mathrm{ff}$.

Darmstadt, Dr.-Ing.-Diss. v. 10. 6. 1910

1211. Just, Karl: Ueber Labyrinthdichtungen für Wasser. Berlin: Dietze 1910. $72 \mathrm{~S} .8^{0}$ T Auch in: Dinglers Polytechn. Journal. Jg. 92. 1911. Bd 326.

Darmstadt, Dr.-Ing.-Diss. v. 24. 1. 1911

1212. Müller, Willy: Über den elektrischen Widerstand von nichtbewehrtemBeton und seinen Einzelbestandteilen. Berlin: Ernst 1911. IV, 69 S. $4^{0}$ - Auch als: Deutseher Ausschuss f. Eisenbeton. H. 6 .

Darmstadt, Dr.-Ing.-Diss. v. 3. 2. 1911

1213. Brake, Ludwig: Werkzeugmaschine und Arbeitszerlegung. Ein Versuch zur Ergänzung der nationalökonomischen Lehre von der Arbeitsvereinigung und Arbeitsteilung. Berlin: Krayn [1911]. 68 S. $8^{0}$

T Auch als: Schriften d. Verbandes Dentscher Diplom-Ingenieure. 7.

Darmstadt, Dr.-Ing.-Diss. v. 15. 5. 1911

1214. Bachmann, Heinrich: Beitrag zur Messung von Luftmengen. 1912. 69 S. $4^{0}$

Darmstadt, Dr.-Ing.-Diss. v. 7. 3. 1912

1215. Mies, 0tto: Untersuchungen über die Knicksicherheit von Kolbenstangen. [1912.] 29 S. $4^{0}$ T Aus: Dinglers Polytechn. Journal. Jg. 93 . Bd 327.

Darmstadt, Dr.-Ing.-Diss. v. 15. 7. 1312

1216. Gompertz, Max: Uber abgesetzte und gekröpfte Wellen. 1912. 88 S., 3 Taf. $4^{0}$ I Auch bei-Simion in Berlin 1913; Ausz. in: Verhandlungen d. Vereins zur Beförderung d. Gewerbfleißes. Jg. 91.

Darmstadt, Dr.-Ing.-Diss. v. 18.12. 1912

\section{ABTEILUNG FÜR ELEKTROTECHNIK}

1217. Möllinger, Julius Adolf: Ueber Drehstrom-Zähler. (1900.) 19 S. $4^{0}$ I Aus: Elektrotechn. Zeitsehrift. Jg. 21.

Darmstadt, Dr.-Ing.-Diss. v. 8. 3. 1902

1218. Hohage, Carl: Ueber einige Anwendungen des Elektrometers bei Wechselstrommessungen. 1903. $30 \mathrm{~S} .8^{0}$ - Auch bei Enke in Stuttgart u. als: Sammlung elektrotechn. Vorträge. Bd 4, H. 7.

Darmstadt, Dr.-Ing.-Diss. v. 21. 7. 1903

1219. Hinden, Heinrieh: Über deformierte Wechselströme mit besonderer $\mathrm{Be}$ rücksichtigung eisengeschlossener Apparate. 1903. IV, 54 S. $8^{0}$. Auch bei Enke in Stuttgart u. als: Sammlung elektrotechn. Vorträge. Bd 4, H. 11/12.

Darmstadt, Dr.-Ing.-Diss. v. 29. 9. 1903
1220. Rossem Nz, A[driaan] C[arel] van: Die Aufnahme von Kommutatordiagrammen. 1903. 31 S. $8^{0}$ T Auch bei Knapp in Halle 1904.

Darmstadt, Dr.-Ing.-Diss. v. 8.10.1903

1221. Herzfeld, Beni: Über die Abhängigkeit des Hysteresisverlustes im Eisen von der Feldwechselzahl. 1903, 69 S., 7 Taf. $8^{\circ}$ - Vgl. Archives des sciences physiques et naturelles. Année 107. Période 4, T. 14. 1902; Comptes rendus hebdomadaires des séances de l' Académie des sciences. T. 136 . S. $957 \mathrm{ff}$; d daraus als: Université de Genève. Laboratoire de Physique. Sér. 1, fasc. 6.

Darmstadt, Dr.-Ing.-Diss. v. 1. 12.1903

1222. Monasch, Berthold: Untersuchungen über den Wechselstromlichtbogen zwischen Metallelektroden bei Hochspannung: 1903. 69 S. $8^{0}$ T Verkürzt in franz. U̇bers. in: L'Éclairage Électrique. T. 34; einige Kapitel aufgenommen in: Monasch: Der elektr. Lichtbogen. Berlin 1904.

Darmstadt, Dr.-Ing.-Diss. v. 1. 12.1903

1223. Siegel, G[ustav]: Die Tarife der Elektrizitätswerke. (Berlin: Springer) 1905. VII, 192 S. $8^{0}$ ๆ Buchausg. u. d. T.: Die Preisstellung beim Verkaufe elektrischer Energie. 1906.

Darmstadt, Dr.-Ing.-Diss. v. 12. 1. 1906

1224. Fischer, Kurt: Untersuchungen über die Pulsationen im Erregerstromkreis von Wechsel- und Drehstrom-Maschinen. 1907. 30 S., 1 Taf. $8^{0}$

Darmstadt, Dr.-Ing.-Diss. v. 16. 2. 1907

1225. Goldschmidt, Rudolf: Die Berechnung des Leerlaufstroms und der Streuung von Asynchronmotoren aus ihren $\mathrm{Ab}$ messungen. 1906. 47, VIII S. $8^{0}$ - Engl. in: The Electrician. Vol. 60. 1908.

Darmstadt, Dr.-Ing.-Diss. v. 8. 6. 1907

1226. Petersen, Waldemar: Eine neue Spannungsregelung. Mitteilung der Arbeitsweise. Theorie des Reguliervorganges. 1907. 48 S. $8^{0}$. Auch bei Enke in Stuttgart.

Darmstadt, Dr.-Ing.-Diss. v. 12. 6. 1907

1227. Schön, Ludwig: Ein Beitrag zur Theorie des Wehneltunterbrechers. 1908. 31 s., 8 Taf. $8^{0}$

Darmstadt, Dr.-Ing.-Diss. v. 21. 4. 1908

1228. Gewecke, Hermann: Ueber die Einwirkung von Strukturänderungen auf die physikalischen, insb. elektrischen Eigenschaften von Kupferdrähten und über die Struktur des Kupfers in seinen verschiedenen Behandlungsstadien. [1909.] 93 S., 11 Taf. $8^{\circ}$ - Aus: Dinglers Polytechn. Journal. Jg. 90. Bd 324 . Darmstadt, Dr.-Ing.-Diss. v. 20. 10. 1909 
1229. Steiner, Desider: Die Hysteresisverluste der ferromagnetisierbaren Manganaluminium-Bronzen in Abhängigkeit von der Frequenz des Wechselfeldes. Mit 5 Taf. Leipzig: J. A. Barth 1911. 49 S., 5 Taf. $8^{0}$ - Erw. aus: Annalen d. Physik. Folge 4, Bd 35. Darmstadt, Dr.-Ing.-Diss. v. 15. 5. 1911

1230. Harbich, Johannes: Glimmverluste paralleler Leiter. 1911. 26 S. $8^{0}$

Darmstadt, Dr.-Ing.-Diss. v. 27. 11. 1911

1231. Krug, Karl: Das Kreisdiagramm der Induktionsmotoren. Berlin: Springer 1911. 68 S. $8^{0}$ I Buchausg. 1912; zum Teil in: Elektrotechniku. Maschinenbau. Jg.28.29. 1910.11.

Darmstaảt, Dr.-Ing.-Diss. v. 29. 11. 1911

1232. Rein, Hans: Der radiotelegraphische Gleichstrom-Tonsender. [Langensalza: Beyer in Komm.] 1912. $62 \mathrm{~S} .8^{0}$

Darmstadt, Dr.-Ing.-Diss. v. 5. 2. 1912

1233. Kuhlmann, Karl: Sicherheits- und Empfindlichkeitsfaktor des Leitungsschutzsystems von Merz \& Price. Berlin: Springer 1912. 31 S. $4^{0}$

Darmstadt, Dr.-Ing.-Diss. v. 24. 4. 1912

1234. Lohmann, P[aul]: Über den Ausgleich periodischer Energieschwankungen in Drehstromnetzen. 1912. 90 S. $8^{0}$

Darmstadt, Dr.-Ing.-Diss. v. 24. 4. 1912

\section{ABTEILUNG FÜR CHEMIE EINSCHLIESZLICH ELEKTROCHEMIE UND PHARMAZIE}

1235. Steiner, 0tto: Studien über das sogenannte ,Glocken-Verfahren " zur Elektrolyse wässeriger Lösungen der Alkalichloride. 1904. 40 S. $8^{0}$ II Aus: Zeitschrift f. Elektrochemie. Jg. 10.

Darmstadt, Dr.-Ing.-Diss. v. 28. 6. 1904

1236. Ans, J[ean] d': Das wasserfreie Ferrosulfat und seine Zersetzung bei höheren Temperaturen. 1905. 51 S., 5 Taf. $8^{0}$ - Gänzlich umgearb. u. erw. in: Zeitschrift f. phys. Chemie. Bd 62. 1908. S. $89 \mathrm{ff}$.

Darmstadt, Dr.-Ing-Diss. v. 26. 1. 1905

1237. Davidson, Emil: Die Zersetzung von Kaliumchlorat durch Salzsäure, eine Reaktion I. Ordnung. 1905. 58 S., 4 Taf. $8^{\circ}$ IVeränd. Utbers. in: Moniteur scientifique. Sér. 4, T. 19, 2; vgl. Zeitschrift f. angewandte Chemie. Jg. 18. Darmstadt, Dr.-Ing.-Diss, v. 8, 3. 1905

1238. Moldenhauer, Wilhelm, Assist. am chem.-techn. u. elektrochem. Inst. d. Techn. Hochsch. Darmstadt: Über Beziehungen zwischen elektrolytischen Vorgängen und der Elektrodentemperatur. 1905. 56 S. $8^{0}$ T Auch in: Zeitschrift f. Elektrochemie. Jg. 11. Darmstadt, Dr.-Ing.-Diss. v. 28. 6. 1905
1239. Siebert, Werner: Über Azofarbstoffe der Triphenylmethanreihe und deren Lackbildungsvermögen. [1906.] $69 \mathrm{~S} .8^{0}$

Darmstadt, Dr.-Ing.-Diss. v. 27. 7. 1906

1240. Hiemenz, Wilhelm: Über Nitroketotetrahydrochinoxaline und deren $\mathrm{Re}$ duktionsprodukte. 1906. 55 S. $8^{0}$

Darmstadt, Dr.-Ing.-Diss. v. 16. 10. 1906

1241. Burger, Paul: Versuche über die Elektrolyse mit Wechselströmen und ihre Anwendung zur Herstellung chemischer Produkte. 1906. 44 S. $8^{0}$

Darmstadt, Dr.-Ing.-Diss. v. 17. 10. 1906

1242. Kranse, Ernst: Versuche zur Oxydation von hydratischem Mangandioxyd in alkalischer Suspension. Leipzig: Fock 1907. 47 S. $8^{0}$

Darmstadt, Dr.-Ing.-Diss. v. 25. 2. 1907

1243. Weber, Karl: Versuche zur elektrolytischen Darstellung von Magnesium. 1907. 24 S. $8^{\circ}$

Darmstadt, Dr.-Ing.-Diss. v. 2. 5. 1907

1244. Kautzsch, J[ohannes]: Die Chlorierung von Äthylchlorid und Äthylidenchlorid im ultravioletten Licht. 1907. 27 S. $8^{0}$ T Ausz. in: Journal f. prakt. Chemie. Bd 188. N. F. Bd 80. 1909.

Darmstadt, Dr.-Ing.-Diss. v. 3. 6. 1907

1245. Nover, Wilhelm: Über Emeraldin. 1907. 32 S. $8^{0}$ ๆ Vgl. Berichte d. Deutschen Chem. Gesellschaft. Jg. 40.

Darmstadt, Dr.-Ing.-Diss. v. 6. 9. 1907

1246. Baumann, Morand Leo: Úber $\mathrm{Hy}$ drazinderivate des Di-p-diamidodiphenylmethans und Di-p-diamidodi-o-tolylmethans. 1908. 47 S. $8^{0}$

Darmstadt, Dr.-Ing.-Diss. v. 4. 4. 1908

1247. Spitz, Carl: Chinolinderivate des 1,5 Naphtylendiamins. 1908. 29 S. $8^{0}$ I Vgl. Journal f. prakt. Chemie. Bd 187. N. F. Bd 79. 1909.

Darmstadt, Dr.-Ing.-Diss. v. 28. 7. 1908

1248. Jochheim, Hermann: Zur Kenntnis der Halogenindigotine und ihrer Sulfosäuren. 1908. $52 \mathrm{~S}$., 1 Taf. $8^{0}$ - Vgl. Berichte d. Deutschen Chem. Gesellschaft. Jg. 41.

Darmstadt, Dr.-Ing.-Diss. v. 10. 8. 1908

1249. Brodal, Peter: Versuche zur Darstellung von Paranitrobenzaldehyd. 1908. $36 \mathrm{~S}$. $8^{0}$

Darmstadt, Dr.-Ing.-Diss. v. 13. 10. 1908

1250. Ahrle, Hermann: Ueber die Bildung und Synthese der Caro'schen Säure. 〈Monosulfopersäure.) 1908. 78 S. $8^{0}$ - Vgl. Journal f. prakt. Chemie. Bd 187. N. F. Bd 79. 1909.

D armstadt, Dr.-Ing.-Diss. v. 28.12. 1908 
1251. Formhals, $\mathbf{R}[$ ichard]: Utber die Calciumsilicide und deren Aufnahmefähigkeit für Stickstoff. 1909. 45 S. $8^{0}$ - Ausz. in: Zeitschrift f. anorgan. Chemie. Bd 64.

Darmstadt, Dr.-Ing.-Diss. v. 1. 8. 1909

1252. Köhres, Hans: Ueber Derivate des m-Brom-o-Amidobenzamids und m-Bromo-Amidobenzhydrazids. 1909. 27 S. $8^{0}$

Darmstadt, Dr.-Ing.-Diss. v. 17. 8. 1909

1253. Siegler, Robert: Ternäre Systeme LLöslichkeit der Erdalkalinitrate in Aethylalkohol-Wassergemengen $\rangle$. 1909. 44 S., 1 Taf. $8^{0}$

Darmstadt, Dr.-Ing.-Diss. v. 6. 10. 1909

1254. Uhlig, 0tto: Versuche zur Darstellung aromatischer Nitrosoverbindungen durch elektrolytische Reduktion mit Wechselstrom. 1909. 44 S. $8^{0}$

Darmstadt, Dr.-Ing.-Diss. v. 15. 10. 1909

1255. Breitwieser, WilheIm: Ueber die Reduktion von Cyanchinolinen mit Natrium und Alkohol. 1909. 19 S. $8^{\circ}$ - Vorläufige Mitteilung in: Journal f. prakt. Chemie. Bd 187. N. F. Bd 79; , ,eine gekürzte Darstellung der Gesamtresultate soll im gleichen Journal erfolgen"; bis N. F. Bd 89, H. $1 / 2$ nicht ersch.

Darmstadt, Dr.-Ing.-Diss. v. 13. 12. 1909

1256. Zeh, Wilhelm: Ueber die Condensation von Imidoäthern mit Amidoestern. 1909. 47 S. $8^{0}$

Darmstadt, Dr.-Ing.-Diss. v. 30. 12. 1909

1257. Schreiner, 0tto: Untersuchungen über die Systeme ,Alkali-Schwefelsäure “ und "Alkali-Phosphorsäure". 1909. 66 S., 7 Taf. $8^{0}$ - Vgl. Zeitschrift f. phys. Chemie. Bd 75. 1911.

Darmstadt, Dr.-Ing.-Diss. v. 26. 1. 1910

1258. Spangenberg, Albert: Zur Erkenntnis des Tongiessens. [1910.] 49 S., 1 Taf. $4^{\theta}$

Darmstadt, Dr.-Ing.-Diss. v. 21. 7. 1910

1259. Wehrheim, 0tto: Über die Oxydation von Ammoniak, Blausäure und Cyan. 1910. 47 S., 1 Taf. $8^{0}$

Darmstadt, Dr.-Ing.-Diss. v. 13.10.1910

1260. Schulz, Walter: Zur Kenntnis der Cellulosearten. 1910. 100 S. $8^{0}$ T Mit Vorw. von G. Schwalbe bei Borntraeger in Berlin 1911; vorläufige Mitteilung in: Berichte d. Deutschen Chem. Gesellschaft. Bd 43.

Darmstadt, Dr.-Ing.-Diss. v. 20. 1. 1911

1261. Schalk, Willem van der: Über Anthrachinon- $\alpha$-carbonsäure. 1911. 57 S. $8^{0}$ If Vorläufige Mitteilung in: Berichte d. Deutschen Chem. Gesellschaft. Bd 44; gekürzt in: J. Liebig's Annalen d. Chemie. Bd 388. 1912.

Darmstadt, Dr.-Ing.-Diss. v. 28. 3. 1911
1262. Andersen, Jörgen Hersleb: Darstellung von Calcium und Calciumlegierungen. [1911.] 35 S. $8^{0}$ Gekürzt in: Zeitschrift f. Elektrochemie. Bd 19. 1913.

Darmstadt, Dr.-Ing.-Diss. v. 3. 8. 1911

1263. Apfel, 0tto: Untersuchungen über die Löslichkeit von Alkaliphosphaten. 1911. 30 S. $8^{0}$

Darmstadt, Dr.-Ing.-Diss. v. 15. 9. 1911

1264. Günzler, Heinrich: Über die Einwirkung von Aminen auf Cyanimidokohlensäureäther und Oxaldiimidoäther. 1911. 43 S. $8^{0}$ - Soll gekürzt ersch. in: Journal f. prakt. Chemie; bis N. F. Bd 89, H. $1 / 2$ nicht ersch.

Darmstadt, Dr.-Ing.-Diss. v. 10.10. 1911

1265. Alefeld, Friedrich: Versuche zur Darstellung von Oxalsäure durch elektrolytische Oxydation von Kohlehydraten. 1911. 37 S. $8^{0}$

Darmstadt, Dr.-Ing.-Diss. v. 11. 10. 1911

1266. Friederich, Walter: Einige Reaktionen und Derivate des Hydroperoxyds. 1911. 46 S. $8^{0}$. In veränderter Form in: Zeitschrift f. anorgan. Chemie. Bd 73. 1912.

Darmstadt, Dr.-Ing.-Diss. v. 22. 11. 1911

1267. Fritsche, Oskar: Über Halogenäthylene und deren Polymerisationsprodukte. 1911. 41 S. $8^{0}$

Darmstadt, Dr.-Ing.-Diss. v. 25. 3. 1912

1268. Robinoff, Michael: Über die Einwirkung ven Wasser und Natronlauge auf Baumwollcellulose. [1912.] 94 S. $8^{0}$ I Auch bei Borntraeger in Berlin; vorläufige Mitteilung in: Zeitschrift f. angewandte Chemie. Jg. 24, 2. 1911 ; „eine gekürzte Darstellung der Gesamtresultate soll in der Ztschr. f. angew. Chem. u. in der Färber-Ztg. erfolgen"; bis Jg. 26 bzw. Jg. 23 nicht ersch.

Darmstadt, Dr.-Ing.-Diss. v. 1. 7. 1912

1269. Bretsch, Eugen: Zur Kenntnis der Imidoäther aus Arylendicyaniden und pCyanbenzoesäureäthylester. 1912. 51 S. $8^{0}$ ๆ ,Eine gekürzte Darstellung soll demnächst im Journal f. prakt. Chemie ersch."; bis N. F. Bd 89 , H. 1/2. nicht ersch.

Darmstadt, Dr.-Ing.-Diss. v. 15. 7. 1912

1270. Roll-Hansen, Cay: Über die Zersetzung von Calcium-Wasserstoffverbindungen. 1912. 28 S. $8^{0}$ I Vgl. Zeitschrift f. anorgan. Chemie. Bd 82. 1913.

Darmstadt, Dr.-Ing.-Diss. v. 18. 12. 1912

1270a. Christiansen, Christian: Utber Natronzellstoff, seine Herstellung und chemischen Eigenschaften. 1912. VIII, 154 S. $8^{0}$ T Mit Vorw. von C. G. Schwalbe als: Schriften d. Vereins d. Zellstoff- u. Papier-Chemiker. H. 6. 1913. Darmstadt, Dr.-Ing.-Diss. v. 23. 10. 1913 


\section{DRESDEN}

Gegründet 1828 als ,Technische Bildungsanstalt", 1851 „Königliche polytechnische Schule“" 1871 „Königlich Sächsisches Polytechnikum", seit 1890 ,Königlich Sächsische Technische Hochschule"

\section{SATZUNGEN ORDNUNGEN BESTIMMUNGEN}

\section{ALLGEMEINES UND VERMISCHTES}

1271. Plan für eine technische Bildungsanstalt in Dresden (v. 1. 2. 1828). $D r^{*}$

1272. Gesetze für die Schüler der technischen Bildungsanstalt zu Dresden (v. 25. 5. 1830).

1273. Organisationsplan für die technische Bildungsanstalt zu Dresden (v. 16.3. 1835). 1835 .

$D r^{*}$

1274. Gesetze für Schüler der technischen Bildungsanstalt zu Dresden (v. 27. 4. 1835). 1835.

1275. Organisationsplan für die technische Bildungsanstalt zu Dresden (v. 26. 2. 1838). [Folgt:] Unterrichtsbeiträge. ๆ Abgedr. in: Programm 1838. 1839 [Nr 1348].

1276. Lehrplan der technischen Bildungsanstalt und der Baugewerkenschule zu Dresden. 1844.

$D r^{*}$

1277. Bekanntmaehung über die Organisation des Unterrichts der $\mathrm{Kgl}$. technischen Bildungsanstalt zu Dresden 1846. [Nebst] Ergänzung zu §3,4,15 v. 28. 2. 1851 . $D r ;$ Erg.: $D r^{*}$

1278. Gesetze für die Schüler der technischenBildungsanstalt zu Dresden [v.1.9.1846].

1279. Den Besuch der Kgl. Sammlungen, öffentlichen Bauten und technischen Etablissements betr., welcher den Schülern der technischen Bildungs-Anstalt zu Dresden zu ihrer Belehrung und Ausbildung gestattet ist. 1849. [Nebst] Neuere Bestimmungen v. 9.5 .1851 .

$D r^{*}$

1280. Kgl. polytechnische Sehule in Dresden. Aufnahmeerfordernisse. [Folgt:] Unterrichtsplan. (Sept.1852.) [Autogr.] $K$

1281. Regulativ für die Schüler der Kgl. polytechnischenSchule zu Dresden. [1853 ?] K

1282. Specialregulativ für die geodätischen Arbeiten. (Unterz.: Dresden, im Juli 1853. Die Direction der Kgl. polytechnischen Schule.)

K

1283. Specialregulativ für die practischchemischen Arbeiten. (Unterz.: Dresden, im Mai 1854. Die Direction der kgl. polytechnischen Schule.)
1284. Organisationsplan der Kgl. polytechnischen Schule zu Dresden genehmigt 14. 3. 1855. [Folgt:] Beil. A. Verordnung die Einrichtung von Maturitätsprüfungen bei $d$. polytechn. Schule zu Dresden betr., v. 17. 1. 1852. B. Lehrziele, welche in Gemässheit des Lehrplanes d. polytechn. Schule bei d. Maturitätsprüfungen vorausgesetzt werden. C. Gesetze für die Schüler d. polytechn. Schule. D. Stiftungsurk unde des Reichsstipendienfonds für Schüler der polytechn. Schule, genehmigt 12.5. u. 8.6. 1853. E. Lehrplan nach d. Ministerialverordnung v. 7. 2. 1855. [Nebst] Veränderungen (ad $\S 4,20,21,23$ v. Dec. 1858).

Dr; Veränd.: Dr*

1285. Regulativ für die Kgl. polytechnische Schule zu Dresden, die Verhältnisse der Angestellten, der allgemeinen Verwaltungsformen und die näheren Bestimmungen über Ausführung des Organisationsplanes vom 14. 3. 1855 enthaltend. 〈Genehmigt 14. 3. u. 19. 6. 1855. $\rangle \quad D r^{*}$

1286. Prospect der Kgl. polytechnischen Schule zu Dresden (v. 30. 6. 1855). - - [Veränderte Fassungen] (v. Dec. 1858; 18. 2. 1862; Oct. 1862).

1287. Prospeet der Abtheilung für Thonmodelliren und Musterzeichnen in der $\mathrm{kgl}$. polytechnischen Schule zu Dresden (v. 30.6. 1855).

$D r^{*}$

1288. K. polytechnische Schule zu Dresden. Specialregulativ für die Uebungen im Feldmessen des allgemeinen Cursus (v. April 1862).

$D r^{*}$

1289. Prospeet der Kgl. polytechnischen Schule zu Dresden (v. Oct. 1862).

1290. Organisationsplan der Kgl. Sächsischen polytechnischen Schule zu Dresden, genehmigt 31. 1. 1865. [Folgt:] Beil. 1. Einrichtung der Abtheilung für Modelliren, Ornament- u. Musterzeichnen. 2. Lehrplan. 3. Auszug aus d. Verordnung v. 24. 12. 1851, die Staatsprüfung der Techniker betr. 4. Disciplinarvorschriften. 5. Den Reisestipendienfond A. betr. 6. Den Fond B. zur Gewährung v. Mittags- 
tischen betr. 7. Gerstkamp-Stiftung. 8. Constantin Novicow-Stiftung. 9. Verordnung v. 2.2. 1865, die Schlussprüfungen betr. [Nebst] Nachtrag (v. 30.9. 1870).

Nachtr.: $D r^{*}$

1291. Einrichtung der mit der Kgl. polytechnischen Schule verbundenen Abtheilung für Modelliren, Ornament- und Musterzeichnen, nach dem Organisationsplane vom 31. 1. 1865.

Dr ÖffB

1292. Studienordnung, Disciplinargesetze und Hausordnung der Kgl. polytechnischen Schule zu Dresden. Vom 13. 2. 1871. Be

1293. Bestimmungen über die Zulassung von Privatdocenten an der Kgl. polytechnischen Schule zu Dresden (v. 28. 3. 1872).

$D r^{*}$

1294. Prospect der kgl. Schule für Modelliren, Ornamenten- und Musterzeichnen zu Dresden (v. 13. 5. 1872).

Dr ÖfB

1295. Statut für das Kgl. Sächsische Polytechnikum zu Dresden (v. 3.4. 1878). T Auch in: Gesetz- u. Verordnungsblatt f. d. Kgr. Sachsen v. J. 1878 . S. $28 \mathrm{ff}$.

1296. Habilitations-0rdnung am Kgl. Polytechnikum Dresden (v. 24. 7. 1878). 1878. - [Dasselbe u. d. T.:] Habilitations Ordnung der K. S. Technischen Hochschule. [Autogr.]

1. Ausg. Dr*

1297. Studienordnung für die Studirenden auf dem $\mathrm{Kgl}$. Polytechnikum Dresden (v. 24. 7. 1878).

1298. Bibliotheksordnung am Kgl. Polytechnikum Dresden (v. 26. 7. 1878). Be

1299. Statuten für die Krankenkasse der Studirenden am Kgl. Polytechnikum Dresden. 1880.

$D r^{*}$

1300. Studienpläne für das Kgl. Polytechnikum Dresden (v. Juli 1882). 1882. Dr*

1301. Bibliotheksordnung am Kgl. Polytechnikum Dresden (v. 23. 1. 1884). 1884. Dr

1302. Statuten für die Krankenkasse der Studirenden am Kgl. Polytechnikum Dresden. 1885. - [Dasselbe mit Änderungen.] 1891. 1895.

$M$

1303. Studienpläne der Kgl. Sächs. Technischen Hochschule. 1890. - [Dasselbe.] [1892.] 22 S. - [Dasselbe mit Änderungen. 1892. 1894. 1896. 1897. - [Dasselbe mit Änderungen. 1899.] 18 S. 1890. 1894: $M$; [1892]: Königsberg UB; 1896. 1897. 1899: Dr*

1304. Statut der $\mathrm{Kgl}$. Sächsischen Technischen Hochschule (v. 3. 2. 1890. Nebst Deckbl. zu $\S \cdot 28$ u. 36 v. 22. 12.1897 u. zu
\$ 31 v. 25. 3. 1898). Auch in: Gesetz- u. Verordnungsblatt f. d. Kgr. Sachsen v. J. 1890. S. 6 ff. 1305. Studienordnung für die Studierenden der Kgl. Sächs. Technischen Hochschule (v. 14. 8. 1890). Ausg. vom Jahre 1890. If Anlage s. $\mathrm{Nr} 1313$.

$D r^{*}$

1306. Verzeichnis der für Studierende bestehenden Stiftungen an der Kgl. Sächsischen Technischen Hochschule zu Dresden. Zsgest. von Dr. Wilhelm Scheffler, ao. Prof. u. ständiger [!] Sekretär a. d. Techn. Hochschule. 1891.

$D r *$

1307. Statuten für die Krankenkasse der Studierenden an der Kgl. Sächs. Technischen Hochschule (v. 24. 2. 1891). 1891.

1308. Habilitationsordnung für die $\mathrm{Kgl}$. Sächs. Technische Hochschule (v. 5. 3. 1895). 1895. [Mehrfach gedr.]

1309. Satzungen für den Verband der Studentenschaft an der $\mathrm{Kgl}$. Technischen Hochschule zu Dresden. Genehmigt 21. 2. 1896. [Folgt:] Geschäftsordnung. Neu aufgelegt S.-S. 1900.

1900: $M$

1310. Allgemeine Grundsätze für die Stipendienerteilung an der Technischen Hochschule zu Dresden. (Als Ms. gedr.) (Diese Grundsätze sollen mit dem 1. 4. 1896 in Kraft treten.)

1311. Bibliotheksordnung der K. S. Technischen Hochschule Dresden (v, 6. 3. 1897). 1897.

1312. Bestimmungen für die Studierenden der Kgl. Sächs. Technischen Hochschule 〈Studienordnung〉 (v. 23. 5. 1898).

1313. K. S. Technische Hochschule zu Dresden. Anlage zu der Studienordnung [Nr 1305]. Bestimmungen über den Honorarerlass bei wiederholtem Hören von Vorlesungen (v. 7.2. 1899). A Aufgenommen in $\S 22$ der Studienordnung v. 30. 9. 1910 [Nr 1326].

1314. [Beg.: Z Zwischen der Technischen Hochschule zu Dresden und der Allgemeinen Renten- Kapital- und Lebensversicherungsbank ,Teutonia" in Leipzig ist nachstehender Kollektiv-Unfall-Versicherungsvertrag abgeschlossen worden. (Dat. 15.9. 1899; genehmigt 20.9.1899.) (1900.) Dr*

1315. Satzungen der Hülfspensions-Kasse bei der Kgl. Technischen Hochschule zu Dresden (v. 11. 5. 1900). 1900.

1316. Promotionsordnung für die Erteilung der Würde eines Doktor-Ingenieurs an der Kgl. Sächs. Technischen Hochschule zu Dresden (v. 29. 5. 1900). I Abgedr. in: Bestimmungen. S. 195. 
1317. Statuten für die Krankenkasse der Studierenden an der Kgl. Sächs. Technischen Hochschule (v. 13. 4. 1901). 1901. [Dasselbe u. d. T.:] Statuten ... der Studentenschaft ... (v. 13. 4. 1901 u. 29. 7 . 1902).

1901: $D r^{*}$

1318. Statut der Kgl. Sächs. Technischen Hochschule (v. 12. 2. 1902; § 30a v. 10. 9. 1903). 1905. (Nebst Deckbl. § 37a.) - Auch in : Gesetz- u. Verordnungsblatt f. d. Kgr. Sachsen v. J. 1902. 1903; daraus auch als S.-A.; Bekanntmachung wegen Ånderung des § 13 v. 22. 6.1908 u. $\S 28$ v. 31. 7. 1908: ebd. 1908.

1319. Bestimmungen für die Studierenden der Kgl. Sächs. Technischen Hochschule 〈Studienordnung〉 (v. 8. 3. 1902). [Dasselbe.] (Mit Nachtrag zu § 22 v. 13. 12. 1902 u. $\S 17,20,24$ v. 10. 9. 1903.) Dr*

1320. Allgemeine Grundsätze für die Stipendienerteilung an der Technischen Hochschule zu Dresden. 〈Als Ms. gedr.) (Diese Grundsätze treten mit dem 1. 10. 1904 in $\mathrm{Kraft}$.)

1321. Satzungen für den Verband der Studentenschaft an der Kgl. Technischen Hochschule zu Dresden. Vom 2. 1. 1905. [Folgt:] Geschäftsordnung.

1322. Satzungen der Friedrich SiemensStiftung an der $\mathrm{Kgl}$. Sächs. Technischen Hochschule. 1905.

1323. Promotions-0rdnung für die Erteilung der Würde eines Doktor-Ingenieurs an der Kgl. Sächs. Technischen Hochschule zu Dresden (v. 7. 7. 1905). [Folgt:] Bekanntmachung, die Erwerbung der Titel ,,Doktor-Ingenieur" und,,Diplom-Ingenieur" betr.; vom 12. 1. 1900. [Nebst] Nachtrag 1 v. 14. 10. 1912. [Folgt:] Bekanntmachung über die Erwerbung der Würde eines Doktors der technischen Wissenschaften; v. 7.9. 1912.

1324. Bibliotheksordnung der K. S. Technischen Hochschule Dresden. Vom 25. 2. 1910. 1910.

1325. Kgl. Sächs. Technische Hochschule. Kollektiv-Unfall-Versicherung der Kgl. Sächsischen Technischen Hochschule bei der Versicherungsaktiengesellschaft Teutonia in Leipzig vom 28.9. 1910.

1326. Studienordnung der Kgl. Säehs. Technischen Hochschule. Vom 30. 9. 1910. [Folgt:] Gebührenordnung. 1910.

1327. Statuten für die Krankenkasse der Studentenschaft an der Kgl. Sächs. Technischen Hochschule (v. 3. 8. bez. 11. 9. 1911). 1912.

\section{PRÚFUNGSORDNUNGEN}

1334. Verordnung, die Einrichtung von Maturitätsprüfungen bei der polytechnischen Schule in Dresden betr., vom17.1.1852. I Aus: Gesetz- u. Verordnungsblatt f. d. Kgr. Sachsen v. J. 1852 . S. 10 ff.

1335. Regulativ für die Absolutorialprüfungen an der Kgl. polytechnischen Schule zu Dresden (v. 15. 5. 1871). [Nebst] Nachtrag. Die Hochbau-Abtheilung betr. (v. 6. 5. 1876 u. 20. 4. 1877; dat. 9. 5. 1878).

Nachtr.: $D r^{*}$

1336. Prüfungsordnung für Candidaten des höhern Lehramtes der technischen und der mathematisch-physikalischen Richtung am Kgl. Polytechnikum Dresden (v. 14. 11 . 1879). 1879. - [Anderer Druck. 1895.] - Auch in: Gesetz- u. Verordnungsblatt f. d. Kgr. Sachsen v. J. 1879. S. $407 \mathrm{ff}$.

1337. Provisorische Geschäftsordnung der Commission für die Prüfungen der Candidaten des höheren Lehramtes am Kgl. Polytechnikum Dresden (v. 10. 7. 1880). [Nebst] Beil. 1880.

Beil.: $S$

1338. Regulativ für die Diplomprüfungen am Kgl. Polytechnikum Dresden [v. 2. 4 . 1883]. 1883. - [Dasselbe] (v. 2.4. 1883). 1885. 1883: $\mathrm{Dr}$ ÖfB; 1885: $\mathrm{Dr}$ *

1339. Regulativ für die Diplomprüfung der Chemischen Abtheilung an der Kgl. Sächs. Technischen Hochschule (v. 29. 1. 1890). 1890.

1340. Regulativ für die Diplomprüfungen an der Kgl. Sächs. Technischen Hochschule. 1892.

1341. Regulativ für die Diplomprüfungen an der Kgl. Sächs. Technischen Hochschule. Allgemeine Bestimmungen. 1894. [Nebst] Besondere Bestimmungen über die Prüfung für das Fach eines Architekten; . . . eines Bau-Ingenieurs; ... eines Vermessungs-Ingenieurs; ... eines Maschinen-Ingenieurs; ... eines Elektro-Ingenieurs; ... eines Fabrik-Ingenieurs; ... eines Chemikers. $\langle$ Neudr. 1898.〉 - 〈Neudr. 1901.〉 [Veränderte Fassung.]

Bes. Best.: M; 1898: M*; 1901: Be ArchVer

1342. Prüfungs-0rdnung für Kandidaten des höheren Lehramtes der mathematischphysikalischen und chemischen Richtung an der Kgl. Technischen Hochschule zu Dresden (v. 20. 10. 1899). T Auch in: Gesetz- u. Verordnungsblatt f. d. Kgr. Sachsen v. J. 1899. S. $452 \mathrm{ff}$. 
1343. Prüfangsordnung für Diplom-Ingenieure an der $\mathrm{Kgl}$. Sächs. Technischen Hochschule zu Dresden (v. 12. 1. 1900). M

1344. Prülungsordnung für Diplom-Ingenieure an der Kgl. Sächs. Technischen Hochschule zu Dresden (v. 2.8. 1901). - Neudr. 1908. - Abgedr. in: Bestimmungen. S. $179 \mathrm{ff}$.

1901: $S$

1345. Prüfungsordnung für Diplom-Ingenieure an der $\mathrm{Kgl}$. Sächs. Technischen Hochschule zu Dresden (v. 17. 2. 1904). [Folgt:] Bekanntmachung, die Erwerbung der Titel ,Doktor-Ingenieur" und „,Diplom-Ingenieur" betr.; v. 12. 1. 1900. [Nebst] Deckbl. Nr. 1 v. Aug. 1907.

Deckbl.: $K$
1346. Prüfungsordnung für Diplom-Ingenieure an der $\mathrm{Kgl}$. Sächs. Technischen Hochschule zu Dresden (v. 23. 4. 1908 u. 19. 4. 1909). 1909. [Folgt:] Bekanntmachung, die Erwerbung der Titel, Doktor-Ingenieur ${ }^{\circ 6}$ und ,Diplom-Ingenieur ${ }^{\text {" }}$ betr.; v. 12. 1. 1900.

1347. Prüfungsordnung für Kandidaten des höheren Schulamtes der mathematischphysikalischen und chemischen Richtung an der Kgl. Technischen Hochschule zu Dresde $n$ (v. 25. 1. 1909). [Nebst] Bekanntmachung über Änderungen der Prüfungsordnung... v. 25. 9. 1911. Aus: Gesetzu. Verordnungsblatt f. d. Kgr. Sachsen v. J. 1909. S. 74 ff.; 1911. S. 187 f.

\section{PERIODISCHE SCHRIFTEN}

1348. Programm zu den am ... erfolgenden Prüfungen der Schüler der technischen Bildungsanstalt (1852 ff.: Kgl. polytechnischen Schule; $1838 \mathrm{ff}$ : : und der Baugewerkschule) zu Dresden. Inh.: 1.) ... [W [iss. Abhandlung.] 2.) Nachricht über die technische Bildungsanstalt (1836. 1837:, deren Organisation, Sammlungen, Lehrer und Schüler; $1838 \mathrm{ff}$.: und über die Baugewerkschule; 1836-1839: nebst Verzeichniß der Jahresprüfungen von Wilhelm Gotthelf Lohrmann, Ober-Insp., Vorsteher d. Anstalt; 1838. 1839: beider Anstalten). [1840 ff.: Titel mehrfach geändert.] 1836-1862. - Forts. der Nachricht s. Jahresbericht [Nr 1349].

Darin:

1836. Schubert, Johann Andreas: Andeutungen über Dampfschiffahrt auf der obern Elbe.

1837. Jähkel, Leopold Ferdinand: Ueber Blitzableiter, nebst Vorschlägen zur wohlfeilern Herstellung derselben.

1838. Jähkel, [Leopold] Ferdinand: Ueber die Behandlung der rohen Seide beim Entschälen und Bleichen.

1839. Schubert, Johann Andreas: Ueber die Anwendbarkeit des Flügel- oder CentrifugalGebläses.

1840. Geinitz, Hanns Bruno: Ueber Braunkohlen Sachsens.

1841. Jähkel, L [eopold] F [erdinand]: Ueber die Fabrikation der Stearinkerzen.

1842. Schubert, J[ohann] A [ndreas]: Versuch einer neuen Begründung der Grundlehren der Mechanik.

1843. Seebeck, A[ugust]: Ueber Zurückwerfung und Beugung des Schalles.

1844. Jähkel, L [eopold] F[erdinan d]: Praktische Winke, die Fabrikation des Argentanblechs betreffend.
1845. Schubert, J[ohann] A[ndreas]: Uéber freie und vorgeschriebene Stützlinien und über die Fortpflanzung des Druckes durch feste Körper, als Einleitung zu einer Theorie der Wölbbögen.

1846. Seebeck, August: Ueber Schwingungen, mit besonderer Anwendung auf die Untersuchung der Elasticität fester Körper.

1847. Heine, G[ustav]: Mittheilungen über den Bau und die Einrichtung des neuen Gebäudes der technischen Bildungsanstalt in Dresden. Nachricht über die Feier zur Eröffnung des neuen Anstaltsgebäudes mit der von Herrn Geh. Regierungsrath [Jacob Heinrich] Thieriot und der vom Director (A[ugust] Seebeck) bei dieser Gelegenheit gehaltenen Rede.

1848. Jähkel, [Leopold] F[erdinand]: 1. Statistische Notizen, die Erzeugung des lohgaren Leders in Sachsen betreffend. 2. Fabrikation des Reitzeugleders in England.

1849. Schubert, J[ohann] A[ndreas]: Theorie der Druckturbinen.

1850. Lösche, [Eduard]: Einige Bemerkungen über den Leitungswiderstand hydroelektrischer Ketten.

1851. Schlömilch, (Oscar): Die Reihenentwickelungen der Differenzial- und Integralrechnung. [Auch bei Schönfeld in Dresden.]

1852. Fort, O(smar): Die Theorie der Tangenten, nebst einem Anhange, einige Summenformeln enthaltend.

1853. Stein, W(ilhelm): Ueber ein neues Farbematerial aus China.

1854. Schubert, J[ohann] A[ndreas]: Ueber die Stützung und Construction der geraden, insbesondere der schiefen Wölbbogen.

1855. Schlömilch, 0skar: Theorie der Kettenbrückenlinien.

1856. Hülsse, Julius (Ambrosius): Ueber Kranken- und Versorgungscassen für die weniger bemittelten Bevölkerungsclassen. 
1857. Schneider, J(ohann) B(ernhard): Ueber die von aussen beaufschlagten horizontalen Turbinen.

1858. Geinitz, Hanns Bruno: Die Leitpflanzen des Rothliegenden und des Zechsteingebirges oder der permischen Formation in Sachsen.

1859. Hülsse, (Julius Ambrosius): Ueber die Einrichtung und Berechnung von Knappschaftsund ähnlichen Unterstützungs-Cassen, welche Sicherheit und Nachhaltigkeit gewähren sollen.

1860. Schneider, (Johann Bernhard): Mittheilungen über die Untersuchungen der Leistungen des Kropfrades in der Schneidemühle des Mühlenbesitzers Herrn Hohlfeld zu Schandau, sowie über den Widerstand beim Schneiden des Holzes.

1861. Nagel, (A ugust): Die Messung der Basis für die Triangulirung des Erzgebirgischen Kohlenbassins.

1862. Stein, W(ilhelm): Ueber das Pflanzengelb〈Phytomelin, Rutinsäure〉 und einige ihm verwandte Körper.

Dr

1349. Jahresbericht über den ... Cursus der Kgl. polytechnischen Schule (35-41: und über den ... Cursus der Kgl. Baugewerkenschule) zu Dresden. 35. [bzw.] 26. 1862-1863 - 42. 1869-1870. A Anfang in: Programm [Nr 1348]. 35. $39-42: D r$

1350. Lections-Verzeichniss der Kgl. Sächsischen Polytechnischen Schule zu Dresden [43.] für das W.-S. 1870-1871. S.-S. 1871. Fortges. u. d. T.:

Programm der Kgl. Polytechnischen Schule zu Dresden für den ... Cursus. [Nebst] Ergänzungen betr. das S.-S. 44. 1871-1872; 45. 1872-1873.

Fortges. u. d. T.:

Programm der Kgl. Sächsischen Polytechnischen Schule zu Dresden für das 46. Lehrj., bzw. für das W.-S. 1873-1874; S.-S. 1874; 47. Lehrj., bzw. für das W.-S. 1874-1875; S.-S. 1875.

Fortges. u. d. T.:

Kgl. Sächsisches Polytechnikum (18751876-1884-1885: zu) Dresden. Programm für das Studienj., bzw. W.-S. : 1875-1876-18881989. [Nebst] Ergänzung enth. das Verzeichniss der Vorlesungen für das S.-S. $1876-1889$.

Fortges. u. d. T.:

Kgl. Sächsisches Polytechnikum Dresden. Ergänzungs-Programm giltig für das W.-S. 1889/1890.

Fortges. u. d. T.:

Verzeichniss der Vorlesungen und Uebungen an der Kgl. Sächs. Technischen Hochschule (1890/91: und Stundenpläne der fachwissenschaftlichen Abteilungen; 1891/92: samt den Stundenplänen der Abteilungen) für das S.-S. 1890. W.-S. 1890/91 [usw.] S.-S. 1899.

Fortges. u. d. T.:

Kgl. Sächs. Technische Hochschule zu Dresden. Verzeiehnis der Vorlesungen und Uebungen samt den Stunden- und Studienplänen. W.-S. 1899/1900. S.-S. 1900 [usw.] -W.-S. 1912/13. 1870-71-1890. 1891. 1891/92. 1892/93-1903/04:Dr; 1871. Nachtr.: Dr*

1351. Berieht über das Kgl. Polytechnikum Dresden 〈Kgl. Sächsische Technische Hochschule> auf das Studienj. 1888/1889 und das W.-S. 1889/1890. 1890. Fortges. u. d. T.:

Berieht über die Kgl. Sächs. Technische Hochschule (1893/94 ff.: zu Dresden) auf (1893/94ff.:für)dasJahr(1891/92. 1892/93ff.: Studienj.) 1890/91-1911/12. 1890-[1912].

Darin: Rede zur Feier d. Geburtstages (1897/98: u. d. Regierungsjubiläums) d. Königs:

1888/89. Fischer, Hugo: Die Entwickelung der Dampfschiffahrt auf der sächsischen Elbe. [Erw. in: Der Civilingenieur. Jg. 1890. N. F. Bd 36.]

18:0/91. Hempel, Walther: Utber den Einfluss der chemischen Technik auf Leben und Sitte. [Auch in: Hempel: Ansgewählte Vorträge u. Aufsätze. Berlin 1913.]

1892/93. Heyn, R(udolph): Die Fortschritte der Neuzeit auf dem Gebiete des Hochbaues.

1893/94. Krause, (Martin): Die Entwickelung der höheren Analysis.

1894/95. Renk, Friedrich: Utber den hygienischen Unterricht auf den technischen Hochschulen. [Auch in: Arbeiten aus d. Kgl. hygien. Institute zu Dresden. Bd 1. 1903.]

1895/96. Stern, Adolf: Die Anfänge der modernen deutschen Litteratur.

1897/98. Meyer, Ernst von: Justus von Liebig als Reformator der Chemie.

1898/99. Mollier, Richard: Ueber die technische Entwickelung der Kriegsflotten seit der Verwendung von Dampfschiffen.

1899/1900. Rohn, Karl: Die Entwickelung der Raumanschauung im Unterricht.

1900/01. Mehrtens, [Georg]: Bilder aus der Geschichte der Technik. [Gekürzt in: Frankfurter Zeitung. Nr 356 v. 24. 12. 1911; zum Teil in erw. Form mit 5 Abb. in: Jung-Deutschland 1913. Kalender d. Jung-Deutschland-Bundes. S. $237 \mathrm{ff}$.]

1901/02. Hempel, Walther: Ueber die Erziehung der jungen Männer. [Auch in: Hempel: Ausgewählte Vorträge u. Aufsätze. Berlin 1913.] 1906/07. Drude, Oscar: Die Rohstoffproduktion der Pflanzen und die Fortschritte der chemischen Synthese in der Weltwirtschaft.

1908/09. Möhlau, Richard: Die Entwicklung und nationalökonomische Bedeutung der deutschen chemischen Industrie im XX. Jahrhundert. kunst.
1909/10. Hartung, Hugo: Einheitliche Bau- 
1910/11. Helm, (Georg): Die Stellung der Theorie in Naturwissensehaft und Technik.

1911/12. Lucas, Georg: Die weitere Entwickelung unserer Eisenbahnen.

Darin ferner:

1898/99. Gess, [Felician]: Rede bei der Gedenkfeier für den Fürsten von Bismarck am 31 . Oktober 1898.

1904/05. Stern, Adolf: Rede zur Gedächtnisfeier am hundertsten Todestage Friedrich Schillers. 1906/07. Schumacher, Fritz: Nachruf für Professor Carl Weichardt.

Darin ferner: Rede (1909/10 ff.: Ansprache) beim Antritt d. Rektorats:

1902/03. Lewicki, Leonidas: ttber die Entwicklung des Studienganges an den Technischen Hochschulen und an der Dresdener Technischen Hochschule insbesondere.

1908/09. Hartung, Hugo: Die wissenschaftlichen Grundlagen des technischen Hochschulunterrichtes.

1909/10. Helm, (Georg): Die Entwickelung der deutschen technischen Hochschulen.

1910/11. Lucas, (Georg): Umgestaltung der Ausbildung an den Technischen Hochschulen.

1911/12. Meyer, (Ernst) von: Stellung der Chemie als Lehrgegenstand an der Universität und an der Technischen Hochschule.

1891/92-1896/97. 1898/99. 1899/1900: Dr
1352. Kgl. polytechnische Schule zu Dresden. Haupt-Liste [der Studirenden, $\mathrm{Zu}$ hörer und Hospitanten], angefertigt am 18. 10. 1872 für das Professoren-Collegium. Dr ÖffB

1353. Verzeichniss der Studirenden, $\mathrm{Zu}$ hörer und Hospitanten (1877/78: Studirenden und Zuhörer; $1878 \mathrm{ff}$.: Studirenden) des Kgl. Polytechnikums (1873/74-1884/85: zu) Dresden. 1873-1874 [nebst] Ergänzungen. Im Studienj. 1874-1875; im Studienj. 1875-76. S.-S. 1876. W.-S. 1876/77 [usw.]W.-S. 1889/90. $1874-90$.

1873/74-1877. 1884. 1884/85. 1886-1889/90: Dr OffB; 1877/78-1883/84. 1885. 1885/86: Dr*

1354. Personal-Verzeichnis der Kgl. Sächsischen (1898 ff.: Sächs.) Technischen Hochschule für das S.-S. 1890. Nr. 1. W.-S. 1890/91. Nr. 2 [usw.]-W.-S. 1912/13. Nr. 46. $1890-1912$.

1-28: $D r^{*}$

1355. Kgl. Sächs. Technische Hochschule zu Dresden. Stundenpläne für das S.-S. 1893. W.-S. 1893/94 [usw.]-S.-S. 1899. - 1890/91. 1891/92. 1899/1900 ff. in: Verzeichnis der Vorlesungen [Nr 1350].

\section{GELEGENHEITS- UND VERMISCHTE] SCHRIFTEN?}

1356. Katalog der Bibliothek der technischen Bildungsanstalt zu Dresden. 1843. XIII, 154 S. [Nebst] Nachtrag bis Ostern 1845. S. $155-179$ [u.] Nachtrag von Ostern 1843 bis Ostern 1851. (1851.) S. 155-296. $8^{0}$

1357. Die polytechnische Schule als Grundlage aller technischen Fachschulen Sachsens von T[raugott] Franke und J[0hann] A[ndreas] Schubert, Prof. an d. techn. Bildungsanstalt zu Dresden. 1849. $30 \mathrm{~S} .8^{0}$

1358. Gedächtnissrede auf Leopold von Buch. Geh. am 23. April 1853 in d. Aula der Polytechn. Schule zu Dresden von Dr. H[anns] B[runo] Geinitz, Prof. an d. Polytechn. Schule zu Dresden. Dresden: Arnoldi 1853. VI, 32 S. $8^{0}$

1359. Die Königliche polytechnische Schule 〈technische Bildungsanstalt〉 zu Dresden während der ersten 25 Jahre ihres Wirkens geschildert von Prof. Dr. (Julius Ambrosius) Hülsse. Zum Besten des neu begründeten Reisestipendienfonds gedr. Dresden: Schönfeld in Comm. 1853. 54 S. $4^{0} \mathrm{Dr}$
1360. Katalog der Bibliothek der Königlichen polytechnischen Schule und der $\mathbf{K o ̈ -}$ niglichen Baugewerkenschule zu Dresden. 1864. Getr. Pag. $8^{0}$

[Sondert. 1-11.] Katalog der Bibliothek der Königlichen polytechnischen Schule zu Dresden. Abth. 1-11.

1. Zeitschriften. Gesellschaftsschriften. 1863.

2. Encyclopaedieen. Sammelwerke. Ausstellungsberichte. Ausstellungskataloge. 1863.

3. Mathematik u. Astronomie einschl. d. angewandten Arithmetik u. Geometrie, nehmlich d. Zahlenrechnens, Versicherungswesens, Maasses u. Gewichtes, d. prakt. Geometrie, descriptiven Geometrie, Perspective. 1863.

4. Physik. Meteorologie. Physische Geographie. Chemie. 1863.

5. Zoologie. Physiologie. Botanik. Mineralogie. Geognosie. Geologie. 1864.

6. Land- u. Forstwirthschaft. Bergbau u. Hüttenwesen. 1864.

7. Technologie. 1864.

8. Mechanik. Maschinenlehre. Ingenieurwissenschaften. Allg. Feuerungskunde. 1864.

9. Baukunst. Kunstgeschichte. Ornamentik. 1864.

10. Länder- u. Völkerkunde. Handelswissenschaften. Staatswissenschaften. Statistik. 1864. 
11. Sprachen. Pädagogik. Unterrichtsanstalten. Biographien. Bibliographie. Musik. Schulprogramme. 1864.

[Sondert. 12.] Katalog der Bibliothek der Königlichen Baugewerkenschule zu Dresden. $D r$ OffB

1361. Mittheilungen der Kön. Sächs. Polytechnischen Schule zu Dresden. (Verf.: Dr. Ernst Hartig, Lehrer (2.3: Prof.) d. mechan.Technologie.) H. 1-3. Leipzig: Teubner $1864-73.4^{\circ}$

$1=$ Hartig, Ernst: Versuche über den Kraftbedarf der Maschinen in der Streichgarnspinnerei und Tuchfabrikation, ausgeführt unter Mitw. der Polytechniker [Hermann Bruno] Arndt, [Hugo] Jüngling, [Ewald Richard] Klien u. [Gustav Adolph] Künzel. 1864. VIII, $72 \mathrm{~S}$.

$2=$ Hartig, Ernst: Versuche über den Kraftbedarf der Maschinen in der Flachs- und Wergspinnerei, ausgeführt unter Mitw. der Polytechniker $\mathrm{F}$ [erdinand] $\mathrm{H}$ [ermann] Becker, E[rnst] E[mil] Freyberg, W[alther] C[äcil] Merkel, Heinrich Judenfeind-Hülsse, Herrmann Judenfeind - Hülsse, E[mil] H[ermann] Nacke u. P[aul] Püschel. Mit 2 Holzschn. u. 13 lithogr. Taf. 1869. 117 S., 13 Taf.

$3=$ Hartig, E[rnst]: Versuche über Leistung und Arbeits-Verbrauch der Werkzeugmaschinen. Ausgeführt unter Mitw. der Studirenden der mechanischen Abtheilung des K. S. Polytechnikum zu Dresden. Mit 24 lithogr. Taf. 1873. 243 S., 24 Taf.

1362. Zur A usstellung der Königlich Sächsischen polytechnischen Schule: (Dat. 1. Mai 1873.) 2 Bi. $4^{0}$

$D r^{*}$

1363. Festsehrift zur Einweihung des neuen K. S. Polytechnikums zu Dresden am 4. November 1875. 1875. 38 S., 6 Taf. $8^{0} \mathrm{Dr}$
1364. Cantate für Männerstimmen mit Orchester. Zur Einweihungsfeier des neuen Polytechnikums zu Dresden am 4. November 1875. Dichtung von Adolf Stern. Musik von Julius Rietz. 2 Bl. $8^{0}$

$D r^{*}$

1365. Katalog der Bibliothek des Königlich Sächsischen Polytechnikums Dresden. (Vorr.: K[arl] Kuschel.) 1876. XII, 612 S. $8^{0}$

1366. Zur Feier des achthundertjährigen Regierungs-Jubiläums des Hauses Wettin. Festrede geh. in d. Aula des Kgl. Polytechnikums zu Dresden am 15. Juni 1889 von Arnold Gaedeke, o. ö. Prof. d. Gesch. Dresden: v. Zahn \& Jaensch. 31 S. $8^{0} \quad$ Be

1367. Katalog der Hochbau-Sammlung in der Kgl. Sächs. Technischen Hochschule zu Dresden. I. Obergeschoss. Saal Nr. 45. Bestand am 1. Nov. 1896. 14 S. $8^{0} \mathrm{Dr}$ 1368. Kirche und Kunst. Rede geh. bei Übernahme des Rektorats der Kgl. Sächsischen Technischen Hochschule am 1. März 1904 von Cornelius Gurlitt. Göttingen: Vandenhoeck \& Ruprecht. 1904. 8 S. $8^{\circ}$ I Aus: Monatsschrift f. Gottesdienst u. kirchl. Kunst. Jg. 9.

1369. Die Neubauten der Königlich Sächsischen Technischen Hochschule zu Dresden. 1905. 56 S., 1 Taf. $4^{0}$ - Teil A. Baubeschreibung. Erw. aus: Deutsche Bauzeitung. Jg. 39. - Teil B. Innere Einrichtung. Aus: Zeitschrift d. Vereines deutscher Ingenieure. Bd 49, Halbj: 1. - Anh. [Hubert Engels:] Die Anstalt zur Prüfung von Schiffswiderständen und hydrometrischen Instrumenten in Dresden-Uebigau. Aus: Zentralblatt d. Bauverwaltung. Jg. 25.

\section{HA BILITATI ON S SCHRIFTEN}

1370. Eggeling, Heinrich: Kant und Fries. Die anthropologische Auffassung der Kritik der Vernunft in ihren wesentlichen Punkten erörtert. 1875. 49 S. $8^{0}$

$\mathrm{Dr}$

Dresden Te. H., Hab.-Schr. v. W.-S. 1875/76

1371. Scheffler, Guillaume, Dr. en phil., membre de l'Inst. Sténogr. Royal de Dresde: Etude littéraire sur Boileau-Despréaux, sa vie et ses écrits. 1875. 45 S. $8^{0}$ - Auch bei Jolowicz in Posen 1876.

Dresden Te. H., Hab.-Schr. v. S.-S. 1876

1372. Koppel, Richard, Dr. phil.: Textkritische Studien über Shakespeare's ,Richard III." und ,King Lear". Dresden: Gilbers 1877. 102 S. $8^{0}$

Be KglB

Dresden Te. H., Hab.-Schr. v. S.-S. 1877
1373. Fischer, H[ugo], Assist. am Polytechn. zu Dresden: Die Stickmaschine. [1877.] 26 S., 3 Taf. $4^{0}$ I Aus: Der Civilingenieur. N. F. Bd 23 . Be

Dresden Te. H., Hab.-Schr. v. S.-S. 1878

1374. Hempel, Walther, Dr.: Über technische Gasanalyse. 1878. 50 S. $8^{0}$ - Erw. u. d. T.: Neue Methoden zur Analyse der Gase. Braunschweig: Vieweg 1880.

Dresden Te. H., Hab.-Schr. v. S.-S. 1878

1375. Steche, Richard, Dr. phil., Architekt: Zur Geschichte des Bucheinbands mit Berücksichtigung seiner Entwickelung in Sachsen. 1877. 62 S. $8^{0}$ I Mit Änderungen u. Zusätzen in: Archiv f. Geschichte d. deutschen Buchhandels. 1. (Publikationen d. Börsenvereins d. Deutschen Buchhändler. 1.) 1878. $\mathrm{Dr}$

Dresden Te. H., Hab.-Schr. v. S.-S. 1878 
1376. Papperitz, Erwin, Dr.: Untersuchungen über die algebraische Transformation der hypergeometrischen Functionen. Mit 2 Figurentaf. 1886.44 S., 2 Taf. $8^{0}$ - Aus: Math. Annalen. Bd 27.

Dresden Te. H., Hab.-Schr. v. W.-S. 1886/87

1377. Vater, Heinrich, gepr. Chemiker, Dr. phil., Geolog d. Kgl. Sächs. Landesuntersuchung: Der Apparat von Warburg und Koch zur Bestimmung der Elasticitätscoefficienten, sowie Anwendung desselben auf zur Axe senkrechte Platten von Kalkspath und Apatit. Mit 1 Taf. Leipzig: W. Engelmann 1886. 40 S., 1 Taf. $8^{0}$ I Aus: Zeitschrift f. Krystallographie u. Mineralogie. Bd 11.

Tharandt Forstakad

Dresden Te. H., Hab.-Schr. v. W.-S. 1886/87

1378. Doss, Bruno, Dr.: Die Lamprophyre und Metaphyre des Plauen'schen Grundes bei Dresden. 〈Mit 2 Taf.> Wien: Hölder 1889. 66 S., 2 Taf. $8^{0}$ T Aus: Tschermak's mineralog. u. petrograph. Mittheilungen. Bd 11.

Dresden Te. H., Hab.-Schr. v. S.-S. 1889

1379. Naetsch, Emil, Dr.: Untersuchungen über die Reduction und Integration von Picard'schen Differentialgleichungen. (1896.) 78 S. $8^{0}$ - Aus: Berichte über d. Verhandlungen d. Kgl. Sächs. Gesellschaft d. Wissenschaften zu Leipzig. Math.-phys. Cl. Bd 48.

Dresden Te. H., Hab.-Schr. v. S.-S. 1896

1380. Schubert, Max, Fabrikdir.: Ueber die Lagerung der Fasern im Papier. [1896.] 13 S., 1 Taf. $4^{0}$ - Aus: Papier-Zeitung. Jg. 21, Halbj. 1.

Dresden Te. H., Hab.-Schr. v. S.-S. 1896

1381. Walther, Reinhold, Dr.: Ueber Reductionen mittelst Phenylhydrazin. Nebst einem Anh.: Einwirkung von Orthoameisensäureäther auf primäre aromatische Amine. Leipzig: J. A. Barth 1896. 48 S. $8^{\circ}$ - Aus: Journal f. prakt. Chemie. Bd 161. N. F. Bd 53 .

Dresden Te. H., Hab.-Schr. v. W.-S. 1896/97

1382. Wolf, Kurt, Dr. med.: Utber die Farbstoffbildung der fluoreszierenden Bakterien des Dresdener Elb- und Leitungswassers. 1897 . 36 S. $8^{0}$ - Auch in: Zeitschrift f. Gewässerkunde. Bd 1. 1898.

Dresden Te. H., Hab.-Schr. v. W.-S. 1897/98

1383. Schlossmann, Arthur, Dr. med. i. Dresden: Ueber einige bedeutungsvolle Unterschiede zwischen Kuh- und Frauenmilch in chemischer und physiologischer Beziehung mit besonderer Berücksichtigung der Säuglingsernährungsfrage. Leipzig: Teubner 1898 . 36 S. $8^{0}$

Dresden Te. H., Hab.-Schr. v. S.-S. 1898
1384. Seefehiner, E[gon] E[wald]: Optische Methoden zu Wechselstromuntersuchungen. 1900. 40 S. $8^{0}$ - Aus: Zeitschrift f. Elektrotechnik. Jg. 18.

Dresden Te. H., Hab.-Schr. v. S.-S. 1900

1385. Toepler, Max: Über die Abhängigkeit des Charakters elektrischer Dauerentladung in atmosphärischer Luft von der dem Entladungsraume continuirlich zugeführten Elektricitätsmenge, nebst einem Anhange zur Kenntnis der Kugelblitze. Leipzig: J. A. Barth 1900. 103 S. $8^{0}$ i Erw. aus: Annalen d. Physik. Folge 4, Bd 2.

Dresden Te. H., Hab.-Schr. v. S.-S. 1900

1386. Bucherer, Hans Theodor, Dr.: Utber die Einwirkung schwefligsaurer Salze auf aromatische Amido- und Hydroxyl-Verbindungen. 1901. 59 S. $8^{0}$ - Ausz. in: Journal f. prakt. Chemie. Bd 177. N. F. Bd 69. 1904.

Dresden Te. H., Hab.-Schr. v. S.-S. 1901

1387. Lottermoser, Alfred, Dr.: Uber anorganische Colloide. 1901. VI, 80 S. $8^{0}$ ๆ Aus: Sammlung chem. u. chem.-techn. Vorträge. Bd 6. Dresden Te. H., Hab.-Schr. v. S.-S. 1901

1388. Müller, Erich, Dr. phil.: Studien über kathodische Polarisation und Depolarisation. 1900.86 S. $8^{0}$ I Auch in: Zeitschrift f. anorgan. Chemie. Bd 26. 1901.

Dresden Te. H., Hab.-Schr. v. S.-S. 1901

1389. Lewicki, E(rnst): Die Anwendung hoher Ueberhitzung beim Betrieb von Dampfturbinen. 1904. 95 S., 1 Taf. $4^{0}$ - Auch als: Mitteilungen über Forschungsarbeiten auf d. Gebiete d. Ingenieurwesens. H. 12; Ausz. in: Zeitschrift d. Vereines deutscher Ingenieure. Bd 47. 1903, Halbj. 1.

Dresden Te. H., Hab.-Schr. v. W.-S. 1902/03

1390. Bruck, Robert, Dr.: Friedrich der Weise als Förderer der Kunst. 1903.241 S. $8^{0}$ - Erw. u. mit Abb. als: Studien zur deutschen Kunstgeschichte. H. 45 .

Dresden Te. H., Hab.-Schr. v. S.-S. 1903

1391. Reuschel, Karl, Dr. phil.: Die deutschen Weltgerichtsspiele des Mittelalters und der Reformationszeit. Literarhistor. Untersuchung. T. 1. 1903. 34 S. $8^{0}$ - Vollst. als: Teutonia. H. 4. 1906.

Dresden Te. H., Hab.-Schr. v. W.-S. 1903/04

1392. Dietz, Rudolf, Dr. phil.: Ueber die Gewinnung von Strohzellstoff nach dem Sulfitverfahren. 1905. 71 S. $8^{0}$ i Ausz. in: Zeitschrift f. angewandte Chemie. Jg. 18, 1. Dresden Te. H., Hab.-Schr. v. S.-S. 1905

1393. König, Walter, Dr.-Ing.: Beiträge zur Chemie der Pyridinfarbstoffe. 1907. 132 S. $8^{0}$ Kleines Bruchstück in: Journal f. prakt. Chemie. Bd 191. N. F. Bd 83. 1911. Dresden Te. H., Hab.-Schr. v. S.-S. 1906 
1394. Nägel, A[dolph], Dipl.-Ing.: Versuche an der Gasmaschine über den Einfluß des Mischungsverhältnisses. 1907. 110 S. $4^{0}$ I Ausz. in: Mitteilungen über Forschungsarbeiten auf d. Gebiete d. Ingenieurwesens. H. 54. 1908; Ausz. in: Zeitschrift d. Vereines deutscher Ingenieure. Bd 51, Halbj. 2.

Dresden Te. H., Hab.-Schr. v. W.-S. 1906/07

1395. Lange, Ludwig, Dr. med., I. Bakteriologe d. K. S. Zentralstelle f. öffentl. Gesundheitspflege: Untersuchungen über Bacterium coli commune und verwandte $\mathrm{Ba}$ zillen. 1907. 62 S., 4 Taf. $8^{0}$ T Aus: Arbeiten aus d. Kgl. hygien. Instituten zu Dresden. Bd 2, H. 1 .

Dresden Te. H., Hab.-Schr. v. S.-S. 1907

1396. Brion, G[eorg], Dr.: Experimen telle Untersuchungen über den Hochspannungs-Lichtbogen. [1907.] S. 761-786, 1 Taf. $4^{0}$ - Aus: Zeitschrift f. Elektrochemie. Jg. 13.

Dresden Te. H., Hab.-Schr. v. W.-S. 1907/08

1397. Thiele, Herm[ann]: Einige Reaktionen im ultravioletten Lichte. 1908. 47 S., 1 Taf. $8^{0}$ Gekürzt in: Zeitschrift $\mathrm{f}$. angewandte Chemie. Jg. 22, 2. 1909; erw. u. übers. in: Annuario di chimica scientifica e industriale. 1910/11.

Dresden Te. H., Hab.-Schr. v. W.-S. 1908/09

1398. Gehler, W[illy]: Beitrag zur Berechnung und Beobachtung von Nebenspannungen eiserner Fachwerkbrücken. 1909. 71 S. $8^{0}$ Um einen Anh. verm. u. d. T.: Die Ermittlung der Nebenspannungen eiserner Fachwerkbrücken $\mathrm{u}$. das prakt. Rechnungsverfahren nach Mohr. Berlin: Ernst 1910.

Dresden Te. H., Hab.-Schr. v. S.-S. 1909

1399. Dember, Harry, Dr.: Erzeugung positiver Strahlen durch ultraviolettes Licht. Leipzig: J. A. Barth 1909. 31 S. $8^{0}$ I Aus: Annalen d. Physik. Folge 4, Bd 30.

Dresden Te. H., Hab.-Schr. v. W.-S. 1909/10
1400. Nußelt, Wilhelm, Dr.-Ing.: Der Wärmeübergang in Rohrleitungen. 1909. 40 S. $4^{0}$ T Auch in: Mitteilungen über Forschungsarbeiten auf d. Gebiete d. Ingenieurwesens. H. 89. 1910; Ausz. in: Zeitschrift d. Vereines deutscher Ingenieure. Bd 53, Halbj. 2.

Dresden Te. H., Hab.-Schr. v. W.-S. 1909/10

1401. Hugershoff, [Reinhard], Dr.-Ing.: Die periodischen Fehler barometrisch bestimmter Höhenunterschiede in der inneren Tropenzone. Dresden: Baensch 1910. 59S. $8^{\circ}$ I Aus: Mitteilungen d. Vereins f. Erdkunde zu Dresden. Bd 2. 1910/12.

Dresden Te. H., Hab.-Schr. v. S.-S. 1910

1402. Rimann, Eberhard, Dr., i. Dresden: Der geologische Bau des Isergebirges und seines nördlichen Vorlandes. Mit 1 Textfig. u. 1 geolog. Übersichtskt. 1910. S. 482533, 1 Taf. $8^{0}$ - Aus: Jahrbuch d. Kgl. PreuB. Geolog. Landesanstalt f. 1910. Bd 31. 1911.

Dresden Te. H., Hab.-Schr. v. S.-S. 1910

1403. Wawrziniok, 0tto, Dipl.-Ing.: Die Ermüdung des Eisenbahnschienenmaterials. Studie. Mit 18 Textfig. Berlin: Springer 1910. 47 S. $8^{0}$. Auch als Buch.

Dresden Te. H., Hab.-Sehr. v. S.-S. 1910

1404. Niedner, [Franz], Dr.-Ing., Stadtbaumeister: Die Strassenreinigung in den deutschen Städten unter besonderer Berücksichtigung der Dresdner Strassenreinigung. Mit $66 \mathrm{Abb}$. im Text u. 5 Tab. Leipzig: W. Engelmann 1911. $99 \mathrm{~S} ., 5$ Taf. $4^{0}$ - Auch als Buch.

Dresden Te. H., Hab.-Schr. v. S.-S. 1911

1405. Neumann, Kurt, Dr.-Ing.: Die Vorgänge im Gasgenerator auf Grund des zweiten Hauptsatzes der Thermodynamik. 1912. $69 \mathrm{~S} .4^{0}$ - Ausz. in: Zeitschrift d. Vereines deutscher Ingenieure. Bd 57. 1913, Halbj. 1; etwas gekürzt als: Mitteilungen über Forschungsarbeiten auf d. Gebiete d. Ingenieurwesens. H. 140. 1913 u. in: Stahl u. Eisen. Jg. 33. 1913, Halbj. 1. Dresden Te. H., Hab.-Schr. v. S.-S. 1912

\section{DOKTOR-INGENIEUR-DISSERTATIONEN}

\section{HOCHBAU-ABTEILUNG}

1406. Muthesius, Hermann: Der Kirchenbau der englischen Secten. 1902.60 S. $8^{0}$ I Mit einigen Ånderungen u. Beifügung v. Abb. in: Muthesius: Die neuere kirchliche Baukunst in England. 1901.

Dresden, Dr.-Ing.-Diss. v. 14. 4. 1902

1407. Fiedler, Wilhelm: Das Fachwerkhaus in Deutschland, Frankreich und England. Berlin: Wasmuth [1902]. 99 S. $4^{0}$ - Auch als: Beiträge zur Bauwissenschaft. H. 1. Dresden, Dr.-Ing.-Diss. v. 19. 7. 1902
1408. Wesser, Rudolf: Der Holzbau mit Ausnahme des Fachwerks. Berlin: Wasmuth [1903]. 73 S. $4^{0}$ - Auch als: Beiträge zur Bauwissensehaft. H. 2.

Dresden, Dr.-Ing.-Diss. v. 15. 11. 1902

1409. Rahtgens, H[ugo]: S. Donato zu Murano und ähnliche venezianische Bauten. Berlin: Wasmuth [1903]. 96 S., 3 Taf. $4^{0}$

- Auch als: Beiträge zur Bauwissenschaft. H. 3. Dresden, Dr.-Ing.-Diss. v. 22. 12. 1902

1410. Holtmeyer, A[loys]: Beiträge zur Baugeschichte der Paulinzeller Kloster- 
kirche. Jena: Fischer 1904. 172 S., 8 Taf. $8^{0}$ T Aus: Zeitschrift d. Vereins f. Thüring. Geschichte u. Altertumskunde. Bd 23. N. F. Bd 15. 1904/05. Dresden, Dr.-Ing.-Diss. v. 13. 3. 1903

1411. Dietrich, Walther: Beiträge zur Entwicklung des bürgerlichen Wohnhauses in Sachsen im 17. und 18. Jahrhundert. 1903. 83 S., 1 Taf. $4^{0}$ - Auch bei Gilbers in Leipzig 1904.

Dresden, Dr.-Ing.-Diss. v. 27. 5. 1903

1412. Mackowsky, Walter: Giovanni Maria Nosseni und die Renaissance in Sachsen. Berlin: Wasmuth 1904. 110 S. $4^{0}$

T Auch als: Beiträge zur Bauwissenschaft. H. 4. 1905. Dresden, Dr.-Ing.-Diss. v. 20. 7. 1904

1413. Hammitzseh, Martin: Der moderne Theaterbau. Der höfische Theaterbau, der Anfang der modernen Theaterbaukunst, ihre Entwicklung und Betätigung zur Zeit der Renaissance, des Barock und des Rokoko. Mit 142 Ill. u. 228 Anm. Berlin: Wasmuth 1906. VIII, 207 S. $4^{0}$ - Auch als: Beiträge zur Bauwissenschaft. H. 8.

Dresden, Dr.-Ing.-Diss. v. 22. 12. 1904

1414. Korn, Richard: Kriegsbaumeister Graf Rochus zu Linar, sein Leben und Wirken. [1905.] XIII, 140 S., 2 Taf. $8^{0}$

- Auch bei Heinrich in Dresden.

Dresden, Dr.-Ing.-Diss. v. 27. 2. 1905

1415. Klopfer, Paul: Weinlig und seine Zeit. Berlin: Wasmuth [1905]. 82 S. $4^{0}$ - Auch u. d. T.: Christian Traugott Weinlig und die Anfänge des Klassizismus in Sachsen, als: Beiträge zur Bauwissenschaft. H. 5 .

Dresden, Dr.-Ing.-Diss. v. 15. 3. 1905

1416. Rauda, Fritz, Architekt: Die mittelalterliche Baukunst Bautzens. Hrsg. von d. Oberlausitzischen Gesellschaft $d$. Wissenschaften zu Görlitz. 1905. XI, 99 S., 6 Taf. $4^{0}$ - Auch bei Tzschaschel in Görlitz.

Dresden, Dr.-Ing.-Diss. v. 6. 7. 1905

1417. Sohrmann, Johannes: Die altindische Säule. Ein Beitrag zur Säulenkunde. Dresden: Kühtmann 1906. VII, 79 S. $4^{0}-$ Auch als Buch.

Dresden, Dr.-Ing.-Diss. v. 26. 7. 1905

1418. Mäkelt, Arthur: Mittelalterliche Landkirchen aus dem Entstehungsgebiete der Gotik. Berlin: Wasmuth 1906. 128 S. $4^{0}$ - Auch als: Beiträge zur Bauwissenschaft. H. 7. Dresden, Dr.-Ing.-Diss. v. 27. 7. 1905

1419. Barth, Alfred: Zur Baugeschichte der Dresdner Kreuzkirche. Studien über den protestantischen Kirchenbau und Dresdens Kunstbestrebungen im 18. Jahrhundert. Mit 120 Abb. Dresden: C. C. Meinhold 1907. 148 S. $4^{0}$

Dresden, Dr.-Ing.-Diss. v. 23. 12. 1905
1420. Goldhardt, Paul: Die heiligen Berge Varallo, Orta und Varese. Berlin: Wasmuth 1908. 89 S. $4^{0}$ - Auch als: Beiträge zur Bauwissenschaft. H. 9.

Dresden, Dr.-Ing.-Diss. v. 24. 7. 1907

1421. Phleps, Hermann: Zwei Schöpfungen des Simon Louis du Ry aus den Schlössern Wilhelmstal und Wilhelmshöhe bei Kassel. Berlin: Ernst 1908. 27 S., 4 Taf. $2^{0}$ - Auch als Buch. Um Vorw. u. Einl. verm. aus: Zeitschrift f. Bauwesen. Jg. 58.

Dresden, Dr.-Ing.-Diss. v. 24. 7. 1907

1422. Sehubert, 0tto, Reg.-Baumeister: Herrera und seine Zeit. [1906.] 58 S., 1 Taf. $4^{0}$ - Teilabdr.; vollst. u. d. T.: Geschichte des Barock in Spanien. (J. Burckhardt: Geschichte d. neueren Baukunst. Bd 8.) Esslingen: Neff 1908. Dresden, Dr.-Ing.-Diss. v. 24. 7. 1907

1423. Lewy, Max: Schloss Hartenfels bei Torgau. Berlin: Wasmuth 1908. 111 S. $4^{0}$ - Auch als: Beiträge zur Bauwissenschaft. H. 10. Dresden, Dr.-Ing.-Diss. v. 27. 11. 1907

1424. Steinberg, Curt: Die sächsische Plastik des XIII. Jahrhunderts im Dienste der Architektur. 1908. 36 S. $4^{0}$

Dresden, Dr.-Ing.-Diss. v. 27. 11. 1907

1425. Rüdiger, Alfred: Die links der Elbe gelegenen Burgen im Königreich Sachsen. Berlin: Wasmuth 1909. VII, 125 S. $4^{0}$ - Auch als: Beiträge zur Bauwissenschaft. H. 14. Dresden, Dr.-Ing.-Diss. v. 15. 5. 1908

1426. Biebrach, Kurt: Die holzgedeckten Franziskaner- und Dominikanerkirchen in Umbrien und Toskana. Berlin: Wasmuth 1908. VI, 70 S. $4^{0}$ - Auch als: Beiträge zur Bauwissenschaft. H. 11.

Dresden, Dr.-Ing.-Diss. v. 9. 7. 1908

1427. Wilde, H(ans): Brussa, eine Entwickelungsstätte türkischer Architektur in Kleinasien unter den ersten Osmanen. Berlin: Wasmuth 1909. 135 S., 4 Taf. $4^{0}$ T Auch als: Beiträge zur Bauwissenschaft. H. 13.

Dresden, Dr.-Ing.-Diss. v. 18. 7. 1908

1428. Andrae, Walter: Der Anu-AdadTempel in Assur. Die ältere Anlage. 1909. 54 S., 3 Taf. $8^{0}-$ Vollst. als: Wissenschaftl. Veröffentlichung d. Deutschen Orient-Gesellschaft. 10. Dresden, Dr.-Ing.-Diss. v. 23. 11. 1908

1429. Böttcher, C[arl]: Altsächsische Wendeltreppen nebst einem Überblick über die Entwicklung des Wendeltreppenbaues im allgemeinen. Dresden: Kühtmann 1909. $\mathrm{XV}, 134$ S. $4^{0}$ T Auch u. d. T.: Die Entwicklung des Wendeltreppenbaues bei eingehender Behandlung der altsächsischen Wendeltreppe. Dresden, Dr.-Ing.-Diss. v. 25. 11. 1908 
1430. Heußinger, Konrad: Das Bauwesen in Alt-Nürnberg erläutert an einigen Beispielen der Ein- und Zweihof-Anlage. Nürnberg: Zerreiss [1909]. 108 S., 1 Taf. $4^{0}$

Dresden, Dr.-Ing.-Diss. v. 25. 11. 1908

1431. Langenegger, Felix, Bauamtmann: Beiträge zur Kenntnis der Baukunst des Irâq 〈heutiges Babylonien〉. Bautechnik, Baukonstruktionen und Aussehen der Baugegenstände unter teilweiser Bezugnahme auf die Baukunst der Vergangenheit des Landes sowie auf die gesamte Baukunst des Islâm. Dresden: Kühtmann 1911. VIII, 200 S., 1 Taf. $4^{0}$ - Auch. u. d. T.: Die Baukunst des Irâq 〈heutiges Babylonien〉...

Dresden, Dr.-Ing.-Diss. v. 3. 12. 1908

1432. Scheerer, Felix: Kirchen und Klöster der Franziskaner und Dominikaner in Thüringen. Ein Beitrag zur Kenntnis der Ordensbauweise. Jena: Fischer 1910. VI, 148 S., 3 Taf. $8^{0}$ - Auch als: Beiträge zur Kunstgeschichte Thüringens. Bd 2.

Dresden, Dr.-Ing.-Diss. v. 3. 12. 1908

1433. Koch, Hugo: Sächsische Gärten. Berlin:Deutsche Bauzeitung (1909). 60 S. $8^{0}$

- Vollst. u. d. T.: Sächsische Gartenkunst. 1910. Dresden, Dr.-Ing.-Diss. v. 13. 1. 1909

1434. Dewitz, Hans: Baupolizeiliche Konstruktionsvorschriften des In- und Auslandes und ihre Anwendung auf Kleinwohnungsbauten. [1909.] 76 S., 4 Taf. $4^{0}$

- Teile in: Baupolizeil. Mitteilungen. Jg. 7. 1910 u. in : Zeitschrift f. Wohnungswesen. Jg. 9. 1910/11.

Dresden, Dr.-Ing.-Diss. v. 17. 2. 1909

1435. Heinemann, Willy: Die Villenbauten des Andrea Palladio. 1909. 135 S. $4^{0}$

Dresden, Dr.-Ing.-Diss. v. 18. 5. 1909

1436. Rannacher, Albert: Das bürgerliche Wohnhaus in Meißen. Meißen: Verein f. Geschichte d. Stadt Meißen; Mosche in Komm. [1910]. 102 S., 24 Taf. $8^{\circ}$ T Aus: Mitteilungen d. Vereins f. Geschichte d. Stadt Meißen. Bd 8, H. 1

Dresden, Dr.-Ing.-Diss. v. 18. 5. 1909

1437. Seheibe, Werner: Die baugeschichtliche Entwickelung von Kamenz in Sachsen. Görlitz: Oberlausitzische Gesellsch. d. Wiss.; Tzschaschel in Komm. 1909. IV, 93 S. $4^{0}$ Dresden, Dr.-Ing.-Diss. v. 18. 5. 1909

1438. Klingenberg, W[alter]: Burgundische Stadt- und Landkirchen. Berlin: Ernst 1910. 98 S. $4^{0}$ - Aus: Zeitschrift f. Bauwesen. Jg. 60 .

Dresden, Dr.-Ing.-Diss, v. 21. 6. 1909

1439. Kösser, Fritz: Holzgedeckte Landkirchen in der Normandie. Dresden: Kühtmann 1909. IV, 139 S. $4^{0}$ - Buchausg. 1910.

Dresden, Dr.-Ing.-Diss. v. 21. 7. 1909
1440. Reuther, 0skar: Das Wohnhaus in Bagdad und anderen Städten des: Irak. Berlin: Wasmuth 1910 . XVI, 119 S. $4^{0}$ - Auch als: Beiträge zur Bauwissensehaft. H.16. Dresden, Dr.-Ing.-Diss. v. 21. 7. 1909

1441. Gerber, William: K. K. Österreichisches Archäologisches Institut. Untersuchungen und Rekonstruktionen an altchristlichen Kultbauten in Salona. 1911. 71 S., 4 Taf. $2^{0}$ - Teildr.; vollst. u. d. T.: Altchristliche Kultbauten Istriens und Dalmatiens. Dresden: Kühtmann 1912.

Dresden, Dr.-Ing.-Diss. v. 20. 7. 1910

1442. Unglaub, Franz: Die Diele im niedersächsischen Bauernhaus und norddeutschen Bürgerhaus. 1911. $177 \mathrm{~S} .8^{0}$ - Auch bei Lübcke \& Nöhring in Lübeck; aus: Zeitschrift d. Vereins f. Lübeckische Geschichte u. Altertumskunde. Bd 13.

Dresden, Dr.-Ing.-Diss. v. 20. 7. 1910

1443. Jordan, Julius: Konstruktions-Elemente assyrischer Monumentalbauten. Berlin: Wasmuth 1910. IV, 42 S. $4^{0}$. Auch als: Beiträge zur Bauwissenschaft. H. 18.

Dresden, Dr.-Ing.-Diss. v. 8.9. 1910

1444. Wiener, Alfred: Das Warenhaus. Berlin: Wasmuth 1911. V, 50 S. $4^{0}$ ๆ Erw. u. d.T.: Das Warenhaus, Kauf-, Geschäfts-, Büro-Haus. 1912. Dresden, Dr.-Ing.-Diss. v. 14. 12. 1910

1445. Koßmann, Walter: Arbeiterwohnhaustypen 〈Einfamilienhäuser〉. Ein Beitrag zum Arbeiterwohnungswesen. Mit 18 Abb. Dresden: Kühtmann 1912. 151 S. $4^{0}$ - Auch als Buch.

Dresden, Dr.-Ing.-Diss. v. 20. 12. 1910

1446. Conert, Herbert: Die sächsischen Terraingesellschaften und ihr Einfluß auf die Stadterweiterung. 1911. $75 \mathrm{~S} ., 2 \mathrm{Pl} .8^{0}$ I Vollst. als: Abhandlungen aus d. volkswirtschaftl. Seminar d. Techn. Hochschule zu Dresden. H. 2. Dresden, Dr.-Ing.-Diss. v. 8. 3. 1911

1447. Krusehwitz, Joh[ann]: Die Baugeldbeschaffung für städtische Wohnhausbauten in Dresden und Bautzen.1911. X,79S.80 - Auch als: Abhandlungen aus d. volkswirtschaftl. Seminar d. Techn. Hochschule zu Dresden. H. 1. Dresden, Dr.-Ing.-Diss. v. 8. 3. 1911

1448. Behrendt, Walter Curt: Die einheitliche Blockfront als Raumelement im Stadtbau. Ein Beitrag zur Stadtbaukunst der Gegenwart. Berlin: B. Cassirer 1911. 108 S., 13 Taf. $8^{0}$ • Als Buch mit Umschlagt. 1912. Dresden, Dr.-Ing.-Diss. v. 26. 7. 1911

1449. Tiseher, Alfred: Der Kampf im deutschen Baugewerbe 1910. 1912. 80 S. $8^{0}$ - Vollst. als: Abhandlungen aus d. volkswirtschaftl. Seminar d. Techn. Hochschule zu Dresden. H. 3. Dresden, Dr.-Ing.-Diss. v. 26. 7. 1911 
1450. Döhring, Karl: Das Phrăchedi in Siam. Berlin: Behrend 1912. 114 S. $8^{0}$ T Aus: Zeitschrift f. Ethnologie. Jg. 44.

Dresden, Dr.-Ing.-Diss. v. 29. 7. 1911

1451. Preusser, Conrad: Nordmesopotamische Baudenkmäler altchristlicher und islamischer Zeit. 1911. 23 S., 33 Taf. $2^{0}$ - Teilabdr.; vollst. als: Wissenschaftl. Veröffentlichungen d. Deutschen Orient-Gesellschaft. 17.

Dresden, Dr.-Ing.-Diss. v. 8.11. 1911

1452. Hoffmann, Paul: Nordische Cistercienserkirchen unter Berücksichtigung der Backsteinbaukunst. Essen-Ruhr: Thaden \& Schmemann 1912. 114 S. $4^{0}$

Dresden, Dr.-Ing.-Diss. v. 24. 1. 1912

1453. Weise, Max: Das bergische Bürgerhaus und der moderne heimische Wohnhausbau. Eine Studie zur Frage der Wiederbelebung alter, bodenständiger Bauweisen. (1912.) 63 S., 19 Taf. $8^{0}$ - Auch bei Schwann in Düsseldorf.

Dresden, Dr.-Ing.-Diss. v. 24. 1. 1912

1454. Sehröder, Karl: Studien über Renaissance-Gärten in Oberdeutschland. Düsseldorf: Schwann (1912.) 76 S. $4^{0}$. Auch als Buch.

Dresden, Dr.-Ing.-Diss. v. 13. 3. 1912

1455. Hiersehe, Waldemar: Pellegrino de' Pellegrini als Architekt. Parchim: Freise 1912. VIII, 115 S., 16 Taf. $4^{0}$ ๑ Auch als Buch.

Dresden, Dr.-Ing.-Diss. v. 12. 6. 1912

1456. Lindner, Werner: Die bäuerliche Wohnkultur in der Provinz Westfalen und ihren nördlichen Grenzgebieten. (Kapitel 1. Das Bauernhaus.) Berlin: Parey 1912. 92 S. $4^{0}$ - Aus: Beiträge zur Geschichte d. westfälischen Bauernstandes. Hrsg. von E. Frhr. v. Kerckerinck zur Borg.

Dresden, Dr.-Ing.-Diss. v. 4. 12.1912

\section{INGENIEUR-ABTEILUNG FÜR BAU- INGENIEURWESEN MIT EINSCHLUSZ DER GEODÄSIE}

\section{Bau-Ingenieurwesen}

1457. Kloss, Max: Analytisch-graphisches Verfahren zur Bestimmung der Durchbiegung zwei- und dreifach gestützter Träger. Mit besonderer Berücksichtigung der Berechnung von Drehstrommotorenwellen. [1902.] 128 S., 4 Taf. $8^{\circ}$ - Auch bei Seydel in Berlin; Ausz. in: Dinglers Polytechn. Journal. Jg. 84. 1903. Bd 318 .

Dresden, Dr.-Ing.-Diss. v. 10. 3. 1902
1458. Thieme, Johannes: Ueber den Einfluss der Gelenke auf den Materialverbrauch in den Gurtungen flusseiserner Bogenbrücken, untersucht an dem Sonderfall des durch einen Parallelträger versteiften $\mathrm{Pa}$ rabelbogens. [1902.] 24 S., 1 Taf. $8^{0}$

Dresden, Dr.-Ing.-Diss. v. 3. 5. 1902

1459. Walloth, C[arl $]$ A[ugust], Reg.-Baumeister: Die Eisenbahnbremsfrage und insbesondere ein Vorschlag zum Abbremsen auf Steilbahnen. Wiesbaden: Bergmann 1903. 48 S. $4^{0}$ I Auch als Buch; aus: Zeitschrift f. d. gesamte Lokal- \& Strassenbahn-Wesen. Jg. 22. Dresden, Dr.-Ing.-Diss. v. 15. 7. 1903

1460. Niedner, Franz, Reg.-Bauführer: Beitrag zur Berechnung von Schiffbrücken. Mit 54 Fig. im Text u. 1 Taf. Leipzig: W. Engelmann 1904. 50 S., 1 Taf. $4^{0}$ - Auch als Buch.

Dresden, Dr.-Ing.-Diss. v. 2. 12. 1903

1461. Fischer, Ewald: Über künstliche Belastungen bei der Aufstellung von Bogenbrücken. 1905. 47 S., 2 Taf. $8^{\circ}$

Dresden, Dr.-Ing.-Diss. v. 16. 3. 1905

1462. Imhoff, K[arl], Reg.-Baumeister, wiss. Mitglied d. Kgl. Prüfungsanstalt $f$. Wasserversorgung u. Abwässerbeseitigung i. Berlin: Die biologische Abwasserreinigung in Deutschland. (Vorr.: [Adolf] Schmidtmann u. [Carl] Günther.) 1906. 157 S., 2 Tab. $8^{0}$ Aus: Mitteilungen aus d. Kgl. Prüfungsanstalt $\mathrm{f}$. Wasserversorgung $\mathrm{u}$. Abwässerbeseitigung. H. 7.

Dresden, Dr.-Ing.-Diss. v. 20. 12. 1905

1463. Kögler, Franz, Reg.-Bauführer: Einflußlinien für beliebig gerichtete Lasten. 1906. 64 S., 6 Taf. $8^{\circ}$ - Auch bei Dressel in Dresden; auszugsweise u. mit Erg. u. Erweiterungen in: Zentralblatt d. Bauverwaltung. Jg. 27. 32. 1907. 12.

$$
\text { Dresden, Dr.-Ing.-Diss. v. 5. 12. } 1906
$$

1464. Trauer, Günther, Reg.-Bauführer: Der günstigste Gurtabstand sowie die Gewichte gegliederter flusseiserner Zweigelenkbogenträger mit nahezu parallelen Gurtungen. Dresden: Dressel 1907. IV, 86 S., 6 Taf. $8^{0}$ - Auch als Buch.

Dresden, Dr.-Ing.-Diss. v. 26. 6. 1907

1465. Schmeitzner, Rudolf, Reg.-Bauführer: Grundzüge der mechanischen $\mathrm{Ab}$ wässerklärung. Leipzig: W. Engelmann 1908. 64 S., 2 Taf. $4^{0}$ Auch als: Fortschritte d. Ingenieurwissenschaften. Gruppe 2, H. 16.

Dresden, Dr.-Ing.-Diss. v. 17. 7. 1907

1466. Speck, Artur, Reg.-Baumeister: Beitrag zur Geschichte und Theorie der Schwebefährbrücken. Mit $36 \mathrm{Abb}$. im Text. 
Leipzig: W. Engelmann 1908. 46 S. $4^{0}$ I Auch als: Fortschritted. Ingenieurwissenschaften. Gruppe 2, H. 18.

Dresden, Dr.-Ing.-Diss. v. 6. 12. 1907

1467. Beyer, Kurt, Reg.-Bauführer: Eigengewicht, günstige Grundmaße und geschichtliche Entwickelung des Auslegeträgers. Mit 70 Fig. im Text. Leipzig: W. Engelmann 1908. VIII, 132 S. $4^{0}$ A Auchals: Fortschritte d. Ingenieurwissenschaften. Gruppe 2, H. 19.

Dresden, Dr.-Ing.-Diss. v. 7. 12. 1907

1468. Gebers, Friedrich: Ein Beitrag zur experimentellen Ermittlung des Wasserwiderstandes gegen bewegte Körper. Berlin: Sehiffbau 1908. 28 S., 3 Taf. $4^{0}$ - Aus: Schiffbau. Jg. 9. 1907/08.

Dresden, Dr.-Ing.-Diss. v. 26. 2. 1908

1469. Platzmann, Ferdinand: Über den Querschnitt der Staumauern. Mit 53 Fig. im Text u. 2 Taf. Leipzig: W. Engelmann 1908. VI, 62 S., 2 Taf. $4^{0}$ Auch als: Fortschritte d. Ingenieurwissenschaften. Gruppe 2, H. 20.

Dresden, Dr.-Ing.-Diss. v. 6. 5. 1908

1470. Schiele, Albert, Kgl. Bauinsp., Mitglied d. Kgl. Prüfungsanstalt f. Wasserversorgung u. Abwässerbeseitigung zu Berlin: Die Abwasserfrage in der englischen Gesetzgebung und Verwaltung mit besonderer Berücksichtigung des gewerblichen Abwassers. Mit 9 Abb. Berlin: (Hirschwald) 1908. VIII, 178 S. $8^{0}$ - Vollst. u. d. T.: Abwasserbeseitigung von Gewerben und gewerbereichen Städten unter hauptsächlicher Berücksichtigung Englands, als: Mitteilungen aus d. Kgl. Prüfungsanstalt f. Wasserversorgung u. Abwässerbeseitigung. H. 11.

Dresden, Dr.-Ing.-Diss. v. 3. 6. 1908

1471. Roch, Hermann: Die Wasserversorgung mittels Talsperren in Deutschland. [1908.] 80 S., 5 Taf. $8^{0}$ - Erhältlich beim Akad. Ingenieur-Verein an d. Techn. Hochsch. Dresden; vgl. Wasser и. Abwasser. Bd 1. 1909. S. $145 \mathrm{ff}$

Dresden, Dr.-Ing.-Diss. v. 16. 7. 1908

1472. Ritter, Max: Beiträge zur Theorie und Berechnung der vollwandigen Bogenträger ohne Scheitelgelenk, insbesondere der Brückengewölbe und der im Eisenbetonbau ïblichen biegungsfesten Rahmen. Berlin: Ernst 1909. 54 S. $4^{0}$ - Auch als: Forscherarbeiten auf d. Gebiete d. Eisenbetons. H. 11.

Dresden, Dr.-Ing.-Diss. v. 30. 7. 1909

1473. Lindboe, Waldemar: Eine neue Formel zur Ermittelung der mittleren Geschwindigkeit in natürlichen Wasserläufen. 1910. $44 \mathrm{~S} .8^{0}$ - Aus: Zeitschrift f. Gewässerkunde. Bd 10. 1911; Referat von H. Engels in: Zentralblatt d. Bauverwaltung. Jg. 30.

Dresden, Dr.-Ing.-Diss. v. 7. 3. 1910
1474. Elsner, Alexander, Reg.-Bauführer: Die Behandlung und Verwertung von Klärschlamm. Mit $30 \mathrm{Abb}$. im Text, Leipzig: W. Engelmann 1910. VIII, 87S. $4^{0} \bullet$ Auch als: Fortschritte d. Ingenieurwissenschaften. Gruppe 2, H.24. Dresden, Dr.-Ing.-Diss. v. 11. 5. 1910

1475. Reichle, Carl, Kgl. Bauinsp. zu Berlin: Die Behandlung und Reinigung der Abwässer. Leipzig: Hirzel 1910. 107 S. $4^{0}$ Dresden, Dr.-Ing.-Diss. v. 2. 6. 1910

1476. Kryzan, Marian, Patentanw.: Über die astatische Äquivalenz der räumlichen Kräftesysteme. 1910. 54 S., 2 Taf. $8^{0}$

Dresden, Dr.-Ing.-Diss. v. 6. 7. 1910

1477. Eifler, Kurt: Úber die Eisenarmierung kreisrunder Betonplatten. 1911. $67 \mathrm{~S}$., 9 Taf. $8^{0}$

Dresden, Dr.-Ing.-Diss. v. 20. 7. 1910

1478. Hauffe, Walter: Gewichte und günstigste Abmessungen der durch Parallelträger versteiften Kabelbrücken. Dresden: Dressel 1910. 43 S. $8^{0}$ - Auch als Buch.

Dresden, Dr.-Ing.-Diss. v. 20. 7. 1910

1479. Nitzsche, Hans: Welche Nebenspannungen entstehen in Gewölben, die in senkrecht zu einer Stirnfläche stehenden Ebenen von äußeren Kräften belastet werden? 1910. 49 S., 2 Taf. $8^{0}$ - Ausz. in : Zement u. Beton. Jg. 10. 1911.

Dresden, Dr.-Ing.-Diss. v. 2. 11. 1910

1480. Müller, Otto, Reg.-Baumeister: Der Einfluß der neuzeitlichen Verkehrssteigerung auf die Durchbildung und Gestaltung der Straßenbahnschienen. Dresden: Dressel 1910. 109 S., 9 Taf. $4^{0}$ - Auch als Buch.

Dresden, Dr.-Ing.-Diss. v. 7. 12.1910

1481. Kleinlogel, Adolf: Über das Wesen und die wahre Größe des Verbundes zwischen Eisen und Beton. Berlin: Springer 1911. 56 S., 6 Taf. $4^{0}$ - Auch als Buch.

Dresden, Dr.-Ing.-Diss. v. 14. 12. 1910

1482. Kirchhoff, R[udolf], Reg. - Baumeister: Der Zweigelenkbogen als statisch unbestimmtes Hauptsystem. Mit 84 Fig. im Text. Berlin:Ernst 1911.62S. $8^{0}$ - Auch alsBuch.

Dresden, Dr.-Ing.-Diss. v. 15. 12. 1910

1483. Bader, [Friedrich], Dr. phil., Reg.Baumeister: Vortrieb und Ausbolzung von Gebirgstunneln. Ein kurzer Abriß der bergmännischen Tunnelbauweisen unter Behandlung und Begründung der neuzeitlichen Änderungen und Verbesserungen. Berlin: Springer 1911. $76 \mathrm{~S} .8^{0}$ \% Auch als Buch.

Dresden, Dr.-Ing.-Diss. v. 26. 7. 1911

1484. Matthias, Franz: Untersuchungen über den Druck und Druckmittelpunkt lotrechter Platten, die recht- und spitzwinklig 
zur Fahrtrichtung durch Wasser geschleppt werden. Berlin: Marfels 1911. $35 \mathrm{~S} .4^{0}$ - Aus: Schiffbau. Jg. 13. 1911/12.

Dresden, Dr.-Ing.-Diss. v. 21. 12. 1911

1485. Bloss, A[dolf] E[rnst], Bauamtmann i. Dresden: Das Eisenbahngleis auf starrem Unterbau. Dresden: Dressel 1912. 70 S., 9 Taf. $4^{0}$ - Auch als Buch.

Dresden, Dr.-Ing.-Diss. v. 31.1. 1912

1486. Katzsch, Karl: Die Bedeutung der IV. Klasse und die Selbstkosten des Personentransportes auf den deutschen Staatsbahnen. [1912.] 83, VII S. $8^{0}$

Dresden, Dr.-Ing.-Diss. v. 14. 2. 1912

1487. Rossin, Richard: Beiträge zur Berechnung mehrstieliger Steifrahmen mit Verwendung eines durchlaufenden Balkens als statisch unbestimmtes Hauptsystem. Berlin: Krayn 1912. 80 S., 1 Taf. $8^{0}$

Dresden, Dr.-Ing.-Diss. v. 14. 2. 1912

1488. Pietsehmann, Eugen: Gewichtsverhältnisse von Hauptträgern durchlaufender eiserner Balkenbrücken über zwei und drei Öffnungen. 1912. 62 S., 11 Taf. $8^{0}$ - Ergebnisse in: Der Brückenbau. Jg. 1.

Dresden, Dr.-Ing.-Diss. v. 28. 2. 1912

1489. Borchers, Richard, Reg. - Baumeister: Beiträge zur Berechnung von Schleusen großen Gefälles. 1912. 97 S. $8^{\circ}$ Dresden, Dr.-Ing.-Diss. v. 13. 3. 1912

1490. Lemberg, Karl: Trinkwasserreinigung durch Schnellsandfiltration. 1912. 54 S. $8^{0}$ - Aus: Journal f. Gasbeleuchtung u. verwandte Beleuchtungsarten. Jg. 56. 1913.

Dresden, Dr.-Ing.-Diss. v. 12. 6. 1912

1491. Alfthan, Bertil v.: Über die Bestimmung der wirtschaftlich günstigsten Durchmesser beiWasser-Druckrohrleitungen. Dresden: Akad. Buchh. 1912. 127 S. $8^{0}$ - Auch als Buch.

Dresden, Dr.-Ing.-Diss. v. 24. 7. 1912

1492. Schubert, Curt, Reg.-Bauführer: Lüftung im Tunnelbau. 1912. 124 S., 8 Bl. Taf. $8^{\circ}$ I Auch bei Wächtler in Dresden-A.

Dresden, Dr.-Ing.-Diss. v. 24. 7. 1912

1493. Gehler, W[illy], Reg.-Baumeister: Beitrag zur Bemessung von Rahmen. Berlin: Ernst 1912. VII, 108 S., 2 Taf. $8^{\circ}$ - Erw. u. d. T.: Der Rahmen. 1913.

Dresden, Dr.-Ing.-Diss. v. 25. 7. 1912

\section{Geodäsie}

1494. Schreiber, Karl Albert, Kgl. Bauinsp.: Beitrag zur Berechnung barometrisch bestimmter Höhenunterschiede. 1907.48 S. $4^{0}$ Dresden, Dr.-Ing.-Diss. v. 13. 2. 1907
1495. Hugershoff, Reinhard: Der $\mathrm{Zu}-$ stand der Atmosphäre als Fehlerquelle im Nivellement. 1907. 71 S. $8^{0}$

Dresden, Dr.-Ing.-Diss. v. 24. 7. 1907

1496. Israel, 0tto: Zur Theorie der einseitig wirkenden Instrumentalfehler an $\mathrm{Re}$ petitionstheodoliten. [Dresden: Dressel in Komm.] 1912. 62 S., 1 Taf. $8^{0}$

Dresden, Dr.-Ing.-Diss. v. 14. 3. 1912

\section{MECHANISCHE ABTEILUNG FÜR MASCHINENBAU, ELEKTROTECHNIK UND FABRIKBETRIEB}

\section{Maschinenbau und mechanische Technologie}

1497. Thümmler, Fritz, staatl. gepr. Bauführer: Fliehkraft und Beharrungsregler. Versuch einer einfachen Darstellung der Regulierungsfrage im Tolleschen Diagramm mit einem Anhange: Über den Einfluß des Reglers auf das Pendeln parallel geschalteter Wechselstrommaschinen. 〈Hierzu 6 Taf.〉 Berlin: (Springer) 1903. 153 S., 1 Taf. $8^{0}$ - Anch als Buch.

Dresden, Dr.-Ing.-Diss. v. 21. 2. 1903

1498. Büchner, Karl: Zur Frage der Lavalschen Turbinendüsen. 1904. 54 S. $4^{0}$ - Auch in: Mitteilungen über Forschungsarbeiten auf d. Gebiete d. Ingenieurwesens. H. 18; Ausz. in: Zeitschrift d. Vereines deutscher Ingenieure. Bd 48, Halbj. 2.

Dresden, Dr.-Ing.-Diss. v. 24. 7. 1903

1499. Herberg, Georg: Untersuchungen über die Exponenten der Ausdehnungslinie im Gasmotorendiagramme hinsichtlich ihrer Grösse und Veränderungen. Aus dem Maschinenlaboratorium B. der Kgl. Sächs. Technischen Hochschule zu Dresden. 1903. $40 \mathrm{~S} .4^{0}$ - Auch bei Calvary in Berlin; aus: Die Gasmotorentechnik. Bd 3. 1903/04.

Dresden, Dr.-Ing.-Diss. v. 24. 7. 1903

1500. Proell, Reinhold: Über den hydraulischen Wirkungsgrad von Turbinen bei ihrer Verwendung als Kraftmaschinen und Pumpen. Berlin: (Springer) 1903. 28 S., 3 Taf. $8^{0}$ - Buchausg. 1904. [Vergriffen.]

Dresden, Dr.-Ing.-Diss. v. 24. 7. 1903

1501. Grießmann, Arnoy staatl. gepr. Bauführer: Beitrag zur Frage der Erzeugungswärme des überhitzten Wasserdampfes und sein Verhalten in der Nähe der Kondensationsgrenze. Mitteilung aus dem Maschinenlaboratorium B der Technischen Hochschule in Dresden.> 1903. $57 \mathrm{~S} .4^{0}$ 
- Auch in: Mitteilungen über Forschungsarbeiten auf d. Gebiete d. Ingenieurwesens. H. 13. 1904; Ausz. in: Zeitschrift d. Vereines deutscher Ingenieure. Bd 47, Halbj. 2.

Dresden, Dr.-Ing.-Diss. v. 2. 12.1903

1502. Klemperer, Herbert: Versuche über den ökonomischen Einfluß der Kompression bei Dampfmaschinen. (Mitteilung aus dem Maschinenlaboratorium B der Technischen Hochschule zu Dresden.) 1904. 46 S. $4^{0}$ - Auch in: Mitteilungen über Forschungsarbeiten auf d. Gebiete d. Ingenieurwesens. H. 24. 1905; gekürzt in: Zeitschrift d. Vereines deutscher Ingenieure. Bd 49. 1905, Halbj. 1.

Dresden, Dr.-Ing.-Diss. v. 2. 12. 1903

1503. Gesell, Carl Walter: Die Leerlaufarbeit der Dampfmaschine. 〈Mitteilung aus dem Maschinenlaboratorium A der Technischen Hochschule in Dresden.> 1904. 74 S. $4^{0}$

Dresden, Dr.-Ing.-Diss. v. 26. 7. 1904

1504. Zeidler, Arthur: Über den Wirkungsgrad der Dampfkessel. 1905. 96 S. $8^{0}$

Dresden, Dr.-Ing.-Diss. v. 15. 3. 1905

1505. Förster, Ernst: Vergleichende Untersuchungen an Kreiselpumpen. 1905. $57 \mathrm{~S}$., 9 Taf. $8^{0}$ - Auch bei Trewendt \& Granier in Breslau.

Dresden, Dr.-Ing.-Diss. v. 28. 7. 1905

1506. Willkomm, otto: Beiträge zur mechanischen Technologie der Wirkerei: Ware und Wirkmuster an Rundstühlen. Leipzig: Martin's Textil-Verl. 1905. 66 S. $4^{0}$ - Aus: Leipziger Monatsschrift f. Textil-Industrie. Jg. 21. 1906.

Dresden, Dr.-Ing.-Diss. v. 28. 7. 1905

1507. Becker, Ernst： Strömungsvorgänge in ringförmigen Spalten und ihre Beziehungen zum Poiseuilleschen Gesetz. <Mitteilung aus dem Maschinenlaboratorium B der Technischen Hochschule zu Dresden.> 1906. 47 S. $4^{0}$ - Auch in: Mitteilungen über Forschungsarbeiten auf d. Gebiete d. Ingenieurwesens. H. 48. 1907; Ausz. in: Zeitschrift d. Vereines deutscher Ingenieure. Bd 51. 1907, Halbj. 2.

Dresden, Dr.-Ing.-Diss. v. 13. 12.1905

1508. Fritzsche, 0tto: Untersuchungen über den Strömungswiderstand der Gase in geraden zylindrischen Rohrleitungen. 1907. 64 S. $4^{0}$ - Auch als: Mitteilungen über Forschungsarbeiten auf d. Gebiete d. Ingenieurwesens. H. 60. 1908; Ausz. in: Zeitschrift d. Vereines deutscher Ingenieure. Bd 52. 1908, Halbj. 1.

Dresden, Dr.-Ing.-Diss. v. 22. 12. 1906

1509. Wobsa, Georg: Zustandsgleichung des Ammoniakdampfes und seine thermischen Eigenschaften. (Mitteilung aus dem
Maschinenlaboratorium B der Technischen Hochschule Dresden.) 1907. 54 S. $8^{0}$ T Ausz. in: Zeitschrift f. d. gesamte Kälte-Industrie. Jg. 14.

Dresden, Dr.-Ing.-Diss. v. 22. 12. 1906

1510. Gies, Heinrich: Der Einfluss des Spinnverfahrens auf die mittlere Haarlänge von Kammgarn. Berlin: (Verl. f. TextilIndustrie) 1907. $47 \mathrm{~S} .4^{0} \cdot$ Aus: Zeitschrift f. Textil-Industrie. Jg. 2.

Dresden, Dr.-Ing.-Diss. v. 13. 2. 1907

1511. Sieglerschmidt, Hermann: Die Wirkungsweise und Berechnung selbsttätiger Pumpen-Hubventile. 1907. 77 S., 3 Taf. $8^{0}$ - Vgl. Zeitschrift d. Vereines deutscher Ingenieure. Bd 52. 1908.

Dresden, Dr.-Ing.-Diss. v. 13. 3. 1907

1512. Heilemann, Walter: Beitrag zur Kenntnis des Wirkungsgrades trockener Luftkompressoren. 1908. 81 S. $4^{0}$ ๆ Auch als: Mitteilungen über Forschungsarbeiten auf d. Gebiete d. Ingenieurwesens. H. 58; Ausz. in: Zeitschrift $d$. Vereines deutscher Ingenieure. Bd 52 , Halbj. 1.

Dresden, Dr.-Ing.-Diss. v. 24. 7. 1907

1513. Nägel, Adolph: Versuche über die Zündgeschwindigkeit explosibler Gasgemische. 1907. $44 \mathrm{~S} .4^{0}$ ' Auch in: Mitteilungen über Forschungsarbeiten auf d. Gebiete d. Ingenieurwesens. H. 54. 1908; Ausz. in: Zeitschrift d. Vereines deutscher Ingenieure. Bd 52. 1908, Halbj. 1.

Dresden, Dr.-Ing.-Diss. v. 24. 7. 1907

1514. Dörffel, Ernst: Untersuchung an einer Kompressions-Kältemaschine an Ḧand der Messung der umlaufenden Ammoniakmengen. 1908. 24 S. $4^{0}$ Aus: Zeitschrift f. d. gesamte Kälte-Industrie. Jg. 15.

Dresden, Dr.-Ing.-Diss. v. 20. 12. 1907

1515. Jasinsky, Wsewolod: Ventilationsverlust in Dampfturbinen mit teilweiser Beaufschlagung. 1908. 64 S. $-4^{0}$ - Auch als: Mitteilungen über Forschungsarbeiten auf d. Gebiete d. Ingenieurwesens. H.67. 1909; Ausz. in: Zeitschrift d. Vereines deutscher Ingenieure. Bd 53. 1909, Halbj. 1.

Dresden, Dr.-Ing.-Diss. v. 12. 2. 1908

1516. Briling. Nikolai: Verluste in den Schaufeln von Freistrahldampfturbinen. 1908. 80 S. $4^{0}$ - Auch als: Mitteilungen über Forschungsarbeiten auf $d$. Gebiete d. Ingenieurwesens. H. 68. 1909; Ausz. in: Zeitschrift d. Vereines deutscher Ingenieure. Bd 54. 1910, Halbj. 1.

Dresden, Dr.-Ing.-Diss. v. 19. 2. 1908

1517. Young, Niels: Einfluß der Appretur auf die Festigkeitseigenschaften eines Kammgarngewebes 〈Serge〉. 1908. 88 S., 17 Taf. $8^{0}$

Dresden, Dr.-Ing.-Diss. v. 13. 3. 1908 
1518. Schneider, Heinrich: Utber die technologische Veränderung der Leinengarne durch den Bleichprozess. Leipzig: Leipz. Monatsschr. f. Textilind. 1908. 38 S. $4^{0}$ - Auch in: Leipziger Monatsschrift f. Textil-Industrie. Jg. 24. 1909.

Dresden, Dr.-Ing.-Diss. v. 3. 6. 1908

1519. Neumann, Kurt: Untersuchung des Arbeitsprozesses im Fahrzeugmotor. 1908. 54 S. $4^{0}$ - Auch als: Mitteilungen über Forschungsarbeiten auf d. Gebiete d. Ingenieurwesens. H. 79. 1909; Ausz. in: Zeitschrift d. Vereines deutscher Ingenieure. Bd 53. 1909, Halbj. 1.

Dresden, Dr.-Ing.-Diss. v. 18. 7. 1908

1520. Schrauff, Georg: Untersuchungen über den Arbeitsvorgang im Injektor. 1908. 56 S. $4^{0}$ Auch als: Mitteilungen über Forschungsarbeiten auf d. Gebiete d. Ingenieurwesens. H. 77. 1909 ; Ausz. in: Zeitschrift d. Vereines deutscher Ingenieure. Bd 53. 1909, Halbj. 1.

Dresden, Dr.-Ing.-Diss. v. 18. 7. 1908

1521. Schuster, Paul: Experimentelle Untersuchung der Strömungsvorgänge in einer Schnelläufer-Francis-Turbine, unter Anwendung einer neuen Methode zur Bestimmung von Stromrichtungen mit Pitotröhren. 1909. 74 S., 1 Taf. $4^{0}$ - Auch als: Mitteilungen über Forschungsarbeiten auf d. Gebiete d. Ingenieurwesens. H. 82. 1910; Ausz. in: Zeitschrift d. Vereines deutscher Ingenieure. Bd 54. 1910, Halbj. 2.

Dresden, Dr.-Ing.-Diss. v. 22. 12. 1908

1522. Arlt, Willy: Untersuchungen über Wetterführung mittels Lutten. 1910. 82 S. $4^{0}$ - Etwas gekürzt als: Mitteilungen über Forschungsarbeiten auf $d$. Gebiete d. Ingenieurwesens. H. 115. 1912; Ausz. in: Zeitschrift d. Vereines deutscher Ingenieure. Bd 56. 1912, Halbj. 2.

Dresden, Dr.-Ing.-Diss. v. 23. 7. 1909

1523. Doblhoff, Walther Freiherr von: Untersuchung von Automobilkühlern. 1910. $68 \mathrm{~S} .4^{0}$ ๆ Auch als: Mitteilungen über Forschungsarbeiten auf d. Gebiete d. Ingenieurwesens. H. 93; Ausz. in: Zeitschrift d. Vereines deutscher Ingenieure. Bd 54. 1910, Halbj. 1.

Dresden, Dr.-Ing.-Diss. v. 23. 7. 1909

15ं24. Plank, Rudolph: Thermodynamische Untersuchung des Vorganges in der Absorptions-Kältemaschine auf Grund der Theorie der binären Gemische. 1910. 31 S. $4^{0}$ - Aus:Zeitschriftf.d. gesamteKälte-Industrie.Jg.17. Dresden, Dr.-Ing.-Diss. v. 24. 11. 1909

1525. Schulze, Walter, Assist. d. Mechan.technolog. Inst. d. Techn. Hochsch. Dresden: Utber den Einfluß der einzelnen Appreturstufen auf die Wasser-, Licht-, Luft- und Wärmedurchlässigkeit eines Tuches. Leipzig: Martins Textil-Verl. 1910. 58 S. $8^{0}$ - Auch in: Leipziger Monatsschrift f.Textil-Industrie. Jg. 25. Dresden, Dr.-Ing.-Diss. v. 6. 7. 1910
1526. Bucher, Willy: Untersuchung über die Verbrennung methanhaltiger Gasgemische. 1910. $50 \mathrm{~S}, 4^{0}$ I Mit unwesentl. Änderungen in: Mitteilungen über Forschungsarbeiten auf $d$. Gebiete d. Ingenieurwesens. H. 117. 1912; Ausz. in: Zeitschrift d. Vereines deutscher Ingenieure. Bd 55. 1911, Halbj. 2.

Dresden, Dr.-Ing.-Diss. v. 20. 7. 1910

1527. Seyrich, Karl Arno: Ueber die Einwirkung des Ziehprozesses auf die wichtigsten, technischen Eigenschaften des Stahls. 1911. $70 \mathrm{~S} .4^{0}$ - A Auch als: Mitteilungen über Forschungsarbeiten auf d. Gebiete d. Ingenieurwesens. H. 119. 1912.

Dresden, Dr.-Ing.-Diss. v. 20. 7. 1910

1528. Lindig, Karl Otto: Über den Lieferungsgrad textiler Arbeitsmaschinen der Jutespinnerei und -Weberei. 1911. 54 S., 2 Taf. $8^{0}$

Dresden, Dr.-Ing.-Diss. v. 11. 3. 1911

1529. Dietr, otto: Über die spezifische Wärme von Faserstoffen. 1911. $69 \mathrm{~S} .8^{0}$ Dresden, Dr.-Ing.-Diss. v. 26. 7. 1911

1530. Kempf, Günther: Strömungsverlauf und einige Energieverluste in der Schiffsschraube. Berlin: Krayn [1911]. 31 S. $4^{0}$

Dresden, Dr.-Ing.-Diss. v. 29.11. 1911

1531. Riehm, Wilhelm: Die experimentelle Bestimmung des Ungleichförmigkeitsgrades. 1912. $48 \mathrm{~S} .4^{0}$ - Etwas gekürzt in: Mitteilungen über Forschungsarbeiten auf d. Gebiete d. Ingenieurwesens. H. 137. 1913; Ausz. in: Zeitschrift d. Vereines deutscher Ingenieure. Bd 57. 1913, Halbj. 2.

Dresden, Dr.-Ing.-Diss. v. 13. 3. 1912

1532. Gellert, Oswald: Eisen und Alteisen in ihren technischen und wirtschaftlichen Beziehungen. 1912. 78 S. $8^{0}$ A Auch als: Abhandlungen aus d. volkswirtschaftl. Seminar d. Techn. Hochschule zu Dresden. H. 4.

Dresden, Dr.-Ing.-Diss. v. 19. 6. 1912

1533. Pfaff, Georg, Kgl. Bauamtmann i. Dresden: Der Nutzen der Gegengewichte in den Rädern der Dampflokomotiven und der vollständige Ausgleich der Triebwerkmassen mit besonderer Berücksichtigung der neueren Schnellzuglokomotiven. [Dresden: Dressel] 1912. VI, 49 S., 5 Taf. $8^{0}$

Dresden, Dr.-Ing.-Diss. v. 10. 7. 1912

\section{Elektrotechnik}

1534. Seefehlner, E[gon] E[wald]: Beitrag zur Theorie der Synchron-Motoren und Wechselstromgeneratoren. Wien: Elektrotechn. Verein 1900. 59 S. $8^{0}$ ๆ Aus: Zeitschrift f. Elektrotechnik. Jg. 18.

Dresden, Dr.-Ing.-Diss. v. 27. 7. 1900 
15็35. Stockhausen, Karl: Untersuchungen über den eingeschlossenen Lichtbogen bei Gleichstrom. Leipzig: J. A. Barth 1907. VII, 97 S. $8^{0}$ Erw. u. d. T.: Der eingeschlossene Lichtbogen bei Gleichstrom.

Dresden, Dr.-Ing.-Diss. v. 6. 3. 1907

1536. Sarfert, W[illiam]: Ueber das Schwingen der Wechselstrommaschinen im Parallelbetrieb. 1908. 60 S. $4^{0}$ - Auch als: Mitteilungen über Forschungsarbeiten auf d. Gebiete d. Ingenieurwesens. H. 61 .

Dresden, Dr.-Ing.-Diss. v. 24. 7. 1907

1537. Wolf, Rudolf: Experimentelle Bestätigung des Vektorendiagramms für den Motor nach Winter-Eichberg-Latour. Mit 41 Fig. im Text. 1910. 63 S. $8^{0}$

Dresden, Dr.-Ing.-Diss. v. 30. 7. 1909

1538. Sehmiedel, Karl: Reibung von Elektrizitätszählern mit rotierendem Anker und der Einfluß der Reibung auf die Fehlerkurve. 1911. 47 S. $4^{0}$ Aus: Verhandlungen d. Vereins zur Beförderung d. Gewerbfleißes. Jg. 89. 90. 1910. 11.

Dresden, Dr.-Ing.-Diss. v. 7. 3. 1910

1539. Weicker, William: Zur Beurteilung von Hochspannungs-Freileitungs-Isolatoren, nebst einem Beitrag zur Kenntnis von Funkenspannungen. 1910. 99 S., 2 Taf. $4^{0}$ - Verkürzt in: Mitteilungen über Forschungsarbeiten anf d. Gebiete d. Ingenieurwesens. H. 100. 1911; Ausz. in: Zeitschrift d. Vereines deutseher Ingenieure. Bd 55. 1911, Halbj. 1.

Dresden, Dr.-Ing.-Diss. v. 30. 7. 1910

1540. Weidig, Paul: Die WechselstromInduktionsmaschine mit einachsiger Sekundärwicklung. $1912.74 \mathrm{~S} .4^{0}$

Dresden, Dr.-Ing.-Diss. v. 8. 3. 1911

1541. Dyhr, Erich: Die Entwicklung der Kommutator-Motoren für Einphasen-Wechselstrom auf Grund der deutschen Patentliteratur. Berlin: Springer 1912. 152 S. $8^{0}$ I Durch Patent-, Literatur- u. Sachverzeichnis erw. u. d. T.: Die Einphasen-Motoren nach den deutschen Patentschriften.

Dresden, Dr.-Ing.-Diss. v. 29. 11. 1911

\section{CHEMISCHE ABTEILUNG FÜR CHEMISCHE TECHNIK UND FABRIK- BETRIEB}

1542. Kegel, Ernst: Zur Frage der Konstitution der Paraoxyazokörper. 1900.58 S. $8^{0}$ - Vgl. Berichte d. Deutschen Chem. Gesellschaft. Jg. 33.

Dresden, Dr.-Ing.-Diss. v. 18. 7. 1900

1543. Krumbiegel, Ernst: Ueber einige Derivate des Triphenyltriazols und die zu ihrer Synthese dienenden Hydrazine und Nitrile. 1901. 79 S. $8^{0}$

Dresden, Dr.-Ing.-Diss. v. 27. 7. 1900

1544. Lax, Wilhelm: Über Abkömmlinge der Phenylhydrazoncyanessigsäureäthylester Leipzig: J. A. Barth 1901. 31 S. $8^{0}$ I Aus: Journal f. prakt. Chemie. Bd 171. N. F. Bd 63.

Dresden, Dr.-Ing.-Diss. v. 17. 11. 1900

1545. Bötteher, H[ans]: Über die Dissociationstemperaturen der Kohlensäure und des Schwefelsäureanhydrites. 1900. 65 S., 4 Taf. $8^{0}$

Dresden, Dr.-Ing.-Diss. v. 19. 12. 1900

1546. Mehner, Hans: Über Abkömmlinge der Anthranilsäure. Leipzig: J. A. Barth 1901. 76 S. $8^{0}$ - Aus: Journal f. prakt. Chemie. Bd 171. N. F. Bd 63.

Dresden, Dr.-Ing.-Diss. v. 8. 3. 1901

1547. Klimmer, Konrad: Über die Farbstoffe der Capriblau- und Phenocyaningruppe, ein Beitrag zur Kenntnis der Oxazinfarbstoffe. 1901. 6, $69 \mathrm{~S} .8^{0}$ ๆ Vgl. Zeitschrift f. Farben- u. Textil-Chemie. Jg. 1. 1902.

Dresden, Dr.-Ing.-Diss. v. 14. 3. 1901

1548. Strohbach, Erich: Ueber Derivate der 2,3-Oxynaphtoësäure. 1901. $51 \mathrm{~S} .8^{0}$ - Vgl. Berichte d. Deutschen Chem. Gesellschaft. Jg. 34 .

Dresden, Dr.-Ing.-Diss. v. 22. 5. 1901

1549. Wünsche, Osear: Zur Kenntnis der Sulfocarbanilide. 1901. 52 S. $8^{0}$

Dresden, Dr.-Ing.-Diss. v. 24. 5. 1901

1550. Sproesser, Ludwig: Über Alkalichlorid-Elektrolyse an Kohlenanoden. 1901. $101 \mathrm{~S} .8^{0}$ - Etwas gekürzt in: Zeitschrift f. Elektrochemie. Jg. 7. 1900/01.

Dresden, Dr.-Ing.-Diss. v. 27. 6. 1901

1551. Raetze, Walther: Beiträge zur Kenntnis des p-Chlorbenzaldehyds. 1901. $57 \mathrm{~S} .8^{0}$ Etwas gekürzt in: Journal f. prakt. Chemie. Bd 173. N. F. Bd 65. 1902.

Dresden, Dr.-Ing.-Diss. v. 23. 7. 1901

1552. Rosenthal, Josef: Ueber Condensation von Aldehyden mit Cyanessigester und Benzylcyanid. 1902. 43 S. $8^{0}$

Dresden, Dr.-Ing.-Diss. v. 24. 7. 1901

1553. Heiduschka, Alfred: Zur Kenntnis des p-Thio-p-tolylanilins. 1901. $55 \mathrm{~S} .8^{0}$ - Etwas gekürzt in: Journal f. praki. Chemie. Bd 176. N. F, Bd 68. 1903.

Dresden, Dr.-Ing.-Diss. v. 15. 11. 1901

1554. Schumacher, Willy: Über Abkömmlinge des Diacetonitrils und Benzoacetodinitrils. 1902.47 S. $8^{\circ}$ - Vgl. Journal f. prakt. Chemie. Bd 186. N. F. Bd 78. 1908. S. $499 \mathrm{ff}$. 532 if.

Dresden, Dr.-Ing.-Diss. v. 14. 3. 1902 
1555. Zimmermann, Max[imilian] Rich[ard]: Benzocyanaldoxim und Abkömmlinge. Leipzig: 'J. A. Barth 1902. 38 S. $8^{0}$ - Aus: Journal f. prakt. Chemie. Bd 174. N. F. Bd 66.

Dresden, Dr.-Ing.-Diss. v. 18. 7. 1902

1556. Rossleben, Alfred: Beitrag zur Kenntnis des Verhaltens von Phenyl-i-cyanat gegen Stickstoffverbindungen. 1902. 57 S. $8^{0}$

Dresden, Dr.-Ing.-Diss. v. 24. 7. 1902

1557. Meyer, Ernst: Zur Kenntnis der Thiotolyltoluidine. 1902. 47 S. $8^{0} \cdot \mathrm{Vgl}$. Journal f. prakt. Chemie. Bd 176. N. F. Bd 68. 1903.

Dresden, Dr.-Ing.-Diss. v. 25. 7. 1902

1558. Sommer, Albert: Über die Einwirkung von Aminen auf Derivate des Trinitro-p-toluidins. 1903. 59 S. $8^{0}$ - Mit unwesentl. Kürzungen in: Journal f. prakt. Chemie. Bd 175. N. F. Bd 67.

Dresden, Dr.-Ing.-Diss. v. 19. 1. 1903

1559. Thode, Carlos J[uan]: Zur Kenntnis des o-Amidobenzhydrazids und einiger Harnstoffderivate. 1903. 38 S. $8^{0}$ - Ausz. des 1. Teils in: Journal f. prakt. Chemie. Bd 177. N. F. Bd 69. 1904.

Dresden, Dr.-Ing.-Diss. v. 21. 1. 1903

1560. Zscheile. Arthur: Beitrag zur Kondensation von Alkyloxysäureestern mit Cyaniden und Ketonen. 1903. 42 S. $8^{0}$

Dresden, Dr.-Ing.-Diss. v. 6. 5. 1903

1661. Lehmann, Hermann: Zur Kenntnis der Polymeren des Acetonitrils. 1903. 40 S. $8^{0}$ - Vgl. Journal f. prakt. Chemie. Bd 186. N. F. Bd 78. 1908. S. $504 \mathrm{ff}$.

Dresden, Dr.-Ing.-Diss. v. 24. 7. 1903

1562. Grossmann, Albert: Beitrag zur Kenntnis der Amidine. 1903. 41 S. $8^{\circ}$ Vgl. Journal f. prakt. Chemie. Bd 186. N. F. Bd 78. 1908. Dresden, Dr.-Ing.-Diss. v. 30. 7. 1903

1563. König. Walter: I. Zur Kenntnis der Einwirkung von Nitrilen auf Karbonsäuren. II. Uber eine neue, vom Pyridin derivierende Klasse von Farbstoffen. Leipzig: J.A. Barth 1904. 72 S. $8^{0}$ Auch in: Journal f. prakt. Chemie. Bd 177. N.F. Bd 69.

Dresden, Dr.-Ing.-Diss. v. 30. 7. 1903

1564. Schönherr. Paul: Über das Verhalten von Abkömmlingen des Phenyl-icyanats zu Stickstoff- und Phosphorverbindungen, sowie über einige sekundäre Sulfochlorphosphine der aromatischen Reihe. 1903. 84 S. $8^{0}$

Dresden, Dr.-Ing.-Diss. v. 11. 11. 1903

1565. Friessner, Alfred: Ueber die elektrolytische Oxydation der schwefligsauren Salze und über die elektrochemische Bildung von Dithionat. 1904. 26 S. $4^{0}$ - Auch in: Zeitschrift f. Elektrochemie. Jg. 10.

Dresden, Dr.-Ing.-Diss. v. 3. 2. 1904

1566. Kretzschmar, Horst: Ueber die Einwirkung von Brom auf Alkali und über die Elektrolyse der Bromalkalien. 1904. 32 S. $4^{0}$ - Auch in: Zeitschrift f. Elektrochemie. Jg. 10.

Dresden, Dr.-Ing.-Diss. v. 17. 3. 1904

1567. Steimmig, Franz: Zur Theorie der Beizfärbungen. 1904. 94 S. $8^{0}$ Verkürzt in: Zeitschrift f. Farben- u. Textil-Industrie. Jg. 3.

Dresden, Dr.-Ing.-Diss. v. 17. 3. 1904

1568. Müller, Richard: Beiträge zur Kenntnis der Phosphorfabrikation. [1904.] 87 S. $8^{0}$ Vgl. Zeitschrift f. angewandte Chemie. Jg. 18, 1. 1905. S. $132 \mathrm{ff}$.

Dresden, Dr.-Ing.-Diss. v. 14. 4. 1904

1569. Hermsdorf, Walter: Zur Kenntnis der Polymerisation von Nitrilen. 1904. 51 S. $8^{0}$ - Vgl. Berichte über d. Verhandlungen d. Kgl. Sächs. Gesellschaft d. Wissenschaften zu Leipzig. Math.phys. Klasse. Bd 57. 1905. S. $324 \mathrm{ff}$.

Dresden, Dr.-Ing.-Diss. v. 27. 4. 1904

1570. Bamberg, Raimund: Über Chinazoline aus Ortho-Amido-meta-Xylyl-paraToluidin. 1904. 69 S. $8^{0}$ - Ausz. in: Journal f. prakt. Chemie. Bd 181. N. F. Bd 73. 1906.

Dresden, Dr.-Ing.-Diss. v. 20. 7. 1904

1571. Markert, Fritz: Über eine neue Methode zur Bestimmung des Sauerstoffs in organischen Körpern. 1904. 67 S. $8^{0}$

Dresden, Dr.-Ing.-Diss. v. 20. 7. 1904

1572. Boericke, Felix: Ueber das elektromotorische Verhalten des Broms und das Anodenpotential bei der Elektrolyse neutraler Bromkaliumlösungen. 1904. 38 S. $4^{0}$ - Auch in: Zeitschrift f. Ellektrochemie. Jg. 11. 1905.

Dresden, Dr.-Ing.-Diss. v. 29. 7. 1904

1573. Groseh, Oskar: Über die Kondensation von Dinitrilen mit Phenolen. 1904. 55 S. $8^{0}$ - Vgl. Journal f. prakt. Chemie. Bd 175. N. F. Bd 67. 1903.

Dresden, Dr.-Ing.-Diss. v. 23. 11. 1904

1574. Neuhäusser, Hans: Über einige Umsetzungen der Diazobenzolcarbonsäuren. 1904. 55 S. $8^{0}$

Dresden, Dr.-Ing.-Diss. v. 17. 12.1904

1575. Bültemann, August: Über den Einfluss des Anodenmaterials auf Anodenvorgänge. 1905. 98 S. $8^{0}$

Dresden, Dr.-Ing.-Diss. v. 20. 12. 1904

1576. Schulze, Armin: Über Abkömmlinge des Ortho-Oxychinolins. $1905.40 \mathrm{~S} .8^{0}$ Dresden, Dr.-Ing.-Diss. v. 28. 1. 1905 
1577. Pohl, Franz: Über Abkömmlinge des Dicyandiamids und die Frage seiner Konstitution. 1905. 63 S. $8^{0}$ Ausz. in: Journal f. prakt. Chemie. Bd 185. N. F. Bd 77. 1908.

Dresden, Dr.-Ing.-Diss. v. 15. 3. 1905

1578. Hofstädter, Erich: Über das Eindringen von Bakterien in feinste Capillaren. Mit 1 Taf. 1905. 63 S., 1 Taf. $8^{0}$ - Aus: Archiv f. Hygiene. Bd 53.

Dresden, Dr.-Ing.-Diss. v. 17. 3. 1905

1579. Greifenhagen, Heinrich: ZurKenntnis der Sulfocarbanilide. 1905. 59 S. $8^{0}$ ๆ Vgl. Journal f. prakt. Chemie. Bd 183. N. F. Bd 75. 1907. S. $188 \mathrm{ff}$.

Dresden, Dr.-Ing.-Diss. v. 28. 6. 1905

1580. Fischer, Paul: Triphenylchlormethan in seinen chemischen Wirkungen als Säurechlorid. 1905. 46 S. $8^{0}$ ๆ Vgl. Journal f. prakt. Chemie. Bd 190. N. F. Bd 82.1910. S. 522 ff.

Dresden, Dr.-Ing.-Diss. v. 26. 7. 1905

1581. Kaiser, Fritz: Kritische Experimentaluntersuchung über die verschiedenen Aufschlussmethoden der Silikate. Untersuchung über die Möglichkeit der Trennung der Magnesia vom Kalium, gegründet auf die verschiedene Löslichkeit der Sulfate in Methylalkohol und Klarlegung der hierbei auftretenden mannigfach verschiedenen Krystalle. $1905.57 \mathrm{~S} ., 1$ Taf. $8^{0}$ - Vgl. Zeitschrift f. analyt. Chemie. Jg. 52. 1913. S. $86 \mathrm{ff}$.

Dresden, Dr.-Ing.-Diss. v. 26. 7. 1905

1582. Lehmann, Arthur: Einwirkung von 2,4 Dinitrochlorbenzol und Pikrylchlorid auf Amidokörper. 1905. 44 S. $8^{0}$

Dresden, Dr.-Ing.-Diss. v. 26. 7. 1905

1583. Scheffler, Wilhelm: Beiträge zur Kenntnis der Westerwaldtone und zur Praxis der Steinzeugindustrie. Mit 32 Tab. 1905. $\mathrm{V}, 110$ S. $8^{\circ}$

Dresden, Dr.-Ing.-Diss. v. 26. 7. 1905

1584. Seidel, Arno: Zur Kenntnis von Mono- und Diaethyl-m-amidophenol und ihren Abkömmlingen. 1905. 46 S. $8^{0}$

Dresden, Dr.-Ing.-Diss. v. 26. 7. 1905

1585. Pinister, Paul: Über Chinazoline aus Thioharnstoffen. 1905. 54 S. $8^{0}$

Dresden, Dr.-Ing.-Diss. v. 24. 11. 1905

1586. Grolée, André: Über Nitrile arylierter Glycine. 1906. $74 \mathrm{~S} .8^{0}$ I Vgl. Berichte d. Deutschen Chem. Gesellschaft. Jg. 39.

Dresden, Dr.-Ing.-Diss. v. 20. 12. 1905

1587. Litter, Hans: Beitrag zur Frage der Konstitution des Murexids und der Purpursäure. 1905. $89 \mathrm{~S} .8^{0}$ T Ausz. in: Journal f. prakt. Chemie. Bd 181. N. F. Bd 73. 1906.

Dresden, Dr.-Ing.-Diss. v. 20. 12. 1905
1588. Sahland, Hugo: Zur Kenntnis des Carbonylaminophenols und Carbonylaminonaphtols sowie einiger Abkömmlinge. 1906. 38 S. $8^{0}$

Dresden, Dr.-Ing.-Diss. v. 21. 2. 1906

1589. Adam, Richard: Utber den Einfluß der Kohlenstoffdoppelbindung auf die Farbe von Azomethinverbindungen. 1906. 60 S. $8^{0}$ $\mid$ Vgl. Zeitschrift f. Farben-u. Textil-Industrie.Jg. 5.

Dresden, Dr.-Ing.-Diss. v. 7. 3. 1906

1590. Lohse, Fritz: Die Bromcyanpyridinreaktion und ihre Anwendung auf einige Arylamin-sulfon- und -carbonsäuren. 1906. 84 S. $8^{0}$

Dresden, Dr.-Ing.-Diss. v. 7. 3. 1906

1591. Sehwalbe, $\mathbf{A}[\mathrm{rthur}]$ : Über $\Omega$-Sulfonsäuren und $\Omega$-Cyanide aromatischer Amine. 1906. $71 \mathrm{~S} .8^{0}$ - Vgl. Berichte d. Deutschen Chem. Gesellschaft. Jg. 39.

Dresden, Dr.-Ing.-Diss. v. 7. 3. 1906

1592. Saring, Georg: Versuche über den Aufschluß von Phosphaten durch Kieselsäure bei hohen Temperaturen. [1906.]42 S. $8^{0}$

Dresden, Dr.-Ing.-Diss. v. 16. 3. 1906

1593. Beyer, Arthur: UUber die elektroanalytische Trennung von Cadmium und Zink. 1906. 86 S. $8^{\circ}$

Dresden, Dr.-Ing.-Diss. v. 18. 7. 1906

1594. Heymann, Osear F[riedrich]: Ueber den Kammerprozeß der Schwefelsäure und die Bestimmung von Stickoxydul in Kammergasen. 1906. $68 \mathrm{~S} .8^{0}$ - Vgl. Zeitschrift f. Elektrochemie. Jg. 12 . S. $600 \mathrm{ff}$.

Dresden, Dr.-Ing.-Diss. v. 18. 7. 1906

1595. Seyde, Franz: Beiträge zur Kenntnis der Sulfitreaktion. 1906. 94 S. $8^{\circ}$ II Ausz. in: Journal f. prakt. Chemie. Bd 183. N. F. Bd 75. 1907.

Dresden, Dr.-Ing.-Diss. v. 19. 7. 1906

1596. Lee, Harry: Über den Wasserstoffgehalt des Elektrolyteisens. 1906. $72 \mathrm{~S}$. $8^{0}$ - Vgl. Zeitschrift f. Elektrochemie. Jg. 13. 1907. S. $561 \mathrm{ff}$; F. Foerster: Beiträge zur Kenntnis d. elektrochem. Verhaltens d. Eisens. Halle 1909. (Abhandlungen d. Deutschen Bunsen-Gesellschaft f. angewande phys. Chemie. Nr. 2.) S. 59 ff.

Dresden, Dr.-Ing.-Diss. v. 24. 7. 1906

1597. Verbeek, Paul: Beiträge zur Kenntnis der Darstellung des Schwefelkohlenstoffs. 1906. $85 \mathrm{~S} .8^{0}$

Dresden, Dr.-Ing.-Diss. v. 24. 10. 1906

1598. Saring, Benno: Versuche über eine Methode zur Bestimmung des Sauerstoffes in organischen Körpern und Zersetzung organischer Körper bei bestimmter konstanter Temperatur. 1907. 36 S. $8^{0}$

Dresden, Dr.-Ing.-Diss. v. 12. 12. 1906 
1599. Näbe, Fritz: Zur Kenntnis des Cyanurbromids. 1907. $51 \mathrm{~S} .8^{\circ} \bullet \mathrm{Vgl}$. Journal f. prakt. Chemie. Bd 186. N.F. Bd 78. 1908. S. $531 \mathrm{ff}$. Dresden, Dr.-Ing.-Diss. v. 6. 2. 1907

1600. Seidel, Friedrich: Studien über den Zellulosedarstellungsprozess und eine Methode für Bestimmung des Reinheitsgrades von Zellulosen. 1907. 81 S. $8^{0}$

Dresden, Dr.-Ing.-Diss. v. 13. 2. 1907

1601. Henning, Wilhelm: Beitrag zur Kenntnis der tri- und dimolekularen Nitrile. 1907. 50 S. $8^{0}$ - Vgl. Journal f. prakt. Chemie. Bd 186. N. F. Bd 78. 1908. S. $516 \mathrm{ff}$.

Dresden, Dr.-Ing.-Diss. v. 6. 3. 1907

1602. Irmseher, Camillo: Utber Kondensation von Dinitrilen mit ungesättigten Ketonen, sowie Ketonsäureestern. 1907. 49 S. $8^{0}$ - Vgl. Journal f. prakt. Chemie. Bd 186. N. F. Bd 78. 1908. S. $524 \mathrm{ff}$.

Dresden, Dr.-Ing.-Diss. v. 6. 3. 1907

1603. Rother, Paul B[ernhard]: Die Bestimmung von Aldehyde und Ketone zur Bewertung ätherischer Öle. 1907. 47 S. $8^{0}$ Dresden, Dr.-Ing.-Diss. v. 13. 3. 1907

1604. Blankenberg, Ferdinand: Uber die elektrolytische Abscheidung von Zink, Nickel und Kupfer aus ammoniakalischer Lösung. Budapest: Weiss [1907]. 95 S., 2 Taf. $8^{0}$ Vgl. Zeitschrift f. Elektrochemie. Jg. 13. S. $561 \mathrm{ff}$.

Dresden, Dr.-Ing.-Diss. v. 17. 7. 1907

1605. Koerner, Theo: Zur Frage der Bildung von Alkohol aus cellulosehaltigen Stoffen. 1907. 53 S. $8^{0}$ - Ausz. in: Zeitschrift f. angewandte Chemie. Jg. 21, 2. 1908. S. $2353 \mathrm{ff}$.

Dresden, Dr.-Ing.-Diss. v. 21. 11. 1907

1606. Nicolaus, Arthur: Zur Kenntnis des Diphenylcarbaminsäurechlorids. 1907. $42 \mathrm{~S} .8^{0}$ - Vgl. Journal f. prakt. Chemie. Bd 190. N. F. Bd 82. 1910. S. $526 \mathrm{ff}$.

Dresden, Dr.-Ing.-Diss. v. 21. 11. 1907

1607. Herrsehel, Paul: Über Kondensationen von Phenoxylessigester mit Cyaniden. 1907. 67 S. $8^{0}$ - Vgl. Journal f. prakt. Chemie. Bd 191. N. F. Bd 83. 1911. S. $173 \mathrm{ff}$.

Dresden, Dr.-Ing.-Diss. v. 6. 12. 1907

1608. Römmler, Willy: Uber den Wasserstoffgehalt des Elektrolytnickels. 1908. 51 S., 1 Taf. $8^{0}$ Vgl. Zeitschrift f. Elektrochemie. Jg. 13. 1907. S. $561 \mathrm{ff}$.

Dresden, Dr.-Ing.-Diss. v. 11. 12. 1907

1609. Rothe, Alfred: Uber die Adsorption von Silbernitrat und Jodkalium durch Jodsilber. 1908. $43 \mathrm{~S} .8^{\circ}$ - Ausz. in: Zeitschrift f. physik. Chemie. Bd 62 . S. $359 \mathrm{ff}$.

Dresden, Dr.-Ing.-Diss. v. 20. 12. 1907
1610. Köhler, Alfred: Uber den Einfluß der Bäuche und Bleiche auf die Kapillarität der Baumwolle. Dresden: Kleinhempel 1908. 56 S. $8^{0}$

Dresden, Dr.-Ing.-Diss. v. 22. 1. 1908

1611. Roch, Heinrich: Zur Kenntnis der Thiazole. 1908. 69 S. $8^{0}$ o Vgl. Journal f. prakt. Chemie. Bd 195. N. F. Bd 87. 1913.

Dresden, Dr.-Ing.-Diss. v. 11. 3. 1908

1612. Ulrich, Henry: Über Isatosäureanhydrid. 1908. $61 \mathrm{~S} .8^{\circ}$ V Vl. Journal f. prakt. Chemie. Bd 117. N. F. Bd 79. 1909.

Dresden, Dr.-Ing.-Diss. v. 11. 3. 1908

1613. Jacoby, Hans: Über die Bildung von Kalkstickstoff. 1908. 86 S. $8^{0}$ - Ausz. in: Zeitschrift f. Elektrochemie. Jg. 13. 15. 1907. 09.

Dresden, Dr.-Ing.-Diss. v. 12. 3. 1908

1614. Koch, Max: Beiträge zur Kenntnis der Einwirkung von nitrosen Gasen und Sauerstoff auf Wasser. 1908. 116 S. $8^{\circ}$ I Ausz. in: Zeitsehrift f. angewandte Chemie. Jg. 21, 2. S. $2161 \mathrm{ff}$.

Dresden, Dr.-Ing.-Diss. v. 13. 3. 1908

1615. Wolf, Johannes: Beiträge zur quantitativen Bestimmung und Trennung von Antimon und Zinn durch Elektrolyse aus den Lösungen ihrer Sulfosalze in Schwefelalkalilösungen. 1908. 142 S. $8^{0}$ - Vgl. Zeitschrift f. Elektrochemie. Jg. 13. 1907.

Dresden, Dr.-Ing.-Diss. v. 29. 4. 1908

1616. Sehmidt, Maximilian P[aul]: Über die Einwirkung von Bisulfit auf Hydrazine spez. Naphtylhydrazine. 1908. 70 S. $8^{0}$ T Ausz. in: Journal f. prakt. Chemie. Bd 187. N. F. Bd 79. 1909.

Dresden, Dr.-Ing.-Diss. v. 30. 6. 1908

1617. Starke, Kurt: Zur Kenntnis der Dinitrile. 1908. 51 S. $8^{0}$ ๆ Vgl. Journal f. prakt. Chemie. Bd 186. N. F. Bd 78 . S. $521 \mathrm{ff}$.

Dresden, Dr.-Ing.-Diss. v. 2. 7. 1908

1618. Schwabe, Erwin: Zersetzungsweisen tetraalkylierter Ammoniumverbindungen. 1908. 53 S. $8^{0}$ - Vgl. Abhandlungen d. math.-phys. Klasse d. Kgl. Sächs. Gesellschaft d. Wissenschaften. Bd 31. (Abhandlungen d. Kgl. Sächs. Gesells. d. Wiss. Bd 58.) 1909. S. $177 \mathrm{ff}$. Dresden, Dr.-Ing.-Diss. v. 16. 7. 1908

1619. Würzner, Kurt: Zur Kenntnis der Oxythiazoline. 1908. 38 S. $8^{0}$

Dresden, Dr.-Ing.-Diss. v. 16. 7. 1908

1620. Böttcher, Martin: Über die Verflüssigung des Tones durch Alkali. 1908. 74 S., 3 Taf. $8^{0}$ ๆ Auch in: Sprechsaal. Jg. 42. 1909, Halbj. 1.

Dresden, Dr.-Ing.-Diss. v. 17. 7. 1908 
1621. Gebauer, Rudolf: Beiträge zur Kenntnis des Aminokaffeïns, des Oxykaffeïns, des Thiokaffeïns und ihrer Derivate. 1908. 74 S. $8^{0}$

Dresden, Dr.-Ing.-Diss. v. 17. 7. 1908

1622. Mustad, Ole: Abscheidungspotential des Eisens aus seinen Sulfat- und Chlorürlösungen bei verschiedenen Temperaturen. 1908. 46 S., 1 Taf. $8^{0}$ - Vgl. F. Foerster: Beiträge zur Kenntnis des elektrochem. Verhaltens d. Eisens. Halle 1909. (Abhandlungen d. Deutschen BunsenGesellschaft f. angewandte phys. Chemie. Nr. 2.) S. $34 \mathrm{ff}$.

Dresden, Dr.-Ing.-Diss. v. 17. 7. 1908

1623. Oettel, Alfred: Über die elektrolytische Abscheidung des Magnesiums. 1908. 71 S. $8^{0}$

Dresden, Dr.-Ing.-Diss. v. 27. 7. 1908

1624. Weger, Kurt: Utber die elektrolytische Reduktion von Chloraten an Eisenkathoden. 1908. $89 \mathrm{~S} .8^{0}$

Dresden, Dr.-Ing.-Diss, v, 28. 7. 1908

1625. Uhlmann, Armin: Über die Anwendung der Sulfitreaktion auf einige Ana〈1.5.)-Derivate des Naphtalins. 1908. $83 \mathrm{~S}$. $8^{0}$ - Ausz. in: Journal f. prakt. Chemie. Bd 188. N. F. Bd 80. 1909 .

Dresden, Dr.-Ing.-Diss. v. 23. 11. 1908

1626. Sonnenburg, Ernest Friedrich: Beiträge zur Anwendung der Sulfitreaktion. 1908. 85 S. $8^{0}$ Vgl. Journal f. prakt. Chemie. Bd 189. N. F. Bd 81. 1910.

Dresden, Dr.-Ing.-Diss. v. 25. 11. 1908

1627. Friese, Walther: Beiträge zur Kenntnis des Staubes in der Stadtluft. 1909. 53 S. $8^{\circ}$ - Etwas abgeändert in: Deutsche Vierteljahrsschrift f. öffentl. Gesundheitspflege. Bd44.1912.

Dresden, Dr.-Ing.-Diss. v. 19. 12. 1908

1628. Richter, Erich: Studien über die Bestimmung von Äthan neben Methan und Wasserstoff. 1909. 56 S. $8^{0}$

Dresden, Dr.-Ing.-Diss. v. 19. 12. 1908

1629. Büttner, Georg: Versuche zur Destillation des Holzes mit überhitztem Wasserdampf. Leipzig: J. A. Barth 1909. 65 S. $8^{0}$ - Mit gekürzter Einl. in: Journal f. prakt. Chemie. Bd 187. N. F. Bd 79.

Dresden, Dr.-Ing.-Diss. v. 5. 2. 1909

1630. Hager, Georg: Kulturversuche mit höheren Pflanzen über die Aufnahme und organische Verteilung von Strontium, Baryum, Magnesium neben und in Vertretung von Calcium. 1909. 100 S. $8^{\circ}$

Dresden, Dr.-Ing.-Diss. v, 5. 2. 1909

1631. Richter, Johannes: Untersuchung von Schwefelsäurekammergasen. $1909.65 \mathrm{~S} .8^{\circ}$

Dresden, Dr.-Ing.-Diss. v. 24. 2. 1909
1632. Lindemann, 0tto: Beiträge zur Kenntnis der Einwirkung von Natronlauge auf Baumwolle. 1909. 56 S., 4 Taf. $8^{0}$

Dresden, Dr.-Ing.-Diss. v. 11. 3. 1909

1633. Sehweitzer, Alexander: Beiträge zur Kenntnis des elektrochemischen Verhaltens des Nickels. 1909. 60 S. $8^{\circ}$ - Ausz. in: Zeitschrift f. Elektrochemie. Jg. 15.

Dresden, Dr.-Ing.-Diss. v. 21. 4. 1909

1634. Eichel, Curt, Kgl. Preuß. Leutnant a. D.: Über das Verhalten des Stickstoffs gegen Silicide. 1909. 74 S., 1 Taf. $8^{0}$

Dresden, Dr.-Ing.-Diss. v. 22. 5. 1909

1635. Ostertag, Aug[ust]: Beiträge zur Kenntnis der Beizenfärbungen. 1909. 66 S. $8^{0}$ - $\mathrm{Zu}$ Abschn. $3 \mathrm{vgl}$. Zeitschrift für angewandte Chemie. Jg. 22, 2. S. $2030 \mathrm{ff}$.

Dresden, Dr.-Ing.-Diss. v. 21. 7. 1909

1636. Zsehimmer, Bodo: Zur Kenntnis der Kondensationen von Alkoholen und Äthern mit Kohlenwasserstoffen und Phenolen. 1909. $48 \mathrm{~S} .8^{0}$ - Vgl. Journal f. prakt. Chemie. Bd 190. N. F. Bd 82. 1910. S, $538 \mathrm{ff}$. Dresden, Dr.-Ing.-Diss. v. 21. 7. 1909

1637. Nobis, Alfred: Die WasserstoffChlorkette. 1909. 102 S. $8^{0}$

Dresden, Dr.-Ing.-Diss. v. 22. 7. 1909

1638. Korn, Rudolph: Untersuchungen über die technisch-mikroskopische Unterscheidung einiger Fasern, insbesondere der Hanf- und Leinenfaser. Berlin: Borntraeger 1910. S. $189-234,1$ Taf. $8^{\circ}$ - Aus: Jahresbericht d. Vereinigung f. angewandte Botanik. Jg. 7. 1909. Dresden, Dr.-Ing,-Diss. v. 23. 7. 1909

1639. Bossel, Gustav N[ikolaus]: Zur Kenntnis der o-Amidosalicylsäure. 1909. 45 S. $8^{0}$

Dresden, Dr.-Ing.-Diss. v. 24. 11. 1909

1640. Ehlert, Hermann: Studien über Salzlösungen. 1909. VI, 75 S. $8^{0}$ - Vgl. Zeitschrift f. Elektrochemie u. angewandte phys. Chemie. Jg. 18. 1912

Dresden, Dr.-Ing.-Diss. v. 24. 11. 1909

1641. Blieh, Julius: Über die Oxydation von Stickoxydluftgemischen und ihre Löslichkeit in Alkalilauge. 1910. VI, $64 \mathrm{~S} .8^{0}$ - Ausz. in: Zeitschrift f. angewandte Chemie. Jg. 23, 2. S. $2017 \mathrm{ff}$.

Dresden, Dr.-Ing.-Diss. v. 21. 12. 1909

1642. Klemperer, Ralph L[eopold]: Über quantitative Spektralanalyse. $1910.75 \mathrm{~S} .8^{0}$ - Kurzer Ausz, in: Zeitschrift f. angewandte Chemie. Jg. 23, 2. S. $1756-59$.

Dresden, Dr.-Ing.-Diss. v. 21. 12. 1909

1643. Pordeseh, Horst: Zur Kenntnis der Thiodiglycolsäure und Thioglycolsäure. 1909. 57 S. $8^{\circ}$

Dresden, Dr.-Ing.-Diss. v. 21. 12. 1909 
1644. Vater, Georg: Studien über die Adsorption von Gasen durch Kohle und einige andere poröse Körper. 1910. 68 S. $8^{\circ} \bullet \mathrm{Vgl}$. Zeitschrift f. Elektrochemie u. angewandte phys. Chemie. Jg. 18. 1912.

Dresden, Dr.-Ing.-Diss. v. 2. 2. 1910

1645. Vetter, Hermann: Über Schwefelfarbstoffe aus 1.2.4-Dinitrophenol. 1910. 70 S. $8^{0}$

Dresden, Dr.-Ing.-Diss. v. 2. 2. 1910

1646. Doleh, Moritz: Das Verhalten von Zinnanoden in Natronlauge. 1911. VI, 109 S. $8^{0}$ - Vgl. Zeitschrift f. Elektrochemie. Jg. 16. 1910. Dresden, Dr.-Ing.-Diss. v. 10. 3. 1910

1647. Sehreckenbaeh, Rudolf: Beiträge zur Kenntnis der Reaktionsfähigkeit von in $\beta$-Stellung nicht substituierten Indolen. 1910. 66 S. $8^{0}-\mathrm{Vgl}$. Journal f. prakt. Chemie. Bd 195. N. F. Bd 87. 1913.

Dresden, Dr.-Ing.-Diss. v. 11. 5, 1910

1648. Grafe, Ernst: Zur Darstellung von Methenylamidinen mittels des o-Ameisensäureäthylesters. 1910. $41 \mathrm{~S} .8^{\circ}$

Dresden, Dr.-Ing.-Diss. v. 12. 5. 1910

1649. Joost, Kurt: Beiträge zur Kenntnis der elektrolytisehen Sauerstoffentwicklung an Kohleanoden. 1910. 73 S. $8^{0}$

Dresden, Dr.-Ing.-Diss. v, 13. 5. 1910

1650. Sprent, Colin: Verhalten von Antimon bei der Kupferraffination. 1910. 70 S. $8^{0}$ I Vgl. Zeitschrift f. Elektrochemie. Jg. 18. 1912. S. 294 f.

Dresden, Dr.-Ing.-Diss. v. 1. 6. 1910

1651. Schildbach, Richard: Über das elektrochemische Verhalten des Kobalts. 1910. 62 S. $8^{0}$ - Ausz. in: Zeitschrift f. Elektrochemie. Jg. 16.

Dresden, Dr.-Ing.-Diss. v. 22. 6. 1910

1652. Neuman, Joseph: Kritische Studien über Hydrolyse der Cellulose und des Holzes. Dresden: Holze \& Pahl 1910. 80 S. $8^{0}$

Dresden, Dr.-Ing.-Diss. v. 15. 7. 1910

1653. Lierg, Friedrich H[ermann]: Beiträge zurChemie des Verkokungsprozesses. 1910. 57 S. $8^{0}$ - Ausz. in: Zeitschrift f. angewandte Chemie. Jg. 24, 2. 1911.

Dresden, Dr.-Ing.-Diss. v. 20. 7. 1910

1654. Weber, Max Gustav: Kritische Studien über die Darstellungsweisen von Selen- und Tellurwasserstoff. 1910.63 S. $8^{0}$ - Vgl. Zeitschrift f. anorgan. Chemie. Bd 77. 1912. Dresden, Dr.-Ing.-Diss. v. 21. 7. 1910

1655. Tedeseo, Hermann: Studien über den Ammoniaksodaprozeß. 1910. 77 S., 2 Taf. $8^{0} \bullet \mathrm{Vgl}$. Zeitschrift f. angewandte Chemie. Jg. 24, 2. 1911 .

Dresden, Dr.-Ing.-Diss. v. 22. 7. 1910
1656. Becker, Georg Albert: Über den Zusammenhang zwischen Farbe und Konstitution der Pyridinfarbstoffe aus sekundären Aminen. 1910. 57 S. $8^{0}$ - Erw. u. verändert in: Journal f. prakt. Chemie. Bd 193. N. F. Bd 85. 1912.

Dresden, Dr.-Ing.-Diss. v. 23. 7. 1910

1657. Eisenreich, Kurt: Über die Verwendung von Silberfluoridlösungen im Silbercoulometer. Leipzig: W. Engelmann 1911. 51 S. $8^{0}$ - Aus: Zeitschrift f. physik. Chemie. Bd 76 .

Dresden, Dr.-Ing.-Diss. v. 25. 7. 1910

1658. Sehauseil, Walther: Versuche um eine für die Kohrener und Frohburger Topfwarenindustrie geeignete Glasur herzustellen, welche allen sanitären Anforderungen genügt. 1910.59 S. $8^{0}$

Dresden, Dr.-Ing.-Diss. v. 25. 7. 1910

1659. Schubert, Carl: Beiträge zur Kenntnis der Dissoziation einiger Oxyde, Karbonate und Sulfide. 1910. 72 S. $8^{0}$. Ermittelte Dissoziationstemperaturen in: Zeitschrift f. Elektrochemie u. angewandte phys. Chemie. Jg. 18. 1912.

Dresden, Dr.-Ing.-Diss. v. 2. 11. 1910

1660. Voigt, Wilhelm: Die Einwirkung von unterbromigsaurem Natron auf organische stickstoffhaltige Verbindungen. 1911. 50 S. $8^{0}$

Dresden, Dr.-Ing.-Diss. v. 7. 12. 1910

1661. Jaeob, Artur: Das Verhalten der salpetrigen Säure in Wasser. 1911. $71 \mathrm{~S} .8^{0}$

Dresden, Dr.-Ing.-Diss. v. 16. 12. 1910

1662. Otto, Johannes: Versuche über die direkte Gewinnung von Aceton 〈Ketonen〉 aus Holzabfällen unter besonderer Berücksichtigung der dabei auftretenden Gase. 1911. 93 S. $8^{0}$

Dresden, Dr.-Ing.-Diss. v. 19. 12. 1910

1663. Früh, Jean: Über die Abscheidung von Eisen und Nickel aus komplexen Oxalatund Laktatlösungen. 1911. $82 \mathrm{~S} .8^{0}$

Dresden, Dr.-Ing.-Diss. v. 20.12. 1910

1664. Näf, Ernst: Versuche zur Theorie der elektrolytischen Weißblechentzinnung. 1911. 101 S. $8^{0}$

Dresden, Dr.-Ing.-Diss. v. 20.12. 1910

1665. Oehler, Rudolf: Über neue $A b-$ kömmlinge von dimolekularen Nitrilen. 1911. 57 S. $8^{0}$

Dresden, Dr.-Ing.-Diss. v. 8. 3. 1911

1666. Engemann, Karl: Uber das Abblättern des Elektrolytnickels. 1911. $109 \mathrm{~S}$., 4 Taf. $8^{0}$ - Ausz. in: Zeitschrift f. Elektrochemie. Jg. 17.

Dresden, Dr.-Ing.-Diss. v. 9. 3. 1911 
1667. Viertel, Artur: Beitrag zur Kenntnis des 1 -Aminoanthrachinons. 1911. $67 \mathrm{~S} .8^{\circ}$ I Vgl. Berichte d. Deutschen Chem. Gesellschaft. Jg. 45. 1918. S. $2233 \mathrm{ff} .2244 \mathrm{ff}$.

Dresden, Dr.-Ing.-Diss. v. 10. 3. 1911

1668. Maetzel, Johannes: Beiträge zur Kenntnis der Farblacke. 1911. $66^{\circ} \mathrm{S} .8^{0}$ I Vgl. Berichte d. Deutschen Chem. Gesellschaft. Jg. 46. 1913. S. $443 \mathrm{ff}$.

Dresden, Dr.-Ing.-Diss. v. 30. 6. 1911

1669. Redlich, Alfred: Über Additionsreaktionen der para-Benzochinone. 1911. 63 S. $8^{0}$

Dresden, Dr.-Ing.-Diss. v. 30. 6. 1911

1670. Friedrich, Richard: Über neue Abkömmlinge der dimolekularen Nitrile. 1911. 64 S. $8^{\circ}$

Dresden, Dr.-Ing.-Diss. v. 5. 7. 1911

1671. Zacharias, Friedrich A[ugust]: Beiträge zur Kenntnis des Koch- und Dämpfprozesses der Pappen-Industrie. [1911.] $99 \mathrm{~S}$., 3 Taf. $8^{0}$ of 2. verb. u. erg. Bearbeitung in: Der Papier-Fabrikant. Jg. 1912.

Dresden, Dr.-Ing.-Diss. v. 5. 7. 1911

1672. Hartmann, Max: Uber p-Toluolsulfonaminoessigsäure und ihre Abkömmlinge. 1911. 34 S. $8^{0}$

Dresden, Dr.-Ing.-Diss. v. 19. 7. 1911

1673. Hofmann, Richard: Die Einwirkungen des Chlorkohlenoxyds bez. Chlorkohlensäurephenylesters auf $\mathrm{Oxy}$ - und Amino-Verbindungen. 1911. 59 S. $8^{\circ}$

Dresden, Dr.-Ing.-Diss. v. 19. 7. 1911

1674. Maffia, Paul: Ueber das Adsorptionsgleichgewicht im Graham'schen Eisenoxydhydrosol. Dresden: Steinkopff 1911. 38 S. $8^{0}$ T Aus: Kolloidchem. Beihefte <Erg. H. zur Kolloid-Zeitschrift〉. Bd 3. 1911/12.

Dresden, Dr.-Ing.-Diss. v. 19. 7. 1911

1675. Freund, Richard: Zur Kenntnis der Chinazoline. 1911. 38 S. $8^{0}$

Dresden, Dr.-Ing.-Diss. v. 26. 7. 1911

1676. Graner, Paul: Utber den physik.chem. Zustand photographischer organischer Entwicklungslösungen, insbesondere die Dissoziationskonstanten des Brenzkatechins, der Kohlensäure und des Phenolphtaleins. 1912. 68 S. $8^{0}$

Dresden, Dr.-Ing.-Diss. v. 26. 7. 1911

1677. Leubner, Adolf: Beitrag zur Chemie der photographischenEntwicklungsvorgänge. 1911. 81 S. $8^{0}$ of Engl. in: The British Journal of Photography. Vol. 59. 1912; vgl. Journal f. prakt. Chemie. Bd 193. N. F. Bd 85. 1912. S. 314 ff.; Zeitschrift f. anorgan. Chemie. Bd 74. 1912.

Dresden, Dr.-Ing.-Diss. v. 26. 7. 1911
1678. Stein, Albreeht: Uber einige fettsaure Ester der Hydrocellulose und ihre Verseifung nebst einer Studie über Verdampfungsgeschwindigkeit von Säuren. 1911. 71 S. $8^{0}$ - T. 1 gekürzt in: Zeitschrift f. angewandte Chemie. Jg. 26. 1913, T. 1; T. 2 gekürzt in: Journal f. prakt. Chemie. Bd 196. N. F. Bd 88. 1913.

Dresden, Dr.-Ing.-Diss. v. 26. 7. 1911

1679. Young, Bernhard: Beiträge zur Kenntnis des Diaphragmenverfahrens. 1911. 63 S. $8^{0}$

Dresden, Dr.-Ing.-Diss. v. 26. 7. 1911

1680. Mühlhaus, Hans: Untersuchungen über das Billiter-Verfahren zur Gewinnung von Alkali und Chlor. 1911. 59 S. $4^{0}$

Dresden, Dr.-Ing.-Diss. v. 15. 11. 1911

1681. Steuding, Alfred: Utber das chemische und spektroskopische Verhalten der Pyridinfarbstoffe aus Amidophenolen und -naphtolen. 1912. 51 S. $8^{0}$

Dresden, Dr.-Ing.-Diss. v. 6. 12. 1911

1682. Bayer, Richard: Utber die Aufspaltung des Pyridins. 1912. 97 S., 2 Bl. Fig. $8^{\circ}$ T Die Ergebnisse der Arbeit wurden zum gröBten Teil veröff. in: Journal f. prakt. Chemie. Bd 191. N. F. Bd 83. 1911.

Dresden, Dr.-Ing.-Diss. v. 21. 12. 1911

1683. Escher, A[rtur] F[ranz] Walter von: Kathodische Vorgänge bei der Elektrolyse gemischter Lösungen von Zink- und Eisensulfat. 1912. 75 S. $8^{0}$

Dresden, Dr.-Ing.-Diss. v. 21. 12.1911

1684. Petsehek, Paul: Studien über Gase. 1911. 77 S. $8^{0}$

Dresden, Dr.-Ing.-Diss. v. 21. 12. 1911

1685. Reiner, Friedrich: Beitrag zur Kenntnis des 2-Aminoanthrachinons. 1912. 74 S. $8^{0}$ - Vgl. Berichte d. Deutschen Chem. Gesellschaft. Jg. 45. S. 2233.

Dresden, Dr.-Ing.-Diss. v. 21. 12. 1911

1686. Reiser, Hans: Über den Einfluß der Mälzungsdauer und des Maischverfahrens auf die Zusammensetzung der Würze in bezug auf Eiweißstoffe, Kohlehydrate und Salze. 1912. $97 \mathrm{~S} .8^{0}$ ot Etwas gekürzt in: Wochenschrift f. Brauerei. Jg. 29.

Dresden, Dr.-Ing.-Diss. v. 21. 12. 1911

1687. Sehneider, Erich: Beitrag zur Kenntnis der Benzimidazole. 1912. 44 S. $8^{0}$ Dresden, Dr.-Ing.-Diss. v. 7. 2. 1912

1688. Esser, Paul Johan Arnout: Uber die Kondensation von Säureestern mit Dinitrilen. 1912. 81 S. $8^{0}$

Dresden, Dr.-Ing.-Diss. v. 13. 3. 1912 
1689. Hering, Georg: Studien über Schwefelsäurefabrikation. $1912.69 \mathrm{~S} .8^{0}$

Dresden, Dr.-Ing.-Diss. v. 13. 3.1912

1690. Nitzelnadel, Konrad Adolf: Versuche über die Verwendbarkeit aus Sulfitzellulose und Strohstoff hergestellter Nitrozellulosen. 1912. 65 S. $8^{0}$. Mit gekürzter Einl. in: Zeitschrift f. d. gesamte SchieB- u. Sprengstoffwesen. Jg. 7.

Dresden, Dr.-Ing.-Diss. v. 13. 3. 1912

1691. Woost, Johannes: Über den Einfluß der Temperatur auf das elektromotorische Verhalten des Eisens in Kalilauge. 1912. 81 S. $8^{\circ}$

Dresden, Dr.-Ing.-Diss. v. 13. 3. 1912

1692. Klingstedt, Adolf: Ein neues Verfahren zur Bestimmung der Zellulose in verholzten Fasern und Studien über die nach diesem Verfahren aus Jute und $\mathrm{Holz}$ isolierten Zellulosen. 1912. 72 S. $8^{0}$

Dresden, Dr.-Ing.-Diss. v. 14. 3. 1912

1693. Zweigler, Fritz: Über die Gewichtszunahme von Papierstoffen beim Erhitzen. 1912. 65 S., 2 Taf. $8^{\circ}$ - Kurzer Ausz. in: Der Papier-Fabrikant. Jg. 10. S. $1364 \mathrm{f}$.

Dresden, Dr.-Ing.-Diss. v. 22. 3. 1912

1694. Raßfeld, Paul: Über die Einwirkung von Säuren und deren Derivaten auf Orthoform. 1912. 57 S. $8^{0}$

Dresden, Dr.-Ing,-Diss. v. 22. 5. 1912

1695. Blumrich, Karl: Über den $\mathrm{Zu}$ sammenhang zwischen Farbe und Konstitution der gemischten Pyridinfarbstoffe aus sekundären Aminen. 1912. 69 S. $8^{\circ}$

Dresden, Dr.-Ing.-Diss. v. 12. 6. 1912

1696. Prausnitz, Paul H[ubert]: Studien über die elektrolytische Herstellung von $\mathrm{Na}$ triumhypochlorit. Halle: Knapp 1912. 56 S. $4^{0}$ - Aus: Zeitschrift f. Elektrochemı. Jg. 18.

Dresden, Dr.-Ing.-Diss. v. 12. 6. 1912

1697. Hübner, Richard: Zur Kenntnis der $\alpha$-Aminosäuren. 1912. 30 S. $8^{0}$

Dresden, Dr.-Ing.-Diss. v. 19. 6. 1912

1698. Jahn, Johannes: Über die Einwirkung von Gasen auf Metalle und Metalllegierungen. 1912. 72 S., 3 Taf. $8^{0}$

Dresden, Dr.-Ing.-Diss. v. 19. 6. 1912

1699. Doleh, Paul: Über die Bedeutung der chlorigsauren Salze bei der Umwandlung von Hypochloriten in Chlorate. 1912. 54 S. $8^{\circ}$

Dresden, Dr.-Ing.-Diss. v. 26. 6. 1912
1700. Lincke, $\mathbf{E}[$ mil] Alexander: Über Kapok. 1912. $98 \mathrm{~S}, 8$ Taf. $8^{0}$ I Soll stark gekürzt ersch. in : Elsäss. Textil-Blatt. Jg. 1914.

Dresden, Dr.-Ing.-Diss. v. 26. 6. 1912

1701. Renner, 0tto: Über die Bestimmung des Fluors. 1912. 84 S. $8^{0}$

Dresden, Dr.-Ing.-Diss. v. 26. 6. 1912

1702. Goebel, Raymond: Über das Verhalten des Wismuts bei der Kupferraffination. 1912. $80 \mathrm{~S} .8^{0}$

Dresden, Dr.-Ing.-Diss. v. 17. 7. 1912

1703. Grieshammer, Willy: Beiträge zur Kenntnis der aromatisch-aliphatischen Diazoaminoverbindungen. 〈Über Arylazodicyandiamide. $>1912.70$ S. $8^{0}$

Dresden, Dr.-Ing.-Diss. v. 24. 7. 1912

1704. Strobach, Waldemar: Abkömmlinge des Diphenylendioxyds. 1912. 32 S. $8^{0}$ Dresden, Dr.-Ing.-Diss. v. 24. 7. 1912

1705. Berge, Paul: Beiträge zur Kenntnis der Dinitrile. 1912. 57 S. $8^{0}$

Dresden, Dr.-Ing.-Diss. v. 25. 7. 1912

1706. Müller, Friedrich: Über die Technik der Probenahme und die Analysenmethoden zur genauen Bestimmung kleiner Mengen von schwefliger Säure und Schwefelsäure in Abgasen. 1912. 71 S. $8^{0}$

Dresden, Dr.-Ing.-Diss. v. 13. 11. 1912

1707. Frenzel, Walther: Über die Gasdurchlässigkeit der gummierten Ballonstoffe. 1912. VIII, 61 S. $8^{0}$ ๆ Aus: Elsäss. Textil-Blatt. Jg. 1913; Nachtrag ebd. S. 1279.

Dresden, Dr.-Ing.-Diss. v. 17. 7. 1912

1708. Schade, Martin: Über die Polarisation bei der elektrolytischen Nickelabscheidung. 1912. 76 S. $8^{0}$

Dresden, Dr.-Ing.-Diss. v. 4. 12. 1912

\section{IN VERBINDUNG MIT DER BERG- AKADEMIE ZU FREIBERG}

1709. NieB, Hermann, Markscheider: Die Bekämpfung der Wassersand-<Schwimmsand- $>$ Gefahr beim norddeutschen Braunkohlenbergbau. Freiberg i. S.: Craz \& Gerlach 1907. 104 S. $8^{\circ}$ T Auch als Buch.

Dresden, Dr.-Ing.-Diss. v. 25. 2. 1907

1710. Pilz, Richard: Die Bleiglanzlagerstätten von Mazarrón in Spanien. Freiberg i. S.: Craz \& Gerlach 1907. 51 S. $8^{0}$ - Umarbeitung u. teilweise Ergänzung eines Aufsatzes in: Zeitschrift f. prakt. Geologie. Jg. 13. 1905.

Dresden, Dr.-Ing.-Diss. v. 25. 2. 1907 
1711. Krause, Carl: Beiträge zur Geschichte der Entwickelung der Instrumente in der Markscheidekunde. 1908. VIII. 49 S. $4^{0}$

Dresden, Dr.-Ing.-Diss. v. 31. 12. 1907

1712. Hagemann, Ferdinand: Bergmännisches Rettungs- und Feuerschutzwesen in der Praxis und im Lichte der BergpolizeiVerordnungen Deutschlands und Oesterreichs. Freiberg i. S.: Craz \& Gerlach 1908. VI, 159 S. $4^{0}$. Auch als Buch.

Dresden, Dr.-Ing.-Diss. v. 11. 3. 1908

1713. Tafel, Victor E[ugen ]: Studie überdie Constitution der Zink-Kupfer-Nickel-Legierungen sowie der binären Systeme KupferNickel, Zink-Kupfer, Zink-Nickel. Freiberg i. S.: Craz \& Gerlach 1908. 50 S., 15 Taf. $4^{0}$ - S. 1-26 auch in: Metallurgie. Jg. 5; Bericht uiber Abschnitt 3: ebd. Jg. 4. 1907.

Dresden, Dr.-Ing.-Diss. v. 2. 4. 1908

1714. Glatzel, Richard, Markscheider: Ein Beitrag zum Elmore'schen Extraktionsverfahren. 〈Aufbereitung von Erzen mit $\mathrm{Zu}$ hilfenahme von Ölen. $\rangle$ 1908. $77 \mathrm{~S} .8^{0}$ I Auch bei Craz \& Gerlach in Freiberg i. S.

Dresden, Dr.-Ing.-Diss. v. 2. 7. 1908

1715. Richter, Paul: Beiträge zur Theorie des Huntington-Heberlein Prozesses und der ihm verwandten Verblaseverfahren. 1909. 84 S. $8^{\circ}$

Dresden, Dr.-Ing.-Diss. v. 31. 7. 1908

1716. Wagner, Percy Albert: Studien an den diamantführenden Gesteinen Südafrikas. Berlin: Borntraeger 1909. XVIII, 132 S., 2 Taf. $8^{0}$ I Auch u. d. T.: Die diamantführenden Gesteine Südafrikas, ihr Abbau und ihre Aufbereitung.

Dresden, Dr.-Ing.-Diss. v. 24. 12. 1908

1717. Lieckfeld, Albert: Autogene Leuchtgas-Schweissmethoden. 1909. 38 S., 4 Bl. Taf. $8^{0}$

Dresden, Dr.-Ing.-Diss. v. 24. 11. 1909

1718. Voigt, Max: Beiträge zur Oxydation des Phosphors im basischen Konverter. 1910. 81 S., 4 Taf. $8^{0}$

Dresden, Dr.-Ing.-Diss. v. 10. 12. 1909

1719. Herzberg, Franz: Beiträge zur geologischen Kenntnis der Preßnitzer Erzlagerstätten. (Mit 5 Taf.) Freiberg i. S.: Craz \& Gerlach 1910. 55 S., 5 Taf. $8^{\circ}$ ๆ Auch als Buch.

Dresden, Dr.-Ing.-Diss. v. 8. 9. 1910

1720. Schertel, Ludwig: Studien über einige Verlustquellen des Blei- und KupferHochofenprozesses. 1910. 49 S., 1 Taf. $8^{0}$ Dresden, Dr.-Ing.-Diss. v. 2. 11. 1910
1721. Leber, Engelbert: Die Frage der Selbstkostenberechnung von Gußstücken in Theorie und Praxis. Aufstellung einer gerechten Stück-Kalkulationsmethode auf vollständig neuer Grundlage, sowie kritische Behandlung der gebräuchlichsten Verfahren. Düsseldorf: Stahleisen 1910. VIII, $126 \mathrm{~S} .4^{0}$ T Auch als Buch; kurzer Ausz. in: Stahl u. Eisen. Jg. 30, Halbj. 1.

Dresden, Dr.-Ing.-Diss. v. 23. 11. 1910

1722. Pütz, 0tto: Die Begutachtung und Wertschätzung von Bergwerksunternehmungen mit besonderer Berücksichtigung der oberschlesischen Steinkohlengruben. Freiberg i. S.: Craz \& Gerlach 1911. 111 S. $8^{0}$ T Auch als Buch.

Dresden, Dr.-Ing.-Diss. v. 14. 12. 1910

1723. Wintgens, Paul: Beitrag zu der Hydrologie von Nordholland. Kerkrade: Alberts 1911. 73 S., 1 Taf. $8^{0}$. Auch als Buch.

Dresden, Dr.-Ing.-Diss. v. 8. 3. 1911

1724. Weidig, Max: Metallurgische und technologische Studien auf dem Gebiete der Legierungs-Industrie, insbesondere über das Ausglühen von Metallen und Legierungen. Berlin: Simion 1911. 123 S. $4^{0}$ - Buchausg. 1912; aus: Verhandlungen d. Vereins zur Beförderung d. Gewerbfleißes. Jg. 90.

Dresden, Dr.-Ing.-Diss. v. 7. 6. 1911

1725. Kühn, Emil: Die chemischen Vorgänge bei der Cyanlaugung von Silbererzen. Mit 34 Fig. Halle: Knapp 1912. 108 S. $8^{0}$ - Auch als Buch.

Dresden, Dr.-Ing.-Diss. v. 3. 1. 1912

1726. Siegfiried, Erich: Die Naphthalagerstätten der Umgebung von Solotwina. Ein Beitrag zur Tektonik des Karpathenrandes in Ostgalizien. Berlin: Verl. f. Fachliteratur 1912. VIII, 72 S., 2 Taf. $4^{0}$ ๆ Auch als Buch. Dresden, Dr.-Ing.-Diss. v. 3. 1. 1912

1727. Dieckmann, Walter: Die geologischen Verhältnisse der Umgebung von Melilla unter besonderer Berücksichtigung der Eisenerz-Lagerstätten des Gebietes von Beni-Bu-Ifrur im marokkanischen Rif. Berlin: Krahmann 1912. 22 S., 2 Taf. $4^{0}$ I Aus: Zeitschrift f. prakt. Geologie. Jg. 20.

Dresden, Dr.-Ing.-Diss. v. 12. 6. 1912

1728. Iseu, Vasile, Dir. d. Bohrmeisterschule aus Câmpina-Rumänien: Die Wasserabsperrung bei Tiefbohrungen auf Erdöl. 1912. $108 \mathrm{~S} .8^{0}$

Dresden, Dr.-Ing.-Diss. v. 13. 11. 1912 


\section{HAN NOVER}

Gegründet 1831 als „Höhere Gewerbeschule", 1847 „Polytechnische Schule“, seit 1879 „,Technische Hochschule“

\section{SATZUNGEN ORDNUNGEN BESTIMMUNGEN}

\section{ALLGEMEINES UND VERMISCHTES}

1729. Bekanntmachung der Direktion der polytechnischen Schule zu Hannover, betr. ihre Vermittelung bei Forderungen hiesiger Einwohner an Studirende dieser Schule (v. 24. 9. 1859).

1730. Sehul-Gesetze für die Schüler und Zuhörer der polytechnischen Schule zu Hannover. Vom 18. u. 27. 6. 1861. [Folgt:] Haus-Ordnung für die polytechnische Schule. Abgedr. in: Verfassung d. Polytechn. Schule zu Hannover. 1863 [Nr 1731].

$H^{*}$

1731. Verfassung der Polytechnischen Schule zu Hannover. Okt. $1863 \quad H^{*}$

1732. Statuten für die Stiftung zur Erinnerung an weiland Leeser Rosenthal und seine Ehefrau Sophie, geb. Blumenthal (v. 18. 6. 1870). 1870.

$H^{*}$

1733. Verfassung der Kgl. Polytechnischen Schule zu Hannover. März 1871. 1871.

1734. Die Karmarseh-Stiftung [an der Polytechnischen Schule] in Hannover (v. 12. 6. 1876). Hannover: Selbstverl. d. Stiftung.

1735. Sehulgesetze für die Studirenden und Zuhörer der Kgl. polytechnischen Schule zu Hannover. [Folgt:] Hausordnung. [1877.]

$H^{*}$

1736. Verfassungs-Statut der Kgl. Technischen Hochschule zu Hannover und Regulativ betr. die Organisation der Abtheilungen an derselben (v. 7. 9. 1880). 1880. - [Neudr.] 1890. 1893. 1895. 1904.

- Abgedr. in: Centralblatt f. d. ges. UnterrichtsVerwaltung in Preußen. Jg. 1881. S. 144 ff. ; Launhardt: Die Kgl. Techn. Hochsch, zu Hann. 1881 [Nr 1775]. S. 81 ff.; Damm 1909. S. $111 \mathrm{ff}$.

1880. 1890. 1893. 1895: $H^{*}$

1737. Bibliothek-0rdnung der Kgl. Technischen Hochschule zu Hannover (v. 12. 7. 1882).

$H^{*}$

1738. Habilitations-0rdnung für die Kgl. Technische Hochschule zu Hannover (v. 24. 4. 1884). Abgedr. in: Damm 1909. S. 131 ff.
1739. Vorschriften für die Studirenden der Kgl. Technischen Hochschule zu Hannover (v. 12. 8. 1884). [Folgt:] Hausordnung v. Jan. 1885. [Nebst] Abweichungen bei der Anwendung auf die Hospitanten v. 1. 4. 1886 .

Abweich: : $H^{*}$

1740. Bestimmungen für den Ausschuss der Studierenden der Technischen Hochschulezu Hannover. Genehmigt 24.1.1888. $H^{*}$

1741. Bestimmungen der C. W. HaseStiftung vom 12. 7. 1892 für Stipendien geeigneter, die Architektur-Abtheilung der Kgl. Technischen Hochschule zu Hannover besuchenden[!] Studirenden[!], Hospitanten oder Zuhörer (v. 12. 7. 1892). $H^{*}$

1742. Satzungen der Krankenkasse der Studentenschaft der Kg]. Technischen Hochschule zu Hannover (v. 8. 8. 1894). I Abgedr. in: Damm 1909. S. 155.

1743. Bestimmungen für den Ausschuss der Studierenden der Technischen Hochschule zu Hannover. Genehmigt 4. 3. 1897. 1902.

$H^{*}$

1744. Promotions-0rdnung für die Ertheilung der Würde eines Doktor-Ingenieurs durch die $\mathrm{Kgl}$. Technische Hochschule zu Hannover (v. 19.6. 1900). A Abgedr. in: Centralblatt f. d. ges. Unterrichts-Verwaltung in Prenßen. Jg. 1900. S. 685 ff.; Bestimmungen. S. 245 .

1745. Satzungen für die Hilfskasse der Kgl. Technischen Hochschule zu Hannover (v. 16. 9. 1902). T Abgedr. in: Damm 1909. S. $149 \mathrm{ff}$ :

1746. Satzungen des Studenten-Verbandes an der Kgl. Techn. Hochschule zu Hannover. [Folgt: 1.] Geschäfts-Ordnung. [2.] Satzungen des Arbeitsamtes. 1905.

\section{PRÜFUNGSORDNUNGEN}

1747. Ordnung der Diplomprüfungen an der Kgl. polytechnischen Schule zu Hannover (v. 20.5. 1873). $1873 . \quad H_{*}$

1748. Erläuterungen zur Ordnung der Diplomprüfungen an der Kgl. Polytechnischen Schule zu Hannover (v. Dez. 1873). $H^{*}$ 
-1749. Vorschriften für die Diplom-Prüfungen an der Kgl. Technischen Hochschule zu Hannover (v. 21. 12. 1887). - [Dasselbe] (v. 21. 12. 1887 u. 30. 11. 1894). - [Neudr.] 1897. Abgedr. in: Centralblatt f. d. ges. Unterrichts-Verwaltung in Preußen. Jg. 1888. S. 190 ff.; Damm 1899. S. 124 ff. 1887: H; 1894. 1897: $H^{*}$

1750. Gesehäfts-0rdnung für die DiplomPrüfungen an der $\mathrm{Kgl}$. Technischen Hochschule zu Hannover (v. 23. 12. 1887). - Abgedr. in: Damm 1899. S. $131 \mathrm{ff}$.

\section{Abteilung für Architektur}

1751. Diplomprüfungs - Ordnung nebst Übergangs-Bestimmungen der Abteilung für Architektur der Kgl. Technisehen Hochschule zu Hannover. Vom 25. 2. 1903. ' Abgedr. in: Bestimmungen. S. $237 \mathrm{ff}$.

1752. Geschäfts-0rdnung für die DiplomPrüfungen der Abteilung für Architektur der Kgl. Technischen Hochschule zu Hannover. Vom 2. 8. 1904.

1753. Diplomprülungs- Ordnung der Abteilung für Architektur der Kgl. Technischen Hochschule zu Hannover. Vom 29. 7. 1908.

1754. Geschäfts-0rdnung für die DiplomPrüfungen der Abteilung für Architektur der Kgl. Technischen Hochschule zu Hannover. Vom 24. 9. 1908.

Abteilung für Bau-Ingenieurwesen

1755. Diplomprüfungs - Ordnung nebst Übergangs-Bestimmungen der Abteilung für Bau-Ingenieurwesen der Kgl. Technischen Hochschule zu Hannover. Vom 25. 2. 1903.

- Abgedr. in: Bestimmungen. S. 230 ff.

1756. Geschäits- Ordnung für die Diplomprüfungen für die Abteilung für Bau-Ingenieurwesen der Kgl. Technischen Hochschule zu Hannover. Vom 2. 8.04. - [Neudr. u. d. T. :] Geschäfts-Ordnung für die Diplom- prüfungen der Abteilung für Bau-Ingenieurwesen an der... Vom 2. 8. 1904. (Mit Anm. zu $\S 7$ betr. Ministerialerlaß v. 14. 3. 1906.)

\section{Abteilung für Maschinen-Ingenieur- wesen}

1757. Diplomprüfungs - Ordnung nebst Übergangs-Bestimmungen der Abteilung für Maschinen-Ingenieurwesen der Kgl. Technischen Hochschule zu Hannover. Vom 25. 2. 1903. - Abgedr. in: Bestimmungen. S. $221 \mathrm{ff}$.

1758. Gesehäfts-0rdnung für die DiplomPrüfungen der Abteilung für Maschinen-Ingenieurwesen der $\mathrm{Kgl}$. Technischen Hochschule zu Hannover. Vom 2.8.04. [Neudr. u. d. T.:] Geschäfts-Ordnung ... Maschinen-Ingenieurwesen an der ... Vom 2. 8. 1904 .

Abteilung für chemisch-technische und elektrotechnische Wissenschaften

1759. Diplomprüfungs - Ordnung nebst Übergangs-Bestimmungen für Elektro-Ingenieure der $\mathrm{Kgl}$. Technischen Hochschule zu Hannover. Vom 25. 2. 1903. - A Abgedr. in: Bestimmungen. S. $215 \mathrm{ff}$.

1760. Diplomprüfungs - Ordnung nebst Ubergangs-Bestimmungen für Ingenieure der Chemie der Kgl. Technischen Hochschule zu Hannover. Vom 13. 3. 1903. A Abgedr. in: Bestimmungen. S. $209 \mathrm{ff}$.

1761. Geschäftsordnung für die Diplomprüfungen der Chemiker an der Kgl. Technischen Hochschule zu Hannover. Vom 2. 8. 04 .

1762. Gesehäfts-0rdnung für die DiplomPrüfungen der Elektro-Ingenieure der Kgl. Technischen Hochschule zu Hannover. Vom 2. 8. 04.

\section{PERIODISCHE SCHRIFTEN}

1763. Programm der höhern Gewerbeschule (1853/54-1878-79: polytechnischen Schule; 1879-80 ff. : Kgl. Technischen Hochschule) zu Hannover (1853/54-1882-83: für das Jahr; 1883-84 ff. : für das Studienj.) 1834. 1853/54. 1857-58-1912-1913. 18[34]-1912.
1889-90-1912-13. Beil.: Zahl der Theilnehmer an den einzelnen Unterrichtsfächern im Studienj. 1888-89-1911-1912.

1834: Göttingen UB; 1853/54. $1857 / 58-1870 / 71 . \quad 1872-1890 / 91$. 1899/1900. Beil. 1902/03. 1910/11. 1911/12: $H^{*}$ 
1764. Adressbueh der Kgl. polytechnischen Schule in Hannover für das Studienj. 1877/78. Hannover: Schmorl \& v. Seefeld Febr. 1878.

$H \mathrm{KglB}$

1765. Kgl. Technische Hochschule zu Hannover. Die Uebergabe des Rektorates am 1. Juli 1892. 1895. 1898. $1892 \quad 98$.

Darin Rede des antretenden Rektors:

1892. Kohlrausch, [Wilhelm]: Die Entwickelung der Elektrotechnik.

\section{GELEGENHEITS- UND}

1767. Die höhere Gewerbeschule in Hannover. Erläuterungen über Zweck, Einrichtung und Nutzen derselben. Von Karl Karmarseh, Dir. dieser Lehranstalt. Hannover: Hahn 1831. 50 S., 1 Taf. $8^{0}$

$\mathrm{Be}$

1768. Die höhere Gewerbeschule in Hannover. Von Karl Karmarseh, 1. Dir. dieser Lehranstalt. 2., sehr erw. Aufl. Mit Abb. des Gebäudes der Anstalt. Hannover: Hahn 1844. VI, 206 S., 2 Taf. $8^{0}$

H

1769. Die polytechnische Schule zu Hannover. Von Karl Karmarseh, 1. Dir. dieser Lehranstalt. Hannover: Hahn 1848. 96 S., 1 Taf. $8^{0}-2$., sehr erw. Aufl. Mit 3 Bl. Abb. d. Gebäudes der Anstalt. Hannover: Hahn 1856. 276 S., 6 Taf. $8^{0}$ 2. Aufl.: Be

1770. Fest-Rede zur Feier des fünfundzwanzigjährigen Bestehens der Polytechnischen Schule in Hannover. Geh. am 2. Mai 1856 vom Dir. [Karl] Karmarsch. 1856. 16 S. $8^{0}$

1771. Katalog der Bibliothek der Königlichen polytechnischen Schule zu Hannover. 1868. XV, $483 \mathrm{~S}$. [Nebst] Nachtrag von 1868 bis 1877 . 1877. IX, 208 S. $8^{0}$

1772. Katalog der Werkzeug-Sammlung an der Königlichen polytechnischen Schule zu Hannover. Von Dr. Karl Karmarseh, Dir. d. hannov. polytechn. Schule. Mit einleitenden Bemerkungen über technolog. Sammlungen im Allg. u. 20 Bl. Zeichn. 1870. XI, 202 S., 20 Taf. $8^{0}$

1773. Auditorium der Vorträge über Technologie des Herrn Direktors und Professors, Geh. Regierungsraths, Dr. [Karl] Karmarsch. 1831. 1875. [Verzeichnis der Teilnehmer in beiden Jahren.] $4 \mathrm{Bl} .4^{0}$

1774. Lieder für den Fest-Commers zur Feier des Einzugs in das neue Gebäude der Königl. Technischen Hochschule zu Han-
1895. Frank, [Albert]: Die Fortschritte auf dem Gebiete des Kraftmaschinenbaues.

1898. Köhler, (Heinrich): Das Wesen und die Bedeutung der Baukunst in der neuesten Zeit. 1892. $1895: H ; 1898: H^{*}$

1766. Personal - Verzeichniss der Kgl. Technischen Hochschule zu Hannover fürs (1905 ff.:fürdas) W.-H. 1900-1901. S.-H. 1901 [usw.]-W.-H. 1912/13. 1900-12.

\section{VERMISCHTE SCHRIFTEN}

nover am Dienstag, den 7. October $1879 \mathrm{im}$ Wallbrecht'schen Concerthause. 15 S. $8^{\circ} \mathrm{H}$ StB

1775. Die Königliche Technische Hochschule zu Hannover von 1831 bis 1881. Zur Jubelfeier des fünfzigjährigen Bestehens der Hochschule bearb. von [Wilhelm] Launhardt, Prof., z. Z. Rektor d. Techn. Hochschule. Mit 4 lithogr. Taf. Hannover: Hahn 1881. IV, 184 S., 4 Taf. $8^{0} \quad H$

1776. Fest-Gedicht zur Jubelfeier des fünfzigjährigen Bestehens der Königlichen Technischen Hochschule zu Hannover. Am 2., 3. und 4. Juni 1881. Im Auftrage des Festausschusses verf. von Ernst Rommel. 1881. 20 S. $4^{0}$

1777. Beschreibung der Jubelfeier des funfzigjährigen Bestehens der Königlichen Technischen Hochschule zu Hannover im Juni 1881 nebst einem Namens-Verzeichniß ehemaliger Studirender der Hochschule, sowie der Hörer des Studienjahres 1880/81. Hannover: Hahn 1881. IV, 112 S., 1 Taf. $8^{0} \mathrm{H}$

1778. Katalog der Bibliothek der Königlichen Technischen Hochschule zu Hannover, 1893. VII, 746 S. [Nebst] Nachtrag von 1893 bis 1904. 1904. VII, 252 S. $4^{0}$

1779. Königliche Technische Hochschule zu Hannover. Die Einweihungsfeier des Neubaues am 24. October 1895. 1895. 14 S. $8^{0}$

1780. Königliche Technische Hochschule zu Hannover. Feier der 25. Wiederkehr des Tages der Neubegründung des Deutschen Reiches am 18. Januar 1896. 1896. 9 S. $8^{0} \mathrm{H}$

1781. Gedächtnissrede zur Feier des 100. Geburtstages Seiner Majestät des hochseligen Kaiser Wilhelms des Grossen geh. am 22. März 1897 in der Königlichen Technischen Hochschule zu Hannover vom Rektor Prof. Albert Frank. 1897. 10 S. $8^{0} \quad H^{*}$ 
1782. Königliche Technische Hochschule zu Hannover. Feier der Jahrhundert-Wende am 9. Januar 1900. 1900. 8 S. $8^{\circ}$ (Darin: [Albert] Frank: Die Entwickelung der Technik im 19. Jahrhundert.)

1783. Königliche Technische Hochschule zu Hannover. Feier des 200jährigen Jubiläums des Königreiches Preussen, der 30jährigen Wiederkehr des Tages der deutschen Kaiser-Proklamation und des Geburtstages Sr. Majestät des Kaisers und Königs am 18. Januar 1901. 1901. 15 S. $8^{0}$ (Darin: [Hubert] Stier: Die Entwickelung der Kunst und ihre Förderung durch die Hohenzollern in den letzten zweiJahrhunderten.) $B e$

1784. Übersicht über die Entwicklung der Königlichen Technischen Hochschule zu
Hannover in den Jahren 1881 bis 1906. 24 S., 6 Bl., 4 Taf. $8^{0}$

1785. Verzeichnis der Festteilnehmer bei der Jubelfeier des 75jährigen Bestehens der Königlichen Technischen Hochschule in Hannover am 25. Mai 1906. 1. Ausg. 21 S. $8^{0}$ - 2. Ausg. 29 S. $8^{0}$

1786. Kommers zur 75jährigen Jubelfeier der Königlichen Technischen Hochschule zu Hannover am 25. Mai 1906 im Festzelte 〈Welfengarten〉. [Liederheft.] 24 S. $4^{0}$

1787. Kulturwerte der Technik. Festrede zur Feier des Geburtstages Sr. Majestät des Kaisers, geh. am 27. Jan. 1912 an d. Kgl. Techn. Hochschule Hannover von Robert Otzen, Prof. Berlin: Springer 1912. 31 S. $8^{0}$

\section{DOKTOR-INGENIEUR-DISSERTATIONEN}

\section{ABTEILUNG FU்R ARCHITEKTUR}

1788. Jänecke, Wilhelm, Reg.-Baumeister : Über die Entwickelung der Akanthusranke im französischen Rokoko dargestellt an Stichen französischer Meister in der Zeit von 1650-1750. Mit 57 nach d. Orig. gez. Abb. Hannover: Jänecke 1902. 31 S. $4^{0}$ I Als Buch u. d. T.: Beiträge zur Geschichte d. Ornamentik. 1.

Hannover, Dr.-Ing.-Diss. v. 29. 6. 1903

1789. Jacobi, Georg v.: Untersuchungen über den Einfluß der Hirsauer Bauschule auf den sächsischen Kirchenbau des XI. und XII. Jahrhunderts. 1904. 40 S., 2 Taf. $8^{0}$

Hannover, Dr.-Ing.-Diss. v. 16. 7. 1903

1790. Michel, Eugen, Reg.-Baumeister: Utber die keramischen Verblendstoffe. Halle: Knapp 1903. 48 S. $4^{0}$ T Als Buch 1904.

Hannover, Dr.-Ing.-Diss. v. 16. 7. 1903

1791. Eichwede, Ferdinand: Beiträge zur Baugeschichte der Kirche des kaiserlichen Stiftes zu Königslutter. 1904. 38 S., 9 Taf. $8^{0}$ Hannover, Dr.-Ing.-Diss. v. 18. 2. 1904

1792. Rowald, Paul, Stadtbauinsp. (i. Hannover): Beiträge zur Geschichte der Grundsteinlegung. 1904. 94 S. $8^{0}$ - Auch u. d. T.: Geschichte der Grundsteinlegung, bei Ernst in Berlin; aus: Zeitschrift f. Bauwesen. Jg. 54 .

Hannover, Dr.-Ing.-Diss. v. 28. 6. 1904

1793. Cube, Gustav von: Über die römische , scenae frons" in den pompejanischen Wandbildern 4. Stils. Berlin: Wasmuth
1906. 42 S., 2 Taf. $4^{0}$ - Auch als: Beiträge zur Bauwissenschaft. H. 6.

Hannover, Dr.-Ing.-Diss. v. 17. 11. 1905

1794. Heinz, W[ilhelm]: Studien über die ehemalige freie Reichsstadt Wetzlar und ihre Bauten. 1907. 59 S., 26 Taf., $1 \mathrm{Pl} .8^{0}$ Hannover, Dr.-Ing.-Diss. v. 17. 11. 1905

1795. Wende, 0skar, Architekt: Wendel Roskopf ,Meister zu Görlitz und in der Schlesy". Ein Beitrag zur Geschichte der Renaissance in Schlesien. 1908. 44 S. $4^{\circ}$

- Etwas gekürzt, aber um eine ganze Reihe v. Abb. verm. in: Schlesiens Vorzeit in Bild u. Schrift. Zeitschrift d. Schles. Altertumsvereins. N. F. Bd 5. (Jahrbuch d. Schles. Museums f. Kunstgewerbe u. Altertümer. Bd 5.) 1909.

Hannover, Dr.-Ing.-Diss. v. 22. 7. 1907

1796. Küster, Heinrich, Reg.-Baumeister a. D., Stadtbauinsp. zu Breslau: Die Belichtung von Aufenthaltsräumen in den Bauordnungen. [Berlin: Heymann 1908.] 79 S. $8^{0}$

Hannover, Dr.-Ing.-Diss. v. 23. 12. 1907

1797. Tilemann, Georg: Der römische Kaiserpalast in Trier. Mit 7 Taf. 1908. 16 S., 3 Taf. $4^{0}$ - Auch u. d. T.: Der römische Kaiserpalast in Trier $\mathrm{u}$. seine Rekonstruktion. 2. [Titel-] Aufl. Hannover: Schmorl \& v. Seefeld 1909.

Hannover, Dr.-Ing.-Diss. v. 27. 8. 1908

1798. Coers, Paul: Die Bautätigkeit der Augustiner in Niedersachsen während des 12. Jahrhunderts. Ein Beitrag zur Baugeschichte. 1909. 51 S., 6 Taf. $8^{0}$

Hannover, Dr.-Ing.-Diss. v. 25. 1. 1909 
1799. Hinrichs, WaltherTh[eodor]: CarlGotthard Langhans, ein schlesischer Baumeister, 1733-1808. Strassburg: Heitz 1909. VI, 88 S., 32 Taf. $8^{0}$ - Auch als: Studien zur deutschen Kunstgeschichte. H. 116.

Hannover, Dr.-Ing.-Diss. v. 25. 1. 1909

1800. Sidow, Hans: Bauregeln und Baugesetze. Ein Beitrag zur Baugeschichte. 1909. $52 \mathrm{~S} .8^{0}$

Hannover, Dr.-Ing.-Diss. v. 25. 1. 1909

1801. Niemann, Richard: Die Grundlagen und Mittel der vorbeugenden Hausschwammbekämpfung auf konstruktivem Wege. 1909. 122 S. $8^{0}$

Hannover, Dr.-Ing.-Diss. v. 24. 7. 1909

1802. Meyer, Franz, Kgl. Militär-Bauinsp.: Handwerkerschutz und Arbeitsbedingungen bei Vergebung öffentlicher Arbeiten, mit besonderer Berücksichtigung des deutschen Baugewerbes und seiner Tarifverträge. Bonn: Georgi 1910. 120 S. $8^{0}$

Hannover, Dr.-Ing.-Diss. v. 7. 3. 1910

1803. Fusch, Gustav: Über HypokaustenHeizungen und mittelalterliche Heizungsanlagen. 1910. V, $116 \mathrm{~S} ., 34$ Taf. $8^{0}$

Hannover, Dr.-Ing.-Diss. v. 21. 11. 1910

1804. Dieckmann, Diedrich: Die Feuersicherheit in Theatern. [1911.] 114 S. $8^{0}$ - Auch bei Jung in München.

Hannover, Dr.-Ing.-Diss. v. 20. 2. 1911

1805. Dunaj, Leon, Reg.-Baumeister: Der Hospitalgedanke im Mittelalter. Mit 3 Taf. 1911. 73 S., 3 Taf. $8^{0}$ - Aus: Zeitschrift f. Architektur u. Ingenieurwesen. Bd 57. N. F. Bd 16. Hannover, Dr.-Ing.-Diss. v. 20. 2. 1911

1806. 0tto, Karl: Über den Anteil der Hygiene an der Entwicklung des deutschen Schulhauses. Hamburg: Boysen \& Maasch 1911. $74 \mathrm{~S} .8^{0}$ Auch als Buch.

Hannover, Dr.-Ing.-Diss. v. 1. 4. 1911

1807. Nonn, Konrad, Reg.-Baumeister: Christian Wilhelm Tischbein, Maler und Architekt 1751-1824. 1911. VIII, 78 S. $8^{0}$ - Um 21 Taf. verm. als: Studien zur deutschen Kunstgeschichte. H. 148.

Hannover, Dr.-Ing.-Diss. v. 26. 6. 1911

1808. Wispler, Hans, Reg.-Baumeister: Utber die Stuckbilder an den Gewölben des Posener Rathauses. Lissa: Eulitz 1912. 25 S., 17 Taf. $4^{0}$

Hannover, Dr.-Ing.-Diss. v. 26. 6. 1911

1809. Fink, Eugen, Bauinsp.: Die Treppenanlagen in den alten Bürgerhäusern der Hansestädte Bremen, Hamburg, Lübeck. Hamburg: Boysen \& Maasch 1912. 28 S., 82 Taf. $4^{0}$ - Auch als Buch.

Hannover, Dr.-Ing.-Diss. v. 25. 7. 1912

\section{ABTEILUNG FÜR BAU-INGENIEUR- WESEN}

1810. Jordan, Hermann, Reg.-Baumeister: Über die Berechnung von Nebenspannungen in Fachwerken mit steifen Knotenverbindungen. 1904. 125 S. $8^{0}$

Hannover, Dr.-Ing.-Diss. v. 30. 3. 1903

1811. Weiske, Paul, kgl. Oberlehrer: Graphostatische Untersuchung der Betonund Betoneisenträger. 〈Hiezu 1 Taf.〉 Wien: Beton \& Eisen 1904. 18 S., 1 Taf. $4^{0}$ - Forscherarbeiten auf d. Gebiete d. Eisenbetons. H. 2.

Hannover, Dr.-Ing.-Diss. v. 5. 5. 1904

1812. Kux, Eduard: Über die elastische Formänderung der Wandungen eiserner Gasbehälterbassins. 1905. 51 S. $8^{0}$ T Aus: Schilling's Journal f. Gasbeleuchtung. Jg. 48.

Hannover, Dr.-Ing.-Diss. v. 26. 9. 1905

1813. Mügge, Carl: Beiträge zur zeichnerischen Lösung technischer Rechnungs-Aufgaben. 1906. VI, 40 S., 19 Taf. $4^{0}$

Hannover, Dr.-Ing.-Diss. v. 11. 1. 1906

1814. Schütz, H[einrich], Reg.-Bauführer: Beiträge zur Bewegungslehre der ebenen statisch bestimmten Fachwerksträger. 1906. 31 S. $4^{0}$ ๆ Aus: Zeitschrift f. Architektur u. Ingenieurwesen. Bd 52. N. F. Bd 11.

Hannover, Dr.-Ing.-Diss. v. 10. 2. 1906

1815. Kinkel, Manfred: Zur Theorie des durchlaufenden Balkens. 1908. 31 S. $8^{0}$

Hannover, Dr.-Ing.-Diss. v. 30. 7. 1907

1816. Müller, Richard: Neue Versuche an Eisenbeton-Balken über die Lage und das Wandern der Nullinie und die Verbiegung der Querschnitte. Versuche über reine Haftfestigkeit. Hrsg. von Rud. Wolle, Zementbaugeschäft, Leipzig. Berlin: Ernst [1909]. VIII, 87 S., 36 Taf. $4^{0}$

Hannover, Dr.-Ing.-Diss. v. 27. 3. 1908

1817. Contag, Hellmut, Reg.-Baumeister, Minden i. W.: Uber die Bodengewinnung bei größeren Erdarbeiten insbesondere Kanalbauten, und über die Wirtschaftlichkeit des Handbetriebs und des maschinellen Betriebs bei diesen Arbeiten. 1909. 82 S., 4 Taf. $8^{0}$ - Ausz. in: Zeitschrift d. Vereines deutscher Ingenieure. Bd 54. 1910, Halbj. 2.

Hannover, Dr.-Ing.-Diss. v. 28. 6. 1909

1818. Remy, Karl, Reg.-Bauführer: Die Grössenbestimmung reiner Versand- und Empfangsschuppen. Wiesbaden: Kreidel 1910. 75 S. $4^{0}$ of Auch als Buch.

Hannover, Dr.-Ing.-Diss. v. 14. 2. 1910

1819. Quietmeyer, Friedrich, Reg.-Baumeister a. D.: Zur Geschichte der Erfindung 
des Portlandzementes. Berlin: TonindustrieZeitung 1911. XX, 188 S. $8^{0}$ ฯ Buchausg. 1912. Hannover, Dr.-Ing.-Diss. v. 4. 7. 1910

1820. Schmidt, W[ilhelm], Stadtbauing. i. Stettin: Die Kosten städtischer Straßen und deren Einfluß auf den Anbau. Berlin: J. Engelmann 1911. 16 S. $4^{0}$ - Aus: Zeitschrift f. Transportwesen u. Strassenbau. Jg. 28.

Hannover, Dr.-Ing.-Diss. v. 12. 5. 1911

1821. Rabbow, Fritz: Über Knickfestigkeit. Die Eulersche Formel für die Knickkraft bei Zugrundelegung verschiedener Dehnungsgesetze. 1912. 47 S., 8 Taf. $8^{0}$

Hannover, Dr.-Ing.-Diss. v. 18. 3. 1912

\section{ABTEILUNG FÜR MASCHINEN- INGENIEURWESEN}

1822. Heidebroek, Enno: Vergleichende Untersuchungen über die hydraulischen Eigenschaften derÜberdruckturbinen. [1902.] 16 S. $4^{0}$ Aus: Dinglers Polytechn. Journal. Jg. 83. Bd 317.

Hannover, Dr.-Ing.-Diss. v. 22. 11. 1901

1823. Handorff, F[ranz] von: Die Ausführung von Kreisteilungen in der Maschinentechnik. Berlin: S. Fischer 1903. 19 S. $4^{0}$ Aus: Zeitschrift f. Werkzeugmaschinen u. Werkzeuge. Jg. 7. 1902/03.

Hannover, Dr.-Ing.-Diss. v. 27. 2. 1903

1824. Diepen, Herman: Die störenden Bewegungen der Dampflokomotive. [1903.] $55 \mathrm{~S} .4^{0}$ - Mit unwesentl. Änderungen in: Annalen f. Gewerbe u. Bawwesen. Bd 54. 1904.

Hannover, Dr.-Ing.-Diss. v. 9. 4. 1903

1825. Wolters, Karl: Die störenden Bewegungen der Ĺokomotive unter Berücksichtigung der auftretenden Reibungswiderstände. (Berlin: Dietze 1903.) 58 S. $8^{\circ}$

- Aus: Dinglers Polytechn. Journal. Jg. 84. Bd 318.

Hannover, Dr.-Ing.-Diss. v. 16. 7. 1903

1826. Griffel, G[eorg]: Die Berechnung der Lasthaken und die sich daraus ergebenden Hakenformen bester Materialausnutzung. Berlin: Dietze 1904. $57 \mathrm{~S} .8^{0}$ - Aus: Dinglers Polytechn. Journal. Jg. 85. Bd 319.

Hannover, Dr.-Ing.-Diss. v. 7. 3. 1904

1827. Scherbius, Arthur: Vorschläge zum Bau eines indirekt wirkenden Wasser-Turbinen-Reglers. [1904.] 44 S., 6 Taf. $8^{0}$

Hannover, Dr.-Ing.-Diss. v. 14. 7. 1904

1828. Schaefer, otto: Theorie eines hydraulischen Maschinenreglers. Berlin: Dietze 1907. 57 S. $8^{0}$ - Aus: Dinglers Polytechn. Journal. Jg. 88 . Bd 322 .

Hannover, Dr.-Ing.-Diss. v. 26. 9. 1905
1829. Anthes, Hugo: Versuchsmethode zur Ermittlung der Spannungsverteilung bei Torsion prismatischer Stäbe. Berlin: Dietze 1906. 75 S. $8^{0}$ - Aus: Dinglers Polytechn. Journal. Jg. 87 . Bd 321.

Hannover, Dr.-Ing.-Diss. v. 10. 2. 1906

1830. Hirschland, Franz Herbert: Ueber die Formänderung von Drahtseilen. Berlin: Dietze 1906. 50 S., 6 Taf. $8^{0}$ - Aus: Dinglers Polytechn. Journal. Jg. 87. Bd 321.

Hannover, Dr.-Ing.-Diss. v. 1. 3. 1906

1831. Adler, Franz: Die Umlaufszahlenreihen bei Werkzeugmaschinen. 1907. $33 \mathrm{~S}$. $4^{0}$ - Auch in: Zeitschrift d. Vereines deutscher Ingenieure. Bd 51, Halbj. 2.

Hannover, Dr.-Ing.-Diss. v. 11. 3. 1907

1832. Berg, Friedrich: Der Spannungszustand einfach geschlungener Drahtseile. Berlin: Dietze 1907. 33 S. $8^{0}$ - Aus: Dinglers Polytechn. Journal. Jg. 88. Bd 322.

Hannover, Dr.-Ing.-Diss. v. 11. 3. 1907

1833. Grun, W[illibald]: Beiträge zur Theorie und Konstruktion der Leit- und Laufvorrichtungen für Turbo-Pumpen, insbesondere für Turbo-Gebläse und -Kompressoren. [1907.] $22 \mathrm{~S} .4^{0}$

Hannover, Dr.-Ing.-Diss. v. 11. 3. 1907

1834. Barten, Hermann, Gewerbe-Refer.: Untersuchungen, betr. die Bewegung der Ventile bei zwangläufigen Dampfmaschinensteuerungen, insbesondere bei der Lentz'schen Ventilsteuerung. 1907. 27 S., 6 Taf. $8^{0}$

Hannover, Dr.-Ing.-Diss. v. 30. 7. 1907

1835. Wach, Hans; Ueber den Wärmedurchgang durch die Zylinderwandungen einer Gasmaschine. 1908. 50 S., 3 Taf. $8^{0}$ Hannover, Dr.-Ing.-Diss. v. 30. 7. 1907

1836. Vormfelde, Karl: Über Milchschleudern, insbesondere über die Lagerung ihrer Trommeln. 1908. 50 S. $4^{0}$ - Aus: Landwirtschaftl. Jahrbücher. Bd 38. 1909.

Hannover, Dr.-Ing.-Diss. v. 3. 4. 1908

1837. Andres, K[arl]: Versuche über die Umsetzung von Wassergeschwindigkeit in Druck. 1909. 36 S. $4^{0}$ - Auch in: Mitteilungen über Forschungsarbeiten auf d. Gebiete d. Ingenieurwesens. H. 76; Ausz. in: Zeitschrift d.Vereines deutscher Ingenieure. Bd 54. 1910, Halbj. 2.

Hannover, Dr.-Ing.-Diss. v. 18. 5. 1908

1838. Dreyer, H[einrich]: Die Berechnung des Arbeitsverbrauches der Griesmühlen 〈Rohrmühlen〉 bei Trockenmahlung. Berlin: Dietze 1908. 24 S. $4^{0}$ - Aus: Dinglers Polytechn. Journal. Jg. 89. Bd 323.

Hannover. Dr.-Ing.-Diss. v. 13. 7. 1908 
1839. Mannes, Hermann: Die Berechnung von Rohrnetzen städtischer Wasserleitungen. 1909. 59 S., 1 Tab. $8^{0}$ - Auch bei Oldenbourg in München; 2. Aufl. 1912; Ausz. in: Gesundheits-Ingenieur. Jg. 32.

Hannover, Dr.-Ing.-Diss. v. 21. 12. 1908

1840. Bock, Ernst: Die Bruchgefahr der Drahtseile. 1909. 42 S. $4^{0}$ - Aus: Glückauf. Jg. 45.

Hannover, Dr.-Ing.-Diss. v. 12. 7. 1909

1841. Neuenhofer, Karl: Der Kettenfadenwächter am mechanischen Webstuhle. Leipzig: Martins Textil-Verl. [1909]. 35 S. $4^{0}$ - Aus: Leipziger Monatsschrift f. Textil-Industrie. Jg. 25. 1910.

Hannover, Dr.-Ing.-Diss. v. 24. 7. 1909

1842. Pape, Martin: Ueber Fahrwiderstände an Laufkranen. Berlin: Dietze 1910. 64 S. $8^{0}$ - Aus: Dinglers Polytechn. Journal. Jg. 91. Bd 325 .

Hannover, Dr.-Ing.-Diss. v. 24. 7. 1909

1843. ter Meer, Gustav: Selbsttätig wirkende Schleudermaschine zur Trocknung der Rückstände städtischer Kanalisationswässer. 1910. 48 S., 3 Tab. $4^{0}$ - Aufsatz über das gleiche Thema, aber in gekürzter Form in: Zeitschrift d. Vereines deutscher Ingenieure. Bd 52. 1908, Halbj. 2.

Hannover, Dr.-Ing.-Diss. v. 20. 12. 1909

1844. Walther, Franz: Versuche über den Arbeitsbedarf und die Widerstände beim Blechbiegen. 1910. 75 S. $4^{0}$ ๆ Auch als: Mitteilungen über Forschungsarbeiten auf d. Gebiete d. Ingenieurwesens. H. 113. 1912; kurzer Ausz. in: Zeitschrift d. Vereines dentscher Ingenieure. Bd 55. 1911, Halbj. 2.

Hannover, Dr.-Ing.-Diss. v. 21. 3. 1910

1845. Zimmermann, Werner: Beiträge zur Beurteilung des Betriebes von Dampfüberhitzern. 1910. 22 S., 2 Tab., 11 Taf. $8^{0}$ - Auch in: Zeitschrift f. Dampfkessel u. Maschinenbetrieb. Jg. 34. 1911; ohne d. graph. Darstellungen in: Der prakt. Maschinen-Konstrukteur. Jg. 45. 1912; gekürzt in: Zeitschrift d. Dampfkesseluntersuchungs- u. Versicherungs-Gesellschaft a. G. Jg. 38. 1911.

Hannover, Dr.-Ing.-Diss. v. 18. 7. 1910

1846. Döhne, Ferdinand: Ueber Druckwechsel und Stöße bei Maschinen mit Kurbeltrieb. 1911. 40 S., 4 Taf. $4^{0}$ - Etwas erw. in: Mitteilungen über Forschungsarbeiten auf $d$. Gebiete d. Ingenieurwesens. H. 118. 1912; Ausz. in: Zeitschrift d. Vereines deutscher Ingenieure. Bd 56 . 1912, Halbj. 1.

Hannover, Dr.-Ing.-Diss. v. 31. 10. 1910

1847. Moog, Otto: Die Globoidschneckengetriebe. 1910. 24 S. $4^{0}$ - Aus: Zeitschrift f. Werkzeugmaschinen u.Werkzeuge. Jg. 15. 1910/11. Hannover, Dr.-Ing.-Diss. v. 14. 11. 1910
1848. Theobald, Wilhelm, Reg.-Rat: Die Herstellung des Blattmetalls in Altertum und Neuzeit. Technologisch-historische Abhandlung. [1912.] X, 121 S. $8^{0}$. Ohne d. Literaturverzeichnis in: Annalen f. Gewerbe u. Bauwesen. Bd 70.

Hannover, Dr.-Ing.-Diss. v. 18. 3. 1912

\section{ABTEILUNG FÜR CHEMISCH-TECH- NISCHE UND ELEKTROTECHNISCHE WISSENSCHAFTEN}

Chemie

1849. Schreiber, Hermann: Ueber Halogenderivate des $\beta$-Aminocrotonsäureesters. 1901. 35 S. $8^{0}$ ๆ Ausz. in: J. Liebig's Annalen d. Chemie. Bd 317.

Hannover, Dr.-Ing.-Diss. v. 5. 6. 1901

1850. Klapproth, W[ilhelm]: Die Fällung des Zinns aus seinen Sulfosalzen und seine Trennung vom Antimon durch Elektrolyse. (1901.) 42 S. $8^{0}$ / Vgl. Zeitschrift f. angewandte Chemie. Jg. 14.

Hannover, Dr.-Ing.-Diss. v. 28. 6. 1901

1851. Thurm, Richard: Über die Konstitution der $\delta$-Methylharnsäure sowie der Alkylderivate des Methyluracils und des Nitrouracils. 1902. 32 S. $8^{0}$ - Vgl. J. Liebig's Annalen d. Chemie. Bd 323.

Hannover, Dr.-Ing.-Diss. v. 11. 7. 1901

1852. Klatt, Hugo F.: Kondensation von Glukose durch Schmelzen mit Chlorammonium. 1903. $23 \mathrm{~S} .8^{0}$ T Auch in: J. Liebig's Annalen d. Chemie. Bd 329.

Hannover, Dr.-Ing.-Diss. v. 18. 7. 1901

1853. Behrens, Wilhelm: Zur Kenntnis des Bisnitrosylbenzyls sowie der bei seiner Bildung entstehenden Nebenprodukte. 1901. 31 S. $8^{0}$ - Vgl. J. Liebig's Annalen d. Chemie. Bd 323. 1902.

Hannover, Dr.-Ing.-Diss. v. 22. 11. 1901

1854. Grünewald, Richard: Die Oxydation des Methyluracils. 1901. 29 S. $8^{0}$ - Vgl. J. Liebig's Annalen d. Chemie. Bd 323. 1902.

Hannover, Dr.-Ing.-Diss. v. 20. 12. 1901

1855. Dierssen, Heinrich : Über die zuckerartigen Abbauprodukte der Stärke bei der Hydrolyse durch Oxalsäure, mit besonderer Berücksichtigung der Lintnerschen Isomaitose. Berlin: Springer 1903. 14 S. $4^{0}$ I Aus: Zeitschrift f. angewandte Chemie. Jg. 16.

Hannover, Dr.-Ing.-Diss. v. 18. 12. 1902

1856. Fricke, Ludwig: Die Oxydation des Trimethyluracils. 1903. 29 S. $8^{0}$ il Vgl. J. Liebig's Annalen d. Chemie. Bd 327.

Hannover, Dr.-Ing.-Diss. v. 18. 12.1902 
1857. Hesse, Paul: Über Condensationsproducte von Aminocrotonsäureester mit Senfölen. 1903. 24 S. $8^{0}$ - Vgl. J. Liebig's Annalen d. Chemie. Bd 329.

Hannover, Dr.-Ing.-Diss. v. 16. 6. 1903

1858. Drewes, Hans: Über die wichtigsten thermischen Daten des Ammoniaks. [1903.] 37 S., 8 Taf. $8^{0}$

Hannover, Dr.-Ing.-Diss. v. 29. 6. 1903

1859. Roth, Paul: UUber die Birotation der Glukose. [1904.] 30 S. $8^{0}$ ・ Vgl. J. Liebig's Annalen d. Chemie. Bd 331.

Hannover, Dr.-Ing.-Diss. v. 10. 12. 1903

1860. Carstens, Johann: Zur Kenntnis der Chromsäure als Oxydationsmittel. (1904.) 49 S., 10 Taf. $8^{0}$ - Ausz. aus einem Teil in: Zeitschrift f. anorgan. Chemie. Bd 50. 1906.

Hannover, Dr.-Ing.-Diss. v. 7. 1. 1904

1861. Siemonsen, Ludwig: Ueber die Constitution des $\beta$-Methylallantoins. (1904.) 45 S. $8^{0}$ - Aus: J. Liebig's Annalen d. Chemie. Bd 333.

Hannover, Dr.-Ing.-Diss. v. 18. 2. 1904

1862. Friedrich, Hermann: Zur Kenntnis der Dialursäure. 1904. 44 S. $8^{0}$ - Vgl. J.Liebig's Annalen d. Chemie. Bd 344. 1906.

Hannover, Dr.-Ing.-Diss. v. 30. 11. 1904

1863. Heikel, Gunnar: Ueber die Birotation der Galactose. (1904.) $38 \mathrm{~S} .8^{0}$ - Aus: J. Liebig's Annalen d. Chemie. Bd 338. 1905.

Hannover, Dr.-Ing.-Diss. v. 15. 12. 1904

1864. Hennicke, Hans: Über die Einwirkung von Senfölen auf Aminocrotonsäureester. 1904. 32 S. $8^{0}$ - Vgl. J. Liebig's Annalen d. Chemie. Bd 344. 1906.

Hannover, Dr.-Ing.-Diss. v. 15. 12.1904

1865. Hufsehmidt, Carl: Die Oxydation der methylierten Methyluracile und die Nitrierung des Trimethyluracils. 1905.34 S. $8^{0}$ - Vgl. J. Liebig's Annalen d. Chemie. Bd 343.

Hannover, Dr.-Ing.-Diss. v. 2. 2. 1905

1866. Offe, Gustav: Die Oxydation von Derivaten des Methyluracils und des Uracils. 1905. $44 \mathrm{~S} .8^{0}$ - Vgl. J. Liebig's Annalen d. Chemie. Bd 353. 1907.

Hannover, Dr.-Ing.-Diss. v. 26. 9. 1905

1867. Osten, Hans: Ueber Nitrierung bei Gegenwart von Phosphorsäureanhydrid und Beiträge zur Kenntnis des Trioxydihydromethyluracils. 1905. 32 S. $8^{0}$ - Vgl. J. Liebig's Annalen d. Chemie. Bd 343.

Hannover, Dr.-Ing.-Diss. v. 26. 9. 1905

1868. Hoebel, Otto: UUber Alkylderivate des Methyluracils. 1906. 34 S. $8^{0}$ - Vgl. J. Liebig's Annalen d. Chemie. Bd 353. 1907.

Hannover, Dr.-Ing.-Diss. v. 19. 10. 1905
1869. Denieke, Gustav: Utber die Oxydation der Harnsäure bei Gegenwart von Ammoniak. 1906. $34 \mathrm{~S} .8^{0}$ - Auch in: J. Liebig's Annalen d. Chemie. Bd 349.

Hannover, Dr.-Ing.-Diss. v. 8. 6. 1906

1870. KieBling, Walther: Über die Condensation von Acetessigester mit Phenylharnstoff. 1906. $31 \mathrm{~S} .8^{0}$ - Aus: J. Liebig's Annalen d. Chemie. Bd 349.

Hannover, Dr.-Ing.-Diss. v. 8. 6. 1906

1871. Rheinfels, C[urt]: Das Maltodextrin $\gamma$ ein Zwischenprodukt der diastatischen Stärkehydrolyse. 1906. 22 S. $8^{0}$ - Autoreferat in: Centralblatt $f$. Bakteriologie, Parasitenkunde u. Infektionskrankheiten. Abt. 2. Bd 17. 1907 u. in: Wochenschrift f. Brauerei. Jg. 23.

Hannover, Dr.-Ing.-Diss. v. 8. 6. 1906

1872. Voigt, otto: Utber die Acetylierung der Zellulose. [1906.] 31 S. $8^{0}$ - Vgl. Zeitschrift f. angewandte Chemie. Jg. 19, 1. S. $998 \mathrm{ff}$.

Hannover, Dr.-Ing.-Diss. v. 29. 6. 1906

1873. Loeser, Carl: Kalkhaltige Tone, ihre Eigenschaften, Verhalten und Färbungen im Feuer. 1906 . VII, 63 S., 3 Taf. $8^{0}$ - Auch bei Nebert in Halle.

Hannover, Dr.-Ing.-Diss. v. 24. 7. 1906

1874. Weitzner, Emil: Versuche zur Synthese des $\alpha$-Methylallantoins. 1907 . 22 S. $8^{0}$ - Gekürzt in: J. Liebig's Annalen d. Chemie. Bd 361. 1908.

Hannover, Dr.-Ing.-Diss. v. 3.12. 1906

1875. Lohr, Friedrich: Über die Phenylhydrazone der Glucose. 1908. 52 S. $8^{0}$ - Vgl. J. Liebig's Annalen d. Chemie. Bd 362.

Hannover, Dr.-Ing.-Diss. v. 23. 3. 1908

1876. Gennerieh, Georg: Utber die Löslichkeit von Oxalaten in Wasser und Salzsäure. [1908.] 46 S., 1 Taf. $8^{0}$

Hannover, Dr.-Ing.-Diss. v. 15. 6. 1908

1877. Niemeyer, Rudolf: Über die Kondensation von Hydantoin mit Formaldehyd. (1908.) 28 S., 1 Taf. $8^{0}$

Hannover, Dr.-Ing.-Diss. v. 30. 11. 1908

1878. Hofmann, Adolf: Utber die Hydrazone der Zucker. (1908.) 61 S. $8^{0}$ - Vgl. J. Liebig's Annalen d. Chemie. Bd 366. 1909.

Hannover, Dr.-Ing.-Diss. v. 21. 12. 1908

1879. Selfultz, Roland: Zur Oxydation der Harnsäure in alkalischer Lösung. 1908. 29 S. $8^{0}$ - Vgl. J. Liebig's Annalen d. Chemie. Bd 365. 1909.

Hannover, Dr.-Ing.-Diss. v. 21. 12. 1908

1880. Voerkelius, G[ustav] A[dolf]: Über die Entstehung der Blausäure aus Ammoniak und Holzkohle, und aus Di- und Trimethylamin. 1909. 32 S., 2 Taf. $8^{0}$ ๆ Ausz. in: Chemiker-Zeitung. Jg. 33 .

Hannover, Dr.-Ing.-Diss. v. 24. 3. 1909 
1881. Lambreeht, Erieh: Ueber die jodometrische Säuremessung und ihre Anwendung zum Nachweis von Hydrolyse. 1909. 55 S. $8^{0}$

Hannover, Dr.-Ing.-Diss. v. 24. 7. 1909

1882. Ludewig, Wilhelm: Ueber die Kondensation von $\beta$-Naphtaldehyd mit Bernsteinsäure und einen neuen Uebergang vom Naphtalin zum Phenanthren. 1910. 51 S. $8^{\circ}$ - Ausz. in: J. Liebig's Annalen d. Chemie. Bd 379. 1911.

Hannover, Dr.-Ing.-Diss. v. 22. 11. 1909

1883. Sehliephacke, Gerhard: Uther die Mutarotation der Maltose. [1910.] 37 S., 2 Taf. $8^{0}-\mathrm{Vgl}$. J. Liebig's Annalen d. Chemie. Bd 377.

Hannover, Dr.-Ing.-Diss. v. 21. 3. 1910

1884. Struve, Karl: Über die Oxydation des Methyluracils. 1910.30 S. $8^{0}$ - Vgl. J. Liebig's Annalen d. Chemie. Bd 378. 1911.

Hannover, Dr.-Ing.-Diss. v. 20. 6. 1910

1885. Schliemann, Wilhelm: Ueber die Cellobiose und die Acetolyse der Cellulose. 1910. 62 S. $8^{0}$ - Vgl. J. Liebig's Annalen d. Chemie. Bd 378. 1911.

Hannover, Dr.-Ing.-Diss. v. 4. 7. 1910

1886. Henkel, Paul: Ueber die Oxydation von 1,4- und 3,4-Dimethyluracil. 1910. $26 \mathrm{~S}$. $8^{0}$ - Vgl. J. Liebig's Annalen d. Chemie. Bd 378. 1911.

Hannover, Dr.-Ing.-Diss. v. 18. 7. 1910

1887. Krausz, Moritz: Beiträge zur fermentativen Fettspaltung. Pées 〈Ungarn〉: (Günsberger) [1910]. 50 S., 1 Taf. $8^{0}$

Hannover, Dr.-Ing.-Diss. v. 18. 7. 1910

1888. Reinsberg, Willy: Über die Phenylhydrazone der Glucose. 1910. 34 S. $8^{0}$ - Mit unwesentl. Änderungen in: J. Liebig's Annalen d. Chemie. Bd 377.

Hannover, Dr.-Ing.-Diss. v. 18. 7. 1910

1889. Hoyer, Heinrich: Über Homopiperonylamin und seine Kondensationsprodukte. 1910. 38 S. $8^{0}$ - Vgl. J. Liebig's Annalen d. Chemie. Bd 395. 1913.

Hannover, Dr.-Ing.-Diss. v. 31. 10. 1910

1890. Mertelsmann, Martin: Über die Sulfonierung des Benzols. 1910. 30 S. $8^{0}$ - Vgl. J. Liebig's Annalen d. Chemie. Bd 378. 1911.

Hannover, Dr.-Ing -Diss. v. 31. 10. 1910

1891. Spillner, Friedrieh G[ustav], Chemiker d. Emschergenossenschaft zu EssenRuhr: Die Trocknung des Klärschlammes. 1910. $63 \mathrm{~S} .8^{0}$ - Auch in: Mitteilungen aus d.
Kgl. Prüfungsanstalt f. Wasserversorgung u. Abwässerbeseitigung zu Berlin. H. 14. 1911.

Hannover, Dr.-Ing.-Diss. v. 31. 10. 1910

1892. Klinckhard, Theodor: Über den $\beta$-Naphtaldehyd und seine Kondensation mit Pyroweinsäure. 1910. 44 S. $8^{0}$ - Vgl. J. Liebig's Annalen d. Chemie. Bd 379. 1911.

Hannover, Dr.-Ing.-Diss. v. 5. 12.1910

1893. Gessner, Ludwig: Stärke-Viskose und Alkalistärke - Xanthogenate. (1910.) 48 S. $8^{0}$ Tgl. J. Liebig's Annalen d. Chemie. Bd 382. 1911.

Hannover, Dr.-Ing.-Diss. v. 19. 12. 1910

1894. Grohmann, Oskar: Über die Oxydation von 3- und 7-Methylharnsäure bei Gegenwart von Ammoniak, 1911. 39 S. $8^{0}$ - Vgl. J. Liebig's Annalen d. Chemie. Bd 382. Hannover, Dr.-Ing.-Diss. v. 19. 12. 1910

1895. Rosenow, Max: Über die Bildsamkeit der Tone. 1911. 49 S. $8^{0}$ - Vgl. SprechSaal. Jg. 46. 1913. S. $445 \mathrm{ff}$.

Hannover, Dr.-Ing.-Diss. v. 19. 12.1910

1896. Westhoff, Franz: Zur Kenntnis der Viscose und der daraus regenerierten Cellulose. 1911. 43 S. $8^{0}$ ๆ Vgl. J. Liebig's Annalen d. Chemie. Bd 382.

Hannover, Dr.-Ing.-Diss. v. 20. 2. 1911

1897. Runte, August: Über Trioxydihydromethyluracil und Trioxydihydrotrimethyluracil. 1911. 29 S. $8^{0}$ Experimenteller Teil etwas gekürzt in: J. Liebig's Annalen d. Chemie. Bd 385 .

Hannover, Dr.-Ing.-Diss. v. 20. 3. 1911

1898. Bückendorff, Oskar: Über Alkylderivate des Methyluracils. 1911. 34 S. $8^{0}$ - Etwas gekürzt in: J. Liebig's Annalen d. Chemie. Bd 385 .

Hannover, Dr.-Ing.-Diss. v. 26. 5. 1911

1899. Kircher, Wilhelm: Über die Kondensation von Methyluracil mit Formaldehyd. 1911. 31 S. $8^{0}$ - Etwas gekürzt in: J. Liebig's Annalen d. Chemie. Bd 385.

Hannover, Dr.-Ing.-Diss. v. 26. 5. 1911

1900. Bretnuitz, Alfred: Über die Untersuchung des Steinsalzes vom Benther Salzgebirge bei Hannover. 1911. 39 S. $8^{0}$ - Ausz. in: Kali. Jg. 5. 1911.

Hannover, Dr.-Ing.-Diss. v. 10. 7. 1911

1901. Löbel, Rudolf: Beiträge zur Kenntnis des Torfteers. 1911. 43 S. $8^{0}$

Hannover, Dr.-Ing.-Diss. v. 10. 7. 1911

1902. Beythien, Rudolf: Über Nitrosodimethyluracilnitriloxyd. 1912. 35 S. $8^{0}$ - Vgl. J. Liebig's Annalen d. Chemie. Bd 389. Hannover, Dr.-Ing.-Diss. v. 5. 2. 1912 
1903. Rinau, Walter: Ueber die Zersetzungsgeschwindigkeit von Monochloramin. 1912. 44 S. $8^{0}$

Hannover, Dr.-Ing.-Diss. v. 5. 2. 1912

1904. Klein, Friedr[ich]: Die Benzoylierung der Zellulose. 1912. VI, 103 S. $8^{0}$ - Vgl. Zeitschrift f. angewandte Chemie. Jg. 26, 1. 1913.

Hannover, Dr.-Ing.-Diss. v. 4. 3. 1912

1905. Greeff, Max: Über die Bildungstemperaturen des Kalziumkarbids. Bonn: Georgi 1912. 42 S., 8 Taf. $8^{0}$

Hannover, Dr.-Ing.-Diss. v. 10. 6. 1912

1906. Kranendieck, $\mathbf{F}[\mathrm{ranz}]$ : Über die Zersetzungsgeschwindigkeit von Ammoniak und von Schwefeltrioxyd. 1912. 61 S. $8^{0}$ - Vgl. Festschrift W. Nernst gewidmet. Halle 1912. S. 99 ff.: Zeitschrift f. phys. Chemie. Bd 80. S. $148 \mathrm{ff}$.

Hannover, Dr.-Ing.-Diss. v. 10. 6. 1912

1907. Becker, Paul: Uber Condensationsprodukte des $\beta$-Phenyläthylformamids und des h-Piperonylformamids. $1912.30 \mathrm{~S} .8^{0}$ - Vgl. Berichte d. Deutschen Chem. Gesellschaft. Jg. 45 .

Hannover, Dr.-Ing.-Diss. v. 15. 7. 1912

1908. ten Doornkaat Koolman, Gerhard: Ueber die Synthese der Muconsäure aus Glyoxalnatriumbisulfit und Malonsäure. 1912. 28 S. $8^{0}$ - Vgl. J. Liebig's Annalen d. Chemie. Bd 393.

Hannover, Dr.-Ing.-Diss. v. 15. 7. 1912.

1909. Oertel, Rudolf: Zur Kenntnis der Oxycellulose. 1912. 46 S. $8^{0}$ - Ausz. in: Zeitschrift f. angewandte Chemie. Jg. 26, 1. 1913.

Hannover, Dr.-Ing.-Diss. v. 18. 12. 1912

\section{Elektrotechnik}

1910. Schüppel, Wilhelm: Über den Einfluss der Beschaffenheit der Oberfläche von elektrischen Maschinen und der Tourenzahl auf die Erwärmung. 1902: 32 S. $8^{0}$ - Ausz. in: Zeitschrift f. Elektrotechnik. Jg. 21. 1903.

Hannover, Dr.-Ing.-Diss. v. 20. 6. 1902

1911. Beckmann, Erich: Untersuchungen über Wirbelstrombremsen. [1903.] $39 \mathrm{~S}$. $4^{0}$

Hannover, Dr.-Ing.-Diss. v. 11. 12. 1902

1912. Schulze, Günther: Über den Spannungsverlust im elektrischen Lichtbogen. 1903. 53 S. $8^{0}$ - Ausz. in: Annalen d. Physik. Bd 317. Folge 4, Bd 12.

Hannover, Dr.-Ing.-Diss. v. 11. 12. 1902
1913. Oldiges, $\mathbf{B}[$ enno]: Ueber den Einfluß der Temperatur auf die Kapazität des Bleiakkumulators. [1904.] 58 S., 4 Taf. $8^{0}$ Hannover, Dr.-Ing.-Diss. v. 9. 12. 1903

1914. Pohl, Robert: Ueber magnetische Wirkungen der Kurzschlussströme in Gleichstromankern. 1905. $62 \mathrm{~S} .8^{0}$ - Auch als: Sammlung elektrotechn. Vorträge. Bd 6, H. 10. Hannover, Dr.-Ing.-Dise. v. 11. 1. 1905

1915. Studniarski, Johann von: Ueber die Verteilung der magnetischen Kraftlinien im Anker einer Gleichstrommaschine. 1905. 36 S. $4^{0}$ - Mit Änderungen in: Mitteilungen über Forschungsarbeiten auf d. Gebiete d. Ingenieurwesens. H. 32. 1906; kurzer Ausz. in: Zeitschrift d. Vereines deutscher Ingenieure. Bd 50. 1906, Halbj. 2.

Hannover, Dr.-Ing.-Diss. v. 11. 1. 1905

1916. Albrecht, Richard, Reg.-Baumeister a. D.: Ueber die Ausnützung des aktiven Materials bei positiven Großoberflächenund Masseplatten des Bleiakkumulators. 1906. 35 S. $4^{0}$ - Ausz. in: Elektrotechn. Zeitschrift. Jg. 28. 1907.

Hannover, Dr.-Ing.-Diss. v. 16. 12. 1905

1917. Brandes, Friedrich: Untersuchungen über Weicheiseninstrumente, insbesondere über den Einfluß der Hysteresis und der Wirbelströme. 1906. 55 S. $8^{0}$

Hannover, Dr.-Ing.-Diss. v. 10. 2. 1906

1918. Rüdenberg, Reinhold: Energie der Wirbelströme in elektrischen Bremsen und Dynamomaschinen. 1906. 102 S. $8^{0}$ - Auch als: Sammlung elektrotechn. Vorträge. $\mathrm{Bd} 10$, H. $8 / 10$.

Hannover, Dr.-Ing.-Diss. v. 24. 7. 1906

1919. Brückmann, Alexander: Erwärmung von Motoren bei aussetzendem Betrieb. Berlin: Dietze 1908. 74 S., 1 Taf. $8^{\circ}$ - Aus: Dinglers Polytechn. Journal. Jg. 89. Bd 323. Hannover, Dr.-Ing.-Diss. v. 23. 12.1907

1920. Grabe, W[alter]: Utber die EnergieÄnderungen und deren Zusammenhang mit den Änderungen der Lichtstärke bei Nebenschluß-Bogenlampen für Gleichstrom. 1908. 78 S., 28 Taf. $4^{0}$

Hannover, Dr.-Ing.-Diss. v. 23. 12. 1907

1921. Meyer-Delius, Heinrich: Beitrag zur Theorie des Mehrphasen-WechselstromKompound-Motors. 1908. 55 S., 17 Taf. $8^{0}$

Hannover, Dr.-Ing.-Diss. v. 23. 12. 1907

1922. Ruths, Johannes: Versuche zur Bestimmung der Widerstände von Förderanlagen. 1909. 38 S., 10 Taf. $4^{0}$ - Auch in: Mitteilungen über Forschungsarbeiten auf $d$. Gebiete d. Ingenieurwesens. H. 85. 1910.

Hannover, Dr.-Ing.-Diss. v. 13. 7. 1908 
1923. Adam, 0tto: Rotierende Anker in rotierenden materiellen Polsystemen. 1909. 74 S. $8^{0}$

Hannover, Dr.-Ing.-Diss. v. 28. 6. 1909

1924. Noeggerath, Jakob E.: Über die Stromabnahme mit besonderer Berücksichtigung hoher Geschwindigkeiten. 1911. 17 S. $4^{0}$ - Aus: Elektr. Kraftbetriebe u. Bahnen. Jg. 9.

Hannover, Dr.-Ing.-Diss. v. 18. 7. 1910
1925. Kühn, Ludwig: Über Spannungsgefahren an geerdeten, eisernen Masten. 1910. 102 S. $8^{0}$

Hannover, Dr.-Ing.-Diss. v. 3. 10. 1910

1926. Linke, W[illy]: Über Schaltvorgänge bei elektrischen Maschinen und Apparaten. Berlin: Springer 1912. 58 S. $4^{0}$ - Ohne wesentl. Kürzung in: Archiv f. Elektrotechnik. Bd 1.

Hannover, Dr.-Ing.-Diss. v. 23. 10. 1911

\section{KARLSRUHE}

Gegründet 1825 als „Polytechnische Schule“, 1885 ,,Technische Hochschule“, 1902 mit dem Beinamen „Fridericiana“"

\section{SATZUNGEN ORDNUNGEN BESTIMMUNGEN}

\section{ALLGEMEINES UND VERMISCHTES}

1927. Gesetze der Großherzogl. Badischen polytechnischen Schule. 1848. - [Veränderte Fassung.] 1849. 1851. 1852. 1854. 1857.

1848. 1851: $K$; 1849: K HofB; 1852: $S$; 1854: $K^{*}$

1928. Die Plenarversammlung an der Polytechnischen Schule zu Carlsruhe (v. 26. 6. 1861).

1929. Gesetze der Großherzogl. Badischen polytechnischen Schule zu Carlsruhe (v. 10. 7. 1861). 1861.

1930. [Dasselbe] (v. 25. 8. 1864). 1864. [Nebst] Anh.: Prüfungs-Ordnung [s. Nr 1950].

1931. Vorsehriften für die Studirenden der Grossherzogl. Badischen Polytechnischen Schule zu Carlsruhe. (Anh.: A. Ordnung für die Prüfung der Studirenden behufs Erlangung eines Zeugnisses über die von ihnen erworbenen Kenntnisse. B. Auszug aus der Ordnung für die Diplomprüfungen und die Ertheilung von Diplomen. C. Auszug aus der Bibliotheksordnung.) 1869. - [Veränderte Fassung.] 1873. 1879. 1879: K 1932. Statuten für die Krankenkasse des Polytechnicums (v. 30. 1. 1874). $K^{*}$ 1933. Statuten für die Krankenkasse der Grossherzogl. Technischen Hochschule in Karlsruhe (v. 28. 12. 1876).

1934. Laboratoriums - Ordnung. [Aug. 1884.]

1935. Ordnung für die Habilitation von Privatdocenten an der Grossh. Technischen Hochschule zu Karlsruhe. Durch Beschluss des grossen Rates festgestellt. 1887.
1936. Vorschriften für die Studierenden der Grossherzogl. Badischen Technischen Hochschule zu Karlsruhe. (Anh.: Auszug aus der Bibliotheksordnung.) 1887. [Veränderte Fassung.] 1894.

1937. Statuten des Ausschusses der Studentenschaft der Grossh. Technischen Hochschule zu Karlsruhe. Genehmigt 25. 6. 1888. [Folgt:] Geschäftsordnung des Ausschusses. 1888.

$M$

1938. Statuten für die Krankenkasse der Grossherzogl. Technischen Hochschule zu Karlsruhe (v. 1. 9. 1892. § 1, Z. 2: 3 Mark.) - [Dasselbe.] (§ 1, Z. 2: 4 Mark 50 Pfennig.) [1995.] — [Dasselbe.] (§ 1, Z. 2: 5 Mark.) [1911.] 1892: $K$

1939. Grossherzogl. Badische Technische Hochschule Karlsruhe. Verfassungs-Statut. Bekanntgemacht durch Erlass v. 17. 8. 1895 <Gesetzes- u. Verordnungsblatt 1895 Nr. 26. S. 347>. - [Dasselbe.] 1904. [Nebst Zusatz zu $\$ 40$ betr. Aufnahme von Frauen als Studierende u. Erg. Bl, enth. veränderte Fassung von $\S 40$; letzteres v. 1911]. 1895: $K$

1940. Grossherzogl. Badische Technische Hochschule zu Karlsruhe. Disciplinar-Vorsehriften für die Studierenden. 1896. - [Dasselbe u. d. T.:] . . Disziplinar-Vorschriften. 1908. 1911. Aus: Vorschriften f. d. Studierenden [ $\mathrm{Nr} 1936]$ 1896: $K$

1941. Grossherzogl. Badische Technische Hochschule zu Karlsruhe. Ordnung für die Habilitation von Privatdocenten. Vom 15. 6. 1896. - [Dasselbe u. d. T. :] Großherzogl.... Privatdozenten ... [1903.] 
1942. Großh. Technische Hochschule Karlsruhe. Hausordnung für das Aulagebäude. [1898.]

1943. [Beg.: ] Aufgrund der ... Landesherrlichen Verordnung vom 11. 11. 1899 ... erlassen Rektor und Senat der Technischen Hochschule die nachfolgende Fundordnung.

1944. Promotions-0rdnung für die Erteilung der Würde eines Doktor-Ingenieurs durch die Technische Hochschule Karlsruhe (v. 28. 6. 1900). - [Neudr.] (Mit Anm. zu $\S 1$ nach Erlaß v. 9. 4. 1906.) I AusführungsBestimmungen zur Promotionsordnung s. Nr 1948.

1945. Mitteilungen über das Studium an der Grossherzogl. Technischen Hochschule zu Karlsruhe. (Karlsruhe [1901]: Malsch \& Vogel. 2027.) - [Dasselbe] auf Grund der neuen Aufnahme-Bedingungen und Prüfungsordnungen. (1910.) - [Veränderte Fassung.] (Karlsruhe [1911]: Malsch \& Vogel. 6675.) - Ausg. Juni 1911.

1901: $K$

1946. Großherzogl. Badische Technische Hochschule zu Karlsruhe. Auszug aus der Bibliotheksordnung. (§ 1-i2.) [Ưm 1904.] - [Veränderte Fassung.] (Okt. 1907.) - Die vollständige Bibliotheksordnung nicht gedruckt. 1904: $K$

1947. Grossherzogl. Badische Technische Hochschule Fridericiana zu Karlsruhe. Ordnung für die Habilitation von Privatdozenten. Vom 12. 5. 1905.

1948. Ausfiihrungs - Bestimmungen zur Promotions-Ordnung für die Erteilung der Würde eines Doktor-Ingenieurs durch die Technische Hochschule Karlsruhe. (Nr 1-5.) [1909.] - [Dasselbe.] (Nr 1-6.) [1910.] [Dasselbe.] (Nr 1-6.) [Beigedr.: Vordruck für Bescheinigung des Referenten. 1911.] [Veränderte Fassung.] 1912.

1949. Technische Hochschule Fridericiana. Bestimmungen für die Benutzung der Bibliothek. 1909.

\section{PRÜFUNGSORDNUNGEN}

1950. Anhang zu den Gesetzen der Grossherzogl. Badischen polytechnischen Schule zu Carlsruhe vom 25. 8. 1864. Prïfungs-0rdnung.

1951. Ordnung der Prüfung zur Erlangung eines Diploms der Grossh. technischen Hochschule zu Karlsruhe für Kenntnisse auf dem Gebiete der Naturwissenschaften (v. 1. 2. 1877).
1952. Ordnung der Diplom- und Fachprüfungen an der Grossherzogl. Badischen Polytechnischen Schule zu Karlsruhe (v. 22. 6. 1883). 1883. - [Neudr.] 1892. 1892: $S$

1953. Diplomprüifungs- Ordnung der Grossherzogl. Badischen Technischen Hochschule Fridericiana zu Karlsruhe. Vom 8. 6. 1903. 1903.

1954. Akademische Schlussprüfungs-Ordnung der Grossh. Badischen Technischen Hochschule Fridericiana zu Karlsruhe. Vom 31. 5. 1904. 1904.

1955. Akademische Fachprüfungs - Ordnung der Grossh. Badischen Technischen Hochschule Fridericiana zu Karlsruhe. Vom 12. 7. 1904.1904.

\section{Abteilung für Architektur}

1956. Grossherzogl. Badische Technische Hochschule zu Karlsruhe. Prüfungs-0rdnung der Abteilung für Architektur. Vom 9. 4. 1896.

1957. Diplomprüfungs-0rdnung der Grossherzogl. Badischen Technischen Hochschule Fridericiana zu Karlsruhe. Vom 25. 9. 1906. Abteilung für Architektur. 1906. - [Dasselbe.] Vom 25. 9. 1906 u. 26. 3. 1910. 1910.

1906: $K$

\section{Abteilung für Ingenieurwesen}

1958. Grossherzogl. Badische Technische Hochschule zu Karlsruhe. Prüfungs-0rdnung der Abteilung für Ingenieurwesen. (Vom 9. 4. 1896.)

K

1959. Diplomprüfungs-0rdnung der Großherzogl. Badischen Technischen Hochschule Fridericiana zu Karlsruhe. Vom 25. 9. 1906. Abteilung für Ingenieurwesen. 1906.

\section{Abteilung für Maschinenwesen}

1960. Grossherzogl. Badische Technische Hochschule zu Karlsruhe. Prüifungs-0rdnung der Abteilung für Maschinenwesen. Vom 9. 4. 1896.

1961. Diplomprüfungs-0rdnung der Grossherzogl. Badischen Technischen Hochschule Fridericiana zu Karlsruhe. Vom 25. 9. 1906. Abteilung für Maschinenwesen. 1906. [Neudr.] 1910. 


\section{Abteilung für Elektrotechnik}

1962. Grossherzogl. Badische Technische Hochschule zu Karlsruhe. Prüfungs-0rdnung der Abteilung für Elektrotechnik. Vom 9. 4. 1896 .

1963. Diplomprüfungs-0rdnung der Grossherzogl. Badischen Technischen Hochschule Fridericiana zu Karlsruhe. Vom 25. 9. 1906. Abteilung für Elektrotechnik. 1906. - [Dasselbe.] Vom 25. 9. 1906 bezw. 30. 4. 1908. 1906/08. - [Dasselbe.] Vom 25. 9. 1907 [vielmehr 1906] ... 1906: $K$

\section{Abteilung für Chemie}

1964. Grossherzogl. Badische Technische Hochschule zu Karlsruhe. Prüfungs-0rdnung der Abteilung für Chemie. Vom 9. 4. 1896. 1896.
1965. Grossherzogl. Badische Technische Hochschule Karlstuhe. Diplomprüfungsordnung der Abteilung für Chemie. Vom 22. 2. 1901.

1966. Diplomprüfungs-Ordnung der Großherzogl. Badischen Technischen Hochschule Fridericiana zu Karlsruhe. Vom 25. 9. 1906. Abteilung für Chemie. 1906.

\section{Abteilung für Forstwesen}

1967. Grossherzogl. Badische Technische Hochschule zu Karlsruhe. Priifungs-Ordnung der Abteilung für Forstwesen. Vom 9. 4. 1896 .

1968. Diplomprüfungs- Ordnung der Grossherzogl. Badischen Technischen Hochschule Fridericiana zu Karlsruhe. Vom 25. 9. 1906 u. 9. 3. 1908. Abteilung für Forstwesen. 1908.

\section{PERIODISCHE SCHRIFTEN}

1969. Programm der (1851-52-1865-66: Anzeige der Vorlesungen an der) Grossherzogl. Badischen Polytechnischen Schule (1885-86 ff.: Technischen Hochschule) zu Carlsruhe für das Jahr (1872-73 ff.: Studienj.) 1832-33-1901/1902. [1832]-1901. Fortges. u. d. T.:

Fridericiana. Grossherzogl. Badische Technische Hochschule zu Karlsruhe. Programm für das Studienj. 1902/1903-1912/1913. 1902-12.

1832 /33: S; 1833/34: Donaueschingen FürstenbB; 1834/35. 1846/47. 1847/48: $\mathrm{K}$ HofB; 1835/361838/39. 1840/41. 1841/42. 1843/44-1845/46. 1849/50 - 1860/61. 1869/70. 1872/73: K; 1839/40. 1848/49: nicht nachweisbar; 1842/43: Kiel UB

1970. Großherzogl. Badische polytechnische Schule zu Karlsruhe. (Aufnahmsbedingungen und Lehrgegenstände der einzelnen Klassen und Fachschulen. Programm der Vorschule.) Studienj. 1843-1844-18581859.

1843/44. 1848/49.

1849/50. 1851/52-1853/54: Donaueschingen Fürstenb B;1844/45: S; 1845/46-1858/59: K HofB

1971. Adreßbuch der (1860-61 ff.: Grossherzogl. Badischen) polytechnischen Schule (1885-86 ff.: Technischen Hoch-Schule) in Carlsruhe. Studienj. (1866-67 : für das Jahr; 1867-68 - 1886/87: für das Studienj.; $1887 / 88$ ff.: für das W.- bzw. S.-S.) $1848-49$ -1902/1903. 1849-1902.
Fortges. u. d. T.:

Fridericiana. Grossherzogl. Badische Technische Hochschule zu Karlsruhe. Adressbuch für das S.-S. 1903. W.-S. 1903/04 [usw.]-W.-S. 1912/13. 1903-12.

$1851 / 52-1859 / 60$.

1861/62. 1869/70. 1871/72. 1885/86. 1906/07: K

1972. (1906/07 ff. : Grossh. Badische Technische Hochschule zu Karlsruhe.) U̇bersicht über die Zahl der Studierenden und Hospitanten im ... getrennt nach Staatsangehörigkeit und Abteilungen. W.-S. 1905/06. S.-S. 1906 [usw.]-W.-S. 1912/13.

1973. Feierlicher Akt des RektoratsWechsels an der Grossh. Technischen Hochschule "Fridericiana" am 21. Nov. 1906. 1906.

Fortges. u. d. T.:

Feierlichkeit anlässlich der Übergabe des Rektorates an der Grossh. Technischen Hochschule „Fridericiana“ zu Karlsruhe. Bericht über das Studienjahr 1906/1907-1908/1909.

Fortges. u. d. T.:

Feier der Grossherzogl. Technischen Hochschule Fridericiana bei Übergabe des Rektorates am 19. Nov. 1910. 25. Nov. 1911. 30. Nov. $1912.1910-[12]$.

Darin Festrede geh. von d. Rektor d. Jahres 1906/07-1912/1913 [auch gesondert ersch.]:

1906. Arnold, E[ngelbert]: Forschen, Erfinden und Gestalten. 
[1907.] Rehbock, Th[eodor]: Der wirtschaftliche Wert der binnenländischen Wasserkräfte unter besond erer Berücksichtigung des Grossherzogtums Baden.

[1908.] Krazer, Adolf: Zur Geschichte des Umkehrproblems der Integrale. [Abgedr. in: Jahresbericht d. Deutschen Mathematiker-Vereinigung. Bd 18. 1909.]

[1909.] Oechelhaeuser, A[dolf] von: Wege, Ziele und Gefahren der Denkmalpflege.

1910. Stäckel, Paul: Geltung und Wirksamkeit der Mathematik. [Abgedr. in: Jahresbericht d. Deutschen Mathematiker-Vereinigung. Bd 20. 1911; Internationale Wochenschrift f. Wissenschaft,
Kunst und Technik. Bd 5. 1911; Zeitschrift d. Vereines deutscher Ingenieure. Bd 55. 1911, Halbj. 1.]

1911. Benoit, Georg: Betrachtungen über die Hebe- und Fördertechnik.

1912. Zwiedineek Edler v. Südenhorst, Otto: Verfassung und Wirtschaftspolitik. [Soll in erweitertem Umfang erscheinen; bis 1913 anderweitig nicht ersch.]

1974. Bibliothek der Technischen Hochschule Fridericiana. Zugangs-Verzeichnis 1909, Halbj. 2-1912, Halbj. 1. Karlsruhe 1910-12. [1894, 1. 2-1909, 1: autogr.]

\section{GELEGENHEITS- UND VERMISCHTE SCHRIFTEN}

\section{REDEN BEIM DIREKTORATS- (1895 fi.: REKTORATS-)WECHSEL}

Die Reden von 1885, 1886, 1887, 1893, 1897 und 1898 wurden nicht gedruckt; die von $1906 \mathrm{ff}$. erschienen vereint mit dem Jahresbericht des abtretenden Rektors [Nr 1973] und gleichzeitig als Sonderausgaben.

1975. Schuberg, K[arl]: Die Forschungsaufgaben im Walde. 1889. [Rede 1888.] 19 S. $4^{0}$

1976. Engler, C[arl]: Der Stein der Weisen. Anh.: Bemerkungen zu Kant's Ansichten über die Chemie als Wissenschaft. 1889. 26 S. $4^{0}$

1977. Schröder, Ernst: Über das Zeichen. 1890. 24 S. $4^{0}$

1978. Wiener, Christian: Die Freiheit des Willens. 1891. $24 \mathrm{~S} .4^{0}$ I Auch bei Fock in Leipzig.

1879. Keller, Karl: Der Charakter der technischen Umwälzungen des 19. Jahrhunderts. 1892. $19 \mathrm{~S} .4^{0}$

1980. Haid, M[atthäus]: Ubber Gestalt und Bewegung der Erde. 1894. 16 S. $4^{0}$

1981. Baumeister, R[einhard]: Wirtschaftliche Aufgaben des Ingenieurs. 1895. 15 S. $4^{0}$

1982. Bunte, H[ans]: Wissenschaftliche Forschung und chemische Technik. 1896. 17 S. $4^{0}$

1983. Brauer, Ernst A[dolf]: Betrachtungen über die Maschine und den Maschinenbau. 1899. $21 \mathrm{~S}: 4^{0}$ T Auch in: Zeitschrift d. Vereines deutscher Ingenieure. Bd44. 1900, Halbj. 1.

1984. Lehmann, 0[tto]: Physik und Politik. 1901. [Rede 1900.] 55 S. $4^{0}$
1985. Haid, M[atthäus]: Die modernen Ziele der Erdmessung. 1901. 20 S. $4^{0}$

1986. Oechelhaeuser, Adolf von: Der kunstgeschichtliche Unterricht an den deutschen Hochschulen. 1902. 34 S. $4^{0}$

1987. Klein, Ludwig: Die botanischen Naturdenkmäler des Grossherzogtums Baden und ihre Erhaltung. 1904. [Rede 1903.] 80 S. $4^{0}$ - Erw. u. d. T.: Bemerkenswerte Bäume im Großherzogtum Baden. Heidelberg: Winter 1908.

1988. Sehur, Friedrieh: Johann Heinrich Lambert als Geometer. 1905. [Rede 1904.] 20 S. $4^{0}$ - Auch in: Jahresbericht d. Deutschen Mathematiker-Vereinigung. Bd 14.

1989. Siefert, Xaver: Der deutsche Wald. sein Werden und seine Holzarten. 1905. $22 \mathrm{~S} .4^{0}$

\section{FESTSCHRIFTEN ZUR FEIER DES GEBURTSTAGES DES GROSZHERZOGS}

1990. Arnold, E[ngelbert], Dr.-Ing.: Experimentelle Untersuchung der Kommutation bei Gleichstrommaschinen. [1908.] 53 S. $4^{0}$ - Auch in: Arbeiten aus d. Elektrotechn. Institut d. GroBherzogl. Technischen Hochschule Fridericiana zu Karlsruhe 1908-1909. 1909 [Nr 2012].

1991. Rehboek, Th[eodor]: Die Ausbildung der Uberfälle beim Abfluss von Wasser über Wehre nebst Beschreibung der Anlage zur Beobachtung von Uberfällen im Flussbaulaboratorium zu Karlsruhe. [1909.] II, 34 S., 3 Taf. $4^{0}$ - T. 1 etwas geändert in: Zeitschrift f. Bauwesen. Jg. 60. 1910. 
1992. Lehmann, O[tto]: Das Kristallisationsmikroskop und die damit gemachten Entdeckungen insbesondere die der flüssigen Kristalle. Mit 48 eingedr. Abb. Braunschweig: Vieweg 1910. IV, 112 S., 1 Taf. $8^{0}$

1993. Zwiedineck Edler von Südenhorst, 0tto, Dr.: Kritische Beiträge zur Grundrentenlehre. 1911. VIII, 71 S. $4^{0}$ - Gekürzt in: Zeitschrift f. d. gesamte Staatswissenschaft. Bd 67.

1994. Pauleke, W[ilhelm]: Das Experiment in der Geologie. 1912. X, $108 \mathrm{~S}$., 19 Taf. $4^{0}$

\section{VERSCHIEDENE SCHRIFTEN}

1995. Grossherzoglich Badische polytechnische Schule. Catalog der Bibliothek. Aufgestellt im August 1850. 1850.72 S. $8^{0} \mathrm{~K}$

1996. Das Chemische Laboratorium an der Grossherzoglichen Polytechnischen Schule zu Carlsruhe. Hrsg. von Dr. C[arl] Weltzien, Prof. d. Chemie u. Vorstand $d$. chem.-techn. Schule, u. H[einrich] Lang, Architekt u. Lehrer an d. Bauschule. Carlsruhe: Ch. F. Müller 1853. 6 S., 3 Taf. gr. $2^{0} \mathrm{~K}$

1997. Grossherzoglich Badische polytechnische Schule. Catalog der Bibliothek. Aufgestellt im März 1854. 1854. 80 S. $8^{0} \quad K$

1998. Statistik des Grossherzoglichen Polytechnikums zu Karlsruhe. Weltausstellung zu Melbourne 1880. 25 Taf. Text, 3 Taf. Grundrisse. $2^{0}$

1999. Die beiden ersten Deutschen Kaiser. Eine akad. Gedächtnißrede geh. an d. Technischen Hochschule in Karlsruhe am 26. Juni 1888 von Arthur Böhtlingk. Karlsruhe: Bielefeld 1888. 23 S. $8^{0} \quad K$

2000. Festgabe zum Jubiläum der vierzigjährigen Regierung Seiner Königlichen Hoheit des Grossherzogs Friedrich von Baden. In Ehrfurcht dargebracht von der Technischen Hochschule in Karlsruhe. SonderAbdr. [d. i. die für den Buchhandel bestimmte Ausg. zum Unterschied von der dem Großherzog überreichten Vorzugsausg. Frankfurt a. M.: Baer.] 1892. XCII, 374 S., 26 Taf. $4^{0}$

Enth.: Entwicklung der Technischen Hochschule von der Gründung bis zur Gegenwart, 1825-1892. - Josef Durm: Zur Baugeschichte des Grossh. Residenzschlosses in Karlsruhe. - Karl Bücher: Die gewerblichen Betriebsformen in ihrer historischen Entwicklung. - Karl Keller: Ferdinand Redtenbacher als
Begründer der Maschinenwissenschaft. - Adolf Weinbrenner: Die Geburtsstätte der Renaissance in Deutschland. - Arthur Böhtlingk: Geschichte und Literatur. - Wilhelm Valentiner: Geschichte der Grossherzogl. Sternwarte. - Wilhelm Lübke: Die Abteikirche Schwarzach. Christian Wiener: Die Zerstreuung des Lichtes durch matte Oberflächen und die Empfindungseinheit zum Messen der Empfindungsstärke. Ludwig Wedekind: Quadratsummen. Parameterdarstellungen quadratischer Mannichfaltigkeiten. Involutorische lineare Transformationen. - Karl Schuberg: Die Wuchsverhältnisse der gemischten Hochwaldbestände in Badens Waldungen. - Otto Lehmann: Geschichte des physikalischen Instituts der Techn. Hochschule Karlsruhe. [Erw. als Festgabe d. Fridericiana zur 83. Versammlung deutscher Naturforscher u. Ärzte. 1911. Nr 2014.] - Heinrich Lang: Geschichte der Gründung der polytechnischen Schule. - Marc Rosenberg: Die Kunstkammer im Grossherzogl. Residenzschlosse zu Karlsruhe. Cosmas Sayer: Ueber die Entwicklung des Flussbaus mit besonderer Rücksicht auf das Grossherzogthum Baden. - Karl Engler: Vier Jahrzehnte chemischer Forschung unter besonderer Rücksicht auf Baden als Heimstätte der Chemie. Eine gedrängte Darstellung.

2001. Statistik der Grossherzoglich Badischen Technischen Hochschule zu Karlsruhe. Weltausstellung zu Chicago 1893. $26 \mathrm{Bl} .2^{0}$

2002. Wilhelm der Glorreiche. Rede zur hundertjährigen Gedächtnisfeier geh. an d. technischen Hochschule zu Karlsruhe am 12. März 1897 von Dr. Arthur Böhtlingk, o. ö. Prof. d. Gesch. Heidelberg: Hörning 1897. 25 S. $8^{0}$

2003. Die Grossherzogliche Technische Hochschule Karlsruhe. Festschrift zur Einweihung der Neubauten im Mai 1899. 88 S., 7 Taf. $4^{0}$

2004. Das elektrotechnische Institut der Grossherzoglichen Technischen Hochschule zu Karlsruhe. Beschreibung des Baues und der inneren Einrichtungen. Von Prof. E[ngelbert] Arnold, Dir. d. Instituts. Mit 31 Textfig., 1 Titelbilde u. 7 Taf. Berlin: Springer. München: Oldenbourg 1899. 59 S., 8 Taf. $4^{0}$ - Seit 1901 alleiniger Verl. v. Springer.

2005. Technische Hochschule Karlsruhe. Bericht über die Feier der Einweihung der Neubauten und der Aula am 17., 18. und 19. Mai 1899. 1899. 32 S. $4^{0}$

2006. Die Entwicklung der Elektrotechnik in Deutschland. Festrede bei d. feierl. Akte der Einweihung des Elektrotechnischen Instituts der Grossherzogl. Technischen 
Hochschule zu Karlsruhe am 18. Mai 1899 geh. von Prof. E[ngelbert] Arnold, Dir. d. Instituts. Karlsruhe: Jahraus 1899. 19 S. $8^{0}$

2007. Die Physiognomie der mitteleuropäischen Waldbäume. Festrede zur Einweihungsfeier des neuen Botanischen Instituts der Technischen Hochschule zu Karlsruhe am 18. Mai 1899 geh. von Prof. Dr. L[udwig] Klein. Mit 10 Taf. in Lichtdr. nach 35 Originalaufnahmen des Verf. Karlsruhe: Jahraus (übergeklebt: Gutsch) 1899. $26 \mathrm{~S}$., 10 Taf. $8^{0}$ - [Titelaufl.] Karlsruhe: Jahraus 1900 .

1900: $K$

2008. Technische Hochschule Karlsruhe. Bericht über die Feier der JahrhundertWende und die Verleihung des Promotionsrechtes am 10. Januar 1900. (Darin: K[arl] Keller: Georg Friedrich Reichenbach.) 1900. 16 S. $4^{0}$

2009. Fünfzigjähriges Regierungs - Jubiläum Seiner Königlichen Hoheit des Grossherzogs Friedrich. Festvortrag und Ansprachen gehalten zur Jubelfeier in der Aula der Technischen Hochschule Fridericiana am 1. Mai 1902. (Darin: [Max] Honsell: [Geschichtlicher Abriss des badischen Ingenieurwesens].) 1902. $32 \mathrm{~S} .4^{0}$

2010. Krankenkasse der Technischen Hochschule zu Karlsruhe. (Belehrung über die Gefahren der Geschlechtskrankheiten. Unterz. : H[ans] Buchner [u. a.].) [1905.] 4 S. $8^{0}$

2011. Akademische Gedächtnisfeier für Seine Königliche Hoheit den verewigten Grossherzog Friedrich von Baden. Gedächtnisrede geh. in der Aula der Grossherzogl. Technischen Hochschule Fridericiana zu Karlsruhe am 21. Okt. 1907 vom z. Rektor Prof. Th[eodor] Rehbock. 14 S. $4^{0}$

2012. Arbeiten aus dem Elektrotechnischen Institut der Großherzoglichen Technischen Hochschule Fridericiana zu Karlsruhe. Hrsg. von Dr.-Ing. E[ngelbert] Arnold, Dir. d. Instituts. Bd [1.] 2. Berlin: Springer 1909. 11.

[1.] 1908-1909. Mit 260 Textfig. (Enth. : E[ngelbert] Arnold: Experimentelle Untersuchung der Kommutation bei Gleichstrommaschinen [Auch als Festschrift zur Feier d. 51. Geburtstages d. Grossherzogs. Nr 1990.] - J[osef] Liska: Die Reibung von Dynamobürsten. [Auch als Diss. Nr 2176.] - Alfred Fraenckel: Der einphasige kompensierte NebenschluBmotor mit besonderer Berücksichtigung des regelbaren Nebenschlußmotors von E. Arnold u. J. L. la Cour. [Auch als
Diss. Nr 2175.] — Edward Jonas: Die experimentelle Untersuchung eines Wechselstromserienmotors nvit besonderer Berücksichtigung der Wendepole. [Auch als Diss. Nr 2177.] - F[riedrich] Schimrigk: Die Wendepolstreuung und ihre Berechnung auf Grund experimenteller Untersuchung. [Auch als Diss. Nr 2178.] — Fr[iedrich] Jordan: Experimentelle Untersuchung der Kommutation mit besonderer Berüeksichtigung der Änderung der Übergangsspannung und der Verteilung des Energieverlustes zwischen Kommutator und Bürste. [Auch als Diss. Nr 2179.] - E[ngelbert] Arnold u. E[mil] Pfiffner: Die UUbergangsspannung von Kohlebürsten in Abhängigkeit von der Temperatur. Aus: E[lektro-] T[echn.] Z[eitschrift. Jg. 28.] 1907.)

2. 1910-1911. Mit 284 Textfig. (Enth.: H[erman] S[ybrand] Hallo: Die Eigensehaften des Kaskadenumformers und seine Anwendung. [Auch als Diss. Nr 2180.] - H[erman] S[ybrand] Hallo: Die Spaltpolumformer. [Auch als Hab.Schrift. Nr 2068.] - Boris von Ugrimoff: Die unipolare Gleichstrommaschine. [Auch als Diss. Nr 2181.] - Oscar Stern: Untersuehung der Statorfelder eines Einphasenmotors. [Auch als Diss. Nr 2184.] - M[artin] Radt: Die Eisenverluste in elliptischen Drehfeldern. [Auch als Diss. Nr 2185.] - A[lexius] Rajz: Die Kaskadenschaltung von dreiphasigen Induktionsmotoren und Kommutatormotoren. [Auch als Diss. Nr 2186.])

2013. Ferdinand Redtenbacher. Bericht über die Feier seines 100. Geburtstages an der Grossh. Technischen Hochschule Fridericiana zu Karlsruhe am 26. Juni 1909. 23 S., 1 Taf. $4^{0}$ - Geändert u. erw. in: Bayerisches Industrie- u. Gewerbeblatt. N. F. Jg. 42. 1910.

2014. Geschichte des Physikalischen Instituts der Technischen Hochschule Karlsruhe von otto Lehmann. Festgabe der Fridericiana zur 83. Versammlung Deutscher Naturforscher und Ärzte. Unter Benutzung der gleichbetitelten Abhandlung des Verf. in der Festschrift der Technischen Hochschule zu Karlsruhe zum 40jähr. Regierungsjubiläum des Großherzogs Friedrich von Baden [Nr 2000]. Karlsruhe: Braun 1911. 99 S. $8^{0}$

2014a. Veröffentlichung des Geodätischen Instituts der Grossherzoglich [!] Technischen Hochschule Fridericiana zu Karlsruhe i. B. Genauigkeitsuntersuchungen über die $\mathrm{Be}$ stimmung der Intensität der Schwerkraft durch relative Pendelmessungen auf 9 Stationen des badischen Oberlandes und auf der schweizerischen Referenzstation zu Basel von Dr. J[osef] Bürgin. Mit 6 Taf. Karlsruhe: Braun 1912. V, 112 S., 6 Taf. $4^{0}$ ฯ Auch als naturw.-math. Diss. v. Heidelberg. 


\section{HABILITATIONSSCHRIFTEN}

2015. Hierholzer, Karl, Dr.: Über Kegelschnitte im Raume. 1870. 24 S. $8^{\circ}$ I Aus: Math. Annalen. Bd 2.

Karlsruhe Te. H., Hab.-Schr. v. 14. 3. 1870

2016. Blankenhorn, A[lfred], Dr.: Geschichte \& Bewirthschaftung des Rebguts Blankenhornsberg bei Ihringen. [1870.] 39 S., 3 Taf. $8^{0}$ Aus: Annalen d. Oenologie. Bd 1. $1869 / 70$.

Karlsruhe Te. H., Hab.-Schr. v. 26. 3. 1870

2017. Just, L(eopold), Dr.: Die Keimung von Triticum vulgare. Ein Beitrag zur Lehre von der Stoffwanderung in den Pflanzen. 1873. 38 S., 1 Taf. $8^{\circ}$ - Aus: Annalen d. Oenologie. Bd 3.

Karlsruhe Te. H., Hab.-Schr. v. 26. 2. 1873

2018. Michaelis, A[ugust], Dr.: Über die Chloride und Oxychloride des Schwefels. 〈Mit 1 lithogr. Taf.〉 1873. 42 S., 1 Taf. $8^{\circ}$ - Aus: J. Liebig's Annalen d. Chemie u. Pharmacie. Bd 170 . N. R. Bd 94.

Karlsruhe Te. H., Hab.-Schr. v. 17.11. 1873

2019. Riffel, Alexander, Dr., pr. Arzt i. Karlsruhe: Uber die anatomischen und physiologischen Eigenschaften der äussern Haut und deren Bedeutung für die Hygieine und Therapie. 1875. $36 \mathrm{~S}$. $8^{\circ}$

Karlsruhe Te. H., Hab.-Schr. v. 1. 5.1875

2020. Wedekind, Ludwig, Dr.: Studien im binären Werthgebiet. Mit 1 lithogr. Taf. 1876. 51 S., 1 Taf. $8^{0}$

Karlsruhe Te. H., Hab.-Schr. v. 4. 4.1876

2021. Bütschli, 0tto, Dr.: Űber die Entstehung des Schwärmsprösslings der Podophrya quadripartita Clp. u. Lchm. Mit 1 lithogr. Taf. 1876. 23 S., 1 Taf. $8^{\circ}$. Aus: Jenaische Zeitschrift f. Naturwissenschaft. Bd 10 . N. F. Bd 3.

Karlsruhe Te. H., Hab.-Schr. v. 23.10.1876 2022. Nüsslin, 0tto, Dr.: Beiträge zur Anatomie und Physiologie der Pulmonaten. I. Das Vorkommen eines Verbindungsganges zwischen Niere und Perikardialraum bei Helix. II. Die Gewichtsveränderungen durch Abgabe und Aufnahme von Wasser bei Helix und Arion. Mit 1 lithogr. Taf. 1879. $47 \mathrm{~S}$., 1 Taf. $8^{0}$

Karlsruhe Te. H., Hab.-Schr. v. 22. 7. 1879

2023. Kelbe, Werner, Dr.: Ueber das Metaisocymol. 1881. 62 S. $8^{\circ}$ - Aus: J. Liebig's Annalen d. Chemie. Bd 210.

Karlsruhe Te. H., Hab.-Schr. v. 9.11. 1881

2024. Cathrein, Alois, Dr.: Über Titaneisen, Leukoxen und Titanomorphit. <Mitteilungen aus dem mineralogischen Labora- torium des Polytechnikum Karlsruhe. I.) Leipzig: W. Engelmann 1882. 16 S. $8^{\circ}$ I Aus: Zeitschrift f. Krystallographie u. Mineralogie. Bd 6 . $1881 / 82$

Karlsruhe Te. H., Hab.-Schr. v. 21. 3. 1882

2025. Kast, Hermann, Dr.: Ueber eine Acetophenondisulfosäure und einige Derivate derselben. 1884.48 S. $8^{0}$

Karlsruhe Te. H., Hab.-Schr. v. 21. 12. 1884

2026. Schleiermacher, August, Dr.: Über die Abhängigkeit der Wärmestrahlung von der Temperatur und das Stefan'sche Gesetz. 1885. 29 S., 2 Taf. $8^{0}$ - Auch in: Annalen d. Physik u. Chemie. N. F. Bd 26.

Karlsruhe Te. H., Hab.-Schr. v. 23. 4. 1885

2027. Wieler, A[rwed], Dr.: Ueber den Antheil des secundären Holzes der dicotyledonen Gewächse an der Saftleitung und über die Bedeutung der Anastomosen für die Wasserversorgung der transpirirenden Flächen. Mit 1 Taf. 1888. 57 S., 1 Taf. $8^{0}$ - Aus: Pringsheim's Jahrbücher f. wiss. Botanik. Bd 19.

Karlsruhe Te. H., Hab.-Schr. v. 12. 5. 1888

2028. Schultheiss, Chr[istoph], Dr.: Die Niederschlags - Verhältnisse des Rheingebietes. Mit $1 \mathrm{Kt}$. $1890.28 \mathrm{~S} ., 1 \mathrm{Kt} .4^{0}$ I Etwas erw. Form des Abschnittes, ,Die klimatischen Verhältnisse" in: Der Rheinstrom u. seine wichtigsten Nebenflüsse. Berlin 1889.

Karlsruhe Te. H., Hab.-Schr. v. 10. 3. 1890

2029. Migula, Walter, Dr.: [1.] Beiträge zur Kenntniss des Gonium pectorale. [2.] Die Artzahl der Bakterien bei der Beurtheilung des Trinkwassers. (1890.) 13 S., 1 Taf. S. $353-361.8^{0}$ - [1.] Aus: Botan. Centralblatt. Bd 43. [2.] Aus: Centralblattf. Bakteriologie u. Parasitenkunde. Bd 8.

Karlsruhe Te. H., Hab.-Schr. v. 23. 12.1890

2030. Dieckhoff, Emil, Dr.: Ueber das Ozon. 1. Abhandlung. [Mehr nicht ersch.] 1891. 68 S. $8^{\circ}$

Karlsruhe Te. H., Hab.-Schr. v. 5. 3. 1891

2031. Scholtz, Max, Dr.: Die Nutationen der Blüthenstiele der Papaver-Arten und der Sprossenden von Ampelopsis quinquefolia Michx. Mit 2 Taf. 1892 . S. $373-406$, 2 Taf. $8^{0}$ ๆ Aus: Beiträge zur Biologie d. Pflanzen. Bd 5 .

Karlsruhe Te. H., Hab.-Schr. v. 4.2. 1892

2032. Delisle, Alfred, Dr.: Ueber die Umwandlung ungesättigter, zweibasischer Säuren in ihre Stereoisomeren. 1892. $31 \mathrm{~S} .8^{0}$ T Aus: J. Liebig's Annalen d. Chemie. Bd 269. Karlsruhe Te. H., Hab.-Schr. v. 31. 10. 1892 
2033. Raseh, G[ustav], Dr., bisher Betriebsleiter d. elektr. Strassenbahn- u. Lichtanlage zu Gera, Reuss j. L.: Ueber die Berechnung der oberirdischen Zuleitung, des Stromverbrauchs und der Leitungsverluste elektrischer Bahnen. 1893. 22 S. $8^{0}$

Karlsruhe Te.H., Hab.-Schr. v. 18. 6. 1893

2034. Engelhorn, Karl: Über die Zeit des Ubergangs aus der mittelalterlichen Bauweise in die der Renaissance in Italien. 1894. 32 S. $4^{0}$

Karlsruhe Te. H., Hab.-Schr. v. 28.10.1893

2035. Behrens, Johannes, Dr.: Physiologische Studien über den Hopfen. 1894. 40 S. $8^{0}$ Aus: Flora oder allg. botan. Zeitung. Bd 78.

Karlsruhe Te. H., Hab.-Schr. v. 29. 6. 1894

2036. Hausrath, Hans, Dr., (großh. bad. Forsttaxator): Die Waldwegbauten des Forstbezirks St. Blasien. 1895. 30 S., 1 Kt. $8^{0}$ - Aus: Forstwissenschaftl. Centralblatt. Jg. 17. Karlsruhe Te. H., Hab.-Schr. v. 5. 2. 1896

2037. Haber, Fritz, Dr.: ExperimentalUntersuchungen über Zersetzung und Verbrennung von Kohlenwasserstoffen. München: Oldenbourg 1896. 116 S. $4^{0}$ ・ Vergriffen.

Karlsruhe Te.H., Hab.-Schr. v. 21. 3. 1896

2038. Wislicenus, Hans 〈Johs. Ad.〉, Dr. : Ueber, activirte" Metalle<Metallpaare> und die Verwendung des activirten Aluminiums zur Reduction in neutraler Lösung. Leipzig: J. A. Barth 1896. 52 S. $8^{0}$ - Aus: Journal f. prakt. Chemie. Bd 162 . N. F. Bd 54 .

Karlsruhe Te. H., Hab.-Schr. v. 5. 2. 1897

2039. Mie, Gustav, Dr.: Entwurf einer allgemeinen Theorie der Energieübertragung. Mit 7 Textfig.) Wien: Gerold in Comm. 1898. 70 S. $8^{0}$ Aus: Sitzungsberichte d. Kais. Akademie d. Wissenschaften in Wien. Math.-nat.Cl. Bd 107. Abth. 2a.

Karlsruhe Te. H., Hab.-Schr. v. 29. 7. 1897

2040. Luggin, H[ans]: Úber die photoelektrischen Erscheinungen und den photographischen Prozess. Mit 3 Fig. im Text. Leipzig: W. Engelmann 1897.61 S. $8^{\circ}$ - Aus: Zeitschrift f. physik. Chemie. Bd 23.

Karlsruhe Te. H., Hab.-Schr. v. 13. 11. 1897

2041. Escherich, K[arl], Dr. med, et phil.: Zur Anatomie und Biologie von Paussus turcicus Friv. Zugleich ein Beitrag zur Kenntniss der Myrmecophilie. Mit 1 lithogr. Taf. u. 11 Abb. im Text. Jena: Fischer 1898. 46 S., 1 Taf. $8^{0}$ - Aus: Zoolog. Jahrbücher. Abt. f. Systematik, Geographie u. Biologie d. Tiere. Bd 12. 1899.

Karlsruhe Te. H., Hab.-Schr. v. 8. 8. 1898
2042. Teichmüller, J[oachim], Ing. Dr.: Die elektrischen Gleichstromleitungen mit Rücksicht auf ihre Elastizität. In Form eines Lehrbuches dargest. 1898. 245 S. $8^{0}$ - Vollst. u. d. T.: Die elektrischen Leitungen. T. 1. Stuttgart: Enke 1899.

Karlsruhe Te. H., Hab.-Schr. v. 28.12. 1898

2043. Lichtenberg, Reinhold, Freiherr von, Dr.: Das antike Grabporträt besonders bei den Etruskern \& Römern. 1900. 97 S. $4^{0}$ - Vollst. als: Zur Kunstgeschichte des Auslandes. H. 3 .

Karlsruhe Te. H., Hab.-Schr. v. 8.11. 1899

2044. May, Walther, Dr., Assist, am zool. Inst. d. Techn. Hochsch. Karlsruhe: Die arktische, subarktische und subantarktische Alcyonaceenfauna. Mit 5 Textfig. Jena: Fischer 1900. 30 S. $4^{0}$ - Aus: Fauna Aretica. Eine Zusammenstellung d. arktischen Tierformen hrsg. v. F. Römer u. F. Schaudinn. Bd 1.

Karlsruhe Te. H., Hab.-Schr. v. 16. 3. 1901

2045. Wöhler, Lothar, Dr. phil.: Die pseudokatalytische Sauerstoffaktivierungdes Platins. 1901. 127 S., 2 Taf. $8^{0}$

Karlsruhe Te. H., Hab.-Schr. v. 25. 2. 1902

2046. Kriemler, Carl J[ohann], Lic. Math., Ingenieur: Labile und stabile Gleichgewichtsfiguren vollkommen elastischer auf Biegung beanspruchter Stäbe mit besonderer Berücksichtigung der Knickvorgänge. Mit 10 Figurentaf. 1902. 56 S., 10 Taf. $4^{0}$

Karlsruhe Te. H., Hab.-Schr. v. 7. 6. 1902

2047. Brunner, Karl, Dr., Assessor am Gr. General-Landes-Archiv zu Karlsruhe: Die Entwicklung des Schulwesens in den badischen Markgrafschaften 〈1453-1803〉. In kurzem UUberblick dargest. (1902.) $38 \mathrm{~S} .8^{\circ}$ - Aus: Monumenta Germaniae Paedagogica. Bd 24.

Karlsruhe Te. H., Hab.-Schr. v. 21. 6. 1902

2048. Bragstad, 0[le] S[ivert]: Theorie des rotierenden Feldes mit Anwendung auf die Bestimmung des Stromdiagrammes der asynchronen Maschinen. Mit 20 Textfig. $u$. 3 graph. Tab. 1902. 71 S. $8^{0}$ - Vollst. als: Sammlung elektrotechn. Vorträge. Bd 3, H. 8/9.

Karlsruhe Te. H., Hab.-Schr. v. 15. 7. 1902

2049. Muth, Franz: Untersuchungen über die Entwickelung der Inflorescenz und der Blüthen, sowie über die angewachsenen Achselsprosse von Symphytum officinale. 1902. 61 S., 7 Taf. $8^{0}$ Aus: Flora oder allg. botan. Zeitung. Bd 91. Erg. Bd.

Karlsruhe Te. H. Hab.-Schr. v. 21. 7. 1902

2050. Eitner, Paul, Dr.: Untersuchungen über die Explosionsgrenzen brennbarer Gase und Dämpfe. 1902 . VIII, 140 S. $8^{0}$ - Aus: Schilling's Journal f. Gasbeleuchtung. Jg. 45.

Karlsruhe Te. H., Hab.-Schr. v. 5.8.1902 
2051. Hamel, Georg, Dr. phil., Assist. f. theoret. Mechanik: Die Lagrange-Euler'schen Gleichungen der Mechanik. 1903. 57 S. $8^{0}$ T Aus: Zeitschrift f. Mathematik u. Physik. Bd 50. 1904.

Karlsruhe Te. H., Hab.-Schr. v. 19.12. 1903

2052. la Cour, Jens Lassen: Leerlaufund Kurzschlußversuch in Theorie und Praxis. 1904. IV, 95 S. $8^{0}$ - Vollst. bei Vieweg in Braunschweig.

Karlsruhe Te. H., Hab.-Schr. v. 25. 3. 1904

2053. Auerbach, Max, Dr., Kustos am Gr. Bad. Naturalienkabinett, zoolog. Abt.: Die Dotterumwachsung und Embryonalanlage vom Gangfisch und der Äsche im Vergleich zu denselben Vorgängen bei der Forelle. 1904. 28 S., 4 Taf. $8^{0}$ - Aus: Verhandlungen d. Naturwiss. Vereins zu Karlsruhe. Bd 17. 1903/04.

Karlsruhe Te. H., Hab.-Schr. v. 25. 5. 1904

2054. Ludwig, Walther, Dr. phil., Assist. d. darstellenden Geometrie: Projektive Untersuchungen über die Kreisverwandtschaften der nichteuklidischen Geometrie. 1904. 54 S., 1 Taf. $8^{0}$

Karlsruhe Te. H., Hab.-Schr. v. 30.6. 1904

2055. Brode, Johannes, Dr. phil.: Über die Oxydation des Stickstoffes in der Hochspannungsflamme. Mit 19 in d. Text gedr. Abb. 1905. 63 S. $8^{0}$ Auch bei Knapp in Halle.

Karlsruhe Te. H., Hab.-Schr. v. 11. 7. 1905

2056. Herzog, R[eginald] $\mathbf{0}[$ liver], Dr.: Chemisches Geschehen im Organismus. 1905. 62 S. $8^{0}$

Karlsruhe Te. H., Hab.-Schr. v. 2. 8.1905

2057. Faber, Georg, Dr. phil.: Über die zusammengehörigen Konvergenzradien von Potenzreihen mehrerer Veränderlicher. 1905. S. $289-324.8^{0}$ - Aus: Math. Annalen. Bd61. Karlsruhe Te. H., Hab.-Schr. v. 13.12. 1905

2058. Sieveking, Hermann, Dr.: Beiträge zur Theorie der elektrischen Entladung in Gasen. Freiburg i. B.: Speyer \& Kaerner 1906. 71 S., 2 Taf. $8^{0}$ - Gekürzt in: Annalen d. Physik. Folge 4, Bd 20.

Karlsruhe Te. H., Hab.-Schr. v. 7. 2.1906

2059. Hellpach, Willy, Dr. phil. et med.: Grundgedanken zur Wissenschaftslehre der Psychopathologie. Leipzig: W. Engelmann 1906. 86 S. $8^{\circ}$ - Aus: Archiv f. d. gesamte Psychologie. Bd 7.

Karlsruhe Te. H., Hab.-Schr. v. 21. 2. 1906

2060. Skita, Aladar, Dr.: Synthesen hydroaromatischer Amidocarbonsäureester. 1906. 104 S. $8^{0}$ - Vgl. Berichte d. Deutschen Chem. Gesellschaft. Jg. 40. 1908.

Karlsruhe Te. H., Hab.-Schr. v. 20. 7. 1906
2061. Hausrath, Herbert, Dr.: Die Untersuchung elektrischer Systeme auf Grundlage der Superpositionsprinzipien. Berlin: Springer 1907. 126 S. $8^{0}$ T Auch als Buch.

Karlsruhe Te. H., Hab.-Schr. v. 4.4.1907

2062. Winkelmann, Max, Dr., Assist. f. theoret. Mechanik: Untersuchungen über die Variation der Konstanten in der Mechanik. 1909. 67 S. $8^{0}$ ๆ Aus: Archiv f. Mathematik u. Physik. Reihe 3, Bd 15.

Karlsruhe Te. H., Hab.-Sehr. v. 15. 11. 1907

2063. Just, Gerhard, Dr. phil: : Physikalisch-chemische Studien an Eisensalzen. Mit 4 Fig. im Text. Leipzig: W. Engelmann 1908. 104 S. $8^{0}$ Aus: Zeitschriftf. physik.Chemie. Bd63.

Karlsruhe Te. H., Hab.-Schr. v. 10. 4. 1908

2064. Mayer, Max, Dr. phil., Dipl.-Ing.: Über einige Gasreaktionen. Methanbildung und Kohlenoxyd-Kohlensäuregleichgewicht. 1908. 136 S. $8^{0}$

Karlsruhe Te. H., Hab.-Schr. v. 29. 5. 1908

2065. Vogt, Wolfgang, Dr. phil., Assist. d. darstellenden Geometrie: Synthetische Theorie der Cliffordschen Parallelen und der linearen Linienörter des elliptischen Raumes. 1909. VII, 58 S. $8^{0}$ Auch bei Teubner in Leipzig.

Karlsruhe Te. H., Hab.-Schr. v. 19. 3. 1909

2066. Steinkopf, Wilhelm, Dr.-Ing. : Beiträge zur Kenntnis des Einflusses negativer Atome und Atomgruppen bei Derivaten des Acetonitrils und Acetamids. Leipzig: J. A. Barth 1910. 116 S. $8^{0}$ I Aus: Journal f. prakt. Chemie. Bd 189. N. F. Bd 81.

Karlsruhe Te. H., Hab.-Schr. v. 23. 12. 1909

2067. Leiser, Richard, Dr. phil.: Elektrische Doppelbrechung der Kohlenstoffverbindungen. Mit 15 als Anh. gedr. Abb. 1910. 71 S., 2 Taf. $8^{0}$ - A Auch als: Abhandlungen d. Deutschen Bunsengesellschaft f. angewandte physik. Chemie. Nr 4.

Karlsruhe Te. H., Hab.-Schr. v. 28.1. 1910

2068. Hallo, H[erman] S[ybrand], Dr.-Ing. : Die Spaltpol-Umformer. Berlin: Springer 1910. 32 S. $8^{0}$ - Auch in: Arbeiten aus d. Elektrotechn. Institut d. Großherzogl. Technischen Hochschule Fridericiana zu Karlsruhe. Bd 2. 1910. 1911. 1911 [Nr 2012].

Karlsruhe Te. H., Hab.-Schr. v. 26. 7. 1910

2069. Mohrmann, Hans, Dr. phil.: Normalflächen und projektive Gruppen. 1911. $32,30 \mathrm{~S} .8^{0}$ - Aus: Rendiconti del Circolo Matem. di Palermo. T. 31.32.

Karlsruhe Te. H., Hab.-Schr. v. 29. 7. 1910

2070. Grisebach, August, Dr. phil.: Der Garten. Eine Geschichte seiner künstlerischen Gestaltung. 1910. V, 98 S. $4^{0}$ I Mit Abb. bei Klinkhardt \& Biermann in Leipzig.

Karlsruhe Te. H., Hab.-Schr. v. 20. 10. 1910 
2071. Henglein, Martin, Dr.: Kristallographische Beiträge zur Kenntnis der Schwerspäte des Freiberger Bergreviers. Stuttgart: Schweizerbart 1911. S. $71-100$, 1 Taf. $8^{0}$ - Aus: Neues Jahrbuch f. Mineralogie, Geologie u. Paläontologie, Beil. Bd 32.

Karlsruhe Te. H., Hab.-Schr. v. 17. 5. 1911

2072. Fuchs, Gilbert, Dr. oec. publ.: Morphologische Studien über Borkenkäfer. I. Die Gattungen Ips De Geer und Pityogenes Bedel. 1911. 45 S. $8^{0}$ - Auch bei Reinhardt in München; II. Die europäischen Hylesinen ebd. 1912; weitere Veröffentlichungen sollen erfỏlgen, bis 1913 mehr nicht ersch.

Karlsruhe Te. H., Hab.-Schr. v. 30. 5. 1911

2073. Sehwaiger, A[nton], Dr.-Ing.: Belastungsausgleich in Kraftwerken. Berlin: Springer 1912. 37 S. $4^{0}$ I Aus: Elektrotechn. Zeitschrift. Jg. 33.

Karlsruhe Te. H., Hab.-Schr. v. 28. 7. 1911
2074. Noether, Fritz, Dr. phil., Assist. f. theoret. Mechanik: Über den Gültigkeitsbereich der Stokesschen Widerstandsformel. 1912. 39 S. $8^{0}$ - Aus: Zeitschrift f. Mathematik u. Physik. Bd 62. 1913.

Karlsruhe Te. H., Hab.-Schr. v. 15. 12. 1911

2074a. Holtzmann, Friedrich, Dr. med., Großh. Bad. Gewerbeinsp.: Gewerbehygiene der Lederfabrikation mit besonderer Berücksichtigung der badischen Industrie. 1912. 28 S. $8^{0}$ - Aus: 'Deutsche Vierteljahrsschrift f. öffentl. Gesundheitspflege. Bd 44.

Karlsruhe Te. H., Hab.-Schr. v. 27. 11. 1912

2074b. Fajans, Kasimir, Dr.: Die Verzweigung der Radiumzerfallsreihe. Mit $9 \mathrm{Abb}$. Heidelberg: Winter 1912. S. 173 -240. $8^{0}$ - Aus: Verhandlungen d. Heidelb. Naturhist.-Med. Vereins. N. F. Bd 12.

Karlsruhe Te. H., Hab.-Sehr. v. 10.1. 1913

\section{DIPLOM-DISSERTATIONEN}

2075. Tatarowiez, Zdzislaw von: Ueber einige Chlorbromsubstitutionsproducte der Methanreihe. 1879.18 S. $8^{0}$

Karlsruhe, Dipl.-Diss, v. 19. 7. 1879

2076. Gaier, Julius: Ueber die Einwirkung von Jod auf die Silbersalze zweibasischer Säuren der Fettkörper. 1. Theil: Einwirkung von Jod auf malonsaures Silber. [Mehr nicht ersch.] [1880.] 31 S. $8^{0}$ - Vgl. Berichte d. Deutschen Chem. Gesellschaft. Jg. 13. Karlsruhe, Dipl.-Diss. v. 23. 7. 1880

2077. Lurie, Gregor: Ein Phenylenkohlensäure-Aether. 1882. 35 S. $8^{0}$

Karlsruhe, Dipl.-Diss. v. 23. 12. 1881

2078. Warth, Constantin: Ueber die im Harzöl vorkommende Capronsäure: 1882. 36 S. $8^{0}$ Vgl. Berichte d. Deutschen Chem. Gesellschaft. Jg. 15 .

Karlsruhe, Dipl.-Diss. v. 24. 5. 1882

2079. Grodnitzky, Boris: Ueber das Elsässer Petroleum. 1884. 38 S. $8^{0}$

Karlsruhe, Dipl.-Diss. v. 30.5. 1884

2080. Edler, Robert: Acetophenon-Derivate. 1884. 44 S. $8^{0}$

Karlsrahe, Dipl.-Diss. v. 27.6.1884

2081. Arnold, August: Ueber die Einwirkung von Brom auf die wässerige Lösung der p-Toluolsulfosäure. 1886. 31 S. $8^{0}$

Karlsruhe, Dipl.-Diss. v. 6.3. 1886

2082. Levin, Ignatz: Beiträge zur Kenntniss des kaukasischen Petroleums. 1886. $68 \mathrm{~S} .8^{\circ}$

Karlsruhe, Dipl.-Diss. v. 6.3.1886
2083. Kosehnitzky, Michael: Ueber die Einwirkung von Brom auf die wässerige Lösung der $\alpha$ - und $\beta$-p-Cymol-sulfosäure. 1888. 31 S. $8^{0}$

Karlsruhe, Dipl.-Diss. v. 25. 7.1887

2084. Pasquay, Carl: Ueber die Einwirkung von Carbonylchlorid auf Ortho- und Para-Nitrophenol und Derivate der erhaltenen Producte. 1888. 27 S. $8^{0}$

Karlsruhe, Dipl.-Diss. v. 3. 2. 1888

2085. 0tten, Gerard: Ueber ein Erdöl aus Argentinien. 1888. 34 S. $8^{0}$ - Etwas gekürzt in: Dingler's Polytechn. Journal. Bd268. Reihe 6, Bd 18.

Karlsruhe, Dipl.-Diss. v. 15. 3.1888

2086. Bauer, Adolf: Ueber die Einwirkung des Acetons auf Ortho- und ParaAmidophenol. 1889. 45 S. $8^{0}$ - Vgl. Berichte d. Deutschen Chem. Gesellschaft. Jg. 22.

Karlsruhe, Dipl.-Diss. v. 19. 7. 1888

2087. Seidner, Salomon: Ueber die Zersetzung der Fettstoffe beim Erwärmen unter Druck. 1889. 58 S. $8^{0}$

Karlsruhe, Dipl.-Diss. v. 29. 1. 1889

2088. Bueb, Julius, Dr.: Beiträge zur Kenntniss einiger Derivate der Chinolin- und Nicotinsäure. 1890.22 S. $8^{0}$

Karlsruhe, Dipl.-Diss. v. 9.7. 1889

2089. Klinge, Constantin: Ueber ein symmetrisches Triphenylpyridin. 1890. 33 S. $8^{0}$ Karlsruhe; Dipl.-Diss. v. 26.11. 1889

2090. Bauer, Franz Wilhelm: Ueber das ๙-Aethylpyridylketon. 1890.36 S. $8^{0}$

Karlsruhe, Dipl.-Diss. v. 10. 6.1890 
2091. Majmon, Heinrich, Assist. am chem. Labor. d. Techn. Hochsch. Karlsruhe: Ueber das $\alpha$-Propylpyridylketon. 1890. 38 S. $8^{0}$

Karlsruhe, Dipl.-Diss. v. 10.6.1890

2092. Sehweitzer, Rudolf: Die Mandelsäuren des Naphtalins und das $\beta$-Naphtylmethylketon. 1890. 41 S. $8^{\circ}$ - Vgl. Berichte d. Deutschen Chem. Gesellschaft. Jg. 24. 1891.

Karlsruhe, Dipl.-Diss. v. 23. 7. 1890

2093. Kronstein, Abraham: Ueber das Imid der $\alpha \beta$-Pyridindicarbonsäure nebst einiger Derivate. 1891. $47 \mathrm{~S} .8^{0}$

Karlsruhe, Dipl.-Diss. v. 21. 1. 1891

2094. Wiehs, Jean: Über das $\beta$-Bromacetonaphton. 1891. 34 S. $8^{0}$

Karlsruhe, Dipl.-Diss. v. 21. 1. 1891

2095. Puhlmann, Ernst: Untersuchungen über die Bildung des Ozons durch die stille elektrische Entladung und die Einwirkung des Ozons auf Benzol und Diphenylamin. 1894. 41 S., 1 Taf. $8^{0}$

Karlsruhe, Dipl.-Diss. v. 16. 12. 1891

2096. Bossung, Eugen, Dr.: Ueber das Erdöl von Montechino. 1892. 34 S. $8^{\circ}$

Karlsruhe, Dipl.-Diss. v. 30.1. 1892

2097. Meyer, 0swald: Úber den Nachweis von Pyridinbasen in dem Teer der Kohle von Messel bei Darmstadt. 1893. 53 S. $8^{\circ}$

Karlsruhe, Dipl.-Diss. v. 30.1. 1892

2098. Feldmann, Jakob: Ueber einige neue substituirte Ketone. 1892. 36 S. $8^{0}$

Karlsruhe, Dipl.-Diss. v. 23. 6. 1892

2099. Tichaner, Heinrich: Untersuchungen über Stickstoffgehalt und AmmoniakAusbeute verschiedener Brennstoffe bei der trocknen Destillation. 1892. 51 S. $8^{0}$. Etwas gekürzt in: Schilling's Journal f. Gasbeleuchtung. Jg. 37. 1894.

Karlsruhe, Dipl.-Diss. v. 20. 7. 1892

2100. Surzycki, Stanislaus: Ueber das a-Isobutylpyridylketon. 1892. 41 S. $8^{0}$

Karlsruhe, Dipl.-Diss.' v. 24. 10. 1892

2101. Jankelewicz, Boris: Ueber das $\alpha^{1}-\alpha^{4}$ Naphtochinonchlorimid. 1892. 42 S. $8^{0}$

Karlsruhe, Dipl.-Diss. v. 28. 10. 1892

2102. Goldstein, Josef: Ueber die Darstellung, das Verhalten und die Constitution einiger Amido-, Nitro- und Oxyazofarbstoffe. 1893. 32 S. $8^{0}$

Karlsruhe, Dipl.-Diss. v. 9. 3. 1893

2103. Heymann, Stanislaw: Ueber die Xylylnitrophenylketone. $1900.27 \mathrm{~S} .8^{0}$

Karlsruhe, Dipl.-Diss. v. 9. 3. 1893
2104. Reinhardt, 0tto: Ueber das Normalamylbenzylketon und dessen Kondensation. 1893. 33 S. $8^{0}$

Karlsruhe, Dipl.-Diss. v. 15. 5. 1893

2105. Zakrzewski, Sigismund von: Ueber 2.3 Naphtalinderivate. 1894. 26 S., 1 Tab. $8^{0}$ - V Vl. Berichte d. Deutschen Chem. Gesellschaft. Jg. 27.

Karlsruhe, Dipl.-Diss. v. 18. 5. 1893

2106. Herzfeld, Robert, Dr.: Ueber $\alpha$ Naphtolsulfosäuren. 1894. 42 S. $8^{0}$ T S. $1-28$ etwas abgeänderter Abdr. der Baseler phil. Diss.: Ueber $\alpha$-Naphtolmonosulfosäuren und einige Derivate derselben. 1893.

Karlsruhe, Dipl.-Diss. v. 22. 7. 1893

2107. Dengler, Leopold: Ueber einige neue Erdöle aus Java. 1893. 51 S. $8^{\circ}$

Karlsruhe, Dipl.-Diss. v. 20.10.1893

2108. Stange, Alfred: Ueber die Constitution der Phtaleïne in alkalischer Lösung. 1894. 40 S. $8^{0}$ ๆ Vgl. Berichte d. Deutschen Chem. Gesellschaft. Jg.26. 1893.

Karlsruhe, Dipl.-Diss. v. 1. 12. 1893

2109. Halperin, Isaak: Zur Kenntniss der Thrane und des Walrathöles. 1895. 54 S. $8^{0}$ Karlsruhe, Dipl.-Diss. v. 31. 1. 1895

2110. Mac Garvey, Frederick: Ueber den Stickstoffgehalt des Bitumens in seiner Beziehung zur Frage der Bildung des Erdöls, Stickstoffbasen des Elsässer Erdöls, Untersuchung eines bituminösen Schiefers aus Texas, die Produkte der trockenenDestillation. von Muscheln und von Fischen. 1896. 35 S. $8^{0}$

Karlsruhe, Dipl.-Diss. v. 22. 5. 1895

2111. Spielvogel, Moritz: Ueber einige Nitronaphtonitrile, -Amide und -Säuren, sowie über die Darstellungen und Nitrirungen von einigen Naphtylaminderivaten. 1895. 45 S. $8^{0}$ i Auch als phil. Diss. v. Bern. Karlsruhe, Dipl.-Diss. v. 27. 6. 1895

2112. Zinberg, Srul: Ueber den Einfluss der Carbonylgruppe bei der Bromirung methylhaltiger aromatischer Ketone. 1895. 37 S. $8^{\circ}$

Karlsruhe, Dipl.-Diss. v. 8. 7. 1895

2113. Schreiber, WilheIm, Dr.: Über einige Nitroderivate des Naphtalins. 1895. 34 S. $8^{0}$

Karlsruhe, Dipl.-Diss. v. 17. 7. 1895

2114. Pollák, Friedrich, Dr.: Über die Condensation der drei Nitrobenzaldehyde mit Acetylaceton. Budapest 1895: Hauptstädt. Druckerei A. G. - [Dasselbe.] Emmendingen 1895: A. Dölter. Je 48 S., 2 Tab. $8^{\circ}$

T Ohne S. $47 / 48$ auch als phil. Diss. v. Basel. Ausg. Emmendingen: Königsberg UB Karlsruhe, Dipl.-Diss. v. 24. 7. 1895 
2115. Sehmidt, C[arl], Dr. phil.: Ueber einen Brandschiefer aus dem Lugauer Kohlenbecken. 1895. 29 S., 1 Taf. $8^{\circ}$

Karlsruhe, Dipl.-Diss. v. 24. 7. 1895

2116. Wild, Wilhelm, Dr. phil.: Über Oxime aus $\boldsymbol{\alpha}$-halogenisierten Aldehyden, Ketonen und Säuren sowie über Oximessigsäuren. 1895. 43 S. $8^{0}$ - Auch als phil. Diss. v. Würzburg; mit unwesentl. Änderungen in: J. Liebig's Annalen d. Chemie. Bd 289. 1896.

Karlsruhe, Dipl.-Diss. v. 21. 12. 1895

2117. Bertelsmann, Heinrich Wilhelm, Dr. phil.: Ueber einige Derivate der Isonicotinsäure sowie über das $\gamma$-Aminopyridin und das $\gamma$-Methylpyridylketon. 1895. $54 \mathrm{~S}$. $8^{0}$ I Auch als phil. Diss. v. Basel.

Karlsruhe, Dipl.-Diss. v. 29.1. 1896

2118. Samoylowiez, Hirseh: Studien über pyrogene Zersetzung des Hexans. 1896. $37 \mathrm{~S} .8^{0}$ T Zum Teil abgedr. in: Schilling's Journal f. Gasbeleuchtung. Jg. 39.

Karlsruhe, Dipl.-Diss. v. 29.1. 1896

2119. Heilbronner, Martin, Dr. phil.: Ueber Jodoniumbasen aus o-Jodtoluol. 1897. 30 S. $8^{0}$ Auch als: naturw.-math. Diss. v. Heidelberg 1896.

Karlsruhe, Dipl.-Diss. v. 26. 7. 1897

2120. Weber, $\mathbf{A}$ [ugust]: Untersuchungen über die Verbrennungsproducte von Leuchtflammen und über die Verbrennung des Leuchtgases an gekühlten Flächen und in Gasmotoren. 1897. 33 S. $8^{0}$ - Vgl. Berichte d. Deutschen Chem. Gesellsehaft. Jg. 29. 1896.

Karlsruhe, Dipl.-Diss. v. 29. 7. 1897
2121. Grüning, Herbert, Dr. phil. nat.: Die Zersetzung schwerer Mineralöle beim Erhitzen. 1898. 69 S. $8^{0}$ ๆ Auch als math.naturw. Diss. v. Freiburg i. S.

Karlsruhe, Dipl.-Diss. v. 25. 3.1898

2122. Luria, Sehmera: Ueber die Einwirkung von Bromwasserstoffsäure auf gebromte Nitrile. 1898. $36 \mathrm{~S}$. $8^{0}$

Karlsruhe, Dipl.-Diss. v. 25. 3. 1898

2123. Rožyński, Janusz: Ueber das mJodacetophenon und dessen Ueberführung in m-Jodmandelsäure. $1899.33 \mathrm{~S} .8^{0}$

Karlsruhe, Dipl.-Diss. v. 30. 6. 1899

2124. Fader, Adolio: Uber Condensationsversuche mit Anthranilsäureester. 1899. $57 \mathrm{~S} .8^{0}$

Karlsruhe, Dipl.-Diss. v. 7. 7. 1899

2125. Frank-Kamenetzky, Albert: Bromide des Isopentans. 1899. 49 S., 1 Taf. $8^{0}$ - Auch als phil. Diss. v. Basel.

Karlsruhe, Dipl.-Diss. v. 8. 7. 1900

2126. Oberländer, Eugen, Dr.: I. Zur Kenntnis der aromatischen Sulfinsäuren. II. Beiträge zur Kenntnis der FriedelCrafts'schen Reaktion. 1898. 32 S. $8^{0}$ - Auch als naturw.-math. Diss. v. Heidelberg $1897 . \quad K$ Karlsruhe, Dipl.-Diss. v. 30.1. 1901

2127. Eggers, F[ranz], Dr.: Zur Kenntnis der aromatischen Aldehyde. 1900. 39 S. $8^{0}$ - Auch als naturw.-math. Diss. v. Heidelberg. $K$ Karlsruhe, Dipl.-Diss. v. 12. 4. 1901

2128. Pierstorff, Hermann, Dr.: Zur Kenntnis der Styrole. Diplom-Arbeit. [Ohne Bezeichnung als Karlsruher Dipl.-Diss.] 1901. 39 S. $8^{0}$

Karlsruhe, Dipl.-Diss. v. 28. 9. 1901

\section{DOKTOR-INGENIEUR-DISSERTATIONEN}

\section{ABTELUNG FÜR ARCHITEKTUR}

2129. Schleuning, W[ilhelm]: Velia in Lucanien. 1902. 28 S., 1 Taf. $4^{0}$ ฯ Aus: Jahrbuch d. Kais. Deutschen Archäolog. Instituts. Bd 4. 1889.

Karlsruhe, Dr.-Ing.-Diss. v, 14. 1. 1902

2130. Friedenthal, Karl Panl: Das kreuzförmige Oktogon. Ein Beitrag zur Entwickelungsgeschichte des Zentral- und Kuppelbaues. 1908. 57 S., 4 Taf. $4^{0}$

Karlsruhe, Dr.-Ing.-Diss. v. 6. 3. 1907

2131. Ehrenberg, Kurt: Baugeschichte von Karlsruhe 1715-1720. Bau- und Bodenpolitik. Eine Studie zurGeschichte des Städtebaues. 1908. $118 \mathrm{~S}$., 3 Taf. $8^{0}$ - Vollst. bei Braun in Karlsruhe u. d. T.: Baugeschichte von Karlsruhe 1715-1870. Bau- u. Bodenpolitik ... Karlsruhe, Dr.-Ing.-Diss. v. 20. 5. 1908
2132. Heiligenthal, Roman Friedrich: Baugeschichte der Stadt Bruchsal vom 13. bis 17. Jahrhundert. Heidelberg: Winter 1909. $149 \mathrm{~S} ., 1 \mathrm{Taf} .4^{0}$ Um Abb. verm. als: Zeitschrift f. Geschichte d. Architektur. Beih. 2.

Karlsruhe, Dr.-Ing.-Diss. v. 15. 7. 1908

2133. Moritz, Eduard: Das antikeTheater und die modernen Reformbestrebungen im Theaterbau. 1910. IV, 114 S. $4^{0}$ - Auch als: Beiträge zur Bauwissenschaft. H. 17.

Karlsruhe, Dr.-Ing.-Diss. v. 19. 5. 1909

2134. Stübinger, 0tto, Reg.-Bauführer: Die römischen Wasserleitungen von Nîmes und Arles. Heidelberg: Winter 1909. 42 S., 2 Taf. $4^{0}$ - Um Abb. verm. als: Zeitschrift $\mathrm{f}$. Geschichte d. Architektur. Beih. 3.

Karlsruhe, Dr.-Ing.-Diss. v. 19. 5. 1909 
2135. Gsell, Martin, Architekt: Eisen, Kupfer und Bronze bei den alten Ägyptern. Archäologisch-metallurgische Abhandlung. [1910.] VIII, 103 S. $8^{0}$

Karlsruhe, Dr.-Ing.-Diss. v. 19. 7. 1910

2136. Gutman, Emil: Das Grossherzogliche Residenzschloss zu Karlsruhe. Heidelberg: Winter 1911. 94 S., 1 Taf. $4^{0}$ - Erw. als: Zeitschrift f. Geschichte d. Architektur. Beih. 5 .

Karlsruhe, Dr.-Ing.-Diss. v. 12. 12.1910

2137. Doerr, Albert: Der Bischofshof in Ladenburg a. N. Eine Darstellung seiner Entwicklung auf Grund geschichtlicher und architektonischer Forschungen unter Beigabe von Abbildungen und geometrischen Aufnahmen. [1912.] 54 S., 12 Taf. $4^{0}$

Karlsruhe, Dr.-Ing.-Diss. v. 5. 12.1912

\section{ABTEILUNG FÜR INGENIEURWESEN}

2138. Krawinkel, Wilhelm: Über städtische Entwässerungskanäle. 1904. 44 S., 5 Taf. $8^{0}$ - Wenig gekürzt in: GesundheitsIngenieur. Jg. 29. 1906.

Karlsruhe, Dr.-Ing.-Diss. v. 2. 7. 1904

2139. Köhler, Emil Johannes: Über einige physikalische Eigenschaften des Sandes und der Methoden zu deren Bestimmung. 1906. 85 S., 1 Taf. $8^{\circ}$. Auch bei Linck in Karlsruhe.

Karlsruhe, Dr.-Ing.-Diss. v. 23. 2. 1906

2140. Ritzmann, Friedrich, Grossh. Fabrikinsp. i. Karlsruhe i. B., früh. Leiter d. Materialprüfungsanst. d. Kais. Werft i. Wilhelmshaven: Untersuchungen über Trass-, Kalk-, Sandmörtel. 1907. 58 S., 2 Taf., $3 \mathrm{Bl}$. Tab. $8^{0}$ - Mit einer andern Einleitung hrsg. u. im Selbstverl. von Anton Hambloch, Dir. in Andernach a. Rh.

Karlsruhe, Dr.-Ing.-Diss. v. 21. 1. 1907

2141. Aichel; Ordulf Georg: Experimentelle Untersuchungen über den Abfluss des Wassers bei vollkommenen Überfallwehren verschiedener Grundrissanordnung. München u. Leipzig: G. Franz 1907. VII, 111 S., $11 \mathrm{Bl}$. Tab., 10 Taf. $8^{0}$ I Kurzer Ausz. in: Zeitschrift d. Vereines deutscher Ingenieure. Bd 52. 1908, Halbj. 2.

Karlsruhe, Dr.-Ing.-Diss. v. 17. 7. 1907

2142. Ludin, Adolf, Grossh. Reg.-Baumeister: Der Ausbau der Niederdruckwasserkräfte. [Frankfurt a. M.: Gesellschaft f. wirtschaftl. Ausbildung in Komm.] 1910. IV, 216 S., 8 Taf. $8^{\circ}$

Karlsruhe, Dr.-Ing.-Diss. v. 19. 5. 1909
2143. Sehachenmeier, Wilhelm: Über mehrfache elastische Gewölbe. Eine theoretische Untersuchung über die statische Wirkungsweise der Übermauerung bei weitgespannten Gewölben, ein Beitrag zur Theorie der Nebenspannungen gewölbter Brücken. Leipzig: W. Engelmann 1910. 84 S. $4^{0}$ I Auch als: Fortschritte d. Ingenieurwissenschaften. Gruppe 2, H. 23.

Karlsruhe, Dr.-Ing.-Diss. v. 17. 7. 1909

2144. Ammann, 0tto: Die Leistungsfähigkeit von Ablaufanlagen auf Verschiebebahnhöfen in ihrer Abhängigkeit von den Gefällsverhältnissen. [1911.] 27 S. $4^{0}$ I Aus: Verkehrstechn. Woche u. Eisenbahntechn. Zeitschrift. Jg. 5. 1910/11.

Karlsruhe, Dr.-Ing.-Diss. v. 24. 2. 1911

2145. Werkmeister, $\mathbf{P}($ aul $)$ : Das württembergische Präzisionsnivellement. 1912. 102 S. $8^{0}$ T Aus: Jahreshefte d. Vereins f. vaterländ. Naturkunde in Württemberg. Jg. 68.

Karlsruhe, Dr.-Ing.-Diss. v. 27. 3. 1912

2146. Sammet, Friedrich: Úber die bauliche Anlage, den Betrieb, die Leistungsfähigkeit und die Betriebskosten des Rangierbahnhofes Karlsruhe. [1912.] 52, 8 S., 5 Taf. $4^{0}$ T Auch bei Lang in Karlsruhe in Komm. Karlsruhe, Dr.-Ing.-Diss. v. 18. 7. 1912 •

\section{ABTEILUNG FÜR MASCHINENWESEN}

2147. Mehlis, Heinrich, Reg.-Baumeister a. D.: Dampfschnellbahnzug für $120 \mathrm{~km}$ mittlere stündliche Geschwindigkeit <150 Km-St. maximal $>1903.40$ S., 10 Taf. $4^{0}$ T 2. Aufl. bei Siemens in Berlin 1904.

Karlsruhe, Dr.-Ing.-Diss. v. 20. 7. 1903

2148. Oesterlin, Hermann, MaschinenIngenieurprakt.: Untersuchungen über den Energieverlust des Wassers in Turbinenkanälen. Berlin: (Springer) 1903. $75 \mathrm{~S}$., 5 Taf. $8^{0}$ ๆ Auch als Buch. [Vergriffen]. Karlsruhe, Dr.-Ing.-Diss. v. 20. 7. 1903

2149. Staus, Anton, Maschinen-Ingenieurprakt.: Einfluß der Wärme auf die Indikatorfeder. 1904. 46 S. $4^{0}$ o Auch in: Mitteilungen über Forschungsarbeiten auf d. Gebiete d. Ingenieurwesens. H. 26/27. 1905.

Karlsruhe, Dr.-Ing.-Diss. v. 22. 7. 1904

2150. Koehler, Georg Wilhelm: Die Rohrbruchventile. Konstruktions - Grundlagen, Untersuchungs-Ergebnisse und Anwendungsregeln. 1906. $81 \mathrm{~S} .4^{0}$ T Auch in: Mitteilungen über Forschungsarbeiten auf d. Gebiete d. Ingenieurwesens. H. 34; Ausz. in: Zeitschrift d. Vereines deutscher Ingenieure. Bd 52. 1908, Halbj. 1.

Karlsruhe, Dr.-Ing.-Diss. v. 11. 3. 1905 
2151. Meuth, Hermann: Kinetik und Kinetostatik des Schubkurbelgetriebes. 1905. 75 S., 6 Taf. $8^{0}$. Aus: Dinglers Polytechn. Journal. Jg. 86 . Bd 320.

Karlsruhe, Dr.-Ing.-Diss. v. 18. 3. 1905

2152. Erlinghagen, C $[$ arl], Obering.: Die Feststellung des Fallens und Streichens von Tiefbohrlöchern durch Messung. 1907. $27 \mathrm{~S}$. $4^{0}$ - Aus: Glückauf. Jg. 43.

Karlsruhe, Dr.-Ing.-Diss. v. 29. 4. 1907

2153. Jacobi, Ernst, Kgl. Preuss. Reg.Baumeister i. Strassburg i. E.: Über die nutzbare Leistung von Güterzug-Lokomotiven und ihr Verhältnis zur Kolbendruck-Leistung. Wiesbaden: Kreidel 1908. 16 S., 2 Taf. $4^{0}$ - Aus: Organ f. d. Fortschritte d. Eisenbahnwesens. N. F. Bd 45.

Karlsruhe, Dr.-Ing.-Diss. v. 28. 6. 1907

2154. Brandt, Paul: Die rotierende Kurbelschleife und die Schleppkurbel als-Antrieb für Propellerrinnen. Berlin: Dietze 908. 46 S. $8^{0}$ I Aus: Dinglers Polytechn. Journal. Jg. 89. Bd 323.

Karlsruhe, Dr.-Ing.-Diss. v. 16. 7. 1907

2155. Hempelmann, August: Versuche über Torsion rechteckig-prismatischer Stäbe. Berlin: Dietze 1907. 13 S., 36 Taf. $4^{0}$ - Ohne Taf. in: Dinglers Polytechn. Journal. Jg. 88. Bd 322.

Karlsruhe, Dr.-Ing.-Diss. v. 16. 7. 1907

2156. Voigt, Alexander: Über die Druckverteilung im Eisen vor einer eindringenden Schneide. 1907. S. $443-535.4^{0}$ o Aus: Verhandlungen d. Vereins zur Beförderung d. Gewerbfleißes. Jg. 86.

Karlsruhe, Dr.-Ing.-Diss. v. 20. 7. 1907

2157. Rehfus, Wilhelm: Schraubengetriebe mit selbsttätiger Druckregulierung. Berlin: Dietze 1910. 74 S. $4^{0}$ I Aus: Dinglers Polytechn. Journal. Jg. 91. Bd 325.

Karlsruhe, Dr.-Ing.-Diss. v. 13. 12. 1907

2158. Grether, Hans, Ingenieurprakt.: Über Potentialbewegung tropfbarer Flüssigkeiten in gekrümmten Kanälen. 1909. $118 \mathrm{~S} .4^{0}$ Auch bei Simion in Berlin; aus: Verhandlungen d. Vereins zur Beförderung d. Gewerbfleißes. Jg. 88 .

Karlsruhe, Dr.-Ing.-Diss. v. 20. 7. 1908

2159. Lutz, R[einhold]: Zur Regelung von Automobilmaschinen. 1909. 68 S. $4^{0}$ - Auch als: Mitteilungen über Forschungsarbeiten auf d. Gebiete d. Ingenieurwesens. H. 69; Ausz. in: Zeitschrift $d$. Vereines deutscher Ingenieure. Bd 53, Halbj. 1.

Karlsruhe, Dr.-Ing.-Diss. v. 25. 11. 1908

2160. Kröner, Hermann, Reg.-Baumeister: Zur Kritik der Turbinen-Regulatoren. 1910. 75 S. $8^{0}$ - Erw. u. d. T.: Die Ge- schwindigkeitsregler der Kraftmaschinen. Berlin u. Leipzig: Göschen 1912. (Sammlung Göschen. 604.)

Karlsruhe, Dr.-Ing.-Diss. v. 30. 1. 1909

2161. Boek, Hermann, Reg.-Bauführer a. D., Ingenieur u. Lehrer am Staatl. Technikum zu Hamburg: Kritische Theorie der freien Riefler-Hemmung. Berlin: Springer 1910. 68 S. $8^{0}$ - Auch als Buch.

Karlsruhe, Dr.-Ing.-Diss. v. 12. 3. 1910

2162. Sternberg, Walther: Über den Ausfluss des Wassers aus kreisförmigen Öffnungen in dünner Wand. 1911. 63 S., 6 Bl. Taf. $8^{0}$

Karlsruhe, Dr.-Ing.-Diss. v. 6. 2. 1911

-2163. Gimbel, otto, Reg.-Bauführer a. D., Oberlehrer am Staatl. Technikum zu Hamburg: Die Verwendung der Dampfturbine als Schiffskreisel. [1911.] 44 S., 1 Taf. $8^{0}$ T Aus: Zeitschrift f. Elektrotechnik u. Maschinenbau. Bd 14; Elektrotechn. Rundsehau. Jg. 28.

Karlsruhe, Dr.-Ing.-Diss. v. 20. 2. 1911

2164. Plieninger, Reginald: Untersuchungen über das Verfahren: Eisen mittels des Sauerstoffstrahles 'zu durchtrennen <autogenes Schneidverfahren $\rangle .1912 .56 \mathrm{~S} ., 8 \mathrm{Taf} .4^{0}$ - Auch in: Zeitschrift f. komprimirte u. flüssige Gase. Jg. 16. 1914.

Karlsruhe, Dr.-Ing.-Diss. v. 26. 2. 1913

2165. Mayer, Rudolf: Über Elastizität und Stabilität des geschlossenen und offenen Kreisbogens. 1912. IV S., S. $245-320$. $8^{0}$ T Aus: Zeitschrift f. Mathematik u. Physik. Bd 61. Karlsruhe, Dr.-Ing.-Diss. v. 9. 4. 1913

\section{ABTEILUNG FÜR ELEKTROTECHNIK}

2166. Gallusser, Hans: Ein Beitrag zur Vorausberechnung der Kommutationsverhältnisse bei Gleichstrommaschinen und des Spannungsabfalls bei Wechselstromgeneratoren. 1902. $60 \mathrm{~S} .8^{0}$ - Auch bei Enke in Stuttgart u. als: Sammlung elektrotechn. Vorträge. Bd 3, H. 10/11.

Karlsruhe, Dr.-Ing.-Diss. v. 1. 3. 1902

2167. Kahn, Max: Der Übergangswiderstand von Kohlenbürsten. 1902 . 57 S. $8^{0}$ T Auch bei Enke in Stuttgart u. als: Sammlung elektrotechn. Vorträge. Bd 3, H. 12.

Karlsruhe, Dr.-Ing.-Diss. v. 1. 3. 1902

2168. Bloch, Leopold: Der Einfluss der Kurvenform auf die Wirkungsweise des Synchronmotors. Mit 34 Abb. 1903. VIII, 76 S. $8^{0}$ - Auch bei Enke in Stuttgart u. als: Sammlung elektrotechn. Vorträge. Bd 5, H. 7/8.

Karlsruhe, Dr.-Ing.-Diss. v. 14. 3. 1903 
2169. Ottenstein, Simon: Das Nutenfeld in Zahnarmaturen und die Wirbelstromverluste in massiven Armatur-Kupferleitern. 1903. 48 S. $8^{\circ}$. Auch bei Enke in Stuttgart u. als: Sammlung elektrotechn. Vorträge. Bd 5, H. 5 .

Karlsruhe, Dr.-Ing.-Diss. v. 19. 12. 1903

2170. Czeija, Karl: Die experimentelle Untersuchung der Kommutationsvorgänge in Gleichstrommaschinen. Mit 31 Abb. 1903. 76 S., 2 Taf. $8^{\circ}$ - Auch bei Enke in Stuttgart u. als: Sammlung elektrotechn. Vorträge. Bd5, H. 9/10. Karlsruhe, Dr.-Ing.-Diss. v. 23. 12. 1903

2171. Marguerre, Fritz: Experimentelle Untersuchungen am Polycyklischen Stromverteilungssystem Arnold-Bragstad - la Cour. 1904. 74 S. $8^{0}$ - Auch bei Enke in Stuttgart u. als: Sammlung elektrotechn. Vorträge. Bd 5,H.11/12.

Karlsruhe, Dr.-Ing.-Diss. v. 23. 12. 1903

2172. Meyer, F[riedrich]W[ilhelm]: Berechnung elektrischer Hochspannungsanlagen auf wirtschaftstheoretischen Grundlagen. Berlin: Springer 1907. 134 S. $8^{0}$ ๆ Erw. u. d. T.: Die Berechnung elektrischer Anlagen auf wirtschaftlichen Grundlagen. 1908.

Karlsruhe, Dr.-Ing.-Diss. v. 29. 1. 1907

2173. Linker, Arthur: Die historische Entwicklung des Einphasen-Wechselstrommotors. Mit $158 \mathrm{Abb}$. Berlin: Dietze 1907. 123, IX S. $8^{\circ}$ - Aus: Dinglers Polytechn. Journal. Jg. 88. Bd 322; Ausz. in: Elektrotechn. u. polytechn. Rundschau. Jg. 24.

Karlsruhe, Dr.-Ing.-Diss. v. 13. 5. 1907

2174. Niebuhr, Herman: Experimentaluntersuchungen über die Selbstinduktion in Nuten gebetteter Spulen bei hoher Frequenz. Berlin: Springer 1907. 59 S. $8^{0}$ - Auch als Buch.

Karlsruhe, Dr.-Ing.-Diss. v. 19. 7. 1907

2175. Fraenckel, Alfred: Der einphasige kompensierte Nebenschlussmotor mit besonderer Berücksichtigung des regelbaren Nebenschlussmotors von E. Arnold und J. L. la Cour. Berlin: Springer 1908. 82 S. $8^{0}$ - Auch in: Arbeiten aus d. Elektrotechn. Institut d. GroBherzogl. Technischen Hochschule Fridericiana zu Karlsruhe 1908-1909. 1909 [Nr 2012].

Karlsruhe, Dr.-Ing.-Diss. v. 20. 7. 1908

2176. Liska, J[osef]: Die Reibung von Dynamobürsten. Berlin: Springer 1908. $38 \mathrm{~S} .8^{0}$ - Auch in: Arbeiten aus d. Elektrotechn. Institut d. GroBherzogl. Technischen Hochschule Fridericiana zu Karlsruhe 1908-1909. 1909 [Nr 2012].

Karlsruhe, Dr.-Ing.-Diss. v. 20. 7. 1908

2177. Jonas, Edward: Die experimentelle Untersuchung eines Wechselstromserienmotors mit besonderer Berücksichti- gung der Wendepole. Berlin: Springer 1908. 37 S. $8^{0}$ - Auch in: Arbeiten aus d. Elektrotechn. Institut d. Großherzogl. Technischen Hochschule Fridericiana zu Karlsruhe 1908 - 1909. 1909 [Nr 2012].

Karlsruhe, Dr.-Ing.-Diss. v. 10. 11. 1908

2178. Schimrigk, F[riedrich]: Die Wendelpolstreuung und ihre Berechnung auf Grund experimenteller Untersuchung. Berlin: Springer 1909. 41 S. $8^{0}$ व Auch in: Arbeiten aus d. Elektrotechn. Institut d. Großherzogl. Technischen Hochschule Fridericiana zu Karlsruhe 1908-1909. 1909 [Nr 2012].

Karlsruhe, Dr.-Ing.-Diss. v. 10. 11. 1908

/2179. Jordan, Friedrich: Experimentelle Untersuchung der Kommutation mit besonderer Berücksichtigung der Änderung der Übergangsspannung und der Verteilung des Energieverlustes zwischen Kommutator und Bürste. Berlin: Springer 1909. 66 S. $8^{\circ}$ - Auch in: Arbeiten aus d. Elektrotechn. Institut d. Großherzogl. Technischen Hochschule Fridericiana zu Karlsruhe 1908-1909. 1909 [Nr 2012].

Karlsruhe, Dr.-Ing.-Diss. v. 14. 6. 1909

2180. Hallo, Herman S[ybrand]: Die Eigenschaften des Kaskadenumformers und seine Anwendung. Berlin: Springer 1910. 99 S., 1 Taf. $8^{\circ}$ I Auch in: Arbeiten aus d. Elektrotechn. Institut d. Großherzogl. Technischen Hochschule Fridericiana zu Karlsruhe. Bd 2. 19101911. 1911 [Nr 2012].

Karlsruhe, Dr.-Ing.-Diss. v. 28. 1. 1910

2181. Ugrimofi, Boris von: Die unipolare Gleichstrommaschine. Berlin: Springer 1910. 98 S. $8^{0}$ - Auch in: Arbeiten aus d. Elektrotechn. Institut d. Großherzogl. Technischen Hochschule Fridericiana zu Karlsruhe. Bd 2. 1910-1911. 1911 [Nr 2012].

Karlsruhe, Dr.-Ing.-Diss. v. 28. 1. 1910

2182. Dexheimer, George: Die Verluste in den Polschuhen von Dynamomaschinen. 1910. 91 S. $8^{0}$

Karlsruhe, Dr.-Ing.-Diss. v. 12. 3. 1910

2183. Wallem, Harald: Die Elektrizität in der Landwirtschaft und deren Beziehungen zu Überlandzentralen. Berlin: Springer 1910. 46 S. $4^{0}$ ๆ Erw. aus: Elektrotechn. Zeitschrift. Jg. 31.

Karlsruhe, Dr.-Ing.-Diss. v. 27. 6. 1910

2184. Stern, 0scar: Untersuchung der Felder eines Einphasen-Repulsionsmotors System Déri. Berlin: Springer 1910. 58 S., 1 Taf. $8^{0}$ T Auch in: Arbeiten aus d. Elektrotechn. Institut d. Großherzogl. Technischen Hochschule Fridericiana zu Karlsruhe. Bd 2. 1910-1911. 1911 [Nr 2012].

Karlsruhe, Dr.-Ing.-Diss. v. 25. 7. 1910 
2185. Radt, Martin: Die Eisenverluste in elliptischen Drehfeldern. Berlin: Springer 1911. 70 S. $8^{0}$ - Ausz. in: Arbeiten aus d. Elektrotechn. Institut d. GroBherzogl. Technischen Hochschule Fridericiana zu Karlsruhe. Bd 2. 1910-1911 [Nr 2012].

Karlsruhe, Dr.-Ing.-Diss. v. 19. 12. 1910

/2186. Rajz, Alexius: Die Kaskadenschaltung von dreiphasigen Induktionsmotoren und Kommutatormotoren. Berlin: Springer 1911. $54 \mathrm{~S} .8^{0}$ I Auch in: Arbeiten aus d. Elektrotechn. Institut d. Großherzogl. Technischen Hochschule Fridericiana zu Karlsruhe. Bd 2. 1910-1911 [Nr 2012].

Karlsruhe, Dr.-Ing.-Diss. v. 27. 5. 1911

2187. Sehumann, Winfried 0[tto]: Uber die Drehmomente der Dämpferwicklung einer Mehrphasen-Synchronmaschine bei kleinen Pendelschwingungen im Parallelbetrieb. 1912. 85 S. $8^{0}$ - Kurz mitgeteilt in: E. Arnold u. J. L. la Cour: Die synchronen Wechselstrom. maschinen. 2. Aufl. Berlin 1913. (Die Wechselstromtechnik. Hrsg. von E. Arnold. Bd 4.) S. $320 \mathrm{ff}$.

Karlsruhe, Dr.-Ing.-Diss. v. 20. 8. 1912

2188. Wirz, Emil: Beitrag zur Theorie und Untersuchung der Ferrarismessgeräte. Berlin: Springer 1912. IV, 129 S. $8^{0}$ ब Auch als Buch. ,Außerdem erscheint ein teilweiser Ausz. in d. Zeitschrift ,Elektrotechnik u. Maschinenbau“" in Wien"; bis Jg. 31. 1913 nicht erseh.

Karlsruhe, Dr.-Ing.-Diss. v. 21. 10. 1912

\section{ABTEILUNG FÜR CHEMIE}

2189. Engler, Adalbert, Dr. phil.: Zur Kenntnis der Kondensationen von Aldehyden mit Ketonen. 1901. 42 S. $8^{0}$ ๆ Vgl. Berichte d. Deutschen Chem. Gesellschaft Jg. 35. 1902.

Karlsruhe, Dr.-Ing.-Diss. v. 1. 7. 1901

2190. Kačer, Filip: I. Über die Einwirkung von Knallquecksilber und Aluminiumchlorid auf Toluol, o-, m- und p-Xylol und auf Mesitylen. II. Úber die Einwirkung von Knallquecksilber auf Phenol. 1901. 46 S. $8^{0}$ T Vgl. zu II: Berichte d. Deutschen Chem. Gesellschaft. Jg. 33. 1900.

Karlsruhe, Dr.-Ing.-Diss. v. 19. 7. 1901.

2191. Witzeck, Rudolf: Über die Schwefelverbindungen im Leuchtgase. 1902. IX, 99 S., 1 Taf. $8^{0}$ ๆ Aus: Schilling's Journal f. Gasbelenchtung. Jg. 46. 1903.

Karlsruhe, Dr.-Ing.-Diss. v. 22. 7. 1902

2192. Sack, Miehael: Über die Entstehung und Bedeutung von Natriumlegierungen bei der kathodischen Polarisation. 1903. $70 \mathrm{~S} .8^{0}$ ๆ Aus: Zeitschrift f. anorgan. Chemie. Bd 34.

Karlsruhe, Dr.-Ing.-Diss. v. 23. 12. 1902
2193. Heymann, Stanislaw: Ueber die Nitrophenylketone des m.- und p.-Xylols, des Mesitylens und Pseudocumols. 1903. 48 S. $8^{\circ}$

Karlsruhe, Dr.-Ing.-Diss. v. 20. 1. 1903

2194. Broniatowski, Heinrich: Zur Kenntnis der Nitrierung des Acetylmetaamidoacetophenons. 1903. $54 \mathrm{~S} .8^{0}$

Karlsruhe, Dr.-Ing.-Diss. v. 2. 2. 1903

2195. Russ, Rudolf: Uber Reaktions-Beschleunigungen und -Hemmungen bei elektrischen Reduktionen und Oxydationen. Mit 11 Fig. im Text. Leipzig: W. Engelmann 1903. $82 \mathrm{~S} .8^{0}$ ๆ Aus: Zeitschrift f. physik. Chemie. Bd 44.

Karlsruhe, Dr.-Ing.-Diss. v. 16. 2. 1903

2196. Krieger, Alfred: Ueber Abkömmlinge des 1.5. Diamidoanthrachinons. 1903. $71 \mathrm{~S} .8^{0}$ T Vgl. Berichte d. Deutschen Chem. Gesellschaft. Jg. 37. 1904.

Karlsruhe, Dr.-Ing.-Diss. v. 16. 3. 1903

2197. Riehardt, Franz: Uber Verbrennungserscheinungen bei Gasen. 1904. 83 S. $8^{0}$ I T. 1 auch in: Sehilling's Journal f. Gasbeleuchtung. Jg. 47.

Karlsruhe, Dr.-Ing.-Diss. v. 23. 7. 1903

2198. Sehiek, Karl: Elektrolyse mit Wechselstrom. 1904. 67 S. $8^{0}$ - Ausz. in: Zeitschrift f. physik. Chemie. Bd 46. 1903.

Karlsruhe, Dr.-Ing.-Diss. v. 23. 7. 1903

2199. Gedel, Louis: Studien über Schwefeleisen mit besonderer Berücksichtigung der Schwefelwasserstoff-Reinigung des Leuchtgases. 1905. $51 \mathrm{~S} .8^{0}$ I Ausz. in: Schilling's Journal f. Gasbeleuchtung. Jg. 48.

Karlsruhe, Dr.-Ing.-Diss. v. 2. 5. 1904

2200. Berblinger, Hans: Untersuchungen über Indanthren. 1904. $83 \mathrm{~S} .8^{\circ}$ ๆ Vgl. Berichte d. Deutschen Chem. Gesellschaft. Jg. 36. 1903.

Karlsruhe, Dr.-Ing.-Diss. v. 15. 7. 1904

2201. Holdermann, Karl: Betrachtungen und Versuche über die Bildung der Harnsäure im tierischen Organismus. 1904. 95 S. $8^{\circ}$ T Vgl. Sitzungsberichte d. Kais. Akademie d. Wissenschaften. Math.-naturw. Kl. Bd 122. Abt. 2 b. Wien 1913. S. 5 ff. (= Monatshefte f. Chemie u. verwandte Teile anderer Wissenschaften. Bd 34 . 1913. S. 623ff.)

Karlsruhe, Dr.-Ing.-Diss. v. 15. 7. 1904

2202. Hollenweger, Wilhelm: Uber die Condensationsfähigkeit der $\beta_{1}$-Amido- $\alpha_{3}$ naphtol- $\beta_{4}$-sulfosäure. $1904.49 \mathrm{~S} .8^{0}$

Karlsruhe, Dr.-Ing.-Diss. v. 15. 7. 1904

2203. Goffin, Oskar: Reduktion von oNitrozimmtsäuremethylketon zu Propylenanthranil. 1904. $58 \mathrm{~S} .8^{\circ}$

Karlsruhe, Dr.-Ing.-Diss. v. 23. 7. 1904 
2204. Räuber, Erwin: Über Derivate der o-o-Dinitrochlorbenzol-p-Sulfosäure. 1905. 58 S. $8^{\circ}$

Karlsruhe, Dr.-Ing.-Diss. v. 23. 7. 1904

2205. Allner, Woldemar: Zur Kenntnis der Bunsenflamme. 1905. 96 S. $8^{0}$ - Aus: Schilling's Journal f. Gasbeleuchtung. Jg. 48.

Karlsruhe, Dr.-Ing.-Diss. v. 19. 12. 1904

2206. Manns, Jaeob: Zur Kenntnis der Kondensation aromatischer $\mathrm{O}$-Amidoketone. Ueber Normal-Propyl-u. Isopropylanthranil. 1905. 88 S. $8^{\circ}$

Karlsruhe, Dr.-Ing.-Diss. v. 11. 3. 1905

2207. Löb, Albert: Elektrolytische Untersuchungen mit symmetrischem und unsymmetrischem Wechselstrom. 1905. $69 \mathrm{~S} .8^{0}$ T Ausz. in: Zeitschrift f. Elektrochemie. Jg. 12. 1906.

Karlsruhe, Dr.-Ing.-Diss. v. 14. 7. 1905

2208. Weis, August: Untersuchungen in der Pyridinreihe. 1905. 56 S. $8^{\circ}$

Karlsruhe, Dr.-Ing.-Diss. v. 14. 7. 1905

2209. Gottlob, Harry: Beitrag zur Kenntnis der Reaktionsenergie bei der Vereinigung von Jod und Wasserstoff. 1906. $51 \mathrm{~S}$, 3 Taf. $8^{\circ}$

Karlsruhe, Dr.-Ing.-Diss. v. 11. 12. 1905

2210. König, James: Die Oxydation und die Oxyde des Palladiums. 1905. $45 \mathrm{~S}$., 1 Taf. $8^{\circ}$ T Ausz. in: Zeitschrift f. anorgan. Chemie. Bd 46.

Karlsruhe, Dr.-Ing.-Diss. v. 11. 12. 1905

2211. Weyl, August: Messung von Diffusions-Potentialen konzentrierter Chloridlösungen. 1905. 33 S. $8^{\circ}$

Karlsruhe, Dr.-Ing.-Diss. v. 11. 12. 1905

2212. Piek, Waldemar: Uber Ferrosilicium. 1906. 97 S. $8^{\circ}$. Weiter ausgestaltet in dem Buch von Pick u. Walter Conrad: Die Herstellung von hochprozentigem Ferrosilizium im elektrischen Ofen. 1909.

Karlsruhe, Dr.-Ing.-Diss. v. 14. 12. 1905

2213. Eberle, Fritz: Uber Abkömmlinge des $\beta$-Amido-Anthrachinons. 1906. $82 \mathrm{~S}$. $8^{0}$

Karlsruhe, Dr.-Ing.-Diss. v. 22. 1. 1906

2214. Steinkopf, Wilhelm: Versuche zur Synthese des Nitroacetonitrils. Ueber die Küpenprodukte des Indanthrens. 1906. 67 S. $8^{\circ}$ T Vgl. Berichte d. Deutschen Chem. Gesellschaft. Jg. 37. 38. 1904. 05.

Karlsruhe, Dr.-Ing.-Diss. v. 22. 1. 1906

2215. Kleiner, Erich G[ünther]: Kritische Untersuchungen über die Härtebestimmung im Wasser und zur Frage der Kesselspeisewasserreinigung. $1906.131 \mathrm{~S} .8^{\circ}$ - Ausz. in: Journal f. Gasbeleuchtung. Jg. 50. 1907.

Karlsruhe, Dr.-Ing.-Diss. v. 18. 6. 1906
2216. Liese, Kurt: Ueber die Messung der Dichtigkeit vagabundierender Ströme im Erdreich. 1906. 27 S. $4^{0}$ I $\$ 2$ ff. auch in: Zeitschrift f. Elektrochemie. Jg. 12.

Karlsruhe, Dr.-Ing.-Diss. v. 18.6. 1906

2217. Seeligmann, Franz: Beiträge zur Kenntnis der Polymerisation. 1906. 75 S. $8^{0}$

Karlsruhe, Dr.-Ing.-Diss. v. 2. 7. 1906

2218. Henseling, Friedrich: Über Methanbildung bei niederen Temperaturen. 1906. 79 S. $8^{0}$ ๆ Vgl. Max Meyer: Utber einige Gasreaktionen. Methanbildung u. Kohlenoxyd-Kohlensäuregleichgewicht. Hab.-Schr. 1908 [Nr 2064]; Journal f. Gasbeleuchtung. Jg. 52. 1909. S. $167 \mathrm{ff}$.

Karlsruhe, Dr.-Ing.-Diss. v. 23. 7. 1906

2219. Fleischmann, Fritz: Untersuchungen über die Knallgaskette bei höherer Tem peratur unter Benützung von Glas und Porzellan als Elektrolyt. 1907. 52 S. $8^{\circ}$ ๆ Außer dem letzten Abschnitt ,Beziehungen zur Groveschen Gaskette" aus: Zeitschrift f. anorgan. Chemie. Bd 51. 1906.

Karlsruhe, Dr.-Ing.-Diss. v. 30. 7. 1906

2220. Niiranen, Weikko: Über die analytische Bestimmung von Stickstoffoxyden und die Gültigkeit des Massenwirkungsgesetzes bei der Stickstoffverbrennung in der Hochspannungsflamme. [1906.]38 S. $8^{0}$. Ausz. in: Zeitschrift f. Elektrochemie. Jg. 13. 1907.

Karlsruhe, Dr.-Ing.-Diss. v. 30. 7. 1906

2221. Baechler, Max: Über ein Oxydationsprodukt des Alizarins mit Ferricyankalium in alkalischer Lösung. 1907. $62 \mathrm{~S} .8^{0}$

Karlsruhe, Dr.-Ing.-Diss. v. 22. 12. 1906

2222. Witzmann, Walter: Úber die Oxyde des Iridiums. 1907. 80 S., 1 Taf. $8^{\circ}$ T Ausz. in: Zeitschrift f. anorgan. Chemie. Bd 57. 1908.

Karlsruhe, Dr.-Ing.-Diss. v. 18. 5. 1907

2223. Schohl, Max: Über Fulvenperoxyde. Ein Beitrag zur Kenntnis der Autoxydation. 1907. 84 S. $8^{0}$

Karlsruhe, Dr.-Ing.-Diss. v. 23. 7. 1907

2224. Wöhler, Paul, wiss. Hilfsarbeiter im Kais. Gesundheitsamt: Die katalytische Wirksamkeit des Chromoxyds und Kupferoxyds im Schwefelsäureprozess. 1907. 53 S. $8^{0}$ T Vgl. Zeitschrift f. phys. Chemie. Jg. 62. 1908. Karlsruhe, Dr.-Ing.-Diss. v. 23. 7. 1907

2225. Albrecht, Rudolf: Über den Ursprung der optischen Aktivität des Erdöles. 1907. 103 S. $8^{0}$

Karlsruhe, Dr.-Ing.-Diss. v. 25. 10. 1907

2226. Koenig, Adolf : Über die Oxydation des Stickstoffes im gekühlten Hochspannungsbogen bei Minderdruck. 1907.76 S. $8^{\circ}$ T Auch bei Knapp in Halle; in kürzerer Fassung in: Zeitschrift f. Elektrochemie. Jg. 13.

Karlsruhe, Dr.-Ing.-Diss. v. 31. 10. 1907 
2227. Seer, Christian: Versuche zur Einführung mehrerer Phtalsäurereste in aromatische Verbindungen und zur Darstellung neuer Küpenfarbstoffe der Anthracenreihe. Anh.: Ueber die Reduktion der o-Benzoylbenzoesäure. 1908. 103 S. $8^{0}$ | Vgl. Berichte d. Deutschen Chem. Gesellschaft. Jg. 44. 1911.

Karlsruhe, Dr.-Ing.-Diss. v. 19. 11. 1907

2228. Neovius, Werner: I. Versuche zur Darstellung von Diphtaloylcarbazolen. II. Ueber zwei neue Reduktionsprodukte des Flavanthrens. 1908. VIII, 51 S. 80 ๆ Vgl, Berichte d. Dentschen Chem. Gesellschaft. Jg. 41

Karlsruhe, Dr.-Ing.-Diss. v. 13. 12. 1907

2229. Plüddemann, Werner: I. Beitrag zur Aufklärung des Schwefelsäurekontaktprozesses. II. Eine neue Methode zur Tensionsbestimmung von Sulfaten. 1907. 79 S, $8^{\circ}$ - Ausz. aus I in: Zeitschrift f. physik. Chemie. Bd 62. 1908.

Karlsruhe, Dr.-Ing.-Diss. v. 13. 12.1907

2230. Adler, Josef: Zur Theorie der Gerbung. 1908. 59 S. $8^{0}$ Vgl. Zeitschrift f. Chemie u. Industrie d. Kolloide. Jg. 2. 1907/08. Suppl. H. 2.

Karlsruhe, Dr.-Ing.-Diss. v. 14. 12. 1907

2231. Stegmüller, Philipp: Beitrag zur Kenntnis der Bildungswärme von Jodwasserstoff aus den Elementen. 1907. 48 S. $8^{0}$

Karlsruhe, Dr.-Ing.-Diss. v. 14. 12. 1907

2232. Kinsky, Josef J[enö]: Die Elektrizitätsleitung in Metallen und Amalgamen. [1908.] $46 \mathrm{~S} .8^{0}$ ๆ Ausz. in: Zeitschrift f. Elektrochemie. Jg. 14.

Karlsruhe, Dr.-Ing.-Diss. v. 8. 5. 1908

2233. Birstein, Gustav: Beitrag zur Elektrolyse der Alkalisalze im festen Zustande. 1909. 86 S., 3 Bl. Taf. $8^{0}$ - Ausz. in: Annalen d. Physik. Bd 26. 1908.

Karlsruhe, Dr.-Ing.-Diss. v. 18. 7. 1908

2234. Levi, Richard: Über hydrocyklische $\alpha$-Aminosäuren. 1908. 69 S. 80 - Vgl. Berichte d. Deutschen Chem. Gesellschaft. Jg. 41.

Karlsruhe, Dr.-Ing.-Diss. v. 18. 7. 1908

2235. Hörth, Franz: Versuche zur Erkenntnis der Milchsäuregärung. 1909. 96 S. $8^{0}$ T Ausz. in: Hoppe-Seyler's Zeitschrift f. physiolog. Chemie. Bd 60 .

Karlsruhe, Dr.-Ing.-Diss. v. 25. 7. 1908

2236. Rodewald, Gustav: Über die Reindarstellung bekannter und neuer Subhaloïde. 1908. 58 S. $8^{0}$ - Ausz. in: Zeitschrift f. anorgan. Chemie. Bd 61. 1909.

Karlsruhe, Dr.-Ing.-Diss. v. 25. 7. 1908
2237. Morden, G[ilbert] W[alworth]: Die Stickoxydbildung aus Luft mit Hilfe einer Gleichstromentladung niedriger Spannung unter vermindertem Drucke. $1909.52 \mathrm{~S} .8^{0}$

Karlsruhe, Dr.-Ing.-Diss. v. 28. 7. 1908

2238. Beutner, Reinhard: Neue galvanische Elemente. 1908. 54 S. $8^{0}$ - Ausz. in: Zeitschrift f. Elektrochemie. Jg. 15. 1909.

Karlsruhe, Dr.-Ing.-Diss. v. 29. 7. 1908

2239. Spanier, Eugen: Zur Kenntnis der Wirkung des Schwefels auf Kohlenwasserstoffe und des Schwefelgehaltes der Erdöle. 1910. 64 S. $8^{0}$

Karlsruhe, Dr.-Ing.-Diss. v. 29. 7. 1908

2240. Altmayer, Viktor: Utber das Methangleichgewicht, die Beziehungen zwischen Nickel und Wasserstoff und einige Methansynthesen mit Calciumhydrür. 1909. $64 \mathrm{~S} .8^{0}$

- Vgl. Journal f. Gasbeleuchtung. Jg. 52. S. 194 ff. Karlsruhe, Dr.-Ing.-Diss. v. 10. 11. 1908

2241. Weber, Friedrich A[ugust]: Ueber die Einwirkung von Kohlenoxyd auf Natronlauge. 1908. $105 \mathrm{~S} .8^{0}$

Karlsruhe, Dr.-Ing.-Diss. v. 10. 11. 1908

2242. Bohrmann, L[udwig]: Das Nitroacetonitril. 1908. 58 S. $8^{0}$ - Vgl. Berichte d. Deutschen Chem. Gesellschaft. Jg. 41.

Karlsruhe, Dr.-Ing.-Diss. v. 1. 12. 1908

2243. Krassa, Paul: Das elektromotorische Verhalten des Eisens mit besonderer Berücksichtigung der alkalischen Lösungen. 1909. 82 S. $8^{0}$ - Ausz. in: Zeitschrift f. Elektrochemie. Jg. 15 .

Karlsruhe, Dr.-Ing.-Diss. v. 19. 12. 1908

2244. Warth, Carl: Kann ein Element sowohl positive wie negative Ionen bilden? [1909.] 48 S., 2 Taf. $8^{0}$

Karlsruhe, Dr.-Ing.-Diss. v. 19. 12. 1908

2245. Heuser, Emil: Utber Oxalmalonsäureester. 1909, 59 S. $8^{0}$ - Gekürzt u. etwas verändert in: J. Liebig's Annalen d. Chemie. Bd397. 1913. S. $301 \mathrm{ff}$.

Karlsruhe, Dr.-Ing.-Diss. v. 12. 3. 1909

2246. Holwech, Wilhelm: Ueber die Beziehung der Stickoxydbildung zu den elektrischen und thermischen Eigenschaften kurzer Gleichstrom-Lichtbögen mit gekühlter Anode. 1910, $22 \mathrm{~S} .4^{0}$ - Aus: Zeitschrift f. Elektrochemie. Jg. 16.

Karlsruhe, Dr.-Ing.-Diss. v. 12. 5. 1909

2247. Routala (Rosenqvist〉, Oskar: Uber die Bildung der Naphtene im Erdöl. 1909. 111 S. $8^{0}$ - Vgl. Berichte d. Deutschen Chem. Gesellschaft. Jg. 42. 43. 1909. 10.

Karlsruhe, Dr.-Ing.-Diss. v. 18, 5. 1909 
2248. Hempel, Hubert: Über Gasöle und Ölgas. 1909. 91 S., 1 Taf., 14 Bl. Tab. $8^{0}$ - Aus: Journal f. Gasbeleuchtung. Jg. 53. 1910. Karlsruhe, Dr.-Ing.-Diss. v. 16. 6. 1909

2249. Martin, Friedr[ieh]: Vier Oxyda tionsstufen des Platins. 1909. 61 S. $8^{0}$ - Vgl. Berichte d. Deutschen Chem. Gesellschaft. Jg. 42 .

Karlsruhe, Dr.-Ing.-Diss. v. 16. 6. 1909

2250. Meier, August: Über Oxydation durch Schimmelpilze. 1909. 94 S. $8^{0}$ - Vorläufige Mitteilungen in: Hoppe-Seyler's Zeitschrift f. physiolog. Chemie. Bd 57. 59. 1907. 09.

Karlsruhe, Dr.-Ing.-Diss. v. 5. 7. 1909

2251. Stuckert, Ludwig: Ueber die Licht ${ }^{-}$ brechung der Gase und ihre Verwendung $\mathrm{zu}$ analytischen Zwecken. 1910. $40 \mathrm{~S} .4^{0}$ T Auch in: Zeitschrift f. Elektrochemie. Jg. 16. Karlsruhe, Dr.-Ing.-Diss. v. 5. 7. 1909

2252. Werkmeister, 0tto: Über Bildung und Zerfall von Eisenkarbid und die gasförmigen Produkte der Einwirkung von Mineralsäuren. 1910.78 S. $8^{0}$

Karlsruhe, Dr.-Ing.-Diss. v. 5. 7. 1909

2253. Becker, Wilhelm: Zur Frage der Erdalkaliperoxydbildung. 1909. 50 S. $8^{0}$

Karlsruhe, Dr.-Ing.-Diss. v. 26. 7. 1909

2254. Halmai, Béla: Beiträge zur Kenntnis der optischen Aktivität und der Entstehung der Naphtene des Erdöls. 1909. 73 S. $8^{0}$ - Vgl. Berichte d. Deutschen Chem. Gesellschaft. Jg. 43. 1910; Das Erdöl. Hrsg. von C. Engler u. H. v. Höfer. Leipzig 1913. Bd 1. S. $195 \mathrm{ff}$.

Karlsruhe, Dr.-Ing.-Diss. v. 26. 7. 1909

2255. Ardan, Alexander: Über Naphtene und Naphtensäuren. 1910. 67 S. $8^{0}$

Karlsruhe, Dr.-Ing.-Diss. v. 27. 7. 1909

2256. Esehmann, Max: Über Bildung und Zersetzung von Calciumcyanamid. 1910. 83 S. $8^{0}$ Ausz. in: Zeitschrift f. Elektrochemie. Jg. 17. 1911.

Karlsruhe, Dr.-Ing.-Diss. v. 27. 7. 1909

2257. Mitrofanoff, Benjamin: Über die Schnellformation von positiven Bleiakkumulatorenplatten. 1909. $70 \mathrm{~S} .8^{0}$ ศ Ausz. in: Zeitschrift f. Elektrochemie. Jg. 15.

Karlsruhe, Dr.-Ing.-Diss. v. 27. 7. 1909

2258. Dyckerhoff, Kurt: Beiträge zur Autoxydation organischer Stoffe. 1910. 82 S. $8^{0}$

Karlsruhe, Dr.-Ing.-Diss. v. 19. 10. 1909

2259. Hirsehkind, Wilhelm: Die umkehrbare Einwirkung von Sauerstoff auf Chlormagnesium. Hamburg u. Leipzig: Voss
1910. 49 S. $8^{0}$ T Ausz. in: Zeitschrift f. anorgan. Chemie. Bd 67.

Karlsruhe, Dr.-Ing.-Diss. v. 17. 12. 1909

2260. Ripke, 0tto: Das Verhalten einiger Fungi imperfecti zu organischen Säuren. 1910. 64 S. $8^{0}$ o Vgl. Hoppe-Seyler's Zeitschrift f. physiolog. Chemie. Bd 73. 1911.

Karlsruhe, Dr.-Ing.-Diss. v. 17. 12. 1909

2261. Terres, Ernst: Synthesen von 1-2Diamidoanthrachinon, Anthrachinonazinen und Indanthren. - Über Nitramine aus $\alpha$ Amidoanthrachinon und 1-5-Diamidoanthrachinon. 1910.42 S. $4^{0}$ I Vgl. betr. T. 1: Berichte d. Deutschen Chem. Gesellschaft. Jg. 46. 1913. S. $1634 \mathrm{ff}$.

Karlsruhe, Dr.-Ing.-Diss. v. 17. 12. 1909

2262. Klonowski, Sigismund: Über die Manganatschmelze und die Überführung von Kaliummanganat in Kaliumpermanganat auf elektrolytischem Wege. 1910. 128 S. $8^{\circ}$ ๆ Vgl. Zeitschrift f. Elektrochemie. Jg. 16.

Karlsruhe, Dr.-Ing.-Diss. v. 25. 1. 1910

2263. Fonda, Gorton R[osa]: Über die Einwirkung von Kohlenoxyd auf Laugen. 1910. 85 S. $8^{\circ}$

Karlsruhe, Dr.-Ing.-Diss. v. 2. 3. 1910

2264. Havas, Béla: Über Eisenblechemaille. Beziehungen zwischen physikalischer Beschaffenheit und chemischer Zusammensetzung derselben. $1910.112 \mathrm{~S} .8^{0}$ I Vgl. Chemiker-Zeitung. Jg. 33. 1909; Sprechsaal. Jg. 43, Halbj. 2. S. 727 f. Jg. 44, Halbj. 1. S. 6 f. $188 \mathrm{f}$. $207 \mathrm{ff} .220 \mathrm{ff}$.

Karlsruhe, Dr.-Ing.-Diss. v. 2. 3. 1910

2265. Platou, Eilif: Calorimetrische Untersuchungen über Stickoxydbildung aus Luft mittels Hochspannungswechselstromentladungen verschiedener Frequenz. 1910. 72 S. $8^{\circ}$ ๆ Vgl. Zeitschrift f. Elektrochemie. Jg. 16.

Karlsruhe, Dr.-Ing.-Diss. v. 11. 5. 1910

2266. Wolokitin, A[rkadij]: Über die Stickoxydbildung bei der Wasserstoffverbrennung. 1910. 59 S., 1 Taf. $8^{0}$ - Ausz. in: Zeitschrift f. Elektrochemie. Jg. 16.

Karlsruhe, Dr.-Ing.-Diss. v. 11. 5. 1910

2267. Speithel, Robert: Über die Vorgänge der Autoxydation bei Aldehyden. 1911. 107 S. $8^{0}$

Karlsruhe, Dr.-Ing.-Diss. v. 12. 5. 1910

2268. Bereza, Stanislaus: Über Darstellung neuer Ketene und über Anlagerung von Diphenylketen an substituierte Chinone. 1910. 119 S. $8^{0}$ T Ausz. in: J. Liebig's Annalen d. Chemie. Bd 380. 1911.

Karlsruhe, Dr.-Ing.-Diss. v. 21. 7. 1910 
2269. Kauko, Yrjö: Kinetische Untersuchung der Reduktion von Permanganatlösungen durch gasförmigen Wasserstoff. 〈Autoreduktion.〉 Leipzig: W. Engelmann 1911. 44 S. $8^{0}$ I Aus: Zeitschrift f. phys. Chemie. Bd 76.

Karlsruhe, Dr.-Ing.-Diss. v. 21. 12. 1910

2270. Makowetzky, Alexander: Über die Bildung von Wasserstoffsuperoxyd, Salpetersäure und Ammoniak bei der Glimmbogenentladung unter Verwendung von Wasser als einer Elektrode. 1911. 19 S. $4^{0}$ - Aus: Zeitschrift f. Elektrochemie. Jg. 17.

Karlsruhe, Dr.-Ing.-Diss. v. 21. 12.1910

2271. Ružiéka, Leopold: Über Phenylmethylketen. 1911. $91 \mathrm{~S} .8^{0}$ - Ausz. in: J. Liebig's Annalen d. Chemie. Bd 380 .

Karlsruhe, Dr.-Ing.-Diss. v. 21. 12. 1910

2272. Engels, William Henry: Über die Wasserstoffgewinnung aus Kohlenoxyd und Kalkhydrat und die Beschleunigung der Wassergasreaktion durch Eisen. 1911. XIII, 55 S. $8^{0}$

Karlsruhe, Dr.-Ing.-Diss. v. 8. 2. 1911

2273. Kon, Norbert: Ueber Einwirkung von Diphenylketen auf carbonylhaltige Verbindungen. [Nebst] Druckfehlerverzeichnis. 1911. 159 S. $8^{0}$ - Vgl. J. Liebig's Annalen d. Chemie. Bd 384.

Karlsruhe, Dr.-Ing.-Diss. v. 13. 6. 1911

2274. Hug, René: Zur Kenntnis der biochemischen Wirkung einiger Säuren. 1911. 138 S. $8^{0}$

Karlsruhe, Dr.-Ing.-Diss. v. 26. 7. 1911

2275. Jürgens, Boris: Úber die Bildung von Hydroxamsäurechloriden aus Nitrokörpern und über die Konstitution der aciNitrokörper. 1911. VIII, 109 S. $8^{0}$ - Vgl. Journal f. prakt. Chemie. Bd 191. N. F. Bd 83.

Karlsruhe, Dr.-Ing.-Diss. v. 27. 7. 1911

2276. Beger, Max: Beitrag zur Wirkung des Ozons auf das Jod und den Schwefel. [1912.] 78 S. $8^{0}$

Karlsruhe, Dr.-Ing.-Diss. v. 31. 1. 1912

2277. Günzburg, Jakob: Über die Darstellung und die Eigenschaften von Ferrobikarbonatlösungen. [1912.] 40 S. $8^{0}$

Karlsruhe, Dr.-Ing.-Diss. v. 31. 1. 1912

2278. Betzel, Richard: Zur Theorie der Desinfektion. 1911. $64 \mathrm{~S} .8^{0}$ ๆ Vorläufige Mitteilung in: Hoppe-Seyler's Zeitschrift f. physiolog. Chemie. Bd 67. 1910.

Karlsruhe, Dr.-Ing.-Diss. v. 13. 2. 1912
2279. Weissmann, Leon: Utber die Abgabe von elektrisch geladenen Teilchen durch einen glühenden Platindraht während der Katalyse von Knallgas. Leipzig: W. Engelmann 1912. 26 S. $8^{0}$ - Aus: Zeitschrift f. physik. Chemie. Bd 79.

Karlsruhe, Dr.-Ing.-Diss. v. 26. 2. 1912

2280. Saladin, 0tto: Ueber das Verhalten einiger Pilze zu Áminosäuren und Oxysäuren. 1911. 120 S. $8^{0}$ - Vgl. Hoppe-Seyler's Zeitschrift f. physiolog. Chemie. Bd 73.

Karlsruhe, Dr-Ing.-Diss. v. 28. 2. 1912

2281. Riehert, Theodor: Utber das Aussalzen von Seifen. 1911. VII, $58 \mathrm{~S} .8^{0}$ - Bruchstücke in: Chemische Revue über die Fett- u. Harz-Industrie. Jg. 17. 1910 u. in: Handbuch d. Chemie u. Technologie d. Öle u. Fette, Wachse u. Harze, hrsg. von Ubbelohde u. Goldschmidt. Bd 3, Abt. 2.

Karlsruhe, Dr.-Ing.-Diss. v. 1. 3. 1912

2282. Sehapringer, Sigismund von: I. Utber die Konstitution der Holzölsäure und die Polymerisation von Holzöl. II. Über die hydrolytische Spaltung wässrigalkoholischer Seifenlösungen. 1912. 67 S. $8^{0}$

Karlsruhe, Dr.-Ing.-Diss. v. 15. 7. 1912

2283. Tausz, Jenö: Beiträge zur Identifizierung und Kenntnis der Kohlenwasserstoffe des Erdöls. 1912. 75 S., 1 Taf. $8^{0}$

Karlsruhe, Dr.-Ing.-Diss. v. 26. 7. 1912

2284. Hansen, David E[rnest]: Über die elektrische Doppelbrechung der Gase. 1912. 35 S. $8^{0}$

Karlsruhe, Dr.-Ing.-Diss. v. 27. 7. 1912

2285. Schlumberger, Ernst: Uber die statische Bestimmung des Ammoniakgleichgewichtes in der Nähe von $500^{\circ}$ C. 1912 . 43 S. $8^{0}$

Karlsruhe, Dr--Ing.-Diss. v. 5. 10. 1912

2286. Meyer, Wilhelm Ad[olf]: Uber katalytische Hydrierungen organischer Verbindungen mit kolloidem Palladium und Platin. 1912. 59 S. $8^{0}$ V Vgl. Berichte d. Deutschen Chem. Gesellschaft. Jg. 45 .

Karlsruhe, Dr.-Ing.-Diss. v. 21. 11. 1912

2287. Hiller, Fritz: Über den Innenkegel gespaltener Kohlenwasserstoffflammen. Leipzig: W. Engelmann 1912. $38 \mathrm{~S} .8^{0}$ T Aus: Zeitschrift f. physik. Chemie. Bd 81.

Karlsruhe, Dr.-Ing.-Diss. v. 20. 12. 1912

2288. Carter, Frederick E[dward]: Über die Verbrennung von Wasserstoff mit Sauerstoff. 1912. 31 S., 1 Taf. $8^{0}$ - Aus: Journal f. Gasbeleuchtung. Jg. 56. 1913.

Karlsruhe, Dr.-Ing.-Diss. v. 13. 1. 1913 


\section{ABTEILUNG FÜR FORSTWESEN}

2289. Seeger, Max, Grossherzogl. Forstprakt.: Beitrag zur Geschichte der Waldungen der Stadt Ettlingen. 1908. 90 S., $1 \mathrm{Kt} .8^{0}$

Karlsruhe, Dr.-Ing.-Diss. v. 6. 12.1907

2290. Stoll, Hermann, Großherzogl. Bad. Forstamtmann: Das Versagen der Weißtannenverjüngung im mittleren Murgtale.
Ein Beitrag zum waldbaulichen Verhalten der Weißtanne. Mit 6 Abb. 1909. 64 S. $8^{0}$ - Aus: Naturwiss. Zeitschrift f. Forst- u. Landwirtschaft. Jg. 7.

Karlsruhe, Dr.-Ing.-Diss. v. 30. 6. 1909

2291. Statz, Paul, Bad. Forstprakt.: Die Abstandszahl, ihre Bedeutung für die Forsttaxation, Bestandeserziehung und Bestandespflege. 1909. 65 S. $8^{0}$

Karlsruhe, Dr.-Ing.-Diss. v. 8. 7. 1909

\section{M Ü N CHEN}

Gegründet 1868 als „Polytechnische Schule“, seit 1877 ,Technische Hochschule ${ }^{\text {“ }}$

\section{SATZUNGEN ORDNUNGEN BESTIMMUNGEN}

\section{ALLGEMEINES UND VERMISCHTES}

2292. -Organische Bestimmungen für die polytechnische Schule in München (v. 12. 4. 1868). München: Fleischmann 1868.

2293. Statuten (5. Abdr.: Satzungen) für die Studirenden der Polytechnischen Schule zu München (v. 5. 10. 1868). 1868. $\langle$ 2. Aufl. $\rangle$ 1871. - 〈3. rev. Abdr. $\rangle$ (mit Abänderungen $\nabla .13 .7$. 1873.) 1873. - <4. rev. Abdr. $\rangle$ (mit Abänderungen v. 13. 7. $1873 \mathrm{u}$. 3. 8. 1875). 1875. - 〈5. rev. Abdr. > (mit Abänderungen v. 13. 7. 1873, 3. 8. $1875 \mathrm{u}$. 14. 8. 1876). 1876. [Folgt:] Allgemeine Gebührenordnung v. 24. 7. 1875. Inscriptions-Ordnung. - [6.-16. Abdr. s. Nr 2299.]

2294. Instruktion für das Dienstpersonale der Kgl. polytechnischen Schule in München. 1869.

$M H \circ{ }^{\prime} B$

2295. Allgemeine Gebührenordnung der $\mathrm{kgl}$. polytechnischen Schule in München (v. 31.5. 1869).

2296. Bibliothek-0rdnung der Polytechnischen Schule zu München (v. 23. 9. 1870). 1870. Abgedr. in: Katalog d. Bibliothek. 1881 [Nr 2353].

2297. Allgemeine Gebührenordnung der kgl. polytechnischen Schule in München. Vom 24. 7. 1875. Abgedr. in: Satzungen f. d. Studirenden. 5. Abdr. [Nr 2293.]

2298. Organische Bestimmungen für die k. bayerische technische Hochschule in München. Vom 6. 8. 1877. 1877.

2299. Satzungen für die Studirenden der (8., 16., 17. Abdr.: K. bayer.) Technischen Hochschule zu München (v. 3. 10. 1877).
〈6. rev. Abdr.〉 1877. [Folgt:] Allgemeine Gebührenordnung v. 3. 10. 1877. Inscriptions-Ordnung. $-\langle 7$. Abdr. $\rangle 1878$. — <8. Abdr. $\rangle$ 1880. - <10. Abdr. $\rangle$ 1883. -

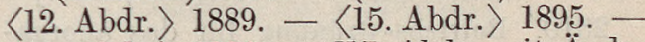
16. Abdr. 1896. - [17. Abdr. mit Änderungen.] 1897. [1. 5. Abdr. s. $\mathrm{Nr} 2293$.] 8. Abdr.: M Minist. d. Innern; 9., 11., 13., 14. Abdr.: nicht nachweisbar; 1\%.Abdr.: $M^{*}$

2300. Bestimmungen über die Habilitation von Privatdocenten an der k. bayer. technischen Hochschule in München (v. 17. 9. 1880). 1880. - 〈2. Abdr. $\rangle$ 1898. - [Neudr.] 1903. 2. $A b d r .: M^{*}$ 2301. Bibliothek- Ordnung der Kgl. Bayer. Technischen Hochschule zu München (v. 6. 10. 1884). 1884.

2302. [Dasselbe] (v. 2. 1. 1892). 1892. - Abgedr. in: Katalog d. Bibliothek. Nachtrag 1. 1892 [Nr 2353].

2303. Verfassung der $K$. bayerischen technischen Hochschule in München. Vom 27. 6. 1899. 1899. [Nebst] Beil. (v. 27. 12. 1902). - [Dasselbe.] Vom 27. 6. 1899 u. 27. 12. 1902. 1910. (Nebst Deckbl. zu $\$ 12$. 15. 18. 21. 30.)

2304. Satzungen für die Studierenden und Zuhörer der K. bayer. technischen Hochschule zu München nebst Gebühren- und Inskriptions-Ordnung. Vom 14. 9. 1899. 1899. - [Dasselbe.] Vom 14. 9. 1899 u. 7. 10. 1901. 1901. - [Neudr.] 1903. 1907. 1899. 1903: $M$; 1901. 1907: $M^{*}$

2305. Laboratoriums - Ordnung für die Praktikantendes elektrotechnischen Instituts der K. Technischen Hochschule zu München (v. 30. 10. 1900). 1900. - [Neudr.] 1907. 
2306. Verfassung der K. bayerischen technischen Hochschule in München. Entwurf als Ms. gedr. [1901.]

M*

2307. Kgl. Allerhöchste Verordnung v. 10. 1. 1901, betr. die Verleihung des Doktortitels durch die K. Bayer. Technische Hochschule zu München, nebst Promotionsordnung (für die Erlangung der Doktorwürde an der K. Bayer. Technischen Hochschule in München). 1901. — [Neudr.] 1908. - [Dasselbe] (v. 10. 1. 1901 u. 2. 8. 1910). 1908 [vielm. 1910.] — [Neudr.] 1911. - Abgedr. in: Bestimmungen. S. $298 \mathrm{ff}$. u. in: C. Walther: Bibliographie der an d. deutschen Techn. Hochschulen erschienenen Dr.-Ing.-Dissertationen in sachl. Anordnung. 1900 bis 1910 . Berlin 1913. 1908: $M^{*} ; 1911: M$

2308. Bibliothek-0rdnung der Kgl. Technischen Hochschule in München (v. 23. 1. 1903). - [Dasselbe u. d. T.: ] BibliotheksOrdnung ... (v. 23. 1. 1903, 28. 5. u. 5. 6. 1908). T Abgedr. in: Katalog d. Bibliothek. Nachtrag 2. 1903 [Nr 2353].

1903: $M$

2309. Inskriptions-0rdnung der K. Technischen Hochschule in München. [1905.]

2310. Betriebs-0rdnung für das anorganisch-chemische und elektrochemische Laboratorium der $\mathrm{K}$. Technischen Hochschule in München (v. 16. 12. 1905).

2311. Allgemeine Gebührenordnung der $\mathrm{K}$. Technischen Hochschule in München (v. Okt. 1906).

$M$

2312. Bestimmungen über die Preisbewerbungen an der K. Bayer. Technischen Hochschule zu München (v. 20. 12. 1906).

2313. Satzungen für die Studierenden und Zuhörer d. K. Bayer. Technischen Hochschule zu München nebst Gebühren- und Inskriptions - Ordnung. [Entwurf.] [Endgültige Fassung] (v. 25.9. 1907). 1907.[Neudr.] (Gebühren-Ordnung v. 20. 8. 1909.) 1909. - [Rev. Ausg.] (Gebühren-Ordnung v. 16. 10. 1911.) 1911. Entwurf: $M^{*} ; 1907: M$

2314. Allgemeine Gebührenordnung der K. Technischen Hochschule in München (v. 20. 8. 1909).

\section{PRÜFUNGSORDNUNGEN}

2315. Bestimmungen über die Abhaltung von Prüfungen für Privatlehrer an der Kgl, polytechnischen Schule zu München (v. 9.2. 1870). 1870.

\section{Absolutorialprüfungen}

2316. Absolutorial-Prüfungs-0rdnung für die Kgl, polytechnische Schule zu München (v. 29. 5. 1869). 1869.
2317. Bestimmungen über die Abhaltung der Absolutorial-Prüfungen der an der Ingenieur-, Hochbau-, mechanisch-technischen und chemisch-technischen Abtheilung der kgl. polytechnischen Schule in München immatriculirten Studirenden (v. 29. 5. u. 26. 8. 1869,4 . 1. u. 13. 7. 1873). 2. rev. Abdr. 1873.

2318. Absolutorial-Prüfungs-Gegenstände der Polytechnischen Schule zu München (v. Febr. 1870). 1870.

2319. Bestimmungen über die Abhaltung der Absolutorial-Prüfungen der an der kgl. polytechnischen Schule zu München studirenden Verkehrs- und Zolldienst-Adspiranten (v. 28. 1. 1871). 1871. - 2. verb. Abdr. (mit Änderungen v. 5. 7. 1872 u. 13. 7. 1873). 1873.

1. $A b d r .: M$ Hof $B$

2320. Bestimmungen über die Abhaltung der Absolutorial-Prüfungen der an der Landwirthschaftlichen Abtheilung der Kgl. polytechnischen Schule in München immatriculirten Studirenden (v. 29. 1. 1874). 1874.

2321. Bestimmungen über die Abhaltung der Absolutorial-Prüfungen der an der kgl. polytechnischen Schule in München studirenden Candidaten des Cultur-Ingenieurfachs (v. 17. 7. 1874). 1874.

2322. Bestimmungen über die Abhaltung der Absolutorialprüfungen an der K. bayerischen technischen Hochschule zu München (v. 18. 6. 1877). 1877.

2323. Bestimmungen über die Abhaltung der Absolutorialprüfungen an der K. bayerischen technischen Hochschule zu München. Entwurf, als Ms. gedr. 1883. - [Dasselbe] (v. 4. 6. 1883). 1883.

Entwurf: $M^{*}$

2324. [Dasselbe] (v. 27. 8. 1887). 1887. [Dasselbe] (v. 27. 8. 1887 u. 22. 7. 1890). 1894. - [Dasselbe] (v. 27. 8. 1887, 22. 7. $1890,11$. u. 29. 7. 1896).

1894: $M^{*}$

\section{Diplomprüfungen}

2325. Bestimmungen über die Abhaltung von Diplom-Prüfungen an der Kgl. polytechnischen Schule zu München (v. 27. 4. 1870). 1870. [Nebst] Nachtrag zur Diplomprüfungsordnung für die Studirenden der Kgl. polytechnischen Schule in München (v. 4. 1. 1873).

MHofB; Nachtr.: $M^{*}$

2326. Bestimmungen über die Abhaltung von Diplomprüfungen an der $\mathrm{K}$, bayerischen technischen Hochschule zu München (v. 30, 9. 1879). 1879. 
2327. Bestimmungen über die Abhaltung der Diplomprüfungen an der K. Technischen Hochschule zu München. 1900.

2328. Bestimmungen über die Abhaltung der Diplomprüfungen an der K. B. Technischen Hochschule zu München [v. 29, 4. 1901]. 1901. [Nebst] Nachtrag betr. Abänderung der $\$ \S 63$ u. 64 v. Mai 1902. [Dasselbe unter Berücksichtigung der Abänderungen. 1903.] 1. Ausg.: $M$

2329. Revidierte Grundlagen für die neuen Diplomprüfungs-Ordnungen der K. Technischen Hochschule zu München. 1904.

\section{Allgemeine Abteilung}

2330. Kgl. Technische Hochschule zu München. Provisorische Prüfungsordnung für das Fach der technischen Physik (v. 30. 7. 1898).

2331. Entwurf. Diplomprüfungs-0rdnung für Studierende der technischen Physik an der K. Technischen Hochschule zu München. [1905.]

2332. Diplomprüfungs-0rdnung für Studierende der technischen Physik an der K. Technischen Hochschule zu München. Vom 12. 7. 1906.1906.

\section{Bauingenieur-Abteilung}

2333. Entwurf. Diplomprüfungs-0rdnung der Bauingenieur-Abteilung der K. Technischen Hochschule zu München. [1905.]

2334. Diplomprüfungs-0rdnung der Bauingenieur-Abteilung der K. Technischen Hochschule zu München. Vom 18. 2. 1906. 1906. [Nebst] Beil. (v. 21. 8. 1909.) - [Dasselbe.] Vom 18. 2. 1906 u. 21. 8. 1909. 1909.

2335. Diplomprüfungs-Ordnung der Bauingenieur-Abteilung der $\mathrm{K}$. Technischen Hochschule in München. Vom 29. 12. 1910 u. 2. 3. 1911. 1911.

\section{Architekten-Abteilung}

2336. Entwurf. Diplomprüfungs-0rdnung der Architekten-Abteilung der K. Technischen Hochschule zu München. [1905.]

2337. Diplompruifungs- Ordnung der Architekten-Abteilung der K. Technischen Hochschule zu München. Vom 10. 1. 1906. 1906. - [Dasselbe.] Vom 10. 1. 1906 u. 5. 9. 1910. 1910.

\section{Maschineningenieur-Abteilung}

2338. Entwurf. Diplomprüfungs-0rdnung der Maschineningenieur-Abteilung der K. Technischen Hochschule zu München. [1905.]

2339. Diplomprüfungs- Ordnung der $\mathrm{Ma}$ schineningenieur-Abteilung der $\mathrm{K}$. Technischen Hochschule zu München. Vom 14. 3. 1906. 1906. - [Neudr.] 1907. — [Dasselbe.] Vom 14. 3. 1906 u. 3. 8. 1909. 1909. [Nebst] Beil. (v. 7. 8. u. 5. 9. 1910.)

\section{Chemische Abteilung}

2340. Entwurf. Diplomprüfungs- Ordnung der chemischen Abteilung der K. Technischen Hochschule zu München. [1905.]

2341. Diplomprüfungs-Ordnung der chemischen Abteilung der K. Technischen Hochschule zu München. Vom 14. 7. 1906. 1906. - [Neudr.] 1907.

\section{Landwirtschaftliche Abteilung}

2342. Entwurf. Diplomprüfungs- Ordnung der Landwirtschaftlichen Abteilung der K. Technischen Hochschule zu München. [1905.]

2343. Diplomprüfungs- Ordnung der Landwirtschaftlichen Abteilung der K. Technischen Hochschule zu München. Vom 23. 11. 1906 u. 13. 1. 1907. 1906.

2344. [Dasselbe.] Vom 22. 2. 1912. 1912.

\section{PERIODISCHE SCHRIFTEN}

2345. Programm der (1872-73 ff.: Kgl. Bayerischen) Polytechnischen Schule (187778 ff.: Kgl. Bayerischen Technischen Hochschule) zu (1910-11 ff.: in) München für (1872-73-1888-89: das Jahr; 1889-90ff. : das Studienj.) 1868-69-1912-1913. 1868-1912.

1871-72. [1. Abdr.] - 〈2., nach d. Stande v. 1. Dez. 1871 rev. Abdr. $\rangle$
1872-73. [1. Aufl.] - 2. Aufl. verm. mit d. Stundenpl. für d. S.-S. 1873.

1893-94. [1. Aufl.] - 2. Aufl.

1894-95. [1. Ausg.] - 2. Ausg.

1899-1900. [1. Ausg.] - 〈2. Ausg. April 1900.〉

1901-02. [1. Ausg.] - 〈2. Ausg. Dez. 1901. $\rangle$

1902-03. [1. Ausg.] - 〈2. Ausg. Jan. 1903.〉

1903-04. [1. Ausg.] — 2. Ausg. Okt. 1903. 3. Ausg. April 1904. 
1904-05. [1. Ausg.] - 2. Ausg. Okt. 1904. 3. Ausg. Nov. 1904. - 4. Ausg. März 1905. 1905-06. [1. Ausg.] - 2. Ausg. Nov. 1905. 3. Ausg. April 1906.

1906-07. [1. Ausg.] - 2. Ausg. Sept. 1906. 3. Ausg. März 1907.

1907-08. [1. Ausg.] - 2. Ausg. Nov. 1907. 3. Ausg. April 1908.

1908-09. [1. Ausg. nebst] Beil. - 2. Ausg. Okt. 1908. - 3. Ausg. März 1909.

1909-10. [1. Ausg.] - 2. Ausg. Okt. 1909. -

3. Ausg. Nov. 1909. - 4. Ausg. März 1910. 1910-11. [1. Ausg.] - 2. Ausg. Okt. 1910.

1911-12. 1. Ausg. Juni 1911. - 2. Ausg. Sept.

1911. - 3. Ausg. Febr. 1912.

1912-13. [1. Ausg.] - 2. Ausg. Okt. 1912. -

3. Ausg. März 1913.

1869/70-1871/72. 1873/74. 1874/75. 1878/79. 1879/80. 1882/83. 1883/84. 1887/88. 1897/98. M; 1871/72. 2. Abdr., 1872/73. 1. A ufl., 1894/95. 1. Ausg.: $M$ HofB; 1904/05. 2. Ausg. 1909/10. 4. Ausg.: $M^{*} ; 1905 / 06$. 3. Ausg.: Königsberg UB 2346. Kgl. polytechnische Schule in München. Verzeichniss der Lehrgegenstände, welche in dem ... gelehrt werden. W.-S. 1868-69. S.-S. 1869. W.-S. 1873/74. S.-S. 1874. W.-S. 1874/75. S.-S. 1875. 1868-75. Fortges. u. d. T.:

Verzeichniss der Vorlesungen, Uebungen und Praktica, welche an der kgl. polytechnischen Schule (1877/78: technischen Hochschule) in München gehalten werden. W.-S. 1875/76. S.-S. 1876 [usw.]-W.-S. 1877/78. $1875-77$.

$1868 / 69$

1869. 1874-1877/78: $M$ HofB; 1873/74: $M^{*}$

2347. Stundenplan der Polytechnischen Schule in München für das W.-S. 1868-69. S.-S. 1869 [usw.]-W.-S. 1872/73. 1868-72.

1869/70. 1870/71. 1871/72. 1872/73: $M$ HofB

2348. Bericht über die Kgl. Polytechnische Schule (1877-78-1905-06: Technische Hochschule) zu München für (1906-07 ff.: $\mathrm{Kgl}$. Technische Hochschule zu München. Bericht über) das Studienj. 1868-1869 1910-1911. 1869-1912. ๆ 1868-69. 1888-89. Ersch. auch ohne die Reden und Vorträge.

Darin :

1868-69. Neureuther, G(ottfried): Der Neubau der polytechnischen Schule in München. (Mit Abb.) - Reden und Vorträge zur Einweihungsfeier der Technischen Hochschule in München. Hrsg. von Carl Max Bauernfeind. (Darunter: Bauernfeind, C(arl) M(ax): Rede über den Einfluss der exacten Wissenschaften auf die allgemeine Bildung und auf die technischen Fachstudien insbesondere.). [Die Reden u. Vorträge sowie die Rede Bauernfeinds auch besonders bei Palm in München.]

1877-78. Kluckhohn, August: Ueber das technische Unterrichtswesen in Bayern bis zur
Gründung der polytechnischen Centralschule in München $\langle 1827\rangle$. Antrittsrede geh. am 22. Dez. 1877.

1878-79. Kluckhohn, August: Ueber die Gründung und bisherige Entwicklung der $\mathrm{k}$. technischen Hochschule zu München. Rede geh. am 26. Juli 1879.

1879-80. Kluckhohn, August v.: Ueber die wissenschaftlichen und künstlerischen Bestrebungen Wittelsbach'scher Fürsten aus dem Hause Pfalz. Festrede zur Vorfeier d. 700 jähr. Regierungs-Jubiläums d. bayer. Herrscherhauses am 24. Juli 1880.

1893-94. Die Gedächtnisfeier für Gottfried von Neureuther.

1894-95. Das Ohm-Denkmal vor der K. technischen Hochschule in München und seine Enthüllung.

1896-97. Stieve, Felix: Festrede zur hundertjährigen Gedenkfeier der Geburt Kaiser Wilhelms I. am 22. März 1897.

1898-99. Du Moulin Eckart, Riehard Graf: Festrede zum hundertjährigen Jubiläum der Wiedervereinigung der bayerischen Lande am 11. März 1899. [Auch in: Beilage zur Allg. Zeitung. Jg.1899, Nr 64.]

1899-1900. Neubau der Landwirtschaftlichen Central-Versuchsstation für Bayern. 4 Taf. (Grundrisse $u$. Schnitte.)

1900-01. Reber, Franz von: Die Anfänge der Kunstpflege des Wittelsbachisehen Hauses. Festrede zur Feier d. 80. Geburtstages des PrinzRegenten am 7. März 1901. [Auch in: Beilage zur Allg. Zeitung. Jg. 1901, Nr 58.]

1904-05. Sulger-Gebing, Emil: Schillers Geistesentwicklung. Festrede bei d. Schillerfeier am 8. Mai 1905.

1905-06. Die Eröffnung der neuen chemischen Institute der K. Technischen Hochschule zu München.

1909-10. Der Neubau der Landwirtschaftlichen Abteilung der $\mathrm{K}$. Technischen Hochschule zu München. (Mit 4 Taf.)

1910-11. Du Moulin-Eckart, Richard Graf: Die Entwicklung des deutsehen Gedankens und des Fürstenideals im bayerischen Königshause. Festrede zur Feier d. 90. Geburtstages d. Prinzregenten Luitpold am 11. März 1911. [Auch in: Nord u. Süd. Jg. 35. Bd 137. 1911.]

Darin ferner Nekrologe:

1872-73. [Bauernfeind, Carl Max v.]: Adolph Döhlemann. [Auch in: Zeitschrift d. bayer. Architekten- u. Ingenieur-Vereins. Bd 5. 1873.]

1873-74. Kluckhohn, A(ugust): Hermann Guthe.

1874-75. Klein, (Felix): Otto Hesse.

1879-80. Frauenholz, W[ilhelm]: Friedrich August Klingenfeld.

1885-86. Kohlrausch, F[riedrich]: Wilhelm v. Beetz. (Aus: Allg. Zeitung. Nr 144 v. 25. Mai 1886.)

1886-87. Geul, Albert: Gottfried v. Neureuther. - Voss, Aurel. Dy(e)k, Walter: Walfried Marx. 
1888-89. Loewe, (Ferdinand): Wilhelm Frauenholz. - Krenter, Franz: Heinrich Manderla.

1890-91. Thiersch, Aug(ust): Rudolph Gottgetreu.

1891-92. Geul, Albert: Joseph Mozet.

1892-93. Banernfeind, Karl Max v.: Johann Nikolaus Bischoff. (Abgeändert aus: Allg. Zeitung. Nr 30 v. 4. Febr. 1893.)

1893-94. Sehröter, Moritz: Johann Bauschinger. - Schmidt, M[ax]: Karl Max von Bauernfeind.

1894-95. Haushofer, Max: Karl von Haushofer. (Verändert u. erg. aus: Biograph. Blätter. Bd 1. 1895.)

1895-96. Braunmühl, A[nton] v.: Ludwig Muggenthaler. - Kiliani, H(einrich): Karl Stölzel.

1897-98. Finsterwalder, (S)[ebastian]. Ebert, (Hermann): Leonhard Sohncke. Thiersch, Aug(ust): Albert Geul. - Günther, S[iegmund]: Felix Stieve. - Loewe, (F) [erdinand]: Gottfried Asimont. - Ammon, L[udwig] v.: Wilhelm von Gümbel.

1898-99. Lipp, A (ndreas): Wilhelm von Miller. - Reber, F[ranz] v.: Konrad von Knoll. - Loewe, (F)[erdinand]: Wilhelm Wittmann. - Bühlmann, J[oseph]: Philipp Sporrer. 1900-01. Soxhlet, F[ranz]: Martin Ewald Wollny. -

1901-02. Haushofer, Max. Sulger-Gebing,

Emil: Wilhelm von Hertz.

1902-03. Loewe, (Ferdinand): Ernst Fischer. - Weber, Maximilian: Franz Bauer. Günther, Siegmund: Carlo Meloier.

1906-07. Du Moulin-Eckart, Richard Graf: Max Haushofer. - Lynen, W(ilhelm): Georg Ultsch. - Giesenhagen, K(arl): Karl Otto Harz. 1907-08. Burckhardt, H(einrich). Finsterwalder, S(ebastian). Günther, S(iegmund): Anton Edler von Braunmühl. - Soxhlet, Franz v.: Karl von Voit. - Günther, S(iegmund): Alfred Blinkhorn.

1908-09. Thiersch, F(riedrich) v.: Karl von Reinhardstoettner. - Lipp, A(ndreas): Emil Erlenmeyer.

1910-11. Günther, S(iegmund): Wilhelm Goetz.

Darin ferner Reden beid. Jahresschluss feier (1889-90: beim Antritt d. Direktorats; 1890-91-1899-1900. 1901-02. 1902-03: bei d. Eröffnungsfeier d. Studienjahres; 1903-04: zur Übernahme d. ersten Wahlrektorates; 1900-01. 1904-05 ff.: bei d. akad. Feier bzw. Jahresfeier):

1880-81. Bauernfeind, Carl Max v.: Johannes Scharrer und seine Bedentung für die Entwicklung der technischen Schulen und der Eisenbahnen in Bayern. (Mit 1 Portr.) [Einzeln bei G. Franz in München in Komm.]

1881-82. Bauernfeind, Carl Max v.: Gedächtnisrede auf Georg Simon Ohm den Physiker. [Einzeln bei G. Franz in München in Komm.; ohne die Anm. in: Bayer. Verkehrsblätter. Jg. 8. 1889.]
1882-83. Bauernfeind, Carl Max v.: Georg von Reichenbach und seine Leistungen auf den Gebieten der Mechanik und des Ingenieurwesens. [Einzeln bei G. Franz in München in Komm.]

1883-84. Bauernfeind, Carl Max v.: Gedächtnisrede auf Friedrich August von Pauli. [Einzeln bei G. Franz in München in Komm.]

1884-85. Bauernfeind, Carl Max v.: Johann Georg von Soldner und sein System der Bayerischen Landesvermessung. [Einzeln bei G. Franz in München in Komm.; etwas gekürzt in: Allg. Deutsche Biographie. Bd 34. 1892.]

1885-86. [s. Nr 2354.]

1886-87. Haushofer, Karl: Ziele und Wege des Studiums an Technischen Hochschulen.

1887-88. (Eine Schlußfeier wurde nicht abgehalten.)

1888-89. Bauernfeind, Carl Max v.: Benjamin Thompson Graf von Rumford. [Einzeln bei G. Franz in München in Komm.; auch in: Allg. Deutsche Biographie. Bd 29. 1889.]

1889-90. Haushofer, Karl: Ueber die Aufgaben der technischen Hochschule auf dem Gebiete der allgemeinen Bildung.

1890-91. Sohncke, Leonhard: Die Umwälzung unserer Anschauungen vom Wesen der elektrischen Wirkungen.

1891-92. Schmidt, Max: Die Methoden der unterirdischen. Orientirung und ihre Entwickelung seit 2000 Jahren. [Etwas erw. u. mit 10 Fig. versehen in: Himmel u. Erde. Jg. 4.1892 u. als: Sammlung populärer Schriften hrsg. von d. Gesellschaft Urania zu Berlin. No. 15.]

1892-93. Schmidt, Heinrich Freiherr von : Die Bangeschichte des Domes St. Peter in Worms und seine geplante Wiederherstellung.

1893-94. Haushofer, K(arl) v.: Rückblicke auf die Entwicklung der K. bayer. technischen Hochschule in den ersten 25 Jahren ihres Bestehens.

1894-95. [Eine Feier wurde nicht abgehalten.]

1895-96. Hoyer, Egbert von: Erörterungen über die Stellungnahme der technischen Hochschulen zu den neuesten Bestrebungen auf dem Gebiete des höheren technischen Unterrichts.

1896-97. Miller, W[ilhelm] v.: Die Entwicklung des Elementbegriffes bis zum Tode Lavoisiers.

1897-98. Haushofer, Max: Die Stellung des Technikers im modernen Staate.

1898-99. Loewe, F[erdinand]: Die geschichtliche Entwicklung der Landstrassen. [Mit etwas veränd. Schluß in: Beilage zur Allg. Zeitung. Jg. 1899, Nr 55. 56.]

1899-1900. Thiersch, Friedrich von: Die Baugeschichte des Klosters Ettal.

1900-1901. Dyck, Walther: Ueber die Beziehungen zwischen dem künstlerischen und dem wissenschaftlichen Erfassen der Natur. [Auch in: Beilage zur Allg. Zeitung. Jg. 1901, Nr 35.]

1901-02. Föppl, August: Die Mechanik im neunzehnten Jahrhundert. [Auch bei Reinhardt in München 1902.]

1902-03. Oebbeke, Konrad: Die Stellung der Mineralogie und Geologie an den Technischen Hochschulen. [Ausz. in: Hochschul-Nachrichten. Jg. 13. 1902/03.] 
1903-04. Dyck, Walther von: Über die Errichtung eines Museums von Meisterwerken der Naturwissenschaft und Technik in München. [Auch in: Beilage zur Allg. Zeitung. Jg. 1904, Nr 68. 69. 1904-05. Kraus, C(arl): Die Pflege der Landwirtschaftswissenschaft in Bayern. [Auch in: Vierteljahresschrift d. Bayer. Landwirtschaftsrates. Jg. 10. 1905; daraus auch als S.-A.]

1905-06. Dyck, Walther von: Die wesentlichen Momente, welche die Entwickelung der Technik in Bayern eingeleitet und Bayerns Leistungen auf technischem Gebiete weit über seine Grenzen hinaus zur Anerkennung gebracht haben. [Etwas gekürzt in: Darstellungen aus d. Geschichte d. Technik. 1906. Nr 2356.] - Burmester, Ludwig: Die geschichtliche Entwicklung der Perspektive in Beziehung zur Geometrie. [Beide Reden in: Beilage zur Allg. Zeitung. Jg. 1906, Nr 6.]

1906-07. Thiersch, Friedrich von: KünstlerischeErziehung. [Auch in: Der Säemann. Jg.3.1907.]

1907-08. Kreuter, Franz: Die wissenschaftlichen Bestrebungen auf dem Gebiete des Wasserbaues und ihre Erfolge. [Auch in: Zentralblatt f. Wasserbau u. Wasserwirtschaft. Jg. 3. 1908.]

1908-09. Schröter, Moritz: Naturwissenschaft und Technik. [Auch in: Münchner Neueste Nachrichten. 1908, Nr 137. 138.]

1909-10. Hocheder, Karl: Gesichtssinn und baukünstlerisches Schaffen. [Auch in: Internat. Wochenschrift f. Wissensehaft, Kunst u. Technik. Bd 4. 1910.]
1910-11. Lynen, Wilhelm: Die Ausbildung zum Konstrukteur und ihre Bedeutung für die Allgemeinheit. [Auch in: Bayer. Industrie- $u$. Gewerbeblatt. Jg. 97. N. F. Jg. 43. 1911.]

1868/69 Ausg. mit u. ohne

Reden sowie d. Sonderausg. d. Reden. 18\%7/78. 1878/79.1880/81-1882/83. 1888/89 erw. Ausg.: M

2349. Personalstand der (1872/73 ff.: Kgl. Bayerischen) Polytechnischen Schule (1877/ 78 ff.: Technischen Hochschule) zu (1910/ $11 \mathrm{ff}$. : in) München im W.-S. 1868/69.S.-S. 1869 [usw.]-W.-S. 1912/13. 1869-1912. 1912. (Nebst Deckbl. zu S. 120.)

1869. $1869 / 70$.

1870/71. 1871. 1874. 187\%-1878/79. 1879/80. 1880. 1881. 1881/82. 1882/83. 1884-1885. 1887/88. 1892/93. 1895/96. 1897/98. 1900/01: M

2350. Specieller Stundenplan der Polytechnischen Schule in München für das Sommersemester 1870. 1870. $1870-71 \mathrm{ff}$. im Programm [Nr 2345].

$M$ Hof $B$

2351. Statistische Uebersicht über die Frequenz der K. Technischen Hochschule in München im W.-S. 1902/03. S.-S. 1903 [usw.]-W.-S. 1912/13. - Mit den endgültigen Frequenzziffern in: Personalstand 1902/03-1912/13 [Nr 2349].

\section{GELEGENHEITS - UND VERMISCHTE SCHRIFTEN}

2352. Joseph von Utzschneider und seine Leistungen auf staats- und volkswirthschaftlichem Gebiet. Rede an die Studirenden der Technischen Hochschule beim Antritte des Rectorats f. d. Jahre 1880 bis 1883 geh. am 18. Dec. 1880 von Carl Max v. Bauernfeind. München: Franz in Comm. 1880. 50 S. $4^{0} M$

2353. Katalog der Bibliothek der Königlichen Technischen Hochschule zu München. Stand Ende Dez. 1880. Zahl der Nummern: 5786, der Bände: 16000. 1881. XV, 501 S. [Nebst] Nachtrag 1. Vom 1. Jan. 1881 bis 1. Juni 1892. 1892. XI, 275 S.; Nachtrag 2. Vom 1. Juni 1892 bis 1. Aug. 1902. (Vorr.: Hermann Brunn.) 1903. XIV, 362 S. $8^{\circ}$

2354. Staatswirtschaftliches Bauwesen und technischer Unterricht in Bayern unter König Ludwig I. Vortrag geh. bei d. Schlussfeier d. techn. Hochschule im hundertsten Jahre nach d. Geburt König Ludwigs des Ersten am 28. Juli 1886 von Carl Max v. Bauernfeind. München: G. Franz in Komm. 1886. 32 S. $4^{0}$
2355. Elektrische Einrichtungen im Elektrochemischen Laboratorium der Kgl. techn. Hochschule zu München ausgeführt im Jahre 1897 nach den Plänen und unter der Leitung des Ingenieur Oscar von Miller in München. Juni 1897. (Umschlagt.: Das elektrochemische Laboratorium der Kgl. Technischen Hochschule München.) $13 \mathrm{~S} ., 5$ Taf. $8^{0}$

2356. Darstellungen aus der Geschichte der Technik, der Industrie und Landwirtschaft in Bayern. Festgabe der Königlichen Technischen Hochschule in München zur Jahrhundertfeier der Annahme der Königswürde durch Kurfürst Maximilian IV. Joseph von Bayern. 1906. XV, $323 \mathrm{~S}$, 21 Taf. $4^{0}$

Enth.: Walther v. Dyck: Die Technik in Bayern zur Zeit der Regierung Maximilian Josephs I. Festrede bei d. akad. Jahresfeier am 8. Jan. 1906. [Auch in: Bericht 1905-06. Nr 2348.] Siegmund Günther: Ein Rückblick auf die Anfänge des technischen Schulwesens in Bayern. Richard Streiter: Münchener Architektur um 1806 und 1906. - Martin Hahn: Sanitäre Zu- 
stände und Einrichtungen in München am Anfang des XIX. Jahrhunderts. - Ernst Voit: Entwicklung der Beleuchtung und Beleuchtungstechnik. Siegmund Günther u. Ferdinand Loewe: Bayerisches Karten- und Straßenwesen sonst und jetzt. - Max Schmidt: Die Messung der Basis München-Aufkirchen und die erste topographische Aufnahme Bayerns zu Beginn des XIX. Jahrhunderts. - Franz Kreuter u. Ernst Henle: Der Wasserbau in Bayern. - Wilhelm Dietz: Die Entwicklung des Brückenbaues und Bayerns Anteilnahme im XIX. Jahrhundert. - Wilhelm Lynen: Die Eisenbahnen und ihre Einrichtungen nach Josef von Baader. - Wilhelm Lynen: Die neuen Schnellzuglokomotiven der Pfälzischen Eisenbahnen. - Rudolf Camerer: Ein Meisterwerk im Bau von Wasserkraftmasehinen. - Ernst Voit: Feinmechanik in Bayern. - Egbert v. Hoyer: Die Faserstoffindustrie 〈Spinnerei, Weberei, Papierfabrikation $\rangle.-$ Gustav Schultz: Die chemische Industrie Bayerns zu Anfang des XIX. und zu Anfang des XX. Jahrhunderts. Gustav Schultz: Über die Glasindustrie Bayerns vor 100 Jahren und in der Gegenwart. - Gustav Schultz: Die Tonindustrie Bayerns in den Jahren 1806 und 1906. - Carl Lintner: Das Brauwesen. - Carl Kraus: Der Zustand der bayerischen Landwirtschaft vor 100 Jahren im allgemeinen. Carl Kraus: Acker- und Pflanzenbau in Bayern vor 100 Jahren. - Emil Pott: Landwirtschaftliche Tierproduktion.

2357. Die neuen chemischen Institute der Königlichen Technischen Hochschule zu München. (Vorr.: Walther von Dyck.) 1909. 64 S., 15 Taf. $2^{0}$

\section{HABILITATION S SCHRIFTEN}

2358. Stadler, Hermann, Dr.: Dioscorides Longobardus. 〈Cod. Lat. Monacensis 337. V. Buch. 1901. S. $161-243.8^{0}$ - Aus: Roman. Forschungen. Bd 13. 1902.

München Te. H., Hab.-Schr. v. 14. 6. 1901

2359. Ewers, Paul, Dr.: Die Spitzenentladung in ein- und zweiatomigen Gasen. Leipzig: J. A. Barth 1905. 82 S. $8^{0}$ ศ Aus: Annalen d. Physik. Folge 4, Bd 17.

München Te. H., Hab.-Schr. v. 23. 2. 1905

2360. Kurz, Karl, Dr. phil.: Radium, Thorium und Aktinium in der Atmosphäre und ihre Bedeutung für die atmosphärische Elektrizität. München: Kgl. Bayer. Akad. d. Wiss. 1909. 56 S., 4 Taf. $4^{0}$ I Aus : Abhandlungen d. K. Bayer. Akademie d. Wissenschaften. Math.-phys. Kl. Bd 25.

München Te. H., Hab.-Schr. v. 22. 7.1909

2361. Aufseß, 0tto Frhr. von und zu, Dr.: Experimentelle Untersuchungen über den Einfluß der Phase und der Rotation auf die Helligkeit von Kugeln und beliebig gestalteten Körpern. 1910. 68 S., 12 Taf. $8^{0}$ - Ausz. in: Astronom. Abhandlungen. Nr 17.

München Te. H., Hab.-Schr. v. 9. 1. 1910

\section{DOKTOR-DISSERTATIONEN}

\section{ALLGEMEINE ABTEILUNG}

2362. Schwaighofer, Hans, Ingenieur: Die Grundlagen der Preisbildung im elektrischen Nachrichten-Verkehr. [1902.] 162 S., 2 Bl. Taf. $8^{0}$ - Auch bei Lindauer in München.

München, Dr.-Ing.-Diss. v. 21. 12. 1901

2363. Haller, Stanislaus: Untersuchung der Brennpunktskurve eines Kegelschnittbüschels mit besonderer Berücksichtigung der gestaltlichen Verhältnisse. 1903. 42 S. $8^{0}$ I Aus : Archiv d. Mathematik u. Physik. Reihe 3, Bd 7. 1904.

München, Dr.-Ing.-Diss. v. 6. 3. 1902

2364. Endrös, Anton, K. Reallehrer i. Traunstein: Seeschwankungen 〈Seiches〉 beobachtet am Chiemsee. Mit 2 Taf. 1903. VIII, 117 S., 2 Taf. $8^{\circ}$. Auch als : Programm d. K. Realschule Traunstein f. 1902/03; Forts. in:
Sitzungsberichte d. math.-phys. Klasse d. K. B. Akademie d. Wissenschaften zu München. Bd 36. 1906.

München, Dr.-Ing.-Diss. v. 21. 12. 1903

2365. Vollkommer, Max: Die Quellen Bourguignon d'Anvilles für seine kritische Karte von Afrika. München: Th. Ackermann 1904. 124 S. $8^{0}$ I Auch als: Münchener geogr. Studien. Stück 16.

München, Dr.-Ing.-Diss. v. 22. 2. 1904

2366. Lutz, Karl Wolfgang: Untersuchungen über atmosphärische Elektrizität mit besonderer Berücksichtigung ihrer technischen Bedeutung. [1904.] 102 S., 9 Taf. $8^{0}$

München, Dr.-Ing.-Diss. v. 3. 3. 1904

2367. Hartig, 0tto: Ältere Entdeckungsgeschichte und Kartographie Afrikas mit Bourguignon d'Anville als Schlußpunkt $\langle 1749\rangle$. Mit 1 Taf. u. 3 Kt. im Texte. Wien: 
Lechner 1905. 101 S., 1 Taf. $8^{\circ}$ ๆ Aus: Mitteilungen d. K. K. Geogr. Gesellschaft in Wien. Bd 48 .

München, Dr.-Ing.-Diss. v. 4. 7. 1904

2368. Ebner, Eduard : Geographische Hinweise und Anklänge in Plutarchs Schrift: de facie in orbe lunae. München: Th. Ackermann 1906. $101 \mathrm{~S} .8^{0}$ - Auch als: Münchener geogr. Studien. Stück 19.

München, Dr.-Ing.-Diss. v. 15. 3. 1905

2369. Fraunberger, Georg: Studien über die jährlichen Niederschlagsmengen des afrikanischen Kontinents. Gotha: Perthes 1906. 12 S., $1 \mathrm{Kt} .4^{0}$ - Aus: Dr. A. Petermanns Mitteilungen aus J. Perthes' geogr. Anstalt. Bd 52.

München, Dr.-Ing.-Diss. v. 7. 8. 1905

2370. Sensburg, Waldemar: Poggio Bracciolini und Nicolò de Conti in ihrer Bedeutung für die Geographie des Renaissancezeitalters. 〈Mit 1 Kartenskizze.> Wien: Lechner 1906. 116 S., 1 Kt. $8^{0}$ - Aus: Mitteilungen d. K. K. Geogr. Gesellschaft in Wien. Bd 49 .

München, Dr.-Ing.-Diss. v. 23.12. 1905

2371. Huber, Franz Josef, Lehramtsassist.: Die Anfänge der alpinen Forsehung in den Ostalpen und im Karstgebiete <bis 1800). 1906. 123 S. $8^{0}$

München, Dr.-Ing.-Diss. v. 12.2. 1906

2372. Attensperger, Albert, Kgl. Reallehrer: Studien zur Morphologie der Vorderpfalz. 1908. $49 \mathrm{~S}$. $8^{0}$

München, Dr.-Ing.-Diss. v. 20. 2. 1907

2373. Rieder, Heinrich, Assist. an d. Techn. Hochsch. München: Untersuchung einer zwei-vierdeutigen kinetographischen Verwandtschaft. 1907. 49 S., 1 Taf. $8^{0}$

München, Dr.-Ing.-Diss. v. 7. 3. 1907

2374. Rothenberg, Siegfried: Geschichtliche Darstellung der Entwicklung der Theorie der singulären Lösungen totaler Differentialgleichungen von der ersten Ordnung mit zwei variablen Grössen. 1908. VIII, 86 S. $8^{0}$ T Auch in: Abhandlungen zur Geschichte d. math. Wissenschaften. H. 20.

München, Dr.-Ing.-Diss. v. 15. 3. 1907

2375. Scheufele, Wilhelm: Die Aufgabe der sechs Punkte in der Photogrammetrie. 1907. 28 S. $8^{0}$ - Aus: Zeitschrift f. Mathematik u. Physik. Bd 55 .

München, Dr.-Ing.-Diss. v. 2. 4. 1907

2376. Müller, Albert, $\mathrm{K}$. Reallehrer i. Kulmbach: Studien über die geographische Lage der Stadt Wasserburg am Inn. 1908. 71 S., 4 Taf. $8^{0}$

München, Dr.-Ing.-Diss. v. 20. 6. 1907
2377. Pfeiffer, Friedrich: Utber die WFlächen mit der Relation $2\left(R_{1}-R_{2}\right)=$ $\sin 2\left(R_{1}+R_{2}\right) 2$ zwischen den Hauptkrümmungsradien $R_{1}$ und $R_{2}$. 1907. 60 S., 5 Taf $8^{0}$

München, Dr.-Ing.-Diss. v. 19. 7. 1907

2378. Erfle, Heinrich: Optische Eigenschaften und Elektronentheorie. Leipzig: J. A. Barth 1907. 83 S. $8^{0}$ i Ausz. in: Annalen d. Physik. Folge 4, Bd 24.

München, Dr.-Ing.-Diss. v. 1. 8. 1907

2379. Jaufmann, Josef, Assist. f. Physik an d. Zentralgewerbesch. München: Untersuchungen über den radioaktiven und elektrischen Zustand der Atmosphäre nach Beobachtungen an der K. B. meteorologischen Hochstation Zugspitze. 1908. 38 S. $4^{0}$ - Auch bei Buchholz in München; aus: Beobachtungen d. meteorolog. Stationen im Kgr. Bayern i. J. 1907. (Deutsches Meteorolog. Jahrbuch f. 1907. Jg. 29. Bayern.)

München, Dr.-Ing.-Diss. v. 16. 3. 1908

2380. Alt, Eugen, Adjunkt d. Kgl. bayer. meteorolog. Zentralstation: Die Doppeloszillation des Barometers insbesondere im arktischen Gebiete. 1909. 22 S. $4^{0}$ - Aus: Meteorolog. Zeitschrift. Bd 26.

München, Dr.-Ing.-Diss. v. 2. 4. 1908

2381. Daunderer, Alois Anton, Prof. f. Math. u. Physik an d. Realsch. Rosenheim: Über die in den unteren Schichten der Atmosphäre vorhandene freie elektrische Raumladung. 1908. 98 S., 6 Taf. $4^{0}$ - Vorläufige Mitteilung in: Physik. Zeitschrift. Jg. 8. 1907. S. $281-286$.

München, Dr.-Ing.-Diss. v. 29. 7. 1908

2382. Gassenmeyer, Eduard: Die Lehre von der Basaltbildung in ihren geologischen und geographischen Konsequenzen bis auf A. G. Werner $\langle 1789\rangle$ und seine Schule. 1908. 58 S. $8^{0}$

München, Dr.-Ing.-Diss. v. 29. 7. 1908

2383. Heydenreich, Adolf: Karl Ernst von Baer als Geograph. Mit 3 Kärtchen. München: Th. Ackermann 1908. 87 S., 3 Taf. $8^{0}$ T Auch als : Münchener geogr. Studien. Stück 23. 1909.

München, Dr.-Ing.-Diss. v. 4. 8. 1908

2384. Schmitt, Theodor: Die Meteorologie und Klimatologie des Albertus Magnus. 1909. 116 S. $8^{0}$

Mănchen, Dr.-Ing.-Diss. v. 4. 8. 1908

2385. Deuerling, 0swald: Die Pflanzenbarren der afrikanischen Flüsse mit Berücksichtigung der wichtigsten pflanzlichen Verlandungserscheinungen. München:Th.Ackermann 1909. $253 \mathrm{~S}$., 14 Taf. $8^{0}$ - Auch als : Münchener geogr. Studien. Stück 24.

München, Dr.-Ing.-Diss. v. 26. 3. 1909 
2386. Förg, Karl, Assist. d. K. KreisOberrealsch. zu Nürnberg: Die Bestimmung des Standpunktes und der äusseren Orientierungselemente in der Photogrammetrie bei bekannter innerer Orientierung. 1909. $67 \mathrm{~S}$., 1 Taf. $8^{0}$ - Auch bei Schrag in Nürnberg $u$. als: Wissenschaftl. Beilage zum Jahresberichte der K. Kreis-Oberrealschule Nürnberg 1908/09.

München, Dr.-Ing.-Diss. v. 26. 3. 1909

2387. Habermehl, Karl: Die mechanischen Ursachen für die regelmässige Anordnung der Teilungswände in Pflanzenzellen. 1909. 48 S. $8^{0}$

München, Dr.-Ing.-Diss. v. 26. 3. 1909

2388. Hübsch, Karl, Assist. an d. Techn. Hochsch. München: Untersuchung einer kinetographischen Verwandtschaft bei speziellen Schleifschiebergetrieben. 1909. 44 S., 2 Taf. $8^{0}$

München, Dr.-Ing.-Diss. v. 26. 3. 1909

2389. Hage, Hans, Assist. an d. Techn. Hochsch. München: Über Begrenzungsflächen unendlich dünner Strahlenbündel, deren Erzeugende gleiche Neigung zum Mittelstrahl haben. 1909. $40 \mathrm{~S} ., 1$ Taf. $8^{0}$ München, Dr.-Ing.-Diss. v. 8. 6. 1909

2390. Endrös, Ludwig, Hauptlehrer I. Klasse f. Physik an d. städt. Gewerbeschulen i. München: Messungen und Registrierungen der dem Erdboden entquellenden Emanationsmengen. 1909. 64 S., 8 Taf. $8^{0}$

München, Dr.-Ing.-Diss. v. 31. 7. 1909

2391. Weinfurtner, Johannes, Kgl. Reallehrer: Die Entdeckung und Erforschung der Insel Neufundland. 1910. 57 S. $8^{\circ}$-Auch bei Koch in Nürnberg.

München, Dr.-Ing.-Diss. v. 31. 7. 1909

2392. Herpich, Hans, Reg.-Baumeister: Die Eisverhältnisse in den südbayerischen Seen. [1910.] 89 S., 5 Taf. $8^{0}$ T Auch als : Münchener geogr. Studien. Stück 26. 1911.

München, Dr.-Ing.-Diss. v. 30. 12. 1909

2393. Hauser, Friedrich: Untersuchung von Bronsonwiderständen. 1911. $59 \mathrm{~S}$., 11 Taf. $8^{0}$ I Ausz. in : Physik. Zeitschrift. Jg. 12. München, Dr.-Ing.-Diss. v. 14. 12.1910

2394. Schoy, Carl, Oberlehrer am städt. Gymn. zu Essen, Ruhr: Die geschichtliche Entwicklung der Polhöhenbestimmungen bei den älteren Völkern. 1911. 33 S., 1 Taf. $4^{0}$ I Auch als: Aus d. Archiv d. Deutschen Seewarte. Jg. 34, Nr. 2.

München, Dr.-Ing.-Diss. v. 17. 2. 1911

2395. Welponer, Anton: Die Betriebskraft als Kostenelement der industriellen Unternehmung. 1912. 68 S., 1 Taf. $8^{0}$

München, Dr.-Ing.-Diss. v. 23. 3. 1911
2396. Rudel, Ernst, Prof. an d. K. Oberrealsch. Ludwigshafen a. Rh.: Die Orientierung photogrammetrischer Aufnahmen bei vertikaler Bildebene unter Benutzung magnetischer Azimute. [1912.] 40 S., 1 Taf. $8^{0}$ - Auch als: Wiss. Beil. zum Jahresbericht d. K. Oberrealsch. Ludwigshafen a. Rh. 1911/12.

München, Dr.-Ing.-Diss. v. 26. 6. 1911

2397. Kimmel, Hermann: Theorie der Luftschrauben auf aerodynamischer Grundlage. 1912. $33 \mathrm{~S} .4^{0}$ I Ausz. in : Zeitschrift f. Flugtechnik u. Motorluftschiffahrt. Jg. 3.

München, Dr.-Ing.-Diss. v. 14. 7. 1911

2398. Heis, Leonhard: Methode zur Bestimmung der Ionenkonstanten. <Beweglichkeiten.> 1912. 41 S, 3 Taf. $8^{0}$

München, Dr.-Ing.-Diss. v. 14. 7. 1911

2399. Kleeberg, Rudolf: Uber die Diskriminantenflächen der Gleichungen A + $\cos \mathrm{x}+\mathrm{B} \sin 2 \mathrm{x}+\mathrm{C} \cos 2 \mathrm{x}+\mathrm{D} \cos 3 \mathrm{x}$ $=0.1911 .87$ S., 5 Taf. $8^{0}$

München, Dr.-Ing.-Diss. v. 15. 7. 1911

2400. Hoffmann, Karl, Assist. am physik. Inst. d. Techn. Hochsch. München: Experimentelle Prüfung der durch verschiedene Messungsanordnungen in einem homogenen Felde hervorgerufenen Störungen <Deformationen > der Niveauflächen. 1911. $67 \mathrm{~S}$, 12 Taf. $8^{0}$

München, Dr.-Ing.-Diss. v. 28. 7. 1911

2401. Weigel, Jakob: Über die gestaltlichen Verhältnisse der Integralkurven einer Differentialgleichung erster Ordnung zweiten Grades in der Umgebung eines Doppelpunktes der Diskriminantenkurve. 1912. S. $279-343.4^{0}$ T Aus: Nova Acta Academiae Caesareae Leopoldino-Carolinae Germanicae Naturae Curiosorum. T. 95.

München, Dr.-Ing.-Diss. v. 28. 7. 1911

2402. Muth, Fritz, gepr. Lehramtskand. i. Augsburg: Über solche Koordinatensysteme auf Flächen, bei denen die eine Schar von Parameterkurven auf der andern gleiche Stücke abschneidet. 1912 . 54 S. $8^{0}$

München, Dr.-Ing.-Diss. v. 14. 12. 1911

2403. Jakob, Georg: Belsazar Hacquet und die Erforschung der Ostalpen und Kar paten. München: Th. Ackermann [1912]. $126 \mathrm{~S} ., 3$ Taf. $8^{0}$ ๆ Auch als : Münchener geogr. Studien. Stück 27.

München, Dr.-Ing.-Diss. v. 24. 5. 1912

2404. Menauer, Jakob, Kgl. Reallehrer an d. Kreisrealsch. I i. Nürnberg: Die Laufänderungen des Gelben Flusses in historischer Zeit. 〈Mit 4 Kt.〉 1912. 72 S., 4 Kt. $8^{0}$ - Auch bei C. Koch in Nürnberg u. als Programm d. Kreisrealschule I in Nürnberg.

München, Dr.-Ing.-Diss. v. 3. 7. 1912 
2405. Müller, Karl, K. Seminarlehrer: Der Polyhistor Konrad Gesner als Freund und Förderer erdkundlicher Studien. 1912. 46 S.80 München, Dr.-Ing.-Diss. v. 4. 7. 1912

2406. Nuber, Ambros, Assist. an d. Techn. Hochsch. München: Untersuchung der Kernkurven spezieller ebener Korrelationen und der damit verbundenen quadratischen Verwandtschaften. 1912.45 S., 1 Taf. $8^{0}$

München, Dr.-Ing.-Diss. v. 5. 7. 1912

2407. Ammann, Hans: Physikalische und biologische Beobachtungen an oberbayerischen Seen. 1912. 70 S., 9 Taf. $4^{0}$ - T. 2. C. Temporalvariationen etwas erw. in: Archiv f. Hydrobiologie u. Planktonkunde. Bd 8. 9. 1912. 13. München, Dr.-Ing.-Diss. v. 16. 7. 1912

\section{BAUINGENIEUR-ABTEILUNG}

2408. Freytag, Ludwig, Obering. d. Vereinigten Maschinenfabrik Augsburg u. Maschinenbauges. Nürnberg, A.-G., Werk Nürnberg: Gesetzmäßigkeiten in der TrägerTheorie. 1904. 46 S. $4^{0}$

München, Dr.-Ing.-Diss. v. 13. 5. 1904

2409. Hohenner, Heinrich, a. o. Prof. d. Geodäsie an d. techn. Hochsch. Stuttgart: Graphisch-mechanische Ausgleichung trigonometrisch eingeschalteter Punkte. Stuttgart: Wittwer 1904. 47 S., 2 Taf. $8^{0}$ - Auch als Buch.

München, Dr.-Ing.-Diss. v. 22. 6. 1904

2410. Herbst, Waldemar, Ingenieur b. d. städt. Wasserversorgung München: Ermittlung einer Beziehung zwischen der Niederschlagsmenge in einem Flußgebiete und der größtmöglichen Abflußmenge in demselben. 1905. 32 S., 3 Taf. $4^{0}$ - Anh. zur Abhandlung d. Kgl. Bayer. Hydrotechn. Bureaus v. J. 1905. 〈GröBte Regenfälle in Bayern u. ihre Verwertung für Hochwasserberechnungen.> Aus: Jahrbuch d. Kgl. Bayer. Hydrotechn. Bureaus. Jg. 6. 1904.

München, Dr.-Ing.-Diss. v. 2. 8. 1904

2411. Weyrauch, Robert, Reg.-Baumeister: Unterlagen zur Dimensionierung städtischer Kanalnetze. Stuttgart u. Berlin: Grub 1904. VI, 67 S. $8^{0}$ - Auch als Buch.

München, Dr.-Ing.-Diss. v. 2. 8. 1904

2412. Bastian, Riehard, Hauptlehrer an d. städt. Baugewerksch. i. Nürnberg: Das elastische Verhalten der Gleisbettung und ihres Untergrundes. Wiesbaden: Kreidel 1906. $38 \mathrm{~S} .4^{0}$ ๆ Aus: Organ f. d. Fortschritte d. Eisenbahnwesens. Jg. 61. N. F. Bd 43. Erg. H.

München, Dr.-Ing.-Diss. v. 21. 3. 1906
2413. Näbauer, Martin, Assist. f. Geodäsie an d. Techn. Hochsch. München: Die Bedeutung der Koordinatengeometrie für die Bauingenieur-Technik. 1907 . VI, 93 S. $8^{0}$ - Aus : Zeitschrift d. Bayer. Geometer-Vereins. Bd 11.

München, Dr.-Ing.-Diss. v. 7. 3. 1907

2414. Schlötzer, Adolf, Assist. f. Geodäsie an d. Techn. Hochsch. München: Der Heliotrop, seine Geschichte, Konstruktion und Genauigkeit. 1909. 58 S. $8^{0}$ - Aus: Zeitschrift d. Bayer. Geometervereins. Bd 13.

München, Dr.-Ing.-Diss. v. 29. 7. 1908

2415. Friedrich, Conrad: Georg Friedrich Brander und sein Werk. München: Selbstverl. [1909]. 55 S., 8 Taf. $4^{0}$

München, Dr.-Ing.-Diss. v. 8. 6.1909

2416. Riesner, Hans: Die Darstellung eines Objektes aus drei photographischen Aufnahmen mit gegebenen Apparatkonstanten bei unbekannten Standpunkten. 1911. 43 S., 2 Taf. $8^{0}$

München, Dr.-Ing.-Diss. v. 5. 8. 1911

2417. Kohlmüller, Franz, Assist. an d. Techn. Hochsch. München: Zur Refraktion im Nivellement. 1912. $101 \mathrm{~S} .8^{0}$ I Aus: Zeitschrift d. Vereins d. Höheren Bayer. Vermessungsbeamten. Bd 16.

München, Dr.-Ing.-Diss. v. 15. 3. 1912

2418. Seheek, Franz: Einfache und stereoskopische Bildmessung im reinen Felsgebiete. 1912. $66 \mathrm{~S} ., 2$ Taf. $8^{0}$. Auch als: Landeskundl. Forschungen hrsg. v. d. geogr. Gesellschaft in München. H. 16. (Aus : Mitteilungen d. geogr. Gesellschaft in München. Bd 7.)

München, Dr.-Ing.-Diss. v. 21. 3. 1912

\section{ARCHITE KTEN-ABTEILUNG}

2419. Fiechter, Ernst R[obert], Architekt u. Assist. an d. Techn. Hochsch. München: Der Tempel der Aphaia auf Aegina. 1904. 57 S., 6 Taf. $4^{0}$ Mit erw. Einl. in : A. Furtwängler: Aegina, das Heiligtum der Aphaia. München 1906.

München, Dr.-Ing.-Diss. v. 20. 7. 1904

2420. Sehmidt, Friedrich, städt. Bauamtsass. i. Regensburg: Über den Ursprung des romanischen Baustils. (1904.) $83 \mathrm{~S} .8^{0}$

München, Dr.-Ing.-Diss. v. 30. 1. 1905

2421. Willieh, Hans: Die Kirchenbauten des Giacomo Barozzi da Vignola. Ein Beitrag zur Entwicklungsgeschichte des Barockstils. 1905. 64 S. $8^{\circ}$ - Erw. u. d. T.: Giacomo Barozzi da Vignola, als: Zur Kunstgeschichte des Auslandes. H. 44. 1906.

München, Dr.-Ing.-Diss. v. 30. 6. 1905 
2422. Sack, Bernhard: Georg Moller. Sein Leben und Wirken. 1908. 79 S. $8^{0}$

München, Dr.-Ing.-Diss. v. 25. 1. 1908

2423. Wagner, Hans, Architekt: Über die romanische Bąukunst in Regensburg. [1909.] 80 S. $8^{0}$ - Auch bei Coppenrath in Regensburg u. d. T.: Studien über...

München, Dr.-Ing.-Diss. v. 21. 12. 1908

2424. Priester, Karl: Bremische Wohnhäuser um 1800. Beiträge zur Baugeschichte der Stadt Bremen. Bremen: Leuwer 1912. 116 S., 1 Taf. $8^{0}$ T Auch als Buch.

München, Dr.-Ing.-Diss. v. 14. 7. 1911

\section{MASCHINENINGENIEUR-ABTEILUNG}

\section{Maschineningenieurwesen}

2425. Döderlein, Gustav: Prüfung und Berechnung ausgeführter Ammoniak-Kompressions-Kältemaschinen an Hand des Indikator-Diagramms. 1903. 113 S., 2 Taf. $8^{\circ}$ - Auch bei Oldenbourg in München; aus: Zeitschrift f. d. gesamte Kälte-Industrie. Jg. 10.

München, Dr.-Ing.-Diss. v. 5. 8. 1902

2426. Uebelacker, Heinrich, K. B. Eisenbahnass. u. Vorstand d. K. Betriebswerkstätte Lichtenfels: Untersuchungen über die Bewegung von Lokomotiven mit Drehgestellen in Bahnkrümmungen. Wiesbaden: Kreidel 1903. 26 S., 3 Taf. $4^{0}$ of Auch als: Organ f. d. Fortschritte d. Eisenbahnwesens. Jg. 58. N. F. Bd 40. Beil.

München, Dr.-Ing.-Diss. v. 19. 12. 1902

2427. Koob, A[ugust], Assist. an d. techn. Hochsch. München: Das Regulierproblem in vorwiegend graphischer Behandlung. 1903. 22 S. $4^{0}$ - Aus: Zeitschrift d. Vereines deutscher Ingenieure. Bd 48. 1904, Halbj. 1.

München, Dr.-Ing.-Diss. v. 22. 6. 1903

2428. Föttinger, Hermann : Effektive Maschinenleistung und effektives Drehmoment, und deren experimentelle Bestimmung. <Mit besonderer Berücksichtigung großer Schiffsmaschinen). 1904. $32 \mathrm{~S}$. $4^{0}$ I Auch in : Mitteilungen über Forschungsarbeiten auf d. Gebiete d. Ingenieurwesens. H. 25. 1905.

München, Dr.-Ing.-Diss. v. 12. 1. 1904

2429. Häusser, F[riedrich], Kgl. Lehrer an d. Industriesch. Kaiserslautern: Untersuchungen über explosible Leuchtgas-Luftgemische. 1905. 41 S., 1 Taf. $4^{0}$ व Auch in: Mitteilungen über Forschungsarbeiten auf d. Gebiete d. Ingenieurwesens. H. 25; Ausz, in: Zeit- schrift d. Vereines deutscher Ingenieure. Bd 50. 1906, Halbj. 1.

München, Dr.-Ing.-Diss. v. 28. 7. 1904

2430. Linde, $\mathbf{R}[$ ichard]: Ueber die thermischen Eigenschaften des gesättigten und überhitzten Wasserdampfes zwischen $100^{\circ}$ und $180^{\circ}$ C. 1904. 44 S., 1 Taf. $4^{0}$. Auch in: Mitteilungen über Forschungsarbeiten auf $\mathrm{d}$. Gebiete d. Ingenieurwesens. H. 21. 1905; Ausz. in : Zeitschrift d. Vereines deutscher Ingenieure. Bd 49. 1905, Halbj. 2.

München, Dr.-Ing.-Diss. v. 28. 7. 1904

2431. Adam, J[ulius], Assist. d. Techn. Hochsch. München: Der Ausfluß von heißem Wasser. 1906. 52 S. $4^{0}$ T Aus: Mitteilungen über Forschungsarbeiten auf d. Gebiete d. Ingenieurwesens. H. $35 / 36$; S. $1-24$ auch in: Zeitschrift d. Vereines deutscher Ingenieure. Bd 50, Halbj. 2.

München, Dr.-Ing.-Diss. v. 23. 12.1905

2432. Dafinger, Eduard, Assist. an d. Techn. Hochsch. München: Graphodynamische Untersuchung einer Heusinger-JoySteuerung. Ein Beitrag zur Erkenntnis der Bewegungsverhältnisse der Steuerungsgetriebe. [Nebst] Berichtigungen. Berlin: Dietze 1906. 34 S. $4^{0}$ T Aus: Dinglers Polytechn. Journal. Jg. 88. 1907. Bd 322.

München, Dr.-Ing.-Diss. v. 20. 7. 1906

2433. Burgmann, Robert: Asbest-Spinnerei. $1906.49 \mathrm{~S} .4^{0}$

München, Dr.-Ing.-Diss. v. 6. 8. 1906

2434. Früh, Michael: Studien über die Bildung des Kötzers beim Selfaktor. Berlin: Dietze 1907. 55 S. $8^{0}$ I Aus: Dinglers Polytechn. Journal. Jg. 88. Bd 322.

München, Dr.-Ing.-Diss. v. 12. 6. 1907

2435. Nußelt, Wilhelm, Assist. an d. Techn. Hochsch. München: Die Wärmeleitfähigkeit von Wärmeisolierstoffen. 1908. $88 \mathrm{~S} .4^{0}$ - Auch in: Mitteilungen über Forschungsarbeiten auf d. Gebiete d. Ingenieurwesens. H. $63 / 64$; Ausz. in: Zeitschrift d. Vereines deutscher Ingenieure. Bd 52, Halbj. 1 u. in: Zeitschrift $\mathrm{f}$. d. gesamte Kälte-Industrie. Jg. 15.

München, Dr.-Ing.-Diss. v. 6. 8. 1907

2436. Dolder, E[ugen]: Über Zustandsverhältnisse strömender Flüssigkeiten und deren Wirkungsweise in Turbinenrädern. (1907.) 49 S., 2 Taf. $8^{0}$ II Auch bei Rascher in Zürich 1908.

München, Dr.-Ing.-Diss. v. 14. 11. 1907

2437. Egerer, Heinrich, Dr. phil.: Beiträge zur Fachwerkstheorie. 1908. 34 S. $8^{0}$ - Wesentlich erw. u. d. T.: Neue Methoden der Berechnung ebener und räumlicher Fachwerke. Berlin: Springer 1909.

München, Dr.-Ing.-Diss. v. 26. 12. 1907 
2438. Gröber, Heinrich, Assist. an d. Techn. Hochsch. München: Physikalische Untersuchungen für die Kältetechnik. I. Die spezifische Wärme derChlornatriumlösungen. II. Die Schmelzwärme der Kryohydrate. III. Die Wärmeleitfähigkeit von Isoliermaterialien bei tiefen Temperaturen. 1908. 38 S. $4^{0}$ - Auch in: Zeitschrift f. d. gesamte Kälte-Industrie. Jg. 16. 1809.

München, Dr.-Ing.-Diss. v. 26. 11. 1908

2439. Baer, Herbert: Die Regelung von Dampfturbinen und ihr Einfluß auf die Energieentwicklung in den einzelnen Druckstufen. 1909. 62 S. $4^{0}$ - Auch als: Mitteilungen über Forschungsarbeiten auf d. Gebiete d. Ingenieurwesens. H. 86. 1910; Ausz. in: Zeitschrift d. Vereines deutscher Ingenieure. Bd 53, Halbj. 2. München, Dr.-Ing.-Diss. v. 18.12. 1908

2440. Deinlein, Wilhelm, Assist. f. theoret. Maschinenlehre an d. Techn. Hochsch. München: Beiträge zur Dampfturbinentheorie. 1909. VIII, 106 S. $8^{0}$. Auch bei Oldenbourg in München u. d. T.: Zur Dampfturbinentheorie. Verfahren zur Berechnung vielstufiger Dampfturbinen.

München, Dr.-Ing.-Diss. v. 21. 12. 1908

2441. Mader, 0tto, Assist. an d. techn. Hochsch. München: Der Resonanz-Undograph. Ein Mittel zur Messung des Ungleichförmigkeitsgrades. Berlin: Dietze 1909. 20 S. $4^{0}$ - Aus: Dinglers Polytechn. Journal. Jg. 90. Bd 324.

München, Dr.-Ing.-Diss. v. 26. 3. 1909

2442. Goetz, Hans, kgl. Gewerbeinsp.: Theoretische Untersuchung einer BonjourLachaus[s]ée-Dampfmaschine auf Massendruck der Steuerung und Resonanz des Regulators. 1909. 66 S. $4^{0}$ - Auch bei Simion in Berlin 1910 u. in: Verhandlungen d. Vereins zur Beförderung d. Gewerbfleißes. Jg. 88.

München, Dr.-Ing.-Diss. v. 1. 7. 1909

2443. Reutlinger, Ernst: Ueber den Einfluß des Kesselsteins und ähnlicher wärmehemmender Ablagerungen auf Wirtschaftlichkeit und Betriebsicherheit von Heizvorrichtungen. 1909. $74 \mathrm{~S} .4^{0}$ T Auch als: Mitteilungen über Forschungsarbeiten auf d. Gebiete d. Ingenieurwesens. H. 94. 1910; Ausz. in: Zeitschrift d. Vereines deutscher Ingenieure. Bd 54. 1910, Halbj. 1.

München, Dr.-Ing.-Diss. v. 1. 7. 1909

2444. Wamsler, Friedrich: Die Wärmeabgabe geheizter Körper an Luft. 1909. VI, $83 \mathrm{~S} .4^{0}$ ๆ Ausz. in: Mitteilungen über Forschungsarbeiten auf d. Gebiete d. Ingenieurwesens. H. 98/99. 1911 u. in: Zeitschriftd. Vereines deutseher Ingenieure. Bd 55. 1911, Halbj.1.

München, Dr.-Ing.-Diss. v. 20. 7. 1909
2445. Sendtner, Albert: Die Bestimmung der Dampffeuchtigkeit mit dem Drosselkalorimeter und die Anwendung desselben zur Prüfung von Wasserabscheidern. 1910. $53 \mathrm{~S} .4^{0}$ T Ausz. in: Mitteilungen über Forschungsarbeiten auf d. Gebiete d. Ingenieurwesens. H. 98/99. 1911 u. in: Zeitschrift d. Vereines deutscher Ingenieure. Bd 55. 1911, Halbj. 2 .

München, Dr.-Ing.-Diss. v. 31. 7. 1909

2446. Kumpimiller, Alexander: Ueber Sulfit-Zellstoff-Ablauge. 1909.74 S. $8^{0}$

München, Dr.-Ing.-Diss. v. 6. 8. 1909

2447. Schneider, Ludwig: Über die Verwertung des Zwischendampfes und des $\mathrm{Ab}$ dampfes der Dampfmaschinen zu Heizzwecken. Eine wirtschaftliche Studie. Mit 85 in d. Text gedr. Fig. u. 1 Taf. Berlin: Springer 1910. VI, 98 S., 1 Taf. $8^{\circ}$. Auch als Buch; 2. bedeutend erw. Aufl. u. d. T.: Die Abwärmeverwertung im Kraftmaschinenbetrieb mit besonderer Berücksichtigung der Zwischen- und Abdampfverwertung zu Heizzwecken. 1912.

München, Dr.-Ing.-Diss. v. 17. 3. 1910

2448. Vogel, Emil: Ueber die Temperaturveränderung von Luft und Sauerstoff. beim Strömen durch eine Drosselstelle bei $10^{\circ} \mathrm{C}$ und Drücken bis zu 150 Atmosphären. 1910. 54 S. $4^{0}$ - Auch in: Mitteilungen über Forschungsarbeiten auf d. Gebiete d. Ingenieurwesens. H. 108/109. 1911; Zeitschrift f. komprimierte u. flüssige Gase. Jg. 14. 1912.

München, Dr.-Ing.-Diss. v. 7. 5.1910

2449. Soennecken, Alfred: Der Wärmeübergang von Rohrwänden an strömendes Wasser. 1910. $106 \mathrm{~S} .4^{0}$ - Auch in: Mitteilungen über Forschungsarbeiten auf d. Gebiete d. Ingenieurwesens. H. 108/109. 1911.

München, Dr.-Ing.-Diss. v. 16. 5. 1910

2450. Hübner, Hans: Das charakteristische Kurvennetz der Ventilatoren im $\mathrm{Zu}$ sammenhang mit dem Widerstandskurvennetz verzweigter Rohrleitungen. 1911. 17 S., 5 Taf. $4^{0}$ ๆ Aus: Zeitschrift f. d. gesamte Turbinenwesen. Jg. 8 .

München, Dr.-Ing.-Diss. v. 2. 6. 1910

2451. Thoma, Dieter, Assist. f. techn. Mechanik an d. Techn. Hochsch. München: Beiträge zur Theorie des Wasserschlosses bei selbsttätig geregelten Turbinenanlagen. 1910. $60 \mathrm{~S} .8^{0}$. Auch bei Oldenbourg in München.

München, Dr.-Ing.-Diss. v. 1. 7. 1910

2452. Losehge, August, Assist. f. theoret. Maschinenlehre an d. Techn. Hochsch. München: Neue Beiträge zur Dampfturbinentheorie. 1910. 54 S. $4^{0}$ i Aus: Zeitschrift f. d. gesamte Turbinenwesen. Jg. 8. 1911. München, Dr.-Ing.-Diss. v. 26. 7. 1910 
2453. Mühlschlegel, G[eorg]: Untersuchungen der Spinnvorgänge. Berlin: Verl. f. Textil-Industrie 1911. 68 S. $4^{0}$ T Auch als Buch.

München, Dr.-Ing.-Diss. v. 26. 7. 1910

2454. Böhm, 0tto, Assist. f. Maschinenbaukunde an d. Techn. Hochsch. München: Beitrag zur Nachrechnung und Auslegung von Bremsversuchen an Wasserturbinen nach dem Diagramm von Prof. Dr. Camerer. 〈Mit Anwendungsbeispiel〉. 1911. 23 S. $4^{0}$ I Aus: Zeitschrift f. d. gesamte Turbinenwesen. Jg. 9. 1912.

München, Dr.-Ing.-Diss. v. 7. 1. 1911

2455. Berger, Richard: Über die Schalldurchlässigkeit. 1911. $24 \mathrm{~S} 4^{0}$ - T. 3 ,Die Versuche" auch in: Gesundheits-Ingenieur. Jg. 34 . München, Dr.-Ing.-Diss. v. 27. 2. 1911

2456. Hofmann, Franz Josef: Die hydraulischen Schmiede-Pressen nebst einer Untersuchung über den Vorgang beim Pressen eines Stahlstückes in geschlossener Matrize. Essen-R.: Deiter [1911]. 58 S., 23 Taf. $4^{0}$ Mit unbedeutenden Änderungen bei Springer in Berlin 1912.

München, Dr.-Ing.-Diss. v. 16. 3. 1911

2457. Kölsch, 0tto, Assist. f. Maschinenbau an d. Techn. Hochsch. München: Über Zylinderzahl und Zylinderanordnung bei Fahr- und Flugzeugmaschinen. Berlin: Springer 1911. VII, 198 S. $8^{0}$ ๆ Buchausg. u. d. T.: Gleichgang u. Massenkräfte bei Fahr- u. Flugzeugmaschinen.

München, Dr.-Ing.-Diss. v. 26. 4. 1911

2458. Dornig, Mario: Beitrag zur Theorie des indirekt wirkenden Beharrungsreglers. 1911. VIII, 62 S., 1 Taf. $8^{0}$

München, Dr.-Ing.-Diss. v. 8. 8. 1911

2459. Nerreter, Andreas, Assist. f. Maschinenbaukunde an d. Techn. Hochsch. München: Graphodynamische Untersuchung einer vierzylindrigen Fahrzeugmaschine mit veränderlichem Hub. 〈Bauart Gill-Aveling, England $\rangle$. Berlin: Krayn 1912. 46 S. $4^{0}$ Aus: Motorwagen. Jg. 15. 16. 1912. 13.

München, Dr.-Ing.-Diss. v. 12. 12. 1911

2460. Heller, Eduard, Assist. f. Maschinenbaukunde an d. Techn. Hochsch. München: Ueber die Formgebung von Steuernocken. Berlin: Verl. f. Fachliteratur 1912. IV, 43 S. $4^{0}$ - Buchausg. 1913; aus: Der Oelmotor. Jg. 1. $1912 / 13$

München, Dr.-Ing.-Diss. v. 9. 2. 1912

2461. Eisner, Gustav: Dampfturbinen mit veränderlicher Tourenzahl. [1912.] 30 S. $4^{0}$

T Aus: Die Turbine. Jg. 9. 1912/13.

München, Dr.-Ing.-Diss. v. 23. 5. 1912
Elektrotechnik

2462. Schenk, Julius: Festigkeitsberechnung grösserer Drehstrommaschinen. Mit 45 Fig. im Text. u. auf 1 Doppeltaf. 1903. 59 S., 1 Taf. $8^{0}$ A Auch bei Teubner in Leipzig.

München, Dr.-Ing.-Diss. v. 5. 8. 1902

2463. Railing, Adolf: Uber Kommutierungsvorgänge und zusätzliche Bürstenverluste. 1903. 58 S. $8^{0}$ व Auch bei Enke in Stuttgart u. als : Sammlıng elektrotechn. Vorträge. Bd 4, H. 8 .

München, Dr.-Ing.-Diss. v. 4. 5. 1903

2464. Jakob, Max: Technisch-physikalische Untersuchungen von AluminiumElektrolyt-Zellen. Mit $32 \mathrm{Abb}$. u. 31 in d. Text gedr. Taf. 1906. IV, 131 S. $8^{\circ}$ Auch bei Enke in Stuttgart u. als: Sammlung elektrotechn. Vorträge. Bd 9 , H. $1 / 3$.

München, Dr.-Ing.-Diss. v. 30. 1. 1905

2465. Linsenmann, Hans, Assist. an d. Techn. Hochsch. München: Die elastische Linie der Gehäuse von Drehstrommaschinen mit grossen Durchmessern. 1906. 32 S. $8^{0}$ T Aus : Zeitschrift f. Mathematik u. Physik. Bd 53.

München, Dr.-Ing.-Diss. v. 18. 12. 1905

2466. Huldschiner, Gottfried: Über das Pendeln parallelgeschalteter Drehstromgeneratoren. 1906. 71 S. $8^{0}$ व Auch bei Enke in Stuttgart u. als: Sammlung elektrotechn. Vorträge. Bd 9, H. $7 / 8$.

München, Dr.-Ing.-Diss. v. 21. 3. 1906

2467. Waldmann, Karl: Beiträge zum Kommutierungsproblem. Mit 13 Figurenbl. Berlin: G. Schade 1907. 54 S., 13 Taf. $8^{0}$ München, Dr.-Ing.-Diss. v. 6. 8. 1906

2468. Schwaiger, A[nton]: Über das Regulierproblem in der Elektrotechnik. 1908. 98 S. $8^{0}$ I Um Vorw. u. Anh. verm. u. d. T.: Das Regulierproblem in der Elektrotechnik. Leipzig: Teubner 1909 .

München, Dr.-Ing.-Diss. v. 26. 7. 1907

2469. Steidle, Hans Carl, K. Oberpostass. i. München: Technische Grundlagen und wirtschaftliche Bedeutung des halbautomatischen Betriebes in Stadt- und Land-Fernsprech-Netzen. 〈Ein Beitrag zur Neuregelung des Telephongebühren-Tarifes. 1909. 70 S. $4^{0}$ A Auch bei Reinhardt in München; T. 2 auch in: Zeitschrift f. Schwachstromtechnik. Jg. 3. S. $67 \mathrm{ff}$.

München, Dr.-Ing.-Diss. v. 14. 12. 1908

2470. Hinlein, Erwin: Ein Beitrag zur Frage der Erwärmung der elektrischen Maschinen. 1909. 73 S. $4^{0}$ If Auch in: Mitteilungen über Forschungsarbeiten auf d. Gebiete d. 
Ingenieurwesens. H. 98/99. 1911; Ausz. in: Zeitschrift d. Vereines deutscher Ingenieure. $\mathrm{Bd} 55$. 1911, Halbj. 1.

München, Dr.-Ing.-Diss. v. 4. 8. 1909

2471. Dreyfus, Ludwig: Die Theorie des Drehstrom-Asynchronmotors in der einachsigen Schaltung und ihre experimentelle Nachprüfung. Berlin: Ernst 1909. 70 S., 3 Taf. $8^{0}$ - Buchausg. 1910.

München, Dr.-Ing.-Diss. v. 6. 8. 1909

2472. Keinath, Georg: Untersuchungen an Meßtransformatoren. 1909. 103 S. $8^{\circ}$ München, Dr.-Ing.-Diss. v. 6. 8. 1909

2473. Vetter, Theodor: Der Perioden-Umformer mit einphasig belastetem Sekundärstromkreise. 1909. 63 S., 10 Taf. $8^{0}$

München, Dr.-Ing.-Diss. v. 6. 8. 1909

2474. Hirschauer, Franz: Die Permeabilität des Fisens bei Magnetisierung durch technischer Wechselströme. 1910. $68 \mathrm{~S}$., 13 Taf. $8^{0}$ Inhaltsangabe des Verf. in: Elektrotechn. Zeitschrift. Jg. 32. 1911.

München, Dr.-Ing.-Diss. v. 17. 3. 1910

2475. Hommel, Gustav: Über das Verhalten des asynchronen Drehstrommotors bei unsymmetrischen Klemmenspannungen. 1910. 49 S., 8 Taf. $4^{0}$ - Auch bei Piloty \& Loehle in München.

München, Dr.-Ing.-Diss. v. 7. 7. 1910

2476. Binder, Ludwig: Úber äußere Wärmeleitung und Erwärmung elektrischer Maschinen. Halle: Knapp 1911.IV, 112 S. $8^{0}$ I Buchausg. u. d. T.: Über Wärmeübergang auf ruhige oder bewegte Luft sowie Lüftung und Kühlung elektrischer Maschinen.

München, Dr.-Ing.-Diss. v. 19. 7. 1910

2477. Wengner, Max: Theoretische und experimentelle Untersuchungen an der synchronen Einphasen-Maschine. 1910. VI, 88 S., 1 Taf. $8^{0}$ I Auch bei Oldenbourg in München.

München, Dr.-Ing.-Diss. v. 25. 7. 1910

2478. Hoerner, Karl: Über den Kraftlinienverlauf im Luftraum und in den Zähnen von Dynamoankern. Berlin: Springer [1910]. 37 S., 3 Taf. $8^{0}$ - Auch als Buch. München, Dr.-Ing.-Diss. v. 5. 8.1910

2479. Humburg, Karl: Das Pendeln bei Gleichstrommotoren mit Wendepolen. Berlin: Springer 1912. 81 S. $8^{0}$ ๆ Auch als Buch. München, Dr.-Ing.-Diss. v. 28. 11. 1911

2480. Hillebrand, Franz: Die Theorie des Drehstrom - Kollektor - Nebenschlußmotors mit getrennter Erreger- und Kompensationswicklung. Berlin: Springer 1912. $42 \mathrm{~S}$., 3 Taf. $4^{0}$ of Aus: Archiv f. Elektrotechnik. Bd 1. München, Dr.-Ing.-Diss. v. 5. 6. 1912
2481. Eismann, Alexander: Der Synchronmotor als Phasenzahl-Umformer. 1912. VIII, 55 S. $8^{\circ}$

München, Dr.-Ing.-Diss. v. 10. 8. 1912

2482. Stokvis, Louis Gustaaf: Der Spannungsabfall des synchronen DrehstromGenerators bei unsymmetrischer Belastung. 1912. VIII, 98 S. $8^{0}$ ๆ Auch bei Oldenbourg in München.

München, Dr.-Ing.-Diss. v. 10. 8. 1912

\section{CHEMISCHE ABTEILUNG}

2483. Hauser, Gottfried: Über die Elektrolyse des Estersalzes der Monobenzylmalonsäure sowie des dibenzylessigsauren Kaliums mit fettsauren Salzen. 1901. 51 S. $8^{0}$

München, Dr.-Ing.-Diss. v. 8. 7. 1901

2484. Purucker, Georg: Ueber dimolekulare Anilverbindungen des i-Valeraldehyds und einen Uebergang derselben in Derivate der Aethylenbasen von A. W. Hofmann. 1901. 47 S. $8^{0}$ i Vgl. Berichte d. Deutschen Chem. Gesellschaft. Jg. 33. 1900.

München, Dr.-Ing.-Diss. v. 8. 7. 1901

2485. Wimmer, Robert: Über $\alpha$-o-Anisidopropionsäure und Derivate derselben. [1901.] 34 S. $8^{0}$

München, Dr.-Ing.-Diss. v. 8. 7. 1901

2486. Bloch, Ignaz: Elektrolyse von Estersalzen ungesättigter und hydroxylierter Dicarbonsäuren mit Kaliumacetat. 1902. 55 S. $8^{0}$

München, Dr.-Ing.-Diss. v. 21. 12. 1901

2487. Flachslaender, Joseph: Ueber Nitroäthylbenzole und daraus hergestellte Tetrazofarbstoffe. Leipzig: J. A. Barth 1902. $24 \mathrm{~S} .8^{0}$ Aus : Journal f. prakt. Chemie. Bd 174. N. F. Bd 66 .

München, Dr.-Ing.-Diss. v. 21. 12. 1901

2488. Herrmann, Max: Ueber die Sulfurierung des m-Nitrotoluols. (1901.) 42 S. $8^{0}$ München, Dr.-Ing.-Diss. v. 21. 12. 1901

2489. König, Roderich: Über Cinchotintoxin und einige Derivate des Cinchotoxins. 1902. 40 S. $8^{0}$

München, Dr.-Ing.-Diss. v. 21. 12. 1901

2490. Klein, Sigmund: Ueber die elektrolytische Oxydation von Anilin und einigen aromatischen Diaminen in alkalischer Lö. sung. 1902. 51 S., 1 Tab. $8^{0}$

München, Dr.-Ing.-Diss. v. 8. 3. 1902

2491. Postius, K[arl] Theodor: Untersuchungen in der Yttergruppe. 1902. 29 S. $4^{0}$

München, Dr.-Ing.-Diss. v. 8. 3. 1902 
2492. Reitinger, Josef: Analytische Untersuchungen über die natürlichen Phosphate der Ceriterden und Yttererden sowie über Zirkon- und Titanmineralien. 1902. $60 \mathrm{~S} .8^{0}-\mathrm{Vgl}$. Zeitschrift f. Krystallographie u. Mineralogie. Bd 37. 1903.

München, Dr.-Ing.-Diss. v. 8. 3. 1902

2493. Staeble, Rupert: Ueber Benzochinon-Sulfosäure. 1902. 44 S. $8^{0}$ ศ Kurzer Ausz. in: Journal f. prakt. Chemie. Bd 177. N. F. Bd 69. 1904.

München, Dr.-Ing.-Diss. v. 8. 3. 1902

2494. Weiss, Ludwig: Ueber die Darstellung der Metalle der Cergruppe durch Schmelzelektrolyse. Mit 4 Taf. 1902. $47 \mathrm{~S}$., 4 Taf. $8^{0}$ T Auch in: J. Liebig's Annalen d. Chemie. Bd 320.

München, Dr.-Ing.-Diss. v. 8. 3. 1902

2495. Merkel, Heinrich: Über Bromprodukte und Alkalimetallverbindungen des Chinophtalons und ein Isomeres desselben. 1902. 50 S., 1 Taf. $8^{0}$ - Vgl. Berichte d. Deutschen Chem. Gesellschaft. Jg. 35.

München, Dr.-Ing.-Diss. v. 5. 8. 1902

2496. Erber, Josef, Assist. an d. Techn. Hochsch. München: Über Amidoalizarine. 1903. 49 S. $8^{0}$ - Vgl. Berichte d. Deutschen Chem. Gesellschaft. Jg. 35. 1902.

München, Dr.-Ing.-Diss. v. 19. 12. 1902

2497. Sehlötter, Max: Ueber die elektrolytische Oxydation von Alkoholen der Fettreihe. $1902.40 \mathrm{~S} .8^{0}$

München, Dr.-Ing.-Diss. v. 19. 12. 1902

2498. Eeker, Karl: Ueber die Elektrolyse organischer Salze. 1903. 68 S., 1 Taf. $8^{0}$

München, Dr.-Ing.-Diss. v. 16. 3. 1903

2499. Kraft, Karl: Untersuchungen über das Cer und das Lanthan. 1903. 34 S. $8^{\circ}$ I Vgl. J. Liebig's Annalen d. Chemie. Bd 325. 1902.

München, Dr.-Ing.-Diss. v. 16. 3. 1903

2500. Rhomberg, Victor: Beiträge zur Kenntnis des Benzylidenanilinnitrils. 1903. 41 S. $8^{0}$

München, Dr.-Ing.-Diss. v. 16. 3. 1903

2501. Sehumann, Ph[ilipp], Assist. an d. Techn. Hochsch. München: Beiträge zur Kenntniss der Schibutter. 1903. 35 S. $8^{0}$ München, Dr.-Ing.-Disss: v. 16. 3. 1903

2502. Sedlmayr, Theodor, Assist. an d. Techn. Hochsch. München: Beiträge zur Chemie der Hefe. 1903. 38 S. $8^{0}$ ๆ Ausz. in: Zeitschrift f. d. gesamte Brauwesen. Jg. 26. München, Dr.-Ing.-Diss. v. 16. 3. 1903

2503. Amann, Max: Zur Frage der Constitution des bimolekularen Propyliden- anilins. Ein Beitrag zur Frage der Existenz stereoisomerer Anilverbindungen. 1903. 43 S. $8^{0}$ I Vgl. J. Liebig's Annalen d. Chemie. Bd 329.

München, Dr.-Ing.-Diss. v. 10. 8. 1903

2504. Hofmann, Karl: Beiträge zur Frage der Konstitution des Chinophtalons und Isochinophtalons. 1903. $59 \mathrm{~S} .8^{0}$ ๆ Vgl. Berichte d. Deutschen Chem. Gesellsehaft. Jg. 37. 1904.

München, Dr.-Ing.-Diss. v. 10. 8. 1903

2505. Kraft, Hermann: Uber die Oxydation methylierter aromatischer Kohlenwasserstoffe mit Cerdioxyd. 1903. 44 S. $8^{0}$

München, Dr.-Ing.-Diss. v. 10. 8. 1903

2506. Mayr, Christian: Ueber die Elektrosynthese aliphatischer und aromatischer Ketoverbindungen. 1904. 51 S. $8^{0}$

München, Dr.-Ing.-Diss. v. 18. 12. 1903

2507. Bosch, Eberhard: Zur Kenntnis des Aethylbenzylanilins. 1904. 48 S. $8^{0}$

I Vgl. J. Liebig's Annalen d. Chemie. Bd 334. München, Dr.-Ing.-Diss. v. 21. 12. 1903

2508. Richard, Isidor, Assist. an d. Techn. Hochsch. München: Ueber die Einwirkung des Formaldehyds auf $\alpha$-Picolin. 1904. 50 s. $8^{0}$ If Vgl. Berichte d. Deutschen Chem. Gesellschaft. Jg. 37.

München, Dr.-Ing.-Diss. v. 21. 12. 1903

2509. Schnell, Josef: Zur Kenntnis der Bitterstoffe des Hoplesn 1904. 51 S. $8^{0}$ T Vgl. Zeitschrift f. d. gesamte Brauwesen. Jg. 27. München, Dr.-Ing.-Diss. v. 21. 12. 1903

2510. Würth, Karl: Untersuchung eines Ölgasteers. 1904. 95 S., 1 Taf. $8^{0}$ - Auch bei Mayer \& Müller in Berlin; vgl. Schilling's Journal f. Gasbeleuchtung. Jg. 48. 1905.

München, Dr.-Ing.-Diss. v. 21. 12.1903

2511. Zorn, Hans: Über Alkoholbildung bei der Elektrolyse fettsaurer Salze. 1904. 51 S. $8^{0}$

München, Dr.-Ing.-Diss. v. 21. 12. 1903

2512. Günther, Ludwig, gepr. Lehramtskand.: Über das farbenempfindliche Chlorsilber und Bromsilber. 1904. 71 S. 80 I Aus : Abhandlungen d. Naturhistor. Gesellschaft zu Nürnberg. Bd 15. 1905.

München, Dr.-Ing.-Diss. v. 3. 3. 1904

2513. Mühlbach, Ernst: Über die Elek trolyse von Cerosalzen. $1903.71 \mathrm{~S} .8^{0}$

München, Dr.-Ing.-Diss. v. 9. 3. 1904

2514. Aichel, Oswald: Die Reduktion von Metalloxyden mit Hilfe von Ceritmetallen. 1904. 41 S. $8^{0}$ I Ausz. in: J. Liebig's Annalen d. Chemie. Bd 337.

München, Dr.-Ing.-Diss. v. 22. 3. 1904 
2515. Fink, Wolfram: Der Flysch im Tegernseer Gebiet mit spezieller Berücksichtigung des Erdölvorkommens. 1904. $30 \mathrm{~S} ., 1 \mathrm{~K}$ t. $4^{0}$ - Aus: Geognost. Jahreshefte. Jg. 16.1903.

München, Dr.-Ing.-Diss. v. 22. 3. 1904

2516. Leher, Ernst: Über die quantitative Bestimmung des Arsens und Antimons als Schwefel-Verbindungen. 1904. 98 S. $8^{0}$

München, Dr.-Ing.-Diss. v. 22. 3. 1904

2517. Mühlhofer, Hans: Über die Einwirkung elektrolytisch erzeugter Halogene auf organische Verbindungen. 1905. 36 S. $8^{0}$

München, Dr.-Ing.-Diss. v. 22. 6. 1904

2518. Mathes, Rudolf: Über die elektrolytische Reduktion von Halogensubstitutionsprodukten der Benzolreihe. 1904.55S. $8^{0}$

München, Dr.-Ing.-Diss. v. 7. 7. 1904

2519. Bauer, Max: Über die Kondensation von Phtalsäureanhydrid mit Phenylmethylpyrazolon. 1905. 60 S., 1 Tab. $8^{0}$ - Vgl. Journal f. prakt. Chemie. Bd 195. N. F. Bd 87. 1913. S. $119 \mathrm{ff}$.

München, Dr.-Ing.-Diss. v. 10. 1. 1905

2520. Bub, Karl: Ueber die elektrolytische Oxydation von Leukobasen. 1905. 59 S. $8^{0}$

München, Dr.-Ing.-Diss. v. 10. 1. 1905

2521. Demeter, Adolf: Der Hochofenbetrieb in Amberg. 1905. 26 S. $8^{0}$

München, Dr.-Ing.-Diss. v. 10.1. 1905

2522. Fraunberger, Fritz: Ueber das elektromotorische Verhalten von Metallen. [1905.] IV, 40 S. $8^{0}$ Mit einigen Änderungen in: Sitzungsberichte d. math.-physik. Klasse d. K. B. Akademie d. Wissenschaften zu München. Bd 34. Jg. 1904.

München, Dr.-Ing.-Diss. v. 10. 1. 1905

2523. Geitz, August: Pyrogene Reaktionen in der Hochspannungsflamme. (Mit 2 Taf.) 1905. 63 S., 2 Taf. $8^{0}$

München, Dr.-Ing.-Diss. v. 10. 1. 1905

2524. Herold, Ignaz: Ueber die Kaustifikation des Kaliumsulfates. 1905. 44 S. $8^{0}$

- Aus: Zeitschrift f. Elektrochemie. Jg. 11.

München, Dr.-Ing.-Diss. v. 10. 1. 1905

2525. Scheidemandel, Julius: Ueber die Gewinnung der seltenen Erdmetalle durch Schmelzelektrolyse. (Mit 2 Taf.) 1905. 52 S., 2 Taf. $8^{0}$ Vgl. J. Liebig's Annalen d. Chemie. Bd 355. 1907.

München, Dr.-Ing.-Diss. v. 10. 1. 1905

2526. Vicari, Ferdinand: Ueber die Konstitution des o-Tolidins. 1905. 39 S. $8^{0}$
- Vgl. Berichte d. Deutschen Chem. Gesellschaft. Jg. 37. 1904.

München, Dr.-Ing.-Diss. v. 10. 1. 1905

2527. Herzog, Gustav: Über Umwandlungen des Hydrocyancarbodiphenylimids. 1905. 40 S. $8^{\circ}$ - Ausz. in: Journal f. prakt. Chemie. Bd 182. N. F. Bd 74. 1906.

München, Dr.-Ing.-Diss. v. 15. 3. 1905

2528. Müller, Bernhard: Untersuchungen über die quantitative Bestimmung einiger seltener Erden. [1905.] 40 S. $8^{0}$

München, Dr.-Ing.-Diss. v. 15. 3. 1905

2529. Hofer, Georg: Kondensation von Phenylanilidoacetonitril und Zimtaldehyd. 1905. 23 S. $8^{0}$

München, Dr.-Ing.-Diss. v. 4. 4. 1905

2530. Schwab, Georg: Beiträge zur Kenntnis des Cinchotoxins und Chinotoxins. [1905.] 32 S. $8^{0}$ Vgl. Berichte d. Deutschen Chem. Gesellschaft. Jg. 38 .

München, Dr.-Ing.-Diss. v. 4. 4. 1905

2531. Zirngibl, Emil: Zur Kenntnis der Einwirkung des Formaldehyds auf $\alpha$-Pikolin. 1905. 49 S. $8^{0}$ - Vgl. Berichte d. Deutschen Chem. Gesellschaft. Jg. 39. 1906.

München, Dr.-Ing.-Diss. v. 4. 4. 1905

2532. Herramhof, H[einrich]: Untersuchungen über Scharffeuerfarben für Hartporzellan und Untersuchung der Spektren einiger seltener Erden insbesondere der Reflexionsspektren ihrer Phosphate. 1905. 55 S., 1 Taf. $8^{0}$ - Vgl. J. Liebig's Annalen d. Chemie. Bd 355. 1907.

München, Dr.-Ing-Diss. v. 30. 7. 1905

2533. Flessa, Franz: Über die Konstitution des Dianilido- $\mu$-cyanhydrobenzoïn und homologe Verbindungen. 1905. $63 \mathrm{~S} .8^{0}$ - Vgl. Berichte d. Deutschen Chem. Gesellschaft. Jg. 43. 1910. S. 2275 ff.

München, Dr.-Ing.-Diss. v. 11. 8. 1905

2534. Schäfer, Ernst: Vergleichende Untersuchung über die Aufschliessung von arsen-, antimon- und schwefelhaltigen Erzen im Chlor- und Brom-〈Kohlensäure >-Strome zum Zwecke der quantitativen Analyse. Wiesbaden: Kreidel 1906. 34 S. $8^{0}$ ๆ Aus: Zeitschrift f. analyt. Chemie. Jg. 45.

München, Dr.-Ing.-Diss. v. 2. 12. 1905

2535. Hermann; Ludwig: Ueber die Trennung der Ytter- und Erbinerden. 1906. 71 S. $8^{0}$

München, Dr.-Ing.-Diss. v. 23. 12. 1905

2536. Ichenhäuser, Ernst: Über einige Disazofarbstoffe aus Phenol und Kresolen. [1905.] 40 S. $8^{0}$

München, Dr.-Ing.-Diss, v. 23. 12.1905 
2537. Regensburger, Paul: Vergleichende Untersuchungen an drei obergärigen Arten von Bierhefe. Jena: Fischer 1906. 60 S., 3 Taf. $8^{\circ}$ Auch in : Centralblatt f. Bakteriologie, Parasitenkunde u. Infektionskrankheiten. Abt. 2, $\mathrm{Bd} 16$.

München, Dr.-Ing.-Diss. v. 23. 12. 1905

2538. Metzger, Josef: Über das Calcium und seine Legierungen. [1906.] 41 S. $8^{0}$ - Ausz. in : J. Liebig's Annalen d. Chemie. Bd 355. 1907.

München, Dr.-Ing.-Diss. v. 12. 2. 1906

2539. Stuchlik, Heinrich, K. Bergmeister i. Traunstein: Die Faciesentwicklung der südbayerischen Oligocänmolasse. Mit 1 geolog. Kt., 2 Profiltaf. u. zahlreichen Textfig. 1906. 68 S., 3 Taf. $4^{0}$ T Auch in: Jahrbuch d. K. K. geolog. Reichsanstalt. Bd 56.

München, Dr.-Ing.-Diss. v. 12. 2. 1906

2540. Beck, Jakob, Oberlehrer an d. Allg. Deutschen Schule i. Antwerpen: Versuche über elektrolytische Oxydation und Reduktion. 1906. 71 S. $8^{0}$

München, Dr.-Ing.-Diss. v. 21. 3. 1906

2541. Mai, Alfred: Über die Darstellung von metallischem Molybdän. 1906. $40 \mathrm{~S} .8^{\circ}$ - Ausz. in: J. Liebig's Annalen d. Chemie. Bd 355. 1907.

München, Dr.-Ing.-Diss. v. 21. 3. 1906

2542. Mayr, Friedrich: Das Bessemern von Kupfersteinen. Freiberg i. S.: Craz \& Gerlach 1906. 40 S., 3 Taf. $4^{0}$ ' Auch als Buch.

München, Dr.-Ing.-Diss. v. 21. 3. 1906

2543. Röhm, Wilhelm: Untersuchung eines Petroleumgasteers. 1906. 55 S. $8^{0}$

München, Dr.-Ing.-Diss. v. 2. 4. 1906

2544. Teichner, Herbert: Über 1,2-Naphtochinon und Derivate desselben. 1906. 48 S. $8^{0}$ Vgl. Berichte d. Deutschen Chem. Gesellschaft. Jg. 38. 1905 .

München, Dr.-Ing.-Diss. v. 2. 5. 1906

2545. Heilbronner, Wilhelm: Über die Sulfurierung des o-Nitro-o-kresols. 1906. 40 S. $8^{0}$

München, Dr.-Ing.-Diss. v. 20. 5. 1906

2546. Kunst, Friedrich: Untersuchung höherer Fraktionen eines Petroleumgasteers. 1906. 57 S. $8^{0}$

München, Dr.-Ing.-Diss. v. 20. 5. 1906

2547. Hauenstein, Leonhard: Beiträge zur Kenntnis des Karbazols. Freiberg i. S.: Craz \& Gerlach 1907. 32 S. $8^{\circ}$ o Ausz. in: Journal f. prakt. Chemie. Bd 184. N. F. Bd 76 . München, Dr.-Ing.-Diss. v. 13. 6. 1906
2548. Weiss, Georg: Ueber die quantitative Bestimmung und Trennung von Zink und Nickel. 1906. 64 S. $8^{0}$

München, Dr.-Ing.-Diss. v. 13. 6. 1906

2549. Kalb, Otto: Ũber Die Nitrosierung von Acetyl-para-amidophenol und Diacetyldiamidophenol $1 \cdot 2 \cdot 4$. 1907 . $50 \mathrm{~S} .8^{0}$

München, Dr.-Ing.-Diss. v. 6. 8. 1906

2550. Laue, Otto: Zur Konstitution der gemischten Azoverbindungen. 1906. 67 S. $8^{0}$ T Vgl. Berichte d. Deutschen Chem. Gesellschaft. Jg. 39.

München, Dr.-Ing.-Diss. v. 6. 8. 1906

2551. Rennebaum, Fritz: Ueber das Verhalten von 3-Nitroparakresol zu Schwefelsäure. $1906.36 \mathrm{~S} .8^{0}$

München, Dr.-Ing.-Diss. v. 6. 8. 1906

2552. Rosenfeld, Joseph: Technische Untersuchungen über rumänisches Petroleum. 1909. 85 S., 2 Taf. $8^{0}$

München, Dr.-Ing.-Diss. v. 27. 11. 1906

2553. Antonar, Aldus: Zur Kenntnis der Chinaalkaloïde. 1906. $33 \mathrm{~S} .8^{0}$. Vgl. Berichte d. Deutschen Chem. Gesellschaft. Jg. 40. 1907.

München, Dr.-Ing.-Diss. v. 31. 12. 1906

2554. Arnoldi, Heinrich: Uber Metallcyanide. 1907. $53 \mathrm{~S} .8^{\circ}$ i Vgl. Berichte d. Deutschen Chem. Gesellschaft. Jg. 39. 1906.

München, Dr.-Ing.-Diss. v. 31. 12. 1906

2555. Beck, Hans: Beiträge zur Kenntnis der Metalle der Cergruppe. 1907. 59 S. $8^{0}$ - Vgl. J. Liebig's Annalen d. Chemie. Bd 331. 1904.

Yünchen, Dr.-Ing.-Diss. v. 31. 12. 1906

2556. Belschner, Gustav: Bestimmung der Stärke in Cerealien durch Polarisation. 1907. 36 S. $8^{0}$ o Vgl. Zeitschrift f. Untersuchung d. Nahrungs- u. Genußmittel, sowie d. Gebrauchsgegenstände. Bd 14. S. $205 \mathrm{ff}$.

München, Dr.-Ing.-Diss. v. 31. 12. 1906

2557. Bernhard, Rudolf: Úber einige zum Dianilido- $\mu$-cyanhydrobenzoïn in Beziehung stehende Verbindungen. 1906. 51 S., 2 Taf. $8^{0}$ - Vgl. Berichte d. Deutschen Chem, Gesellschaft. Jg. 43. 1910. S. 2276 ff.

München, Dr.-Ing.-Diss. v. 31. 12. 1906

2558. Kohlhaus, Wilhelm: Beiträge zur Kenntnis der Farbstoffe der Kongoreihe. 1907. 64 S. $8^{0}$

München, Dr.-Ing.-Diss. v. 31. 12. 1906

2559. Leibu, Josef: Über enzymatische Prozesse beim Weichen, Keimen und darauffolgenden Trocknen der Gerste, des Weizens und des Roggens. 1907. 62 S. $8^{\circ}$

München, Dr.-Ing.-Diss. v. 31. 12. 1906 
2560. Löbering, Max: Zur Konstitution des Chinonaphtalons und Beiträge zur Frage der Konstitution des Pyrophtalons. 1907. 63 S. $8^{0}$ Vgl. Berichte d. Deutschen Chem. Gesellschaft. Jg. 39. 1906.

München, Dr.-Ing.-Diss. v. 31. 12. 1906

2561. Mendheim, Hans: Untersuchungen über Gips. [1906.] 78 S. $8^{0}$

München, Dr.-Ing.-Diss. v. 31. 12.1906

2562. Riedelbauch, Rudolf: Untersuchungen über metallisches Vanadin, Niob und Tantal. 1907. 43 S. $8^{0}$ I Mit unwesentl. Anderungen in: J. Liebig's Annalen d. Chemie. $\mathrm{Bd} 355$.

München, Dr.-Ing.-Diss. v. 31. 12. 1906

2563. Wagner, Ludwig, gepr. Lehramtskand.: Krystallographisch-chemischeUntersuchung der Halogenide aliphatischer Ammoniumbasen. Leipzig: W. Engelmann 1907. 56 S. $8^{0}$ Aus : Zeitschrift f. Krystallographie u. Mineralogie. Bd 43.

München, Dr.-Ing.-Diss. v. 31.12. 1906

2564. Weiss, Ludwig: Beiträge zur Kenntnis der in Gerste und Malz vorkommenden Phosphorverbindungen. 1907. 63 S. $8^{0}$

München, Dr.-Ing.-Diss. v. 31. 12. 1906

2565. Schuster, Matthäus, Assist. an d. Geognost. Abt. d. Kgl. Oberbergamts zu München: Beiträge zur mikroskopischen Kenntnis der basischen Eruptivgesteine aus der bayerischen Rheinpfalz. 1907. $69 \mathrm{~S}$., 1 Kt. $4^{0}$ I Aus: Geognost. Jahreshefte. Jg. 19. 1906.

München, Dr.-Ing.-Diss. v. 21. 2. 1907

2566. Hiendlmayr, Adolf: Beiträge zur Chemie der Chrom- und Kobalt-Ammoniake. 1907. 36 S. $8^{0}$ - Vgl. Berichte d. Deutschen Chem. Gesellschaft. Jg. 37. 1904. 38. 1905.

München, Dr.-Ing.-Diss. v. 15. 3. 1907

2567. Lehrburger, Karl: Ueber die Sulfurierung des o-Nitro-m-Kresols. 1907. 34 S: $8^{\circ}$

München, Dr.-Ing.-Diss. v. 15. 3. 1907

2568. Mandelbaum, Robert: Ueber Calciumborat und über die Bestimmung der Borsäure. 1907. 50 S. $8^{0}$ - Ausz. in: Zeitschrift f. anorgan. Chemie. Bd 62. 1909.

München, Dr.-Ing.-Diss. v. 15. 3. 1907

2569. Röhler, Robert: Zur Konstitution der Chinophtalone. 1907. 61 S. $8^{0}$

München, Dr.-Ing.-Diss. v. 15. 3. 1907

2570. Baumann, Fritz: Die quantitative Bestimmung des Arsens als Arsentrisulfid. 1907. 68 S. $8^{0}$

München, Dr.-Ing.-Diss. v. 13. 6. 1907
2571. Gutmann, S[alomon]: Über die Einwirkung von Aminen auf die Chinonsulfosäure. [1907.] 31 S. $8^{0}$

München, Dr.-Ing.-Diss. v. 13. 6. 1907

2572. Scheller, Emil: Ueber die Kondensation von Propylenbromid mit Natracetessigester. 1907. 47 S. $8^{0}$

München, Dr.-Ing.-Diss. v. 13. 6. 1907

2573. Höfle, Jakob: Die Moore der bayerischen Hochebene als Folgeerscheinung der Eiszeit. 1909. 41 S., 3 Taf. $8^{0}$ München, Dr.-Ing.-Diss. v. 6. 8. 1907

2574. Martin, Alfred: Ueber die Darstellung von metallischem Wolfram. 1908, 62 S. $8^{0}$ - Vgl. Zeitschrift f. anorgan. Chemie. Bd 65. 1910.

München, Dr.-Ing.-Diss. v. 6.12. 1907

2575. Mitscherlich, Harbord: Ueber die Darstellung flüssiger und fester Chloride in der Wechselstrom-Hochspannungsflamme. 1908. 46 S., 7 Taf. $8^{0}$

München, Dr.-Ing.-Diss. v. 6. 12. 1907

2576. Schäffer, Adolf: Ueber die Einwirkung konzentrierter Schwefelsäure auf Chinolin bei höherer Temperatur und Gegenwart von Quecksilbersulfat. 1908. 31 S. $8^{0}$

München, Dr.-Ing.-Diss. v. 27. 12. 1907

2577. Übelhör, Fritz: Vergleichende Untersuchungen über die gewichtsanalytische Bestimmung des Kobalts. 1908. 23 S. $8^{0}$ München, Dr.-Ing.-Diss. v. 27. 12. 1907

2578. Herzfeld, Eugen: Beiträge zur Kenntnis des Pseudocumols. 1908. 42 S. $8^{\circ}$ - Vgl. Berichte d. Deutschen Chem. Gesellschaft. Jg. 42.1909.

München, Dr.-Ing.-Diss. v. 15. 1. 1908

2579. Höhn, Fritz: Untersuchungen über Wasserstoffpersulfid. 1909. 48 S. $8^{0}$ of Vgl. Berichte d. Deutschen Chem. Gesellschaft. Jg. 41. 1908.

München, Dr.-Ing.-Diss. v. 2. 3. 1808

2580. Schieder, Heinrich: Die quantitative Bestimmung des Antimons als Antimontrisulfid. 1908 . $45 \mathrm{~S} .8^{0}$

München Dr.-Ing.-Diss. v. 2. 3. 1908

2581. Dachs, Julius: Beiträge zur Kenntnis der Torulaceen. 1908. 66 S. $8^{0}$ - Vgl. Centralblatt f. Bakteriologie, Parasitenkunde u. Infektionskrankheiten. Abt. 2, Bd 21. S. $386 \mathrm{ff}$. München, Dr.-Ing.-Diss. v. 20. 3. 1908

2582. Wirth, Christian: Untersuchungen über die Bestimmung der diastatischen Kraft des Malzes und von Malzextrakten. 1908. 52 S. $8^{0}$ Ausz. in: Zeitschrift f. d. gesamte Brauwesen. Jg. 31.

München, Dr.-Ing.-Diss. v. 20. 3. 1908 
2583. Faehr, Paul: Ueber die Reduktion der Metalloxyde der Chromgruppe mittelst Wasserstoffs in der Hochspannungsflamme. 1908. 54 S., 3 Taf. $8^{0}$

München, Dr.-Ing.-Diss. v. 28. 3. 1008

2584. Jakob, Fritz: Uber die partielle elektrolytische Reduktion aromatischer Polynitrokörper bei Gegenwart von $\mathrm{Va}$ nadinsalzen. 1908. $85 \mathrm{~S}$, 1 Taf. $8^{0}$ ๆ Vgl. Berichte d. Deutschen Chem. Gesellschaft. Jg. 41 München, Dr.-Ing.-Diss. v. 27. 5. 1 C08

2585. Michel, Alfred: Versuche über Darstellung von Magnesium-Baryum-Legierungen durch Schmelzelektrolyse. 1908. $35 \mathrm{~S}$. $8^{0}$

München, Dr.-Ing.-Diss. v. 27. 5. 1c08

2586. Schwarz, Willy: Uber die Sulfonierung von p-Nitro-m-Kresol. 1908. 44 S. $8^{0}$

München, Dr.-Ing.-Diss. v. 27. 5. 1908

2587. Kohn, Franz: Photo-elektromotorische Untersuchungen über Mischungen von Ammonoxalat mit Merkurichlorid und Ferrichlorid. 1908. 67 S. $8^{0}$

München, Dr.-Ing.-Diss. v. 1. 7. 1؟08

2588. Beyschlag, Heinrich: Úber die Einwirkung von Schwefel auf Meta-Toluylendiamin. 1908. VIII, 71 S. $8^{0}$ I Vgl. Berichte d. Deutschen Chem. Gesellschaft. Jg. 42. 1909.

München, Dr.-Ing.-Diss. v. 29. 7. 1908

2589. Lehmann, Richard: Untersuchungen über Zirkonoxyd und seine Verwendung. 1908. 48 S. $8^{0}$ - Vgl. Zeitschrift f. anorgan. Chemie. Bd 65. 1910.

München, Dr.-Ing.-Diss. v. 29. 7. 1908

2590. Münichsdorier, Franz: Mineralogisch-petrographische Studien am Silberberg bei Bodenmais. 1908. $32 \mathrm{~S} .4^{0}$ T Aus: Geognost. Jahreshefte. Jg. 21.

München, Dr.-Ing.-Diss. v. 29. 7. 1908

2591. Strell, Martin, gepr. Lehramtskand.: Ueber die Fällungsbedingungen für Kupfer und Blei durch Schwefelwasserstoff aus neutralen und salzsauren Lösungen und die Anwendung der gewonnenen Ergebnisse zu einer quantitativen Trennung der beiden Metalle. 1908. 50 S. $8^{0}$

München, Dr.-Ing.-Diss. v. 29. 7. 1808

2592. Kaufmann, Ludwig: Ueber die Einwirkung von unterchloriger Säure auf Olefinphenole und Olefinphenoläther. 1908. 22 S. $8^{0}$

München, Dr.-Ing.-Diss. v. 14. 12. 1808

2593. Kuhn, Eugen: Ueber GheddaWachs 〈Ostindisches Wachs〉. 1909. 42 S. $8^{0}$ - Vgl. Journal f. prakt. Chemie. Bd 194 . N. F. Bd 86. 1912

München, Dr.-Ing.-Diss. v. 14. 12. 1908
2594. Schärtel, Georg, gepr. Lehramtskand.: Über einige Kondensationsprodukte aus Salicyliden- und Hydrocyansalicylidenanilin. 1908. $54 \mathrm{~S} .8^{0}$ I Vgl. Berichte d. Deutschen Chem. Gesellschaft. Jg. 43. 1910.

München, Dr.-Ing.-Diss. v. 14. 12. 1908

2595. Söllner, Fritz: Zur Kenntnis des Dimethylol $\alpha$ Pikolins. 1909. 34 S. $8^{0}$

München, Dr.-Ing.-Diss. v. 14. 12. 1908

2596. Herrle, Hanns: Ueber Elektrolysen mit Wechselstrom. 1909. 71 S. $8^{0}$

München, Dr.-Ing.-Diss. v. 22. 12. 1808

2597. Herrmann, Georg: Über die elektrolytische Darstellung von Chromosalzen. 1909. 83 S. $8^{0}$

München, Dr.-Ing.-Diss. v. 22. 12.1908

2598. Vestner, Hans: Über die quantitative Trennung des Zinks von Mangan, Eisen, Nickel und Kobalt durch Schwefelwasserstoff in schwach mineralsaurer Lösung. 1909. 84 S. $8^{0}$

München, Dr.-Ing.-Diss. v. 22. 12. 1908

2599. Schleicher, Alwin: Unterschiede in der Rostneigung einiger Eisenmaterialien. 1909. 41 S. $4^{0}$ T Ohne die Belegzahlen u. d. tabellarischen Auszug in: Metallurgie. Jg. 6.

München, Dr.-Ing.-Diss. v. 24. 12. 1908

2600. Stimmelmayr, Anton: Ueber die Darstellung und Untersuchung von regulinischem Wolframmetall. 1909. $41 \mathrm{~S} .8^{\circ}$ München, Dr.-Ing.-Diss. v. 22. 2. 1909

2601. Vervuert, Gottfried: Die quantitative Bestimmung des Zinns als Zinndioxyd. 1909. 45 S. $8^{0}$.

München, Dr.-Ing.-Diss. v. 22. 2. 1909

2602. Harster, Richard: Vergleichende Untersuchungen über die Ermittelung des Wirkungswertes einer salzsauren Zinnchlorürlösung unter Anwendung verschiedener Urprüfungssubstanzen. 1909. $75 \mathrm{~S} .8^{0}$ München, Dr.-Ing.-Diss. v. 28. 2. 1909

2603. Neumann, Eugen: Darstellung und Untersuchung regulinischen Zirkoniums. 1909. 42 S., 3 Taf. $8^{0}$ I Etwas gekürzt in: Zeitschrift f. anorgan. Chemie. Bd 65. 1910.

München, Dr.-Ing.-Diss. v. 28. 2. 1909

2604. Kaiser, Hans: Ueber metallisches Titan. 1909. 103 S. $8^{0}$ T Ausz. in: Zeitschrift f. anorgan. Chemie. Bd 65. 1910.

München, Dr.-Ing.-Diss. v. 1. 3. 1909

2605. Müller, Heinrich: Ueber ein Kondensationsprodukt aus Phtalsäureanhydrid und 1-Phenyl-3-methyl-5-Pyrazolon und Umwandlungen desselben. $1909.38 \mathrm{~S} .8^{0}$ o Vgl. Journal f. prakt. Chemie. Bd 195. N. F. Bd 87. 1913. S. 132 ff.

München, Dr.-Ing.-Diss. v. 1. 3. 1909 
2606. Engelhardt, Theodor: Über die Stickstoffverbindungen des Siliciums. 1909. 84 S., 2 Taf. $8^{0}$ - Etwas gekürzt in : Zeitschrift f. anorgan. Chemie. Bd 65. 1910.

München, Dr.-Ing.-Diss. v. 10. 3. 1909

2607. Frentzel, Alexander: Das Passauer Granitmassiv. Petrographisch-geologische Studie. 1911. S. 105-192, 2 Taf. $4^{0}$ 'Aus : Geognost. Jahreshefte. Jg. 24.

München, Dr.-Ing.-Diss. v. 10. 3. 1909

2608. Landecker, Max: Untersuchungen über die quantitative Bestimmung der Erdsäuren. 1909. 50 S. $8^{0}$ I Ausz. in: Zeitschrift f. anorgan. Chemie. Bd 64 .

München, Dr.-Ing.-Diss. v. 10. 3. 1909

2609. Surabekoff, Georg: Ueber die Einwirkung von Nitrobenzol auf Monoketone bei Gegenwart von Natriumalkylat. 1909. $38 \mathrm{~S}$. $8^{0}$

München, Dr.-Ing.-Diss. v. 10. 3. 1909

2610. Vlachos, Alkibiades: Ueber Kondensation des Formaldehyds mit Acetophenon. 1909. $43 \mathrm{~S} .8^{0}$

München, Dr.-Ing.-Diss. v. 10. 3. 1909

2611. Jwanowski, Waclaw: Ueber Bestimmungen des Wertes und des Preises der Brennereikartoffelschlempe. 1909. 43 S. $8^{0}$ - Ausz. in: Zeitschrift f. Spiritusindustrie. Jg. 43. N. F. Jg. 32; Referat des Verf. in: Journal f. Landwirtschaft. Jg. 57.

München, Dr.-Ing.-Diss. v. 26. 3. 1909

2612. Leberle, Hans: Beiträge zur Kenntnis der Gattung·Mycoderma. 1909. 106 S., 2 Taf. $8^{0}$ Ausz. in : Centralblatt f. Bakteriologie, Parasitenkunde u. Infektionskrankheiten. Abt. 2, Bd 28. 1910.

München, Dr.-Ing.-Diss. v. 26. 3, 1909

2613. Miller, Martin: Beiträge zur chemischen Kenntnis der Weizenmehle. [1909.] 37 S. $8^{0}$ - Auch in: Zeitschrift f. d. gesamte Getreidewesen. Jg. 1.

München, Dr.-Ing.-Diss. v. 5. 6. 1909

2614. Müller, Julius: Bromderivate des o-Amido- und o-Oxybenzaldehyds. 1909. 31 S. $8^{0}$ - Vgl. Berichte d. Deutschen Chem. Gesellschaft. Jg. 42 .

München, Dr.-Ing.-Diss. v. 5. 6. 1909

2615. Siller, Rudolf: Zur Chemie des Hopfens. Berlin: Springer 1909. 31 S. $8^{\circ}$ - Aus: Zeitschrift f. Untersuchung d. Nahrungsu. Genußmittel. Bd 18.

München, Dr.-Ing.-Diss. v. 5. 6. 1909

2616. Feistmann, Fritz: Über Erythrodextrin II $\alpha$ und Amylose. 1909. 37 S. $8^{0}$ München, Dr.-Ing.-Diss. v. 1. 7. 1909
2617. Perl, Alfred: Ueber einige im Steinkohlenteer neuentdeckte Kohlenwasserstoffe. 1909. 50 S. $8^{0}$ - Vgl. Berichte d. Deutschen Chem. Gesellschaft. Jg. 42.

München, Dr.-Ing.-Diss. v. 8. 7. 1909

2618. Frei, August, Kgl. Reallehrer i. Bamberg: Untersuchungen über die Bestandteile der Haferkörner unter dem Einfluss verschiedener Witterungs- und Anbauverhältnisse. 1910. 150 S., 11 Taf. $8^{0}$ ๆ Aus : Die landw. Versuchs-Stationen. Bd 72.

München, Dr.-Ing.-Diss. v. 4. 8. 1909

2619. Kayser, Eberhard: Ueber die neutralen Kohlenwasserstoffe des Imprägnieröls. 1909. 70 S., 1 Taf. $8^{0}$

München, Dr.-Ing.-Diss. v. 4. 8. 1909

2620. Bartz, Rudolf: Über die Bromierung des Cinchotoxins. 1909. 47 S., 1 Taf. $8^{0}$

München, Dr.-Ing.-Diss. v. 6. 8. 1909

2621. Elia, Alceo D': Ueber Nitro- und Amidoderivate des Chinolins. 1909. 27 S. $8^{0}$ München, Dr.-Ing.-Diss. v. 6. 8. 1909

2622. Pabstmann, Georg: Ueber die quantitative Bestimmung der Phosphorsäure als Magnesiumpyrophosphat. [1909.] 74 S. $8^{0}$

München, Dr.-Ing.-Diss. v. 6. 8. 1909

2623. Rothschild, Max: Úber Synthesen ringförmiger Verbindungen mittelst Dipropylmalonylchlorid und Succinylchlorid. 1909. 44 S. $8^{0}$

München, Dr.-Ing.-Diss. v. 6. 8. 1909

2624. Sander, Alexander: Über das Äthylbenzol und seine Nitroderivate. 1909. $22 \mathrm{~S}$. $8^{0}$ Tgl. Berichte d. Deutschen Chem. Gesellschaft. Jg. 42.

München, Dr.-Ing.-Diss. v. 6. 8. 1909

2625. Treubert, Franz, Adjunkt d. landw. Zentralversuchsstation and. Techn. Hochsch. München: Ueber Wismutoxydul und seine Salze. Mit einem Anh.: Neue quantitative Methoden. 1909. 71 S. $8^{0}$ - Vgl. Berichte d. Deutschen Chem. Gesellschaft. Jg. 31. 1898.

München, Dr.-Ing.-Diss. v. 6. 8. 1909

2626. Franz, Friedrich: Beiträge zum Nachweis und zur Kenntnis des Erdnußöles. 1910. 47 S. $8^{0}$

München, Dr.-Ing.-Diss. v. 20. 12. 1909

2627. Geys, Karl: Beiträge zur chemischen Kenntnis der Gerstenspelzen. 1910. 40 S. $8^{0}$ Ausz. in: Zeitschrift f. d. gesamte Brauwesen. Jg. 33.

München, Dr.-Ing.-Diss. v. 20. 12. 1909 
2628. Reidenbach, Rudolf: Ueber die quantitative Bestimmung des Magnesiums als Magnesiumpyrophosphat nebst einem Anhang: Ueber die Bestimmung des Magnesiums als Magnesiumsulfat. 1910. 109 S. $8^{0}$

München, Dr.-Ing.-Diss. v. 20. 12. 1909

2629. Schenk, Eduard, gepr. Lehramtskand.: Über die Reduktion von Pinen zu Pinan, Eigenschaften und chemisches Verhalten des letzteren. 1910. 44 S. $8^{0}$

München, Dr.-Ing.-Diss. v. 20. 12. 1909

2630. Dorfmuiller, Gustav: Ueber die Constitution des B.Bromkarmins. 1910. 30 S. $8^{0}$ Vgl. Berichte d. Deutschen Chem. Gesellschaft. Jg. 43 .

München, Dr.-Ing.-Diss. v. 30. 12. 1909

2631. Kohl, Julius Philipp: Ueber die elektrolytische Reduktion von Titansalzen. Deren Anwendung als Ueberträger bei der elektrolytischen Reduktion organischer Körper. 1910. 81 S. $8^{0}$

München, Dr.-Ing.-Diss. v. 30. 12. 1909

2632. Schertel, Wilhelm: Über die Einwirkung von rauchender Schwefelsäure auf Chinolin bei Wasserbadtemperatur und Gegenwart von Quecksilbersulfat. 1910. 39 S. $8^{\circ}$

München, Dr.-Ing.-Diss. v. 30. 12. 1979

2633. Löw, 0skar: Studien über das Verhalten des 3-Nitro-p-Kresols zu Schwefelsäure nebst einem Anhange: Über das Verhalten der 3-Nitro-p-Oxybenzoesäure zu Schwefelsäure. 1910. 46 S. $8^{0}$ - Vgl. Berichte d. Deutschen Chem. Gesellschaft. Jg. 42 . 43. 1909. 10.

München, Dr.-Ing.-Diss. v. 22. 1. 1910

2634. Vetter, Heinrich: Beiträge zur Kenntnis der Chinhydrone und Phenochinone. 1910. 58 S. $8^{0}$

München, Dr.-Ing.-Diss. v. 1. 2. 1910

2635. Diekert, Eugen, gepr. Lehramtskand.: Untersuchungen über die quantitative Bestimmung des Antimons als Trisulfid und als Tetroxyd. 1910. 76 S. $8^{0}$

München, Dr.-Ing.-Diss. v. 2. 3. 1910

2636. Freitag, Max: Ueber die quantitative Bestimmung des Arsens als Trisulfid und Pentasulfid. 1910. 124 S. $8^{0}$

München, Dr.-Ing.-Diss. v. 2. 3. 1910

2637. Geiger, Arthur: Beiträge zur Kenntnis der Sprosspilze ohne Sporenbildung. Mit 5 Fig. im Text, 1 Taf. u. 14 Tab. im Anh. Jena: Fischer 1910. 53 S., 1 Taf. $8^{0}$ - Aus: Centralblatt f. Bakteriologie, Parasitenkunde u. Infektionskrankheiten. Abt. 2, Bd 27. München, Dr.-Ing.-Diss. v. 2. 3. 1910
2638. Metzger, Karl, gepr. Lehramtskand.: Über Zirkon- und Wolframlegierungen. 1910. 55 S. $8^{0}$

München, Dr.-Ing.-Diss. v. 2. 3. 1910

2639. Hartmann, August: Zirkonemail. 1910. 58 S. $8^{0}$ - Ausz. in: Keram. Rundschau. Jg. 19. 1911. S. $118 \mathrm{ff}$.

München, Dr.-Ing.-Diss. v. 15. 3. 1910

2640. Lay, Emil: Über Silicium-Stickstoff-Wasserstoff-Verbindungen. 1910.79S. $8^{0}$

München, Dr.-Ing.-Diss. v. 15. 3. 1910

2641. Reich, Jakob: Elektrolytische Reduktion von Nitrokörpern mit Molybdän als Wasserstoffüberträger. 1910. 74 S., 1 Taf. $8^{0}$

München, Dr.-Ing.-Diss. v. 15. 3. 1910

2642. Lahrmann, 0tto: Über Magnesiumoxychlorid und seine Beziehung zu Sorels Magnesiazement. 1910. 29 S. $8^{0}$

München, Dr.-Ing.-Diss. v. 1. 7. 1910

2643. Weckbach, Franz: Über die elektrolytische Oxydation von Manganosalzen in stark saurer Lösung. 1910. $89 \mathrm{~S} .8^{0}$

München, Dr.-Ing.-Diss. v. 1. 7. 1910

2644. Loewenstein, Ernst: Über die elektrolytische Reduktion aromatischer Mononitrokörper bei Gegenwart von Vanadinsalzen. 1910. 84 S. $8^{0}$

München, Dr.-Ing.-Diss. v. 19. 7. 1910

2645. Waldmann, Anton: Elektrolytische Reduktionsversuche von Kaliumferricyanid mittelst Wechselstrom. 1910. 72 S. $8^{0}$

München, Dr.-Ing.-Diss. v. 19. 7. 1910

2646. Kohn, Rudolf: Ueber einen Anthracenrückstand. 1910. 44 S. $8^{0}$

München, Dr.-Ing.-Diss. v. 6. 8. 1910

2647. Yowak, Alfred, Ingenieur: Ueber die chemische Wirkung dunkler elektrischer Entladungen auf Kohlenoxyd und kohlenoxydhaltige Gasgemenge. 1910. 68 S. $8^{\circ}$

München, Dr.-Ing.-Diss. v. 6. 8. 1910

2648. Szèkely, Alexander: Untersuchung der rohen Mesitylensulfosäure und Auffindung des Isopropylbenzols im Steinkohlenteer. 1910. 47 S. $8^{0}$ o Vgl. Berichte d. Deutschen Chem. Gesellschaft. Jg. 43.

München, Dr.-Ing.-Diss. v. 6. 8. 1910

2649. Wetsch, Karl: Über galvanostegische Schwarzbadniederschläge und das kathodische Verhalten von Rhodansalzen. 1910. 81 S. $8^{0}$

München, Dr.-Ing.-Diss. v. 6. 8. 1910

2650. Frangopol, Dumitru: Zur Kenntnis der Naphtensäuren des rumänischen Erdöls. 1910. 84 S. $8^{0}$

München, Dr.-Ing.-Diss. v. 18. 11. 1910 
2651. Heydenreich, 'Karl: Photo-elektromotorische Untersuchungen von Chlorsilber und Bromsilber. 1911. 108 S., 4 Taf. $8^{0}$

München, Dr.-Ing.-Diss. v. 18. 11. 1910

2652. Lifschütz, Alexander: Studien über die Bildung der isomeren Nitrokörper bei dem Nitrierungsprozesse der monosubstituierten Benzole. 1910. 52 S. $8^{0}$

München, Dr.-Ing.-Diss. v. 24. 11. 1910

2653. Schaidhauf, Alois: Untersuchungen über Natriumamid nebst einem Anhang über quantitative Bestimmung der Salpetersäure. [1911.] $68 \mathrm{~S} .8^{0}$

München, Dr.-Ing.-Diss. v. 24. 11. 1910

2654. Bussjäger, Hermann: Über den Oxaethylacetessigester $\langle\gamma-O x y-\alpha$-acetylbuttersäureester $>$ und seine Spaltungsprodukte. 1910. 49 S. $8^{0}$

München, Dr.-Ing.-Diss. v. 30. 11. 1910

2655. Stumpi, Carl: Ueber die Elektrolyse der Estersalze der $\Delta_{2,6}$ Dihydro-sowie der $\Delta_{2}$ Tetrahydro-Phtalsäure mit fettsauren Salzen. 1910. 62 S. $8^{0}$.

München, Dr.-Ing.-Diss. v. 30.11. 1910

2656. Weinstein, Jancu: Zur Kenntnis der Koagulationsverhältnisse der löslichen Eiweißstoffe des Malzes und der Einwirkung der proteolytischen Enzyme auf das koagulierbare Eiweiss. 1911. 68 S. $8^{0}$ ๆ Ausz. in: Zeitschrift f. d. gesamte Brauwesen. Jg. 34.

München, Dr.-Ing.-Diss. v. 9. 12. 1910

2657. Zehetmaier, Heinrich: Über die Einwirkung von Stickstoffdioxyd auf kristallwasserhaltige Salze. 1911. 48 S. $8^{0}$ München, Dr.-Ing.-Diss. v. 9. 12. 1910

2658. Gatterbauer, Josef: Zur Kenntnis des sogenannten Gallisins im technischen Stärkezucker. Berlin: Springer 1911. 28 S. $8^{0}$ T Aus: Zeitschrift f. Untersuchung d. Nahrungsu. Genußmittel. Bd 22.

München, Dr.-Ing.-Diss. v. 21. 12. 1910

2659. Kunz, Eduard: I. Untersuchungen über die Hydrolyse der Stärke durch FlussSäure. II. Über Pentosane u. die sog. Furfuroïde. 1910. 56 S. $8^{0}$

München, Dr.-Ing.-Diss. v. 21. 12. 1910

2660. Lederer, Wilhelm: Darstellung und Untersuchung reinen, geschmolzenen Molybdäns. 1911. $43 \mathrm{~S} .8^{\circ}$

München, Dr.-Ing.-Diss. v. 23. 12. 1910

2661. Luftschitz, Heinrich: Synthesen und Umwandlungsprodukte in der Gruppe des Phenylmethylpyrazolons. 1910. $28 \mathrm{~S}$, 1 Taf. $8^{0}$

München, Dr.-Ing.-Diss. v. 23. 12. 1910
2662. Redlich, Béla: Beiträge zur Kenntnis von para-Aethyltoluol. 1911. $36 \mathrm{~S} .8^{0}$

München, Dr.-Ing.-Diss. v. 23. 12. 1910

2663. Reindel, Robert, gepr. Lehramtskand.: Über die quantitative Bestimmung des Wismuts mit einem Anhang: Úber die Umwandlung des Bleichlorids in das Sulfat. 1911. $60 \mathrm{~S}$. $8^{0}$

München, Dr.-Ing.-Diss. v. 23. 12. 1910

2664. Schuh, Heinrich: Beiträge zur Kenntnis der Unterphosphorsäure. 1911. 57 S., 1 Taf. $8^{0}$

München, Dr.-Ing.-Diss. v. 23. 12.1910

2665. Winckler, Curt oscar: Über die Einwirkung der salpetrigen Säure auf einige primäre aliphatische Amine. 1911. 69 S. $8^{0}$

München, Dr.-Ing.-Diss. v. 23. 12. 1910

2666. Althen, Albert: Versuche zur Herstellung von metallischem Beryllium. 1911. 32 S. $8^{0}$

München, Dr.-Ing.-Diss. v. 21. 1. 1911

2667. Scharrer, Andreas: Über das Verhalten salzsaurer Antimon- und Zinnlösungen gegen Schwefelwasserstoff und die quantitative Trennung der beiden Metalle. 1911. 104 S. $8^{0}$

München, Dr.-Ing.-Diss. v. 21. 1. 1911

2668. Ehrengut, Leopold: Ueber Metoxazine aus den drei isomeren Hydrocyansalicylidentoluidinen bzw. deren Komponenten. 1911. $44 \mathrm{~S} .8^{0}$

München, Dr.-Ing.-Diss. v. 7. 2. 1911

2669. Ott, Ludwig, gepr. Lehramtskand. : Elektrolyse geschmolzener Molybdate und Vanadate. 1911. 48 S., 1 Taf. $8^{0}$

München, Dr.-Ing.-Diss. v. 10. 2. 1911

2670. 0tt, Friedrich: Elektrolytische Reduktion der Niobsäure. 1911. V, 73 S. $8^{0}$ T Ausz. in: Zeitschrift f. Elektrochemie. Jg. 18. 1912.

München, Dr.-Ing.-Diss. v. 18. 3. 1911

2671. Picard, Martin: Ueber Nachweis und Bestimmung kleiner Mengen Kohlenoxyd. 1911. 69 S. $8^{0}$

München, Dr.-Ing.-Diss. v. 18. 3. 1911

2672. Raeithel, Oskar: Uber die Elektrolyse von Estersalzen mehrbasischer, ungesättigter Säuren. 1911. 43 S. 80

München, Dr.-Ing.-Diss. v. 17. 5. 1911

2673. Sgalitzer, Friedrich: Über Hydrazinverbindungen des Isatins und deren Reduktion. 1911. 55 S. $8^{0}$

München, Dr.-Ing.-Diss. v. 17. 5. 1911 
2674. Bub, Friedrich: Ueber die Kondensation von Acenaphtenchinon mit aromatischen Hydrazinsulfosäuren. 1911. 63 S. $8^{0}$

München, Dr.-Ing.-Diss. v. 13. 6. 1911

2675. Schimon, 0tto: Beiträge zur Kenntnis rot gefärbter niederer Pilze. 1911. $127 \mathrm{~S}$., 2 Taf. $8^{0}$ Vgl. Centralblatt f. Bakteriologie, Parasitenkunde u. Infektionskrankheiten. Abt. 2, Bd 35. S. $81 \mathrm{ff}$.

München, Dr.-Ing.-Diss. v. 16. 6. 1911

2676. Schmiedel, Maximilian: Über das as-m- ̈̈thylxylol $\langle 1,3,4\rangle$ und über das 0 Äthylxylol $\langle 1,2,4\rangle$ mit besonderer Berücksichtigung der Sandmeyerschen und der Fittigschen Synthese. 1911. 99 S. $8^{0}$

München, Dr.-Ing.-Diss. v. 16. 6. 1911

2677. Führer, Eugen: Ueber das Normalpropylbenzol aus Steinkohlenteer. 1911. 58 S. $8^{0}$

München, Dr.-Ing.-Diss. v. 20. 6. 1911

2678. Koopmann, Johann: Beiträge zur Kenntnis der Amidophenolsulfonsäuren IV und I. 1911. 33 S. $8^{0}$

München, Dr.-Ing.-Diss. v. 20. 6. 1911

2679. Kugel, Egolf : Beiträge zur Kenntnis der Amidophenolsulfosäuren III und II. 1911. 34 S. $8^{0}$

München, Dr.-Ing.-Diss. v. 20. 6. 1911

2680. Saigger, Eugen: Herstellung des Brom- bezw. Jodxylols aus para-Xylidin, sowie des Aethylxylols und seiner Derivate. [1911.] 48 S. $8^{\circ}$

München, Dr.-Ing.-Diss. v. 20. 6. 1911

2681. Bajai, Ernst: Über Hexahydroparatoluidin. 1911. $53 \mathrm{~S} .8^{0}$

München, Dr.-Ing.-Diss. v. 30.6.1911

2682. Pohl, Theodor: Über die Elektrolyse der Esterkaliumsalze der $\Delta^{1}$-Tetrahydroterephtalsäure mit Kaliumazetat. 1911. 85 S. $8^{0}$

München, Dr.-Ing.-Diss. v, 30. 6.1911

2683. Reiss, Ludwig: Über die Bromierung des Chinotoxins. 1911. 43 S., 1 Taf. $8^{0}$

München, Dr.-Ing.-Diss. v. 30.6. 1911

2684. Schweisgut, Ferdinand: Über Kupfersalzhydroxylaminverbindungen. 1911.52S. $8^{0}$

München, Dr.-Ing.-Diss. v. 30. 6. 1911

2685. Huber, Eugen: Über die partielle elektrolytische Reduktion aromatischer Polynitrokörper bei Gegenwart von Vanadinsalzen. [1911.] $53 \mathrm{~S}$. $8^{0}$

München, Dr.-Ing.-Diss. v. 8. 7. 1911

2686. Wempe, Georg: Beiträge zur Kenntnis der Molybdate. 1911. 76 S. $8^{0}$ T Ausz. in: Zeitschrift f. anorgan. Chemie. Bd 78. 1912.

München, Dr.-Ing.-Diss. v. 8. 7, 1911
2687. Lehmann, Eugen: Utber die indifferenten Kohlenwasserstoffe des Anthracenöles. 1911. 65 S. $8^{0}$

München, Dr.-Ing.-Diss. v. 10. 7. 1911

2688. Flor, Konrad: DasVanadin als elektrolytischer Sauerstoffüberträger. 1911. 89 S. $8^{0}$

München, Dr.-Ing.-Diss. v. 13. 7.1911

2689. Peissakowitsch, Victor: Untersuchungen über anodische Metallfärbungen in ammoniakalischen Bädern. 1911. 55 S. $8^{0}$ of Ohne die Einleitung auch in: Elektrochem. Zeitschrift. Jg. 18. 19. 1911/12. 12/13.

München, Dr.-Ing.-Diss. v. 13. 7. 1911

2690. Fürst, Bernhard: Über diè Einwirkung der schwefligen Säure auf aromatische Hydroxylamine. 1912. $29 \mathrm{~S} .8^{0}$

München, Dr.-Ing.-Diss. v. 28. 7. 1911

2691. Groebl, Anton: Über die Salpetersäurebestimmung nach Pelouze-Fresenius. 1911. 52 S. $8^{\circ}$

München, Dr.-Ing.-Diss. v. 28. 7. 1911

2692. Kretzer, Heinrich, Assist.: Beiträge zur Petrographie der Oberpfalz: Das Gebiet zwischen Weiden und VohenstrauB. 1912. 45 S., 1 Kt., 1 Taf. $8^{0}$ - Aus: Berichte d. naturwiss. 〈früher zoolog.-mineralog.) Vereines zu Regensburg. H. 13. f. d. J. 1910 u. 1911.

München, Dr.-Ing.-Diss. v. 28. 7. 1911

2693. Kritzenthaler, Fritz: Ưber die elektrolytische Oxydation von Kohlenwasserstoffen der Benzolreihe bei Gegenwart von Mangansulfat als Sauerstoffüberträger. 1911. 65 S. $8^{0}$

München, Dr.-Ing.-Diss. v. 28. 7. 1911

2694. Meyer, Hans: Über ein Nebenprodukt der Oxydation von -m-Xylol mit verdünnter Salpetersäure nebst einem Beitrag zur Kenntnis von Toluylnitromethan. [1911.] 67 S. $8^{0}$

München, Dr.-Ing.-Diss. v. 28. 7. 1911

2695. Minnich, Carl: Die Fortschritte der Goldaufbereitung und ihre Beziehung zur Lagerstättenlehre. 1911. 34 S. $4^{0}$ I Auch in: Zeitschrift f. prakt. Geologie. Jg. 19.

München, Dr.-Ing.-Diss. v. 5. 8. 1911

2696. Niklas, Hans, Assist. an d. chem.bodenkundl. Abt. d. Kgl.-Forstl. Versuchsanst.: Untersuchungen über den Einfluss von Humusstoffen auf die Verwitterung der Silikate. Berlin: Verl. f. Fachliteratur 1912. 31 S. $8^{0}$ Aus: Internat. Mitteilungen f. Bodenkunde. Bd 1.

München, Dr.-Ing.-Diss. v. 5. 8. 1911

2697. Rück, Ulrich: Úber die Darstellung von reinem, wasserfreiem Rhodanwasserstoff. 1911. 70 S., 3 Taf. $8^{0}$ - Ausz. in: Zeitschrift f. anorgan. Chemie. Bd 77. 1912.

München, Dr-Ing.-Diss. v. 5. 8. 1911 
2698. Sasinowski, Michael von: Über die Elektrolyse der Estersalze einiger aliphatischer Polycarbonsäuren. 1911. $95 \mathrm{~S} .8^{0}$

München, Dr.-Ing.-Diss. v. 9. 8. 1911

2699. Siegler, Max: Ueber Dimethylund Diaethylanilin-Meta-Sulfosäuren. [1912]. 48 S. $8^{\circ}$

München, Dr.-Ing.-Diss. v. 9. 8. 1911

2700. Teltscher, Friedrich: Über das elektrometrische Verhalten von Quecksilbersalzlösungen. 1912. 68 S., 4 Taf. $8^{0}$

München, Dr.-Ing.-Diss. v. 21. 11. 1911

2701. Kieninger, Max: Ueber Umwandlungen des Isosafroldibromids und Derivate desselben. 1911. $53 \mathrm{~S} .8^{0}$

München, Dr.-Ing.-Diss. v. 2. 12. 1911

2702. Sandlar, Emil: Über die Darstellung von Cersilicium. 1912. 54 S. $8^{0}$

München, Dr.-Ing.-Diss. v. 2. 12. 1911

2703. Adler, Ludwig: Über organische und anorganisch gebundene Phosphorsäure im Bier und ihre Beziehung zu Gerste und Malz. 1912. $58 \mathrm{~S} .8^{\circ}$ ๆ Mit Änderungen in: Zeitschrift f. d. gesamte Brauwesen. Jg. 35.

München, Dr.-Ing.-Diss. v. 11. 12. 1911

2704. Hilpoltsteiner, Johann: Beiträge zur Kenntnis der Enzyme des Roggenmalzes. 1912. 50 S. $8^{0}$

München, Dr.-Ing.-Diss. v. 11. 12. 1911

2705. László, Alexander: Kondensation von Phtalimid mit Phenylmethylpyrazolon. 1911. 54 S., 1 Taf. $8^{0}$

München, Dr.-Ing.-Diss. v. 12. 12. 1911

2706. Lechner, Arnulf: Über die Nitrierung des Chinolins und die Herstellung von Chinolythydroxylaminen. 1912. $48 \mathrm{~S} .8^{\circ}$

München, Dr.-Ing.-Diss. v. 15. 12. 1911

2707. Fischer, Hans: Elektrolytische Reduktion von Azoxy-, Azo-, Nitrosobenzol und Phenylhydroxylamin bei Gegenwart von Vanadinsalzen als Wasserstoffüberträger. 1912. 62 S. $8^{0}$

München, Dr.-Ing.-Diss. v. 6. 2. 1912

2708. Gillitzer, Georg, Berg- u. Salinenprakt.: Der geologische Aufbau des Reiteralpgebirges im Berchtesgadener Land. 1912. S. $161-227,4$ Taf. $4^{0}$ I Aus: Geognost. Jahreshefte. Jg. 25.

München, Dr.-Ing.-Diss. v. 24. 2. 1912

2709. Steinberger, Daniel: Beitrag zur Kenntnis des Berberins. 1912. 52 S. $8^{0}$ I Vgl. J. Liebig's Annalen d. Chemie. Bd 397. 1913.

München, Dr.-Ing.-Diss. v. 24. 2. 1912

2710. Schmidt, Otto: Über Cumylindigo und Cumylisatin. 1912. 71 S. $8^{0}$

München, Dr.-Ing.-Diss. v. 1. 3. 1912
2711. Schoeller, Viktor: Über Vergilben von Papier. 1912. 42 S. $8^{0}$

München, Dr.-Ing.-Diss. v. 1. 3. 1912

2712. Beyersdorfer, Paul: Beiträge zur Analyse der Kohlehydrate des Bierextraktes. 1912. $63 \mathrm{~S} .8^{0}$ I Vgl. Zeitschrift f. d. gesamte Brauwesen. Jg. 35 . S. $556 \mathrm{ff}$.

München, Dr.-Ing.-Diss. v. 14. 3. 1912

2713. Burger, Eugen: Über die Einwirkung von Monomethylresorcin auf Phtalsäureanhydrid. 1912 . 30 S. $8^{0}$

München, Dr.-Ing.-Diss. v. 14. 3. 1912

2714. Dreyer, Gustav: Beiträge zur Chemie der Hefe. 1912. 31 S. $8^{0}$ ๆ Ausz. in: Zeitschrift f. d. gesamte Brauwesen. N. F. Jg. 36. 1913.

München, Dr.-Ing.-Diss. v. 14. 3. 1912

2715. Kováes, Eugen: Über die Säuren des Gheddawachses. 1912. 33 S. $8^{0}$

München, Dr.-Ing.-Diss. v. 21. 3. 1912

2716. Gerstacker, Ludwig: Ueber Eisencyanide. 1912. 87 S. $8^{0}$ - Vgl. Farben-Zeitung. Jg. 17. 1911/12, Hälfte 2. S. 2272 ff.; ChemikerZeitung. Jg. 37. 1913. S. $137 \mathrm{ff}$.

München, Dr.-Ing.-Diss. v. 22. 3. 1912

2717. Moser, Wilhelm: Ueber die Einwirkung von salpetriger Säure auf $\beta$ Phenyläthylamin und n-Hexylamin. 1912. 70 S. $8^{\circ}$

München, Dr.-Ing.-Diss. v. 22. 3. 1912

2718. Muggenthaler, Hans: Ueber die Bestimmung der Hexabromidzahlen des Leinöles und einiger anderer fetter trocknender Oele, sowie die Bestimmung von Rüböl in ersterem. 1912. 104 S. $8^{0}$ - Vgl. FarbenZeitung. Jg. 18. 1912/13, Hälfte 1. S. $131 \mathrm{ff}$. München, Dr.-Ing.-Diss. v. 22. 3. 1912

2719. Kaden, Johannes: Ueber Stärke und Stärkekleister. 1912 . 70 S. $8^{0}$

München, Dr.-Ing.-Diss. v. 7. 5. 1912

2720. Miller, Paul: Úber partielle Verseifung von Triglyceriden. 1912. $52 \mathrm{~S} .8^{0}$ T Vgl. Journal f. prakt. Chemie. Bd 196. N. F. Bd 88 .

München, Dr.-Ing.-Diss. v. 7.5. 1912

2721. Strohmeyer, Franz, gepr. Lehramtskand.: Über die Vestner'sche Fällung von Eisen, Kobalt, Nickel und Mangan sowie über die direkte Überführung der Sulfide dieser Metalle in wägbare Sauerstoffverbindungen bezw. Metall. 1912. 64 S. $8^{0}$ München, Dr.-Ing.-Diss. v. 23. 5. 1912

2722. Wiliseh, Karl: Ueber die Thermozahlen fetter Oele. 1912. $80 \mathrm{~S} .8^{0}$ \% Vgl. Farben-Zeitung. Jg. 19. 1913/14, Hälfte 1. S. 861 ff. München, Dr.-Ing.-Diss. v. 23. 5. 1912 
2723. Fleischhacker, David: Das Maltoseanilid und die Anwendung des Anilins zur Isolierung der Maltose aus Dextringemischen. 1912. $75 \mathrm{~S}$. $8^{0}$

München, Dr.-Ing.-Diss. v. 12. 6. 1912

2724. Häussler, Robert: Über Methylendigallussäure. 1912. 91 S. $8^{0}$

München, Dr.-Ing.-Diss. v. 12. 6. 1912

2725. Reichel, Wilhelm, gepr. Lehramtskand.: Ueber die elektrolytische Darstellung der Ueberschwefelsäure und deren Ueberführung in Wasserstoffsuperoxyd. 1912. 88 S. $8^{0}$

München, Dr.-Ing.-Diss. v. 10. 7. 1912

2726. Stiegler, Hans: Über eine neue Methode der Rohfaserbestimmung. [1912.] 101 S. $8^{0}$ T Etwas gəkürzt in: Journal f. Landwirtschaft. Bd 61. 1913.

München, Dr.-Ing.-Diss. v. 10. 7. 1912

2727. Bleicher, Karl: Über aethan- und bromaethansulfonsaure Salze. Leipzig: W. Engelmann 1912. 35 S. $8^{0}$ - B. Krystallograph. Teil. (S. 17 ff.) aus: Zeitschrift f. Krystallographie u. Mineralogie. Bd 51. 1913.

München, Dr.-Ing.-Diss. v. 16. 7. 1912

2728. Hans, Wilhelm, gepr. Lehramtskand.: Photochemische Untersuchungen über Bromsilber. 1912. 91 S. $8^{0}$

München, Dr.-Ing.-Diss. v. 16. 7. 1912

2729. Laubmann, Ernst: Über Elektrolysen von Estersalzen der Camphersäure sowie von Kaliumsalzen der campholytischen Säuren mit Kaliumazetat. 1912. 80 S. $8^{0}$

München, Dr.-Ing.-Diss. v. 31. 7. 1912

2730. Kißkalt, Konrad: Einige Beiträge zur chemischen Kenntnis des Castor〈Bohnen-〉 Mehls. 1912. 65 S. $8^{0}$ I Soll demnächst ersch. in: Zeitschrift f. d. gesamte Getreidewesen.

München, Dr.-Ing.-Diss. v. 7. 8.1912

2731. Wartburg, Oswald Rudolph v.: Über die elektrolytische Darstellung von metallischem Natrium. 1912. 66,4 S. $8^{0}$

München, Dr.-Ing.-Diss. v. 7. 8. 1912

2732. Bauer, Karl: Über Kondensationsprodukte aus Salicylaldehydcyanhydrin und secundären Aminen. 1912. 48 S. $8^{0}$

München, Dr.-Ing.-Diss. v. 16. 11. 1912

2733. Noldin, Fritz: Beiträge zur Kenntnis der sogenannten schwarzen Hefen. 1912. 71 S., 4 Taf. $8^{0}$ Vgl. Centralblatt f. Bakteriologie, Parasitenkunde u. Infektionskrankheiten. Abt. 2. Bd 39. 1913. S. 1 ff.

München, Dr.-Ing.-Diss. v. 6.12.1912

\section{LANDWIRTSCHAFTLICHE ABTEILUNG}

2734. Kleemann, Andreas: Untersuchungen über Malzdiastase. 1905. $42 \mathrm{~S} .8^{0}$ T Aus: Die landw. Versuchs-Stationen. Bd 63. 1906.

München, Dr.-Ing.-Diss. v. 21. 6. 1905

2735. Schultz, Eduard: Untersuchungen über die Beziehungen der Blutbeschaffenheit 〈Erythrocyten u. Hämoglobin〉 zur Leistungsfähigkeit von Milchkühen. 1906. 51 S., 2 Taf. $8^{0}$ T Ausz. in: Fühlings Landw. Zeitung. Jg. 55 .

München, Dr.-Ing.-Diss. v. 10. 8. 1905

2736. Kießling, Ludwig, $K$. Adjunkt d. K. Saatzuchtanstalt i. Weihenstephan: Untersuchungen über die Trocknung der Getreide, mit besonderer Berücksichtigung der Gerste. 1906. 127 S. $8^{0}$ I Aus: Vierteljahresschrift d. Bayer. Landwirtschaftsrates. Jg. 11.

München, Dr.-Ing.-Diss. v. 23. 1. 1906

2737. Raum, Johannes, Noviz im Benediktinerstifte Weltenburg: Zur Kenntnis der morphologischen Veränderungen der Getreidekörner unter dem Einflusse klimatischer Verhältnisse. (Mit 30 sachl. $u$. 8 meteorolog. Tab. außerhalb d. Textes, 3 Taf. f. graph. Darstellungen in Lithogr. u. 3 Lichtdruckbildern.) [1906.] 137 S., 6 Taf., 4 Bl. Tab. $8^{0}$ I Referat in: Naturwiss. Zeitschrift f. Land- u. Forstwirtschaft. Jg. 5. 1907. München, Dr.-Ing.-Diss. v. 20. 7. 1906

2738. Niggl, Ernst: Untersuchungen über die Wachstumsvorgänge bei den Getreiden unter dem Einfluß verschiedener Saattiefen. 1907. 68 S. $8^{0}$ - Aus: Vierteljahresschrift d. Bayer. Landwirtschaftsrates. Jg. 12.

München, Dr.-Ing.-Diss. v. 6. 8.1907

2739. Prohaska, Ludwig: Untersuchungen über die Bedeutung der physiologischen Blutbeschaffenheit <Erythrocyten, Leukocyten, und Hämoglobin > von Milchkühen. 1908. 92 S. $8^{0}$

München, Dr.-Ing.-Diss. v. 30.12. 1907

2740. Ferneke B, Karl: Die Haferrispe nach Aufbau und Verteilung der Kornqualitäten <Korngewichte und Spelzengehalte $\rangle$. 1908. $106 \mathrm{~S}$., 5 Taf. $8^{0}$ Referat in: Fühlings landw. Zeitung. Jg. 58. 1909.

München, Dr.-Ing.-Diss. v. 7. 8. 1908

2741. Frankau, August: Untersuchungen über die Beziehungen der physikalischen Bodeneigenschaften zueinander und zur mechanischen Bodenanalyse. 1909. 46 S. $8^{0}$ München, Dr.-Ing.-Diss. v. 1. 3. 1909 
2742. Bauer, Otto, k. Obergeometer d. bayer. Flurbereinigungs-Kommission i. München: Bonitierungsversuch auf agronomisch-naturwissenschaftlicher Grundlage, angewendet auf die Flurbereinigungs-Gebiete von Lindflur, Zellingen a. M. und Gundelfingen. 1909. 100 S., 3 Taf. $8^{0}$

München, Dr.-Ing.-Diss. v. 8. 7. 1909

2743. Suter, Josef, Landwirtschaftslehrer i. Sursee 〈Schweiz〉: Die reine Graswirtschaft in der Hügelregion des nordost- und zentralschweizerischen Alpenfusslandes.

1910. $126 \mathrm{~S} .4^{0}$ T Aus: Landw. Jahrbücher. Bd 39.

München, Dr.-Ing.-Diss. v. 30. 12. 1909

2744. Moertlbawer, Fritz, landw. techn. Hilfsarb. im Bayer. Landwirtschaftsrat: Ueber den Einfluss verschiedenzeitiger Salpeterdüngung auf Ausbildung und Ertrag der Getreidepflanze. München 1910. III, 152 s. $8^{\circ}$

München, Dr.-Ing.-Diss. v. 5. 8. 1910

2745. Baumann, Edmund: Untersuchungen über Ausbildung, Wachstumsweise und mechanische Leistung der Koleoptile der Getreide. [Diessen:] Huber 1911. 85 S. $8^{\circ}$ München, Dr.-Ing.-Diss. v. 7. 1. 1911

2746. Ahr, Joseph, Prof. i. Weihenstephan: Untersuchungen über Rentabilitätsfragen der Düngung. München: Gerber 1911. 94 S. $4^{0}$ Auch als: Landw. Jahrbuch f. Bayern. Jg. 1, Nr 7.

München, Dr.-Ing.-Diss. v. 17. 2. 1911

2747. Warthiadi, Demeter : Veränderungen der Pflanze unter dem Einfluss von Kalk und Magnesia. München: Gais 1911. VI, 154 S. $8^{\circ}$

München, Dr.-Ing.-Diss. v. 3. 5. 1911

2748. Ziegler, August: Untersuchungen über die Basalborste der zweizeiligen Gerste. 1911. 82 S., 7 Taf. $8^{0}$ T Ein Teil in: Zeitschrift f. d. gesamte Brauwesen. Jg. 34 .

München, Dr.-Ing.-Diss. v. 22. 5. 1911

2749. Gümbel, Hermann: Untersuchungen über die Keimungsverhältnisse verschiedener Unkräuter. 1912. 107 S. $4^{0}$. Aus: Landw. Jahrbücher. Bd 43.

München, Dr.-Ing.-Diss. v. 13. 2. 1912

\section{STUTTGART}

Gegründet 1829 als „Vereinigte Kunst-, Real- und Gewerbeschule“, 1832 „,Gewerbeschule“, 1840 erweitert als "Polytechnische Schule", 1862 neu organisiert mit Ausbildung zur Technischen Hochschule, 1876 „Polytechnikum“, 1890 ,,Technische Hochschule“ genannt

\section{SATZUNGEN ORDNUNGEN BESTIMMUNGEN}

\section{ALLGEMEINES UND VERMISCHTES}

2750. Organische Bestimmungen für die Kgl. politechnische[!] Schule zu Stuttgart. Vom 16. 4. 1862. T Abgedr. in: RegierungsBlatt f. d. Kgr. Württemberg v. J. 1862 . S. 109 ff. u. in: Zur Feier des Geburtsfestes des Königs. 1863 [Nr 2824]; geändert u. ergänzt 18. 7. 1870 (Reg.Bl. S. 339 ff.).

2751. Statuten für die Studirenden der technischen Abtheilung der $\mathrm{K}$. polytechnischen Schule zu Stuttgart. 1862. - [Veränderte Fassung.] 1865. 1862: $S^{*} ; 1865: S$

2752. Statuten für die Schüler an der mathematischen Abtheilung und der Handels-Classe der $K$. polytechnischen Schule zu Stuttgart. 1862.

2753. Statuten für die Studirenden der K. polytechnischen Schule zu Stuttgart. 1876. [Folgt:] Auszug aus der BibliothekOrdnung.
2754. Preis-Statut des Kgl. Polytechnikums in Stuttgart. Vom 11. 7. 1876.

2755. Organische Bestimmungen für das Kgl. Polytechnikum zu Stuttgart. Vom 21. 8. 1876. I Abgedr. in: Regierungs-Blatt f. d. Kgr. Württemberg v. J. 1876 . S. 345 ff. u. in: Jahres-Bericht f. 1876/77 [Nr 2827].

2756. [Beg. :] Stuttgart, Euer habe ich die Ehre, in der Anlage im Auftrage des Comités die Urkunde, bezw. das Statut der im Herbst vorigen Jahres ... gegründeten Stipendienstiftung zu überreichen ... C [hristia n Friedrich] Leins, Vorsitzender. (Darin: Urkunde der Stipendien-Stiftung zur Feier des 50jährigen Jubiläums der K. technischen Hochschule zu Stuttgart. 1880.)

2757. Statuten für die Studirenden des K. Polytechnikums zu Stuttgart. 1880. [Folgt:] Auszug aus der BibliothekOrdnung. [Nebst] Nachtrag zu S. 24 bis 26. - [Veränderte Fassung.] 1885. 
2758. Organische Bestimmungen für das Kgl. Polytechnikum zu Stuttgart. Vom 17. 6. 1885, Regierungsbl. S. 281 ff. . [Teilweise abgeändert u. ergänzt 25. 2. 1890 (Reg.-Bl. S. 67), 22. 12. 1891 (Reg.-Bl. S. 351), 25. 2. 1900 (Reg.-Bl. S. 151) u. 16. 10. 1902 (Reg.-Bl. S. 352).]

2759. Kgl. Polytechnikum Stuttgart. ordnung für die Habilitation von Privatdocenten. Vom 3. 7. 1885.

$M$

2760. Auszug aus der Habilitationsordnung der K. Technischen Hochschule zu Stuttgart v. 3. 7. 1885.

2761. Die Unfallversicherung für Studierende und Hospitanten der K. Technischen Hochschule in Stuttgart (seit 1. 10. 1890, auf 1. 10. 1903 erw.). - [Dasselbe] (auf 1. 10. 1908 ergänzt).

2762. Preis-Statut der $\mathrm{Kgl}$. Technischen Hochschule Stuttgart. Vom 19. 1. 1892.

2763. K. Technische Hochschule in Stuttgart. Verfügung der Ministerien der auswärtigen Angelegenheiten, Abteilung für die Verkehrsanstalten, des Innern und der Finanzen, betr. Vorschriften über die praktische Ausbildung der Regierungsbauführer des Hochbau- und Bauingenieurfachs. Vom 30. 6. 1893 . 〈Reg.-Bl. S. 213.〉

$S$

2764. Statuten für die Studierenden der K. Technischen Hochschule in Stuttgart. [1894.] 32 S. - [Dasselbe. Mit Zusatz zu den Schlußbemerkungen auf S. 30. 1895.] $33 \mathrm{~S}$.

$S L B$

2765. K. Technische Hochschule in Stuttgart. Laboratoriums-0rdnung. Vom 19. 12. 1895.

2766. Vorsehriften für die Studierenden der K. Technischen Hochschule in Stuttgart. 1898. [Folgt:] Auszug aus der BibliothekOrdnung - [Veränderte Fassung.] Stuttgart [1900]: Metzler. 27 S. [S. 11 beg. : bis zu 12 Stunden ....] - [Veränderte Fassung.] Stuttgart [1904]: Metzler. 27 S. [S. 11 beg.: Semester erhoben ...] - [Veränderte Fassung.] 1909. 1912. 1900. 1904: K

2767. Bestimmungen über die Semesterund Schlußzeugnisse der Kgl. Technischen Hochschule in Stuttgart. Vom 30. 5. 1900 bzw. 15. 2. 1908.

2768. Promotions-0rdnung für die Erteilung der Würde eines Doktor-Ingenieurs durch die $\mathrm{Kgl}$. Technische Hochschule zu Stuttgart. Vom 7. 8. 1900. Abgedr. in: Regierungsblatt f. d. Kgr. Württemberg v. J. 1900. S. 645 ff.; Jahresbericht f. $1899 / 1900$ [Nr 2827]; Bestimmungen. S. $316 \mathrm{ff}$.
2769. Verzeichnis der für Studierende bestehenden Stipendien an der K. Technischen Hochschule in Stuttgart. Aufgestellt im Sept. 1900.

2770. Satzungen für die Krankenkasse der Studierenden der K. Technischen Hochschule Stuttgart (จ. 11. 1. bezw. 8. 2. 1901). 1901.

2771. [Dasselbe] (v. 19. 9. 1 1. 24. 10. 1902). 1902.

$S L B$

2772. [Dasselbe] (v. 23. 9. 1903). 1903. $S L B$

2773. Verfassung der Kgl. Technischen Hochschule in Stuttgart (v. 28. 9. 1903). 1903.

2774. Satzungen für die Krankenkasse der Studierenden der K. Technischen Hochschule Stuttgart (v. 19. 3. 1906). 1906. [Dasselbe.] 1908. - [Dasselbe mit kleinen Änderungen.] 1912.

1906: $S$ LB

2775. Bestimmungen über die akademischen Preise der Kgl. Technischen Hochschule Stuttgart (v. 12. 2. 1907).

2776. K. Technische Hochschule Stuttgart. Verfassung der Adolf von ErnstStiftung. [1908.]

2777. Bekanntmachung des Rektorats der Technischen Hochschule, betr. die Erteilung des Grads eines Diplom-Ingenieurs an württembergische Regierungsbauführer. Vom 26. 3. 1909. T Aus: Amtsblatt des K. Württ. Ministeriums d. Kirchen- u. Schulwesens. 1909. S. 49.

2778. K. Technische Hochschule in Stuttgart. Verfassung der Robert-Bosch-Stiftung. [1911.]

2779. Mitteilungen über die Aufnahmebestimmungen und Einrichtungen der $\mathrm{Kgl}$. Technischen Hochschule in Stuttgart. Studienj. 1912/13.

\section{PRÚFUNGSORDNUNGEN}

2782. Stuttgart. Kgl. Polytechnische Schule. Bestimmungen über die Abhaltung von Diplom-Prüfungen an den Fachschulen für Architektur, Ingenieurwesen, Maschinenbau, chemische Technik, Mathematik und Naturwissenschaften. 1875.

2783. Zusammenstellung der für die Studirenden des Kgl. Polytechnikums wichtigsten Bestimmungen über die Staatsprüfungen, welche auf Grund der am Polytechnikum gemachten Studien abgelegt werden. 1883. [Nebst] Anm. zu A., B. u. C. v. Dez. 1892. 
2784. K. Technische Hochschule in Stuttgart. Verfügung des K. Ministeriums des Kirchen- und Schulwesens vom 23, 5. 1883 $\langle$ Reg.-Bl. S. 73 ff. $\rangle$, betr. die am Polytechnikum in Stuttgart abzuhaltende mathematisch-naturwissenschaftliche Vorprüfung für Kandidaten des Bau- und Maschinen-Ingenieurfachs. [Neudr.; Anm. zu Polytechnikum: „Die Bezeichnungen „Polytechnikum“" ... sind jetzt ersetzt durch die Benennungen ,Technische Hochschule“"...]

2785. Kgl. Technische Hochschule in Stuttgart. Bestimmungen über die Staatsprüfungen im Baufache $\langle$ Hochbau-, Bauingenieur- und Maschineningenieurfache $\rangle$ in Württemberg. 1892.

2786. Die wichtigsten Bestimmungen über die Staatsprüfungen im Baufache <Hochbau-, Bauingenieur- und Maschineningenieurfache $>$ in Württemberg. Für die Studierenden der Technischen Hochschule zsgest. auf Grund der Kgl. Verordnung v. 13. 4. 1892 〈Reg.-Bl. S. 149 ff.〉 u. der Ministerial-Verfügungen v. 10.5. 1892 bezw. 13. 6. 1892 〈Reg. Bl. S. 162-192.〉 - [Neudr.] 1903.

2787. Statut für die Diplomprüfungen der Kgl. Technischen Hochschule in Stuttgart v. 22. 12.1898 mit Ergänzungen v. 8. 8. 1899. ๆ Abgedr. in: Bestimmungen. S. 308 ff. $S^{*}$

\section{Abteilung für Architektur}

2788. Kgl. Württembergisches Polytechnikum zu Stuttgart. Statut für die Diplomprüfung an der Fachschule für Architektur. Vom 7. 7. 1871, 19. 1. 1877 u. 14. 5. 1881.[Dasselbe u. d. T.:] Kgl. Technische Hochschule in Stuttgart. Statut für die Diplomprüfung an der Abteilung für Architektur.

2789. Statut für die Diplomprüfung der AbteilungfürArchitektur an derKgl.Technischen Hochschule in Stuttgart. Vom 16. 1. 1894.

2790. Ordnung für die Diplomprüfung an der Abteilung für Architektur der $\mathrm{Kgl}$. Technischen Hochschule in Stuttgart. Vom 14. 10. 1908.

2791. Vorschriften für die Diplomprüfungen für Architekten an der Kgl. Technischen Hochschule in Stuttgart. Vom 25. 1. 1912. (Enth.: I. Diplomprüfungsordnung. II. Geschäftsordnung.)

2792. Diplomprüfungs- Ordnung für Architekten an der Kgl. Technischen Hochschule in Stuttgart. Vom 25. 1. 1912. I Aus: Vorschriften f. d. Diplomprüfungen ... [Nr 2791].

2793. Geschäftsordnung zu den Diplomprüfungen für Architekten an der Kgl. Tech- nischen Hochschule in Stuttgart. Vom 25. 1. 1912. A Aus: Vorschriften f. d. Diplomprüfungen ... [Nr 2791].

\section{Abteilung für Bauingenieurwesen}

2794. Kgl. Württembergisches Polytechnikum zu Stuttgart. Statut für die Diplomprüfung an der Fachschule für Bauingenieurwesen. Vom 7. 7. 1871, 19. 1. 1877 u. 14. 5. 1881. - [Dasselbe u. d. T.:] Kgl. Technische Hochschule in Stuttgart. Statut für die Diplomprüfung an der Abteilung für Bauingenieurwesen.

2795. Statut für die Diplomprüfung der Abteilung für Bauingenieurwesen an der Kgl. Technischen Hochschule in Stuttgart. Vom 16. 1. 1894.

2796. Ordnung für die geodätische Diplomprüfung an der Abteilung für Bauingenieurwesen der Kgl. Technischen Hochschule in Stuttgart. Vom 17. 10. 1906.

2797. Diplomprüfungs- Ordnung für Bauingenieure an der Abteilung für Bauingenieurwesen der $\mathrm{Kgl}$. Technischen Hochschule in Stuttgart. Provisorisch genehmigt 11. 1. 1908.

2798. [Dasselbe.] Vom 14. 10. 1908.

2799. Vorschriften für die Diplomprüfungen für Bauingenieure an der Kgl. Technischen Hochschule in Stuttgart. Vom 25. 1. 1912. (Enth.: I. Diplomprüfungsordnung mit Änderungen v. 4. 9. 1912. II. Geschäftsordnung.)

2800. Diplomprüfungs- Ordnung für Bauingenieure an der Abteilung für Bauingenieurwesen der Kgl. Technischen Hochschule in Stuttgart. Vom 25. 1. 1912. T Aus: Vorschriften f. d. Diplomprüfungen ... [Nr 2799]. 2801. Geschäftsordnung zu den Diplomprüfungen für Bauingenieure an der Kgl. Technischen Hochschule in Stuttgart. Vom 25. 1. 1912. I Aus: Vorschriften f. d. Diplomprüfungen ... [ $\mathrm{Nr} 2799]$.

Abteilung für Maschineningenieurwesen einschließlich der Elektrotechnik

2802. Kgl. Württembergisches Polytechnikum in Stuttgart. a) Statut für die Diplomprüfungen an der Fachschule für Maschineningenieurwesen. Vom 1. 6. 1886. [Folgt:] b) Prüfungs-Instruktion. I Abgedr. in: Jahres-Bericht f. 1885/86 [Nr 2827].

2803. Statut für die Diplomprüfungen der Abteilung für Maschineningenieurwesen an der Kgl. Technischen Hochschule in Stutt- 
gart. Vom 7. u. 26. 6. 1893. [Nebst Deckbl. zu $§ 5$ u. 9.]

2804. K. Technische Hochschule in Stuttgart. Verfügung der Ministerien der auswärtigen Angelegenheiten, Abteilung für die Verkehrsanstalten, des Innern, des Kirchenund Schulwesens und der Finanzen, betr. Vorschriften über die Werkstattthätigkeit der Kandidaten des Maschineningenieurfachs vor Ablegung der Vorprüfung und über die praktische Ausbildung der Regierungsbauführer dieser Fachrichtung. Vom 30. 6. 1893 〈Reg.-Bl. S. 223.〉

2805. Statut für die Diplomprüfungen der Abteilung für Maschineningenieurwesen einschliesslich Elektrotechnik an der Kgl. Technischen Hochschule in Stuttgart. Vom 22. 12. 1898. - [Dasselbe] mit Ergänzungen v. 8. 8.1899 .

1898: $S L B$

2806. Diplomprüfungs-0rdnung für $\mathrm{Ma}$ schinen- und Verwaltungsingenieure an der Abteilung für Maschineningenieurwesen einschließlich der Elektrotechnik der $\mathrm{Kgl}$. Technischen Hochschule in Stuttgart. Provisorisch genehmigt 11. 1. 1908.

2807. [Dasselbe.] Vom 14. 10. 1908.

2808. Vorschriften für die Diplomprüfungen für Maschinen- und Verwaltungsingenieure an der $\mathrm{Kgl}$. Technischen Hochschule in Stuttgart. Vom 25. 1. 1912. (Enth.: I. Diplomprüfungsordnung. II. Geschäftsordnung.)

2809. Diplomprüfungs-Ordnung für Maschinen- und Verwaltungsingenieure an der Abteilung für Maschineningenieurwesen einschl. der Elektrotechnik der Kgl. Technischen Hochschule in Stuttgart. Vom 25. 1. 1912. T Aus: Vorschriften f. d. Diplomprüfungen... [Nr 2808].

2810. Gesehäftsordnung zu den Diplomprüfungen für Maschinen- und Verwaltungsingenieure an der Abteilung für Maschineningenieurwesen einschl. der Elektrotechnik der Kgl. Technischen Hochschule in Stuttgart. Vom 25. 1. 1912. I Aus: Vorschriften f. d. Diplomprüfungen ... [Nr 2808].

2811. Ordnung für die elektrotechnische Diplomprüfung an der Abteilung für Maschineningenieurwesen einschließlich der Elektrotechnik der Kgl. Technischen Hochschule in Stuttgart. Vorläufig genehmigt 4. 10. 1907.

2812. [Dasselbe.] Vom 7. 7. 1908.

2813. Vorschriften für die Diplomprüfungen für Elektroingenieure an der $\mathrm{Kgl}$.
Technischen Hochschule in Stuttgart. Vom 25. 1. 1912. (Enth.: I. Diplomprüfungsordnung. II. Geschäftsordnung.)

2814. Diplomprüfungs- Ordnung für Elektroingenieure an der Abteilung für $\mathrm{Ma}$ schineningenieurwesen einschl. der Elektrotechnik der Kgl. Technischen Hochschule in Stuttgart. Vom 25. 1. 1912. - Aus: Vorschriften f. d. Diplomprüfungen ... [Nr 2813].

2815. Geschäftsordnung zu den Diplomprüfungen für Elektroingenieure an der $\mathrm{Ab}$ teilung für Maschineningenieurwesen einschl. der Elektrotechnik derK gl. TechnischenHochschule in Stuttgart. Vom 25. 1. 1912. I Aus: Vorschriften f. d. Diplomprüfungen ... [Nr 2813].

Abteilung für Chemie einschließlich des Hüttenwesens und der Pharmazie

2816. Kgl. Württembergisches Polytechnikum in Stuttgart. a) Statut für die Diplomprüfung an der Fachschule für chemische Technik. Vom 27. 8. 1872. [Folgt:] b) Prüfungs-Instruktion der Fachschule für chemische Technik. Vom 11. 8. 1875.

2817. Statut für die Diplomprüfungen der Abteilung für Chemie einschliesslich Hüttenwesen etc. an der $\mathrm{Kgl}$. Technischen Hochschule in Stuttgart. Vom 20. 3. 1896.

2818. Diplomprüfungsordnung der $\mathrm{Ab}$ teilung für Chemie einschl. des Hüttenwesens und der Pharmazie an der Kgl. Technischen Hochschule zu Stuttgart. Vom 13. 7. 1904. [Nebst] Änderungen zu § 11 u. 15 v. 18. 2. 1910 . Änderungen: $S$

\section{Abteilung für Mathematik und Naturwissenschaften}

2819. Statut für die Diplomprüfungen der Abteilung für Mathematik und Naturwissenschaften an der $\mathrm{Kgl}$. Technischen Hochschule in Stuttgart. Vom 9. 10. 1895.

2820. Statut für die geodätische Diplomprüfung an der Abteilung für Mathematik und Naturwissenschaften der Kgl. Technischen Hochschule in Stuttgart. Vom 13.2.1897.

\section{Abteilung für allgemein bildende Fächer}

2821. Stuttgart. Kgl. Polytechnische Schule. Statut für die Diplomprüfungen an der Fachschule für allgemein bildende Fächer. Vom 4. 4. 1876.

2822. Statut für die Diplomprüfungen der Abteilung für allgemein bildende Fächer an der Kgl. Technischen Hochschule in Stuttgart. Vom 21. 3. 1896. 


\section{PERIODISCHE SCHRIFTEN}

2823. Programm der Kgl. polytechnischen Schule zu Stuttgart. 1840.

2824. Einladungs-Schrift (1846 ff. : der k. polytechnischen Schule in Stuttgart) zu der Feier des Geburtsfestes Seiner Majestät des Königs Wilhelm von Würtemberg (1844. 1845: in der $\mathrm{Kgl}$. polytechnischen Schule zu Stuttgart) am 27. Sept. 1844-1861. (Enth. auch Schulnachrichten für 1843/441860/61.)

1845. 1847. 1850. [Umschlagt.:] Zur Feier des Geburtsfestes S. Majestät des Königs Wilhelm von Württemberg die k. polytechnische Schule zu Stuttgart.

Fortges. u. d. T.:

Zur Feier des Geburtsfestes Seiner Majestät des Königs Wilhelm von Württemberg. Eine Denkschrift der Kgl. polytechnischen Schule zu Stuttgart, den 27. Sept. 1862. 1863. (1863 enth. auch Schulnachrichten für 1862/63.)

1863: $S$

Darin :

1844. Pro ß, Friedrich : Über praktische Geometrie.

1845. Kurr, Joh[ann] Gottlob: Beiträge zur fossilen Flora der Juraformation Württembergs. (Mit 2 Taf.)

1846. Reusch, Friedrich Eduard: Die gezwungene Bewegung des Atoms.

1847. Fehling, Hermann: Chemische Untersuchung der Soolen, des Stein- und Kochsalzes, sowie der Mutterlaugen der $K$. württembergischen Salinen. Mit besonderer Berücksichtigung der concentrirten Mutterlaugen.

1848. Fischer, F(erdinand): Ueber die Vortheile architektonischer Kenntnisse für Laien.

1849. Mauch, J[ohann] M[atthäus]: Abhandlung über die mittelalterlichen Baudenkmale in Württemberg, mit 4 lithogr. Taf. begleitet.

1850. Proß, Friedrich: Abhandlung über das einfache und das vollständige Viereck. Nebst einem Anh. über den Nutzen und die Anwendbarkeit des Legendrischen Lehrsatzes bei sphärischterristrischen [!] Triangulirungen. (Mit 3 Taf.)

1851. Breymann, G(ustav): Die Bedeutung des Eisens für die Baukunst der neueren Zeit.

1852. Gugler, (Bernhard): Ueber die Anwendung der allgemeinen Gleichung einer Kegelschnittstangente.

1853. Müller, Chr(istian): Abhandlung über einige durch Kreisexcenter bewegte Dampischieber. (Mit 1 Taf.)

1854. Hänel, Ad(olph): Abhandlung über die Constructionsverhältnisse eiserner Gitterbalken. Mit 2 Figurentaf.

1855. Egle, J(oseph): Abhandlung über das Schattiren der Oberflächen regelmässiger Körper. Mit 1 Figurentaf.
1856. Holtzmann, Carl: Ueber die Vertheilung des Drucks im Innern eines Körpers.

1857. Baur, C(arl) W(ilhelm): Die Ausgleichung der Richtungen in einem Dreiecksnetz mit unvollständigen Centralsystemen. Mit $1 \mathrm{Fi}$ gurentaf. u. $3 \mathrm{Tab}$.

1858. Holtzmann, Karl: Die Wellenbewegung tropfbarer Flüssigkeiten.

1859. Müller, Chr(istian): Abhandlung über Kolben- und Schieber-Diagramme. (Mit 4 Taf.)

1860. Hänel, Adolph: Die inneren Kräfte rechtwinklig belasteter Balken und die Berechnung eiserner I-Balken.

1861. Holtzmann, Karl: Ueber die Theorie der Erscheinungen der Capillarität.

1862. Baeumer, Wilh[elm]: Das bürgerliche Wohnhaus der Stadt bei den Griechen und Römern, im deutschen Mittelalter, im 16. bis 19. Jahrhundert. Mit 6 Figurentaf. u. 3 Holzschn.

1863. Schröter, Moritz: Beschreibung der Aufstellung der Eisenbahnbrücke bei Neckarelz. Mit 5 Figurentaf.

$$
\text { 1844: } S^{*} ; 1845-1848.1850-1861: S
$$

2825. Programm der Kgl. Württembergischen Polytechnischen Schule (1877/781889/90: des Kgl. Württembergischen Polytechnikums; 1890/91 ff. : der Kgl. Württembergischen Technischen Hochschule) zu (1892/93 ff.: in) Stuttgart für das Jahr (1891/92ff.: Studienj.) 1863 auf 1864-19121913. $1863-1912$.

1867/68. $1872 / 73 . \quad 1882 / 83 . \quad 1885 / 86 . \quad 1887 / 88$. 1889/90. 1890/91. 1892/93-1895/96. 1899/1900: $S$

2826. Kgl. Polytechnische Schule inStuttgart. Bekanntmachung der Ergebnisse der Preisbewerbung vom Jahr 1863-64 und der für das Jahr 1864-65 gestellten Preisaufga ben. 1862-63 bzw. 1863-64 in: Denkschrift zur Feier d. Einweihung d. neuen Gebäudes [Nr 2838].

1864/65. Ergebnisse in: Einladungsschrift zu d. Ausstellung v. Arbeiten [Nr 2842].

1865-66-1880-81. Ergebnisse in: Jahres-Bericht f. 1865/66-1880/81 [Nr 2827]. Be

2827. Jahres-Bericht (1901/1902 ff.: Bericht) der Kgl. Polytechnischen Schule (1876/77-1888-89: des Kgl. Polytechnikums; 1889-90 ff. : der Kgl. Technischen Hochschule) zu (1892/93 ff.: in) Stuttgart für das Schulj. (1866/67 ff.: Studienj.) 1865/66-1909/10. 1866-[1911].

1843/44 1860/61 in: Einladungsschrift zu d. Feier d. Geburtsfestes d. Königs. 1844-1861 [Nr 2824].

1861/62. [Kein Jahresbericht veröffentlicht.] 1862/63 in: Zur Feier d. Geburtsfestes d. Königs. 1863 [Nr 2824].

1863/64 in: Denkschrift zur Feier d. Einweihung d. neuen Gebäudes [Nr 2838]. 
1864/65 in: Einladungs-Schrift zu d. Ausstellung v. Arbeiten [Nr 2842].

1865/66. Mit einer Abhandlung über den Umbau eines städtischen Wohnhauses in Stuttgart von Alexander Tritschler. [Die Abh. auch einzeln bei Fues in Tübingen.]

1866/67. Mit einer Abhandlung über die Schwingungsbewegungen der Lokomotiven von [Paul] Zech. [Die Abh. auch einzeln bei Fues in Tübingen.]

1867/68. Mit einer Abhandlung „Zur Theorie der Tonnengewölbe" von [Adolph] v. Hänel. [Die Abh. auch einzeln bei Fues in Tübingen.]

1868/69. Mit dem Abdr. eines Vortrags über das ehemalige Lusthaus in Stuttgart als Monument des früheren Renaissancestyls von Wilhelm Bäumer. Mit 4 Fig. u. 3 Holzschn. [Die Abh. auch einzeln bei Fues in Tübingen.]

1880/91. Mit einem Anh.: Statistik der Studierenden für die letzten 20 Studienjahre.

1895/96. Mit einem Anh.: Die neuen Gebäude für das elektrotechnische Institut und das chemische Laboratorium. (Mit 1 Ansicht u. 3 Grundrissen.)

1800/01. Mit einem Anh.: Das Ingenieurlaboratorium der Technischen Hochschule Stuttgart. (Nach den Aufzeichnungen des Laboratoriumsvorstands [Carl] v. Bach.) 1801. [Der Anh. auch als Sonderabdr.]

1906/07. Mit einem Anh.: Die Materialprüfungsanstalt der Technischen Hochschule Stuttgart. (Nach den Aufzeichnungen des Vorstandes der Materialprüfungsanstalt C[arl] v. Bach.) 1908. [Der Anh. auch als Sonderabdr.]

1910/11 ff. in: Programm f. 1911/12 ff. [Nr 2825]. 1865/66-1880/81. 1883/84-1899/1900.

1900/01 S.-A. des Anh., 1906/07 S.-A. des Anh.: S
2828. K. Polytechnikum (1889 ff. : Kgl. Technische Hochschule: 1892 ff. : in) Stuttgart. Ergebniss (1891 ff.: Ergebnisse) der Preisbewerbung vom Jahre ... (1904 ff. : Veröff. bei d. Feier d. Geburtsfestes d. Königs am 25. 2. [des folgenden Jahres]). $1881-$ 1911. 1881-1904 auch in: Jahresbericht $f$. 1881/82-1903/04 [Nr2827]. 1891-1899. 1903: $S^{*}$

2829. Kgl. Technische Hochschule (1893 u. $1894 \mathrm{ff}$. : in) Stuttgart. Preisaufgaben für ... 〈veröff. am 25. 2. [des zuerst genannten Jahres]〉. (1892 u. 1893.) 1893 u. 1894 [usw.]-1912 u. 1913.

1892 u. $1893-1906$ u. 1907: $S$

2830. Verzeichnis der Studierenden der Kgl. Technischen Hochschule Stuttgart sowie deren (1898ff.: mit Angabe ihrer) Wohnungen $>$ (1901 ff. : Personal-Verzeichnis der Kgl. Technischen Hochschule zu Stuttgart) für das W.-S. 1897/98. S.-S. 1898 [usw.]-W.-H. 1912/13. 1898-1912. 1898/99-1901/02. 1905/06: $S$

2831. Kgl. Technische Hochschule Stuttgart. Aufgaben für die Diplomprüfungen (1901/02-1907/08: sowie die Staatsprüfungen im Baufache) vom Studienj. 1901/02 -1910/1911. 1902-11.

1872/73-1884/85 in: Jahres-Bericht f. 1872/73 $-1884 / 85$ [Nr 2827].

1872/73-1874/75. [Nur f. d. Maschinenbaufachschule.]

1875/76. [Nur f. d. math.-naturw. Fachschule.] 1885/86-1908/09. [Autogr]. 1885/86-1887/88. 1889/90-1900/01: S*;1888/89: nicht nachweisbar

\section{GELEGENHEITS - UND VERMISCHTE SCHRIFTEN}

\section{REDEN ZUM GEBURTSFEST DES KöNIGS}

2833. Bäumer, [Wilhelm]: Die Bedeutung des kunstgewerblichen Unterrichts für Württemberg. $1870.15 \mathrm{~S} .8^{0}$

2834. Ernst, Adolf: Kultur und Technik. Berlin: Springer 1888. 35 S. $8^{0}$ - Auch in: Zeitschrift d. Vereines deutscher Ingenieure. Bd 32. $S^{*}$

2835. Häussermann, [Carl]: Die Elektricität im Dienste der chemischen Industrie. Stuttgart: Wittwer (1895). 16 S. $8^{0}$ S

2836. Bantlin, A[lbert]: Die deutsche Industrie und die Arbeiterversicherung. Mit 14 Taf. Stuttgart: Bergsträsser 1901. 48 S. $8^{0} \mathrm{~S}$

\section{VERSCHIEDENE - SCHRIFTEN}

2837. Entwurf einer erweiterten Organisazion der technischen Zentralschule zu
Stuttgart. (Durch amtl. Auftrag veranlasste Arbeit.) Von Dr. K[arl] M[arcell] Heigelin, Prof. an d. Gewerbesch. Stuttgart: Metzler 1831. IV, $32 \mathrm{~S} .8^{0}$

2838. Denkschrift zur Feier der Einweihung des neuen Gebäudes der Königl. Polytechnischen Schule begangen im ersten Jahre der Regierung Seiner Majestät des Königs Karl von Württemberg am 30. September und 1. Oktober 1864 mit einem Beitrag zur Kenntniss der vaterländischen Kirchenbauten von C[hristian] F[riedrich] Leins. (Enth. auch den Jahresbericht für das Schulj. 1863/64.) 46 S., 7 Taf. $4^{0} \quad S$

2839. Beschreibung der Einweihung des neuen Gebäudes der K. polytechnischen Schule in Stuttgart, vom 29. September bis 1 . Oktober 1864. Hrsg. von der Fest-Commission. Stuttgart: Nitzschke 1864. 47 S., 2 Taf. $4^{0} S$ 
2840. Katalog der Bibliothek der Polytechnischen Schule in Stuttgart. 1865. IV, 139 S. $8^{0}$

2841. Zur Erinnerung an Karl Alexander Holtzmann, R. d. O. d. württ. Krone, Director der K. polytechnischen Schule in Stuttgart. Geb. den 23. Okt. 1811, gest. den 25. April, beerdigt den 27. April 1865. $23 \mathrm{~S} .8^{0}$

$S^{*}$

2842. Einladungs-Schrift der Königl. Poly technischen Schule zu Stuttgart zu der am 30. und 31. Juli 1865 stattfindenden Ausstellung von Arbeiten ihrer Studirenden und Schüler vom Schuljahr 1864/65. Inh.: 1) eine Abhandlung über den Bewegungsmechanismus des Parr-Curtis Selfactors von Prof. Carl Heinrich Schmidt, mit 2 Taf. Zeichn.; 2) Jahresbericht der Königl. Polytechnischen Schule. 1865. 19 S., 2 Taf. $4^{0} \mathrm{~S}$

2843. Katalog der Bibliothek der Polytechnischen Schule in Stuttgart. 1871. 237 S. $8^{0}$

2844. Denkschrift zur Enthüllungsfeier des Epitaphiums, welches die Königliche Polytechnische Schule in Stuttgart ihren im deutsch-französischen Kriege 1870-71 gefallenen Zöglingen in der Aula des Schulgebäudes errichtet hat. Begangen am 2. Dez. 1873. Stuttgart: Spemann 1874. 19 S., 1 Taf. $4^{0}$

2845. Architekturbild der Universitätsstadt Tübingen und ihrer Umgebung von C[hristian] F[riedrich] v. Leins, Prof. d. Architektur am K. Polytechnikum zu Stuttgart. Mit 62 Holzschn. u. 1 Pl. der Stadt. (Umschlagt.: Festschrift zur Feier des vierhundertjährigen Bestandes der Eberhard-
Karls-UniversitätTübingen vom Königlichen Polytechnikum zu Stuttgart.) [1877.] 55 S., 1 Pl. $4^{0}$

2846. Katalog der Bibliothek des Königlichen Polytechnikums in Stuttgart (Nachtr.: Polytechnikums Stuttgart). 1879. $430 \mathrm{~S}$. [Nebst] Nachtrag 1. 1879-1884. 1885. IV, 188 S.; Nachtrag 2. 1884 -89. 1889. VII, 187 S. $8^{0}$

Nachtr. 2: $S$

2847. Festschrift zur Feier der Einweihung des neuen Flügelanbaues sowie des fünfzigjährigen Jubiläums der K. technischen Hochschule zu Stuttgart am 20. bis 25. Octo ber 1879 (mit einer urkundlichen Geschichte der Entwicklung der Anstalt von Dr. P[aul] Zech. Mit 1 Photolithogr. u. 1 Lithogr.) 42 S., 2 Taf. $4^{0}$

2848. Übersicht aller Polytechniker von 1829 bis 1879 alphabetisch geordnet. $94 \mathrm{~S}$. $2^{0}$ [Lithogr.]

2849. Die Hoflager und Landsitze des Württembergischen Regentenhauses von Dr. C[hristian] F[riedrich] v. Leins, Prof. d. Architektur. Mit 115 Abb. Stuttgart: Greiner \& Pfeiffer [1889]. (Umschlagt.: Festschrift zur Feier der 25jährigen Regierung Seiner Majestät des Königs Karl von Württemberg. Vom Lehrerkollegium der Königl. Technischen Hochschule in Stuttgart.) IV, 107 S., 3 Taf. $4^{0}$

2850. Katalog der Bibliothek der Königlichen Technischen Hochschule in Stuttgart. (Vorr.: E[mil] Koller.) 1902. VII, 618 S. $4^{0}$

2851. Der Neubau des physikalischen Instituts der Technischen Hochschule Stuttgart. Von K[arl] R[ichard] Koch. (1911.) 14 S. $4^{0} \uparrow$ Aus: Physik. Zeitschrift. Jg. 12.

\section{HABILITATION S SCHRIFTEN}

2852. Kaim, Franz, Dr.: Shakespeares Macbeth. Eine Studie. 1888. 34 S. $8^{0}$ Stuttgart Te. H., Hab.-Schr. v. 19. 9. 1888

2853. Einbeck, J[ohannes], Obering.: Theorie der Heisswasserheizung. Stuttgart: Wittwer 1887. 35 S., 2 Taf. $8^{0}$

Stuttgart Te. H., Hab.-Schr. v. 26.11. 1888

2854. Seelig, Eduard, Dr.: Über Benzylacetat und ähnliche Körper, insbesondere ihr Verhalten gegen Chlor \& Brom. 1889. 31 S. $8^{0}$ A Aus: Journal f. prakt. Chemie. Bd 147. N. F. Bd 39.

Stuttgart Te. H., Hab.-Schr. v. 19.1. 1889
2855. Philip, Max, Dr.: Das Pyridin und seine nächsten Derivate. Stuttgart: Metzler 1889. $68 \mathrm{~S} .8^{0}$

Stuttgart Te. H., Hab.-Schr. v. 8. 6.1889

2856. Westenholz, Friedrich von, Dr.: Ueber Byrons historische Dramen. Ein Beitrag zu ihrer ästhetischen Würdigung. Stuttgart: Frommann 1890. 64 S. $8^{\circ}$ If Auch als Buch.

Stuttgart Te. H., Hab.-Schr. v. 26. 12. 1889

2857. Diez, Max, Prof. Dr.: Theorie des Gefühls zur Begründung der Aesthetik. 
Stuttgart: Frommann 1892. XII, 172 S. $8^{0}$

- Auch als Buch.

Stuttgart Te. H., Hab.-Schr. v. 25. 7. 1891

2858. Endriss, K[arl], Dr., i. Stuttgart: Zur Geologie der Höhlen des Schwäbischen Albgebirges. 1. Der Bau des Gutenberger Höhlensystems. Mit 1 Taf. 1892. S. 49-83, 1 Taf. $8^{0}-$ Aus: Zeitschrift d. Deutschen Geolog. Gesellschaft. Bd 44; mehr nicht ersch.

Stuttgart Te. H., Hab.-Schr. v. 7.11. 1891

2859. Fränkel, Ludwig, Dr.: Shakespeare und das Tagelied. Ein Beitrag zur vergleichenden Litteraturgeschichte der germanischen Völker. Hannover: Helwing 1893. 47 S. $8^{0}$ - Nur Abschnitt I, 1 u. III, 3; im Buchh. vollst.

Stuttgart Te. H., Hab.-Schr. v. 9. 6. 1893

2860. Johannsen, Otto, Dir. d. Fachsch. f. Spinnerei, Weberei, Wirkerei u. Färberei i. Reutlingen: Studien über den Wickelkörper der Mulemaschine. Leipzig: Th.Martin [1895]. 31 S., 2 Taf. $8^{0}$ - Wenig erweitert u. d. T.: Studien über den Wickelkörper des Selfactors.

Stuttgart Te. H., Hab.-Schr. v. 7. 11. 1894

2861. Wölffing, Ernst, Dr.: Die singulären Punkte der Flächen. 1896. 25 S. $8^{0} \mathrm{~S}$

Stuttgart Te. H., Hab.-Schr. v. 2. 5. 1895

2862. Haussmann, [Karl], Assist.: Untersuchung einiger Methoden der Grubenmessung. 1897. $32 \mathrm{~S} ., 3$ Taf. $8^{0}$

Stuttgart Te. H., Hab.-Schr. v. 13. 3. 1896

2863. Spindler, H[einrich], Dr., chem.techn. Mitglied d. K. W. Medizinalkollegiums: Die Unschädlichmachung der Abwasser in Württemberg. Stuttgart: Enke 1896. VI, 157 S. $8^{0}$. Auch als Buch. S

Stuttgart Te. H., Hab.-Schr. v. 20.3. 1896

2864. Kauffmann, Hugo, Dr., Assist. am Labor. f. allg. Chemie d. Techn. Hochsch. Stuttgart: Über elektrisch konjugierte Valenzen und über Eigenschaften derselben. 1898. 48 S. $8^{0}$

Stuttgart Te. H., Hab.-Schr. v. 6.12.1897

2865. Pfeiffer, Gustav, Dr. phil.: Ein Problem der romanischen Wortforschung. [1.]. Stuttgart: Greiner \& Pfeiffer 1900. 40 S. $8^{0}-$ Auch als Buch; 2. (S. $41-60$.) 1900; die auf S. 60 angekündigte weitere Forts. erschien nicht.

Stuttgart Te. H., Hab.-Schr. v. 11. 3. 1898

2866. Ensslin, Max: Explosionsmotor und Verbrennungsmotor <0ttomotor und Dieselmotor $\rangle$. 1900. 35 S. $8^{0}$

Stuttgart Te. H., Hab.-Schr. v. 12.1.1900

2867. Schmidt, Julius, Dr., Assist. am Chem.-technolog. Labor. d. Techn. Hochsch.
Stuttgart: Ueber Tautomerie- und Isomerieerscheinungen bei Oximen der allgemeinen Formel $\stackrel{\mathrm{R}}{\mathrm{R}}>\mathrm{C}=\mathrm{N}-\mathrm{OH}$ beziehungsweise ihren Acylderivaten. 1900. 48 S. $8^{0}$

Stuttgart Te. H., Hab.-Schr. v. 22. 1. 1900

2868. Mäule, C[hristian]: Das Verhalten verholzter Membranen gegen Kaliumpermanganat, eine Holzreaktion neuer Art. Stuttgart: Zimmer 1901. 22 S. $8^{0}$ S

Stuttgart Te. H., Hab.-Schr. v. 27. 10. 1900

2869. Engliseh, W[ilhelm] Eugen, Dr. sc. nat.: Das Schwärzungsgesetz für Bromsilbergelatine. Halle: Knapp 1901. 45 S. $4^{0}$ - Auch als Buch; S. 11-25 mit Ausnahme zweier Versuchsreihen auch in: Archiv f. wissenschaftl. Photographie. Bd 1. 1899. S. 117 ff. Bd 2. 1900. S. $131 \mathrm{ff}$; S. $25-45$ ebd. Bd 2. 1900 . S. 243 ff. $S$ Stuttgart Te. H., Hab.-Schr. v. 12.1. 1901

2870. Gastpar, A[lfred], Dr. med. i. Stuttgart: Die Abwasserfrage in Stuttgart. Mit 14 Fig. Stuttgart: Wittwer 1902. 109 S. $8^{0} \mathrm{~S}^{*}$ Stuttgart Te. H., Hab.-Schr, v. 1. 3. 1902

2871. Seel, Eugen, Dr., Stuttgart: Untersuchungen über Aloe. [1903.] 61 S. $8^{0} \mathrm{~S}$ Stuttgart Te. H., Hab.-Schr. v. 24. 3. 1902

2872. Rohland, Paul, Dr.: Studien über den Portland-Zement. 1904. 39 S. $8^{0}$ - Aus: Rohland: Der Portland-Zement vom physikal.chem. Standpunkte. 1903.

Stuttgart Te. H., Hab.-Schr. v. 3. 11.1902

2873. Kaulla, Rudolf, Dr. jur. et oec. publ.: Die Lehre vom gerechten Preis in der Scholastik. 1904. S. 579-602. $8^{0}$ - Aus: Zeitschrift f. d. gesamte Staatswissenschaft. Jg. 60.

Stuttgart Te. H., Hab.-Schr. v. 10.1. 1903

2874. Bauer, Ludwig, Dr. med. i. Stuttgart: Der Zug nach der Stadt und die Stadterweiterung. Eine rassenhygienische Studie. Berlin. Stuttgart. Leipzig: Kohlhammer 1904. VI, 171 S. $8^{0}$ - Auch als Buch.

Stuttgart Te. H., Hab.-Schr. v. 29. 3. 1904

2875. Stïbler, E[ugen], Dr.: Der Impuls bei der Bewegung eines starren Körpers. 1906. 39 S. $8^{0}$ - Aus: Zeitschrift f. Mathematik u. Physik. Bd 54. 1907.

Stuttgart Te. H., Hab.-Schr, v. 14. 11. 1905

2876. Baumann, R[ichard]: Die Festigkeitseigenschaften der Metalle in Wärme und Kälte. Mit $46 \mathrm{Abb}$. 1907. 72 S. $4^{0}$ If Auch bei Kröner in Stuttgart.

Stuttgart Te. H., Hab.-Schr. v. 17. 3.1906

2877. Sehmidt, Martin: Das Wellengebirge der Gegend von Freudenstadt. Mit 8 Textfig. u. 2 Taf. [1907.] 99 S., 2 Taf. $8^{0}$ 
- Mitteilungen d. Geolog. Abteilung d. Kgl. württemb. Stat. Landesamtes. No. 3.

Stuttgart Te. H., Hab.-Schr. v. 26. 11. 1906

2878. Brunner, Erich: Die kathodische und anodische Stromspannungskurve bei der Elektrolyse von Jod-Jodkaliumlösungen. Mit 6 Fig. im Text. Leipzig: W. Engelmann 1907. 126 S. $8^{0}$ I Aus: Zeitschrift f. physik. Chemie. Bd 58.

Stuttgart Te. H., Hab.-Schr. v. 3.1. 1907

2879. Hilzheimer, Max, Dr.: Beitrag zur Kenntnis der nordafrikanischen Schakale nebst Bemerkungen über deren Verhältnis zu den Haushunden insbesondere nordafrikanischen und altägyptischen Hunderassen. <Aus d. Sammlung d. Zoolog. Instituts zu Strassburg.> Mit 10 Taf. u. 4 Tab. Stuttgart: Schweizerbart 1908. 111 S., 4 Tab., 10 Taf. $4^{0}$ T Auch als: Zoologica. H. 53.

Stuttgart Te. H., Hab.-Schr. v. 15. 11. 1907

2880. Obermiller, Jul[ius], Dr.: Die orientierenden Einflüsse und der Benzol- kern. Mit experimentellem Anh.: Einige Studien über die Sulfonsäuren des Phenols. Leipzig: J. A. Barth 1909. 144, 46 S. $8^{0}$

- Buchausg. ohne den Anh. Stuttgart Te. H., Hab.-Schr. v. 23. 2. 1909

2881. Wallot, J[ulius], Dr.: Stehende elektrische Wellen an ungleichartigen Drähten. Leipzig: J. A. Barth (1909). S. 495 -578. $8^{0}$ - Aus: Annalen d. Physik. Folge 4, Bd 30 .

Stuttgart Te. H., Hab.-Schr. v. 10. 11. 1909

2882. Bawer, Hugo, Dr. phil.: Einwirkung von Organomagnesiumverbindungen auf Dikarbonsäureanhydride. Ein Beitrag zur Kenntnis der Phthalide. 1911. IV, 86 S. $8^{0}$ T Kurzer Ausz. in: Archiv d. Pharmazie. Bd 247. 1909.

Stuttgart Te. H., Hab.-Schr. v. 11. 3. 1910

2883. Baum, Julius, (Dr.): Die Ulmer Plastik um 1500. Stuttgart: Hoffmann 1911.

XVI, 176 S. $4^{0}$ o Buchausg. mit 58 Taf. Stuttgart Te. H., Hab.-Schr. v. 5. 1. 1912

\section{DOKT OR-INGENIEUR-DIS SERTATI ONEN}

\section{ABTEILUNG FÜR ARCHITEKTUR}

2884. Eberbach, 0tto, Reg.-Baumeister: Die deutsche Höhenburg des Mittelalters in ihrer baulichen Anlage, Entwicklung und Konstruktion. [1903.] 63 S., 6 Taf. $8^{0}$

Stuttgart, Dr.-Ing.-Diss. v. 29. 1. 1903

2885. Klaiber, Christoph, Reg. - Baumeister: Die Grundrißbildung der deutschen Stadt im Mittelalter unter besonderer $\mathrm{Be}$ rücksichtigung der schwäbischen Lande. Berlin: Wasmuth 1912. 67 S. $4^{0}$ Durch Einleitung, Voraussetzungen u. Schluß verm. als: Beiträge zur Bauwissenschaft. H. 20. Stuttgart, Dr.-Ing.-Diss. v. 26.1. 1912

\section{ABTEILUNG FÜR BAUINGENIEUR- WESEN}

2886. Frank, W[ilhelm], Reg.-Bauführer: Uber die analytische Bestimmung der elastischen Verrückungen von Fachwerken und vollwandigen Trägern mit Anwendung auf die Berechnung von statisch unbestimmten Systemen. 1901. 58 S. $4^{0}$

Stuttgart, Dr.-Ing.-Diss. v. 22. 2. 1901

2887. Thiem, G[ünther], Ziviling. i. Leipzig: Hydrologische Methoden. Leipzig: Gebhardt 1906. 56 S., 8 Taf. $4^{0}$

Stuttgart, Dr.-Ing.-Diss. v, 25. 1. 1906

2888. Färber, R[ichard]: Der rationelle Entwurf gewölbter Bögen mit drei Gelenken.
Stuttgart: Wittwer 1907. 95 S., 3 Taf. $8^{0}$ - Um einen 2. Teil verm. u. d. T.: Dreigelenkbogenbrücken und verwandte Ingenieurbauten. 1908.

Stuttgart, Dr.-Ing.-Diss. v. 27. 11. 1907

2889. Heyd, Th[eodor]: Die Planung wirtschaftlicherStädte-Kanalisationen. 1908. 104 S., 2 Taf. $8^{0}$ - Erw. bei Haas in Mannheim u. d. T.: Die Wirtschaftlichkeit bei den StädteEntwässerungsverfahren.

Stuttgart, Dr.-Ing.-Diss. v. 11. 3. 1908

2890. Klein, Albert: Die Zeit- und Breitenbestimmung durch Beobachtung gleicher Zenitdistanzen mit Hilfe des kleinen Nonienuniversales. Mit 2 Diagr. u. 15 Tab. 1908. 132 S., 9 Taf. $8^{0}$

Stuttgart, Dr.-Ing.-Diss. v. 3. 6. 1908

2891. Heintel, Karl, Reg.-Baumeister : Die Berechnung der Einsenkung von Eisenbetonplatten und Plattenbalken. Berlin: Springer 1908. IV, 45 S., 1 Taf. $8^{0}$ I Buchausg. u. d. T.: Berechnung der Einsenkung ... 1909. Stuttgart, Dr.-Ing.-Diss. v. 27. 7. 1908

2892. Zimmermann, Karl, Reg.-Bauführer i. Stuttgart: Der Dreigelenkbogen aus Stein, Beton oder Eisenbeton. Rechnerische und zeichnerische Verfahren; Näherungsformeln und Tabellenwerte; allgemeine Formeln zur Dimensionierung rechteckiger Fugen. 1909. 109 S. $4^{0}$ - Auch bei d. Deutschen Verlags-Anstalt in Stuttgart.

Stuttgart, Dr.-Ing.-Diss. v. 11. 3. 1909 
2893. Pilgrim, Heinrich: Gewölbe- und Rahmenberechnung für Eisenbetonkonstruktionen. Mit $40 \mathrm{Abb}$. im Texte. Wiesbaden: Kreidel 1910. 43 S. $2^{0}$ - Vollst. u. d. T.: Gewölbe-, Rahmen- und kon tinuierliche Berechnung von Eisenbeton- und Eisenkonstruktionen mit Anwendung auf praktische Beispiele. 1911.

Stuttgart, Dr.-Ing.-Diss. v. 13. 1. 1910

2894. Grunsky,CarlEwald: Hydrometrische Messungs-Verfahren in den Vereinigten Staaten Amerikas. 1910. 50 S. $8^{0}$ - Aus : Zeitschrift f. Gewässerkunde. Bd 10. 1911.

Stuttgart, Dr.-Ing.-Diss. v. 7. 7. 1910

2895. Schaechterle, K(arl) W(ilhelm), Reg.-Baumeister i. Stuttgart: Beiträge zur Theorie und Berechnung der im Eisenbetonbau üblichen elastischen Bogen, Bogenstellungen und mehrstieligen Rahmen mit Beispielen aus der Praxis. Mit $91 \mathrm{Abb}$. im Text. Berlin: Ernst [1912]. VI, 117 S. $4^{0}$ - Auch als: Forscherarbeiten auf d. Gebiete d. Eisenbetons. H. 17; 2. neubearb. u. erw. Aufl. 1914. Stuttgart, Dr.-Ing.-Diss. v. 19. 7. 1911

2896. Breitung, W[ilhelm]: Auswertung von Regenbeobachtungen und Bestimmung der Regenabflussmengen für städtische $\mathrm{Ka}$ näle. [1912.] 86 S., 9 Bl. Taf. $8^{0}$ ๆ Auch bei Leineweber in Leipzig.

Stuttgart, Dr.-Ing.-Diss. v. 19. 12. 1911

2897. Locher, Hugo, Reg.-Baumeister bei d. Kgl. Württemb. Ministerialabt. f. d. Strassen- u. Wasserbau: Die Behandlung des Abwassers aus Schlachthöfen und deren Nebenbetrieben. [1912.] 63 S. $4^{0}$ - Auch im Bauzeitungs-Verl. in Stuttgart als: Neue bautechn. Literatur. H. 2.

Stuttgart, Dr.-Ing.-Diss. v. 12. 3. 1912

2898. Kommerell, otto, Kais. Baurat im Reichsamt f. d. Verwaltung d. Reichseisenbahnen: Grundlagen für die statische Berechnung von Tunnelmauerwerk. Berlin: Ernst 1912. VII, 100 S., 1 Taf. $4^{0}$ - Buchausg. vollst. u. d. T.: Statische Berechnung von Tunnelmauerwerk.

Stuttgart, Dr.-Ing.-Diss. v. 10. 7. 1912

\section{ABTEILUNG FÜR MASCHINEN- IN GENIEURWESEN EINSCHLIESZLICH DER ELEKTR OTECHNIK}

\section{Maschineningenieurwesen}

2899. Roser, E[dmund], Maschineninsp. am Ingenieurlabor. d. Techn. Hochsch. Stuttgart: Untersuchung des Grissongetriebes. Mit $53 \mathrm{Abb}$. Stuttgart: Bergsträsser 1901. 40 S., 8 Taf. $4^{0}$

Stuttgart, Dr.-Ing.-Diss. v. 13. 6. 1901
2900. Ensslin, Max, Privatdoc. an d. Techn. Hochsch. Stuttgart: Mehrmals gelagerte Kurbelwellen mit einfacher und doppelter Kröpfung. Ihre Formänderung und Anstrengung. Mit $74 \mathrm{Abb}$. 1902. VI, 154 S. $4^{0}$ - Auch bei Bergsträsser in Stuttgart. Stuttgart, Dr.-Ing.-Diss. v. 12. 3.1902

2901. Berner, Otto: Untersuchungen über den Einfluss der Art und des Wechsels der Belastung auf die elastischen und bleibenden Formänderungen. Mit 5 Fig. im Text u. 5 lithogr. Taf. Berlin: (Springer) 1903. 72 S., 5 Taf. $8^{0}$ - Auch als Buch.

Stuttgart, Dr.-Ing.-Diss. v. 18. 12.1902

2902. Menzel, Alfred, Reg.-Bauführer: Untersuchungen über das bei Bestimmung der Druckelasticität übliche Verfahren, die Dehnungen an der Mantelfläche der Versuchskörper zu messen. [1903.] $44 \mathrm{~S} .4^{0}$

Stuttgart, Dr.-Ing.-Diss. v. 18. 12.1902

2903. Stahl, Hugo: Untersuchung des Auslaufweges elektrischer Aufzüge. 1904. 46 S. $4^{0}$ - Auch in: Mitteilungen über Forschungsarbeiten auf d. Gebiete d. Ingenieurwesens. H. 20; Ausz. in: Zeitschrift d. Vereines deutscher Ingenieure. Bd 49. 1905, Halbj. 1.

Stuttgart, Dr.-Ing.-Diss. v. 18. 5. 1904

2904. Pfleiderer, Carl: Dynamische Vorgänge beim Anlauf von Maschinen mit besonderer Berücksichtigung von Hebemaschinen. Stuttgart: Wittwer 1906. IV, 84 S. $8^{0}$ - Auch als Buch.

Stuttgart, Dr.-Ing.-Diss. v. 21. 2. 1906

2905. Moser, Max: Die Beziehungen zwischen dem Eindringwiderstand und den kritischen Punkten der Eisenkohlenstofflegierungen. 1906. 56 S., 3 Taf. $8^{0}$

Stuttgart, Dr.-Ing.-Diss. v. 23. 7. 1906

2906. Desterlen, Fritz, Obering. i. Heidenheim a. d. Brenz: Beitrag zur Theorie der Francis-Turbinen. Mit Versuchen an einer 300 pferdigen Turbine. Berlin: Springer 1908. IV, 106 S., 19 Taf. $8^{0}$ I Buchausg. u. d. T.: Zur Theorie der Francis-Turbinen.

Stuttgart, Dr.-Ing.-Diss. v. 17. 7. 1907

2907. Mayer, F[riedrich]: Die Wärmetechnik des Siemens-Martinofens. 1908. 123 S., 20 Taf. $8^{0}$ Auch bei Knapp in Halle 1909; erste Veröffentlichung in: Stahl u. Eisen. Jg. 28, Halbj. 1.

Stuttgart, Dr.-Ing.-Diss. v. 14. 7. 1908

2908. Daiber, E[rnst]: Die Formänderung rechter Winkel. 1909. $78 \mathrm{~S} .8^{0}$

Stuttgart, Dr.-Ing.-Diss. v. 27. 7. 1908

2909. Kirner, J[osef]: Optischer Interferenzindikator. Untersuchung über das selbsttätige Aufzeichnen des zeitlichen Ver- 
laufes sich sehr schnell ändernder und sehr hoch ansteigender Drucke, im besonderen des Gasdruckes beim Schuß. 1909. 55 S. $4^{0}$ T Auch als: Mitteilungen über Forschungsarbeiten auf d. Gebieted. Ingenieurwesens. H. 88.1910; Ausz. in: Zeitschrift d. Vereines deutscher Ingenieure. Bd 53, Halbj. 2.

Stuttgart, Dr.-Ing.-Diss. v. 20. 1. 1909

2910. Braun, Ernst: Druckschwankungen in Rohrleitungen mit Berücksichtigung der Elastizität der Flüssigkeit und des Rohrmaterials. Stuttgart: Wittwer 1909. 48 S. $8^{0}$ - Auch als Buch.

Stuttgart, Dr.-Ing.-Diss. v. 18. 6. 1909

2911. Schneider, Richard, Reg.-Bauführer: Die Erzeugung der Stirnräder-Evolventen nach dem Wälzverfahren. 1911. 62 S., 4 Taf. $8^{0}$

Stuttgart, Dr.-Ing.-Diss. v. 13. 1.1910

2912. Bretschneider, 0tto: Versuche über die Verdrehung von Stäben mit rechteckigem Querschnitt und zur Ermittlung der Längs- und Querdehnung auf Zug beanspruchter Stäbe. 1911. 40 S. $4^{0}$ I Auch in: Mitteilungen über Forschungsarbeiten auf $d$. Gebiete d. Ingenieurwesens. H. 121. 1912; Ausz. in: Zeitschrift $d$. Vereines deutscher Ingenieure. $\mathrm{Bd}$ 56. 1912, Halbj. 1.

Stuttgart, Dr.-Ing.-Diss. v. 25. 1. 1911

2913. Gugel, Christian, Reg.-Baumeister: Uber Materialzuführungsvorrichtungen an Exzenter- und Ziehpressen. Berlin: Springer 1912. 117 S. $8^{0}$ - Auch als Buch.

Stuttgart, Dr.-Ing.-Diss. v. 12. 3. 1912

\section{Elektrotechnik}

2914. Wild, J[ulius]: Die Ursache der zusätzlichen Eisenverluste in umlaufenden glatten Ringankern. Beitrag zur Frage der drehenden Hysterese. 1911. $58 \mathrm{~S} .4^{0}$ I Auch als: Mitteilungen über Forschungsarbeiten auf d. Gebiete d. Ingenieurwesens. H. 125. 1912; Ausz. in: Zeitschrift d. Vereines deutscher Ingenieure. Bd 56. 1912, Halbj. 2.

Stuttgart, Dr.-Ing.-Diss. v. 31. 5. 1911

\section{ABTEILUNG FÜR CHEMIE EIN- SCHLIESZLICH DES HÜTTENWESENS UND DER PHARMAZIE}

\section{Chemie}

2915. Kämpf, Ad[olf]: Über Nitroderivate des Phenanthrenchinons und über deren Abkömmlinge. 1903. 76 S. $8^{0}$ Vgl. Berichte d. Deutschen Chem. Gesellschaft. Jg. 35. 36. 1902.03.

Stuttgart, Dr.-Ing.-Diss. v. 9. 11. 1903
2916. Leipprand, Fritz: Ueber Trimethyı aethylen- und Tetramethylaethylennitrosobromid. 1904. VIII, 60 S. $8^{0}$ - Vgl. Berichte d. Deutschen Chem. Gesellschaft. Jg. 37.

Stuttgart, Dr.-Ing.-Diss. v. 22. 12. 1903

2917. Pay, Erwin de: Über Nitroresorcin: 1904. 58 S. $8^{0}$ Vgl. Berichte d. Deutschen Chem. Gesellschaft. Jg. 37.

Stuttgart, Dr.-Ing.-Diss. v. 22. 12. 1903

2918. Pulvermüller, Carl: Ueber Nitroderivate des -Diphenols. 1904. 41 S. $8^{0}$ Stuttgart, Dr.-Ing.-Diss. v. 22. 12. 1903

2919. Junghans, Erhard: Ueber Bromderivate des Phenanthrenchinons. 1904. 57 S. $8^{0}$ I Vgl. Berichte d. Deutschen Chem. Gesellschaft. Jg. 37.

Stuttgart, Dr.-Ing.-Diss. v. 27. 7. 1914

2920. Unger, Carl: Entwicklung der Zement-Forschung nebst neuen Versuchen auf diesem Gebiet. [1904.] II, 65 S. $8^{0}$ I Auch bei Wittwer in Stuttgart.

Stuttgart, Dr.-Ing.-Diss. v. 27. 7. 1904

2921. Ladner, Gustav: Ueber Halogenund Halogennitroderivate des Phenanthrens. 1905. VI, 32 S. $8^{0} \cdot$ Vgl. Berichte d. Deutschen Chem. Gesellschaft. Jg. 37. 1904.

Stuttgart, Dr.-Ing.-Diss. v. 15. 12. 1904

2922. Stockmayer, Hugo: Über das Anisyl-phenyl-propen und andere aus dem Anisyl-phenyl-keton mittels der Grignardschen Methode erhaltene Verbindungen. 1905. VIII, 42 S. $8^{0}$ I Vgl. Berichte d. Deutschen Chem. Gesellschaft. Jg. 37. 1904. S. 225 ff.

Stuttgart, Dr.-Ing.-Diss. v. 26. 1. 1905

2923. Wiegandt, Friedrich: Ueber $\alpha$ Methyl- und $\alpha$-Phenylstilben, sowie den Methylenäther des 3.4.Dioxystilbens und deren Bromide. 1905. 59 S. $8^{0}$ - Vgl. Berichte d. Deutschen Chem. Gesellschaft. Jg. 37. 1904. S. $1429 \mathrm{ff}$.

Stuttgart, Dr.-Ing.-Diss. v. 22. 2. 1905

2924. Grieb, Karl: Einwirkung magnesiumorganischer Verbindungen auf Aldehyde, Ketonalkohole u. $\langle 1,2\rangle$ Diketone. 1905. 50 S. $8^{0}$ Vgl. Berichte d. Deutschen Chem. Gesellschaft. Jg. 37. 1904. S. $453 \mathrm{ff}$.

Stuttgart, Dr.-Ing.-Diss. v. 26. 7. 1905

2925. Grombach, Adolf: Über fluorogene Chromophore. 1906. 94 S., 2 Tab. $8^{0}$ ๆ Vgl. J. Liebig's Annalen d. Chemie. Bd 344. S. $45 \mathrm{ff}$. Stuttgart, Dr.-Ing.-Diss. v. 25. 1. 1906

2926. Leypold, Karl: Úber Metaanethol und 3-Methoxystilben sowie den Einfluss der Metastellung der Methoxylgruppe auf die Eigenschaften derartiger Verbindungen und ihrer Derivate. 1906. 101 S. $8^{0}$ Stuttgart, Dr.-Ing.-Diss. v. 21. 2. 1906 
2927. Schall, Richard: Über synthetische Versuche mit Amidodiphensäuren und Studien in der Karbazolreihe. $1906.50 \mathrm{~S} .8^{0}$

Stuttgart, Dr.-Ing.-Diss. v. 20. 6. 1ऽ06

2928. Jetter, Wilhelm: Über das 1,3 Diphenylpropenol und Derivate. 1906. 53 S. $8^{0}$

Stuttgart, Dr.-Ing.-Diss. v. 23. 7. $1 \subseteq 06$

2929. Hofmann, Alexander: Über das o-Anethol nebst einigen Nachträgen zur Kenntnis des p-Anethols. 1906. 64 S. $8^{0}$ - Vgl. Berichte d. Deutschen Chem. Gesellschaft. Jg. 37. 38. 1904. 05.

Stuttgart, Dr.-Ing.-Diss. v. 26. 7. $1 \subseteq 06$

2930. Frank, Hermann: Ueber das pNitrobenzolazoresorcin, einige seiner Derivate sowie sein physikalisch-chemisches Verhalten als Farbstoff. 1907. 78 S. $8^{0}$

Stuttgart, Dr.-Ing.-Diss. v. 19.12. 1906

2931. Mezger, Robert: Beiträge zur Chemie des Phenanthrens und Fluorens. 1907. VI, 56 S., 4 Tab. $8^{0}$ Vol. Berichte d. Deutschen Chem. Gesellschaft. Jg. 40.

Stuttgart, Dr.-Ing.-Diss. v. 17. 7. $1 \subseteq 07$

2932. Franck, Willy: Über sterische Hinderung bei Derivaten des Nitroresorcindimethyläthers. 1908. 62 S. $8^{0}$ - Vgl. Berichte d. Deutschen Chem. Gesellschaft. Jg. 40. 1907.

Stuttgart, Dr.-Ing.-Diss. v. 19. 12. 1907

2933. Moser, Hans: Über die Addition von Brom an Stilben, $\alpha$-Methylstilben, Benzalmalonester u. seine Derivate. 1908. $61 \mathrm{~S}$. $8^{0}$ - Vgl. Berichte d. Deutschen Chem. Gesellschaft. Jg. 40. 1907.

Stuttgart, Dr.-Ing.-Diss. v. 19. 12.1907

2934. Bogisch, Alfred: Über die Einwirkungsprodukte magnesiumorganischer Verbindungen auf das o-methoxy-tolyl-phenylketon und das o-methoxy-tolyl-methylketon sowie den Einfluss der Parastellung der Methoxylgruppe auf die Eigenschaften derartiger Verbindungen. 1908.91 S. $8^{0}$

Stuttgart, Dr.-Ing.-Diss. v. 20. 5. 1908

2935. Fritz, Immanuel: Über Derivate des Hydrochinons. 1908. 120 S., 1 Taf. $8^{0}$

Stuttgart, Dr.-Ing.-Diss. v. 3. 6. 1908

2936. Schaal, Oscar: Über die Verwendung von Cyclohexylmagnesiumjodid zur Synthese von Verbindungen der alicyklischen Reihe. Beiträge zur Kenntnis der Grignard'schen Reaktion. 1909. 58 S. $8^{0}$ - Vgl. Berichte d. Deutschen Chem. Gesellschaft. Jg. 42.

Stuttgart, Dr.-Ing.-Diss. v. 4. 11. 1908

2937. Söll, Julius: Ueber die Gewinnung von Morpholchinon aus Phenanthren. 1908. $54 \mathrm{~S} .8^{0}$ - Vgl. Berichte d. Deutschen Chem. Gesellschaft. Jg. 41.

Stuttgart, Dr.-Ing.-Diss. v. 4. 11. 1908
2938. Stuitzel, Hermann: Studien in der Fluorenreihe. 1909. $43 \mathrm{~S} .8^{0}$ I Mit einigen Änderungen in: J. Liebig's Annalen d. Chemie. $\mathrm{Bd} 370$.

Stuttgart, Dr.-Ing.-Diss. v. 20. 1. 1909

2939. Widmann, Karl Th[eodor]: Ueber die Einwirkung von Salpetersäure und von salpetriger Säure auf Diketone und Ketonsäureester. 1909. 88 S. $8^{0}$ Vgl. Berichte d. Deutschen Chem. Gesellschaft. Jg. 42. S. 497 ff. $1886 \mathrm{ff}$.

Stuttgart, Dr.-Ing.-Diss. v. 14. 5. 1ᄃ09

2940. Lumpp, Hermann: Über Oxyphenanthrene und deren Abkömmlinge. 1909. 66 S. $8^{0}$ Vgl. Berichte d. Deutschen Chem. Gesellschaft. Jg. 43. 1910.

Stuttgart, Dr.-Ing.-Diss. v. 23. 7. 1909

294i. Pannwitz, Paul: Beiträge zur Chemie des Triphenylcarbinols. 1910. $76 \mathrm{~S} .8^{0}$ Stuttgart, Dr.-Ing.-Diss. v. 13. 1. 1910

2942. Reinert, Eugen: Ueber das 1-Methyl-3-cyclohexanon und Derivate desselben. 1910. VIII, 72 S. $8^{0}$

Stuttgart, Dr.-Ing.-Diss. v. 13. 1. 1910

2943. Schairer, otto: Ueber die Gewinnung von 2-Oxy-morpholchinon $\langle 2,3$, 4-Trioxy-phenanthrenchinon> aus 4-Nitrophenanthrenchinon. 1910. 63 S. $8^{0}$

Stuttgart, Dr.-Ing.-Diss. v. 11. 5. 1910

2944. Schumacher, Friedrich: Die Erzlagerstätten am Schauinsland im südwestlichen Schwarzwalde. Eine Untersuchung auf dem Gebiete der chemischen Geologie. Berlin: Krahmann 1911. 56 S., 4 Taf. $4^{0}$ I Aus: Zeitschrift f. prakt. Geologie. Jg. 19.

Stuttgart, Dr.-Ing.-Diss. v. 11. 5. 1910

2945. Meyer, Emil: Beiträge zur Auxochromtheorie. 1910. 87 S. $8^{\circ}$

Stuttgart, Dr.-Ing.-Diss. v. 7. 7. 1910

2946. Fischer, Ernst: Uber Bromnitroderivate des Phenanthrenchinons. Jena: Fischer 1910. $61 \mathrm{~S} .8^{0}$

Stuttgart, Dr.-Ing.-Diss. v. 26. 7. 1910

2947. Geiger, Otto: Beiträge zur Kenntnis der Oxyphenanthrene und Oxyphenanthrenchinone. 1910. $61 \mathrm{~S} .8^{0}$

Stuttgart, Dr.-Ing.-Diss. v. 26. 7. 1910

2948. Gentner, Carl: Über die beiden sogenannten Diphenyldinitrosacyle. 1910. 127 S. $8^{0}$

Stuttgart, Dr.-Ing.-Diss. v. 26. 7. 1910

2949. Heinle, Eugen: Über Nitro- und Aminophenanthrene und deren Abkömmlinge. 1910. $70 \mathrm{~S} .8^{0}$ I Vgl. Berichte d. Deutschen Chem. Gesellschaft. Jg. 44. 1911.

Stuttgart, Dr.-Ing.-Diss. v. 26. 7. 1910 
2950. Lamparter, $\mathbf{0}$ [skar]: Über die Einwirkung magnesiumorganischer Verbindungen auf das p-Methoxytolylphenylketon und dasp-Methoxytolylmethylketon. 1910.54 S. $8^{0}$

Stuttgart, Dr.-Ing.-Diss. v. 26. 7. 1910

2951. Spoun, Otto: Ueber die Gewinnung von Phenanthrenchinon- und Phenanthrenabkömmlingen aus 2-Nitrophenanthrenchinon. $1910.52 \mathrm{~S} .8^{0}$

Stuttgart, Dr.-Ing.-Diss. v. 26. 7. 1910

2952. Weissel, Leopoldo, Assist. am Labor. f. reine u. pharm. Chemie: Die AuxochromGesetze bei Derivaten der Terephtalsäure. 1910. 69 S. $8^{0}$ - Vgl. J. Liebig's Annalen d. Chemie. Bd 393. 1912.

Stuttgart, Dr.-Ing.-Diss. v. 26. 7. 1910

2953. Koppe, Paul: Über die elektrolytische Reduktion von Acetophenon und Benzophenon. 〈Beitrag zur Theorie der Überspannungswirkung.> 1911. 70 S. $8^{0}$ - Vgl. Zeitschrift f. Elektrochemie. Jg. 16. 1910. S. $236 \mathrm{ff}$.

Stuttgart, Dr.-Ing.-Diss. v. 15. 12. 1910

2954. Haid, August: Ueber Oxy- und Nitro-Derivate des Fluorens und Fluorenons. 1911. $48 \mathrm{~S} .8^{0}$

Stuttgart, Dr.-Ing.-Diss. v. 31. 5. 1911

2955. Dieterle, $\mathbf{H}[$ ugo ], Assist. am Labor. f. reine u. pharm. Chemie: Über die Reaktionsfähigkeit der Kohlenstoff-Doppelbindungen in mehrfach ungesättigten Ketonen. 1911. 80 S. $8^{0}$ ๆ Vgl. Berichte d. Deutschen Chem. Gesellschaft. Jg. 44.

Stuttgart, Dr.-Ing.-Diss. v. 19. 7. 1911

2956. Sauer, Eberhard: Uebergänge von der Phenanthrenchinon- zur Phenanthrenreihe. 1911. VII, $57 \mathrm{~S} .8^{0}$ ๆ Vgl. Berichte d. Deutschen Chem. Gesellschaft. Jg. 44.

Stuttgart, Dr.-Ing.-Diss. v. 19. 7. 1911

2957. Wagner, Hans: Ueber Halogenderivate des Fluorens und Bis-diphenylenäthens. 1911. VII, $44 \mathrm{~S} .8^{\circ}$ - Vgl. J. Liebig's Annalen d. Chemie. Bd 387. 1912.

Stuttgart, Dr.-Ing.-Diss. v. 19. 7. 1911

2958. Bauerle, Richard: Über das Verhalten des Pinacolins bei Grignard-Synthesen. 1911. $61 \mathrm{~S} .8^{0}$

Stuttgart, Dr.-Ing.-Diss. v. 31. 7. 1911

2959. Burr, Karl: Über Derivate des 2,5-Dimethoxybenzaldehyds. 1911. 44 S. $8^{0}$ T Vgl. Berichte d. Deutschen Chem. Gesellschaft. Jg. 40. 1907.

Stuttgart, Dr.-Ing.-Diss. v. 31. 7. 1911
2960. Renschler, Eugen: Ein Beitrag zum elektrochemischen Verhalten der Eisenoxyduloxydelektrode. 1911. 56 S. $8^{0}$

Stuttgart, Dr.-Ing.-Diss. v. 31. 7. 1911

2961. Sigwart, August: Über Hydrierung des Carbazols und Kondensationen von Chinonen mit Hexahydrocarbazol und sonstigen heterocyklischen Stickstoffverbindungen. 1911. 84 S. $8^{0}$ I Vgl. Berichte d. Deutschen Chem. Gesellschaft. Jg. 45. 1912.

Stuttgart, Dr.-Ing.-Diss. v. 31. 7. 1911

2962. Diefenthäler, 0tto: Berlinerblau und Turnbullsblau. 1912. 68 S. $8^{0}$ - Vgl. Journal f. prakt. Chemie. Bd 192. N. F. Bd 84. 1911. S. $353 \mathrm{ff}$.

Stuttgart, Dr.-Ing.-Diss. v. 19.12. 1911

2963. Aeckerle, Emil: Uebergänge von Mono- und Diketochloriden der Phenanthrenchinonreihe zur Phenanthrenreihe. Darstellung bimolekularer Phenanthrenverbindungen. 1912. VII, 55 S. $8^{0}$

Stuttgart, Dr.-Ing.-Diss. v. 12. 3. 1912

2964. Glatz, Ernst: Ueber Kondensationsprodukte von Phenanthrenchinonen mit Hydroxylamin, Phenylhydrazin, Semiearbazid und Amidoguanidin. 1912. VII, 50 S. $8^{0}$ Stuttgart, Dr.-Ing.-Diss. v. 12. 3. 1912

2965. Müller, Otto: Einfluss der Stromkonzentration auf die elektrolytische Chloratbildung und elektrochemische Bestimmung der Reaktionskonstanten der chemischen Chloratbildung. 1912. VII, 39 S. $8^{0}$ - Vgl. Festschrift. W. Nernst zu seinem 25 jähr. Doktorjubiläum gewidmet v. seinen Schülern. 1912. Stuttgart, Dr.-Ing.-Diss. v. 16. 3. 1912

2966. Burekhardt, Hermann: Beiträge zur Halochromie. 1912. 48 S. $8^{0}$ I Vgl. Berichte d. Deutschen Chem. Gesellschaft. Jg. 46. 1913.

Stuttgart, Dr.-Ing.-Diss. v. 24. 7. 1912

2967. Pay, Albrecht de: Über Derivate des Benzophenons. 1912. 46 S. $8^{0}$

Stuttgart, Dr.-Ing.-Diss. v. 24. 7. 1912

2968. Weipert, Hugo: Ueber Bromderivate des Phenanthrens und Phenanthren chinons. 1912. 62 S. $8^{0}$

Stuttgart, Dr.-Ing.-Diss. v. 24. 7. 1912

\section{Hüttenwesen}

2969. Matt, M[aximilian] F[riedrich]: Über die Herstellung von Ferrosilicium. 1911. 67 S. $8^{0}$

Stuttgart, Dr.-Ing.-Diss. v. 19. 5. 1911 


\section{PERSONENREGISTER}

Die Ziffern mit vorgesetzem S. verweisen auf die Seiten, die übrigen auf die Nummern des Verzeichnisses

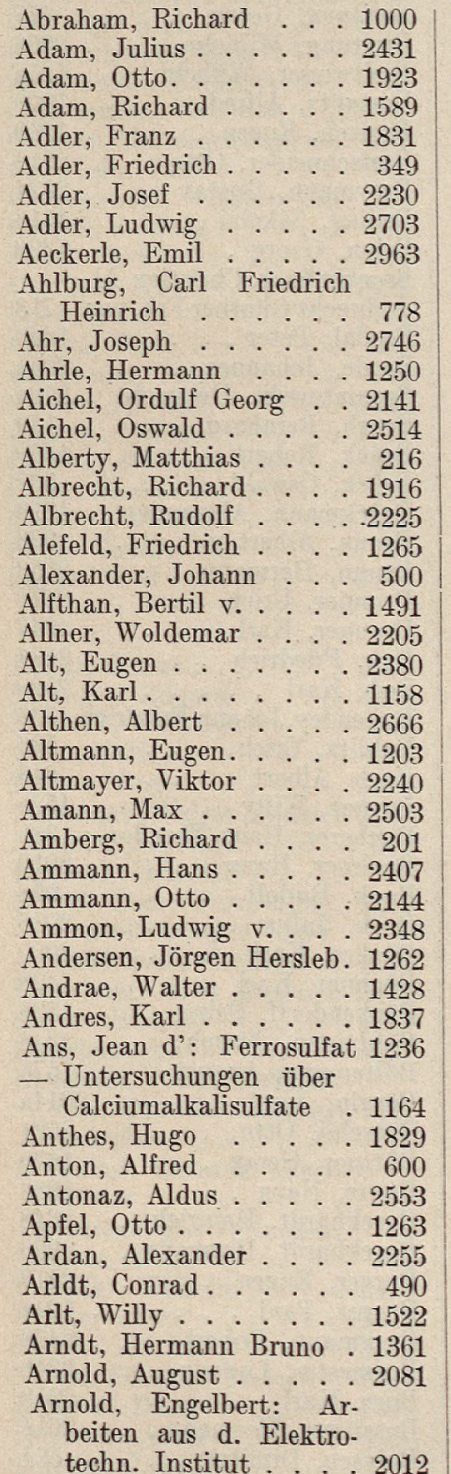

- Entwicklung d. Elektrotechnik

- Forschen, Erfinden . . 1973

- Elektrotechn. Institut. 2004

- Übergangsspannung v. Kohlebürsten

- Untersuchung d. Kommutation . 1990. 2012

Arnoldi, Heinrich . . . 2554

Asthöwer, Walter . . . 522

Attensperger, Albert . . . 2372

Auerbach, Herbert. . . 550

Auerbach, Max . . . 2053

AufseB, Otto . . . . 2361

Bach, Carl v.: Ingenieurlaboratorium ... 2827

- Materialprüfungsanstalt 2827 Bachmann, Heinrich . 1214

Bader, Friedrich. . . . 1483

Baechler, Max . . . 2221

Baer, Herbert: Maschinenlaboratorium ... 923

- Regelung v. Dampfturbinen . . . . . . 2439

Baerwald, Hans . . . 1168a

Baeumer, Wilhelm: Bedeutung d. kunstgewerbl. Unterrichts .... 2833

- Lusthaus in Stuttgart. 2827

- Bürgerl. Wohnhaus . . 2824

Bajai, Ernst . . . 2681

Baldamus, Max . . 838

Bamberg, Raimund . . 1570

Bandow, Erich . . . . 532

Bantlin, Albert . . . . 2836

Barten, Ernst $\quad . \quad . \quad . \quad 452$

Barten, Hermann . . . 1834

Barth, Alfred . . . . . 1419

Barth, Curt . . . . 114

Barth, Otto. . . . . 192

Bartz, Karl . . . . 121

Bartz, Rudolf . . . 2620

Bastian, Richard . . . 2412

Bauer, Adolf . . . . . 2086

Bauer, Franz Wilhelm . . 2090

Bauer, Hugo . . . . . 2882

Bauer, Karl. . . . . . 2732

Bauer, Ludwig . . . . 2874
Bauer, Max . . . 2519

Bauer, Otto. . . . 2742

Bauerle, Richard . . . 2958

Bauernfeind, Carl Max v.: Staatswirtsch. Ballwesen ...... 2354

- Bischoff . . . . . . 2348

- Döhlemann . . . . 2348

- Gedächtnisrede auf Ohm ...... 2348

- Gedächtnisrede auf Pauli . . . . . 2348

- Rede üb. d. Einfluss d. exacten Wissenschaften 2348

- Reden z. Einweihungsfeier. . . . . 2348

- Reichenbach . . . . 2348

- Scharrer . . . . 2348

- Soldner . . . . . 2348

- Thompson Graf von Rumford. . . . . . 2348

- Utzschneider . . . . 2352

Bauersfeld, Walther . . 421

Baum, Julius . . . . . 2883

Baumann, Edmund . . 2745

Baumann, Fritz . . . 2570

Baumann, Morand Leo 1246

Baumann, Richard . . 2876

Baumeister, Reinhard . . 1981

Baur, Carl Wilhelm . . . 2824

Baur, Ludwig . . . 1140

Bauwens, Franz . . . 120

Bayer, Richard . . . . 1682

Beck, Erich . . . . . 169

Beck, Hans . . . . . . 2555

Beck, Jakob . . . . . . 2540

Becker, Eduard . . . . 361

Becker, Erich . . . . . 930

Becker, Ernst . . . . 1507

Becker, Ferdinand Hermann . . . . . 1361

Becker, Gabriel : . . 481

Becker, Georg Albert . 1656

Becker, Hermann . . . . 174

Becker, Leonhard . . . 496

Becker, Paul . . . . . 1907

Becker, Wilhelm . . . 2253

Beckmann, Erich . . . 1911

Beckmann, Ernst: Hammer u. Presse . . . 118 
Beckmann, Ernst: Untersuchungen über Aluminate d. Bariums .

Beckurts, Heinrich : Arzneimittelsynthesen. . . . 682

- Fest-Schrift . . . . 795

- Pharmazeut. Institut . 800

- Öl d. Angosturarinde . 795

- Centrale Wasserversorgung ...... 795

Beger, Max . . . . . 2276

Behr, Heinrich v. . . . . 382

Behrendt, Walter Curt . 1448

Behrens, Johannes. . . 2035

Behrens, Wilhelm . . . 1853

Belschner, Gustav . . 2556

Bendemann, Friedrich . . 423

Benoit, Georg . . . 1973

Berblinger, Hans . . . 2200

Bereza, Stanislaus . . . 2268

Berg, Friedrich . . . 1832

Berge, Paul . . . . . 1705

Berger, Arnold Erich . 1102

Berger, Richard . . . . 2455

Berghoff-Ising, Franz . . 1096

Bergmann, Arnold . . . 115

Bergmann, Georg . . . 478

Bergwitz, Karl ... . 806

Berlowitz, Max . . . . 461

Berndt, Otto . . . . 1091

Berner, Otto . . . . 2901

Bernhard, Friedrich . . 401

Bernhard, Rudolf . . . 2557

Bertelsmann, Heinrich Wilhelm ...... 2117

Bertschinger, Hermann. . 432

Besig, Karl . . . . . 531

Betzel, Richard . . . . 2278

Beuth, Peter Christian Wilhelm . . . . . . 348

Beutner, Reinhard . . 2238

Beyer, Arthur. . . . . 1593

Beyer, Johannes . . . 582

Beyer, Kurt . . . . . 1467

Beyersdorfer, Paul . . 2712

Beyschlag, Heinrich . . 2588

Beythien, Rudolf . . . 1902

Biebrach, Kurt . . . . 1426

Bieder, Curt . . . . . 810

Biehringer, Joachim . . . 803

Biel, Rudolf ..... 422

Bieńkowski, Stanislaw von 463

Billig, Gerhard . . . 577

Binder, Ludwig . . . . . 2476

Birkenstock, Otto . . 405

Birstein, Gustav. . . . 2233

Blaeß, Viktor: Strömung in Röhren . . . . . 1167

- Zentrifugalpumpen . . 1199

Blanke, Johann Heinrich. 739

Blankenberg, Ferdinand . 1604

Blankenhorn, Alfred . . . 2016

Blasius, Johann Heinrich . 778

Blasius, Rudolf . . . 795

Blasius, Wilhelm ... 795
Bleicher, Karl . . . . 2727

Blich, Julius . . . . . 1641

Bloch, Ignaz . . . . 2486

Bloch, Leopold . . . . 2168

Blome, Hermann . . . 616

Blome, Otto v. . . . 724

Bloss, Adolf Ernst. . . . 1485

Blum, Otto . . . . 394

Blumrich, Karl . . . 1695

Bock, Ernst . . . . 1840

Bock, Hermann . . . . 2161

Bock, Paul . . . . 860

Bode, Albert . . . . 792

Bodenstedt, Friedrich . 792

Böhler, Otto . . . . 595

Böhm, Otto. . . . . 2454

Böhtlingk, Arthur: Geschichte u. Literatur . 2000

— Deutsche Kaiser . . . 1999

- Wilhelm d. Glorreiche. 2002

Boericke, Felix . . . 1572

Boeters, Oskar . . . 535

Böttcher, Hans . . . . 1545

Böttcher, Karl . . . . 1429

Böttcher, Martin . . . 1620

Boetticher, Karl . . . 354

Bogisch, Alfred . . . . 2934

Bohlmann, Robert . . 800

Bohny, Friedrich . . . 1182

Bohrmann, Ludwig . . 2242

Book, Gilbert. . . . . . 541

Boos, Eduard . . . . . 1180

Borchers, Richard . . . 1489

Borchers, Wilhelm: Einrichtungen d. Laborator. f. Metallhüttenw. .

- Institut für Metallhüttenwesen .

- Otto Intze †. . .

- Ist d. Hüttenwesen ein Zweig d. Chemie? .

- Versuche zu höchsten Wärmegraden zu gelangen. . . . . . .

Borght, Richard van der: Friedenspolitik.

- Krieg u. Volkswirtschaft .

- Rede anf Bismarck.

Bormemann, Ferdin

Borrmann, Ferdinand 1022

Borth, Walther . . . 1003

Bosch, Eberhard . . . 2507

Bosch, Johann Baptist. . 1186

Bossel, Gustav Nikolans . 1639

Bossung, Eugen. . . . 2096

Bräuler, Ludwig: Bilder aus d. Eisenbahnwesen

- Die Techn. Hochschule

Bräutigam, Max. . . . 599

Bragstad, Ole Sivert . . 2048

Brake, Ludwig . . . . 1213

Brandes, Friedrich . . 1917

Brandt, Paul: Kurbelschleife

2154
Brandt, Paul: Rathaus zu Danzig. . . . . . .

Brandt, Paul : Verblasen von Kupferstein . .

Brauer, Ernst Adolf: Berechnung von Förder-

Drahtseilen . B atrachtungen über

Maschine .. . . . 1983

Braunmühl, Anton r. . 2348

Braun, Ernst . . . . . 2910

Braun, Otto . . . . 574

Bredt, Julius: Doctor-Promotion ..... 65

- Organ. Laboratorium . 95

Breitung, Georg . . . 477

Breitung, Wilhelm . . 2896

Breitwieser, Wilhelm . . 1255

Bretnütz, Alfred. . . . 1900

Bretsch, Eugen . . . . 1269

Bretschneider, Otto . . 2912

Breymann, Gustar . . 2824

Briling, Nikolai . . . 1516

Brion, Georg . . . . 1396

Brockenburg, Christian Al-

brecht Günther Frh. von 713

Brodal, Peter . . . . . 1249

Brode, Johannes . . . 2055

Broniatowski, Heinrich. 2194

Bruch, Reinhard . . . 149

Bruck, Robert .... 1390

Brück, Oswald . . . . 537

Brüçkmann, Alexander. . 1919

Brunn, Albert v. . . . 993

Brunn, Hermann ... 2353

Brunner, Erich . . . . 2878

Brunner, Karl. . . . . 2047

Bub, Friedrich . . . . 2674

7 Bub, Karl . . . . . 2520

Bubendey, Johann Friedrich 336

Bublitz, Erich. . . . 596

Buch, Albert . . . . . 848

Bucher, Willy . . . 1526

Bucherer, Hans Theodor . 1386

Buchner, Hans . . . . 2010

Buck, Rudolf . . . . . 928

Bueb, Julius . . . . . 2088

Bücher, Karl . . . . . 2000

Büchner, Karl . . . . 1498

Bückendorff, Oskar . . . 1898

Bühlmann, Joseph. . . 2348

Bültemann, August . . . 1575

Bürgin, Josef . . . . .2014a

Bütschli, Otto. . . . . 2021

Büttner, Georg . . . . 1629

Bunte, Hans . . . . . 1982

Burckhardt, Heinrich . 2348

Burckhardt, Hermann . . 2966

Burger, Eugen . . . . 2713

Burger, Paul . . . . . 1241

Burgmann, Robert . . 2433

Burmester, Ludwig . . . 2348

Burr, Karl . . . . . . 2959

Bussjäger, Hermanı . . . 2654

Butescu, Dimitrie . . . 591 
Camerer, Rudolf: Diagramme zur Turbinentheorie . . . . . 1195

- Meisterwerk v. Wasserkraftmaschinen . . . 2356

- Versuche über Regulirung d. Rider-Steuerung 1147

Canaris, Carl . . . . 929

Carius, Arthur . . . 1176

Carl als Taufname s. Karl

Carsten, Albert: Festschrift . . . . . 984

- Gartenkunst . . . . 983

Carstens, Johann . . 1860

Carter, Frederick Edward. 2288

Cathrein, Alois . . . . 2024

Cauwenberghe, Robert Van 1015

Celichowski, Kasimir. . . 549

Christiansen, Christian . .1270a

Christie, Malcolm Grahame 204

Christlein, Paul . . . . 472

Chrzanowski, Wieslaw von 466

Claisen, Ludwig . . . . 86

Claus, Carl . . . . . 454

Claußner, Paul . . . 1021

Coers, Paul . . . . . 1798

Collett, Emil . . . . 536

Commentz, Carl . . . . 524

Conert, Herbert . . . . 1446

Contag, Hellmut . . . 1817

Cordier, Wilhelm von .. 467

Crain, Rudolf . . . . . 424

Cranz, Hermann. . . . 470

Cube, Gustav von . . 1793

Czeija, Karl . . . . 2170

Dachs, Julius . . . . 2581

Dafinger, Eduard . . . 2432

Dahmen, Reiner . . . 555

Dahn, Felix. . . . . 792

Daiber, Ernst. . . . 2908

Dammann, Walter Heinrich 1166

Danaila, Negoitza. . . 560

Danneel, Heinrich . 96

D'Ans, Jean: Ferrosulfat. 1236

- Untersuchungen über Calciumalkalisulfate $\cdot 1164$

Daunderer, Alois Anton . 2381

David, Richard . . . . 1013

Davidson, Emil . . . . 1237

Dedekind, Julius Levin Ulrich: Handelslehranstalt 697

- Lehrplan . . . . . 778

Dedekind, Richard: Festschrift . . . . 790

- Zerlegungen v. Zahlen. 795

Degener, Paul . . . 795

Deinlein, Wilhelm . . . 2440

D'Elia, Alceo . . . . . . 2621

Delisle, Alfred. . . . . 2032

Delius, Heinrich Meyer- . 1921

Dember, Harry . . . . 1399

Demeter, Adolf . . . 2521

Dengler, Leopold . . . 2107
Denicke, Gustav . . 1869

Denker, Wilhelm ... 125

Desamari, Kurt . . . . 880

Deuerling, Oswald . . . 2385

Dewitz, Hans . . . . . 1434

Dexheimer, George ... 2182

Dickert, Eugen . . . . 2635

Dieckhoff, Emil . . . . 2030

Dieckmann, Diedrich. . . 1804

Dieckmann, Theodor . 623

Dieckmann, Walter . . 1727

Diefenthäler, Otto . . 2962

Dieffenbach, Otto . . 1136

Diepen, Herman. . . . 1824

Dierssen, Heinrich . . 1855

Dieterle, Hugo . . . . . 2955

Diethelm, Hans . . . . . 814

Dietrich, Alfred . . . . 349

Dietrich, Emil . . . . 349

Dietrich, Max . . . 396

Dietrich, Walther . . . 1411

Dietz, Otto ..... 1529

Dietz, Rudolf . . . . . 1392

Dietz, Wilhelm . . . 2356

Diez, Max .... 2857

Dingeldey, Friedrich: Anwendung d. Mathemat. 1091

- Topolog. Process . . . 1135

Dittmann, Kurt Emil . . 143

Dobbert, Eduard: Bauakademie . . . 349. 359

- Gedächtniss-Feier. . . 364

- Kunstgeschichte .. 322

- Rede z. 25j. Regierungsjubiläum.

Doblhoff, Walther Frh. von 1523

Döderlein, Gustav . . . . 2425

Döhne, Ferdinand . . . 1846

Doehne, Konrad ... 435

Döhring, Karl … 1450

Dörffel, Ernst . . . . 1514

Doergens, Richard . . 327

Doerr, Albert . . . . . 2137

Doerrien, Melchior Carl. . 760

Dolch, Moritz . . . 1646

Dolch, Paul. . . . . . 1699

Dolder, Eugen . . . . 2436

Dondorff, Jakob. . . . 102

Doornkaat Koolman, Gerhard ten .... 1908

Dorfmüller, Gustav . . . 2630

Dorn, Ernst . . . . 1116

Dornig, Mario . . . . 2458

Doss, Bruno . . . . 1378

Drawe, Rudolf .... 430

Drewes, Hans

Dreyer, Gustav . . . . . 2714

Dreyer, Heinrich . . . 1838

Dreyfus, Ludwig . . . 2471

Drude, Oscar . . . . 1351

Duczmal, Casimir . . . 900

Dürre, Ernst Friedrich: Entwicklung d. Hüttenwesens ..... - Rede auf Wilhelm I. .
Du Moulin-Eckart, Richard Graf: Entwicklung d. deutschen Gedankens : 2348

- Festrede z. Jubiläum . 2348

- Haushofer . . . . 2348

Dunaj, Leon . . . 1805

Durm, Josef . . . . . 2000

Dyck, Walther von: Beziehungen im Erfassen d. Natur . . . . 2348

- Errichtung eines $\mathrm{Mu}$ seums ...... 2348

- Neue chem. Institute . 2357

- Marx . . . . . 2348

- Momente d. Entwicke-

lung d. Technik . . 2348

- Technik in Bayern . . 2356

Dyckerhoff, Kurt . . . 2258

Dyhr, Erich. . . . 1541

Eberbach, Otto . .. 2884

Eberle, Fritz . . . . . 2213

Eberlein, Wilhelm . . . 855

Ebert, Hermann. . . . 2348

Ebert, Johann Arnold . . 678

Ebinghaus, Hugo . . . 812

Ebner, Eduard . . . . 2368

Eckart, Richard Graf Du Moulin-: Entwicklung d. deutschen Gedankens . 2348

- Festrede z. Jubiläum . 2348

- Haushofer . . . . 2348

Ecker, Karl. . . . . . 2498

Edler, Robert . . . . 2080

Egerer, Heinrich. . . . . 2437

Eggeling, Heinrich . . 1370

Eggers, Franz . . . . 2127

Egle, Joseph . . . . . 2824

Ehlers, Hermann . . 820

Ehlert, Hermann . . . 1640

Ehrenberg, Kurt . . . 2131

Ehrengut, Leopold. . . . 2668

Eichel, Curt . . . . 1634

Eichwede, Ferdinand. . . 1791

Eifler, Kurt. . . . . 1477

Eiler, Karl ..... 219

Einbeck, Johannes . . . 2853

Einhorn, Alfred . . . . . 1132

Eisenreich, Kurt . . . 1657

Eisenwerth, Adolf Schmoll von . . . . 1196

Eismann, Alexander . . 2481

Eisner, Gustav . . . . 2461

Eitner, Paul . . . . 2050

Elia, Alceo D' . . . . 2621

Ellon, Kurt . . . . . 449

Elsner, Alexander . . . 1474

Emde, Hermann . . . . 800

Emperius, Adolf: Observationes in Lysiam . . 756

- De oratione Corinth. . 755

Emperius, Joh. Ferd. Friedr.: Den Manen d. Hofrathes Emperius . 
- Nothwendigkeit d. Kirchen-Reformation . . 773 Endriss, Karl . . . . . 2858 Endrös, Anton . . . . 2364 Endrös, Ludwig . . . . 2390 Engelhardt, Theodor . 2606 Engelhorn, Karl . . . 2034 Engels, Hubert . . . . 1369 Engels, William Henry. . 2272 Engelskirchen, Peter . 542 Engemann, Karl . . . 1666 Engler, Adalbert . . . . 2189 Engler, Karl: Vier Jahrzehnte chem. Forschung 2000

- Stein d. Weisen . . 1976

Englisch, Wilhelm Eugen. 2869

Ensslin, Max: Explosionsmotor. . . . 2866

- Kurbelwellen . . . 2900

Erb, Friedrich . . . 849

Erbe, Albert . . . . . 388

Erber, Josef . . . . . 2496

Erfle, Heinrich . . . . 2378

Erlinghagen, Carl . . . 2152

Ernst Friedrich Erbprinz v. Sachsen-Saalfeld 713. 714

Ernst, Adolf . . . . . 2834

Esch, Rudolf Julius . . . 1190

Escher, Artur Franz Walter von . . . . . 1683

Escherich, Karl . . . . 2041

Eschmann, Max . . . 2256

Esser, Friedrich . . . . 128

Esser, Paul Johan Arnout 1688

Essich, Eugen .... 109

Essich, Otto .... 835

Eulenstein, Fritz . . . 194

Euler, Karl . . . . . 506

Ewers, Paul ..... 2359

Faber, Georg . . . . 2057

Faber, Paul. . . . . 547

Fabricius, Johann Andreas 731

Fader, Adolfo . . . . 2124

Faehr, Paul. . . . . 2583

Färber, Richard . . . 2888

Fajans, Kasimir . . . .2074b

Fehling, Hermann . . 2824

Fehrmann, Karl . . . 485

Feinmann, Isidor . . . 875

Feistmann, Fritz . . . 2616

Feldmann, Clarence . . 1151

Feldmann, Jakob . . . 2098

Felser, Hans Ludwig . 180

FernekeB, Karl . . . . 2740

Feyerherm, Paul . . . 817

Fiechter, Ernst Robert. . 2419

Fiedler, Wilhelm . . . 1407

Fink, Eugen . . . . . 1809

Fink, Karl . . . . . . 349

Fink, Wolfram . . 2515

Finkelstein, Alfons. . . 460

Finsterwalder, Sebastian:

Braunmühl . . . 2348
Sohncke . . . . 2348

Fischer, Arthur . . . . 200

Fischer, Ernst. . . . 2946

Fischer, Ewald . . . . 1461

Fischer, Ferdinand . . 2824

Fischer, Friedrich . . . 998

Fischer, Hans . . . . 2707

Fischer, Hugo: Entwickelung d. Dampfschiffahrt 1351 - Stickmaschine . . . 1373 Fischer, Kurt . . . . . 1224

Fischer, Otto . . . . 901

Fischer, Paul . . . . 1580

Flachslaender, Joseph . . 2487

Flamm, Oswald ... 340

Fleig, Eduard . . . . . 924

Fleischhacker, David. . . 2723

Fleischmann, Fritz . . 2219

Flessa, Franz . . . . 2533

Flor, Konrad . . . . . 2688

Fluhr, Robert . . . . 132

Fodor, Otto. ..... 578

Föppl, August . . . . 2348

Föppl, Otto. . . . . 215

Förg, Karl . . . . . 2386

Foerster, Ernst: Bedeutung d. flüss. Feuerung 512

Förster, Ernst: Untersuchungen an Kreiselpumpen. . . . . 1505

Föttinger, Hermann . . 2428

Fonda, Gorton Rosa . . 2263

Forbát, Emerich . . . 1171

Forch, Carl . . . . . 1148

Former, Alexander . . 811

Formhals, Richard. . . 1251

Forstmann, Richard . . . 134

Fort, Osmar . . . . 1348

Fraenckel, Alfred . 2012. 2175

Fränkel, Ludwig . . . 2859

Franck, Willy . . . . 2932

Frangopol, Dumitru . . 2650

Frank, Albert: Entwickelung d. Technik . . 1782

- Fortschritte d. Kraftmaschinenbaues . . 1765

- Gedächtnissrede auf Wilhelm d. Grossen . . 1781

Frank, Hermann . . . . 2930

Frank, Wilhelm . . . . 2886

Frank-Kamenetzky, Albert 2125

Frankau, August . . . 2741

Franke, Max . . . . 874

Franke, Traugott . . . 1357

Franz, Friedrich. . . . 2626

Frauenholz, Wilhelm . 2348

Fraunberger, Fritz . . 2522

Fraunberger, Georg . . . 2369

Frei, August . . . . . 2618

Freise, Friedrich. . . . 127

Freitag, Max .... . 2636

Frentzel, Alexander . . 2607

Frentzen, Georg .. . . S. 4

Frenzel, Walther . . 1707

Frerichs, Heinrich . . . 800
Freund, Richard . . . 1675

Freyberg, Ernst Emil . . 1361

Freytag, Ludwig . . . 2408

Fricke, Ludwig . . . 1856

Fricke, Robert: Bedeutung d. allg. Abteilung 682 - Arithmet. Charakter. . 795

Friedenthal, Karl Paul. . 2130

Friederich, Walter .. 1266

Friedmann, Ignatz. . . 609

Friedrich, Hermann . . 1862

Friedrich, Konrad . . . 2415

Friedrich, Richard . . 1670

Friese, Walther . . . 1627

Friessner, Alfred ... 1565

Fritsch, Carl . . . 1160

Fritsche, Oskar . . . 1267

Fritz, Immanuel. . . . 2935

Fritzsche, Otto . . . 1508

Früh, Jean . . . . . 1663

Früh, Michael . . . . 2434

Fuchs, Gilbert . . . . 2072

Führer, Eugen . . . . 2677

Fürst, Bernhard . . . 2690

Fusch, Gustav . . . . 1803

Gaedeke, Arnold . . . 1366

Gärtner, Carl Christian: Discours à l'anniversaire de Philippine Charlotte 708

- Elegie. . . . . . . 764

- Gesetze d. Colleg. Car. 637

- Laws of the Car. Coll.. 639

- Loix du College Car. . 638

- Rede auf d. Geburtsfest Carls 1750 ......

- Rede auf d. Geburtsfest Carls 1752 ..... .

- Rede b. d. Geburtsfeste d. Philippine Charlotte 1749

- Rede b. d. Geburtsfeste d. Philippine Charlotte 1751

- Rede bei d. Vermählung v. Ernst Friedrich.

- Sammlung v. Reden. S. 40

Gaier, Julius . . . . 2076

Gallusser, Hans . . . . 2166

Gassenmeyer, Eduard . . 2382

Gasser, Max .... . 1163

Gast, Paul . . . . . 1159

Gastpar, Alfred . . . . 2870

Gatterbauer, Josef. . . 2658

Gebauer, Rudolf . . . 1621

Gebers, Friedrich . . . 1468

Gebhardt, Adolf. . . . 587

Gebing, Emil Sulger-:

Schillers Geistesentwicklung. . . . . 2348

- Hertz . . . . . . . 2348

Gedel, Louis

Gehler, Willy: Bemessung v. Rahmen .... 1493 
- Berechnung v. Nebsnspannungen . . . . 1398

Geiger, Arthur . . . . 2637

Geiger, Carl . . . . 151

Geiger, Otto . . . . . 2947

Geilenkirchen, Theodor. . 152

Geinitz, Hanns Bruno:

Braunkohlen .... 1348

- Gedächtnissrede auf L. Buch ...... 1358

- Leitpflanzen d. Rothliegenden ..... 1348

Geitmann, Hans. ... . 462

Geitz, August . . . . 2523

Gelbke, Milan .. . . 214

Geldermann, Arthur . . 1011

Gellert, Oswald . . . 1532

Gennerich, Georg . . 1876

Gensecke, Wilhelm .. . 427

Gentner, Carl . . . . . 2948

Gentzen, Felix . . . . 996

Genzmer, Ewald . . . 977

Gerber, William . . . 1441

Gerecke, Karl ‥ 819

Gerstacker, Ludwig . . 2716

Gersten, Ewald . . . . 1031

Gesell, Carl Walter . . . 1503

Gess, Felician . . . . 1351

Gessner, Ludwig . . . 1893

Geul, Albert: Mozet . 2348

- Neureuther . . . . 2348

Gewecke, Hermann . . 1228

Geyer, Georg . . . . . 1194

Geys, Karl . . . . . 2627

Giech, Carl Ludwig Graf von: Manibus .

- Rede b. d. Geburtsfeste d. Philippine Charlotte

Giech, Carl Wilhelm Graf von

Giesenhagen Karl * 1510

Giller, Willy . . . . 417

Gillhausen, Werner Giesbert . . . . . 181

Gillitzer, Georg . . 2708

Gimbel, Otto . . . 2136

Giseke, Nikolas Dietrich . 723

Glatz, Ernst . . . . . 2964

Glatzel, Richard . . . 1714

Glawe, Alfred . . . . 534

Gleye, Rudolf . . . 822

Glinz, Karl . . . . . 135

Glücksberg, Rachmil : 891

Göbel, Heinrich . . . . 1174

Goebel, Raymond . . . 1702

Goecke, Otto . . . . 1027

Goerens, Paul . . . 163

Goering, Adolf: Ansprache z. Gedächtniss v. Winkler

353

- Formen d. Eisenbahnw. 334

Goetz, Franz ... . 1193

Goetz, Hans . . . . 2442

Goffin, Oskar . . . 2203
Goldhardt, Paul . . . 1420

Goldschmidt, Rudolf . . 1225

Goldstein, Josef . . . 2102

Goldstein, Julius . . . 1152

Gompertz, Max . . . 1216

Gottlob, Harry . . . 2209

Grabe, Waiter . . . 1920

Graefe, Friedrich . . 1116

Grafe, Ernst . . . . . 1648

Gramberg, Anton . . . 1202

Graner, Paul . . . . . 1676

Grantz, Max . . . . 341

Graumann, Carl Artur . . 161

Graun, Karl Heinrich:

Passionsmusik . . . 765

- Te Deum laudamus . 767

- Der Tod Jesu . . 766. 769

Greeff, Max. . . . . 1905

Greifenhagen, Heinrich 1579

Greim, Georg . . . . . 1137

Greineder, Friedrich . . 217

Grell, Albert . . . . 320

Grether, Hans . . . 2158

Grieb, Karl . . . . 2924

Grieshammer, Willy . . 1703

Grießmann, Arno . . 1501

Griffel, Georg . . . 1826

Grisebach, August . . 2070

Grodnitzky, Boris . . . 2079

Gröber, Heinrich . . . 2438

Groebl, Anton . . . . 2691

Grohmann, Oskar . . . 1894

Grolée, André . . . 1586

Grombach, Adolf . . . 2925

Grosch, Oskar. . . . 1573

Grossmann, Albert . . 1562

Grotrian, Otto: Zum Gedächtnis v. Herrmann. 98b

- Liste der Veröffentlichungen

95

Grünewald, Richard . . 1854

Grüning, Herbert . . . 2121

Grun, Willibald . . . 1833

Grunsky, Carl Ewald . . . 2894

Gsell, Martin . . . . 2135

Gümbel, Hermann . . . 2749

Gümbel, Ludwig . . . 516

Günther, Emil . . . . 146

Günther, Karl . . . . 1462

Günther, Ludwig . . . 2512

Günther, Siegmund: Blinkhorn

- Braunmühl. . . . 2348

- Goetz ... 2348

- Bayer. Kartenwesen. . 2356

- Meloier . . . . . . 2348

- Rückblick auf techn. Schulwesen . . . . 2356

- Stieve . . . . . 2348

Günzburg, Jakob . . . 2277

Günzler, Heinrich . . . 1264

Guilielmus als Taufname s. Wilhelm

Gugel, Christian

2913

Guggiari, Pedro Bruno 590
Gugler, Bernhard . . . , 2824

Gurlitt, Cornelius . . . 1368

Gutermuth, Max Friedrich:

Einfluss v. Wissenschaft

auf $d$. Technik.

1091

- Einrichtungen d. Kraftwerkes

Guth, Max .. . . . 378

Gutman, Emil . . . 2136

Gutmann, Salomon .. 2571

Gutowsky, Nikolaus . . 175

Haake, Heinrich . . . 1209

Haber, Fritz . . . . . 2037

Habermehl, Karl . . . . 2387

Händel, Georg Friedrich · 729

Hänel, Adolph v.: Ab.

handlung über Gitter-

balken .

2824

- Kräfte belasteter

Balken ......

- Theorie der Tonnengewölbe . . . . . . 2827

Häusser, Friedrich . . . 2429

Häussermann, Carl . . . 2835

Häussler, Robert . . . 2724

Hage, Hans . . . . . . 2389

Hagemann, Ferdinand . . 1712

Hager, Georg . . . . . 1630

Hahn, Martin ... . . 2356

Haid, August . . . . . 2954

Haid, Matthäus: Gestalt

d. Erde. . . . . 1980

- Ziele d. Erdmessung 1985

Haimberger, Paul Frh. von 1002

Haller, Stanislaus . . . 2363

Hallmann, Karl . . . . 212

Hallo, Herman Sybrand:

Eigenschaften d. Kas-

kadenumformers 2012. 2180

- Spaltpolumformer 2012. 2068

Halmai, Béla . . . . 2254

Halperin, Isaak . . . . 2109

Hamel, Georg . . . 2051

Hammitzsch, Martin . . 1413

Handorff, Franz von . . 1823

Hanemann, Heinrich . 611

Hans, Wilhelm .. . . 2728

Hansen, Adolph . . . 1134

Hansen, Cay Roll-. . . 1270

Hansen, David Ernest . . 2284

Hanszel, Hubert. . . . 444

Harbich, Johannes . . 1230

Hardebeck, Carl . . 207

Harenberg, Johann Christoph: Disputatio de Tatarorum vestigiis . . 750

- Stirpis Estensis origines 747

- De parabolanis . . 748

- Programma Brunsvicense primum ....

- Theologia Christianorum ..... 741

Harkort, Hermann ... 166 
Harnack, Otto: Goethe's Verhältnis zu Shakespeare

- Verwendung hist. Stoffe 1097

Harnickell, Wilhelm . . . 932

Harster, Richard . . . . 2602

Hartig,Ernst: Mittheilungen 1361

- Versuche über Kraftbedarf. . . . 1361

- Versuche über Leistung 1361

Hartig, Otto . . . . 2367

Hartmann, August . . 2639

Hartmann, Ernst . . . 862

Hartmann, Max . . 1672

Hartmann, Robert. ... 813

Hartung, Hugo: Baukunst 1351

- Grundlagen d. Hochschulunterrichtes . . 1351

Hartwig, Ludwig . . . . 861

Harwig; Gerhard . . . 397

Haslinger, Carl . . . . 552

Hauck, Guido: Innere Anschauung .... 332

- Perspektiv. Apparat . 349

- Enthüllungsfeier des Hauck-Denkmals . . 370

- Grenzen zwischen Malerei und Plastik

- Rede z. Gedäch tnisfeier 368

Hauenstein, Leonhard . . 2547

Hauffe, Walter ... . 1478

Hauser, Friedrich . . . 2393

Hauser, Gottfried . . . 2483

Haushofer, Karl v.: Aufgaben d. techn. Hochschule . . . . . 2348

- Rückblicke . . . 2348

- Ziele u. Wege . . . . 2348

Haushofer, Max: Karl von Haushofer . . . 2348

- Hertz . . . . . 2348

- Stellung d. Technikers 2348

Hausrath, Hans . . . . 2036

Hausrath, Herbert . . . 2061

Haussmann, Karl . . . 2862

Havas, Béla . . . . 2264

Havestadt, Christian: Verhalten d. Tidewelle :

Havestadt, Christian: Verwendung v. Heberverschlüssen. . . . . 399

Hecht, Hermann . . . 379

Hecht, Leopold . . . 1023

Hecker, Hermann . . . 100

Heidebroek, Enno. . . 1822

Heiduschka, Alfred . . 1553

Heigelin, Karl Marcell . . 2837

Heikel, Gunnar . . . . 1863

Heilbronner, Martin . . 2119

Heilbronner, Wilhelm . . 2545

Heilemann, Walter . . 1512

Heiligenthal, Roman Friedrich . . . . . . 2132

Heiliger, Ernst Anton . 706

Heine, Bruno . . . . 418
Heine, Gustav . . . 1348

Heinel, Carl . . . . . 428

Heinemann, Willy . . 1435

Heinle, Eugen . . . . 2949

Heintel, Karl . . . . 2891

Heinz, Wilhelm .... 1794

Heinzelmann, Alfred . . 1029

Heinzerling, Friedrich: Entwickelung d. Brükkenbaues ...... Sprengwerke .... 95

- Treppendächer . . . 95

- Vermittelungs-Gesetz . 60

Heis, Leonhard . . . 2398

Hellenschmidt, Georg . . 112

Heller, Eduard .... . 246

Heller, Wilhelm .... 380

Helling, Hermann . . . 1019

Hellmann, Emil . . 124

Hellpach, Willy . . . 2059

Helm, Georg: Entwickelung d. techn. Hochschulen ...... 1351

— Stellung d. Theorie . 1351

Hempel, Hubert . . . 2248

Hempel, Walther: Einfluss $d$. Technik . 1351

— Erziehung . . . . . 1351

- Gasanalyse. . . . . 1374

Hempelmann, August . . 2155

Henglein, Martin . . 2071

Henkel, Paul . . . . 1886

Henkelmann, Josef . 1009

Henle, Ernst . . . . . 2356

Henneberg, Lebrecht . . 1116

Henneking, Carl .. . 409

Hennicke, Hans . 1864

Henning, Wilhelm . . 1601

Henseling, Friedrich . . 2218

Hensen, Caspar . . . . 206

Herberg, Georg . . . . 1499

Herbst, Waldemar . . 2410

Hercher, Ludwig . . . . 1172

Hering, Georg. . . . 1689

Hermann, Ludwig. . . 2535

Hermsdorf, Walter . . 1569

Herold, Ignaz .... 2524

Herpich, Hans . . . . 2392

Herramhof, Heinrich . . 2532

Herrle, Hanns. . . . . 2596

Herrmann, Georg . . . 2597

Herrmann, Gustav: Gewerbl. Arbeit

- Ausstellung für Unfallverhütung.

- EinfluB d. techn. Wiss.

Zum Gedächtnis .

- Reibungswinkel. . . . 81

Herrmann, Max 2488

Herrschel, Paul . . . 1607

Hertwig, August . . . 70

Herwegen, Leo .... 140

Herz, Paul . . . . . 544

Herzberg, Franz 1719

Herzfeld, Beni . . . 1221
Herzfeld, Eugen . . . 2578

Herzfeld, Robert . . . 2106

Herzog, Gustav . . . 2527

Herzog, Reginald Oliver . 2056

Hesse, Paul . . . . . 1857

Hesse, Robert . . . 187

Hettner, Georg . . . . 338

Heumann, Hermann . . 1006

Heumann, Johann David. 733

Heuser, Emil . . . . 2245

Heußinger, Konrad . . 1430

Heyd, Theodor . . . 2889

Heydenreich, Adolf . . 2383

Heydenreich, Karl . . 2651

Heyl, Georg . . . . . 1146

Heymann, Oscar Friedrich 1594

Heymann, Stanislaw:

Nitrophenylketone .. 2193

- Xylylnitrophenylketone 2103

Heyn, Rudolph . . . 1351

Hiemenz, Hans . . . . 1204

Hiemenz, Wilhelm .. 1240

Hiendlmayr, Adolf. . . . 2566

Hierholzer, Karl . . . . 2015

Hiersche, Waldemar . . 1455

Hilbing, Wilhelm ... 211

Hilgenstock, Karl . . . . 133

Hillebrand, Franz . . . 2480

Hiller, Fritz . . . . . 2287

Hilpert, August . . . . 440

Hilpert, Georg .. . . 493

Hilpoltsteiner, Johann . . 2704

Hilzheimer, Max . . . . 2879

Hinden, Heinrich . . . 1219

Hinlein, Erwin . . . . 2470

Hinrichs, Walther Theodor 1799

Hinz, Richard Reinhold . 808

Hirsch, August . . . . 71

Hirschauer, Franz . . . 2474

Hirschkind, Wilhelm . . 2259

Hirschland, Franz Herbert 1830

Hirschwald, Julius: Bau-

techn. Gesteinsunter-

suchungen .... $378 \mathrm{c}$

- Mineralog. Museum . . 374

Hocheder, Karl . . . . 2348

Hochschild, Heinrich. . . 455

Hoebel, Otto . . . . 1868

Hoefer, Kurt . . . . 482

Höfle, Jakob . . . . 2573

Höhle, Heinrich . . . . . 99

Höhn, Fritz. . . . . . 2579

Hölscher, Uvo . . . . 384

Höniger, Walter ... . 465

Hoening, Carl . . . . 457

Hoerner, Karl . . . . . 2478

Hörth, Franz . . . . 2235

Hofer, Georg . . . . . 2529

Hoffmann, Karl . . . 2400

Hoffmann, Paul: Cistercienserkirchen ... 1452

Hoffmann, Paul: Prüfung von Geschwindigkeitsmessern ...... 1004

Hofmann, Adam ... 834 
Hofmann, Adolf . . . 1878

Hofmann, Alexander . . 2929

Hofmann, Franz Josef . 2456

Hofmann, Karl: Beiträge zum Chinophtalon . 2504

Hofmann, Karl: Dom zu Worms ..... 1095

Hofmann, Richard. . . 1673

Hofstädter, Erich . . . 1578

Hohage, Carl . . . . 1218

Hohenner, Heinrich . . 2409

Holdermann, Karl . . 2201

Hollaender, Josef . . . 561

Hollenweger, Wilhelm . . 2202

Holm, Fritz. . . . 507

Holtmeyer, Aloys . . . 1410

Holtzmann, Friedrich . . .2074a

Holtzmann, Karl: Zur Erinnerung . . . . . 2841

- Theorie d. Capillarität. 2824

- Vertheilung d. Drucks. 2824

— Wellenbewegung . . 2824

Holwech, Wilhelm . . 2246

Holz, Nikolaus .... 92

Hommel, Gustav . . . 2475

Honsell, Max . . . . 2009

Horn, Fritz . . . . 518

Horn, Jakob . . . . 1104

Hoyer, Egbert von: Erörterungen betr. techn. Unterricht

2348

— Faserstoffindustrie . 2356

Hoyer, Emil . . . . . 533

Hoyer, Heinrich . . . 1889

Huber, Eugen . . . . . 2685

Huber, Franz Josef . . . 2371

Hübner, Hans. . . . . . 2450

Hübner, Richard . . 1697

Hübsch, Karl . . . . . 2388

Hülsse, Heinrich Judenfeind- . . . . . . 1361

Hülsse, Herrmann Judenfeind- . . . . . .. 1361

Hülsse, Julius Ambrosius: Einrichtung v. Knappschaftscassen ... 1348

- Krankencassen . . . . 1348

- Polytechn. Schule . 1359

Hüser, Friedrich. . . . 933a

Hufschmidt, Carl . . . 1865

Hug, René . . . . . 2274

Hugershoff, Reinhard:

Fehler v. Höhenunterschieden . . . . 1401

- Zustand d. Atmosphäre 1495

Huldschiner, Gottfried . . 2466

Humburg, Karl . . . . 2479

Huppertz, Wilhelm . . 153

Jacob, Artur . . . . 1661

Jacobi, Ernst . . . . 2153

Jacobi, Georg v. . . . 1789

Jacobsthal, Eduard: Araceenformen ... 349
- Boettichers Leistungen 354

- Rückblicke auf die Prinzipien Schinkels ... 325

Jacoby, Hans . . . . 1613

Jaeger, Hans . . . . 1205

Jaeger, Paul . . . . . 854

Jähkel, Leopold Ferdinand: Behandlung d. Seide. . 1348

- Blitzableiter . . . . 1348

- Fabrikation des Reitzeugleders . . . . 1348

- Fabrikation d. Stearin-

kerzen . Notizen betr. Leder. . 1348

- Winke betr. Argentanblech ..... 1348

Jänecke, Wilhelm . . . 1788

Jännicke, Wilhelm. . . 1138

Jahn, Johannes: Einwirkung v. Gasen. . . 1698

Jahn, Johannes: Ventilsteuerungen . . . 528

Jakob, Fritz . . . . 2584

Jakob, Georg . . . . 2403

Jakob, Max. . . . . . 2464

Jankelewicz, Boris . . 2101

Jasinsky, Wsewolod . . . 1515

Jastrow, Fritz . . . . 438

Jaufmann, Josef . . . 2379

Ichenhäuser, Ernst . . 2536

Idaszewski, Kasimir Sigismund .

Jerusalem, Johann Friedrich Wilhelm: Nachricht v. d. Colleg. Car.

- Vorläuffige Nachricht.

Jetter, Wilhelm . . . 2928

Imhoff, Karl . . . 1462

Immenkötter, Theodor . 105

Intze, Otto: Ausführungen auf wasserwirtsch. Gebiete .......

— Gedächtnisrede . . . 97

- Welche Mittel bietet d. Techn. Hochschule? .

Jochheim, Hermann . 1248

Johannsen, Otto . . . 2860

Joisten, Anton . . . . 189

Jonas, Edward . . 2012. 2177

Joost, Kurt . . . . . 1649

Jordan, Franz . . . . 412

Jordan, Friedrich . 2012. 2179

Jordan, Hermann . . . 1810

Jordan, Julius . . . . 1443

Josse, Emil: Ausbildung d. Ingenieurs . . . 372

- Mittheilungen aus d.Maschinen-Laboratorium . 376 Irmscher, Camillo . . . 1602 Iscu, Vasile . . . . 1728 Ising, Franz Berghoff- . . 1096 Israel, Otto . . . 1496 Judenfeind-Hülsse, Heinrich . . . . . 1361
Judenfeind-Hülsse, Herrmann ....... 1361

Jüngling, Hugo . . . 1361

Jürgens, Boris ... . . 2275

Jürgens, Enno: Auflösung linearer Gleichungssysteme ...... 83

- Stellung d. Mathematik 67

Jung, Adalbert . . . 618

Junghans, Erhard . . . 2919

Just, Gerhard . . . . 2063

Just, Karl . . . . . . 1211

Just, Leopold ... . . 2017

Jwanowski, Waclaw . . 2611

Kačer, Filip .. . . . 2190

Kaden, Johannes : . . 2719

Kähler, Wilhelm: Zweijähr. Kursus f. Handelswiss.

- Nationalökonomie und Ingenieurbildung... .

- Staat u. Vaterland . . 73

- Stand d. Handelshochschul-Bewegung . $\quad 95$

- Wie studiert man an e. Handelshochschule?. . 94

Kämpf, Adolf . . . . 2915

Kahn, Max . . . . . . 2167

Kahnert, Paul . . . 614

Kaim, Franz . . . . . 2852

Kaiser, Fritz . . . . . 1581

Kaiser, Hans . . . . . 2604

Kalb, Otto . . . . . 2549

Kallmorgen, Walther ... 1001

Kamenetzky, Albert Frank- 2125

Kammerer, Otto: Ansprache bei d. Feier f. Wiebe . 361

- Aufgaben d. Dipl.-Ing. 366

- Schillers Bedeutung . 369

- Ist d. Unfreiheit d. Kultur eine Folge d. Ingenieurkunst? . . : :

- Werkzeug u. Arbeits-

- Zusammenhang d. Maschinentechnik mit Wissenschaft u. Leben

360

Karbe, Werner . . . . 475

Karl I. Herzog v. Braunschweig 631. 634. 699703. $705-707.710 .715-720$. 722. $723.725-727$

Karl Wilhelm Ferdinand Erbprinz zu Braunschweig . . . 704. 724

Karmasch, Karl: Auditorium ..... 1773

- Festrede ... 1770

- Gewerbeschule . 1767. 1768

- Katalog d. WerkzeugSammlung ..... 1772

— Polytechn. Schule . 1769

Kassel, Georg . . . 601

Kast, Hermann . . . . 2025 
Katzseh, Karl

Kauder, Hanns Julius Graf von Schweinitz und Krain Frh. von . . . 725

Kauffmann, Hugo . . . 2864

Kaufmann, Ludwig . . . 2592

Kauko, Yrjö . . . . 2269

Kaulla, Rudolf . . . . 2873

Kautzsch, Johannes . . 1244

Kautzsch, Rudolf . . . . S. 64

Kaven, August von . . 77

Kayser, Eberhard . . . . 2619

Kayser, Heinrich . . . 1105

Kayser, Otto . . . . 104

Kegel, Ernst . . . . 1542

Keinath, Georg . . . . 2472

Kelbe, Werner . . . 2023

Keller, Karl: Charakter d. techn. Umwälzungen . 1979

- Redtenbacher .. . 2000

- Reichenbach . . . . 2008

Kellermann, Heinrich . . 570

Kempf, Günther . . . 1530

Keppeler, Gustav . . . 1153

Kerner, Fritz . . . . . 468

Kieninger, Max . . . . 2701

Kießling, Ludwig . . . . 2736

Kießling, Walther . . . 1870

Kiliani, Heinrich . . . 2348

Kimmel, Hermann. . . 2397

Kinkel, Manfred . . . 1815

Kinsky, Josef Jenö . . 2232

Kircher, Wilhelm . . . 1899

Kirchhoff, Rudolf . . . 1482

Kirner, Josef . . . . . . 2909

Kirsch, Albert . . . 1008

Kirschmann, Walter . . . 145

Kissin, Schmul-Juda . 876

Kißkalt, Konrad . . 2730

Klaas, Adolph . . . 1116

Klaiber, Christoph . . 2885

Klapproth, Wilhelm . . 1850

Klatt, Hugo F. . . . . 1852

Kleeberg, Rudolf . . . . 2399

Kleemann, Andreas . . 2734

Klein, Albert . . . . 2890

Klein, Felix. . . . . . 2348

Klein, Friedrich . . . . 1904

Klein, Georg . . . . 450

Klein, Joseph: Anwendbarkeit d. Ammons . . 1133

- Bestimmungd.Ameisensäure ....... 1116

Klein, Ludwig: Naturdenkmäler .... 1987

- Physiognomie . . . 2007

Klein, Sigmund . . . . 2490

Kleiner, Erich Günther. . 2215

Kleinlogel, Adolf: Versuche mit Beton . . 1168

- Wesen d. Verbundes . 1481

Klemm, Gustav . . . . 1139

Klemperer, Herbert . . 1502

Klemperer, Ralph Leopold 1642

Klien, Ewald Richard . . 1361
Klimmer, Konrad . . . 1547

Klinckhard, Theodor . . 1892

Klinge, Constantin. . . 2089

Klingenberg, Walter . . 1438

Klingstedt, Adolf . . . . 1692

Klönne, Theodor . . . 617

Klonowski, Sigismund . 2262

Kloos, Johan Hermann: Hermannshöhle.

- Verhältnisse d. Schollengebirges . . . . 795

Klopfer, Paul . . . . 1415

Klose, Georg . . . . 398

Kloss, Max . . . . . 1457

Kluckhohn, August: Bestrebungen Wittelsbach'. scher Fürsten .....

- Gründung der techn. Hochschule . . . 2348

- Guthe ...... 2348

- Techn. Unterrichtswesen in Bayern . . . 2348

Knapp, Friedrich . . . 795

Koch, Alexander . . . 1091

Koch, Franz . . . . 1024

Koch, Gottlieb von . . 1116

Koch, Hugo . . . . . 1433

Koch, Karl Richard . . 2851

Koch, Max . . . . . 1614

Koch, Richert von .. 494

Koch, Waldemar . . . 459

Kock, Friedrich . . . . 473

Köcher, Johann Christoph: Commentatio de eruditione

- Gebet fürs Carolinum . 632

- Theologiae liturg. specimen .

Köchy, Otto . . . $98 \mathrm{~b}$

Kögler, Franz . . . 1463

Köhler, Alfred . . . 1610

Köhler, Emil Johannes . 2139

Koehler, Georg Wilhelm . 2150

Köhler, Gustav . . . . . 126

Köhler, Heinrich . . . 1765

Köhres, Hans . . . . . 1252

Kölsch, Otto . . . . 2457

Koenig, Adolf . . . 2226

König, August . . . . 1198

König, James . . . . 2210

König, Roderich. . . 2489

König, Walter: Beiträge zu Pyridinfarbstoffen . 1393

- Kenntnis v. Nitrilen . 1563

Köppen, Karl von . . 856

Körner, Carl . . . . . 779

Koerner, Theo. . . . 1605

Kösser, Fritz . . . 1439

Kohl, Julius Philipp . . 2631

Kohlhaus, Wilhelm ... 2558

Kohlmeyer, Ernst Justus. 615

Kohlmüller, Franz . . 2417

Kohlrausch, Friedrich .2348

Kohlrausch, Wilhelm . . 1765

Kohn, Franz
Kohn, Rudolf . . . . 2646

Kolb, Adelbert . . . . 1142

Koller, Emil . . . . . 2850

Kollmann, Ernst . . . . 539

Kommerell, Otto

Kon, Norbert . . . . . 2273

Koob, August . . . . 2427

Koolman, Gerhard ten Doornkaat. . . . 1908

Koopmann, Johann . . 2678

Koppe, Paul .... . 2953

Koppel, Richard . . . 1372

Korn, Richard . . . . 1414

Korn, Rudolph . . . . 1638

Korthals, Wilhelm . . 447

Koschnitzky, Michael . . 2083

Koßmann, Walter . . . 1445

Kováes, Eugen . . . 2715

Kraft, Hermann . . . . 2505

Kraft, Karl . . . . . 2499

Krain Frh. von Kauder, Hanns Julius Graf von Schweinitz und. . . 725

Kramer, Erwin . . . . 487

Kranendieck, Franz . . 1906

Krassa, Paul . . . . 2243

Kraus, Carl: Acker- u. Pflanzenbau . . . . 2356

- Pflege d. Landwirtschaftswissenschaft . . 2348

- Zustand d. Landwirtschaft

Krause, Ernst $\quad * \quad \cdot 1249$

Krause, Karl . . . . . . 1711

Krause, Martin . . . . 1351

Krausz, Moritz .... 1887

Krawinkel, Wilhelm . . 2138

Krazer, Adolf . . . . . 1973

Kretzer, Heinrich . . . 2692

Kretzschmar, Horst . . . 1566

Kreuter, Franz: Wiss. Bestrebungen ... 2348

- Manderla . . . . 2348

- Wasserbau in Bayern . 2356

Krieger, Alfred . . . . 2196

Kriemler, Carl Johann . . 2046

Kritzenthaler, Fritz . . . 2693

Kröner, Hermann . . . . 2160

Krohn, Reinhold: Berufstätigkeit d. Ingenieurs. 978

- Entwicklung d. Brükkenbaues .... 974

Kroll, Adolphe Victor . 583

Kronstein, Abraham . . . 2093

Kropf, Fritz ... . . 545

Kroseberg, Wilhelm . . 898

Krüger, Walter . . . . . 445

Krug, Karl . . . . . . 1231

Krumbiegel, Ernst . . 1543

Krupp, Otto . . . . . 184

Kruschwitz, Johann . . 1447

Kryzan, Marian . . 1476

Kučera, Gottlieb . . 1155

Kühle, Josef . . . . . 122

Kühn, Emil. . . . 1725 
Kühn, Ludwig . . . . 1925

Külp, Edmund . . . . 1110

Künzel, Gustav Adolph . 1361

Kürth, Alfred . . . . 436

Küster, Heinrich . . . 1796

Kugel, Egolf . . . . . . 2679

Kuhlmann, Karl . . . 1233

Kuhlow, Kurt. . . . . 389

Kuhn, Eugen . . . . . 2593

Kumpfmiller, Alexander . 2446

Kumpmann, Walter . . 602

Kunschert, Franz . . . 857

Kunst, Friedrich . . 2546

Kunz, Eduard . . . . 2659

Kurek, Franz . . . . . 622

Kurr, Johann Gottlob . . 2824

Kurz, Karl . . . . . . 2360

Kusch, Max . . . . . 431

Kuschel, Karl . . . 1365

Kux, Eduard . . . . . 1812

Kyrieleis, Wilhelm. . . 406

Lachmann, Robert . . 892

la Cour, Jens Lassen. . . 2052

Ladner, Gustar . . . . 2921

Lahrmann, Otto . . . 2642

Lambertin, Gustav . . 1017

Lambrecht, Erich . . . 1881

Lambrecht, Justus Ludwig Daniel . . . . . 745

Lambris, Gustav . . . 208

Lamparter, Oskar . . . 2950

Lampe,Emil: Entwickelung der Mathematik . . . 328

- Hauck. . . . . . . . 368

- Rede bei der Gedenkfeier der Proklamirung 355

Lampe, Erich Hermann . 568

Landecker, Max . . . 2608

Landsberg, Theodor: Beitrag z. Theorie d. Fachwerks

- Entwickelung d. Techn. Hochschule ....

- Untersuchung über d. Raumverhältnisse . . 1123

Landsberger, Felix. . . . 567

Láng, Georg . . . . . 185

Lang, Heinrich: Geschichte der polytechn. Schule. 2000

- Chem. Laboratorium . 1996

Lange, Ludwig . . . . 1395

Lange, Theodor . . . . . 139

Langenegger, Felix . . 1431

László, Alexander . . . 2705

Lattorff, Johann Friedrich von ....... 711

Latzke, Ernst Gottlieb. . 750

Laubmann, Ernst . . . 2729

Laue, Otto . . . . . . 2550

Launhardt, Wilhelm . . 1775

Laval, Leo .... . . 170

Lawaczeck, Franz . . . 828

Lax, Wilhelm . . . . 1544
Lay, Emil _. . . . 2640

Leber, Engelbert . . . . 1721

Leberle, Hans . . . . . 2612

Lechner, Arnulf . . . . 2706

Lederer, Wilhelm . . . 2660

Lee, Harry . . . . . . 1596

Leher, Ernst . . . . . 2516

Lehmann, Arthur . . . 1582

Lehmann, Eugen . . . . 2687

Lehmann, Hermann . . 1561

Lehmann, Max . . . 144

Lehmann, Otto: Geschichte des physikal. Instituts . . 2000. 2014

- Kristallisationsmikroskop . . . . . 1992

- Physik u. Politik. . . 1984

Lehmann, Richard. . . . 2589

Lehmer, Carl . . . . . 159

Lehrburger, Karl . . . . 2567

Leibu, Josef . . . . . . 2559

Leiner, Franz . . . . . 818

Leins, Christian Friedrich v. : Architekturbild von Tübingen ...... .

- Denkschrift zur Einweihung . . . . . . 2838

- Hoflager . . . . . 2849

- Urkunde d. Stipendienstiftung . . . . 2756

Leipprand, Fritz . . . 2916

Leiser, Richard . . . . . 2067

Lemberg, Karl . . . . 1490

Lengemann, August . . . 68

Lepiarezyk, Victor. . . 610

Lepsius, Richard: Neue Gebäude d. Hochschule 1124

- Methoden des Unterrichts

1125

— Rheinstrom . . . . 1093

Lessing, Julius . . . . . 349

Leubner, Adolf . . . . 1677

Levi, Richard . . . . 2234

Levin, Ignatz . . . . . . 2082

Lewicki, Ernst . . . . 1389

Lewicki, Leonidas . . . 1351

Lewy, Max . . . . . . 1423

Leypold, Karl . . . . . 2926

Lichtenberg, Reinhold Frh. von ....... 2043

Lichtenstein, Leon . . 498

Lickfett, Herbert . . . 1026

Lieber, Max . . . . 488

Lieckfeld, Albert . . . 1717

Liedgens, Josef . . . . 627

Liemke, Otto . . . . 390

Liepe, Siegfried . . . . 113

Lierg, Friedrich Hermann 1653

Liesching, Theodor . . 177

Liese, Kurt . . . . . 2216

Lifschütz, Alexander . . 2652

Limmer, Fritz. . . . . 805

Lincke, Emil Alexander . 1700

Lindboe, Waldemar . . 1473

Linde, Otto . . . . 800
Linde, Richard . . . . 2430

Lindemann, Otto ... 1632

Lindemann, Wilhelm. . . 826

Lindig, Karl Otto . . . 1528

Lindner, Bernhard. . . . 878

Lindner, Werner . . . 1456

Linke, Willy . . . . 1926

Linker, Arthur . . . . 2173

Linnenbrügge, Hans . . . 529

Linsenmann, Hans. . . 2465

Lintner, Carl . . . . . 2356

Lipp, Andreas: Erlenmeyer . . . . . . 2348

- Miller . . . . . . . 2348

Lipp, Ludwig . . . . . 1173

Lipski, Jakob . . . . 873

Liska, Josef . . 2012. 2176

Litter, Hans … . . 1587

Locher, Hugo . . . . . . 2897

Löb, Albert . . . . . 2207

Loebel, Avram . . . . . 571

Löbel, Rudolf . . . . . 1901

Loebell, Richard . . . . 1143

Löbering, Max . . . . 2560

Lösche, Eduard . . . . . 1348

Loeser, Carl . . . . . 1873

Löw, Oskar . . . . 2633

Loewe, Ferdinand: Asimont . . . . . 2348

- Entwicklung d. Landstrassen . . . . . . . 2348

- Fischer . . . . . 2348

- Frauenholz. . 2348

- Bayer. Kartenwesen . 2356

- Wittmann . . . . . 2348

Loewenstein, Ernst . . 2644

Lohmann, Paul . . . . 1234

Lohr, Friedrich . . . 1875

Lohrmann, Wilhelm Gotthelf ....... 1348

Lohse, Fritz . . . . 1590

Lorenz, Hans . . . . . 982

Loschge, August. . . 2452

Lottermoser, Alfred . . 1387

Lucas, Georg: Entwickelung d. Eisenbahnen . 1351

- Umgestaltung d. Hochschulausbildung . . . 1351

Ludewig, Wilhelm . . . 1882

Ludin, Adolf . . . . . 2142

Ludwig, Hans . . . . 479

Ludwig, Walther ... 2054

Lübke, Wilhelm . . . 2000

Lüdicke, Arthur . . . 682

Lütke, Heinrich . . . . 925

Luft, Max . . . . 546

Luftschitz, Heinrich . . 2661

Luggin, Hans . . . . . 2040

Lukaszezyk, Jacob . . . 593

Lulofs, Warner .... 843

Lumpp, Hermann . . . . 2940

Luria, Schmera . . . 2122

Lurie, Gregor . . . . . 2077

Luther, Gerhard. . . 1206

Lutz, Karl Wolfgang . . 2366 
Lutz, Reinhold . . . . 2159

Lutzau, Gustav v. . . . 853

Lux, Emil . . . . . 871

Lynen, Wilhelm: Ausbildung z. Konstrukteur. 2348

- Eisenbahnen . . . . 2356

- Schnellzuglokomotiven 2356

- Ultsch . . . . . 2348

Mac Garvey, Frederick. . 2110

Mackowsky, Walter . . 1412

Mader, Otto ..... 2441

Mäkelt, Arthur . . . 1418

Maetzel, Johannes . . 1668

Mäule, Christian . . . 2868

Maffia, Paul . . . . 1674

Mai, Alfred . . . . . . . 2541

Maier, Johann .. . . 852

Majerczik, Wilhelm . . 501

Majmon, Heinrich . . . 2091

Makowetzky, Alexander . 2270

Mandelbaum, Robert . . 2568

Mangold, Georg . . . . . 1005

Mangoldt, Hans von: Anschauungen üb. d. Wesen d. Elektrizität ..

- Bilder aus d. Entwickelung d. Mathematik .

- Denkschrift üb. d. Er öffnungsfeier .

- Entladung einer Leidener Flasche ....

- Die Techn. Hochschule

- Rüekblick auf d. Gesch. d. Rechenkunst .. S. 4

Mann, Ludwig . . . . 403

Mannes, Hermann . . . 1839

Manns, Jacob ... . 2206

Marcus, Henri. . . . . 408

Mardus, Georg . . . 553

Marguerre, Fritz. . . 2171

Markert, Fritz . . . 1571

Markgraf, Henry . . . . 933

Martens, Adolf . . . . 378

Martens, Hans Alfred . . 1187

Martin, Alfred . . . 2574

Martin, Friedrich . . . 2249

Martin, Walter ... 1030

Marx, Karl . . . . . . 868

Marx, Karl Michael: Physikal. Sammlung ..

- Verhältniß d. Mischung zur Form .....

Masur, Tobias . . . 870

Mathes, Rudolf . . . 2518

Mathesius, Walter: Entwicklung d. Eisenindustrie.

- Imponderabilien des Hochschulstudiums .

Mathesius, Walter: Studie über Manganstahl . : . 624

Matt, Maximilian Friedrich 2969

Mattersdorff, Wilhelm . . 433
Matthaei, Adelbert: Lage d. Baukunst . 974

- Schillers Ringen . . . 986

Matthaei, Wilhelm Otto. . 510

Matthias, Franz . . . 1484

Mauch, Johann Matthäus 2824

Maurer, Eduard . . . 172

Mauvillon, Eleazar. . . . 678

May, Richard . . . . 209

May, Walther . . . 2044

Mayer, Friedrich . . . 2907

Maver, Max. . . . . 2064

Mayer, Rudolf .... 2165

Mayr, Christian . . . 2506

Mayr, Friedrich . . . 2542

Meer, Gustav ter . . . 1843

Mehlis, Heinrich . . . 2147

Mehmke, Rudolf . . . 1116

Mehner, Hans . . . . 1546

Mehrtens, Georg. . . 1351

Meier, August . . . . 2250

Memmel, Christian. . . 588

Menauer, Jakob . . . 2404

Mendheim, Hans .. . 2561

Mentz, Walter . . . . 980

Menzel, Alfred . . . 2902

Merkel, Heinrich . . . 2495

Merkel, Walther Cäcil . . 1361

Mertelsmann, Martin . 1890

Metz, Norbert . . . 620

Metzger, Josef .... 2538

Metzger, Karl … . 2638

Meuth, Hermann . . . 2151

Meuthen, Adam . . . . 196

Meyer, Alfred Gotthold: Techn. Hochschule . . 359

- Hundertjahrfeier . . . 362

Meyer, Emil . . . . . 2945

Meyer, Ernst: Thiotolyltoluidine

Meyer, Ernst von: Liebig 1351 - Stellung d. Chemie . . 1351

Meyer, Franz . . . . 1802

Meyer, Friedrich ... . 448

Meyer, Friedrich Wilhelm. 2172

Meyer, Georg: Rede auf Kaiser Friedrich

- Rede auf Kaiser Wilhelm

Meyer, Georg Isidor . . 495

Meyer, Hans . . . . . 2694

Mever, Johann Friedrich. 761

Meyer, Karl: AugustinerKlosterkirche.

Meyer, Karl: Phtaleïne. . Mever, Kurt

Meyer, Oswald ..... .

Mever, Richard: Beziehungen $z w$. Fluorescenz u. chem. Constitution.

- Probleme der organ. Chemie

- Ziele u. Aufgaben der Elektrochemie ... .

Meyer, Wilhelm Adolf . . 2286
Meyer-Delius, Heinrich . . 1921

Mezger, Robert . . . . 2931

Michaelis, August . . . 2018

Michaelis, Kurt . . . 559

Michel, Alfred. . . . 2585

Michel, Eugen ... . 1790

Mie, Gustav . . . . 2039

Mies, Otto . . . . 1215

Miethe, Adolf . . . . 339

Migula, Walter . . . 2029

Miller, Martin . . . . 2613

Miller, Paul . . . . . 2720

Miller, Oscar von: Elektr. Einrichtungen ... 2355

Miller, Wilhelm v.: Entwicklung d. Elementbegriffes .

Minnich, Carl . 2695

Mitrofanoff, Benjamin . . 2257

Mitscherlich, Harbord . . 2575

Möhlau, Richard . . . 1351

Möller, Max. . . . . . 795

Möller, Paul . . . . 426

Möllinger, Julius Adolf. . 1217

Moertlbauer, Fritz . . 2744

Mohrmann, Hans . . . 2069

Moldenhauer, Erich . . 116

Moldenhauer, Wilhelm: Beziehungen $\mathrm{b}$. elektrolyt.

Vorgängen . . . . 1238

- Einwirkung v.Sauerstoff 1161

Moll, Friedrich .... 515

Mollier, Richard . . . 1351

Monasch, Berthold. . . 1222

Monasch, Bruno . . . 1010

Monnartz, Philipp . . . 183

Moog, Otto..... 1847

Morek, Emanuel. . . . 492

Morden, Gilbert Walworth 2237

Morgenstern, Bodo Heinrich ........ . 732

Moritz, Eduard . . 2133

Moser, Hans . . . . . 2933

Moser, Max . . . 2905

Moser, Wilhelm ... . 2717

Mosler, Hugo . . . 804

Mügge, Carl .. . . . 1813

Mühlbach, Ernst . . . 2513

Mühlhaus, Hans . . . 1680

Mühlhofer, Hans . . . 2517

Mühlschlegel, Georg . . . 2453

Müller, Albert: Darstellung d. Elektrolyteisens . . 171

Müller, Albert: Studien über Wasserburg . . 2376

Müller, Arno Otto . . . 434

Müller, Bernhard . . . 2528

Müller, Christian: Abhandlung üb. Dampfschieber 2824 - Abhandlung über Kolben-Diagramme. . . 2824

Müller, Erich . . . . 1388

Müller, Friedrich . . . 1706

Müller, Heinrich: Kondensationsprodukt . . 2605 
Müller, Heinrich: Vom Kriege hinter d. Front Müller, Julius : Beiträge zur Kenntnis d. Metaphosphate ......

Müller, Julius: Bromderivate ...... 2614

Müller, Karl . . . . 2405

Müller, Max . . . . . 793

Müller, Otto: Angosturaalkaloide ..... .

Müller, Otto: Einfluss d. Stromkonzentration . . 2965

Müller, Otto: Einfluß d. Verkehrssteigerung . . 1480

Müller, Paul: Beitrag z. Theorie d. Nebenspan-

nungen
Müller, Paul: Gegenstrombremsung . . . . .

Müller, Paul: Leitfähigkeit
d. Metallegierungen .

Müller, Reinhold: Ausbildung d. Perspektive. . 682

- Beiträge zur Theorie d. Gelenkvierecks . . . 795

- Reliefperspektive . . 1103

Müller, Richard: Beiträge z. Phosphorfabrikation 1568

Müller, Richard: Versuche an Eisenbeton-Balken 1816

Müller, Willy . . . . . 1212

Müller-Breslau, Heinrich . 331

Münichsdorfer, Franz . . 2590

Münker, Emil . . . . 191

Muggenthaler, Hans . . 2718

Mustad, Ole .... . 1622

Muth, Franz . . . . . 2049

Muth, Fritz . . . . 2402

Muthesius, Hermann . 1406

Mysz, Ernst . . . . 1207

Nachtweh, Alwin ... 825

Nacke, Emil Hermann . . 1361

Nádai, Árpád . . . . . 469

Näbauer, Martin . . 2413

Näbe, Fritz . . . . . 1599

Näf, Ernst . . . . . 1664

Nägel, Adolph: Versuche an d. Gasmaschine . 1394

- Versuche über Zündgeschwindigkeit ... 1513

Naetsch, Emil. . . . 1379

Nagel, August. . . . 1348

Nagel, Willibald . . . 1144

Nagelschmidt, Ernst. . . 1025

Natalis, Friedrich . . . 845

Nathusius, Hans . . 157

Neovius, Werner . . 2228

Nerreter, Andreas . . 2459

Nettmann, Paul . . . 117

Neuenhofer, Karl . . 1841

Neugebohrn, Karl . . . 841

Neuhäusser, Hans . . . 1574
Neuman, Joseph . . . 1652

Neumann, Eugen . . . 2603

Neumann, Kurt: Untersuchung d. Arbeitsprozesses . . . . . .

- Vorgänge im Gasgenerator..... 1405

Neureuther, Gottfried von: Gedächtnisfeier . . . 2348

- Neubau d. polytechn. Schule. . . . . 2348

Nicolaus, Arthur . . . 1606

Nicolaus, Georg . . . . 453

Nicolescu, Cristea Otin . 579

Niebuhr, Herman . . . . 2174

Niedner, Franz: Beitrag $z$. Berechnung v. Schiffbrücken . . . . . 1460

- Strassenreinigung . 1404

Niedt, Hermann . . . . 188

Nielsen, Otto . . . . 628

Niemann, Richard . . 1801

Niemeyer, Rudolf . . . 1877

Nieb, Hermann . . . . 1709

Niggl, Ernst . . . . . . 2738

Niiranen, Weikko . . 2220

Niklas, Hans . . . . . 2696

Nitzelnadel, Konrad Adolf 1690

Nitzsche, Hans ... . . 1479

Noack, August . . . . . 1116

Nobis, Alfred . . . . . 1637

Noeggerath, Jakob Ernst. 1924

Noether, Fritz . . . 2074

Noldin, Fritz . . . . . 2733

Nonn, Konrad . . . 1807

Nottebohm, Friedrich Wilhelm ...... 318

Nova, Max ...... 381

Nover, Wilhelm . . . 1245

Nowak, Alfred . . . . 2647

Nuber, Ambros . . . . 2406

Nüsslin, Otto . . . . 2022

Nugel, Karl.

605

NuBelt, Wilhelm: Wärmeleitfähigkeit .... 2435

— Wärmeübergang . . . 1400

Oberhoffer, Paul ... 165

Oberländer, Eugen. . . . 2126

Obermiller, Julius . . . 2880

Oberschuir, Ewald . . 137

Oder, Moritz . . . . 395

Oebbeke, Konrad . . . 2348

Oechelhaeuser, Adolf von: Kunstgesch. Unterricht 1986

- Wege d. Denkmalpflege 1973

Oeder, Johann Ludwig: De mensura virium

- De vibratione chordarum

Oertel, Rudolf . . . . 1909

Oesterlen, Fritz . . . 2906

Oesterlin, Hermann .. . 2148
Oettel, Alfred ...... 1623

Offe, Gustar . . . . 1866

Okada, Harukichi . . 885

Oldiges, Benno . . . . 1913

Osten, Hans

Ostertag, Angust ... . 1635

Osthoff, Max Heinrich Phi-

lipp . . . . . . . 439

Otin, Cristea Nicolescu. . 579

Ott, Friedrich . . . 2670

Ott, Gotthilf ..... 154

Ott, Ludwig . . . . . 2669

Otten, Gerard.$\cdots .2085$

Ottenstein, Simon . . . 2169

Otto, Johannes ... . 1662

Otto, Karl . . . . . 1806

Otzen, Robert . . 1787

Ozorovitz, Naftaly . . 538

Pabstmann, Georg ... 2622

Paglianti, Pietro. . . . 193

Pannwitz, Paul . . . . 2941

Pape, Martin . . . . . 1842

Papperitz, Erwin . . . 1376

Parisius, Adolf $\quad 368$

Pasquay, Carl . . . . 2084

Paulcke, Wilhelm . . . 1994

Pay, Albrecht de . . . 2967

Pay, Erwin de . . . 2917

Pedersen, Harald . . . 195

Peetz, Ludwig . . . . 155

Peiseler, Gottlieb . . . 464

Peissakowitsch, Victor . . 2689

Peppermüller, Hermann:

Bibliothek . ... 95

- Nachtrag-Katalog . . 79

Perl, Alfred ..... 2617

Petersen, Otto . . 162

Petersen, Waldemar: Elektrostat. Maschinen . 1162 - Spannungsregelung . . 1226

Petri, Victor Friedrich Lebrecht: Anthologia Pindarica. . . . .

- Commentationes in Jobum . . . 757. 758

- Observationes in Pindarum ..... 753

- Wesen d. Collegii Car. 694 Petschek, Paul . . . . 1684 Pfaff, Georg . . . . . 1533

Pfahl, Werner . . . 480

Pfarr, Adolf . . . . . 1091

Pfeifer, Hermann . . 796

Pfeiffer, Friedrich . . . . 2377

Pfeiffer, Gustar . . . . 2865

Pfeil, Josef ...... 199

Pfiffner, Emil

Pfister, Hermann von . . 1116

Pfleiderer, Carl . . . . 2904

Pfnister, Paul . . . 1585

Pfotenhauer, Hermann . 866

Philip, Max . . . . 2855

Philippi, Erich 1014 
Philippi, Heinrich . . . 607

Philippine Charlotte Herzogin zu Braunschweig 708. 709. 711. 712. 721

Philips, Moritz . . . . 598

Phleps, Hermann . . . 1421

Picard, Martin . . . . 2671

Pick, Waldemar : . . . 2212

Pierstorff, Hermann . . 2128

Pietschmann, Eugen . . 1488

Pilgrim, Heinrich . . . 2893

Pilz, Richard . . . . . 1710

Pirlet, Joseph . . . . 103

Plank, Rudolph . . . . 1524

Plato, Wilhelm . . . . 991

Platou, Eilif . . . . 2265

Platzmann, Ferdinand . . 1469

Plenske, Ernst . . . . 205

Plieninger, Reginald . . 2164

Plotho, Wilhelm Julius

Edler von: Cantate. . 709

- Discours ... . 708

Plüddemann, Werner . . 2229

Pockrandt, Willy . . . 458

Pöthig, Otto . . . . . 809

Pohl, Franz . . . 1577

Pohl, Robert ..... 1914

Pohl, Theodor. . . . . 2682

Polis, Peter . . . . . 95

Pollák, Friedrich . . 2114

Pordesch, Horst . . . 1643

Postius, Karl Theodor . . 2491

Pott, Emil ... . . . 2356

Praetorius, Paul . . . 527

Prausnitz, Paul Hubert. . 1696

Preuss, Ernst: Festigkeit v. Nickelstahlnieten. - 1165

- Geschwindigkeit der Durchbiegungen . . 1200

Preusser, Conrad . . . 1451

Priester, Karl: . . . . . 2424

Probst, Emil . . . . . 402

Prochnow, Adolf . . . 877

Pröll, Arthur: Beiträge z. Schiffsschraube

- Kraftverhältnisse bei Schiffsmaschinen-Steuerungen ..... . 525

Proell, Reinhold . . . 1500

Prohaska, Ludwig . . . . 2739

ProB, Friedrich: Abhandlung über d. Viereck . 2824

- Prakt. Geometrie . . 2824

Püschel, Paul . . . . . 1361

Pütz, Otto . . . . . 1722

Pütz, Paul . . . . . 160

Pützer, Friedrich . . . 1098

Puhlmann, Ernst . . . 2095

Pulvermüller, Carl . . . 2918

Puppe, Johann . : . . 612

Purper, Emil . . . . . . 441

Purucker, Georg. . . . 2484

Putsch, Albert . . . . . 129

Puttkammer, Georg . . 869
Quasebart, Karl . . 167

Quensell, Hermann . . 563

Quietmeyer, Friedrich . . 1819

Rabbow, Fritz . . . 1821

Radt, Martin . . 2012. 2185

Raeithel, Oskar . . . . 2672

Raetze, Walther .. . 1551

Räuber, Erwin . . . . 2204

Rahtgens, Hugo . . . 1409

Railing, Adolf . . . . 2463

Rajz, Alexius . . . 2012. 2186

Ranck, Christoph . . . 388

Randhahn, Walther . . 131

Rannacher, Albert . . 1436

Rappaport, Philipp August 387

Rasch, Gustav . . . . 2033

Raßfeld, Paul .... 1694

Rauda, Fritz . . . . 1416

Raum, Johannes . . . 2737

Rauschenplat, Günzel von 197

Reber, Franz von: Anfänge

d. Kunstpflege . . . 2348

- Knoll . . . . . 2348

Recke, Oskar . . . . . 1197

Redlich, Alfred . . . . 1669

Redlich; Béla . . . . . 2662

Redtenbacher, Ferdinand. 2013

Redwitz, Oscar von . . . 792

Regensburger, Paul . . 2537

Rehbock, Theodor: Ausbildung d. Überfälle . 1991

— Gedächtnisrede . . . 2011

- Wert d. Wasserkräfte. 1973

Rehfus, Wilhelm ... 2157

Reich, Jakob . . . . 2641

Reichard, Elias Caspar: De finibus licentiae poetic.

- Natalitia Caroli . . . 702

- Ode auf d. Geburtsfest Carls .

- Redeaufd. Vermählung Friedrich V......

- Der Dänen Trost. . . 751

Reichel, Franz . . . . 82

Reichel, Walter: Bemerkungen z. elektrotechn. Unterricht . . . 378b

- Betrachtungen über Drehstrom .... 486

Reichel, Wilhelm . . . 2725

Reichle, Carl . . . . . 1475

Reidenbach, Rudolf . . . 2628

Rein, Hans . . . . . . 1232

Reindel, Robert . . . . 2663

Reiner, Friedrich . . . 1685

Reinert, Eugen . . . . 2942

Reinhardt, Otto . . . . 2104

Reinke, Otto ..... 789

Reinsberg, Willy ... 1888

Reiser, Hans . . . 1686

Reiss, Ludwig . . . 2683

Reissner, Hans . . . . 393

Reitinger, Josef . . . 2492
Remy, Karl . . . . 1818

Renk, Friedrich . . . . 1351

Renker, Max . . . . . 564

Rennebaum, Fritz . . . 2551

Renner, Otto . . . . 1701

Renschler, Eugen . . . 2960

Retschy, Curt . . . 1007

Reuleaux, Franz ... 326

Reusch, Friedrich Eduard 2824

Reuschel, Karl . . . . 1391

Reuther, Oskar . . . . 1440

Reutlinger, Ernst . . . 2443

Rheinfels, Curt . . . 1871

Rhomberg, Victor . . . 2500

Richard, Isidor . . . . 2508

Richardt, Franz . . . . 2197

Richert, Theodor . . . 2281

Richter, Erich . . . 1628

Richter, Fritz . . . . 824

Richter, Johannes . . . 1631

Richter, Paul . . . . 1715

Riebensahm, Paul . . . 446

Riedelbauch, Rudolf . . 2562

Rieder, Heinrich . . . . 2373

Riedler, Alois: Ansprache bei d. Feier f. Wiebe . 361

- Bedeutung d. Technik. 335

- Techn. Hochschulen. . 358

- Rede zur Jahrhundertwende ..... 363

Riehm, Wilhelm . . 1531

Rieppel, Paul . . . . . 429

Riesner, Hans . . . 2416

Rietschel, Hermann: Rede z. 100. Geburtstag Kaiser Wilhelms

- Stand der WohnungsHygiene . . . . . 329

Rietz, Julius . . . . . 1364

Riffel, Alexander . . . 2019

Rimann, Eberhard. . . . 1402

Rinau, Walter . . . 1903

Rinckleben, Paul . . . 896

Rindl, Max. . . . . 562

Ripke, Otto. . . . . 2260

Ritmeier, Theodor Wilhelm: De scholarum evocatione . . . . 735

- Exercitatio Carolina . 743

Ritter, Max . . . 1472

Rittershans, Emil . . . 792

Ritzmann, Friedrich . . 2140

Robinoff, Michael . . . 1268

Roch, Eugen . . . . 520

Roch, Heinrich . . . . 1611

Roch, Hermann . . . 1471

Roderburg, Alexander . . 213

Rodewald, Gustav . . 2236

Röhler, Robert . . . . 2569

Röhm, Wilhelm . . . . 2543

Roemmelt, Josef . . . 497

Römmler, Willy . . . 1608

Roessler, Ludwig von . . 1101

Rötscher, Felix . . . 425

Rogowski, Walter . . 1012 
Rohland, Paul .... 2872

Rohn, Karl . . . . . . 1351

Roll-Hansen, Cay . . . 1270

Rommel, Ernst . . . 1776

Roquette, Otto . . . 1116

Rosenberg, Marc . . . 2000

Rosenfeld, Joseph . . . 2552

Rosenow, Max . . . 1895

Rosenqvist, Oskar Routala 2247

Rosenthal, Josef. . . . 1552

Roser, Edmund . . . . . 2899

Rosintal, Josef . . . . 391

Rossem, Adriaan Carel van 1220

Rossin, Richard . . . . 1487

Rossleben, Alfred . . . 1556

Roth, Paul: Birotation der Glukose .... 1859

Roth, Paul: Festigkeitstheorien . . . . . . 411

Rothe, Alfred . . . . 1609

Rothe, Johannes . . . 519

Rothenberg, Siegfried . 2374

Rother, Paul Bernhard . 1603

Rothschild, Max. . . . 2623

Routala Rosenqvist, Oskar 2247

Rowald, Paul ..... 1792

Rožyński, Janusz . . . 2123

Rudel, Ernst . . . . . 2396

Rudolphi, Max . . . . 1149

Rück, Ulrich . . . . . 2697

Rüdenberg, Reinhold . . 1918

Rüdiger, Alfred . . . . . 1425

Rüdorff, Friedrich . . . 323

Rülf, Benno . . . . . 410

Rummel, Kurt . . . 106

Runne, Ernst . . . . . . 888

Runne, Hermann . 8 800. 889

Runte, August . . . . 1897

Ruppel, Friedrich ... 385

Russ, Rudolf . . . . . 2195

Ruths, Johannes . . . 1922

Rutowsky, Friedrich August Graf . . . . . 764

Ružička, Leopold . . . 2271

Sachs, Leon . . . 1181

Sachsenberg, Ewald . . 511

Sack, Bernhard . . . . . 2422

Sack, Michael . . . . 2192

Sadlon, Alfred ..... 594

Sahland, Hugo . . . . 1588

Saigger, Eugen . . . . 2680

Saklatwalla, Byramji. . . 608

Saladin, Otto . . . 2280

Saller, Heinrich . . . . 1189

Salm, Eduard ... . 203

Sammet, Friedrich. . . 2146

Samoylowicz, Hirsch. . . 2118

Sander, Alexander . . . 2624

Sandkuhl, Hermann . . . 210

Sandlar, Emil . . . 2702

Sanio, Paul ..... 471

Sarfert, William . . 1536

Saring, Benno . . . 1598
Saring, Georg . . . 1592

Sasinowski, Michael von . 2698

Sauer, Bruno . . . . . 792

Sauer, Eberhard. . . 2956

Saver, Cosmas . . . 2000

Schaal, Oscar . . . . 2936

Schachenmeier, Wilhelm . 2143

Schacht, Theodor: Ein-

ladung z. d. Prüfungen 1107

- Nachrichten üb. d. Gewerbschule

- Zweck d. Gewerbschule

1106. 1109

Schade, Martin . . . 1708

Schaechterle, Karl Wilhelm 2895

Schäfer, Ernst . . . . 2534

Schaefer, Georg. . . . 1116

Schaefer, Otto . . . 1828

Schäfer, Rudolf . . . . 626

Schäffer, Adolf . . . . 2576

Schärtel, Georg . . . . 2594

Schaidhauf, Alois ... . 2653

Schairer, Otto. . . . 2943

Schalk, Willem van der. . 1261

Schall, Richard . . . 2927

Schaller, Ludwig . . . 815

Schaper, Carl . . . . . 886

Schapira, Carl.

Schapringer, Sigismund von .

Scharrer Andreas * * 2662

Schatz, Desiderius . . 850

Schauseil, Walther. . . 1658

Scheck, Franz. . . . 2418

Scheerer, Felix . . . . 1432

Scheffers, Georg . . . 346

Scheffler, Wilhelm: Beiträge zur Kenntnis d. Westerwaldtone ...

Scheffler, Wilhelm: Étude sur Boileau

- Verzeichnis d. Stiftungen

1583

Scheibe, Werner . . 1437

Scheibner, Richard . . 383

Scheidemandel, Julius . . 2525

Schellbach, Johannes . . 887

Scheller, Alfred . . . . 865

Scheller, Emil . . . . 2572

Schellhaass, Hans Walther Hugo.

Schenck, Heinrich . . . 1091

Schenck, Rudolf: Beiträge zur Bestimmung d. Erweichungskoeffizienten

Schenck, Rudolf: Denkschrift üb. d. Einweihungsfeier.

Schenk, Eduard ... 929

Schenk, Julius . . . 2462

Scherbius, Arthur . . . 1827

Scherenberg, Ernst . . 792

Schering, Karl . . . . . 1091

Schertel, Ludwig . . . . 1720

Schertel, Wilhelm . . . 2632
Scheufele, Wilhelm . . 2375

Schick, Karl . . . . . 2198

Schieder, Heinrich . . 2580

Schiele, Albert . . . . 1470

Schildbach, Richard . . 1651

Schiller, Emil . . . . . 1028

Schiller, Herbert . . . 895

Schilling, August Jakob . 1141

Schimon, Otto ... . 2675

Schimrigk, Friedrich 2012. 2178

Schinkel, Max . . . . 1184

Schleicher, Alwin . . . 2599

Schleiermacher, Angust . 2026

Schlesinger, Georg .. 414

Schleuning, Wilhelm . . 2129

Schlichting, Julius . . . 324

Schlie, Klaus . . . . . 899

Schliemann, Wilhelm . . 1885

Schliephacke, Gerhard . . 1883

Schliestedt, Friedrich Wilhelm von: Empfindungen bey $d$. Tode . . 771

- Klagen über d. Verlust 770

Schlink, Wilhelm . . . 1156

Schlömilch, Oskar: Reihenentwickelungen . . 1348

- Theorie der Ketten. brückenlinien. . . . 1348

Schlösser, Paul . . . . 150

Schlötter, Max . . . 2497

Schlötzer, Adolf : . . 2414

Schlossmann, Arthur . . 1383

Schlumberger, Ernst . . 2285

Schmeitzner, Rudolf . . 1465

Schmid, Max: Friedrich d. Grosse als Bauherr . . 61

- Kaisergeburtstagsrede S. 4

Schmidt, Friedrich . . 2420

Schmidt, Heinrich Frhr. von ....... 2348

Schmidt, Julius . . . 2867

Schmidt, Karl: Berechnung der Luftpumpen.

Schmidt, Karl: Brandschiefer...... 2115

Schmidt, Karl Heinrich. . 2842

Schmidt, Martin. . . 2877

Schmidt, Max: Bauernfeind ..... 2348

- Messung d. Basis . . 2356

- Methoden d. snterirdischen Orientirung. . . 2348

Schmidt, Maximilian Paul 1616

Schmidt, Otto . . . 2710

Schmidt, Reinhold. . . 521

Schmidt, Robert . . . 101

Schmidt, Rudolf . . . 530

Schmidt, Wilhelm . . 1820

Schmidtmann, Adolf : 1462

Schmiedel, Karl . . . 1538

Schmiedel, Maximilian . 2676

Schmiedt, Friedrich . . . 565

Schmitt, Franz . . . 1178

Schmitt, Theodor . . . . 2384

Schmitz, Otto Heinrich. . 832 
Schmoll von Eisenwerth, Adolf . . . . . . 1196 Schneider, Erich. . . 1687 Schneider, Heinrich . . 1518 Schneider, Johann Bernhard: Mittheilungen üb. d. Kropfrad . . . 1348

- Turbinen ..... 1348

Schneider, John Joseph . 456

Schneider, Ludwig . . 2447

Schneider, Richard . . . 2911

Schnell, Josef . . . . 2509

Schoeller, Viktor . . . 2711

Schön, Ludwig . . . . 1227

Schoeneich, Hugo . . . 517

Schönherr, Paul ... 1564

Schönhut, Franz Karl Anton . . . .... 775

Schöppe, Willi .... 136

Schohl, Max . . . . 2223

Scholtz, Max . . . . 2031

Schossberger, Endre . . . 926

Schoy, Carl . . . . . 2394

Schrader, Ernst . . . . 1157

Schraube, Gustav . . . 625

Schrauff, Georg . . . 1520

Schreckenbach, Rudolf . . 1647

Schreiber, Hermann . . 1849

Schreiber, Karl Albert . . 1494

Schreiber, Wilhelm . . 2113

Schreiner, Otto . . . . 1257

Schrodt, Johann Heinrich: Historia belli tricennalis 749

- De scholarum rectore iudicia.

- Programma ... 746

Schröder, Ernst . . . . 1977

Schröder, Herbert . . . . 823

Schröder, Karl . . . . 1454

Schröter, Moritz: Bauschinger . . . . . 2348

- Naturwiss. u. Technik. 2348

Schröter, Moritz: Beschreibung d. Aufstellung d.

Eisenbahnbrücke bei

Neckarelz . . . . 2824

Schroth, Walter . . 1032

Schuberg, Karl: Forschungsaufgaben .. 1975

- Wuchsverhältnisse . . 2000

Schubert, Johann Andreas: Andeutungen über Dampfschiffahrt . . 1348

- Anwendbarkeit d. Flügelgebläses . . . . 1348

- Polytechn. Schule. . 1357

— Stützlinien. . . . 1348

- Stützung d. Wölbbogen 1348

- Theorie der Druckturbinen . . . . . 1348

- Versuch einer Begründung d. Mechanik . 1348

Schubert, Karl . . . 1659

Schubert, Kurt . . . . 1492

Schubert, Max . . . 1380
Schubert, Otto ... 1422

Schüppel, Wilhelm . . 1910

Schürmann, Eugen . . 419

Schütz, Ernst . . . . 164

Sehütz, Heinrich ... 1814

Schütz, Renatus. . . . 1185

Schütz, Wilhelm . . 186

Schuh, Heinrich . . . 2664

Schuller, Aladár . . . . 584

Schulte, Wilhelm ... . 554

Schultheiss, Christoph . . 2028

Schultz, Clarence Benjamin 508

Schultz, Eduard. . . 2735

Schultz, Gustav: Glas-

industrie Bayerns. . . 2356

- Chemische Industrie

Bayerns .

- Tonindustrie Bayerns 2356

Schultz, Roland ... 1879

Schulz, Erdmann . . . 931

Schulz, Walter . . . 1260

Schulz, Wilhelm. .57

Schulze, Armin . . . 1576

Schulze, Günther . . . 1912

Schulze, Walter. . . . 1525

Schumacher, Friedrich . . 2944

Schumacher, Fritz . . 1351

Schumacher, Willy . . 1554

Schumann, Philipp . . . 2501

Schumann, Winfried Otto. 2187

Schur, Friedrich. . . 1988

Schuster, Matthäus ... 2565

Schuster, Paul . . . . 1521

Schuster, Siegfried. . . . 897

Schwab, Georg ... . 2530

Schwabach, Max Carl Günther . . . 420

Schwabe, Erwin . . . 1618

Schwaiger, Anton: Belastungsausgleich . . 2073

- Regulierproblem . . 2468

Schwaighofer, Hans . . . 2362

Schwalbe, Arthur . . . 1591

Schwalbe, Carl Gustav. 1154

Schwarz, Willy ... . 2586

Schwarze, Bruno . 836

Schwarzenau, Hans Christian Frh. von: Cantate

- Rede auf d. Geburtsfest Carls

Schweinitz und Krain Frh. von Kauder, Hanns Julius Graf von

Schweisgut, Ferdinand . . 2684

Schweitzer, Alexander . . 1633

Schweitzer, Rudolf . . . 2092

Schwetje, Heinrich . . 483

Sedlmayr, Theodor . . . 2502

Seebeck, August: Rede b. Eröffnung d. neuen Anstaltsgebäudes . . . 1348

- Schwingungen . . 1348

- Zurückwerfung des Schalles......

1348
Seefehlner, Egon Ewald:

Beitrag zur Theorie d.

Synchron-Motoren . .

- Methoden z. Wechsel-

stromuntersuchungen . 1384

Seeger, Max . . . . . 2289

Seel, Eugen . . . . 2871

Seelig, Eduard . . . . . 2854

Seeligmann, Franz . . 2217

Seer, Christian . . . . 2227

Seidel, Arno . . . . . 1584

Seidel, Friedrich. . . . 1600

Seidler, Johann Wilhelm. 761

Seidner, Michael. . . . 502

Seidner, Salomon . . . 2087

Seiler, Carl . . . . . . 130

Sell, Hans . . . . . . 575

Selter, Fritz . . . . . . 476

Sendtner, Albert . . . . 2445

Sensburg, Waldemar .2370

Seyde, Franz . . . . 1595

Seyrich, Karl Arno . . 1527

Sgalitzer, Friedrich . . 2673

Sichling, Konrad . . . 890

Sidow, Hans . . . . . 1800

Siebert, Werner . . . . 1239

Siebert, Wilhelm . . . . 844

Siedler, Eduard Jobst . . 1177

Siefert, Xaver. . . . 1989

Siegel, Gustav . . . 1223

Siegfried, Erich . . . 1726

Siegler, Max . . . . 2699

Siegler, Robert . . . . . 1253

Sieglerschmidt, Hermann. 1511

Siemann, Richard . . . . 514

Siemonsen, Ludwig . . . 1861

Sieveking, Hermann . . 2058

Sievers, Otto . . . . . 792

Sigwart, August $\quad . \quad . \quad 2961$

Siller, Rudolf . . . . . . 2615

Singer, Felix . . . . . . 569

Skita, Aladar . . . . . 2060

Skutsch, Rudolf . . . 833

Slaby, Adolf: Ansprache b. d. Boetticher-Feier. 354

- Gesetz von d. Erhaltung d. Energie . . .

Sleumer, Hermann Josef Smissen, Heinrich van der 551 Sobbe, Carl . . . . . 442 Söll, Julius . . . . . . . 2937

Söllner, Fritz . . . . . 2595

Soennecken, Alfred . . . 2449

Sohncke, Leonhard . . 2348

Sohrmann, Johannes. . 1417

Sommer, Albert . . . . 1558

Sommerfeld, Arnold . . . 95

Sonne, Eduard: Entwickelung d. Techn. Hochschule . . . . . 1116

- Schiffswiderstand . 1116

Sonnenburg, Ernest Friedrich . ..... 1626

Sophia Antoinette Prinzessin von Braunschweig 713. 714 
Soxhlet, Franz v.: Voit . 2348 - Wollny . . . . 2348 Spangenberg, Albert. . . 1258 Spanier, Eugen . . . . 2239 Speck, Artur . . . . . 1466 Speer, Oskar . . . . . 837 Speithel, Robert . . . 2267 Spengler, Oscar . . . . . 858 Spielberg, Hermann . . . 353 Spielmann, Friedrich. . . 119 Spielvogel, Moritz . . . 2111 Spies, Fritz . . . . 879 Spillner, Friedrich Gustav 1891 Spindler, Heinrich . . 2863 Spitz, Carl . . . . . 1247 Spoun, Otto . . . . 2951 Sprent, Colin . . . . 1650 Springorum, Friedrich . . 176 Sproesser, Ludwig . . . 1550 Stadeler, August . . . 168

Stadler, Hermann . . . 2358

Staeble, Rupert . . . . 2493

Stäckel, Paul . . . . . 1973

Stahl, Hugo

Stange, Alfred . . . . 2108

Stark, Johannes . . . 75

Starke, Kurt . . . . . . 1617

Statz, Paul . . . . . . 2291

Stauber, Georg . . . . 416

Stauch, Adolf.. .526

Staus, Anton . . . . . 2149

Steche, Richard . . . 1375

Stecher, Emil . . . . . . 586

Steffe, Hermann . . . 604

Stegmüller, Philipp . . 2231

Steidle, Hans Carl . . . 2469

Steil, Edmund . . . . . 504

Steimmig, Franz . . . 1567

Stein, Albrecht . . . . 1678

Stein, Wilhelm: Farbematerial . . . 1348

— Pflanzengelb . . . . 1348

Steinberg, Curt . . . . 1424

Steinberger, Daniel . . . 2709

Steiner, Desider . . . . 1229

Steiner, Otto . . . . 1235

Steinkopf, Wilhelm: Beiträge z. Acetonitril . . 2066

- Versuche zur Synthese d. Nitroacetonitrils . . 2214

Steinweg, Eugen . . . 621

Stenzel, Georg . . . . 829

Stern, Adolf: Anfänge d. Litteratur . . . . 1351

- Cantate . . . . 1364

- Rede zur Schillerfeier 1351

Stern, Oscar . . . 2012. 2184

Sternberg, Walther .. 2162

Steuding, Alfred . . . 1681

Steuer, Karl . . . . . 1020

Stiefelhagen, Hans. . . 1131

Stiegler, Hans. . . . . 2726

Stier, Hubert . . . . . 1783

Stieve, Felix . . . 2348

Stimmelmayr, Anton . 2600
Stockem, Lorenz . . . 148

Stockfisch, Karl . . . 572

Stockhausen, Karl . . 1535

Stockmayer, Hugo. . . . 2922

Stokvis, Louis Gustaaf. . 2482

Stoll, Hermann . . . . 2290

Strach, Georg . . . 392

Streiter, Richard . . . 2356

Strell, Martin . . . . . . 2591

Strieboll, Erich . . . . 1183

Strobach, Waldemar . 1704

Strohbach, Erich . . . . 1548

Strohmeyer, Franz . . . 2721

Struck, Emil . . . . . 55

Struve, Henry .... 407

Struve, Karl . . . . . . 1884

Stuchlik, Heinrich . . 2539

Stuckert, Ludwig . . . 2251

Studniarski, Johann von . 1915

Stübinger, Otto . . . . 2134

Stübler, Eugen . . . . 2875

Stützel, Hermann . . . 2938

Stumpf, Carl . . . . 2655

Styri, Haakon . . . . 198

Subkis, Solomon .. . 847

Südenhorst, Otto Zwiedineck Edler von: Beiträge zur Grundrentenlehre

- Verfassung . . . . 1973

Sulger-Gebing, Emil: Schillers Geistesentwicklung. . . . . 2348

- Hertz . . . . . 2348

Surabekoff, Georg. . . 2609

Surzycki, Stanislaus . . . 2100

Suter, Josef . . . . . 2743

Sy, Louis Philippe. . . 780

Szèkely, Alexander . . 2648

Szitnick, Robert. . . . 484

Tafel, Victor Eugen . . 1713

Taitelbaum, Itzek . . . 884

Tatarowicz, Zdzislaw von. 2075

Tausz, Jenö . . . . 2283

Tecklenburg, Kurt. . . 1192

Tedesco, Hermann .. . 1655

Teichmïller, Joachim . . 2042

Teichner, Herbert . . . 2544

Telemann, Georg Philipp. 768

Teltscher, Friedrich . . 2700

ten Doornkaat Koolman, Gerhard

Teodorin, Joan . . . 585

ter Meer, Gustav . . . 1843

Terres, Ernst . . . . . 2261

Thele, Walter . . . . . 509

Thelen, Karl . . . . . 111

Theobald, Wilhelm . . 1848

Theusner, Martin . . . 606

Thiel, Georg . . . . 927

Thiel, Karl . . . . . 1116

Thiele, Hermann . . . 1397

Thielsch, Max ... 558
Thiem, Günther . . 2887

Thieme, Johannes . . . 1458

Thieriot, Jacob Heinrich . 1348

Thiersch, August: Geul . 2348 - Gottgetreu . . . . 2348

Thiersch, Friedrich v. : Baugeschichte v. Ettal . . 2348

- Künstler. Erziehung . 2348

- Reinhardstoettner . . 2348

Thode, Carlos Juan . . 1559

Thoma, Dieter . . . . 2451

Thomas, Felix . . . . 179

Thomas, Friedrich . . 147

Thomsen, Kurt . . . . 619

Thümmler, Fritz . . . 1497

Thurm, Richard . . 1851

Tichauer, Heinrich . . . . 2099

Tilemann, Georg . . . 1797

Tillmann, Max . . . . 523

Timerding, Heinrich Emil 799

Tischer, Alfred . . . . . 1449

Titlestad, Nicolay . . . . 883

Tögel, Karl : . . . . 863

Toepler, Max . . . . . 1385

Trauer, Günther. . . . 1464

Treubert, Franz . . . 2625

Tritschler, Alexander . . 2827

Troeger, Julius: Beiträge $\mathrm{zu}$ Angosturaalkaloiden 800

— Einwirkung v. Kaliumsulfid

- Öl d. Angosturarinde . 795

Trommsdorff, Paul . . 988

Trucksaess, Hans . . . 556

Tuckermann, Ernst . . 451

Uebelacker, Heinrich. . . 2426

Übelhör, Fritz . . . . 2577

Ürményi, Dezsö . . . . 589

Ugrimoff, Boris von 2012. 2181

Uhde, August . . . 696

Uhde, Constantin . . . 779

Uhlig, Otto. . . . . 1254

Uhlmann, Armin ... 1625

Ulrich, Christoph . . . . 894

Ulrich, Henry . . . . 1612

Unger, Carl . . . . 2920

Unger, Otto . . . . 597

Unglaub, Franz . . . 1442

Utard, Anton . . . . . 1208

Valentiner, Wilhelm . . 2000

Vater, Georg . . . . 1644

Vater, Heinrich . . . . 1377

Vaubel, Wilhelm . . 1145

Verbeek, Paul . . . 1597

Vervuert, Gottfried . . 2601

Vestner, Hans. . . . 2598

Vetter, Heinrich . . . 2634

Vetter, Hermann . . . 1645

Vetter, Theodor . . . . 2473

Vetterlein, Ernst: Aufnahme d. Chores . . 1150

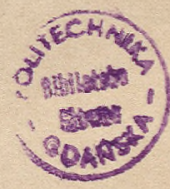


Vetterlein, Ernst: Auftreten d. Gotik ... 1169

Vicari, Ferdinand . . . 2526

Vierkandt, Alfred: Entstehungsgründe neuer Sitten . . . . . 795

- Volksdichte . . . . 802

Vierschilling, Aloys . . 138

Viertel, Artur . . . . . 1667

Vlachos, Alkibiades . . 2610

Voege, Willi . . . . 839

Völker, Philipp . . . . 1179

Voerkelius, Gustav Adolf. 1880

Vogel, Emil . . . . . 2448

Vogel, Hermann Wilhelm. 349

Vogt, Wolfgang . . . . 2065

Vogts, Hans . . . . 1175

Voigt, Alexander . . . 2156

Voigt, Johann August . . 703

Voigt, Max . . . . . 1718

Voigt, Otto . . . . 1872

Voigt, Wilhelm . . . 1660

Voissel, Peter . . . . 110

Voit, Ernst: Entwicklung

d. Beleuchtung ... 2356

- Feinmechanik . . . 2356

Vollkommer, Max . . . 2365

Vollmer, Karl . . . . 1016

Vormfelde, Karl . . . 1836

Voss, Aurel . . . . . . 2348

Waag, Hans

1170

Wach, Hans

1835

Wäser, Bruno

581

Wagener, August . . . . 979

Wagner, Gustav

Wagner, Hans: Baukunst in Regensburg . . . 2423

Wagner, Hans: Halogenderivate d. Fluorens. . 2957

Wagner, Heinrich: Kreuzigungsgruppen . 1116

- Zusammenstellung der Raumverhältnisse . . 1123

Wagner, Ludwig . . . . 2563

Wagner, Perey Albert . . 1716

Walbe, Heinrich: Bankunst 1091

- Bauordnungen . . . 1100

Walbrecker, Walther .. 141

Waldeck, Karl .. . . 592

Waldmann, Anton ... 2645

Waldmann, Ernst . . . . 1018

Waldmann. Karl .... 2467

Wallem, Harald . . . . 2183

Wallichs, Adolf . . . . 74

Wallot, Julius. . . . . 2881

Walloth, Carl August . . 1459

Walther, Franz . . . . 1844

Walther, Reinhold . . 1381

Wamsler, Friedrich . . . 2444

Wark, Nicolas Jean . . 190

Warlimont, Felix . . . 173

Wartburg, Oswald Rudolph v.

2731
Warth, Carl . . . . 2244

Warth, Constantin. . . 2078

Warthiadi, Demeter . . 2747

Wasmus, Adolf . . . 846

Watzinger, Adolf: Einrichtungen d. Kraftwerkes 1131

- Wert d. Zwischenüberhitzung . . . . 1210

Wawrziniok, Otto . . 1403

Weber, August . . . . 2120

Weber, Friedrich August. 2241

Weber, Heinrich: Ableitung d. Gleichgewichtsgleichung

Weber, Heinrich: Theorie d.Modular-Gleichungen 349

Weber, Karl . . . . 1243

Weber, Max Gustav . 1654

Weber, Maximilian . . 2348

Weckbach, Franz . . . 2643

Wecken, Wilhelm . . . . 842

Wedekind, Ludwig: Quadratsummen . . . . 2000

- Studien im Werthgebiet 2020

Wedemeyer, Otto . . 827

Weeren, Julius . . . . 349

Wegele, Hans . . . . . 1099

Weger, Kurt . . . . 1624

Wehrheim, Otto ... 1259

Weicker, William ... 1539

Weidig, Max . . . . . 1724

Weidig, Paul . . . . . 1540

Weidmann, Carl . . . 107

Weigel, Jakob . . . . 2401

Weihs, Gustav . . . 218

Weil, Robert . . . . 505

Weiller, Paul . . . . 613

Weinbrenner, Adolf . . 2000

Weinfurtner, Johannes . . 2391

Weingarten, Julius . . 349

Weinstein, Jancu . . . 2656

Weipert, Hugo . . . . . 2968

Weis, August . . . . . 2208

Weise, Max ..... 1453

Weishaupt, Carl . . . 999

Weiske, Paul . . . . . 1811

Weiss, Georg . . . . . 2548

Weiss, Ludwig: Beiträge zur Kenntnis d. Phosphorverbindungen . . 2564

Weiss, Ludwig: Darstellung d. Metalle d. Cergruppe 2494

Weiss, Richard . ... 1188

Weissel, Leopoldo . . . 2952

Weissmann, Leon . . . 2279

Weitbrecht, Martin . . 513

Weitzner, Emil . . . . . 1874

Weiwers, Johann . . . 202

Welponer, Anton . . . 2395

Weltzien, Carl . . . 1996

Wempe, Georg . . . . 2686

Wende, Oskar ... 1795

Wendt, Karl . . . . . 413

Wenger, Albert . . . . 437

Wengner, Max . . . . 2477
Wentzel, Fritz. . . . 557

Wenzel, Georg . . . 540

Werkmeister, Otto . . 2252

Werkmeister, Paul ... 2145

Werner, Christian Albert . 123

Werner, Paul ..... 576

Werner, Siegfried Georg . 415

Wesser, Rudolf . . . . 1408

Westenholz, Friedrich von 2856

Westerkamp, Arthur . . 882

Westermann, Heinrich . . 142

Westhoff, Franz . . 1896

Wetsch, Karl . . . . 2649

Weyl, August . . . . . 2211

Weyl, Fritz . . . . 178

Weyrauch, Robert . . 2411

Wickop, Georg . . . . 1091

Widmann, Karl Theodor . 2939

Wiebe, Hermann . . . 361

Wiegandt, Friedrich . . 2923

Wiehs, Jean . . . . 2094

Wieler, Arwed: Antheil d. Holzes an d. Saftleitung 2027

- Das Botan. Institut . 95

Wienecke, Carl . . . . 400

Wiener, Alfred . . . . . 1444

Wiener, Christian: Freiheit d. Willens . . 1978

- Zerstreuung d. Lichtes 2000

Wild, Julius . . . . . 2914

Wild, Wilhelm . . . 2116

Wilde, Hans . . . . . 1427

Wilhelm Herzog von

Braunschweig .. . 777

Wilisch, Karl . . . . 2722

Wille, Heinrich . . . 840

Willich, Hans . . . . . 2421

Willkomm, Otto . . 1506

Willmann, Erich von . 1191

Wilms, Otto ... 603

Wimmer, Robert . . . 2485

Winckler, Curt Oscar . . 2665

Winkelmann, Max . . 2062

Winkler, Emil: BelastungsGleichwerthe . . . 349

- Feier zur Enthüllung d.

Büste . . . . 353

Wintgens, Paul . . . 1723

Wirth, Christian . . . 2582

Wirz, Emil . . . . . 2188

Wislicenus, Hans . . . 2038

Wispler, Hans. . . . . 1808

Witt, Otto Nikolaus: Lebensbedingungen . . 333

- Rede auf Bismarck . . 357

— Rückblicke ... . 345

Witte, Karl . . . . . 864

Witzeck, Rudolf . . . 2191

Witzmann, Walter . . 2222

Wobsa, Georg . . 1509

Wöhler, Lothar . . . . 2045

Wöhler, Paul". . . . 2224

Wölffing, Ernst . . . 2861

Wohl, Alfred . . . . . . 981

Wolf, Hermann . . . 474 
Wolf, Johannes :... 1615

Wolf, Josef . . . . . 821

Wolf, Kurt . . . . . 1382

Wolf, Rudolf ..... 1537

Wolff, Albert ..... 543

Wolff, Fritz: Ansprache z. Gedächtniss v. Spielberg 353 Berlin....... 365

Wolff, Justus . . . . 566

Wolff, Paul ..... 156

Wolfsleben, Kurt . . . 881

Wolokitin, Arkadij . . 2266

Wolters, Karl . . . . 1825

Wommelsdorf, Heinrich. . 491

Woost, Johannes . . . 1691

Wüllner, Adolph : Entwicklung d. Grundanschauungen in d. Physik. .

- Festrede auf Wilhelm I.

- Festschrift . . . . .

- Friedrich III . . . - 85

- Verwendung d. Physik 50

Wünsche, Oscar . . . 1549

Würth, Karl . . . . 2510

Würzner, Kurt . . . . . 1619

Wüst, Fritz: Entwicklung d. Eisenindustrie . . .
- Mitteilungen aus d. Eisenhütt. Institut . . 98a Wulff, Constantin . . . 1201

Young, Bernhard . . . 1679

Young, Niels . . . . 1517

Zablinsky, Karl . . . . 573

Zachariä, Friedrich Wilhelm . . . . . . 768

Zacharias, Friedrich August 1671 Zacharias, Ludwig . . 831

Zahn, Walter . . . . . 108

Zakrzewski, Sigismund von 2105

Zech, Paul: Abhandlung über Schwingungsbewegungen . . . . . 2827

- Festschrift zur Einweihung d: Flügelanbaues 2847

Zedlitz, Friedrich Gotthard Frh. von . . . 722

Zedlitz, Karl Abraham Frh. von: Cantate . 717

- Rede b. Geburtsfeste Carls . . . . . . .
Zeh, Wilhelm .... 1256

Zehetmaier, Heinrich • 2657

Zeidler, Arthur . . . . 1504

Zeidler, Georg. . . . . 682

Zeising, Hermann . . . 851

Zeissig, Konrad . . . . . S. 64

Ziegler, August . . . . . 2748

Zillgen, Joseph . . . . . 443

Zimmermann, Karl . . . 2892

Zimmermann, Maximilian

Richard . . . . 1555

Zimmermann, Werner . 1845

Zinberg, Srul . . . . . 2112

Zincke, Georg Heinrich. . 744

Zinke, Carl Friedrich Wilhelm ..... 726

Zirngibl, Emil

Zorn, Hans . . . . 2511

Zscheile, Arthur . . 1560

Zschimmer, Bodo .. . 1636

Zsuffa, Milan .... 580

Zweigler, Fritz . . . . 1693

Zwiedineck Edler von Südenhorst, Otto: Beiträge zur Grundrentenlehre ...... 1993

716 - Verfassung . . . 1973 
Monotypesatz und Druck:

Adolf Gertz G.m.b. H., Charlottenburg

Danckelmannstrasse 3

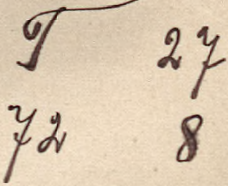



Biblioteka Główna

\section{(ii) $7175 \mathrm{~s}$}

Politechniki Gdańskiej 